Matthias Krups

Marketing innovativer Dienstleistungen am Beispiel elektronischer Wirtschaftsinformationsdienste 
Matthias Krups

\section{Marketing innovativer Dienstleistungen am Beispiel elektronischer Wirtschaftsinformationsdienste}

Mit dem Aufkommen und der verbreiteten Durchsetzung neuer Technologien entstehen heutein allen hochindustrialisierten Ländern neue Dienstleistungsmärkte. Die vorliegende Arbeit hat sich zum Ziel gesetzt, ein Konzept zur Erschließung dieser innovativen Märkte zu entwickeln. Als exemplarischer Analysebereich liegt dabei der Markt elektronischer Wirtschaftsinformationsdienste zugrunde. Parallel zur theoretischen Diskussion erfolgt eine partielle Überprüfung der Tragfähigkeit des vorgeschlagenen Konzepts anhand einer explorativen, empirischen Studie.

Matthias Krups, geboren 1955, studierte Betriebswirtschaftslehre an der Universität Münster von 1974 bis 1981. Examen als Diplom-Kaufmann 1981 in Münster. Seither Assistent am Institut für Marketing der Westfälischen Wilhelms-Universität Münster. Seit 1983 Geschäftsführer der Wissenschaftlichen Gesellschaft für Marketing und Unternehmensführung e.V. 
Marketing innovativer Dienstleistungen am Beispiel elektronischer Wirtschaftsinformationsdienste 


\section{Schpiften zu Marketine und Manacement}

Herausgegeben von Prof.Dr. Heribert Meffert

\section{Band 10}

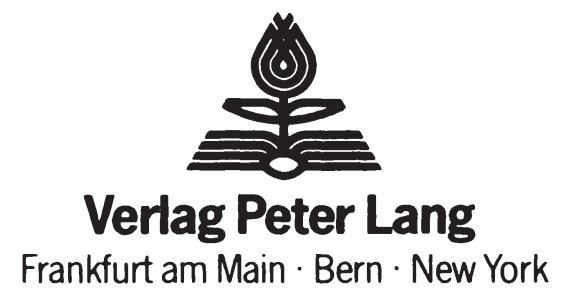




\section{Matthias Krups}

\section{Marketing innovativer Dienstleistungen am Beispiel elektronischer Wirtschafts- informationsdienste}

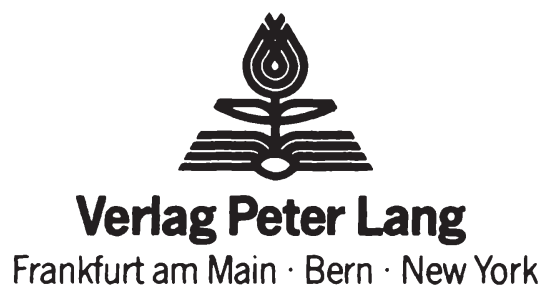


CIP-Kurztitelaufnahme der Deutschen Bibliothek

\section{Krups, Matthias:}

Marketing innovativer Dienstleistungen am Beispiel

elektronischer Wirtschaftsinformationsdienste /

Matthias Krups. - Frankfurt am Main ; Bern ; New

York: Lang, 1985.

(Schriften zu Marketing und Management ; Bd. 10)

ISBN 3-8204-5692-9

NE: GT

Open Access: The online version of this publication is published on www.peterlang.com and www.econstor.eu under the international Creative Commons License CC-BY 4.0. Learn more on how you can use and share this work: http://creativecommons.org/licenses/by/4.0.

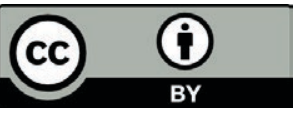

This book is available Open Access thanks to the kind support of ZBW - Leibniz-Informationszentrum Wirtschaft.

ISSN 0176-2729

ISBN 3-8204-5692-9

ISBN 978-3-631-75074-2 (eBook)

(C) Verlag Peter Lang GmbH, Frankfurt am Main 1985

Alle Rechte vorbehalten.

Nachdruck oder Vervielfältigung, auch auszugsweise, in allen Formen wie Mikrofilm, Xerographie, Mikrofiche, Mikrocard, Offset verboten.

Druck und Bindung: Weihert-Druck GmbH, Darmstadt 
Vorwort des Herausgebers

Fragen der strategischen Markteintrittsplanung gewinnen in den letzten Jahren zunehmend an Bedeutung. In besonderem Maße gilt dies für den Dienstleistungssektor. Im Zuge eines an Dynamik gewinnenden Strukturwandels erfährt dieser Bereich gegenwärtig eine bemerkenswerte Aufwärts entwicklung. Getragen ist diese Entwicklung nicht zuletzt durch das Entstehen neuer Märkte im Gefolge tiefgreifender Neuerungen im Feld der Informations- und Kommunikationstechnologien.

Vor diesem Hintergrund nimmt es Wunder, daB in der betriebswirtschaftlichen Literatur bislang die strategischen Probleme des Markteintritts in innovative Dienstleistungsmärkte nahezu unberücksichtigt geblieben sind. Insbesondere mangelt es an einer fundierten und systematischen Auseinandersetzung mit den strategischen Entscheidungsproblemen, die seit einigen Jahren unter dem Schlagwort "defining the business" für den Konsum- und Investitionsgüterbereich diskutiert werden.

Der Verfasser der vorliegenden Arbeit will zum Abbau dieses Forschungsdefizits beitragen. Er greift hierfür bewährte Konzepte aus dem Bereich der strategischen Planung ebenso auf wie er neuere Ansätze zum Dienstleistungsmarketing kritisch überprüft. Letztlich verfolgt der Verfasser mit seiner Arbeit die Zielsetzung., mit einem Konzept zur Lokalisierung, Auswahl und Bearbeitung strategischer Geschäftsfelder verbesserte Entscheidungshilfen für den Eintritt in innovative Dienstleistungsmärkte zu entwickeln. Konkretes Untersuchungsobjekt ist dabei die Einführung eines bereichsubergreifenden elektronischen wirtschaftsinformationsdienstes durch einen kommerziellen Informationsanbieter. 
Ausgehend von einer Charakterisierung des Marktes für elektronische Informationsdienste entwickelt und prift der Verfasser alternative Selektionsprozesse der Geschäftsfeldwahl. Zur Verdeutlichung des Entscheidungsablaufs entwirft er einen dreidimensionalen Bezugsrahmen, der die Wahl des künftigen Geschäftsfeldes über eine sukzessive Festlegung der zu leistenden Abnehmerfunktion, der einzusetzenden Informationstechnologie und der zu bedienenden Abnehmergruppe vorsieht. SchlieBlich diskutiert der Verfasser alternative strategische StoBrichtungen der Bearbeitung des so selektierten Dienstleistungsgeschäftsfeldes.

Einige Aspekte der Arbeit verdienen eine besondere Heraushebung. Dies gilt einmal für die grundsätzliche Problematisierung des strategischen Selektionsprozesses. Hier kommt der Verfasser zu dem zentralen Ergebnis, daB zur Reduzierung der mit dem Markteintritt verbundenen Risiken die Geschäftsfeldwahl zunächst und insbesondere von der spezifischen "Kompetenz" der Unternehmung ausgehen sollte.

Im Zuge der weiteren Erörterung entwickelt der Verfasser ein Modell der Marktsegmentierung, das er unter Bezugnahme auf Daten aus einer eigenen, sorgfältig angelegten Befragung in überzeugender Weise empirisch überprüf. Mit Hilfe statistischer Testverfahren sowie der Diskriminanzanalyse zeigt der Verfasser auf, wie potentielle Abnehmer mit grundsätzlich höherer Bereitschaft zur Inanspruchnahme eines elektronischen Informationsdienstes anhand konkreter Merkmalskombinationen selektiert werden können. Diese Vorgehensweise bringt gegenüber den bekannten diffusionstheoretischen Modellen und Segmentierungsansätzen im Investitionsgüterbereich interessante weiterführende Aspekte. 
SchlieBlich entwickelt der Verfasser interessante Ansätze für die Systematisierung und Ausgestaltung von Strategien der Bearbeitung innovativer Dienstleistungsmärkte sowie für eine kreative Umsetzung dieser Strategien unter Einsatz einer differenzierten Marketing-Matrix.

Insgesamt gesehen leistet der Verfasser einen beachtlichen Beitrag zur Strukturierung und Analyse eines bislang wenig bearbeiteten komplexen Problembereichs der Marketingplanung. Die Arbeit enthält eine Vielzahl eigenständiger und innovativer Gedanken, die eine Bereicherung für das strategische Marketing im Dienstleistungsbereich bedeuten. Damit gibt sie für die Verbesserung der Planung von Marktwahl- und Marktbearbeitungsentscheidungen - nicht nur bezogen auf elektronische Informationsdienstleistungen - wertvolle Hinweise.

Münster, im Januar 1985 Prof. Dr. Heribert Meffert 
M. Krups - 978-3-631-75074-2

Downloaded from PubFactory at 01/11/2019 08:37:05AM

via free access 
Mit dem Aufkommen und der verbreiteten Durchsetzung neuer Technologien entstehen heute in allen hochindustrialisierten Ländern eine Vielzahl neuer Dienstleistungsmärkte. Diese neuen Märkte gezielt zu erschlieBen birgt für Unternehmen mit entsprechender Innovationsorientierung weitreichende Chancen. Gleichwohl zeigt sich, daB bei der Auswahl, Bewertung und erfolgreichen Besetzung neuer Dienstleistungsgeschäftsfelder die betroffenen Marktpioniere derzeit nur auf eine schwache, theoretisch fundierte Hilfestellung von seiten der Wissenschaft zurückgreifen können.

Die vorliegende Arbeit hat sich zum Ziel gesetzt, diese Lücke in der strategischen Marketingplanung zu schlieBen. Auf der Grundlage eines auf Abell zurückgehenden, die Technologie explizit einbeziehenden Ansatzes der Geschäftsfeldplanung wird ein Konzept zur Lokalisierung, Auswahl und Bearbeitung strategischer Geschäftsfelder im Bereich innovativer Investivdienstleistungen entwickelt. Eine partielle oberprüfung der Tragfähigkeit einzelner Komponenten des Konzepts erfolgt dabei anhand einer explorativen, empirischen Studie. Entsprechend ihrer theoretischen und empirischen Anlage versteht sich die Arbeit letztlich als Basisuntersuchung, die den Weg zur Beantwortung detaillierter und differenzierter Fragestellungen eröffnet.

Die Abfassung der vorliegenden Arbeit war nur mit vielfältiger Unterstützung verschiedener Personen möglich. Mein besonderer Dank gilt an dieser Stelle meinem akademischen Lehrer, Herrn Professor Dr. Heribert Meffert, der die Anregung für die Themenstellung gab und die Fertigstellung der Arbeit in allen Phasen umfassend förderte. 
Die konzeptionelle Vorbereitung sowie die Durchführung der empirischen Untersuchung erfolgte mit Unterstützung des Druck - und Verlagshauses Gruner + Jahr AG \& Co. Die Initiative zur Durchfuhrung des Forschungsprojektes ging aus von Herrn Professor Dr. Jan Hensmann, dem ich hier meinen herzlichen Dank ausspreche. Darüber hinaus standen Mitarbeiter der Marktforschung sowie der Abteilung Informationssysteme jederzeit als kompetente Gesprächspartner bereit. Dafür danke ich besonders Herrn Gunter Pratz, Herrn Heiner Eggert sowie Herrn Lutz Glandt.

Nicht zuletzt möchte ich allen Kolleginnen und Kollegen am Institut für Marketing danken, die mich von anderer Arbeit entlastet und vielfältig unterstuitzt haben. Dies gilt in besonderem Maße für Herrn Dr. Michael Walters und Herrn Dipl.-Kfm. Frank Schubert, die jederzeit zur kritischen Diskussion bereit waren und wertvolle Hinweise gaben. Frau Eva-Maria Figge danke ich für die sorgfältige, reibungslose und allzeit unermüdliche Durchführung der Schreibarbeiten. Schließlich gebiuht meiner Frau ein herzliches Dankeschön für das entgegengebrachte Verständnis sowie die vielfältigen Entlastungen während der Abfassung dieser Arbeit.

Muinster, im Januar $1985 \quad$ Matthias Krups 


\section{Inhaltsverzeichnis}

Se ite

A. Bedeutung der Markteintrittsentscheidung in innovativen Investivdienstleistungsmärkten

1. Gegenstand und Probleme des investiven Dienstleistungsmarketing

1.1 Zur aktuellen Bedeutung investiver Dienstleistungen

1.2 Stand des Marketing investiver Dienstleistungen

1.3 Informationen als innovative Investivdienstleistungen

2. Der Markt elektronischer Wirtschaftsinformationsdienste als Untersuchungsbereich

2.1 Struktur des Marktes für externe Wirtschafts informationen

2.2 Anbieter und Angebotsformen externer Informationsdienste

2.3 Nachfrager und Nachfragepotentiale im Bereich elektronischer Informationsdienste

3. Problemstellung und Zielsetzung der Arbeit

3. 1 Konzept eines bereichsübergreifenden elektronischen Wirtschaftsinformationsdienstes

3.2 Paradigma der Markteintrittsplanung im Bereich elektronischer Informationsdienste

$3.3 \mathrm{Ziel}$ und Gang der Untersuchung

B. Auswahl strategischer Geschäftsfelder im Bereich elektronischer Informationsdienste

1. Definition strategischer Geschäftsfelder

1.1 Ansatzpunkte zur strategischen Geschäftsfelddefinition in der Literatur

1.11 Darstellung alternativer Ansätze

1.12 Würdigung der Obertragbarkeit auf den Markt elektronischer Wirtschaftsinformationsdienste

1.2 "Informationsfunktion", "Obertragungstechnologie" und "Abnehmer" als Bestimmungsgrößen dreidimensionaler Geschäftsfelder 
Seite

1.21 Operationalisierung der Dimensionen am Beispiel eines institutionellen Informationsanbieters

1.211 Operationalisierung der Funktionsdimension

1.212 Operationalisierung der Technologiedimension

1.213 Operationalisierung der Abnehmerdimension

1.3 Strategischer Suchraum als Ausgangsraster der Geschäftsfeldwahl

2. Wahl der Geschäftsfeldstrategie und Bestimmung des Auswahlprozesses

2. 1 Begrenzungsfaktoren der Geschäftsfeldselektion

2.2 Alternative Geschäftsfeldstrategien als Ausgangspunkt

2.3 Konzept einer mehrstufigen Geschäftsfeldwahl

2.31 Darstellung möglicher ProzeBalternativen

2.32 Auswahl der geeigneten Prozeßalterna$t$ ive

2.4 Kennzeichnung eines ressourcenorientierten Auswahlprozesses

2.41 Entscheidungsablauf der Geschäftsfeldwahl

2.42 Entscheidungskriterien der Geschäftsfeldwahl

2.43 Bezugsrahmen der Geschäftsfeldwahl

3. Auswahl strategischer Angebotskombinationen

3.1 Bestimmung der Informationsfunktion

3.11 Anbieterbezogene Determinanten

3. 111 Unternehmensressourcen

3.112 Unternehmensfähigkeiten

3.12 Marktbezogene Determinanten

3.121 Abnehmerbedarf

3.122 Konkurrenzsituation 
3.13 Durchführung der Funktionsauswahl

105

3.2 Zuordnung der Technologiedimension als Feinauswahl

3.21 Funktionsunabhängige Determinanten der Technologiewahl

3.211 Ressourcenstärke

3.212 Technologieattraktivität

3.22 Funktionsbezogene Determinanten der Technologiewahl

3.221 Zentrale Anforderungen an die Informationsversorgung als $\mathrm{Be}$ stimmungsfaktoren der Technologie

3.2211 Bestimmung relevanter Anforderungskriterien der Informationsversorgung

3.2212 Relative Bedeutung relevanter Anforderungskriterien

3.2213 Funktionsabhängigkeit relevanter Anforderungskriterien

3.222 Leistungscharakteristika alternativer ubertragungstechnologien

3.2221 Bestimmung relevanter Leistungsmerkmale

3.2222 Beurteilung relevanter Leistungsmerkmale

3.23 Durchführung der Technologieauswahl

3. 3 Selektion geschäftsfeldrelevanter Angebotskombinationen

3. 31 Determinanten der Angebotsauswahl

3.311 Ressourcenbedarf

3.312 Skaleneffekte

3.313 Komplexität der Dienstleistung

3.314 Abnehmerverhalten

3.32 Darstellung exemplarisch ausgewählter Angebotskombinationen 
Seite

4. Auswahl angebotsadäquater Abnehmergruppen

4.1 Marktsegmentierung als Instrument zur Abgrenzung relevanter Abnehmergruppen

4. 11 Ansätze zur Marktsegmentierung im Bereich investiver Dienstleistungen

4.12 Modell der Marktsegmentierung für elektronische Informationsdienstleistungen

4.2 Kriterien zur Auswahl industrieller Abnehmer elektronischer Informationsdienste

4.21 Informationsbedarf als Kriterium der Kaufveranlassung

4.211 objektive Indikatoren des Informationsbedarfs

4.2111 Branche

4.2112 Internationale Ausrichtung

4.212 Subjektive Indikatoren des Informationsbedarfs

4.2121 Häufigkeit von Informationsbedarfssituationen

4.2122 Zufriedenheit mit der Informationsversorgung

4.2123 Zufriedenheit mit unternehmensexternen Informationsquellen

4.2124 Kostensensitivität

4.22 Dispositionsspielraum als Kriterium der Kauffähigkeit

4.221 Organisationsdemographische Kriterien

4.2211 Unternehmensgröße

4.2212 Informationstechnologische Ausstattung

4.222 Informationsdemographische Kriterien

4.2221 Existenz eines Budgets für unternehmensexterne Informationen 
Seite

4.2222 Verfügbarkeit von Informationen aus verbundenen Unternehmen

192

4.2223 Aufwand für die Beschaffung unternehmensexterner Informationen

4.2224 Nutzungsgrad unternehmensexterner Informationen

4.2225 Nutzer unternehmensexterner Informationen

194

4.23 Merkmale des Buying Center

4.24 Zusammenfassende Darstellung relevanter Auswahlkriterien

4.3 Durchführung der Abnehmerauswahl am Beispiel eines elektronischen Brancheninformationsdienstes

5. Darstellung und Würdigung des ausgewählten Geschäftsfeldes

C. Strategien der Geschäftsfeldbearbeitung im Bereich elektronischer Informationsdienste

1. Besonderheiten der Geschäftsfeldbearbeitung be $i$ investiven Dienstleistungen

1.1 Zentrale Merkmale des Absatzes investiver Dienstleistungen

1.11 Immaterialität der Dienstleistung

1.12 Notwendigkeit der Integration eines Fremdfaktors

1.13 Simultaneität von Dienstleistungsproduktion und -konsumtion

1.14 Interdependenzen $z$ wischen Dienstleistungserstellung und -marketing

1.2 ProzeB der Bearbeitung investiver Dienstleistungsmärkte

2. Segmentierung der ausgewählten Abnehmer als Grundlage von Marktbearbeitungsstrategien

2.1 Beschaffungsverhalten bei elektronischen Informationssystemen als Ausgangspunkt

2.11 Typologisierung der Beschaffungsentscheidung

2.12 Darstellung des Entscheidungsprozesses 
2.2 Problemevidenz als zentrale Determinante des Beschaffungsverhaltens

2.21 Begriff und Abgrenzung der Problemevidenz

2.22 Ansatz zur Operationalisierung der Problemevidenz in bezug auf elektronische Informationsdienste

2.221 Operationalisierung der Problemwahrnehmung

2.222 Operationalisierung der Dienstleistungsidentifikation

2.223 Verknüpfung von Problemwahrnehmung und Dienstleistungsidentifikation

2.23 Messung der Problemevidenz am Beispiel eines elektronischen Brancheninformationsdienstes

2.24 Leistungsfähigkeit und Grenzen des Konzepts der Problemevidenz

2.241 Realitätsgehalt des Problemevidenzkonstrukts

2.242 Aussagewert der Problemevidenz für die Marktsegmentierung

2.243 Zusammenfassende Bewertung des Problemevidenzkonzepts

3. Bearbeitung ausgewählter Abnehmergruppen am Beispiel elektronischer Informationsdienste

3.1 Ableitung von Basisstrategien der Marktbearbeitung

3.11 Strategien bei fehlender Problemevidenz

3.12 Strategien bei vorhandener Problemevidenz

3.2 Umsetzung der Basisstrategien durch Einsatz der Marketing-Matrix

3.21 Struktur und Merkmale der MarketingMatrix

3.221 Marketing-Matrix bei fehlender Problemevidenz

3.222 Marketing-Matrix bei vorhandener Problemevidenz 
D. Implikationen der Untersuchung

1. Zusammenfassende Würdigung der Untersuchungsergebnisse

2. SchluBfolgerungen für die Praxis

3. Auswirkungen auf die weitere Forschung

270

Anhang 1: Anlage der empirischen Untersuchung zur Akzeptanz elektronischer Wirtschaftsinformationsdienste in der Bundesrepublik Deutschland

Anhang 2: Tabellen

Anhang 3: Fragebogen 283

Literaturverzeichnis 296 


\section{Abbildungsverzeichnis}

Abb. 1: Systematik der Wirtschaftsguter

Seite

Abb. 2: Kreislauf von Informationsangebot und

-nachfrage

Abb. 3: Struktur des Marktes für Wirtschaftsinformationen

Abb. 4: Charakteristika zweier entgegengesetzter Idealtypen von Informationsvermittlungstellen

Abb. 5: Arten der Informationsvermittlungsste1len

Abb. 6: Orientierungsraster zur Definition strategischer Geschäftsfelder im Markt für Informationsdienstleistungen

Abb. 7: Der Markt für elektronische Wirtschaftsinformationen als Ausgangspunkt zur Festlegung strategischer Geschäftsfelder

Abb. 8: Systematik relevanter Telekommunikationsformen

Abb. 9: Strategische Technologiealternativen eines elektronischen Informationsdienstes

Abb. 10: Strategischer Suchraum zur Bestimmung und Auswahl strategischer Geschäftsfelder im Markt fur elektronische Wirtschaftsinformationen

Abb. 11: Abgrenzung des Handlungsfeldes für die Planung der Dienstleistungsinnovation

Abb. 12: Idealtypen möglicher Geschäftsfeldstrategien

Abb. 13: Alternative ProzeBabfolgen der Geschäftsfeldwahl als Ausdruck unterschiedlicher Prioritätensetzung im Rahmen der Innovationsplanung

Abb. 14: Bewertung der alternativen ProzeBabfolgen der Geschäftsfeldwahl

Abb. 15: Entscheidungsablauf einer iterativen Geschäftsfeldwahl 
Seite

Abb. 16: Entscheidungskriterien zur iterativen Geschäftsfeldwahl

Abb. 17: Bezugsrahmen der Geschäftsfeldwahl

Abb. 18: Alternative Ansätze zur Operationalisierung des Informationsbedarfs

Abb. 19: Nutzungshäufigkeit externer Informationsquellen durch Unternehmen

Abb. 20: Punktbewertungsverfahren zur Auswahl der Geschäftsfeld-Funktion

Abb. 21: Nachfrage-Technologie-Zyklus datenbankgespeicherter Fachinformationen

Abb. 22: Ermittlung der Ressourcenstärke

Abb. 23: Ermittlung der Technologieattraktivität

Abb. 24: Technologie-Lebenszyklus und Stufen der Weiterentwicklung des Bildschirmtextsystems

Abb. 25: Diffusionsverlauf telekommunikativer Obertragungsformen (1975-1985)

Abb. 26: Ausstattungsstand und Stand der Planung hinsichtlich der Einrichtung von Telekommunikationsanschliussen in der Bundesrepublik Deutschland

Abb. 27: Anwendungsspektrum alternativer Telekommunikationsformen

Abb. 28: Dimensionen der Konkretisierung von Anforderungen an die Informationsversorgung

Abb. 29: Bedeutungsinhalt der Faktoren F1 bis F5

Abb. 30: Anforderungen an die Versorgung mit unternehmensexternen Informationen

Abb. 31: Aufgabenbezogenheit der Technik

Abb. 32: Entwicklung von Kriterien der Leistungsfähigkeit alternativer Geschäftsfeldtechnologien

Abb. 33: Gegenüberstellung von Anforderungsmerkmalen der Informationsversorgung und Leistungscharakteristika einsetzbarer Technologien 
Abb. 34: Punktbewertung zur Auswahl der Geschäftsfeldtechnologie

Abb. 35: Vor-ausgewählte Angebotskonstellationen als Funktions-/Technologie-Kombinationen

Abb. 36: Managementrelevante Charakterisierung alternativer Informationsdienstleistungen

Abb. 37: Kostensensitivität potentieller Nutzer eines elektronischen Informationsdienstes

Abb. 38: Hierarchische Grundstruktur von Kriterien zur Abnehmersegmentierung

Abb. 39: Bezugsrahmen zur Auswahl relevanter Abnehmergruppen eines elektronischen Informationsdienstes

Abb. 40: Angewandte Verfahren zur Oberprifung der Relevanz von Kriterien der Abnehmerauswahl

Abb. 41: Relevante Einzelfaktoren zur Selektion von Abnehmern eines elektronischen Informationsdienstes

Abb. 42: Ergebnis der diskriminanzanalytischen Klassifikation der befragten Unternehmen als Adopter/Nichtadopter eines elektro$n$ ischen Brancheninformationsdienstes

Abb. 43: Schematische Darstellung des ausgewählten Dienstleistungsgeschäftsfeldes

Abb. 44: Zusammenhang zwischen Leistungserstellung, Marketing und Konsumtion

Abb. 45: Phasen des Marktbearbeitungsprozesses bei investiven Dienstleistungen

Abb. 46: Typologie von Investitionsentscheidungen

Abb. 47: Phasen des Kaufentscheidungsprozesses bei der Beschaffung investiver Dienstleistungen

Abb. 48: Abgrenzung der Problemevidenz einer Dienstleistung

Abb. 49: Abgrenzung der Problemwahrnehmung hinsichtlich der Versorgung mit Brancheninformationen 
Abb. 50: Konstellation vorhandener und fehlender Problemevidenz

Abb. 51: Operationalisierte Abgrenzung der Problemevidenz

Abb. 52: Bewertung des Problemevidenzkonzeptes in der Anwendung auf elektronische Informationsdienste

Abb. 53: Strategie der Marktbeeinflussung im Fall fehlender Problemevidenz

Abb. 54: Modell der Beziehungen im Servuctionssystem eines elektronischen Informationsdienstes

Abb. 55: Marketing-Matrix für elektronische Wirtschaftsinformationsdienste

Abb. 56a: Ausgestaltung der Marketing-Matrix für

elektronische Informationsdienste im

Fall fehlender Problemevidenz

Abb. 56b: Ausgestaltung der Marketing-Matrix für elektronische Informationsdienste im Fall vorhandener Problemevidenz

Abb. 57 : Organisationsdemographische Merkmale der Stichprobe

Abb. 58 : Merkmale der Fragenbeantworter 


\section{Tabellenverzeichnis}

Seite

Tab. 1 : Quantifizierung des Informationsbedarfs am empirischen Beispiel

Tab. 2 : Zufriedenheit mit externen Informationsquellen in bezug auf alternative Informationsbereiche

Tab. 3 : Ergebnisse der faktorenanalytischen Verdichtung von Anforderungen an Informationen alternativer Informationsbereiche

Tab. 4 : Faktormuster der Anforderungen an Informationen zum Thema "Gesamtwirtschaft", "Branche" und "Export"

Tab. 5 : Beziehungszusammenhang zwischen funktionsspezifischen Anforderungen $(i=1-14)$ und funktionsübergreifenden Anforderungsfaktoren $(j=1-10)$

Tab. 6 : Relevanz objektiver Bedarfsmerkmale

Tab. 7 : Relevanz subjektiver Bedarfsmerkmale

Tab. 8 : Relevanz einer geringen Informationszufriedenheit vs. hoher Informationsanforderungen hinsichtlich der Bereitschaft zur Adoption eines elektronischen Brancheninformationsdienstes

Tab. 9 : Relevanz organisationsdemographischer Kriterien

Tab. 10a: Relevanz informationsdemographischer Merkmale (I)

Tab. 10b: Relevanz informationsdemographischer Merkmale (II)

Tab. 11 : Relevanz von Merkmalen des Buying Center

Tab. 12 : Ergebnis der Diskriminanzanalyse zur Ermittlung relevanter Kriterien zur Auswahl potentieller Abnehmer eines elektronischen Brancheninformationsdienstes 
Seite

Tab. 13: Kreuztabellarische Gegenüberstellung der Wichtigkeit (ANWICHT) und des Erfüllungsgrades (ANERF) von Anforderungen an die Versorgung mit Brancheninformationen

Tab. 14 : Kreuztabellarische Gegenüberstellung der Wahrnehmung eines Informationsversorgungsproblems (PROD) und der Bereitschaft, einen elektronischen Brancheninformationsdienst in Anspruch zu nehmen (Dienstleistungsidentifikation:

(BBEREIT)

Tab. 15 : Faktorenstruktur der Anforderungen an die Versorgung mit Brancheninformationen

Tab. 16 : Faktorenstruktur der Anforderungen an die Versorgung mit Gesamtwirtschaftlichen / Konjunkturinformationen

Tab. 17 : Faktorenstruktur der Anforderungen an die Versorgung mit Exportinformationen 


\section{Abkürzungsverzeichnis}

a.a.0.

Abb.

Anm.d.Verfassers

asw

Auf1.

BAf I

$B d$.

$B d d W$

BFUP

BH

BMFT

$B$ tx

bzw.

ca.

DB

DBW

d. $h$.

Diss.

DU

durchges.

EJOM

et al.

etc.

$f$.; ff.

$\mathrm{FAZ}$

GID

$H$.

HBR

$H d B$

HdO

HM

HWA

Hrsg.

i.d.R.

i.e. am angegebenen Ort

Abbildung

Anmerkung des Verfassers

Absatzwirtschaft

Auflage

Bundesstelle für AuBenhandels informationen

Band

Blick durch die Wirtschaft

Betriebswirtschaftliche Forschung und Praxis

Business Horizons

Bundesministerium für Forschung und Technologie

Bildschirmtext

beziehungsweise

circa

Der Betrieb

Die Betriebswirtschaft

das heiBt

Dissertation

Die Unternehmung

durchgesehen

European Journal of Marketing

et alteri

et cetera

folgend; folgende

Frankfurter Allgemeine Zeitung

Gesellschaft für Information und Dokumentation

Heft

Harvard Business Review

Handwörterbuch der Betriebswirtschaft

Handwörterbuch der Organisation

Harvard Manager

Handwörterbuch der Absatzwirtschaft

Herausgeber

in der Regel

id est 
IHK

I11.

IMM

insbes.

I U D

i.s.

IVS

JdAV

Jg.

JoCR

JOM

JOMR

JPMM

jr.

Kap.

Mass.

MJ

MM

MS

NFD

N.J.

No.

$\mathrm{Nr}$.

o.Nr.

o.S.

$0 . V$.

rev. ed.

$S$.

SfS

SMJ

Sp.

SPSS

Tab.
Industrie- und Handelskammer

111 inois

Industrial Marketing Management

insbesondere

Information und Dokumentation

im Sinne

Informationsvermittlungs - und Beratungstelle $(n)$

Jahrbuch der Absatz- und Verbraucherforschung

Jahrgang

Journal of Consumer Research

Journal of Marketing

Journal of Marketing Research

Journal of Purchasing and Materials

Management

junior

Kapitel

Massachusetts

Marketing Journal

Manager Magazin

Management Science

Nachrichten für Dokumentation

New Jersey

number

Nummer

ohne Nummer

ohne Seite

ohne Verfasser

revised edition

Seite

Sektion für Systemanalyse

Strategic Management Journal

Spalte

Statistical Package for the Social

Sciences

Tabelle 


$\begin{array}{ll}\text { u.a. } & \text { und andere } \\ \text { u.ä. } & \text { und ähnliche } \\ \text { u.U. } & \text { unter Umständen } \\ \text { usw. } & \text { und so weiter } \\ \text { vgl. } & \text { vergleiche } \\ \text { Vol. } & \text { Volume } \\ \text { Wist } & \text { Wirtschaftswissenschaftliches Studium } \\ \text { WW } & \text { Wirtschaftswoche } \\ \text { Z. B. } & \text { zum Beispiel } \\ \text { ZfB } & \text { Zeitschrift für Betriebswirtschaft } \\ \text { ZfbF } & \text { Zeitschrift für betriebswirtschaft- } \\ \text { ZFO } & \text { liche Forschung } \\ \text { ZFP } & \text { Zeitschrift für Organisation } \\ \text { z.T. } & \text { (Zeitschrift Führung + Organisation) }\end{array}$


A. Bedeutung der Markteintrittsentscheidung in innovativen Investivdienstleistungsmärkten

1. Gegenstand und Probleme des investiven Dienstleistungsmarketing

1.1 Zur aktuellen Bedeutung investiver Dienstleistungen

Im Zeichen anhaltend hoher Arbeitslosigkeit, stagnierender Märkte und eines wachsenden internationalen Wettbewerbsdrucks richten sich die Blicke in Wirtschaft, Politik und Gesellschaft in der juingeren Vergangenheit zunehmend auf den Dienstleistungs bereich.' Mit dem Begriff der Dienstleistung verbindet sich heute mehr denn je die "große Hoffnung des 20. Jahrhunderts", die bereits im Jahre 1954 von dem französischen Nationalökonomen Jean Fourastié beschworen wurde. 2

1 Zur Charakterisierung der aktuellen Situation stagnierender Märkte vgl. insbes. Meffert, H., Strategische Planungskonzepte in stagnierenden und gesättigten Märkten, in: DBW, 43. Jg., 1983, H. 2, S. $193 \mathrm{f}$. (im folgenden zitiert als: Meffert, H., Strategische Planungskonzepte) sowie derselbe, Marketingstrategien in stagnierenden und schrumpfenden Märkten, in: Betriebswirtschaftliche Entscheidungen be $i$ Stagnation, Festschrift für Edmund Heinen zum 65. Geburtstag, Hrsg.: Pack, L., Börner, D., Wiesbaden 1984, S. $41 \mathrm{ff}$.

$2 \mathrm{Vgl}$. Fourastié, J., Die große Hoffnung des 20 . Jahrhunderts (deutsche obersetzung: von Le grand espoir du XXe siècle, Paris 1963), 2. Aufl., Köln 1969; vgl. in diesem Sinne auch: Deutscher Sparkassenverlag (Hrsg.), Die Dienstleistungsgesellschaft Ausdruck des Strukturwandels in einer Volkswirtschaft, in: Wirtschaftskunde, Beilage zu "Kleiner Wirtschaftsspiegel", $\mathrm{Nr}$. 1, Januar 1983, S. $1 \mathrm{ff}$. Zur wachsenden Bedeutung der Dienstleistung aus absatzwirtschaftlicher Sicht der Unternehmen vgl. insbes. Meffert, H., Perspektiven des Marketing in den 80er Jahren, in: DBW, 40. Jg., 1980, H. 1 , S. 75 (im folgenden zitiert als: Meffert, H., Perspektiven); Raffée, H., Marketingperspektiven der 80 er Jahre, in: Marketing.ZFP, 4. Jg., 1982, H. 2, S. 85 . 
Tatsächlich ist der historische Trend zur Dienstleistungsgesellschaft unverkennbar. 'Ihre besondere Aktualität gewinnt die nicht selten als "revolutionär"2 bezeichnete sekuläre Strukturverschiebung der Wirtschaftssektoren durch Einflüsse, die seit der letzten 01krise 1979 in verstärktem Maße wirksam geworden sind und die seither dem Dienstleistungsbereich zusätzliche Entwicklungsimpulse verleihen. Hierzu zählen vor allem

- die aufgrund jahrelanger Stagnation und Rezession rapide angestiegene Arbeitslosigkeit im giterproduzierenden Sektor ${ }^{3}$,

1 Vgl. U.a. Statistisches Bundesamt (Hrsg.), Statistisches Jahrbuch für die Bundesrepublik Deutsch1 and, Wiesbaden 1983, S. 25; Statistisches Bundesamt (Hrsg.), Umsatzsteuerstatistik, Wiesbaden 1983, Fachserie L, Reihe 7, bzw. Fachserie 14, Reihe 8.

$2 \mathrm{Vgl}$. zu dieser Qualifizierung des Bedeutungszuwachses des Dienstleistungsbereichs insbes. Regan, W.J., The Service Revolution, in: JoM, Vol. 27 (1963), July, S. $57 \mathrm{ff}$.; Grönroos, Chr., Strategic Management and Marketing in the Service Sector, Report No. 83-104 des Marketing Science Institute (Hrsg.), Cambridge, Mass., May (1983), S.1 (im folgenden zitiert als: Grönroos, Chr., Strategic Management). Im Gegensatz dazu vgl. Meyer, P.W., Trostmann, Th., Die Revolution findet nicht statt, in: asw, 22. Jg., 1979, H. 10, Sonderaus gabe, S. $22 \mathrm{ff}$. (im folgenden zitiert als: Meyer, P.W., Trostmann, Th., Die Revolution); Rasmussen, Th., Aspekte einer verschlafenen Revolution, in: asw, 22. Jg., 1979, H. 10, Sonderausgabe, S. 21.

3 Statistiken weisen nach, daß sich in Zeiten konjunktureller Tiefs der säkulare Trend eines stetig schrumpfenden Anteils der "statischen" Produktionsbetriebe zugunsten zunehmender Anteile der "dynamischen" Dienstleistungsbereiche wesentlich beschleunigt. Vgl. hierzu Claus, F.J., Rezession beschleunigt Trend zur Dienstle istungsgesellschaft in den USA, in: Ifo-Schnelldienst, 34. Jg., 1982, H. 28, S. 14 . 
- das Aufkommen neuer Mikro-Technologien, die zum einen Automatisierungs - und damit Rationalisierungstendenzen im produzierenden Gewerbe auslösten, zum anderen aber auch den weg in neue, innovative Dienstleistungsbereiche freigaben ${ }^{1}$,

- Veränderungen in den Lebensgewohnheiten und Wertestrukturen der Verbraucher mit einem beobachtbaren Trend zu mehr Lebensqualität, die zu einem wachsenden Bedarf an Dienstleistungen im konsumtiven Bereich fuhren2, sowie

- die Tatsache, daß Dienstleistungsmarken seit 1979 eintragungs- und damit schutzfähig sind; auf diese Weise verlor der Einwand an Bedeutung, Dienstleistungen seien grundsätzlich kopierbar. 3

$1 \mathrm{Vgl}$. in diesem Sinne Rasmussen, Th., Aspekte, a.a.0., S. 21; Berekoven, L., Die Dienstleistungs-Generation - Erbin der Industriegesellschaft, in: asw, 22. Jg., 1979, H. 10, Sonderausgabe, S. 6 (im folgenden zitiert als: Berekoven, L., Dienstle istungs-Generation); Schuh, C.F., Die Büroautomation wartet auf neue Dienstle istungsangebote, in: asw, 22. Jg., 1979, H. 10, Sonderausgabe, S. $120 \mathrm{ff}$.; Zahn, E., Mikroelektronik in der Informationsgesellschaft, in: HM, o. Jg., 1983, H. 3, S. 10 .

2 Vgl. U.a. Nüssle, G., Goldene Zeiten für Dienstleister, in: asw, 22. Jg., 1979, H. 10, Sonderausgabe, S. 28; Raffée, H., a.a.0., S. 81 ; Langeard, E., Bateson, I.E.G., Lovelock, Chr. H., Eiglier, P., Services Marketing: New Insights from Consumers and Managers, Report No. 81-104 des Marketing Science Institute, Cambridge, Mass., August 1981 , S. 9.

$3 \mathrm{Vgl}$. Berekoven, L., Dienstleistungs-Generation, a.a.0., S. 6. Die Bedeutung, die dem warenrechtlichen Schutz der Dienstleistung in der Praxis eingeräumt wird, dokumentiert sich in der Tatsache, daß im Zeitraum zwischen dem 1. April 1979 und 31. August 1982 dem Deutschen Patentamt $r$ d. 17.000 Anmeldungen von Dienstleistungen zur Eintragung in die Warenzeichenrolle vorgelegt wurden. Vgl. Graumann, I., Die Dienstleistungsmarke, Muinchen 1983, S. 1. Zur Dienstleistungsmarke vgl. ferner Heil, G., Die Dienstleistungsmarke - erste Erfahrungen und geplante Praxis, in: Markenartikel, 1979, H. 5, S. $237 \mathrm{ff}$.; Schreiner, P., Die Dienstleistungsmarke, in: Markenartikel, 1982 , H. 10, S. $436 \mathrm{ff}$.; Messing, H.W., "Dienstleistungs marke" - eine neue Variante im Markenangebot, in: Markenartike1, 1983, H. 10, S. $496 \mathrm{ff}$. 
Obgleich in Fachkreisen die wachsende Bedeutung der Dienstleistung nahezu einhellig betont wird', konnte die Wissenschaft bislang zu keiner einheitlichen Auffassung über den Begriff der Dienstleistung finden. 2 Insbesondere besteht kein Konsens daruber, welche Bereiche zum Dienstleistungssektor zu zählen sind. ${ }^{3}$ Im Rahmen dieser Arbeit soll der Dienstleistungs-Definition von Engelhardt und Schwab gefolgt werden. Danach sind unter Dienstleistungen alle selbständigen, marktfähigen Tätigkeiten zu verstehen, die auf die Bereitstellung und/oder den Einsatz von Potentialfaktoren gerichtet sind, wobei an den Ergebnissen des Erstellungsprozesses kein Eigentum im juristischen Sinne erworben werden kann. ${ }^{4}$

$1 \mathrm{Vgl}$. U.a. Berekoven, L., Dienstleistungs-Generation, a.a.0., S. 12. Rasmussen spricht beispielsweise von einer langfristigen Stabilisierung des Anteils der im Dienstleistungssektor Beschäftigten im Bereich zwischen 60 und 70 Prozent; vgl. Rasmussen, Th., a.a.0., S. 21. Signalfunktion für die Bundesrepublik Deutschland hat nicht zuletzt die rasante Entwicklung, die der tertiäre Sektor in den USA genommen hat. Im Jahre 1982 vereinigte dort der Dienstle istungssektor insgesamt $70,6 \%$ der Beschäftigten auf sich. Vgl. US-Department of Commerce (Hrsg.), Statistik der Beschäftigtenanteile nach Wirtschaftssektoren für die USA, zitiert in: ClauB, F.J., a.a.0., S. 12.

$2 \mathrm{Vgl}$. Z. B. Grönroos, Chr., Strategic Management, a.a.0., S. 2; Hilke, W., Dienstleistungs-Marketing aus der Sicht der Wissenschaft, gekürzte Fassung des Grundsatzreferats vom 28. November $1983 \mathrm{im}$ Rahmen des Workshops "Dienstleistungs-Marketing", durchgeführt von der Wissenschaftichen Geselischaft für Marketing und Unternehmensführung e.V., Münster, $\mathrm{S} .1 \mathrm{f}$.

3 Einige Autoren, unter innen vor allem Berekoven, vertreten beispielsweise die Auffassung, "daB die Bereiche Handel, Banken und Versicherungen keine typischen Dienstleistungszweige darstellen ..." (Berekoven, L., Dienstle istungsmärkte - Die Wachs tumshoffnung der $80 \mathrm{er}$ Jahre?, in: Markenartikel, 1983, H. 6, S. 315 (im folgenden zitiert als: Berekoven, $L$., Dienstleistungsmärkte).

4 Vgl. Engelhardt, W.H., Schwab, W., Die Beschaffung von investiven Dienstleistungen, in: DBW, 42. Jg., 1982 , Nr. 4, S. 504; vgl. in diesem Sinne auch Judd, R., The Case of Redefining Services, in: JoM, 28. Jg., 1964, H. 1, S. 59; Wyckham, R.G., Fitzroy, P.T., Mandry, G.D., Marketing of Services. An Evolution of the Theory, in: EJoM, 9. kquips-978-3-631-75d $74-2$ S. 63 . 
Unterscheidet man in Analogie zum Sachgüterbereich Dienstleistungen danach, ob sie für private $\mathrm{Nach}$ frager oder Unternehmen bereitgestellt werden (vgl. Abbildung 1$)^{\uparrow}$, so rücken gegenwärtig die sog. investiven Dienstleistungen als an Wichtigkeit gewinnende Marktleistungen zunehmend in den Mittelpunkt des Interesses.

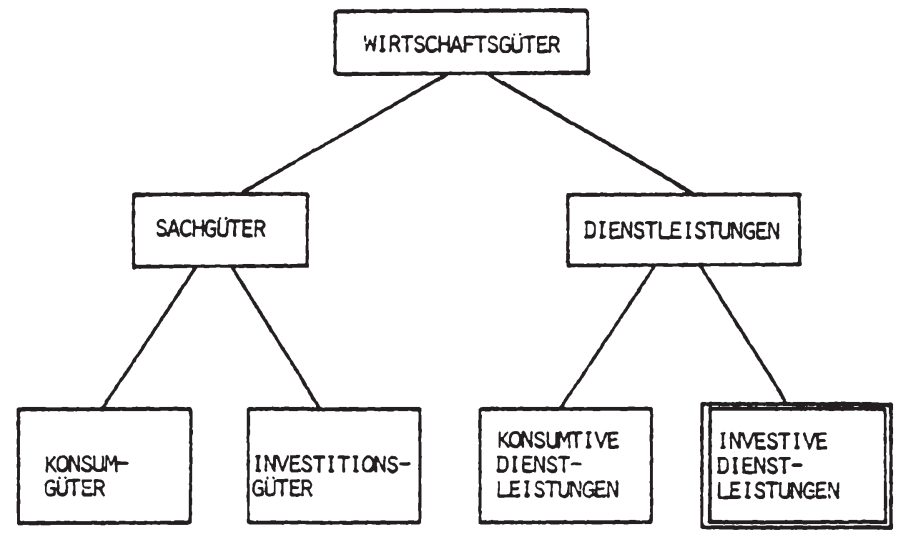

Abb. 1: Systematik der Wirtschaftsgüter

$1 \mathrm{Vgl}$. zu dieser Unterscheidung auch Engelhardt, W.H., Schwab, W., a.a.0., S. 505 sowie Hilke, W., a.a.0., S. 3. Kaufmann nimmt die gleiche Systematisierung vor, bezeichnet jedoch die investive Dienstleistung als Produktivdienstleistung; vgl. Kaufmann, E.J., Marketing für Produktivdienstleistungen, Frankfurt/Main, Zürich 1977, S. 30. Da sich im wissenschaftlichen Sprachgebrauch der Terminus "Investivdienstleistung" ( in Analogie zu dem Begriff "Investitionsgut") durchgesetzt hat, soll im folgenden an der Bezeichnung "investive Dienstleistung" (engl.: "industrial service" oder "business service") als Pendant zur "konsumtiven Dienstleistung" festgehalten werden. 
Unter investiven Dienstleistungen werden generell solche Dienstleistungen verstanden, die von organisationen gekauft werden, um mit dem Einsatz dieser Dienstleistungen anschließend Sachleistungen oder weitere Dienstleistungen für die Fremdbedarfsdeckung zu erstellen.' Als Beispiele solcher Investivdienstleistungen sind $u$. a. von Organisationen beschaffte Beratungsleistungen, Transporte, Instandhaltungen, Gutachten, Rechtsbeistand, Montageleistungen, Marktforschung, etc. zu nennen.

Statistiken über die steuerbaren Umsätze von Dienstleistungsunternehmen in den letzten zwei Jahrzehnten belegen deutlich, daß der Bereich der investiven Dienstleistungen ein überdurchschnittliches Umsatzwachstum erzielen konnte. So wiesen zwischen 1962 und 1976 zum Beispiel die Unternehmen der Rechts- und Wirtschaftsberatung, Architektur- und Ingenieurbiros, die Wirtschaftswerbung und die mit Vermietung, Verwaltung und Vermittlung beschäftigten Unternehmen durchschnittliche Umsatzzuwächse von 13 bis 16 Prozent pro Jahr auf. 2 Ursache ist hierfür nicht zuletzt der anhaltende

1 Vgl. Engelhardt, W.H., Schwab, W., a.a.0., S. 505; im gleichen Sinne Hilke, W., Dienstleistungs-Marketing, a.a.0., S. 3. Kaufmann definiert die Investivdienstleistung, indem er ausführlich auf ihre absatzspezifischen Besonderheiten eingeht: "Produktivdienstleistungen werden in Form eines Leistungsversprechens angeboten und verkauft sowie im Verlauf eines Erstellungsprozesses von einer Produktivdienstleistungsunternehmung in Zusammenarbeit mit einer nachfragenden Unternehmung produziert. Die Beteiligung der Kundenunternehmung am Erstellungs proze $B$ besteht in der Bereitstellung des Leistungsobjektes und im Zurverfügungstellen von Informationen. Gleichzeitig mit der Dienstleistungserstellung ... findet auch die Nutzung der Produktivdienstleistung statt, wobei der Nutzen der Produktivdienstleistung für die Kundenunternehmung wesentlich vom Ausma $B$ und der Qualität der Bereitstellung der externen Komponenten durch die Kundenunternehmung abhängt. Die Ergebnisse des Produktivdienstleistungserstellungsprozesses sind größtenteils erklärungsbedurftig, in ihrer qualitativen Ausführung schwer zu bewerten und immateriell, sie können jedoch auf materiellen Trägermedien festgehalten und gespeichert werden".

2 Vgl. Nüssen, G., a.a.0., S. 34 . 
Trend zur Spezialisierung und zum Kostenmanagement in den Abnehmerunternehmen, in dessen Folge investive Dienstleistungen als Vorleistungen auf allen Stufen wirtschaftlicher Aktivität zunehmend an Boden gewinnen.

\subsection{Stand des Marketing investiver Dienstleistungen}

Vor dem Hintergrund dieser Entwicklung muß es erstaunen, daB die wissenschaftliche Erforschung des gesamten Dienstleistungsbereichs heute noch in ihren Anfängen steckt. Sowohl die deutsche Betriebswirtschaftslehre als auch die amerikanische Managementlehre haben sich der spezifischen Problematik der Dienstleistungen und des Dienstleistungsmanagements bisher nur eher beiläufig gewidmet. Auch die in jüngerer Zeit zunehmende $Z a h l$ der Veröffentlichungen vermögen diesen Eindruck nicht nachhaltig zu revidieren. Allgemein besteht in der Literatur insbesondere eine ubereinstimmende Auffassung über den unbefriedigenden Stand der Forschung zu den Problemen des Marketing von Dienstleistungen. ' In besonderem Maße betrifft dieser den Bereich investiver Dienstleistungen. In der Tat dokumentiert sich in kaum einem Feld der Absatzwirtschaft die Diskrepanz zwischen wissenschaftlicher Durchdringung und praktischer Bedeutung in gleicher Eindringlichkeit. ${ }^{2}$ während sich amerikanische Wissenschaftler

1 Vgl. Engelhardt, W.H., Schwab, W., Die Beschaffung, a.a.0., S. 503; Hilke, W., a.a.0., S. 1 f.; Klaus, P., Auf dem Weg zu einer Betriebswirtschaftslehre der Dienstleistungen: der Interaktionsansatz, Entwurf eines Beitrags fuir die $D B W$, vorgelegt im $\mathrm{Fe}$ bruar 1984, S. 1 (im folgenden zitiert als: Klaus, P., Dienstieistungen).

2 Grönroos faBt als einer der führenden Vertreter der "Nordic School" of service marketing seine Auffassung hierzu wie folgt zusammen: "... marketing academicians may have failed in developing marketing theory in such a direction that it can be successfully adopted by service firms" (Grönroos, Chr., Strategic Management, a.a.0., S. 2) und: "One could say that service marketing now stands at the same point as industrial marketing did some ten years ago" (Grönroos, Chr., An Applied Service Marketing Theory, in: EJoM, Vol. 16, 1982, S. 83 (im folgenden zitiert als: Grönroos, Chr., 
und die Vertreter der "Nordic School" of Service Marketing seit etwa Mitte der 70er Jahre diesem Themenbereich widmen ${ }^{1}$, ist in der deutschen Literatur das Problem des Marketing investiver Dienstleistungen bis in die jüngste Vergangenheit faktisch nicht aufgegriffen worden. 2

Definiert man investives Dienstleistungsmarketing als die Planung, Koordination und Kontrolle aller auf die aktuellen und potentiellen investiven Dienst-

Applied Theory II)). Shostack stellt ergänzend fest: "... marketing offers no guidance, terminology, or practical views that are clearly relevant to services". Shostack, L.G., Breaking Free from Product Marketing, in: JoM, Vol. 41, 1977, April, S. 73 (im folgenden zitiert als: Shostack, L.G., Breaking Free); vgl. ebenso Bateson, I.E.G., Why we need service marketing, in: Ferre11, 0.C., Brown, St.W., Lamb, C.W. Jr. (Hrsg.), Conceptual And theoretical Developments in Marketing, Chicago, 111., 1979, S. 131.

$1 \mathrm{Vgl.Z}$. B. Wittreich, W.J., How to Buy/Sell Professional Services, in: HBR, Vol. 44, March-April 1966, S. $127 \mathrm{ff}$. ; Turner, E.B., Marketing Professional Services, in: JoM, Vol. 33, October 1969, S. 56 ff.; Wilson, A., Marketing of Professional Services, London 1972; Kotler, Ph., Connor, R.A. Jr., Marketing Professional Services, in: JoM, Vol. 41, 1977, No. 1, S. $71 \mathrm{ff}$.; Gummesson, E. Toward a Theory of Professional Service Marketing, in: IMM, Vol. 7, 1978, S. $79 \mathrm{ff} .$, Grönroos, Chr., An Applied Theory for Marketing Industrial Services, in: IMM, 1979, S. $45 \mathrm{ff}$. (im folgenden zitiert als: Grönroos, Chr., Applied Theory I). Es fällt auf, daß sich die Mehrzahl der Beiträge vor allem mit einem engeren Teilbereich der "industrial services" auseinandersetzt, nämlich mit den sog. "professional" (oder "consultancy") services". Hierunter werden individuell erbrachte Beratungsleistungen im weitesten Sinne des Wortes verstanden. Zu zählen sind hierzu u. a. EDVDienstleistungen, Unternehmens-, und Werbeberatung, Rechtsbeistand etc., als Gegensatz zu Transportdiensten, Reinigungs - und Instandhaltungsdiensten etc. Vgl. zu dieser Unterscheidung insbes. Grönroos, Chr., Applied Theory I, a.a.0., S. 45 f.

2 Zwei der wenigen Ausnahmen bilden die Arbeiten von Kaufmann und Engelhardt/Schwab. Vgl. Kaufmann, E.J., a.a.0. und Engelhardt/Schwab, a.a.0. 
leistungsmärkte gerichteten Unternehmensaktivitäten ${ }^{\uparrow}$, so stehen vor allem folgende Fragestellungen im Mittelpunkt der aktuellen wissenschaftlichen Diskussion: ${ }^{2}$

- Wie lassen sich Investivdienstleistungen als wesentliche Bestandteile der von Unternehmen erworbenen Marktleistungen erfassen, beschreiben und klassifizieren?

- Welche Schlußfolgerungen ergeben sich aus den spezifischen Merkmalen der Investivdienstleistung für die Gestaltung des Marketinginstrumentariums?

- Lassen sich Managementprinzipien und Methoden industrieller (i. S. von Sachmittel produzierender) Betriebe auf das Management von Dienstleistungsbetrieben übertragen?

- Von welchen Faktoren hängt die Ubertragbarkeit gegebenenfalls im einzelnen ab?

- Welche Kriterien können für die Beurteilung der wirtschaftlichen Effektivität der Dienstleistungserstellung angewandt werden?

Bemerkenswert erscheint, daß sich die Gesamtheit der aufgezählten Fragestellungen allein auf die Bearbeitung solcher Dienstleistungsfelder bezieht, die die Unternehmung bereits im weitesten Sinne erschlossen hat. Völlig unberücksichtigt bleiben dabei die gerade im Zeichen vieler aktueller technologischer Neue-

$1 \mathrm{Vgl}$. zu dieser Definition Meffert, H., Marketing. Einführung in die Absatzpolitik, 6., durchges. Aufl., Wiesbaden 1982 (im folgenden zitiert als: Meffert, H., Marketing). Zu Bedeutungsinhalt und Merkmalen des Marketing vgl. ferner z. B. Kotler, Ph., Marketing-Management: Analyse, Planung und Kontrolle, Stuttgart $1977, \mathrm{~S} .11 \mathrm{ff}$.

2 Vgl. Klaus, P., Dienstleistungen, a.a.0., S. 2. 
rungen ${ }^{1}$ besonders brisanten Probleme der Auswahl, Bewertung und erfolgreichen Besetzung neuer Dienstleistungs-Geschäftsfelder. ${ }^{2}$ Zahllose Beispiele aus der Praxis belegen, welche weitreichenden Marktchancen sich derzeit beispielsweise aus der Einführung never Telekommunikationsdienste in der Bundesrepublik Deutschland gerade für den Dienstleistungsbereich ergeben. ${ }^{3}$ An dieser Stelle versagt jedoch die Wissen-

1 Gedacht ist hierbei vor allem an die Innovationen im Bereich der Mikroelektronik. Vgl. insbes. Meffert, H., Unternehmensführung und neue Informationstechnologien - Akzeptanz und geplanter Wandel im Unternehmen, 11 kommentierte Thesen von Prof. Dr. H. Meffert, in: Unternehmensführung und neue Informationstechnologien. Dokumentation des 6 . Münsteraner Führungsgesprächs, Arbeitspapier Nr. 14 der Wissenschaftichen Gesellschaft für Marketing und Unternehmensführung e.V., Hrsg.: Meffert, H., Wagner, H., Münster 1983, S. $27 \mathrm{f}$. (im folgenden zitiert als: Meffert, H., Neue Informationstechnologien); vgl. ferner: Hupe, R., Wettlauf um die Super-Märkte, in: MM, 13. Jg., 1983, Nr. 6, S. 112 ff.; Schwerdtner, H.E., Schliusseltechnologien/Weg zu neuem Wachstum. Chancen auch für kleinere und mittlere Unternehmen, in: Handelsblatt, $\mathrm{Nr}$. 125, 4. Juli 1983, S. B1 und B4; Bues, M., Wirtschaftsinformatik der 90 er Jahre, in: Büro + Verkauf, 53. Jg., Februar 1984, Nr.2, S. $18 \mathrm{ff}$..

2 In ersten groben Ansätzen findet sich dieser Aspekt lediglich bei Langeard wieder. Vgl. Carman, I.M., Langeard, E., Growth Strategies of Services Firms, in: SMJ, Voi. 1, 1980, H. 1, S. 7 ff.; Langeard, E., Grundlagen des Dienstleistungsmarketing (ins Deutsche ubertragen und bearbeitet von Pardey, R.), in: Marketing.ZFP, 3. Jg., 1981, H. 4, S. $233 \mathrm{ff.}$, insbes. S. $238 \mathrm{ff}$.

3 Zu denken ist hier $z$. B. im Zusammenhang mit dem neuen Medium Bildschirmtext an neue Dienstleistungen von Zeitungsverlagen, wie "elektronic publishing", Blitzmeldungen, Archivdienste etc., aber auch von Werbeagenturen ( $B i$ )dschirmtext-Beratung-, Programmgestaltung) oder Auskunfteien (InformationsbrokerDienste). Zu Gegenstand, Einsatzmöglichkeiten und Auswirkungen des Bildschirmtextes vgl. insbes. Meffert, H., unter Mitwirkung von Althans, J., Krups, M., Middelhoff, Th., Schnetkamp, G., Bildschirmtext als Kommunikationsinstrument. Einsatzmöglichkeiten im Marketing, Stuttgart 1983; Meffert, H., Stichwort Bildschirmtext, in: DBW, 43. Jg., 1983, S. 315 f.; Krups, M., Schubert, F., Zum Stand des Bildschirmtextes in der Bundesrepublik Deutschland, Arbeitspapier Nr. 31 des Instituts für Marke- 
schaft den betroffenen Unternehmen eine theoretisch fundierte Hilfestellung; den Firmen bleibt es selbst uberlassen, "strategische Fenster" 1 zu erkennen und in geeigneter Weise aufzustoßen.

Als ein in der aktuellen Diskussion vielzitiertes Beispiel eines solchen strategischen Fensters erscheint die Entwicklung und "Vermarktung" elektronisch gestutzter Informationsdienstleistungen.

\subsection{Informationen als innovative Investivdienst- leistungen}

Informationen nehmen heute im Prozeß der betrieblichen Leistungserstellung eine vorrangige Bedeutung ein. Allerorts wird auf die ökonomische Relevanz der Information hingewiesen; vielfach bezeichnet man sie als den "vierten Inputfaktor" 2 . Definiert man Infor-

ting der Universität Münster, Hrsg.: Meffert, H., Münster 1983 sowie Meffert, H., Schubert, F., Der Einfluß von Telekommunikationsmedien, insbesondere Bildschirmtext auf die Non-Store-Marketinginstrumente von Hersteller-, Handels- und Dienstleistungsunternehmen in der Bundesrepublik Deutschland, Arbeitspapier $\mathrm{Nr} .32$ des Instituts für Marketing der Universität Münster, Hrsg.: Meffert, H., Münster 1984.

1 Abell, D.F., Strategic Windows, in: JoM, Vol. 42 , July 1978, No. 3, S. $21 \mathrm{ff}$.

$2 \mathrm{Vgl}$. hierzu u. a. Mag, W., Informationsbeschaffung, in: HdB, 4. Aufl., Hrsg.: Grochla, E., Wittmann, W., Stuttgart 1975, Sp. $1882 \mathrm{f.}$; Bearcheli, C.A., Information handling - as product or as service, in:

MJ, 13. Jg., 1980, H. 3, S. 226; derselbe, Information as Resource - Its Importance is Growing Ever Greater, in: MJ, 16. Jg., 1983, H. 1, S: 24; Hennemann-Böckels, B., Bertelsmann will auch "DatenbankVerleger" werden, Teil XVII der Beitragsreihe zum Umgang mit Datenbanken, in: BddW, 0.N., 15.02.84, S. 5 (im folgenden zitiert als: Hennemann-Böckels, B., Teil XVII). 
mation als "zweckorientiertes Wissen"1, so rücken aus der Marketingperspektive dieser Untersuchung jene Informationen in den Vordergrund, die industriellen Abnehmern von unternehmensexternen Anbietern gegen Entgelt zur Verfügung gestellt werden. Informationen genaver: Informationsdienstleistungen ${ }^{2}$ - sollen damit im folgenden als Wirtschaftsguter aufgefaßt werden, die auf Märkten angeboten und nachgefragt werden. ${ }^{3}$

1 Berthel, I., Information, in: HdB, 4. Aufl., Hrsg.: Grochla, E., Wittmann, W., Stuttgart 1975, Sp. 1866 (im folgenden zitiert als: Berthel, I., Information); zum Begriffsinhalt der Information vgl. ferner Bössmann, E., Information, in: HdW, Hrsg.: Albers, W. et al., Stuttgart, New York etc. 1978, S. 185; Wittmann, W., Information, in: HdO, 2. Aufl., Hrsg.: Grochla, E., Stuttgart 1980, Sp. 894 .

2 Der Verfasser ordnet damit Informationen im oben definierten Sinne den Dienstleistungen zu. Vgl. u.a. Muiller, W., Nickel, H., Das Marketing von Informationsprodukten, am Beispiel von Versicherungen, in: Marktorientierte Unternehmensführung: Wissenschaftliche Tagung des Verbandes der Hochschullehrer für Betriebswirtschaft, Wirtschaftsuniversität Wien 1983 , Hrsg.: Mazanec, I., Scheuch, F., Wien 1984, S. $331 \mathrm{ff..}$ Gleichwohl sei darauf hingewiesen, daB in der Literatur höchst divergente Auffassungen darüber bestehen, ob Informationen uberhaupt Dienstleistungen darstellen, unter welchen speziellen Voraussetzungen dies sinnvoll sein könnte und welche Konsequenzen für ihr Marketing sich daraus ableiten lassen könnten; vgl. Müller, W., Nickel, H., a.a.0., S. 736 f.; Kaufmann beispielsweise grenzt Information explizit von Dienstleistungen ab. Andererseits bezieht er aber "Produktivdienstleistungen der Informationsbeschaffung und Beratung", unter ihnen explizit auch "Informationsdienste", in die Dienstleistungsdiskussion mit ein. Vgl. Kaufmann, E.J., a.a.0., S. $25 \mathrm{ff}$. und $\mathrm{S}$. 38 . Vgl. auch einen entsprechenden Hinweis bei Miilier, W., Nickel, H., a.a.0., S. 732 .

$3 \mathrm{Vgl}$. zu dieser Interpretation der Information $z$. B. Berthel, I., Information, a.a.0., Sp. 1869, und Bössmann, E., a.a.0., S. 185. Müller und $\mathrm{Nickel}$ sprechen in diesem Zusammenhang von "Informationsprodukt". Vgl. Müller, W., Nickel, H., a.a.0., S. $732 \mathrm{ff}$. Zur allgemeinen Beschreibung und Abgrenzung von Informationsprodukten vgl. ebenda. 
Zweck der Informationen aus der Sicht des Anbieters ist ihr Absatz auf dem Informationsmarkt. Hierbei wird der Begriff "Informationsmarkt" in einem relativ engen Sinne definiert: Der Informationsmarkt ist der ökonomische Ort, an dem das Angebot von Informationsdienstleistungsbetrieben ${ }^{1}$ auf seine Nachfrage $t r i f f t^{2}$.

Abbildung 2 gibt schematisch den Marktkreislauf von Informationsangebot und -nachfrage wieder. ${ }^{3}$

1 Informationsdienstleistungsbetriebe sollen dabei als solche erwerbswirtschaftlich tätigen Unternehmen verstanden werden, deren Leistung in der Erzeugung, Bearbeitung und Verarbeitung von Informationen besteht. "Zu diesem Zweck vollzieht sich ihr DienstleistungsprozeB in den elementaren Schritten Informationsaufnahme, Informationsverarbeitung und Informationsabgabe. Diese drei Aufgabenmerkmale... stellen gemeinsam den wesentlichen Bestandteil der betrieblichen Zielsetzung .. von Informationsdienstleistungsbetrieben dar". Höring, K., Organisation der Informationsdienstle istungsbetriebe, in: HdO, 2. Aufl., Hrsg.: Grochla, E., Stuttgart 1980, S: 914. Eine Systematisierung der verschiedenen Arten von Informationsdienstleistungsunternehmen findet sich ebenda, Sp. $915 \mathrm{f}$.

2 Zur volkswirtschaftlich-statischen Definition des Marktes, der diese Begriffsfassung zugrunde liegt, vgl. U.a. Meffert, H., Marketing, a.a.0., S. 54; vgl. ferner Helmstädter, E., Wirtschaftstheorie, Bd. 1, München 1974, S. 26 und $0 t t$, A.E., Preistheorie, in: Kompendium der Volkswirtschaftslehre, 5., uberarbeitete und ergänzte Auf1., Hrsg.: Ehrlicher, W., Esenwein-Rothe, I., Jürgensen, H., Rose, K., Bd. 1, Göttingen 1975, S. 118.

3 Explizit aus der Betrachtung ausgeklammert werden in diesem Zusammenhang die "Informationsangebote" von Versicherungen, Kreditinstituten, etc. Im Gegensatz dazu: Müller, W., Nickel, H., a.a.0. 


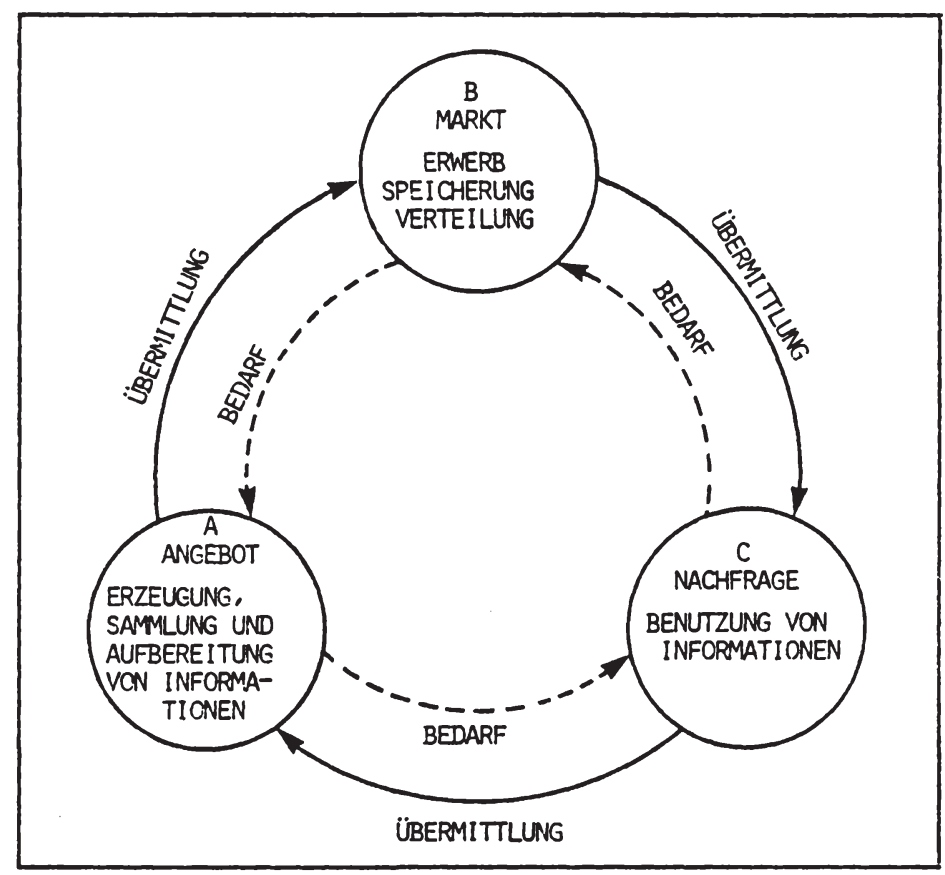

Abb. 2: Kreislauf von Informationsangebot und -nachfrage

(Quelle: in Anlehnung an Luhn, H.P., Automation und Feedback in einem umfassenenden wissenschaftlichen Kommunikationssystem, in: NfD, 10.Jg., 1959, H.4, S. 174)

Ein Informationsmarkt, dem in jüngerer Zeit eine wachsende Aufmerksamkeit zuteil wird, stellt der Markt unternehmensexterner Wirtschaftsinformationsdienste dar. Dieser Markt soll im Rahmen der vorliegenden Arbeit als Bezugsobjekt im Mittelpunkt der Betrachtung stehen. Mit der Darstellung seiner wesentlichen strukturellen Besonderheiten wird zunächst die Grundlage geschaffen, auf der die Problemstellung der Arbeit aufbaut. 
2. Der Markt elektronischer Wirtschaftsinformationsdienste als Untersuchungsbereich

Ausgehend von der zunehmenden Komplexität und Dynamik der Umwelt, in deren Folge die Unternehmen eine immer steigende Menge von Informationen gewinnen, verarbeiten und speichern müssen, erstaunt die Tatsache wenig, daß Entscheidungsträger heute für die Handhabung solcher Informationen einen überragenden Teil ihrer Arbeitszeit aufwenden. Etwa 80 \% der verfügbaren Zeit verwenden Unternehmensmitglieder der strategischen und der funktionalen Managementebene für den Umgang mit Informationen. Dabei investieren die Unternehmen im Durchschnitt $12 \%$ vom Umsatz für das Informationswesen. 1

Im Kontrast zu diesem hohen Bedeutungsgehalt betrieblicher Information steht die weitbeklagte Situation einer unbefriedigenden Informationsversorgung in den Unternehmen. Kennzeichnend für diese Situation ist das Stichwort vom "Paradoxon der Informationsarmut im Informationsüberfluß"2; die wachsende Fülle immer schwerer zu handhabender und häufig redundanter Information steht in krassem Gegensatz zu dem Mangel an tatsächlich benötigten, unmittelbar problembezogenen und direkt umsetzbaren Informationen.

1 Die Angaben entstammen einer Untersuchung der Firma IBM bei 7.200 Unternehmen der Branchen Elektro und Elektronik sowie Leicht- und Schwermaschinenbau in Frankreich. Vgl. IBM (Hrsg.), Das Management des Informationssystems: BISM (Business Information Systems Management), Informationsbroschüre, Frankfurt 1981.

2 Diller, H., Marketing-Informationssysteme, in: WiSt, 5. Jg., 1976, Nr. 3, S. 97. Exakter wäre die Bezeichnung "Nachrichtenüberfluß" bzw. "DatenüberfluB"; vgl. ebenda; vgl. im gleichen Sinne Heussler, R., Die Information als Produktionsfaktor, Teil 2 einer Beitragsreihe unter dem Titel "Die Informationselite", in: BddW, 0.N., 02.03.83, 0.S. 
Vor diesem Hintergrund ist das Aufkommen eines Marktes zu verstehen, der hier als Markt für externe Wirtschaftsinformationen definiert werden soll. Anbieter auf diesem Markt sind die Gesamtheit institutioneller Dienstleistungsunternehmen, die entgeltich für Wirtschaftsorganisationen externe (von außerhalb des nachfragenden Unternehmens stammende), betrieblich benötigte Informationen bereitstellen. In ihrer Bedeutung haben diese "Information Providers" durch die Fortschritte im Bereich der Computertechnik in den vergangenen Jahren einen rasanten Aufschwung genommen. Datenbankgestützte Informationsdienste bestimmen zunehmend das Bild. 1983 erwirtschafteten solche Datenbankanbieter weltweit einen Umsatz von 4 bis 4,5 Mrd. DM. 1

Es ist kennzeichnend für den aktuellen Entwicklungsstand des Informationsmarktes in der Bundesrepublik Deutschland, daß von diesem Umsatz nur ein Teil von $1 \%$ auf den deutschen Markt fällt. ${ }^{2}$ So stellt Stukenbröker fest, daß in der Bundesrepublik Deutschland der Markt für Wirtschaftsinformationen, was Anzahl und Struktur der potentiellen Abnehmer, Bedarf und Bedarfsstrukturen, Beziehungen zu alternativen Informationsprodukten und Wettbewerbsaspekțe anbetrifft, als weitgehend unerschlossen gelten muß. 3

Ursächlich ist dieser Zustand auf folgende Faktoren zurückzuführen:

- Zum einen ist das Angebot externer Wirtschaftsinformationen, insbesondere unter Einsatz der elektronischen Telekommunikation, in der Bundesrepublik Deutschland noch relativ jung und wenig bekannt von daher konnte sich bislang noch in kaum nennenswertem Umfang eine Nachfrage entwickeln.

$1 \mathrm{Vgl}$ o.V., Bertelsmann bietet Informationsdienste an. Datenbankdienste GmbH können eine Marktlücke schließen, in: FAZ, Nr. 284, 07.12.83, S. 16 (im folgenden zitiert als: o.V., Bertelsmann).

$2 \mathrm{Vgl}$. ebenda.

$3 \mathrm{Vgl}$. Stukenbröker, B., Zur Angebotssituation der Onl ine-Dienste in Deutschland, in: NfD, 30. Jg., $1979, \mathrm{Nr} .1, \mathrm{~S} .4$. 
- Zum anderen besteht trotz der Fülle des bereitstehenden Datenmaterials insgesamt noch eine beachtliche Diskrepanz zwischen dem Informationsbedarf in weiten Teilen der Wirtschaft und dem Angebot vieler Information Providers.1

- Darüber hinaus ist gerade in der Bundesrepublik Deutschland eine verbreitete, nur zögerliche Bereitschaft in den Unternehmen zur Beschaffung entgeltiicher Informationen $z u$ beobachten. ${ }^{2}$

- Schließlich bestehen nicht zuletzt noch erhebliche Vertriebssçhwächen auf seiten der Informationsanbieter. 3

Angesichts dieser Situation bietet der Markt für Wirtschaftsinformationen in den kommenden Jahren beachtliche Marktchancen für innovative Dienstleistungsunternehmen. Insofern eignet er sich in besonderer Weise fur die Untersuchung solcher Markteintrittsstrategien, die die Erschließung investiver Dienstleistungsmärkte zum Ziel haben. Um ein tieferes Verständnis für den Informationsmarkt und die auf ihm vertriebenen Informationsangebote zu schaffen, wird ini folgenden zunächst eine morphologische Beschreibung der Marktstruktur vorgenommen. Im AnschluB sind die Anbieter- und Nachfragerseite insoweit zu beleuchten, als es für eine Diskussion der besonderen Markteintrittsproblematik unerläßlich erscheint. ${ }^{4}$

$1 \mathrm{Vgl}$. Schwab, H., Das IuD-Programm aus heutiger Sicht. Voraussetzungen, Mängel, positive Ergebnisse, in: $\mathrm{NfD}, 32 . \mathrm{Jg} ., 1981, \mathrm{Nr} .4 / 5, \mathrm{~S} .167$.

2 Vgl. Hennemann-Böckels, B., Teil XVII, a.a.0.

$3 \mathrm{Vgl}$. O.V., Aufbruch in die Zukunft, in: WW, Nr. 17, 20.04.84, S. 41 (im folgenden zitiert als: 0.V., Aufbruch).

$4 \mathrm{Vgl}$. zu den folgenden Ausführungen insbes. Krups, M., Situationsanalyse zum Markt für Wirtschaftsinformationen. Eine Bestandsaufnahme auf der Basis von Sekundärinformationen, Arbeitspapier des Instituts für Marketing der Universität Muinster, Hrsg.: Meffert, H., o.N., Münster 1982 . 


\subsection{Struktur des Marktes für externe Wirtschafts- informationen}

Zahlreiche Beiträge in der Literatur ${ }^{1}$, Ergebnisse von Forschungsstudien ${ }^{2}$ sowie Ausführungen in wichtigen internationalen Verzeichnissen informationsanbietender oder -vermittelnder Unternehmen ${ }^{3}$ erlauben die These, daß der Markt für Wirtschaftsinformationen im wesentlichen durch vier Arten von Anbietern bestimmt wird:

- Datenbank-Verleger (database publishers)

- Datenbank-Vermittler (online vendors; hosts)

- Informations- und Dokumentationsstellen (IuD-Stellen) (information collection and analysis centers)

- Informationsvermittlungs - und Beratungsstellen (IVS) (information brokers; information consultants).

1 Vgl. insbes. Comer, I.M., Chakrabarti, A.K., The Information Industry for the Industrial Marketer, in: IMM, Vol. 7,1978, S. $65 \mathrm{ff} .$.

2 Vgl. insbes. Rieth, D., Eckert, G., Erhebung be $i$ faktischen und potentiellen Informationsanbietern und Terminalbetreibern, Teilbericht 4 der ODINSYSTEMANALYSE. Systemanalyse, Konzeption und technische Realisierung des nationalen Informationsverbundes mit AnschluB an EURO-NET, Teilprojekt der GID/SfS, Heidelberg 1979; Bernhardt, U., Bestandsaufnahme in der Bundesrepublik Deutschland existierenden Stellen mit Informationsvermittlungsund -beratungsfunktion, Forschungsbericht 10 80-011 des Bundesministeriums für Forschung und Technologie, August 1980.

$3 \mathrm{Vgl}$. Learned Information Ltd. (Hrsg.), Information Industry Market Place 1981. An International Directory of Information Products and Services, New York, London 1980. 
Ausgehend von dieser Unterteilung $1 \ddot{B}$ t sich die Struktur des Marktes für Wirtschaftsinformationen vereinfachend, wie in Abbildung 3 skizziert, darstellen. 1

\subsection{Anbieter und Angebotsformen externer Informa- tionsdienste}

Eine zunehmend bedeutsame Stellung im Markt für externe Wirtschaftsinformationen nehmen "elektronische Informationsdienste" ein. Hierunter sind solche Dienstleistungsunternehmen zu subsummieren, die den direkten Zugriff auf ihr datenbankgespeichertes Informationspotential unter Einsatz der elektronischen Datenubertragung im Sinne eines online-Verfahrens ermöglichen. Im wesentlichen sind dies sog. DatenbankVerleger sowie Datenbank-Vermittler.

1 Die vorgenommene Unterteilung darf nicht darüber hinwegtäuschen, daß der Markt für Wirtschaftsinformationen gerade in der Bundesrepublik Deutschland noch weitgehend in seinen Anfängen steckt und einer großen Dynamik unterliegt. Dies impliziert, da $B$ die zweifelsfreie Zuordnung von Informationsanbietern zu bestimmten Anbieterklassen nur mit großen Einschränkungen möglich ist. So treten beispielsweise IuD-stellen vereinzelt bereits selbst als Datenbank-Produzenten bzw. Datenbank-Vermittler auf, indem sie ihren Kunden den Onl ine-Zugriff auf ihr Informationspotential zu ermöglichen. Daneben bilden sich aus der Gesamtheit der elektronischen Informationsanbieter immer häufiger organisatorische Integrationsformen heraus. SchlieBlich differieren auch vielfach die selbstgewählten Unternehmensbezeichnungen der einzelnen Informationsanbieter untereinander, selbst dann, wenn sie von dritter Seite als gleichartig angesehen werden können. "Some information providers classify themselves as computer companies; others see themselves as conventional publishers; a third group sees itselfs as part of a "new" information industry". Learned Information Ltd. (Hrsg.), a.a.0., S. 5. 


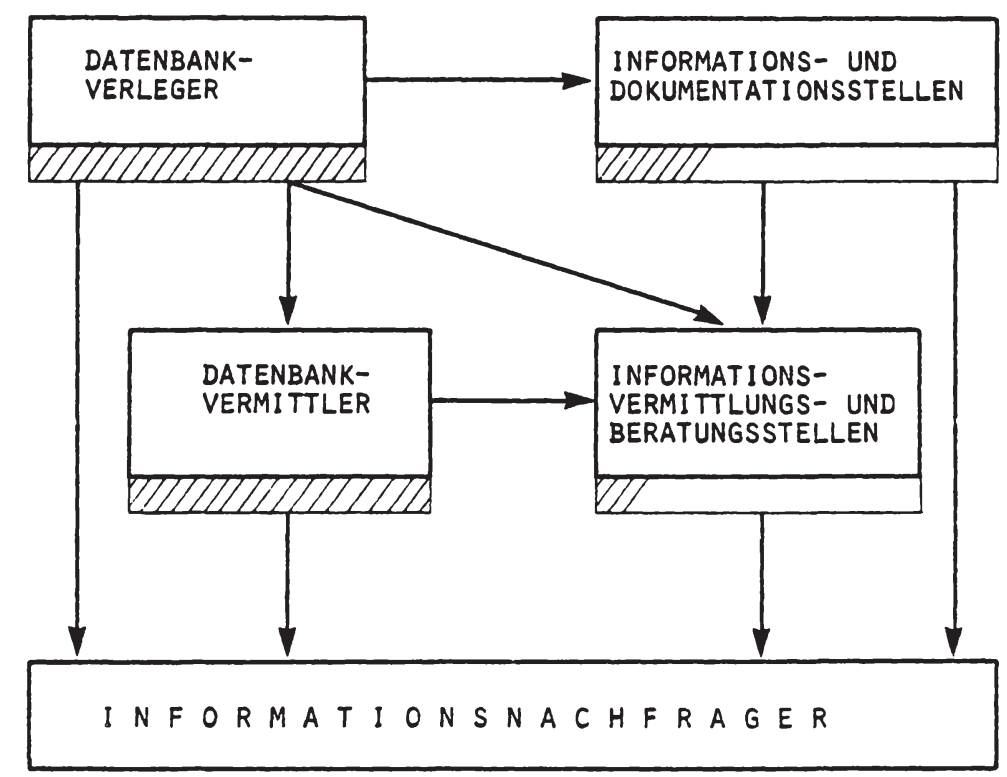

77 RELATIVE BEDEUTUNG DES ZUGRIFFS ÜBER ELEKTRONISCHE MEDIEN

$\longrightarrow \quad$ INFORMATIONSABSATZ-BEZIEHUNGEN

Abb. 3: Struktur des Marktes für Wirtschaftsinformationen

(Quelle: in Anlehnung an Krups, M., a.a.0., S. 5) 
Unter Datenbank-Verlegern (data base publishers) sind Institutionen und Unternehmen zu verstehen, die zu bestimmten Fach- und Wissensgebieten alle relevanten Informationen sammeln, aufbereiten und zu elektronisch gespeicherten Datenbeständen (machine-readable data bases) zusammenfuigen, um diese online direkt oder über Datenbankvermittler (online vendors) indirekt Dritten als entgeltliche Marktleistung anzubieten. Entscheidend ist bei dieser Anbieterkategorie die Funktion der Erstellung der Datenbank bzw. Datenbanken (information production).

Das Angebot der Datenbank-Verleger läßt sich grob nach sogenannten

- Hinweisdatenbanken (bibliographic data bases) ${ }^{1}$ oder

- Faktendatenbanken (nonbibliographic data bases)

1 Hinweisdatenbanken geben auf Anfrage Auskunft über Literaturfundstellen, die zu dem jeweils angesprochenen Problemfeld mehr oder weniger detailitierte Informationen liefern können. Der Datenbank-Verleger stellt die hierzu notwendige Literaturquellen-Datenbasis zusammen, indem er laufend

- aus einer Vielzahl von Veröffentlichungen, wie be ispielsweise Wochenzeitungen, Zeitschriften, Magazinen, Diensten u. a., eine Auswahl relevanter Artikel trifft (Lektorat),

- diese durch die Zuordnung bestimmter Suchworte bzw. Suchprofile (Deskriptoren) indexiert (Erschließung),

- i.d.R. zudem eine Zusammenfassung (abstract) des Inhaltes des zu archivierenden Artikels verfaBt und schlieBlich

- Fundstelle und Abstract auf einem elektronischen Datenträger (Magnetbank, Magnetplatte) abspeichert. 
unterteilen. ' Während die Hinweisdatenbank letztlich "nur" die Ortung von Literaturbeiträgen zu bestimmten Problemkreisen aus einer Fülle dokumentierter Publikationen ermöglicht, eröffnet ihr Gegenpart die Faktendatenbank - dem potentiellen Nutzer den Zugang zu umfassenden Beständen "harter Fakten". Im wesentlichen handelt es sich hierbei um Informationen in Form von Zahlen (Statistiken) oder in weiterverarbeiteter Form von Graphiken, Diagrammen u. ä.

Beim Absatz des Datenbankangebotes agieren zwischen Datenbank-Verleger und Datenbanknutzer vielfach Vermittlungsunternehmen, die im geläufigsten Sprachgebrauch als "Hosts", genauer als "Online Vendors" bezeichnet werden. ${ }^{2}$ Es handelt sich hierbei um Unternehmen, in deren Rechner die Suchprogramme für den Abruf von Datenbanken aus eigenen oder dritten Datenbanken aktiviert sind, und die als Absatzmittler den Vertrieb der Informationen auf der Basis von Anschlußstunden-Kostensätzen (connect-hour charges) uibernehmen. 3

1 Vielfach erfolgt die identische Unterscheidung unter Anwendung der Begriffe "qualitative" (bibliographische) und "quantitative" (Fakten-)Datenbanken. Vgl. Hennemann-Böckels, B., Ausländische Hosts teilen sich den Markt auf. Noch Lücken im Angebot, Teil III der Beitragsreine zum Umgang mit Datenbanken, in: BddW, O.N., 29.08.83, 0.S. ( im folgenden zitiert als: Hennemann-Böckels, B., Teil III).

2 "Online vendors are those organisations that sell access to their own or other producers' data bases on their own computer systems". Learned Information Ltd. (Hrsg.), a.a.0., S. 7.

3 Man kann drei Typen von Online Ventors unterscheiden. Die erste Gruppe bilden die sog. "time-sharing bureaus", die zusätzlich zu ihrem traditionellen Angebot der Datenfernverarbeitung ihren Kunden den Zugang zu verschiedenen Datenbanken ermöglichen. Die zweite Gruppe umfaßt $0 n l$ ine Vendors im engeren Sinne, also Organisationen, deren ausschließliche Unternehmensaufgabe im Verkauf von Anschlußstunden an ihr Datenbankprogramm liegt. In diesen Fällen, wo Datenlieferant und Computerbetreiber verschiedene Organisationen sind, spricht man vom Computerbetreiber als Host. Die dritte Gruppe von Online Vendors betrifft schlieBlich jene Datenbankanbieter, die sowohl die Datenbanken erstellen als auch selbst über den eigenen Computer zur Verfügung stellen. Vgl. Learned Information Ltd. (Hrsg.), a.a.0., S. 7 . 
Beide Anbietertypen, Datenbank-Verleger und -Vermittler, betreiben, so sie in direkten kontakt mit dem Informationssuchenden treten, einen Dienst, der sich unter der Bezeichnung "online information retrieval" in den USA bereits weitgehend durchgesetzt hat. In der Bundesrepublik Deutschland steht dieser Markt erst am Anfang seiner Entwicklung. ' GröBere Bedeutung hinsichtlich des Angebots wirtschaftsrelevanter Information kommt den IUD-Stellen sowie den Informationsvermittlungstellen $z u$.

Das Aufgabenprofil der IuD-Stellen umfaBt

- die systematische Sammlung von Informationen,

- ihre inhaltiche Analyse und Zuordnung zu bestimmten Sachbegriffen,

- die Speicherung in einer Form, die den einfachen Zugriff und das rasche Wiederfinden ermöglicht sowie - die Vermiţtlung dieser Informationen an Inter-

Ausgehend von dieser Definition könnten solche Stellen als optimale Lieferanten externen Wissens für Unternehmen in der Wirtschaft dienen. In der Tat war dies eines der Ziele, das die Bundesregierung 1974 bei der Verabschiedung ihres Planes zum Aufbau eines "Fachinformationszentrums Wirtschaft" vor Augen hat-

$1 \mathrm{Vgl.zu}$ dieser Einschätzung u.a. Stukenbröker, B., Onl ine-Dienste, a.a.0., S. $3 \mathrm{f}$.; Hennemann-Böckeis, B., Teil III, a.a.0.

$2 \mathrm{Vgl}$. GID (Gesellschaft für Information und Dokumentation mbH) (Hrsg.), Zugang zur Fachinformation für jedermann, Informationsbroschüre, Frankfurt am Main 1981 , S. 18. 
te. ' Zwischenzeitlich wurde von staatlicher Seite jedoch dieser Plan aus Gründen fehlender Umsetzbarkeit zurückgenommen. ${ }^{2}$ Damit fällt den wenigen auf Wirtschaftsinformationen spezialisierten IuD-Stellen in der Bundesrepublik Deutschland die Aufgabe zu, für Informationsanfragen aus der Wirtschaft bereitzustehen. Die Erfahrung zeigt jedoch, daß die nur auf konventionellem Wege zugänglichen Datenbestände dieser IuD-Stellen kaum genutzt werden, weil der Zugang zu schwierig ist und die Rechercheergebnisse zu lange auf sich warten lassen. 3

Die aus der Sicht der unternehmerischen Praxis vergleichsweise größte Bedeutung kommt der vierten Gruppe von Informationsanbietern, den Informationsver mittlungs - und -beratungsstellen (IVS) zu.

1 Der Aufbau des sog. "FIZ Wirtschaft" war integrierter Bestandteil des "Programms zur Förderung der Information und Dokumentation (IUD-Programm) 19741977". (BMFT (Bundesministerium für Forschung und Technologie)) (Hrsg.), Programm der Bundesregierung zur Förderung der Information und Dokumentation (IUD-Programm) 1974-1977, 0.0., 0.J.). Dieses Programm war als ambitioniertes und weitreichendes Vorhaben konzipiert worden mit der Zielsetzung, auf dem Wege der Rationalisierung und Reorganisation der Informations - und Dokumentationseinrichtungen "das auf der Welt vorhandene Wissen der Allgemeinheit uneingeschränkt zur Verfügung zu stellen, so daß es zur Lösung der wissenschaftlichen, technischen, ökonomischen und politischen Aufgaben unserer Zeit eingesetzt werden kann. Dem Programm lag die Vorstellung zugrunde, da $\beta$ die oberlebensfähigkeit eines relativ rohstoffarmen Industrielandes, wie der Bundesrepublik Deutschland, nur gesichert werden könne, wenn die weltweit vorhandenen Ressourcen an Wissen und Informationen optimal genutzt werden" (Schwab, H., a.a.0., S. 166).

$2 \mathrm{Vgl}$. BMFT-Leistungsplan 1982-1984, zitiert nach Hennemann-Böckels, B., Teil XVII, a.a.0.

$3 \mathrm{Vgl}$. Beyer, W., Informationsvermittlung in der Bundesrepublik Deutschland. Ein Oberblick, Bd. 12 der Reihe Aktuelle Beiträge und Berichte, Hrsg.: Gesellschaft für Information und Dokumentation $\mathrm{mbH}$ (GID), Frankfurt am Main $1982, \mathrm{~S} .37$. 
Informationsvermittlungsstellen haben im weitesten Sinne die Funktion einer Zwischenstation zwischen denjenigen, die Informationen benötigen und Anfragen stellen und denjenigen, die Informationen bereithalten, z. B. IuD-Stellen, aber auch im Online-Bereich Datenbank-Anbieter oder -Hosts (vgl. Abbildung 3 ).

Die Vielfalt der Institutionen, die im weitesten Sinne Informationsvermittlung betreiben, erschwert eine klare Differenzierung einzelner Arten von IVS. Grundsätzlich lassen sich, wie in Abbildung 4 verdeutlicht, "klassische IVS" und "nichtklassische IVS" unterscheiden. Hierbei können die klassischen IVS ${ }^{1}$ kurz als "Vermittler zu externen Informationsquellen" charakterisiert werden; die nichtklassischen IVS teilen sich wiederum in "Literaturdokumentationsstellen" und "Informations- und Beratungsstellen" auf. Unter letzteren werden weiter "Pressestellen" und "Auskunfts- und Beratungstellen" unterschieden.

Differenziert man diese IVS in einem zweiten Schritt nach ihrem Informationszweck bzw. ihren Informationsadressaten, so kristallisieren sich zwei entgegengesetzte IVS-Typen heraus. Auf der einen Seite stehen die forschungsorjentierten, auf der anderen die praxisorientierten IVS ${ }^{2}$ (vgl. Abbildung 4 ).

Die Charakteristika dieser beiden IVS-Typen (vgl. Abbildung 5) machen deutlich, daB insbesondere die Auskunfts - und Beratungsstellen von Relevanz für die informationssuchende Wirtschaft sind. Das Hauptmerkmal

\footnotetext{
1 "Klassische IVS" wurden hierbei definiert als Einrichtungen,

- die es als regelmäBige Aufgabe übernehmen,

- fuir Informationsendnutzer,

- unter Einbeziehung auch institutionsexterner Informationsquellen

- zu recherchieren

- und die recherchierte Information für den Benutzer aufzubereiten oder

- Hinweise auf externe Informationsmöglichkeiten zu geben.

Vgl. Bernhardt, U., a.a.0., S. 7.

$2 \mathrm{Vgl}$. ebenda, S. $55 \mathrm{ff}$..
} 
FORSCHUNGSORIENTIERTE IVS

PRAXISORIENTIERTE IVS

FORSCHUNGSRELEVANTE INFORMATION

TÄTIGKEITSRELEVANTE INFORMATION

WI SSENSCHAFTLICHE INFORMATION

INFORMATION AUS DER PROBLEMSITUATION

LITERATURHINWEISE

SACHVERHALTE

IN IUD-SYSTEMEN DOKUMENTIIERTE INFORMATION VON ALLGEMEININTERESSE

SPEZIFISCHE INFORMATION FUR EINZELINTERESSENTEN, NICHT DOKUMENTIERT

FUR FORSCHER, WI SSENSCHAFTLER

FUR PRAKTISCH

BERUFSTÄTIGE

Abb. 4 : Charakteristika zweier entgegengesetzter Idealtypen von Informationsvermittlungsstellen

(Quelle: in enger Anlehnung an Bernhardt, U., a.a.0., S. 56)

\begin{tabular}{|c|c|c|c|}
\hline IVS & IM WEITESTEN S & VNE & \\
\hline KLASSISCHE IVS & \multicolumn{3}{|c|}{ NICHTKLASSISCHE IVS } \\
\hline \multirow[b]{2}{*}{$\begin{array}{l}\text { VERMITTLER ZU } \\
\text { EXTERNEN } \\
\text { QUELLEN }\end{array}$} & \multirow[b]{2}{*}{$\begin{array}{l}\text { LITERATUR } \\
\text { DOKUMENTATIONS- } \\
\text { STELLEN }\end{array}$} & \multicolumn{2}{|c|}{$\begin{array}{l}\text { INFORMATIONS - UND } \\
\text { BERATUNGSSTELLEN }\end{array}$} \\
\hline & & $\begin{array}{l}\text { PRESSE- } \\
\text { STELLEN }\end{array}$ & $\begin{array}{l}\text { AUSKUNFTS- } \\
\text { UND BERATUNGS- } \\
\text { STELLEN }\end{array}$ \\
\hline \multicolumn{2}{|c|}{ FORSCHUNGSOR I ENT IERT } & \multicolumn{2}{|c|}{ PRAXISORIENTIERT } \\
\hline
\end{tabular}

Abb. 5: Arten der Informationsvermittlungsstellen

(Quelle: in Anlehnung an Bernhardt, U., a.a.0., S. 58) 
dieser Stellen liegt in der Ausrichtung ihrer Dienstleistungen auf die Problemlösung in der alltäglichen Berufspraxis. Dabei orientiert sich die angebotene Information primär an den Interessensbereichen der jeweils vertretenen Nutzergruppe. Gleichwohl steht die Vermittlung entscheidungsrelevanter Ad-hoc-Informationen auch bei diesen Informationsstellen noch weitgehend in ihren Anfängen; dies trifft in besonderem Maße auf den Einsatz elektronischer Medien zu. ${ }^{1}$

Umfangreiche Erfahrungen mit der (Online- und offline-)Vermittlung von Informationen sind demgegenüber in den USA im Rahmen des sog. "information brokerage" gesammelt worden. 2

1 So greifen heute von den Innovationsberatungsabteilungen der etwa 90 Industrie- und Handelskammern kaum mehr als zwanzig über Datenterminals auf Online-Datenbanken zurück. In den AuBenwirtschaftsabteilungen der IHKs erfolgt die Informationsbeschaffung sogar aus nahmslos auf konventionellem Wege (Korrespondenz, Telefon). Vgl. Beyer, W., a.a.0., S. 32 .

2 Für den Terminus "Informationsvermittlung" existieren im amerikanischen Sprachgebrauch mehrere artähnliche Begriffe, die jedoch jeweils unterschiedliche Dienstleistungen bezeichnen. Man unterscheidet vor allem

- information referral,

- technology transfer,

- information analysis und

- information brokerage.

Die meisten dieser Dienste bestehen bereits rd. zwei Jahrzehnte und können damit als weitgehend etabliert gelten. Im Zusammenhang mit der Vermittlung von "information on demand" kommt jedoch dem Information Broker als vergleichsweise jüngtem Dienst die gröBte Bedeutung zu. Seine Funktionen sind vergleichsweise mit denen der Auskunfts- und Beratungsstellen, jedoch spielt das Prinzip der unternehmerischen Gewinnerzielung sowie die Nutzung des online information retrieval bei der Informationsgewinnung eine weit bedeutendere Rolle. Zum Information Brokerage vgl. Bellomy, F.0., Die privatwirtschaftliche Informationsversorgungsszene (information brokerage) in den USA, in: NfD, 30. Jg., 1979, Nr. 1, S. $17 \mathrm{ff}$.; Seeger, Th., Strauch, D., a.a.0., S. 9f.; Ettel, W., Informationsvermittlung in den Vereinigten Staten, in: NfD, 31. Jg., 1980, ifr. 415, S. $185 \mathrm{ff}$.; Potter, A.R., Information Brokers and their Role in the Online Industry, in: 4 th International Online Information Meeting, London 9-11, December 1980, Hrsg.: Learned Information (Europe) Ltd., Oxford-New Jersey 1980, S. $295 \mathrm{ff..}$ 
Information Brokers betreuen grundsätzlich Informationsendnutzer, die mit gezielten, sporadischen oder regelmäßigen Informationswünschen an sie herantreten. Ihren Kunden bieten sie ein umfangreiches Dienstleistungsprogramm, das von speziellen Informationsauskünften über Informationsberatung bis hin zu abonnierbaren Berichtsserien im Sinne von "current awareness"Diensten reicht.

Ihre Informationen beziehen Information Brokers aus maschinenlesbaren Datensammlungen (maschine readable data bases) der Datenbankanbieter bzw. -hosts, aber auch von Primärliteraturherausgebern und Bibliotheken, die sie vertraglich oder durch entsprechende urheberrechtliche Regelungen dazu berechtigen, ihre Informationen mit finanziellem Gewinn weiterzuverkaufen. Es erscheint bemerkenswert, $d a B$ in der Bundesrepublik Deutschland dieses "Informationsmakler"-Konzept faktisch nicht aufgegriffen worden ist; derzeit sind weniger als ein halbes Dutzend Informationsvermittler bekannt, die nach angloamerikanischer Broker-Konzeption tätig sind. 1

\subsection{Nachfrager und Nachfragepotentiale im Bereich elektronischer Informationsdienste}

Die bisherigen Ausführungen machen deutlich, daB die Anbieterseite im Markt für externe Wirtschaftsinformationen bislang in keiner Weise hinreichend den Bedarf potentieller Nachfrager an schneller, problemgerechter Informationsversorgung zu befriedigen vermochte. Insofern mangelt es auch in der einschlägigen Literatur nicht an Stimmen, die auf den Umfang des latent vorhandenen Nachfragepotentials - gerade im Bereich elektronisch ubermittelter Ad-hoc-Information - hinweisen. ${ }^{2}$ Auch wenn bis dato keine Unter-

1 Vgl. Beyer, W., a.a.0., S. 47.

2 "... Dabei ist der Informationsbedarf der KMU (Kleinen und Mittleren Unternehmen, Anm. d. Verfassers) nahezu unbegrenzt. Doch fehlt die aktive Nachfrage, weil über die Informationsmöglichkeiten Unkenntnis herrscht. In den KMU präsentiert sich dem Informationsmarkt ein beträchtliches Benutzerpotential, das der ErschlieBung harrt" (Beyer, W., a.a.0., S. 28 ). 
suchung bekannt geworden ist, die dieser Frage explizit nachgegangen wäre ${ }^{1}$, erscheinen die Aussagen über die beachtliche Marktlücke, die sich potentiellen Informationsanbietern in diesem Bereich eröffnet, in ihrer konsistenten Tendenz plausibel. Bestätigt wird diese Tendenz z. B. auch durch den "Eurodata Report 1979". 2 Dieser prognostiziert, daß der Anteil der zum Information Retrieval eingesetzten Terminals an der Gesamtheit aller europäischen Datenterminals sich im Zeitraum von 1979 bis 1987 nahezu verdreifachen wird. Die absolute Zahl der Terminals wird dabei (von 11.200 auf 191.000) um das 17-fache zunehmen. 3

Wegbereiter für den hinter diesen Zahlen stehenden Nachfragezuwachs nach elektronischen Informationsdiensten ist dabei vor allem die zunehmende $Z a h l$ von Kommunikationsnetzen und Datenbanken. Nicht zuletzt wird - zumindest für den Bereich der mittelständischen und Kleinindustrie - der Bildschirmtext als

1 Eine solche generelle Nachfrageranalyse wäre auch insoweit von der Methodik und hinsichtlich ihrer Aussagekraft (Validität und Reliabilität) höchst problematisch. Vieles spricht für die Annahme, daB die befragten Unternehmen überfordert wären, sollten sie ihren allgemeinen Bedarf an externen Online-Informationen quantifizieren.

2 Logica Ltd. (Hrsg.), Eurodata '79, study synopsis (Zusammenfassung des Eurodata-Reports über Datenkommunikation in West Europa 1979-1987), London 1979.

$3 \mathrm{Vgl}$. ebenda, S. 9. 
neues "Datenterminal für jedermann" diesen Entwicklungsprozeß beschleunigen. 1

Für ein Dienstleistungsunternehmen, das an dem neuen Informationsmarkt partizipieren will, ist es letztlich entscheidend, fruhzeitig ein nachfragegerechtes Informationsdienstleistungs-Angebot zu entwickeln. ${ }^{2}$ Dies führt zu der Problemstellung, die im Mittelpunkt der vorliegenden Arbeit stehen soll.

1 Zur Relevanz des Bildschirmtextes für die elektronisch gestuitzte Informationsversorgung der Wirtschaft vgl. Taylor, K.H., Onl ine Business Databases and Viewdata, in: 3rd International Online Information Meeting, London 4-6 December 1979, Hrsg.: Learned Information (Europe) Lts., Oxford-New York 1979, 0.S.; Strauch, D., Vowe, G., Bildschirmtext und Zweiwegkabelkommunikation als neue Medien für Informationssysteme: Relevanz für den IuD-Bereich, in: Erstes deutsches Online-Informationstreffen, Köln, 5.-8. Mai 1980; Rauch, W., Rüdel, W., Simon, C., Zimmermann, H., Zum Einsatz von Bildschirmtext im I UD-Bereich (Teil I), in: Das Inforum, $\mathrm{Nr} .6$, 1980, 0.S.; Rosenthal, K., Bildschirmtext: Datenbank mit Mickymaus-Image, in: Online-adi-nachrichten, $\mathrm{Nr} .11,1980, \mathrm{~S} .905 \mathrm{ff}$.; Diebold Deutschland GmbH (Hrsg.), Btx-Rettungsformel für IuD? Neue Aspekte furr Oni ine-Datenbanken, in: Diebold Management Report, August/September 1981, S. 1; Ockenfeld, M., Einsatz von Videotex (Bildschirmtext) für Information und Dokumentation - drei Beispiele, in: NfD, 31. Jg., 1980, Nr. 1, S. $27 \mathrm{ff}$.

$2 \mathrm{Vgl}$. insbesondere Ela, I.D., Irwin, M.R., Technology Changes Market Boundaries, in: IMM, Vol. 12, 1983, S. 156 und Alber, A.F., Get Ready for Electronic Publishing, in: BH, Vol. 26, 1983, JanuaryFebruary, S. 55 . 
3. Problemstellung und Zielsetzung der Arbeit

3.1 Konzept eines bereichsübergreifenden elektronischen Wirtschaftsinformationsdienstes

Ausgangspunkt der Untersuchung bildet das Problem eines kommerziellen Informationsanbieters ${ }^{1}$, das derzeitige Betätigungsfeld über den Bereich der periodischen Bereitstellung papiergebundener Informationen hinaus durch den Einsatz elektronischer Medien systematisch auszuweiten. Grundlage der oberlegungen bildet das Konzept eines bereichsubergreifenden ${ }^{2}$

1 Mit dem Attribut "kommerziell" soll festgestellt werden, daB der Fall eines nach erwerbswirtschaftlichen Prinzipien handelnden Informationsanbieters im Mittelpunkt der Betrachtung steht.

2 Bereichsubergreifend meint in diesem Zusammenhang, da $B$ grundsätzlich Informationen aus unterschiedlichen betrieblichen Entscheidungsbereichen in die Betrachtung einbezogen werden sollen, so z. B. Informationen zum Thema Personal, Aus- und Weiterbildung, Export/AuBenhandel, Forschung und Entwicklung, etc. Gedacht ist jedoch insbesondere an Informationen, die den gesamten Marketingbereich betreffen, so beispielsweise Brancheninformationen, Produktinformationen, Marktstatistiken, Länderinformationen, etc. Vgl. zum Konzept bereichsübergreifender Marketinginformationsdienste vor allem Greiner, P., Zentrale oder dezentrale Informationsversorgung?, in: HM, o. Jg., 1984, H. 1, S. 77 sowie Heinzelbecker, K., Aufbau und Implementierung eines Planungs- und Informationssystems für das Marketing in der Investitionsgiterindustrie (POM), Forschungsbericht ID 83-005 des BMFT Mannheim, Mai 1983 (im folgenden zitiert als: Heinzelbecker, K., POM). Eine aktuelle obersicht uber Art und Inhalt der datenbankgestiutzten Informationsangebote zum Themenbereich Marketing in der Bundesrepublik Deutschland findet sich in: 0.V., Informationen ohne Grenzen, in: asw, 25. Jg., 1982, H. 9, S. 84. 
elektronischen ${ }^{1}$ Auskunftssystems ${ }^{2}$ für Wirtschaftsinformationen. Dieses Konzept fuBt auf der Erkenntnis, da $B$ der Informationsbedarf unterschiedlicher Wirt-

1 Die Konzentration auf "elektronische" Auskunftssysteme beinhaltet die gezielte Ausrichtung auf innovative Formen der Informationsbereitstellung. Elektronisch bedeutet hierbei, daB Informationen aus datenbankgespeicherten Beständen unter Einsatz der Mittel der Telekommunikation abgerufen bzw. uibermittelt werden sollen. Unter einer Datenbank wird in diesem Zusammenhang die "Zusammenfassung verschiedener Daten Liverstanden /, die mit Hilfe spezieller Software ... so gespeichert und verwaltet werden, daß die Benutzer die Daten nach unterschied1 ichsten Ordnungskriterien ... unmittelbar (d. h. ohne aufwendige Sortiervorgänge) abrufen können". Heinzelbecker, K., Ausbaustufen eines EDV-Marketing-Informationssystems, in: Management-Zeitschrift io, 47. Jg., 1978, Nr. 9, S. 407 ( $i m$ folgenden zitiert als: Heinzelbecker, K., EDV-MIS). Zur näheren inhaltlichen Kennzeichnung des Datenbankbegriffs vgl. ferner z. B. Meffert, H., Informations systeme. Grundbegriffe der EDV und Systemanalyse, TübingenDüsseldorf 1975, S. $33 \mathrm{ff}$.; derselbe, Computergestuitzte Marketing-Informationssysteme. Konzeptionen, Modellanwendungen, Entwicklungsstrategien, Schriftenreihe "Unternehmensfürung und Marketing", Bd. 1, Hrsg.: Meffert, H., Wiesbaden 1975, S. $29 \mathrm{ff}$. (im folgenden zitiert als: Meffert, H., Computergestützte MIS); Scheer, A.-W., Datenbanksysteme im Marketing, in: Marketing.ZFP, 2. Jg., 1980, März, H. 1, S. $40 \mathrm{ff}$. Telekommunikation bedeutet im oben zitierten Zusammenhang die "Kommunikation zwischen Menschen, Maschinen und anderen Systemen mit $\mathrm{Hilfe}$ von nachrichtentechnischen übertragungsverfahren". Fellbaum, K.-R., Telekommunikation von $A-Z$, Ausgabe 1981/82, Berlin 1981, S. 106.

2 Mit der Planung eines "Auskunftssystems" wird die Festlegung getroffen, daß dem potentiellen Benutzer auf Anfrage Informationen von auskunftsbereiten Datenbeständen übermittelt werden sollen. Von solchen "benutzeraktiven Informationssystemen" zu unterscheiden sind sog. Berichtssysteme und Entscheidungssysteme. Vgl. Szyperski, N., Informationssysteme, in: HdB, 4. Aufl., Hrsg.: Grochla, E., Wittmann, W., Stuttgart 1975, Sp. 1907 (im folgenden zitiert als: Szyperski, N., Informationssysteme); derselbe, Computergestuitzte Informationssysteme, in: Hd0, 2 . Aufl., Hrsg.: Grochla, E., Stuttgart 1980, Sp. 923 und 924 (im folgenden zitiert als: Szyperski, N., Computergestuitzte Informationssysteme); Heinzelbecker, K., Marketing-Informationssysteme heute, in: MJ, Jg., 1978, S. 138 (im folgenden zitiert als: Heinzelbecker, K., Marketing-Informationssysteme); Köhler, R., Heinzelbecker, K., Informationssysteme für die Unternehmensführung, in: DBW, 37. Jg., 1977, S. 272. 
schaftsunternehmen $z$. T. große Oberschneidungen aufweist, und daB dieser unternehmensubergreifende Informationsbedarf - insbesondere bei kleinen und mittleren Unternehmen - bislang in noch nicht befriedigender Form gedeckt wird. 1

Aus dieser Perspektive geben vor allem die folgenden Problemaspekte den AnstoB zur Schaffung eines zentralen Informationsdienstes:

- die aus den bisherigen externen Informationsquellen (IHKs, Verbände, Fachzeitschriften) gewonnenen Informationen werden tendenziell passiv aufgenommen, nicht gut genug ausgewertet und verarbeitet;

- die Informationen sind im Entscheidungsfalle vielfach veraltet bzw. nur bedingt zuverlässig;

- im Rahmen der Informationsverwaltung fällt ein ho: her Aufwand für die Archivierung, Aktualisierung, Wiederauffindung und Weiterverarbeitung der Daten an;

- beim Einsatz innerbetrieblicher Datenbanken treten Probleme der Datenerfassung und -pflege auf;

- eine entscheidungsorientierte Aufbereitung und Darstellung der Daten ist sehr arbeitsaufwendig. 2

Für die Fuille dieser Probleme kann ein elektronisch gestüztes externes Informationszentrum eine Lösung anbieten. Gleichwohl kann es dies nicht für alle Informationsbereiche und alle Informationsprobleme gleichzeitig und im gleichen AusmaB. Angesichts dieser Situation stellt sich dem Unternehmen das stra-

$1 \mathrm{Vgl}$. hierzu insbesondere die unter Fußnote 1, S. 38 dieser Arbeit zitierten Untersuchungen.

2 Vgl.Greiner P., a.a.0., S. 77; Beyer, W., a.a.0., S. $31 \mathrm{f}$. ; Pfaffhausen, A., Zur Informationsversorgung der Absatzführung in mittelständischen Betrieben. Eine empirische Analyse in ausgewählten Branchen der Konsumgüterindustrie, Beiträge zur Mittelstandsforschung, Hrsg.: Institut für Mittelstandsforschung - Forschungsgruppe Bonn -, H. 80 , Göttingen 1981 , S. 79 . 
tegische Planungsproblem, über die Festlegung der zu erbringenden Problemlösungen das Geschäftsfeld zu definieren, das es mit seiner innovativen Informationsdienstleistung besetzen will. 1

\subsection{Paradigma der Markteintrittsplanung im Bereich elektronischer Informationsdienste}

Die betriebswirtschaftliche Literatur hat sich bislang nur in wenigen Randaspekten dem Problem der strategischen Planung für investive Dienstleistungen gewidmet. Insoweit fehlt bis dato der Versuch, die vielfältigen Problemschichten der Markteintrittsplanung in diesem Bereich auf eine theoretisch fundierte Basis zu stellen. Vor diesem Hintergrund empfiehlt es sich, die einzelnen Problemfelder durch ein Paradigma der Markteintrittsplanung systematisch zu erfassen. ${ }^{2}$ Hierbei treten folgende Fragestellungen in den Vordergrund:

1 Diese Fragestellung bezüglich der Markteintrittsentscheidung und der damit verbundenen Auswahl des Geschäftsfeldes der Unternehmung wird in der amerikanischen Literatur seit einiger Zeit unter der Problemstellung des "defining the business" diskutiert und als "the starting point of strategic planning" angesehen. Vgl. dazu Abell, D.F., Defining the Business. The Starting Point of Strategic Planning, Englewood Cliffs, N.J. 1980 (im folgenden zitiert als: Abell, D.F., Defining the Business); Abell, D.F., Hammond, J.S., Strategic Planning, Englewood Cliffs, N.J. 1979, S. 389 ff.; Köhler, R., Grundprobleme der strategischen Marketingplanung, in: Die Führung des Betriebes, Festschrift für Curt Sandig zum 80. Geburtstag, Hrsg.: Geist, M., Köhler, R., Stuttgart 1981, S. 272.

$2 \mathrm{Vgl.zu}$ diesem Vorgehen z. B. Walters, M., Marktwiderstände als Problem der Marketingplanung, dargestellt am Beispiel der Marktwahl und Marktbearbeitung im Bereich der Textverarbeitung, Diss. Minster 1983, S. 8. 
In WELCHER PLANUNGSRANGFOLGE? sollen

WELCHE INFORMATIONEN?

unter Einsatz

WELCHER TECHNOLOGIE?

WELCHER ZIELGRUPPE?

angeboten werden,

und

WELCHE TEILSEGMENTE?

dieser Zielgruppe sollen

WIE?

bearbeitet werden?

Jede der Fragen des Paradigmas eröffnet ein weites Spektrum von Einzelaspekten, die zweckmäßigerweise im Rahmen der Markteintrittsplanung Berücksichtigung. finden sollten. Hierbei betreffen die ersten vier Fragestellungen den Bereich der Marktwahl ${ }^{1}$, während die zwei folgenden Fragen sich auf die Marktbearbei-

1 Die Marktwahl beinhaltet hierbei die Erfassung und Bewertung einzelner Marktalternativen im Hinblick auf ihre Zielerfuillung. Vgl. Meffert, H., Marktsegmentierung und Marktwahl im internationalen Marketing, in: DBW, 37. Jg., 1977, Nr. 3, S. 441 (im folgenden zitiert als: Meffert, H., Marktsegmentierung) und Meffert, H., Althans, J., Internationales Marketing, Stuttgart, Berlin, Köln, Mainz 1982, S. $69 \mathrm{ff}$. Der Begriff Markt soll im folgenden aus der Sicht des planenden Unternehmens synonym mit den Begriffen "Geschäftsfeld", "Geschäftseinheit", "Betätigungsfeld" bzw. "business" oder "strategic business unit" verwandt werden. 
$\underline{\text { tung }}^{1}$ des ausgewählten Geschäftsfeldes beziehen.

Am Anfang der Geschäftsfeldwahl steht zunächst das Problem, einen geeigneten PlanungsprozeB zu bestimmen. Hinter der Frage "welche Planungsrangfolge" verbirgt sich dabei das Dilemma, daB eine simultane Festlegung der drei strategischen Planungsvariablen Informationsfunktion, Bereitstellungstechnologie und relevante Zielgruppe ${ }^{2}$ nicht möglich ist. Von daher gilt es, einen "optimalen" ProzeB der sukzessiven Auswahl der zu erbringenden Funktion, der einzusetzenden Technologie und der zu bedienenden Abnehmergruppe zu entwerfen.

Nach der Festlegung einer geeigneten ProzeBalternative vertieft sodann der Aspekt "welche Informationen" die Frage nach dem Zweck bzw. Sachziel der anzubietenden Dienstleistung. Das Unternehmensmanagement muß bestimmen, für die Unterstuitzung welcher Entscheidungen es den potentiellen Informationsnachfragern Informationen anbieten will. Das bedeutet: es muß eine Eingrenzung der Zweckbestimmung seines Informationsangebotes (Informationsfunktion) auf bestimmte Klassen solcher Entscheidungsprobleme vornehmen, für deren Lösung es den erforderlichen Informationsbedarf zu decken bestrebt und in der Lage ist. Besondere Bedeutung kommt hierbei dem Problem der angestrebten Qualität der In-

1 Im Mittelpunkt der Marktbearbeitung steht die Entwicklung segmentspezifischer Marktbeeinflussungsstrategien. DefinitionsgemäB stellt die Marktbearbeitung einen integralen Bestandteil des Segmentierungskonzepts dar, das die Aufteilung des Marktes in homogene Käufergruppen-bzw. - segmente vorsieht. Vgl. Meffert, H., Marketing, a.a.0., S. $213 \mathrm{ff}$. Zur Marktsegmentierung vgl. vor allem Frank, R.E., Massy, W.F., Wind, Y., Market Segmentation, Englewood Cliffs, N.J. 1972; Bauer, E., Markt-Segmentierung als Marketing-Strategie, Berlin 1976; Böhler, H., Methoden und Modelle der Marktsegmentierung, Stuttgart 1977; Meffert, H., Marktsegmentierung, a.a.0., und Freter, H., Marktsegmentierung, Stuttgart usw. 1983 (im folgenden zitiert als: Freter, H., Marktsegmentierung).

2 Vgl. Abell, D.F., Defining the Business, a.a.0. 
formationsbedarfsdeckung zu. Eine Vielzahl empirischer Untersuchungen ${ }^{1}$ weist darauf hin, daB wichtige Anforderungen an die Qualität der Informationen - Aktualität, Schnelligkeit der Bereitstellung, Zuverlässigkeit und Genauigkeit, Vollständigkeit, Verdichtungsgrad etc. - bislang durch die bisher verfügbaren Informationsquellen nicht erfüllt sind. Hier ist es Aufgabe des Managements, die zentralen Informationsanforderungen in ihrem angestrebten Ausprägungsgrad und in der Rangfolge ihrer Priorität festzulegen.

Mit der Frage "welche Technologie?" wird ein Kernproblem der vorliegenden Markteintrittsentscheidung angesprochen. Die neuen Möglichkeiten, die sich aus der Verbindung von Computertechnik und elektronischer Nachrichtenübertragung ergeben, erschlieBen dem Unternehmen im Markt für Wirtschaftsinformationen bislang nur in wenigen Ansätzen genutzte, innovative Wege der Informationsaufbereitung, -speicherung, -übertragung und -manipulierung. Das Management hat hier zu beurteilen, welche Technologie( $n)$ unter Berücksich-

$1 \mathrm{Vgl.Z}$. B. die Ergebnisse von Untersuchungen zu den "Problemen der Informationsversorgung kleiner und mittlerer Unternehmen", die im Rahmen eines Projektes der GID durchgeführt wurden. Vgl. hierzu Beyer, W., a.a.0., S. $27 \mathrm{ff}$.. Vgl. ferner Dirlam, H.-W., Langheim, J., Anforderungen an die Informationsvermittlung in der Fleischindustrie als Beispiel für die mittelständische Wirtschaft, in: $N f D, 31$. Jg., 1980 , S. $177 \mathrm{ff}$.; Hofer, P., Wolff, H. et al., Wirksamkeitskriterien einer branchenorientierten Informationsvermittlungs - und Beratungsstelle für den Maschinenbau, Forschungsbericht ID 78-01 des BMFT, Basel, April 1978; Schwarting, U., Thoben, C., Wittstock, M., Nachfrageverhalten kleiner und mittlerer Unternehmen nach AuBenhandels information und -beratung. Eine Umfrage im Bereich der Industrieund Handelskammer zu Münster, Beiträge zur Mittelstandsforschung, Hrsg.: Institut für Mittelstandsforschung - Forschungsgruppe Bonn -, H. 83, Göttingen 1982; Pfaffhausen, A., a.a.0.. 
tigung vorhandener Ressourcen und unternehmensspezifischen know-hows zur bestmöglichen Erfüllung der vorgegebenen Informationsanforderungen einzusetzen ist (sind).

Die Fragestellung "welche Zielgruppe?" betrifft als vierte im Problembereich der Geschäftsfeldwahl die zentrale Unternehmensentscheidung, für welche in sich möglichst homogene Gruppe( $n$ ) industrieller Abnehmer die Informationsdienstleistung konzipiert und bereitgestellt werden sol1. Das Management muß hierbei im einzelnen entscheiden, nach welchen Kriterien die zur Auswahl stehenden Zielgruppen bestmöglich abzugrenzen und nach welcher Selektionsregel einzelne potentielle Abnehmerunternehmungen in die Marktbearbeitungsaktivitäten einzubeziehen sind.

Das Ausgangsproblem im Rahmen der sich an die Marktwahl anschließenden Marktbearbeitung umreiBt die Frage der Differenzierung von "Teilsegmenten" innerhalb der selektierten Zielgruppe. Die Unterscheidung von Subsegmenten erhält ihre Legitimation aus der Erkenntnis, daB die Erfolgschancen des Marketingprogramms eines Anbieters investiver Dienstleistungen in dem Maße steigen, "in dem es ihm gelingt, dem Bedarf, den restriktiven Bedingungen und den Erwartungen der Abnehmer zu entsprechen. Damit steht die Analyse der industiellen Abnehmer und ihres Einkaufsverhaltens im Mittelpunkt der Oberlegungen. Hier stellt man fest, daß sich die in Frage kommenden Unternehmen bezüglich einer Vielzahl von Merkmalen unterscheiden". 1

1 Gröne, A., Marktsegmentierung bei Investitionsgïtern, Schriftenreihe "Unternehmensführung und Marketing", Bd. 9, Hrsg.: Meffert, H., Wiesbaden 1977, S. 20 . 
Eine zentrale Rolle spielt gerade im vorliegenden Fall einer hochinnovativen Dienstleistung, ob diese Dienstleistung für den einzelnen potentiellen Nachfrager "problemevident" ist. "Damit ist gemeint, daB der Nachfrager Einsicht in die Tatsache hat, daB er die angebotene Dienstleistung zur Lösung seiner Probleme benötigt. 2 Hinter der Frage "warum?" verbirgt sich in diesem Zusammenhang das Problem des Managements, aus dem Vorliegen bzw. dem Fehlen von Problemevidenz bei einzelnen Abnehmerklassen deren erwartetes Kaufverhalten zu prognostizieren und hieraus situationsadäquate Marktbearbeitungsprogramme zu entwickeln.

Die Frage des "wie?" einer solchen Marktbearbeitung verweist schließlich auf das Problem der optimalen Gestaltung des Marketingmix. ${ }^{3}$ Beantworten muß das Management hier insbesondere die Frage, ob für das Marketing von investiven Dienstleistungen das tradierte Instrumentarium des Konsum- und Investitionsgütermarketing noch Gültigkeit hat, oder ob nicht effizientere Methoden der Klassifizierung und Anwendung der einzelnen Marktbearbeitungsmaßnahmen bestehen.

\footnotetext{
1 Der Aspekt der Problemevidenz wurde als innovatives Konstrukt zur Erklärung des Beschaffungsverhaltens bei investiven Dienstleistungen von Engelhardt und Schwab in die wissenschaftliche Diskussion eingebracht. Vgl. Engelhardt, H., Schwab, W., a.a.0., S. $506 \mathrm{ff}$.

乞. Vgl. Engelhardt, H., Schwab, W., a.a.0..

3 Der Ausdruck "Marketingmix" bezeichnet dabej "die zu einem bestimmten Zeitpunkt betroffene Auswahl von Marketingaktivitäten in einer bestimmten Aus prägung". Meffert, H., Marketing, a.a.0., S. 87.
} 


\subsection{Ziel und Gang der Untersuchung}

Ausgehend von der paradigmatischen Eingrenzung der Problemstellung liegt das Ziel der vorliegenden Untersuchung in der Entwicklung eines Konzepts zur Lokalisierung, Auswahl und Bearbeitung strategischer Geschäftsfelder im Bereich innovativer Investivdienstleistungen. Der normative Charakter des Konzepts drückt sich in dem Bestreben aus, Hinweise für die "optimale"Gestaltung des Prozesses der Abgrenzung und Selektion erfolgversprechender Geschäftsfelder zu liefern. Einen mehr deskriptiven Anspruch erhebt der zweite Teil der Arbeit, in welchem der Versuch unternommen wird, das Konstrukt der Problemevidenz einer investiven Dienstleistung durch geeignete Indikatoren zu operationalisieren und alternativen Marktbearbeitungstrategien zugrunde zu legen.

Eine partielle oberprïfung und Illustrierung der analysierten Einflußfaktoren, Zusammenhänge und Verhaltensweisen erfolgt anhand einer explorativen, empirischen Studie.

Aus den Zielen der Untersuchung ergeben sich in Anlehnung an das oben entwickelte Paradigma die folgenden forscherischen Schwerpunkte dieser Arbeit:

(1) Der Entwurf eines problemadäquaten Prozesses zur Auswahl strategischer Geschäftsfelder im Bereich elektronischer Informationsdienstleistungen.

(2) Die exemplarische Anwendung und Bewertung eines Konzepts der iterativen, die Technologie explizit einbeziehenden Geschäftsfeldwahl.

(3) Die Herausarbeitung und Prüfung geeigneter Kriterien zur Identifizierung und Abgrenzung der anzubietenden Informationsdienstleistung und der zu bearbeitenden Zielgruppe.

$1 \mathrm{Vgl}$. Anhang 1, S. 273 dieser Arbeit. 
(4) Die Operationalisierung des Konstrukts der Problemevidenz von investiven Dienstleistungen am vorliegenden Beispiel eines elektronischen Informationsdienstes und die Prüfung seiner Anwendbarkeit auf die Mikrosegmentierung einer ausgewählten Zielgruppe.

(5) Die Entwicklung eines geeigneten Marketinginstrumentariums für die Bearbeitung einzelner $Z$ ielsegmente des Informationsdienstes.

Mit dieser Ziel- und Schwerpunktsetzung ist bereits ein grobes Raster für den Gang der Untersuchung abgesteckt:

Kapitel B befaBt sich mit dem Problemkomplex der Geschäftsfeldwahl. Ausgehend von der Lokalisierung und Abgrenzung alternativer Geschäftsfelder in ihrer funktionalen, technologischen und abnehmerbezogenen Dimension werden mögliche Alternativen der Gestaltung des Auswahlprozesses diskutiert. Unter Zugrundelegung einer iterativen Vorgehensweise erfolgt sodann die Geschäftsfeldselektion.

Von dem somit bestimmten Geschäftsfeld ausgehend stellt Kapitel C die Marktbearbeitung in den Mittelpunkt. Die ausgewählten, potentiellen Abnehmergruppen werden nach Maßgabe der bei ihnen zu erwartenden Problemevidenz in Teilsegmente zerlegt. Diese bilden dann die Grundlage differenzierter Marktbearbeitungsprogramme.

In Kapitel D erfährt das Gesamtkonzept der Auswahl und Bearbeitung investiver Dienstleistungsmärkte abschließend eine zusammenfassende Würdigung. 
B. Auswahl strategischer Geschäftsfelder im Bereich

1. Definition strategischer Geschäftsfelder

Im Rahmen der strategischen Unternehmensplanung nimmt die Geschäftsfelddefinition qua ihrer Bedeutung und Position im Planungsproze $B$ eine vorrangige Stellung ein.' Versteht man die strategische Unternehmensplanung als "umfassenden, iterativen und dynamischen Prozeß"2, dann bildet die Abgrenzung und Festlegung der relevanten Produkt/Markt-Kombinationen ein erstes strategisches Teilproblem, das in interdependenter Beziehung zu den nachfolgenden ProzeBphasen steht.

1 Die strategische Unternehmensplanung hat in den vergangenen anderthalb Dekaden im Mittelpunkt des Interesses sowohl der Wissenschaft als auch der Praxis gestanden. Eine vergleichende Analyse der Vielzahl von Interpretationen und zugewiesenen Begriffsinhalten findet sich bei Steffenhagen (vgl. Steffenhagen, H., Der Strategiebegriff in der Marketingplanung. Ein literaturkritisch gestüzter Vorschlag zur Verwendung des Ausdrucks "Marketing-Strategie", Arbeitspapier des Instituts für Marketing der Universität Muinster, Hrsg.: Meffert, H., Nr. 29, Münster 1983). Allgemein soll an dieser Stelle unter strategischer Unternehmensplanung die Ziel- und Maßnahmenplanung zur globalen Analyse der unternehmensbezogenen Erfolgsquelien und der Entwicklung langfristig angelegter Konzepte zur Zukunftssicherung verstanden werden. Vgl. zu dieser Definition vor allem Meffert, H., Wehrle, F., Strategische Unternehmensplanung, in: HM, 0.Jg., 1983, Nr. 2, S. 50 sowie Gälweiler, A., Unternehmensplanung, Frankfurt, New York 1974, S. $133 \mathrm{ff}$. und S. $229 \mathrm{ff}$. Ein materiell ähnliches Verständnis der strategischen Unternehmensplanung ist inzwischen bei den meisten Autoren, insbesondere auch im anglo-amerikanischen Raum, zu finden. Vgl. Wehrle, F., Strategische Marketingplanung in Warenhäusern. Anwendung der Portfolio-Methode, Schriften zum Marketing, Hrsg.: Meffert, H., Bd. 1, Frankfurt und Bern 1981, S. 1 .

2 Meffert, H., Wehrle, F., a.a.0., S. 50. 
Die empirische Forschung hat in ihrer Konzentration auf Planungsprozesse, -methoden und -techniken, die die Analyse bestehender Geschäfte zum Gegenstand hat, geraume Zeit die Frage der Neu-Definition vorhandener Geschäftsfelder und der Definition neu zu besetzender Geschäftsfelder vernachlässigt. 'Diese Tatsache muß insofern erstaunen, als Einigkeit darüber besteht, daß für die langfristigen Unternehmensdispositionen weniger die gegenwärtige situation bzw. die langfristigen Trends als die Brüche in diesen Trends von ausschlaggebender Bedeutung sind. ${ }^{2}$ Eben solche Diskontinuitäten geben aber in vielen Fällen zumindest implizit AnlaB zur Neudeterminierung der Unternehmenstätigkeit. ${ }^{3}$ Explizit und zwingend ergibt sich die Notwendigkeit zur Geschäftsfelddefinition immer dann, wenn das Unternehmen innovative Betätigungsbereiche erschlieBt. ${ }^{4}$

Die vorstehende Charakterisierung des Marktes für elektronische Wirtschaftsinformationen macht deutlich, daB dieser Markt sich durch einen hohen Innovationsgrad, d. h. durch einen hohen Neuigkeitsgrad

$1 \mathrm{Vgl}$. vor allem Abell, D.F., Defining the Business, a.a.0., S. 244; vgl. im gleichen Sinne Köhler, R., a.a.0., S. 266 f. und Meffert, H., Wehrle, F., a.a.0., S. 52 .

2 Auf diesen Tatbestand hat bereits Peter Drucker 1968 hingewiesen. Vgl. Drucker, P.F., The Effective Executive, London 1966, S. 17. Vgl. im gleichen Sinne: Pfeiffer, W., Innovationsmanagement als know-How-Management, in: Führungsprobleme industrieller Unternehmungen, Festschrift für Friedrich Thomée zum 60. Geburtstag, Hrsg.: Hahn, D., Berlin, New York 1980 , S. 440.

3 Als Anlässe für eine solche Neudefinition der unternehmerischen Geschäftstätigkeit nennt Abell beispielhaft "strategic issues" wie die Einführung neuer Produkte, die Elimination veralteter Produkte, die Erschließung neuer Kundensegmente, die Diversifikation im Wege der Firmenakquisition und die Aufgabe bestimmter Geschäfte durch Verkauf. Vgl. Ábel1, D.F., Defining the Business, a.a.0., s. 6 .

$4 \mathrm{Vgl}$. ebenda, S. 7. 
der angebotenen Dienstleistungen aus Anwendersicht auszeichnet. Insofern hat sich das Unternehmen mit dem Problem eines "pioneering entry" 1 auseinanderzusetzen, im Rahmen dessen es zunächst die Frage nach der geeigneten Definition des in Frage kommenden Problemlösungsbereiches beantworten muB. Ein solcher Fall steht im vorliegenden Fall im Mittelpunkt der Betrachtung. Es ist zu prüfen, welchen Beitrag hierbei die bisher gelieferten Ansätze der Geschäftsfeldplanung leisten können. ${ }^{2}$

\subsection{Ansatzpunkte zur strategischen Geschäftsfeld- definition in der Literatur}

\subsection{Darstellung alternativer Ansätze}

In der historischen Entwicklung der Diskussion um die Definition des Betätigungsfeldes einer Unternehmung bestand zunächst lange Zeit die Konvention, den Unternehmenszweck durch die Festlegung der Produktart zu fixieren. Mit dem Hinweis Levitts ${ }^{3}$ auf die Kurzsichtigkeit einer solch engen Definition der Geschäftstätigkeit wich die produktorientierte Be-

1 "Typically, a pioneer enters the market with s single innovative technology aimed at satisfaction of a particular customer function". Abell, D.F., Defining the Business, a.a.0., S. 220.

2 Ein konziser Oberblick über die Verfahren der Geschäftsfelddefinition findet sich bei Köhler, R., a.a.0., S. $266 \mathrm{ff}$. Vgl. hierzu auch Meffert, H., Wehrle, F., a.a.0., S. $52 \mathrm{f}$.

3 In seinem vielfach als "klassisch" bezeichneten Aufsatz "Marketing Myopia" hat Th. Levitt 1960 auf die Gefahr der "Marketing-Kurzsichtigkeit" einer Geschäftsfeldplanung hingewiesen, die nicht die technologische Entwicklung bei der Formulierung des Unternehmenszweckes einbezieht. Vgl. Levitt, Th., Marketing Myopia, in: HBR, Vol. 38, July-August 1960 , S. $45 \mathrm{ff} .$. Vgl. hierzu auch Levitts retrospektive Betrachtungen aus dem Jahre 1975: Levitt, Th., Marketing Myopia, in: HBR, Vol. 53, September-october 1975, S. 26 ff.. 
schreibung des Geschäftsfeldes einer Definition, die sich an den für die Unternehmung grundsätzlich relevanten Problemen und den daraus resultierenden Problemlösungsmöglichkeiten orientierte. Den Vorwurf des mangelnden Konkretisierungsgrades einer solchen Geschäftsfelddefinition setzte Ansoff ${ }^{1}$ die vielzitierte ProduktMarkt-Matrix entgegen, mit Hilfe derer er den Unternehmensgegenstand als Kombination von Produktarten und $A b-$ nehmergruppen kennzeichnete.

Eine richtungweisende Weiterentwicklung dieses Ansatzes erfolgte durch Abe $11^{2}$, der die Technologiedimension in die Geschäftsfelddiskussion einbrachte. Ausgangspunkt Abells ist die These, daB das Produkt einer Unternehmung als das physische Gegenstück der Anwendung einer Technologie zur Realisierung bestimmter Problemlösungen für eine bestimmte Zielgruppe zu interpretieren sei. Entsprechend definierte er das Geschäftsfeld einer Unternehmung als eine bestimmte Kombination

- der Funktion, die das Unternehmen im Sinne einer zu erbringenden Marktleistung wahrnehme,

- der Zielgruppe, für die diese Funktion wahrgenommen werde sowie

- der Technologie, unter Verwendung derer die Funktionserfiullung erfolge. 3

$1 \mathrm{Vgl}$. Ansoff, H.I., Corporate Strategy, New York u. a. 1965, S. 109. Die Produkt-Markt-Matrix wurde später von Kotler durch die Einbeziehung von Differenzierungsaspekten weiterentwickelt. Vgl. Kotler, P., Marketing Management. Analysis, Planning and Control, 4. Aufl., Englewood CTiffs, N.J. 1980 , S. 82 ff.; vgl. ferner Meffert, H., Marketing, a.a.0., S. 37 .

2 Vgl. Abe1l, D.F., Defining the Business, a.a.0..

$3 \mathrm{Vgl}$. ebenda, S. 17. 
Aus dieser Perspektive werden "'Märkte' näher nach Nachfragesektoren und Merkmalen der bedarfskonstituierenden Probleme beschrieben, während an die Stelle von 'Produkten' die Angabe des Problembezugs ('Functions') und der dafür in Frage kommenden Technologien tritt".

Letztlich bilden damit

- segmentspezifische Bedarfsmerkmale im Rahmen bestimmter Funktionserfüllungen

- die Gestaltungsart der anwendbaren Technologien und grundsätzliche Gestaltungsschwerpunkte der sonstigen Marketing-Mix-Bestandteile sowie

- Marktsegmente i. S. relativ homogener Käufergruppen

den Bezugsrahmen zur Identifikation und Abgrenzung alternativer Felder der Geschäftstätigkeit.

\subsection{Würdigung der Obertragbarkeit auf den Markt elektronischer Wirtschaftsinformationsdienste}

Ohne im einzelnen auf eine sukzessive Prüfung der skizzierten Ansätze einzugehen, erscheint mit hoher Plausibilität die These zulässig, daß beim Eintritt in einen innovativen Dienstleistungsmarkt die Suche nach dem geeigneten strategischen Geschäftsfeld unter Zugrundelegung einer dreidimensionalen

1 Köhler, R., a.a.0., S. 268. 
Betrachtung die präzisesten Ergebnisse liefert. ${ }^{1}$ Dabe $i$ eröffnet das dreidimensionale Raster dem Planenden den erforderlichen kreativ-gestalterischen Spielraum, den er für seine weitreichenden Diversifikationsüberlegungen benötigt. Die Einführung der Technologie als dritte Dimension liegt hierbei um so näher, als der betrachtete Markt für Wirtschaftsinformationen gerade aus den aktuellen technologischen Entwicklungen im Bereich der Nachrichtenübertragung seine neue, für das planende Unternehmen entscheidende Perspektive bezieht.

In Anlehnung an Abell empfiehlt es sich, in der Ausgangsphase der Geschäftsfeldplanung zunächst von einem relativ hohen Abstraktionsniveau auszugehen. In diesem Sinne kann als Ausgangsraster ein Bezugsrahmen aufgespannt werden, innerhalb dessen die Informationsdienstleistung

- durch die zu erfüllende Informationsfunktion,

- die einzusetzende Obertragungstechnologie sowie

- die zu berücksichtigenden Abnehmergruppen

definiert wird (vgl. Abbildung 6). Eine Konkretisierung dieser Dimensionen läßt sich erreichen, indem man die Achsenbezeichnungen problemgerecht spezifiziert. Danach besteht im vorliegenden Fall die Informationsfunktion des Unternehmens in der Befriedigung des externen Informationsbedarfs bestimmter Abnehmergruppen, spezifiziert nach den jeweils abnehmeradäquaten Informationsanforderungen. Auf diese Funktion ist als dritte Dimension der Modus der Informationsversorgung abzustimmen.

1 Im Rahmen einer zweidimensionalen Produkt/MarktBetrachtung müßte die Frage des einzusetzenden Obertragungsmediums entweder offengelassen oder aber vorzeitig, d. $h$. vor einer fundierten Prijfung alternativer Geschäftsfelder, festgelegt werden ( $z$. B. Datenfernubertragung). 


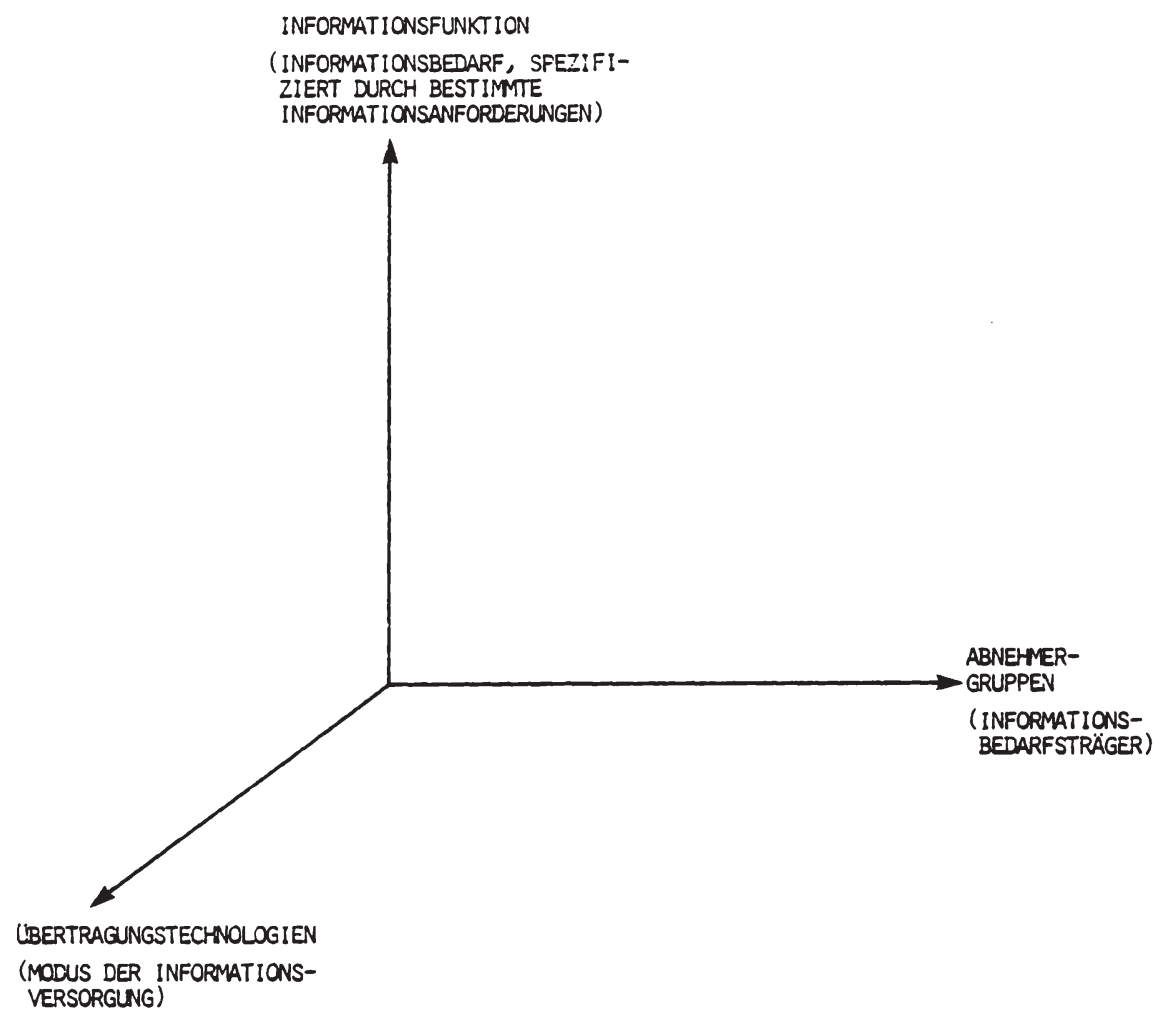

INFORMATIONSFUNKTION

INFORMATIONSBEDARF, SFEZIFI-

ZIERT DURCH BESTIMMTE

INFORMATIONSANFORDERUNGEN）

ABNEHMER-

(INFORMATIONS-

BEDARFSTRÄGER）

Abb. 6 : Orientierungsraster zur Definition strategischer Geschäftsfelder im Markt für Informationsdienstleistungen

1.2 "Informationsfunktion", "Obertragungstechnologie" und "Abnehmer" als BestimmungsgröBen dreidimensionaler Geschäftsfelder

Informationsfunktion, Obertragungstechnologie und $A B$ nehmergruppen stellen drei "primitive Dimensionen" 1 dar, innerhalb derer sich die Geschäftsfeldplanung vollzieht. Ein erster Schritt zur konkreteren Definition des geplanten Dienstleistungsspektrums besteht in der Festsetzung des Umfangs (scope) der einzubeziehenden Funktionen, Technologien und Abnehmergruppen. In einem zweiten Schritt ist darüber zu bestim-

1 Abel1, D.F., Defining the Business, a.a.0.0. S. 
men, welchen Differenzierungsgrad das anzubietende Dienstleistungspaket entlang seiner drei Dimensionen aufweisen und welcher Grad der Differenzierung gegenüber konkurrierenden Anbietern durchgesetzt werden soll.

Aus der Interdependenz dieser drei Entscheidungstatbestände und ihrer Abhängigkeit von einer Vielzahl von Einflußfaktoren erklärt sich die besondere Komplexität der Geschäftsfeldbestimmung. Der Planende steht vor dem Problem, entweder früzeitig strategische Festlegungen zu treffen, die die Zahl der in Frage kommenden Geschäftsfeldalternativen stufenweise eingrenzt, oder auf schwerpunktbildende Vorentscheidungen zu verzichten und sich so in den nachfolgenden Analyseschritten möglichst viele Entscheidungsalternativen offenzulassen. Letzteres steht jedoch in Konflikt mit dem Bestreben, die Komplexität der Planungsarbeit durch geeignete Heuristiken schrittweise zu reduzieren. ${ }^{2}$ Für die vorliegende Problemstellung soll vor diesem Hintergrund ein mittlerer Weg eingeschlagen werden.

\subsection{Operationalisierung der Dimensionen am Bei- spiel eines institutionellen Informationsan- bieters}

Es sei beispielhaft davon ausgegangen, daß das Management der Unternehmung den Suchraum für sein zukünftiges Betätigungsfeld als den Problemlösungsbereich umrissen habe, in dem der Ad-hoc-Informationsbedarf von industriellen Organisationen nach unternehmensexter-

$1 \mathrm{Vgl}$ Abell, D.F., Defining the Business, a.a.0., S. $185 \mathrm{ff.}$; vgl. dazu auch Köhler, R., a.a.0., S. 270 .

2 Vgl. hierzu vor allem Köhler, R., a.a.0., S. 270. 
nen Informationen unter Einsatz telekommunikativer Obertragungsmöglichkeiten zu decken ist. Durch diese grundsätzliche Festlegung trifft das; Unternehmen zugleich die Entscheidung zur Exklusion anderer Problemlösungsbereiche. So verzichtet es beispielsweise darauf, andere Informationsfunktionen ( $z$. B. periodische Berichterstattung zu bestimmten Themen) zu übernehmen oder andere Abnehmergruppen ( $z$. B. wissenschaftliche Institutionen, Verbände, etc.) zu berücksichtigen. Des gleichen sollen (grundsätzlich) nicht-elektronische Bereitstellungsformen (rein papiergestützte Informationen oder persönliche Informationsberatung) aus dem zu erbringenden Leistungsspektrum ausgeklammert bleiben. Das so begrenzte Suchfeld der Unternehmung im Markt für Wirtschaftsinformationen gibt Abbildung 7 wieder. Es gilt nunmehr, den gegebenen Entscheidungsraum entlang seiner drei Dimensionen aufzugliedern.

\subsection{Operationalisierung der Funktionsdimension}

Mit der näheren Beschreibung der Funktionsdimension stellt sich dem Planenden die Aufgabe, festzulegen, welche Informationsfunktionen $i$. S. der Lösung ad hoc auftretender Informationsprobleme sein Unternehmen als extern agierende Dienstleistungsinstitution grundsätzlich für Wirtschaftsorganisationen erfüllen kann. Konkret ist das maximale Angebotsspektrum an unternehmensexternen Informationen zu benennen und aufzuschlusseln. Hierbei ergeben sich zwei hochgradig interdependente Probleme:

1. Die Wahl geeigneter Kriterien zur Unterscheidung einzelner Informationsbedarfsbünde 1 .

2. Die Festlegung des Aggregationsniveaus der einzelnen Informationsbedarfsbündel. 


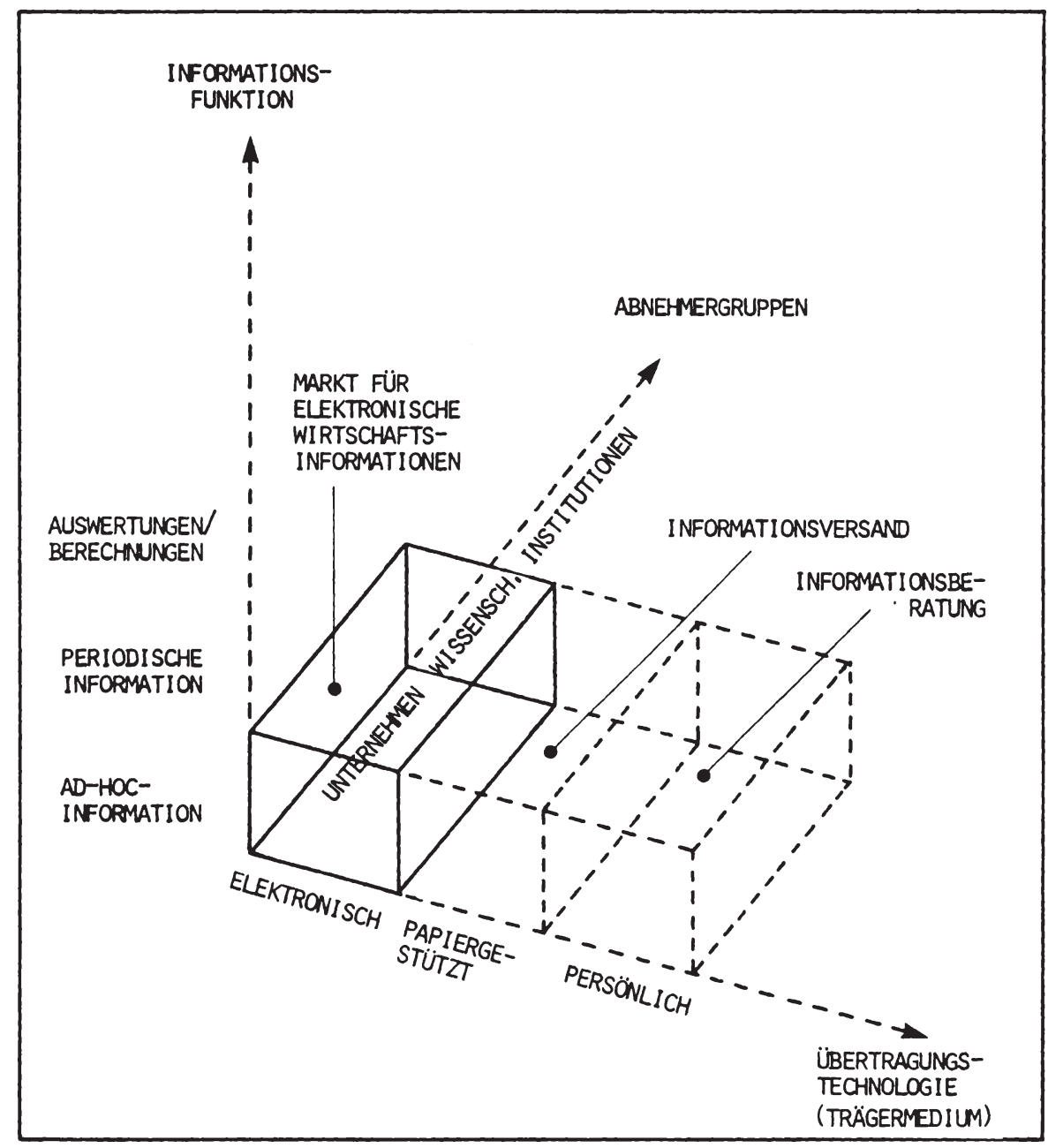

Abb. 7: Der Markt für elektronische Wirtschaftsinformationen als Ausgangspunkt zur Festlegung strategischer Geschäftsfelder 
Eine pragmatische Lösung beider Probleme kann darin bestehen, von einer Unterscheidung der Informationen nach inhaltlichen Gesichtspunkten auszugehen und die Informationen $z$. B. nach betrieblichen Funktionen (Personal, Finanzen, $F+E$, Marketing, etc.) zu untergliedern. 1 Dabei bleibt es grundsätzlich unbelassen, die so gebildeten Informationsbedarfsbündel weiter zu disaggregieren. Entsprechend müBten z. B. marketingrelevante Informationen in einzelne Komponenten wie Produktinformationen, Kundeninformationen, Informationen über Absatzwege etc. entflochten werden.

Eine zweite grundsätzlich denkbare Gliederungssystematik kann darin bestehen, die Informationen nach MaBgabe bestimmter Anforderungen zu unterscheiden, so z. B. nach der erforderlichen Schnelligkeit der Bereitstellung ("Sofortinformationen" vs. "innerhalb einer gesetzten Frist bereitzustellende Informationen"), der Aktualität ("tagesaktuelle Informationen" vs. "historische Informationen") oder des Verdichtungsgrades ("Kurzinformationen" vs. "umfassende Informationen").

Besonders konsequent, weil auf den eigentlichen Informationszweck abstellend, wäre schließlich die Untergliederung der Informationsfunktion nach Entscheidungseinheiten. Solche Entscheidungseinheiten lassen

1 Eine solche Unterteilung findet sich vielfach bei der Unterscheidung alternativer Formen betrieblicher Informationssysteme, so z. B. im Rahmen einer empirischen Untersuchung Köhlers und Heinzelbeckers, die sich mit dem Stand der Verbreitung und den Einstellungen deutscher Unternehmen zu Management- und Marketinginformationssystemen befaßt. Vgl. Köhler, R., Heinzelbecker, K., a.a.0., S. 271. 
sich als Klassen relativ homogener Entscheidungsprobleme interpretieren und durch die drei Dimensionen "Entscheidungsebene" (wer?), "Entscheidungsphase" (wann?) und "Entscheidungsbereich" (wofür?) beschreiben. 1 Nachteilig an einer solchen Unterscheidung einzelner Entscheidungseinheiten ist die abermalige Erhöhung der Problemkomplexität. Sie besteht darin, daB statt einer nunmehr drei Subdimensionen zu operationalisieren sind.

Zur Reduzierung der Problemkomplexität empfiehlt sich vor diesem Hintergrund eine verhältnismäßig einfache Heuristik, die die Aufmerksamkeit auf zwei strategische Elemente der geplanten Informationsdienstleistung lenkt:

- die Deckung von ad hoc auftretendem Informationsbedarf,

- die Beschränkung auf unternehmensexterne Informationen.

Unter Berücksichtigung dieser zwei Elemente reduziert sich die $Z a h l$ der in Frage kommenden Informationsbereiche "automatisch" um jene unternehmerischen Funktionen, die nur in sehr begrenztem Umfang externe Informationen benötigen. Vielfach sind dies gleichzeitig solche Entscheidungsbereiche, wo die kontinuierliche Informationsversorgung durch bereits implementierte hausinterne systeme gewährleistet ist.

$1 \mathrm{Vgl}$. in diesem Sinne Heinzelbecker, K., EDV-MIS, a.a.0., S. 405 und derselbe, Marketing-Informationssysteme, a.a.0., S. 136. 
Als Ergebnis intensiver Expertengespräche ${ }^{1}$ und der eingehenden Analyse von Sekundärinformationen ${ }^{2}$ soll an dieser Stelle eine Aufgliederung der für die Unternehmung in Frage kommenden Informationsfunktionen nach folgenden Wissensbereichen erfolgen: ${ }^{3}$

- Personal/Aus - und Weiterbildung,

- AuBenhande1/Export,

- Wirtschaftsrecht,

- gesamtwirtschaftliche Entwicklung/Konjunktur,

- Branchen/Märkte,

- Anschriften/Firmenauskünfte,

- Finanzen/Steuern,

- Technologie/Produktinnovationen.

1 Die Gespräche fanden in Form von Expertenrunden im Zeitraum zwischen Oktober 1981 und Juni 1983 statt. Beteiligt waren Medienfachleute, Marktforscher und Experten aus dem Bereich der Textdokumentation eines namhaften deutschen Verlagshauses.

2 Die Analyse konzentrierte sich schwerpunktmäßig auf das derzeit international vorhandene Angebot an externer Wirtschaftsinformation und Beratung. Die gewählte Unterteilung spiegelt in wesentlichen Eckpunkten die inhaltichen Strukturen dieses Angebots wieder. Dabei wurden Bereiche, die durch international etablierte Datenbanken bereits weitgehend abgedeckt werden ( $z$. B. Patent-Datenbanken) bewuBt aus der weiteren Betrachtung ausgeklammert.

3 Abgrenzungsprobleme der Bereiche untereinander können in diesem Stadium der Planung vernachlässigt werden, da die konkrete Zuordnung der Informationen zu einzelnen Bereichen erst mit dem Aufbau der entsprechenden Datenbanken virulent wird. 


\subsection{Operationalisierung der Technologiedimension}

Die Spezifizierung der verwendbaren Technologien hängt wesentlich von der Beantwortung mehrerer interdependenter Einzelfragen ab. Geht man davon aus, daß definitionsgemäB ausschlieBlich Formen der Telekommunikation zum Einsatz kommen sollen, ergibt sich zunächst das Problem der zweckmäßigen Systematisierung bzw. Aggregation. Es besteht z. B. die Möglichkeit, grundsätzlich zwischen Text-, Festbild- und Sprachkommunikation oder aber zwischen Online- (Direkt-) und offline-Kommunikation zu unterscheiden ${ }^{1}$ (vgl. Abbildung 8). Die Heterogenität der einzelnen Telekommunikationsdienste hinsichtlich ihrer Verfügbarkeit und Leistungscharakteristika ${ }^{2}$ läBt in diesem Zusammenhang jedoch keine der genannten Aggregationsmöglichkeiten als sinnvoll erscheinen.

1 Weitere Unterscheidungen können z. B. hinsichtlich der Gleichzeitigkeit der Kommunikation seitens Sender und Empfänger (synchrone vs. asynchrone Telekommunikation) oder der Betriebsart (Richtung des Nachrichtenflusses: simplex, halbduplex, duplex) vorgenommen werden. Zur näheren Charakterisierung und Systematisierung unterschiedlicher Telekommunikationsformen siehe z. B. Fellbaum, K.-R., a.a.0., S. 108; Musiol, A., Einheit der Büroarbeit und Vielfalt der Büromaschinen - eine Analyse der heutigen und eine Prognose der künftigen Bürosituation - 1 . Teil, in: ZfO, 50. Jg., 1981, H. 2, S. 75 ff.; Picot, A., Anders, W., Telekommunikationsnetze als Infrastruktur neuer Entwicklungen der geschäftlichen Kommunikation, in: WiSt, 12. Jg., April 1983, H. 4, S. $187 \mathrm{ff}$. (im folgenden zitiert als: Picot, A., Anders, W., Netze); Wagner, H., Unternehmensführung und neue Informationstechnologien. Auswirkungen auf Unternehmensstrategie und Unternehmensorganisation, Arbeitspapier der Wissenschaftlichen Gesell schaft für Marketing und Unternehmensführung e.V., Hrsg.: Meffert, H., Wagner, H., Nr. 13, Münster 1983, insbes. S. $8 \mathrm{ff}$. Formen der Bewegtbild-(Fernsehen) und Datenkommunikation (Fernsteuern, Fernüberwachen, Datenfernüberwachen) werden beim vorliegenden Entscheidungsproblem aufgrund mangelnder Relevanz bewuBt aus geklammert.

2 Vgl. hierzu insbesondere die Ausführungen unter Punkt 3.222 dieses Kapitels. 
Als zweite Frage ist zu klären, ob grundsätzlich alle Telekommunikationsmöglichkeiten berücksichtigt werden sollen. Diskussionen mit Medienexperten bestätigen hier die Zweckmäßigkeit der Entscheidung, das Kabelfernsehen und den Kabeltextabruf auszuschiließen, da mit einem mittelfristig ausreichenden Distributionsgrad dieser Kommunikationskanäle nicht zu rechnen ist. 1

\begin{tabular}{|l|c|c|}
\hline & ONLINE & OFFLINE \\
\hline & - BILDSCHIRMTEXT* & $-\begin{array}{c}\text { FERNSCHREIBER } \\
\text { (TELEX) }\end{array}$ \\
$\begin{array}{l}\text { TEXT- } \\
\text { KOMMUNIKATION }\end{array}$ & $\begin{array}{c}\text { KABELTEXTABRUF* } \\
\text { DATENFERNUBER- } \\
\text { TRAGUNG } \\
\text { (TEXTUBERTRAGUNG } \\
\text { PER STAND- ODER } \\
\text { WÄHLLEISTUNG) }\end{array}$ & $\begin{array}{c}\text { BUROFERNSCHREIBER } \\
\text { (TELETEX) }\end{array}$ \\
\hline $\begin{array}{l}\text { FESTBILD- } \\
\text { KOMMUNIKATION }\end{array}$ & $\begin{array}{c}\text { KABELFERNSEHEN } \\
\text { MIT RUCKKANAL }\end{array}$ & $-\begin{array}{c}\text { TELEKOPIERER } \\
\text { (TELEFAX) }\end{array}$ \\
\hline $\begin{array}{l}\text { SPRACH- } \\
\text { KOMMUNIKATION }\end{array}$ & - TELEFON & \\
\hline
\end{tabular}

- bei dialog mit einem angeschlossenen externen rechner

Abb. 8 : Systematik relevanter Telekommunikationsformen

1 Nach Expertenschätzung ist in der Bundesrepublik Deutschland mit einem öffentlichen Fernmeldedienst "Kabeltext" nicht vor den neunziger Jahren zu rechnen. Vgl. z. B. Picot, A., Anders, W., Telekommunikationsdienste für den Geschäftsbereich, in: Wist, 12. Jg., Juni 1983, H. 6, S. 277 (im folgenden zitiert als: Picot, A., Anders, W., Dienste); Kaiser, W., Kabeltext und Kabeltextabruf, in: Elektronische Textkommunikation. Vorträge des vom 12. - 15. Juni 1978 in München abgehaltenen Symposiums, Hrsg.: Kaiser, W., Berlin, Heidelberg, New York 1978, S. $70 \mathrm{ff}$. 
Zudem handelt es sich hier um Medien, deren Nutzung nahezu ausschlieblich auf privater Seite stattfinden wird. 1

SchlieBlich bleibt die Frage zu beantworten, welcher Bedeutungsinhalt dem Terminus "verwendbare Technologie" als Dimension des strategischen Suchraumes zugewiesen werden soll. Versteht man ihn allgemein als Synonym des Begriffes "eingesetztes Telekommunikationsmedium"2, so ist zu berücksichtigen, da B Informationsanfrage und Informationsübermittlung über un-

1 Diese Auffassung bestätigt u. a. das Ergebnis einer Erhebung über "Neue Medien im Management-Urteil", die 1982 vom Institut für Marketing der Universität Münster durchgeführt wurde. Vgl. Meffert, H., Neue Medien im Management-Urteil, in: asw, 25. Jg., 1982, H. 6, S. $72 \mathrm{ff}$, insbes. S. 75; vgl. ferner Middelhoff, Th., Walters, M., Akzeptanz neuer Medien Eine empirische Analyse aus Unternehmersicht. Arbeitspapier des Instituts für Marketing der Universität Münster, Hrsg.: Meffert, H., Nr. 27, Münster 1981, und Meffert, H., Bruhn, M., Middelhoff, Th., Neue Medien. Situation und Stand der Diskussion in der Bundesrepublik Deutschland, Arbeitspapier der Wissenschaftlichen Gesellschaft für Marketing und Unternehmensführung e.V., Hrsg.: Meffert, H., Wagner, H., Münster 1981, S. $45 \mathrm{ff}$.

2 Der Begriff "Telekommunikationsmedium" soll hier wie im folgenden synonym für "Telekommunikationsdienst" verwandt werden. Unter Telekommunikationsdienst versteht man die "Leistungen von nachrichtentechnischen Systemen, die Teilnehmern von öffentlichen oder privaten. Unternehmern angeboten werden, um ihre Telekommunikationsbediurfnisse zu erfiullen" (Fellbaum, K.R., a.a.0., S. 106). In der Bundesrepublik Deutschland hat die Deutsche Bundespost das Angebotsmonopol für 311 e nennenswarten Telekommunikationsdienste inne. Das kennzeichnende Merkmal dieser Dienste besteht darin, daB der Nachrichtenaustausch auf der Grundlage öffentlich anerkannter, meist internationaler Standards erfolgt, daß die Dienstgüte vom Träger des Netzes garantiert wird und daB alle Teilnehmer in einem öffentlichen Teilnehmerverzeichnis erfaBt sind. Vgl. hierzu Picot, A., Anders, W., Netze, a.a.0., S. 187 . 
terschiedliche Medien abgewickelt werden können. So ist es ohne weiteres denkbar, daB Nutzer des Informationsdienstes $z$. B. uber $B i l d s c h i r m t e x t$ Informationen anfordern, deren Umfang die Obertragungskapazität dieses Mediums überfordert. Hier ist grundsätzlich zu klären, ob die Anforderung eines postalischen Versandes von Informationen ( $z$. B. Originalquellen Btx-gespeicherter Abstracts) als Option einbezogen werden soll.

Vor dem Hintergrund dieses Problems erscheint es zweckmäBig, die Technologie als Kombination sinnvoller Informationsabruf-und -übermittlungsmodi zu interpretieren. Die Gesamtzahl der plausiblen Kombinationsmöglichkeiten gibt Abbildung 9 wieder. ${ }^{1}$ Die einzelnen Kombinationen 2.1 bis 7.7 stellen damit die insgesamt einzubeziehenden technologischen Alternativen im Raster des strategischen Suchfeldes dar.

\subsection{Operationalisierung der Abnehmerdimension}

Im Rahmen der Konkretisierung der Abnehmerdimension steht die Aufgabe im Vordergrund, die Gesamtheit der potentiellen industriellen Abnehmer in Marktsegmente zu unterteilen, die hinsichtlich ihres Bedarfs und ihres Kaufverhaltens weitgehend homogen sind. ${ }^{2}$ Mit dieser Erfassung einzelner relevanter Teilmärkte wird

1 Der Beurteilung der Plausibilität der einzelnen Felder liegt dabei der Maßstab vernüntigen unternehmerischen Verhaltens in einer spezifischen Informationsbedarfs-Situation zugrunde. In diesem Sinne ist es z. B. nicht plausibel, daß das Unternehmen angeforderte Informationen per Bildschirmtext uibermittelt, wenn eine Anfrage per Telex vorliegt. Vielmehr wird die Obermittlung per Bildschirmtext sinnvollerweise nur im Wege des Abrufs von Informationen aus Btx-gespeicherten Datenbeständen erfolgen.

2 Vgl. Abell, D.F., Defining the Business, a.a.0., S. 170; Hinterhuber, H.H., Wettbewerbsstrategie, Berlin, New York 1982, S. 41; Köhler, R., a.a.0., S. 271. 


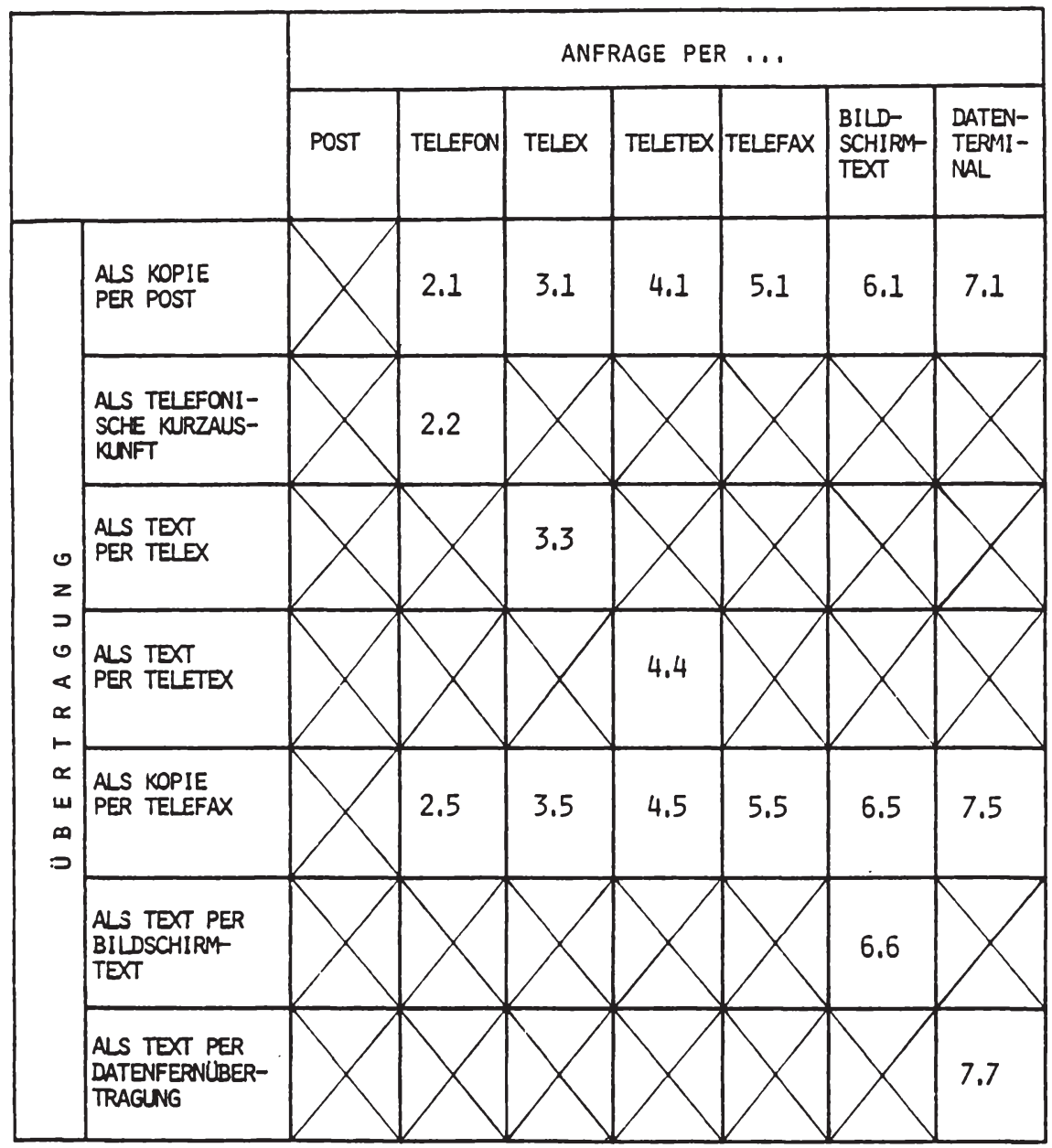

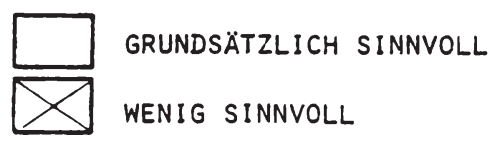

Abb. 9: Strategische Technologiealternativen eines elektronischen Informationsdiens tes 
bereits in einer frühen Phase des Planungsprozesses ein wichtiger Schritt zur Makrosegmentierung des Gesamtmarktes vorweggenommen. ${ }^{1}$ Die Anforderungen an eine solche Aufteilung der potentiellen Abnehmerschaft verlangen, daB das zugrunde gelegte Segmentierungskriterium

- einen engen Bezug zum Kaufverhalten hat und zu einer Trennung der Kaufverhaltensweisen führt,

- meBbar ist,

- im Zeitablauf stabil bleibt,

- einen Bezug zur Marktbearbeitung aufweist,

- die Ansprechbarkeit der Segmente gewährleistet und

- ökonomisch vertretbare Segmentgrößen produziert. ${ }^{2}$

Bereitet eine Makrosegmentierung unter Berücksichtigung dieser Kriterien im ("normalen") Falle einer bereits vorhandenen Marktleistung nicht unbeträcht1 iche Schwierigkeiten ${ }^{3}$, so treten diese in gesteigerter Form auf, wenn die Abnehmersegmentierung für eine noch nicht präzisierte bzw. nur in groben Zügen umrissene Dienstleistung vorgenommen werden soll. Es

1 Zur Unterscheidung von Makro- und Mikrosegmentierung vgl. vor allem Wind, Y., Cardozo, R., Industrial Market Segmentation, in: IMM, Vol. 3, 1974, S. $153 \mathrm{ff}$. ; vgl. hierzu auch Webster, jr., F.E., Industrial Marketing Strategy, New York u. a. 1979, S. $80 \mathrm{f}$. und S. 124 f.; Engelhardt, W.H., Günther, B., Investitionsgitermarketing, Stuttgart u. a. 1981, S. $89 \mathrm{ff}$. ; Backhaus, K., Investitionsgüter-Marketing, München 1982 , S. $80 \mathrm{f}$.

$2 \mathrm{Vgl}$. dazu vor allem Gröne, A., a.a.0., S. $43 \mathrm{ff}$. sowie Freter, H., Marktsegmentierung, a.a.0., S. $43 \mathrm{ff}$. ; eine Auswahl dieser Kriterien findet sich z. B. auch bei Frank, R.E., Massy, W.F., Wind, Y., Market Segmentation, Englewood Cliffs, N.J. 1972, S. 27 f.; Böhler, H., Methoden und Modelle der Marktsegmentierung, Stuttgart 1977 , S. $36 \mathrm{ff}$.; Kotler, Ph., a.a.0., S. 164 f..

3 Die Schwierigkeiten resultieren aus der Tatsache, daß $z$ wischen den eher robusten Kriterien der Makrosegmentierung einerseits und dem Kaufverhalten bzw. der Marktbearbeitung der industriellen Abnehmer andererseits i.d.R. nur sehr begrenzte Bezüge bestehen. So läßt sich aus der Untergliederung von Unternehmen nach ihrer GröBe, Branche, Nutzungsintensität, regionale Lage etc. allenfalls grobe Hinweise für die Bearbeitung der so unterteilten Unternehmensgruppen ableiten. 
leuchtet unmittelbar ein, daB die Abnehmersegmentierung zum Beispiel ganz andere Formen annehmen wird, wenn es um das Angebot von AuBenhandelsinformationen über Bildschirmtext geht, als dann, wenn das Angebot von Firmenauskünften im Wege des "on-line information retrieval" betrachtet wird. Der Planende steht somit vor der Situation, die gemeinsamen zentralen Merkmale seiner geplanten Dienstleistung im Sinne eines kleinsten gemeinsamen Nenners der möglichen Angebotsalternativen zu finden und diese als Ausgangspunkt für die Segmentierung $z u$ nehmen. Entsprechend müssen für das unterstellte fiktive Globalprodukt die Kaufvoraussetzungen ermittelt, das Kaufverhalten antizipiert, die Instrumente der Marktbearbeitung festgelegt werden usw., um an diesen MaBstäben die "Güte der Segmentierungskriterien gemessen durch die Nähe des Bezuges - zu beurteilen.

Diese Ausführungen machen deutlich, da $B$ eine endguiltige, differenzierte Unterscheidung der Abnehmergruppen in der Phase der Geschäftsfelddefinition wenig sinnvoll - sofern überhaupt möglich - ist. Aufgrund des zwangsläufig niedrigen Informationsstandes kann die Unternehmung mögliche Unterscheidungsmerkmale nur tentativ und vorläufig festlegen.' Solche Unterscheidungskriterien beziehen sich geeigneterweise zunächst auf direkt beobachtbare, äußere Merkmale. ${ }^{2}$ Als solche werden in der Literatur am häufigsten die organisationsdemographischen Merkmale "Unternehmensgröße" und "Branche" herangezogen. 3

$1 \mathrm{Vgl}$. in diesem Sinne auch Köhler, R., a.a.0., S. 272.

2 Kriterien zur Makrosegmentierung finden sich z. B. bei Gröne, A., a.a.0., S. 51 ff.; Frank, R.E., Massy, W.F., Wind, Y., a.a.0., S. 95; Scheuch,.F., Investitionsguiter-Marketing, Opladen 1975, S. 67 sowie Böhler, H., a.a.0., S. $63 \mathrm{ff}$. Abgrenzungskriterien im Bereich investiver Dienstieistungen benennt Kaufmann, E.J., a.a.0., S. 101.

$3 \mathrm{Vgl}$. vor allem Gröne, A., a.a.0., S. 52; Wind, Y., Cardozo, R., a.a.0., S. 156 f. sowie Kaufmann, E.J., a.a.0., S. $100 \mathrm{f}$. 
Wenn im folgenden eine Untergliederung der Abnehmerdimension nach Unternehmensgrößen (gemessen durch Umsatz) vorgenommen wird, so soll dies nicht auf eine oberlegenheit einer solchen Segmentierung im Markt elektronischer Wirtschaftsinformationsdienste hinweisen, sondern ist vielmehr beispielhaft zu verstehen. An späterer Stelle im ProzeB der Geschäftsfeldplanung hat - im Rahmen der Abnehmerselektion - an die Stelle dieses einzelnen Merkmals eine Kombination von Kriterien zu treten, die bestmöglich imstande ist, erfolgsträchtige Segmente von weniger erfolgsträchtigen zu trennen. Eine solche Unterscheidung wird um so eher möglich sein, "je besser es mit Hilfe einer innovativen Segmentierung gelingt, Marktnischen aufzudecken bzw. durch eine möglichst homogene Zusammenfassung von Abnehmerbedürfnissen die Grundlagen für eine möglichst bedarfsadäquate, die Kauffähigkeit und -bereitschaft der Abnehmer berücksichtigende Gestaltung der Problemlösungen zu schaffen". 1

\subsection{Strategischer Suchraum als Ausgangsraster der Geschäftsfeldwahl}

Mit der Konkretisierung der drei Dimensionen des strategischen Entscheidungsraumes ist die Voraussetzung zur Lokalisierung und Auswahl des aus der Sicht der Unternehmung optimalen Geschäftsfeldes geschaffen. Abbildung $10 \mathrm{gibt}$ für das beispielhaft betrachtete Unternehmen den relevanten Suchraum als Markt für elektronische Wirtschafts informationen wieder. Dieser Markt setzt sich aus einer Vielzahl für das Unternehmen grundsätzlich in Frage kommender Funktions-/ Technologie-/Abnehmersegmente (FTA-Segmente) zusammen.

1 Walters, M., a.a.0., S. 71. 


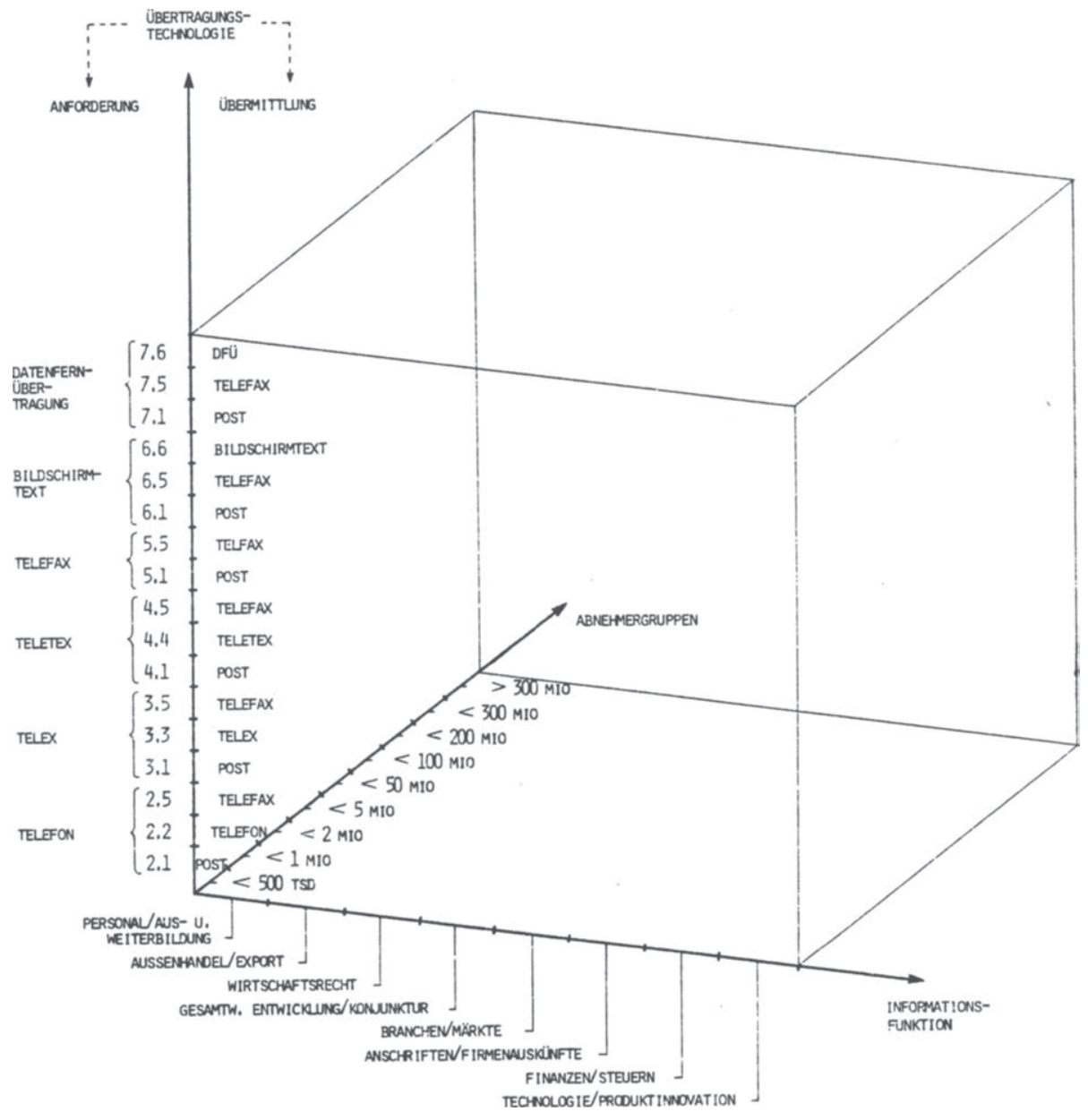

Abb. 10: Strategischer Suchraum zur Bestimmung und Auswahl strategischer Geschäftsfelder im Markt für elektronische Wirtschafts informationen 
Eine beliebige Kombination solcher Segmente kann aus dem nachfolgenden SelektionsprozeB als das für das Unternehmen geeignetste Geschäftsfeld hervorgehen.

In der Literatur wird an dieser Stelle mit Recht auf das besondere Problem der dreidimensionalen Geschäftsfeldplanung hingewiesen, das in dem unvermeidbaren Einbeziehen einer oft nicht mehr handhabbaren Anzahl von FTA-Segmenten besteht. 1 In der Tat wächst mit zunehmender Differenzierung der Dimensionen die Anzahl der Analyseeinheiten, für die strategische Daten (Marktpotentiale, Konkurrenzintensität etc.) gesammelt und ausgewertet werden müssen, exponentiell an. Hinzu kommt, daB im Rahmen der Analyse keine Unabhängigkeit zwischen den einzelnen FTA-Segmenten zu unterstellen ist. Insofern müssen zur Berücksichtigung möglicher komplementärer oder konfliktärer Effekte streng genommen neben den einzelnen Segmenten auch alle möglichen Segmentkombinationen in die Bewertung einbezogen werden.

Aus Gründen der Planungsökonomie ergibt sich vor diesem $H$ intergrund notwendig der Zwang zu einem vereinfachenden Vorgehen. Eine Möglichkeit könnte hier in dem Verzicht auf die explizite Analyse einer der Dimensionen bestehen.2 Eine solche Verkürzung des Problems der Geschäftsfeldplanung erscheint jedoch nur dann zulässig, wenn keine Unabhängigkeit zwischen den Dimensionen besteht. Eben diese Unabhängigkeit unter stellt jedoch der dreidimensionale Ansatz, indem er die drei Dimensionen orthogonal, d. h. im rechten Winkel aufeinanderstoßen 1 äßt. Dies bedeutet, daß es keine systematischen Abhängigkeiten z. B. zwischen Funktion und Technologie derart geben kann bzw. darf, daB durch die Funktion immer auch schon die Technologie vorbestimmt ist. Solche Abhängigkeiten lassen sich im Markt elektronischer Informationsdienste nicht feststellen; eine bestimmte Information erfordert nicht zwangsläufig den Einsatz einer bestimmten übertragungsform. Vielmehr liegt die Zuordnung im Ermessen des Planenden.

Als Fazit der Oberlegungen ergibt sich, daß mit der expliziten Berücksichtigung der drei relevanten Geschäftsfelddimensionen die Frage der Reduktion der Problemkomplexität zunächst ungelöst bleibt. In dem Problem, die Gesamtheit möglicher Analyseeinheiten

$1 \mathrm{Vgl}$. vor allem Walters, M., a.a.0., S. $78 \mathrm{ff}$.

$2 \mathrm{Vgl}$. ebenda, S. 79. 
bewältigen zu müssen ${ }^{1}$, offenbart sich dem Planenden das Dilemma, entlang der Funktionsdimension eine Informationsdienstleistung auswählen zu müssen, deren geeigneter Nutzer (entlang der Abnehmerdimension) ex ante nicht bekannt ist, deren Markterfolg jedoch maBgeblich von den Merkmalen und Bedürnissen dieses spezifischen Nutzers abhängt. Die faktische Undurchführbarkeit einer simultanen und somit optimalen Lösung dieses Problems macht eine Problemdekomposition erforderlich ${ }^{2}$, die die Aufgabe der Bewertung des gesuchten Geschäftsfeldes auf eine Bewertung einzelner Segmente herunterbricht. Damit wird jedoch das Problem der großen Zahl von Analyseeinheiten geschaffen.

Ein praktikabler Ausweg aus diesem Dilemma kann nun darin bestehen, über eine sukzessive Auswahl der zu erbringenden Funktion, der einzusetzenden Technologie und der zu bedienenden Abnehmergruppe zu einer Bestimmung des bestgeeignetsten Geschäftsfeldes zu gelangen. Hierzu ist jedoch zuvor die strategische Festlegung darüber zu treffen,

- welche grundlegende Geschäftsfeldstrategie angewandt und

- in welcher Abfolge die einzelnen.Dimensionen im Rahmen des strategischen Auswahlprozesses berücksichtigt werden sollen.

1 Im vorliegenden Fall handelt es sich um $(8 \times 17 \times 9)=$ 1224 FTA-Segmente (vgl. auch Abbildung 10).

2 Vgl. hierzu Kirsch, W., Die Handhabung von Entscheidungsproblemen, München 1978, S. $189 \mathrm{ff} .$. 
2. Wahl der Geschäftsfeldstrategie und Bestimmung des Auswahlprozesses

\subsection{Begrenzungsfaktoren der Geschäftsfeldselektion}

Mit der Auswahl einzelner Funktionen, Technologien und Abnehmergruppen zur Bestimmung eines neuen Geschäftsfeldes steht das Unternehmen vor dem Problem, im Sinne einer quasi-optimalen Innovationsplanung die grundsätzlichen Begrenzungsfaktoren seiner Handlungs möglichkeiten zu erfassen und in geeigneter Weise zu gewichten.

Ausgehend von der öberlegung, da $B$ eine erfolgreiche Dienstleistungsinnovation letztlich mit der Akzeptanz der Dienstleistung am Markt endet, stellt der Marktbedarf nach der geplanten Dienstleistung einen entscheidenden Begrenzungsfaktor für die Wahl des Geschäftsfeldes dar. Andererseits hat sich das Unternehmen an den ihm gesetzten Grenzen des "Machbaren" zu orientieren. Die Nähe der geplanten Innovation zum bisherigen Angebotsprogramm, produktions- und entwicklungstechnische Synergien, verfügbare Ressourcen und vorhandenes know-how determinieren den "Aktionsradius", der dem Unternehmen für seine Planung gegeben ist. Damit begrenzen Marktpotential und Unternehmenspotential das Feld, innerhalb dessen der InnovationsprozeB zu planen ist.

$1 \mathrm{Vgl}$. hierzu und zum folgenden insbes. Hübner, H., Produktinnovation und Produktion. Möglichkeiten und Grenzen der Nutzung von Erfolgsfaktoren der Produktion für die marktorientierte Innovation, in: Marktorientierte Unternehmensführung: Wiss. Tagung des Verbandes der Hochschullehrer für Betriebswirtschaft, Wirtschaftsuniversität Wien 1983, Hrsg.: Mazanec, I., Scheuch, F., Wien 1984, S. 377 ff.. 
Abbildung 11 kennzeichnet das Aktionsfeld einer Unternehmung, das die Einführung eines innovativen elektronischen Informationsdienstes plant. Um das Innovationsrisiko auf einem möglichst niedrigen $\mathrm{Ni}$ veau zu halten, muB sich das Unternehmen auf der einen Seite auf die Basis seiner besonderen Ressourcen und Fähigkeiten beziehen. Diese können sowohl die möglichen, vorhandenen Informationsbasen als auch die Technologien betreffen, mit denen das Unternehmen bereits Erfahrungen gesammelt hat. Schließlich kommt als dritte Ressourcenquelle einer gefestigten Abnehmerbasis eine wesentliche Bedeutung $z u$. Gerade im Dienstleistungsbereich ebnet ein vorteilhaftes und ausreichend verbreitetes Unternehmensimage oftmals neuen Marktangeboten den Weg zu einer schnellen Akzeptanz. 1

Neben dem Vorhandensein entsprechender Ressourcen ist die Realisierbarkeit der neuen Informationsdienstleistung abhängig von dem dienstleistungstechnischen know-how der Unternehmung. Dieses betrifft im vorliegenden Fall sowohl redaktionelle und dokumentarische Fähigkeiten als auch die Verfügbarkeit der nötigen Software und Orgware. Beide sind erforderlich, um das Leistungspotential zur Realisierung der Dienstleistungsvorhaben aufzubauen und die dauernde (technische) Funktionsfähigkeit des Dienstleistungssystems ("Servuctions-Systems" ${ }^{2}$ ) zu garantieren.

1 Vgl. Thomas, D.R.E., Strategie in Dienstleistungsunternehmen, in: HM, o. Jg., 1983, H. 2, S. 45; Levitt, Th., Marketing success through differentiation - of anything, in: HBR, Vol. 58, JanuaryFebruary 1980 , S. 84 (im folgenden zitiert als: Levitt, Th., Marketing success).

2 Langeard, E., Grundfragen des Dienstleistungsmarketing (ins Deutsche ubertragen und bearbeitet von Pardey, R.), in: Marketing.ZFP, 3. Jg., 1981, H. 4, S. $234 \mathrm{ff} .$. Der Begriff "Servuctions-System" ergibt sich dabei aus der Verknüpfung von "Dienstleistung" (Service) und (Dienstleistungs-) "Produktion", die synchron erfolgen. 


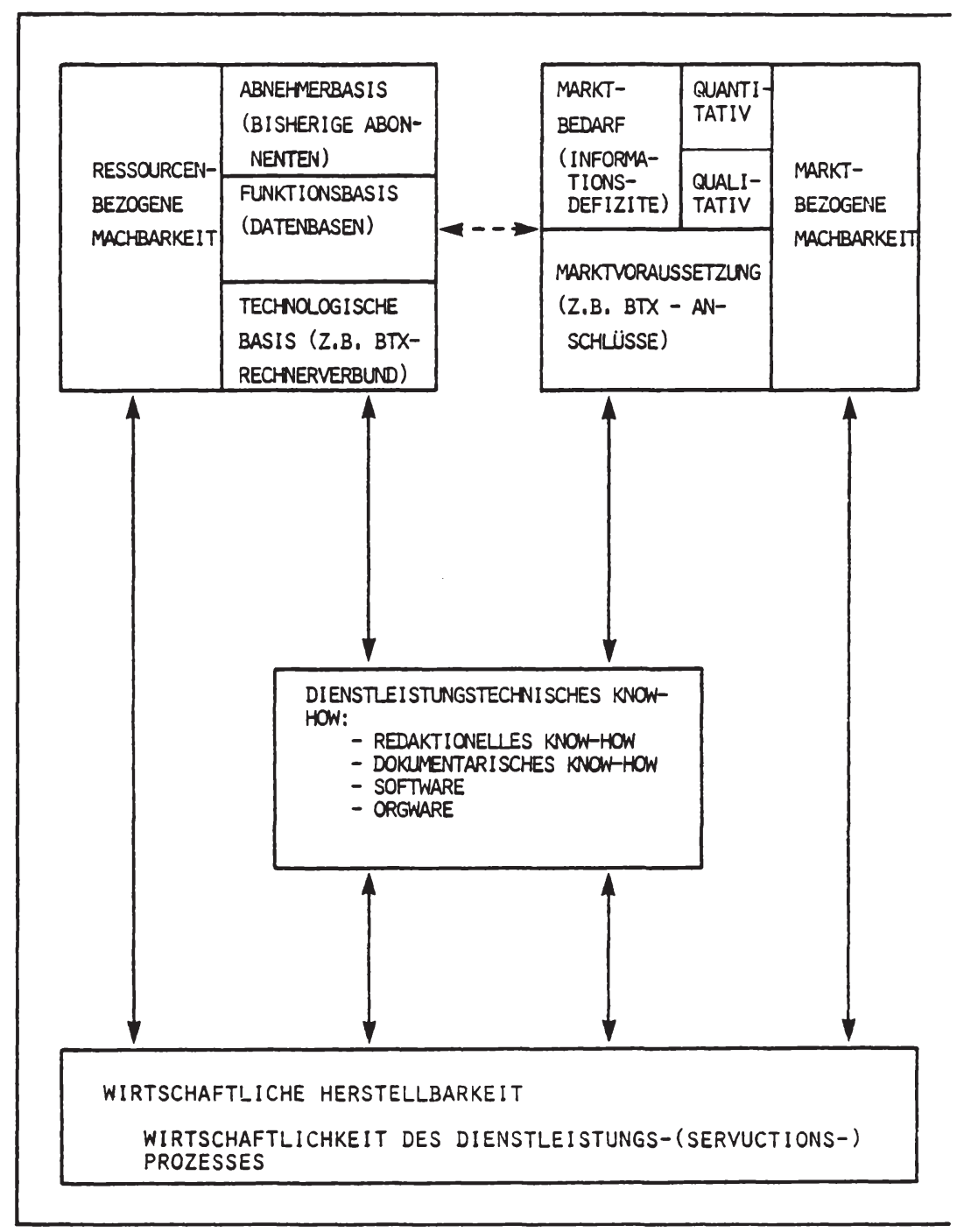

Abb. 11 : Abgrenzung des Handlungsfeldes für die Planung der Dienstleistungsinnovation

(Quelle: in Anlehnung an Hübner, H., a.a.0., S. 383 ) 
Seitens des Marktpotentials sind es der qualitative und quantitative Marktbedarf, die das Feld der Innovationsplanung entscheidend determinieren. Der qualitative Marktbedarf stellt dabei auf den Bedarf nach der jeweiligen Abnehmerfunktion ab, die mit der Dienstleistung erbracht werden soll. Mit der quantitativen Dimension ist das realisierbare mengenmäBige Absatzvolumen der Unternehmung angesprochen. Darüber hinaus sind die Voraussetzungen des Marktes zur Inanspruchnahme eines vom Unternehmen als vorteilhaft erkannten Informationsdienstes von Bedeutung ( 2 . B. das Vorhandensein der entsprechenden informationstechnologischen Infrastruktur).

Im Spannungsfeld zwischen markt- und unternehmensbezogener Machbarkeit bestimmen als interdependenter EinfluBfaktor die Aussichten hinsichtlich einer wirtschaftlichen Durchführbarkeit des Dienstleistungspro-zesses die Innovationsplanung. Die zentrale Frage richtet sich in diesem Zusammenhang auf das erforderliche Investitionsvolumen und die voraussichtijche Amortisationsdauer. 1

Der skizzierte Beziehungszusammenhang hat in zweierlei Hinsicht unmittelbare Relevanz für die Planung der Dienstleistungsinnovation: Zum einen gibt er Hinweise auf die Ausgestaltung der Geschäftsfeldstrategie der Unternehmung. Damit ist die strategische Festlegung darüber gemeint, welchen Umfang und welchen Grad der Differenzierung das strategische Geschäfts-

1 Untersuchungen haben ergeben, da $B$ zwischen dem $B e-$ ginn des Aufbaus eines neuen Geschäftsfeldes und dem Heraustreten dieses Geschäftsfeldes aus der Verlustzone durchschnittlich rund acht Jahre verstreichen. Vgl. Briggadike, R., The risky business of diversification, in: HBR, Vol. 57, May-June 1979, S. 106. Ein weiteres kritisches Moment ergibt sich durch die erforderliche Vorlaufzeit für den Aufbau geeigneter Datenbanken, der zwischen fünf und zehn Jahre in Anspruch nehmen kann. Vgl. o.V., Aufbruch, a.a.0., S. 50 . 
feld der Unternehmung aufweisen soll. Zum anderen öffnet der Beziehungszusammenhang den Blick für die Notwendigkeit, die marktorientierte Innovation durch eine ressourcenorientierte zu ergänzen. Damit ist die Frage angesprochen, welchen Dimensionen des Geschäftsfeldes im Rahmen der Planung welche Priorität eingeräumt werden soll.

\subsection{Alternative Geschäftsfeldstrategien als Aus - gangspunkt}

Die Entscheidungstatbestände bei der Festlegung der Geschäftsfeldstrategie lassen sich in ihrem Kern auf die grundsätzliche Frage reduzieren, wie viele und welche FTA-Segmente das Unternehmen als sein Geschäftsfeld definieren sollte. 'Mit der Festlegung des Grades der Segmentabdeckung (Zahl der ausgewählten Segmente) wird gleichzeitig durch die Zielrichtung der Segmentauswahl eine Entscheidung über den Grad der Differenzierung von Konkurrenzangeboten sowie der Differenzierung innerhalb des Geschäftsfeldes getroffen.

Unterstellt man zum Zweck der Komplexitätsreduktion, daß je Dimension des Geschäftsfeldes nur zwischen den zwei Alternativen "Spezialisierung" und "volle Abdeckung der Dimension" gewählt werden kann, so lassen sich idealtypischerweise folgende $(2 \times 2 \times 2=)$ acht Geschäftsfeldstrategien unterscheiden ${ }^{2}$ (vgl. Abbildung 12):

1 Vgl. Walters, M., a.a.0., S. 82 .

2 Vgl. zu ähnlich gearteten Typologien von Geschäftsfeldstrategien Abell, D.F., Defining the Business, a.a.0., S. $192 \mathrm{ff}$.; Kotler, Ph., a.a.0., S. 84 . 


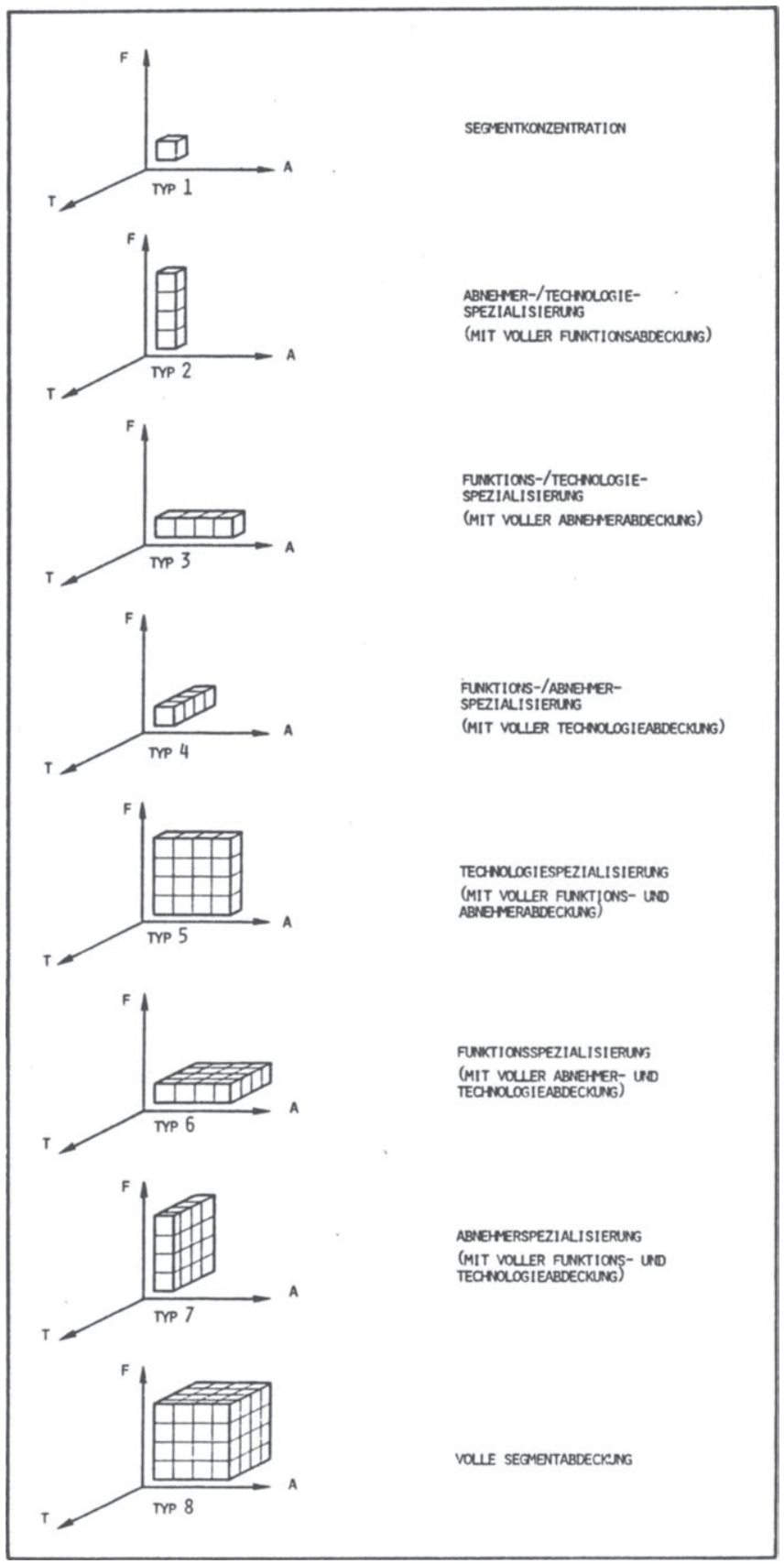

Abb. 12: Idealtypen möglicher Geschäf tsfeldstrategien $75074-2$

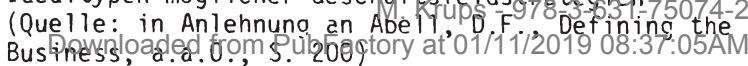


1. Segmentkonzentration

Bereitstellung relativ eng umrissener Informationen ( $z$. B. Exportinformationen) über eine Technologie ( $z$. B. Bildschirmtext) für eine relativ eng umrissene Zielgruppe ( $z$. B. exportierende, mittelständische Industrieunternehmen)

2. Abnehmer-/Technologiespezial isierung Bereitstellung eines umfassenden Informationsangebots, zugeschnitten für eine relativ eng umrissene Zielgruppe ( $z$. B. Großunternehmen mit über 1.000 Beschäftigten) und abzurufen über eine einzige Technologie ( $z$. B. Datenfernübertragung)

\section{Funktions-/Technologiespezial isierung}

Bereitstellung eines relativ eng umrissenen Informationspaketes ( $z$. B. aktuelle Daten zur gesamtwirtschaftlichen Entwicklung) über eine einzige Technologie ( $z$. B. Bildschirmtext) für eine nicht begrenzte $Z a h l$ von Informationsinteressenten

4. Funktions-/Abnehmerspezialisierung

Bereitstellung von Informationen $z u$ einem relativ eng umrissenen Themenkreis ( $z$. B. Firmenauskünfte) für eine begrenzte Zielgruppe (z. B. Investitionsguterunternehmen) über eine Vielzahl von obertragungswegen (Telexanfrage/-auskunft, Onl ine-Datenbank, Bildschirmtext, etc.)

5. Technologiespezialisierung

Bereitstellung eines umfassenden Informationsangebotes für eine vorab nicht begrenzte Abnehmerschaft über eine Technologie ( $z$. B. Btx-Universal-Wirtschaftsinformationsdienst) 
6. Funktionsspezial is ierung

Bereitstellung eines thematisch begrenzten Fachinformationsdienstes unter Einsatz verschiedenster obertragungstechnologien für eine Vielzahl von Interessentengruppen

7. Abnehmerspezialisierung

Bereitstellung eines Full Service-Wirtschaftsinformationsdienstes für eine ausgewählte Zielgruppe

8. Volle Segmentabdeckung

Bereitstellung eines Universal-Informationsdienstes für elektronische Wirtschaftsinformationen

Neben diesen idealtypischen Strategiealternativen ist eine nahezu unbegrenzte Zahl von Mischstrategien denkbar, so z. B. eine Vielfalt von Strategien der selektiven Abdeckung einzelner Dimensionen.

Zur Auswahl einer bestimmten Strategiealternative sind in der Literatur eine Vielzahl von Kriterien entwickelt worden. ' Eine ubergeordnete Rolle nimmt dabei nach ibereinstimmender Auffassung das Marktstadium ein. ${ }^{2}$ In Abhängigkeit von der jeweiligen Phase eines Marktes im Verlauf seines Lebenszyklus-

1 Vgl. 2. B. Shapiro, B., Industrial Products Policy, Marketing Science Institute, Cambridge, Mass. 1977, S. $112 \mathrm{ff} . ;$ Abell, D.F., Defining the Business, a.a.0., S. $179 \mathrm{ff}$.; Kotier, Ph., a.a.0., S. 209.

2 "The implications for management entering a new business area depend heavily whether the entrant is entering a completely new market, a growth market, or an established mature market". Abell, D.F., Defining the Business, a.a.0., S. 220. 
ses führen die einzelnen Kriterien zu bestimmten typischen, jeweils unterschiedlichen (Norm-)Strategien. 1

Betrachtet man beispielsweise den Fall des Eintritts in einen Pioniermarkt, so wird sich ein Anbieter hier typischerweise auf eine bestimmte Technologie konzentrieren, um mit dieser eine bestimmte Abnehmerfunktion zu erfüllen. Offen bleibt dabei zunächst, ob es sinnvoller ist, ein breites Abnehmerspektrum zu bearbeiten (Marktdurchdringungsstrategie) oder auf eine eng definierte Abnehmergruppe abzuzielen (Marktabschöpfungsstrategie). Die erstgenannte Alternative bietet sich an, wenn mit einer relativ hohen Elastizität der Nachfrage in Abhängigkeit vom eingesetzten Marketingaufwand gerechnet werden kann. Der zweite Weg ist dann angebracht, wenn die Nachfrageelastizität insofern als gering anzusehen ist, als auch langfristig nur solche Unternehmen gewonnen werden können, für die die Dienstleistung einen unmittelbaren und wesentlichen Nutzenvorteil verspricht.

Tendenziell wird vor diesem Hintergrund auch für den Eintritt in den Markt elektronischer Wirtschaftsinformationsdienste ein - hinsichtlich der Funktion und Technologie - konzentriertes Vorgehen naheliegen. Die Frage, auf welche Funktion, welche Technologie und welche Abnehmergruppen das Unternehmen sich konzentrieren soll, rückt dabei wieder das Problem der Interdependenz der Dimensionen in den Vordergrund. Will man dieses Problem auflösen, so führt der Weg notwendig über die sukzessive Analyse der einzelnen Dimensionen. Hierzu ist es jedoch unumgänglich, zunächst eine Entscheidung hinsichtlich der Planungspriorität der jeweiligen Dimensionen zu treffen.

1 Vgl.Abell, D.F., Defining the Business, a.a.0., S. 220 . 


\subsection{Konzept einer mehrstufigen Geschäftsfeldwahl}

2.31 Darstellung möglicher Prozeßalternativen

Ausgehend von dem in Abbildung 9 skizzierten dreidimensionalen Suchraum sind im Rahmen der schrittweisen Durchführung der Geschäftsfeldselektion $(3 !=3 \times 2 \times 1=$ ) sechs alternative Rangfolgen der Analyse der einzelnen Dimensionen denkbar (vgl. Abbildung 13):

\section{Abnehmer - Funktion - Technologie}

In dieser Abfolge der Analyseschritte findet sich der klassische Marketingansatz wieder, der die Abnehmerbedürfnisse an den Anfang aller Oberlegungen stellt. ${ }^{1}$ Diesem Ansatz folgend läge es zunächst nahe, auch beim Eintritt in den Markt für Wirtschaftsinformationen von den Abnehmern auszugehen. Es leuchtet jedoch unmittelbar ein, daB das anbietende Unternehmen mit der umfassenden Befriedigung der Vielfalt von Informationsbedurfnissen einer wie auch immer gearteten Abnehmergruppe überfordert wäre. Zudem stellt sich zwangsläufig die Frage, nach welchen Kriterien einer Abnehmergruppe der Vorrang vor allen anderen eingeräumt werden sollte. SchlieBlich läBt sich nicht von vornherein feststellen, ob eine Technologie, die der obertragung bestimmter Informationsinhalte angemessen ist, auch von der ins Auge gefaBten Abnehmergruppe akzeptiert wird.

\section{Abnehmer - Technologie - Funktion}

Ein zweiter Ansatz kann darin bestehen, sich zunächst auf eine bestimmte Abnehmergruppe und die'von dieser bevorzugten Technologie zu konzentrieren. So könnten $z$. B. umweltbewußten Konsumentenschichten Produkte angeboten werden, die auf die Nutzung von Sonnenener-

$1 \mathrm{Vgl.zur}$ Philosophie dieses Ansatzes insbes. Meffert, H., Marketing, a.a.0., S. 35 . 


\begin{tabular}{|c|c|c|}
\hline & ALLGEMEINE MARKETINGBEISPIELE & $\begin{array}{l}\text { BEISPIELE AUS DEM BEREICH ELEK- } \\
\text { TRONISCHER WIRTSCHAFTSINFORMA- } \\
\text { TIONEN }\end{array}$ \\
\hline $\begin{array}{l}\text { ABNEHMER } \\
\text { L FUNKTION } \\
\qquad \text {-TECHNOLOGIE }\end{array}$ & $\begin{array}{l}\text { JUNGE LEUTE/VIEL FREIZEIT } \\
\text { LEIGENAKTIVE UNTERHALTUNG, SPIELE } \\
\text { LVIDEOTECHNIK(VIDEOSPIELE) }\end{array}$ & $\begin{array}{l}\text { INGENIEURBUROS, ENTWICKLUNES- } \\
\text { ABTEILUNGEN } \\
\text { LPATENTINFOPMATIONEN } \\
\text { LONLINE RETRIEVAL }\end{array}$ \\
\hline $\begin{array}{l}\text { ABNEHMER } \\
\text { TECHNOLOGIE } \\
\longrightarrow \text { FUNKTION }\end{array}$ & $\begin{array}{l}\text { UMELTBEWUSSTE/'ALTERNATIVE' } \\
\text { LSOLARJECHNIK } \\
\text { SONNENENERGIEBETRIEBENE } \\
\text { HEIZUNG }\end{array}$ & $\begin{array}{l}\text { KLEINERE UND MITTLERE UNTER- } \\
\text { NEHMEN } \\
\text { LELEX } \\
\text { LKURZINFORMATIONEN }\end{array}$ \\
\hline $\begin{array}{l}\text { FUNKTION } \\
\text { DTECHNOLOGIE } \\
\text { LABNEHER }\end{array}$ & $\begin{array}{l}\text { 'WEICHE WÄSCHE' } \\
\text { - GETRENWT EINZUSETZENDER } \\
\text { WEICHSPÜLER } \\
\text { 'PULOVERTRÄGER' } \\
\text { Z.B. JUNGE FRAUEN, STRICK- } \\
\text { BEGEISTERTE }\end{array}$ & $\begin{aligned} & \text { AKTUEULE GESAMTWIRTSCHAFTLICHE } \\
& \text { INFORMATIONEN } \\
& \text { L } \\
& \text { BTX } \\
&$\[ \text { (STABSSTEUEN DER) GE- } \]$ \\
& \text { SCHAFTSLEITUNG, VOLKSWIRT- } \\
& \text { SCHAFTLICHE ABTEILUNGEN }\end{aligned}$ \\
\hline $\begin{array}{l}\text { FUNKTION } \\
\text {-ABNEEMER } \\
\text { DTECHNOLOIE }\end{array}$ & $\begin{array}{l}\text { SCHNEUE ZUBEREITUNG VON WARMEN } \\
\text { ESSEN } \\
\text { VOLL-BERUFSTÄTIGE,JUNGGESEUEN } \\
\text { \MIKROWEULNTECHNIK }\end{array}$ & $\begin{array}{l}\text { EXPORTINFORMATIONEN } \\
\text { LUNTERNEMEN MIT WACHSENDER } \\
\text { EXPORTINTENSITÄT } \\
\text { L BTX MIT ERGÄNZENDEM } \\
\text { INFORMATIONSABRUF }\end{array}$ \\
\hline $\begin{array}{l}\text { TECHNOLOGIE } \\
\text {-ABNEHER } \\
\text {-FUNKTION }\end{array}$ & $\begin{array}{l}\text { TIEFGEFRIEREN } \\
\text { D BESITZER VON GEFRIERSCHRÄNKEN } \\
\text { \TIEFKUHLKOST }\end{array}$ & $\begin{array}{l}\text { DATENFERNVERARBEITUNG } \\
\text { GROSSUNTERNEHMEN } \\
\text { BRANCHEN - UND PRODUKT- } \\
\text { ZEITREIHEN }\end{array}$ \\
\hline $\begin{array}{l}\text { TECHNOLOGIE } \\
\text { DFUNKTION } \\
\text { \ABNEMER }\end{array}$ & $\begin{array}{l}\text { TEFLON } \\
\text { - BRATEN OHE FESTBRENEN(PFANWEN) } \\
\text {-HAUSHALTSVORSTÄNDE }\end{array}$ & $\begin{array}{l}\text { TELEFAX } \\
\text { LZEICHNUNGEN,DIAGRAMME,BILDER } \\
\text { LMARKTFORSCHUNGSABTEILUNGEN }\end{array}$ \\
\hline
\end{tabular}

Abb. 13: Alternative Prozeßabfolgen der Geschäftsfeldwahl als Ausdruck unterschiedlicher Prioritätensetzung im Rahmen der Innovationsplanung 
gie abzielen. Als so erbrachte Abnehmerfunktionen sind z. B. Stromgeneratoren, Warmwasserheizungen usw. denkbar, deren gemeinsames Merkmal im Einsatz einer spezifischen, abnehmeradäquaten Technologie (hier: Solartechnik) liegt. Bezogen auf den Markt für Wirtschaftsinformationen liefert dieser Ansatz jedoch keine operationaler Lösungen. Wählt man z. B. eine Abnehmergruppe (beispielsweise Kleinunternehmen) und paßt diesei die Technologie an (z. B. Telex), so ergibt sich hieraus weder ein Hinweis auf geeignete Informationen, dic auf diesem Wege bereitgestellt werden sollten, noch auf die Akzeptanz des Dienstleistungsangebots insgesamt.

\section{Funktion - Technologie - Abnehmer}

Dieser Ansatz, der von einer bestimmten Abnehmerfunktion ausgeht und die Technologie auf diese abstimmt, orientiert sich relativ stark an der Machbarkeit der Innovation aus der Sicht der planenden Unternehmung. Produktionstechnische Voraussetzungen und vorhandenes Know-how sind in der Regel die Initiatoren der Entwicklung und Einführung solcher neuen Marktleistungen. Ein ausreichender Marktbedarf wird hierbei grundsätzlich unterstellt oder in einer getrennten Analyse sichergestellt. Bezogen auf die Marktwahl im Bereich elektronischer Wirtschaftsinformationen würde ein Unternehmen im Rahmen eines solchen Vorgehens prïfen, welche Ressourcen und besonderen Stärken es im Hinblick auf alternative Formen und Inhalte denktarer Informationsdienste besitzt, um hiervon ausgehend die geeignete Obertragungstechnologie festzulegen und für das so geschaffene Marktangebot eine erfolgversprechende Abnehmergruppe zu suchen. Dem Vorteil der besonderen Kompetenz und des hieraus resultierenden Wettbewerbsvorsprungs steht dabei die UngewiBheit gegenuiber, ob mit dem eigenen Angebot die besonders lukrativen Abnehmer-bzw. Funktionssegmente erreicht bzw. abgedeckt werden. 


\section{Funktion - Abnehmer - Technologie}

Der Ansatz, der eine bestimmte Abnehmerfunktion als Ausgangspunkt der Planung wählt, findet seine frühe Fürsprache bereits bei Levitt, der forderte, daß bestimmte Problemlösungen, zugeschnitten auf unterschiedliche Abnehmergruppen, die Planung der unternehmerischen Geschäftsfelder bestimmen sollten. ${ }^{1}$ Für das Unternehmen, das einen elektronischen Informationsdienst einzuführen plant, erscheint ein solches Vorgehen zunächst attraktiv, kann es doch seine Ressourcen den Marktbedürnissen gegenüberstellen und so zu dan zweckmäßigsten Abnehmerfunktionen gelangen. Probleme entstehen jedoch, wenn zur Erbringung dieser Funktion Technologien erforderlich sind, die das Unternehmen nicht beherrscht bzw. nicht zu nutzen bereit ist. Beispielsweise ist es denktar, daB das Unternehmen Exportinformationen bereithält, die Abnehmer jedoch im Rahmen der Informationsnachfrage eine persönliche Betreuung wünschen und die Abfrage datenbankgespeicherter Informationen ( $z$. B. über Bildschirmtext) ablehnen.

\section{Technologie - Abnehmer - Funktion}

Eine Planung von Innovationen von der Technologie her kommend wird geläufig als "technology push" bezeichnet. ${ }^{2}$ Es besteht Einigkeit unter den Experten, daB gerade der Markt für Wirtschaftsinformationen gegenwärtig einen solchen "Technologieschub" zu erfahren beginnt, indem nämlich auf der Suche nach erfolgsträchtigen Nutzungsmöglichkeiten solch neuer Ober-

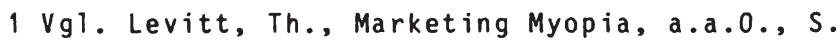
$45 \mathrm{ff.} ; \mathrm{vgl}$. auch derselbe, Marketing success, a.a.0., S. $83 \mathrm{ff}$.

$2 \mathrm{Vgl.Z}$. B. Rupp, M., Produkt/-Markt-Strategien, 2. Auf1., Zürich 1983, S. 183; Uhlmann, L., Typen industrieller Innovationsprozesse, in: ifo-Schnelldienst, 30. Jg., 1978, Nr. 33, S. $5 \mathrm{ff} . ; \mathrm{vgl}$. insbes. S. 9 . 
tragungswege wie Bildschirmtext Anbieter mit neuartigen Informationsangeboten unter Einsatz eben dieser obertragungswege auf den Markt stoßen. Wenig zweckmäßig erscheint es jedoch, zu stark auf die bei diesem Ansatz angestrebte Kompatibilität von Technologie und Abnehmern abzustellen, da die Technologie - hier die Form der Informationsabfrage und -übermittlung - nur als Mittel zum Zweck - hier: der Informationsversorgung - verstanden werden kann. Die eigentliche Abnehmerfunktion läuft Gefahr, bei einer solchen Prioritäterisetzung letztlich zu kurz zu kommen. Dies wird deutlich, wenn man beispielsweise das neue Medium Bildschirmtext zum Einsatz bringen möchte, hierzu prüf, welche Unternehmen in absehbarer Zukunft einen Btx-AnschluB haben werden, und erst dann fragt, welche Informationen fur diesen Abnehmerkreis geeigneterweise bereitgestellt werden sollten.

\section{Technologie - Funktion - Abnehmer}

Diese letzte Vorgehensalternative läßt sich an vielen Beispielen aus der Raumfahrtechnik nachvollziehen. Hier werden für einzelne Projekte neue technische Lösungen entwickelt (z. B. Teflon, "moonboots"), für die dann später ein breiterer Absatzmarkt gesucht wird. Dabei wird die Frage gestellt, welche neuen Abnehmerfunktionen mit der neuen Technologie erbracht werden können, und wer die potentiellen Nachfrager solcher Problemlösungen sein werden. Im Markt für Wirtschaftsinformationen bedeutet ein solches Vorgehen, daB das Ziel der Nutzung einer bestimmten Technologie ( $z$. B. Bildschirmtext) den Ausgangspunkt der Planung bildet. Auf diesem Ausgangspunkt basierend fragt das Unternehmen, für welche Informationsfunktion die Technologie besonders prädestiniert ist, um dann nach erfolgversprechenden zielgruppen für eben diese Informationsfunktion zu suchen. 


\subsection{Auswahl der geeigneten ProzeBalternative}

Die Auswahl der adäquaten ProzeBalternative kennzeichnet insofern einen kritischen Punkt im Rahmen der Geschäftsfeldplanung, als hier bereits bestimmte Selektionskriterien für die spätere, eigentliche Geschäftsfeldauswahl präjudiziert werden. Während funktions- und technologieorientierte Vorgehensweisen stärker auf den Aspekt der unternehmensbezogenen Machbarkeit abstellen, zielen die abnehmerorientierten Planungsansätze verstärkt auf die Lokalisierung und Ausnutzung von Marktchancen. In der Literatur unterscheidet man in diesem Zusammenhang neuerlich zwischen autonomen und marktinitiierten Innovationen. 1 Es setzt sich zunehmend die Erkenntnis durch, daB erfolgreiche Innovationen zwar marktorientiert, aber nicht notwendig marktinitiiert sein muissen. "Die Bedeutung sog. autonomer Innovationen liegt in einem vergleichsweise geringeren Risiko, da bei dieser Vorgehensweise versucht wird, vorhandene Ressourcen und Potentiale für Innovationen zu nutzen". 2

In Abbildung 14 wird versucht, die sechs möglichen ProzeBalternativen der Geschäftsfeldwahl anhand einer groben Heuristik einer vergleichenden Bewertung zu unterziehen. Unter der Prämisse, daß die Bewertungskriterien in etwa als gleichgewichtig anzusehen sind, ergibt sich, daB Alternative $3 z u$ bevorzugen ist, die Alternative also, die die Funktion und die Technologie an den Ausgangspunkt der überlegung stellt. Bei durchschnittlichem Marktrisiko zeichnet sich diese Alternative dadurch aus, daß in hohem Maße die eigenen Ressourcen genutzt und die Planungsschritte mit relativ klaren Inhalten bzw. Vorgehensvorschriften gefüllt werden können.

1 Vgl. Staudt, E., Innovationsplanung, Vorlesungsunterlagen, Wien 1983, zitiert in: Hubner, H., a.a.0., S. 380 .

2 Hübner, H., a.a.0., S. 380. 


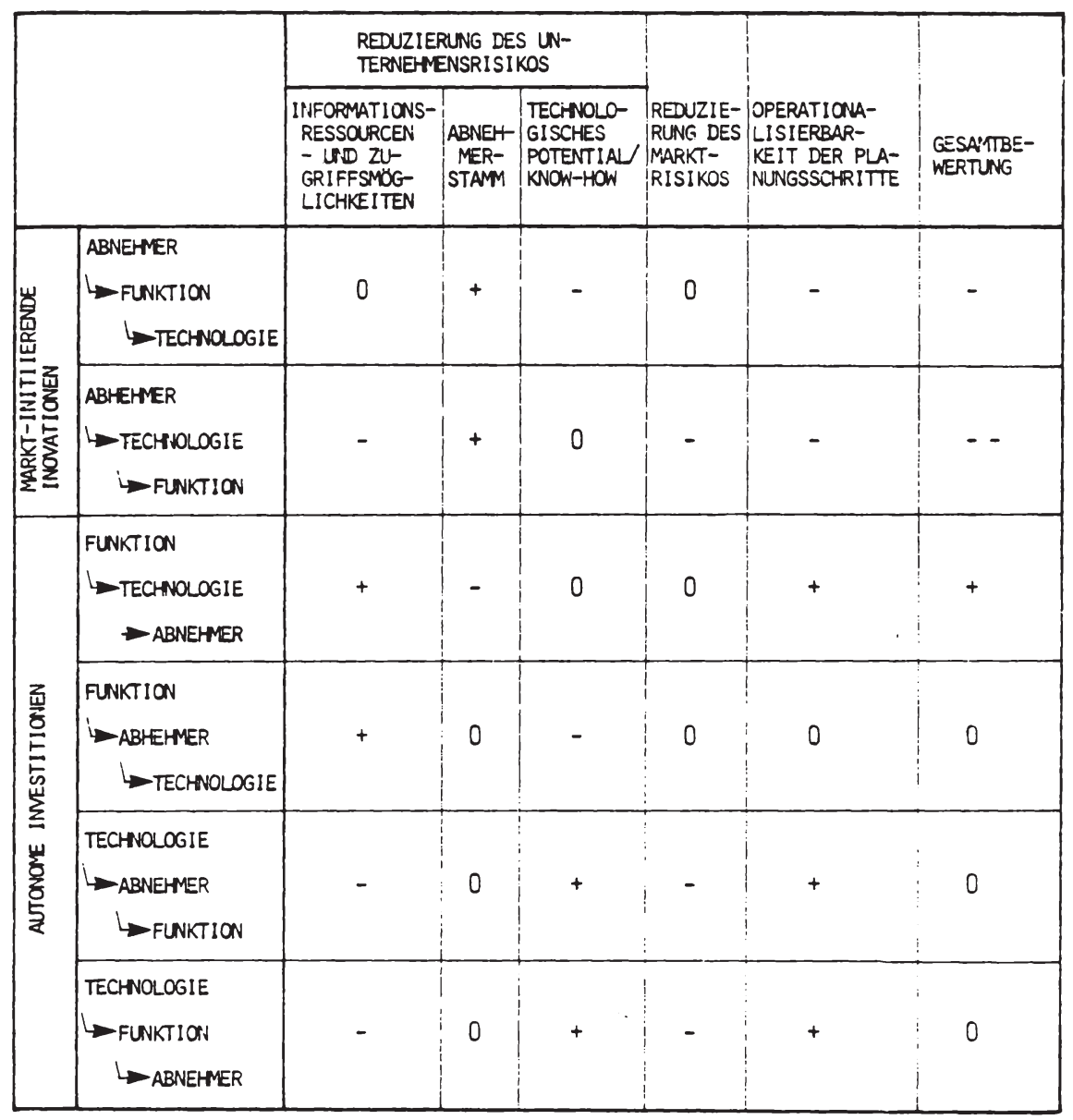

Legende: ++ sehr vorteilhaft

+ vorteilhaft

0 indifferent

- nachteilig

-. sehr nachteilig

Abb. 14: Bewertung der alternativen Prozeßabfolgen der Seschäftsfeldwahl 
Die Bestätigung dieser Bewertung durch die Realität der Praxis dokumentiert sich in verschiedenen empirischen Untersuchungen, die sich mit dem auslösenden Moment betrieblicher Innovationsprozesse befaßt haben. Unter Beruicksichtigung der in begrenztem Umfang empirisch validierten Tatsache, daß es keinen allgeme in gültigen Innovationsproze $\beta$ gibt ${ }^{1}$, lieferte die Analyse von 567 erfolgreichen Innovationsfällen aus den USA das Ergebnis, daB in mehr als $50 \%$ der Fälle die Produktion und vorhandenes technisches know-how den AnstoB zur Innovation gibt. 2

In die gleiche Richtung zielen die Ergebnisse einer jüngeren Erhebung bei Schweizer Unternehmen, die sich mit den MiBerfolgsursachen gescheiterter Innovationsprojekte befaßte. 3 Hier wurde festgestellt, daß in je $20 \%$ der untersuchten Fälle die "falsche Beurteilung der Herstellungs- und Fabrikationskosten" sowie die "falsche Beurteilung der technischen Anforderungen (Stand der Technik, technologische Trends") den Ausschlag für das Scheitern der Innovation gab.4

Schließlich zeigt auch die Geschichte von ca. 100 in der Bundesrepublik Deutschland in den letzten Jahren neugegruindeten Unternehmen den hohen Beitrag der Produkt- und Produktionstechnologie für die erfolgreiche Besetzung neuer Geschäftsfelder. 5 So war die "Beherrschung einer spezifischen Produkttechnologie" in $42 \%$ der Fälle der Initiator für die Neugründung, in $38 \%$ der Fälle gab die "Beherrschung einer spezifischen Produktionstechnologie" den entscheidenden AnstoB.

1 Vgl. Uhlmann, L., a.a.0., S. 6.

2 Vgl. Widmer, S., Erfolg mit Lizenzen, Zürich 1980, S. 12 .

$3 \mathrm{Vgl}$. Brehm, E., Von der Idee zum Produkt. Vorlesungsunterlagen, Zürich 1983, zitiert in: Hübrer, H., Produktinnovation, a.a.0., S. 385 .

$4 \mathrm{Vgl}$. ebenda. Eine frühere Untersuchung von Mißerfolgsursachen bei der Neueinführung investiver Güter ergab, da $B$ vor allem die mangelnde MarketingProfessionalität des verantwortlichen Managements zum Scheitern des Neuproduktes führte. Ressourcenbezogene Schwächen, wie fehlendes Produktions-oder Entwicklungs-know-how, hatten demgegenüber keinen nennenswerten Einfluß auf Erfolg oder Mißerfolg des Produktes. Vgl. Cooper, R.G., Why New Industrial Products Fail, in: IMM, Vol. 4, 1975, S. $315 \mathrm{ff}$.

5 Vgl.Ohem, K. (Hrsg.), Mit Ideen zum Erfolg, Frankfurt/Main 1980. 
Für eine schwerpunktartige und vorrangige orientierung der Geschäftsfeldplanung an den Ressourcen und Fähigkeiten der Unternehmung spricht zusammenfassend vor allem die damit verbundene gezielte Nutzung von Entwicklungs-, Herstellungs- und Marketing-Synergien. 1 Die Bedeutung solcher Synergien für den langfristigen Erfolg einer Unternehmung konnte im Rahmen einer empirischen Studie in Kanada eindrucksvoll nachgewiesen werden. 2 Die Studie zeigte, daß der Erfolg des Gesamtprogramms einer Unternehmung signifikant korreliert mit dem Grad, in dem neve Produkte zu den Ressourcen und technischen Leistungsfähigkeiten der Unternehmung "passen". ${ }^{3}$ Gleichzeitig bestätigte sich damit, daß "technology-push ideas are as likely to succeed as market-pull products". 4 Nicht zuletzt wies die Studie nach, daB gerade Firmen, die hochinnovative Produkte unter Einsatz neuester Technologien entwickeln, deren Produkte zudem in starkem Maße das Nu.tzungsverhalten der Abnehmer beeinflussen und den $A b-$ nehmern einen besonderen Nutzenvorteil - auch relativ zur Konkurrenz - stiften, insgesamt einen besonders positiven Erfolgssaldo aufweisen. ${ }^{5}$ Damit ist der Hinwe is verbunden, daB die ressourcenorientierte Geschäftsfeldplanung in keiner Weise den Innovationsgrad der Neuprodukteinführung und damit die "Aggressivität" 6 des Markteintritts vorbestimmt.

1 Zum Synergiekonzept vgl. Ansoff, H.I., Corporate Strategy. An Analytic Approach to Business Policy for Growth and Expansion, New York etc. 1965, S. $75 \mathrm{ff}$. ; vgl. ferner Ulrich, H., Unternehmenspolitik, Bern, Stuttgart 1978, S. $178 \mathrm{f}$.; Pümpin, C., Management strategischer Erfolgspositionen, Bern, Stuttgart 1982 , S. 65 .

$2 \mathrm{Vgl}$. Cooper, R.G., The Impact of New Product Strategies, in: IMM, Vol. 12, 1983, S. $243 \mathrm{ff}$. (im folgenden zitiert als: Cooper, R.G., New Product Strategies).

$3 \mathrm{Vgl}$. ebenda, S. 250.

4 Cooper, R.G., Identifying Industrial New Product Success: Project New Prod, in: IMM, Vol. 8, 1979, S. 130 (im folgenden zitiert als: Cooper, R.G., Project New Prod).

$5 \mathrm{Vgl}$. Cooper, R.G., New Product Stratgies, a.a.0., S. 247.

6 Ebenda. 
2.4 Kennzeichnung eines ressourcenorientierten Auswahlprozesses

\subsection{Entscheidungsablauf der Geschäftsfeldwahl}

Vor dem Hintergrund der obigen Oberlegungen ist das generelle Plädoyer für ein ressourcenorientiertes Vorgehen bei der Planung des Eintritts in innovative Investivdienstleistungsmärkte $z u$ verstehen, das der Verfasser den folgenden Ausführungen zur Geschäftsfeldwahl im Markt für Wirtschaftsinformationen zugrunde legt. Abbildung 15 gibt den Entscheidungsablauf der schrittweisen Geschäftsfeldselektion im Oberblick wieder.

Der Ablauf macht deutlich, daß zunächst in einer ersten Grobselektion die möglichen Informationsfunktionen zu analysieren sind. Aus einer Zahl von $i=1-n$ in Frage kommenden Funktionen $F_{i}$ wird eine Zahl geeigneter Funktionen anhand eines Kataloges von Auswahlkriterien selektiert. Anschließend sind - abgestimmt auf die ausgewählten Funktionen, die Technologien $T_{j} z u$ bestimmen, die je Funktion $F_{i}$ zum Einsatz kommen sollen. Die so ermittelten, strategisch relevanten Angebotskombinationen $\mathrm{F}_{j} / \mathrm{T}_{j}$ werden dann einer Feinselektion unterzogen. Für die aus dieser zweiten Selektionsstufe hervorgehenden Angebotskombinationen sind schließlich adäquate Abnehmergruppen $A_{k}$ auszuwählen. Das Geschäftsfeld ergibt sich damit aus einer - vorab unbekannten - Zahl von $F_{j} / T_{j} / A_{k}$-Kombinationen (FTASegmenten).

Je nachdem, ob das gefundene Geschäftsfeld einer abschließenden Wirtschaftlichkeitsanalyse standhält, sind erforderlichenfalls die jeweiligen Auswahlkriterien zu modifizieren und der SelektionsprozeB zu wiederholen. 


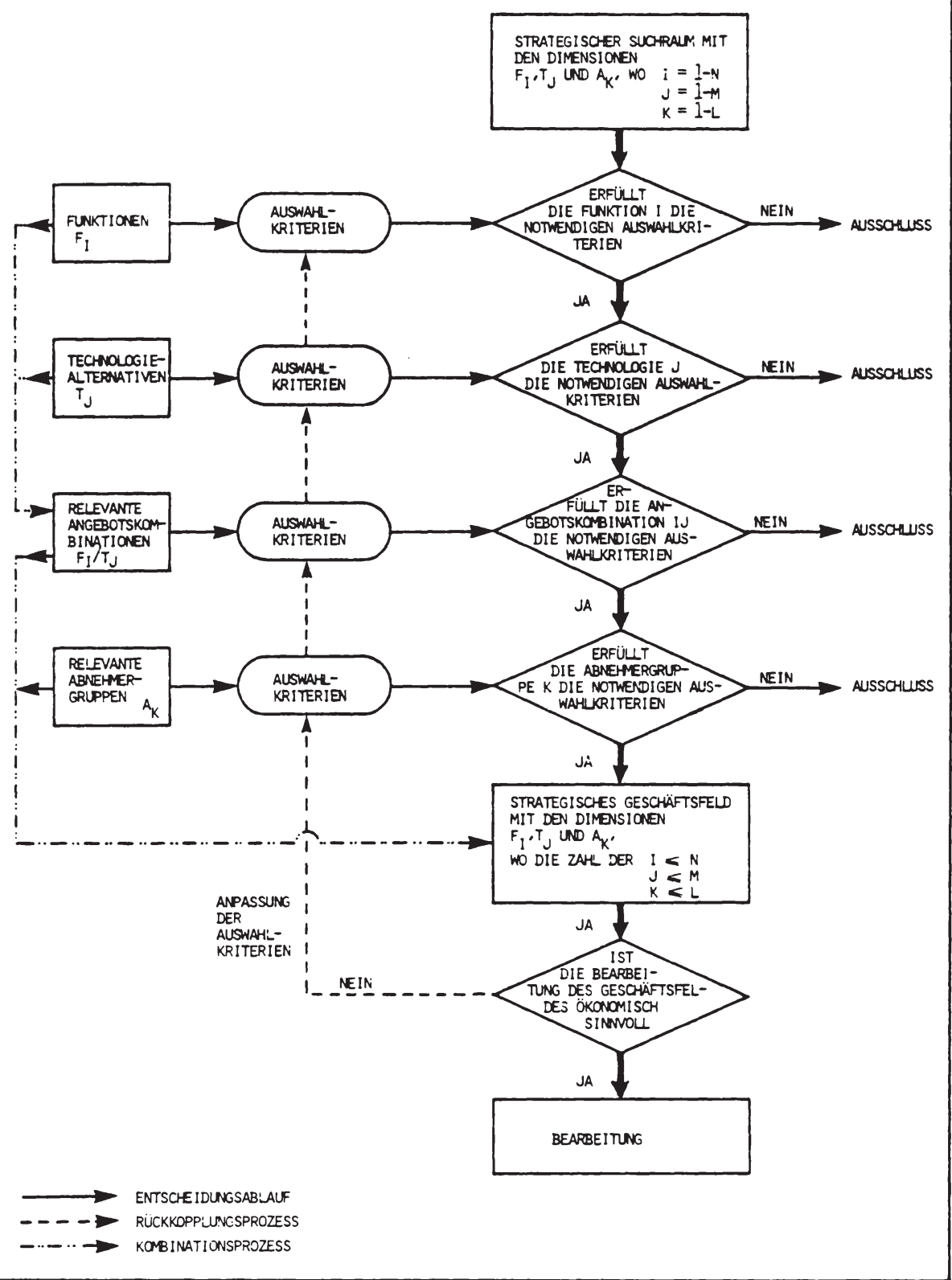

Abb. 15: Entscheidungsablauf einer iterativen Geschätsfeldwahl 


\subsection{Entscheidungskriterien der Geschäftsfeldwahl}

In der Literatur findet sich eine Vielzahl von Kriterien, die klassischerweise der Geschäftsfeldwahl zugrunde gelegt werden. Hierzu zählen insbesondere

- unternehmensbezogene Kriterien wie

- Unternehmensressourcen

- Fähigkeiten der Unternehmung und

- marktbezogene Kriterien wie

- Realisierbarkeit von Skaleneffekten

- Ressourvenbedarf

- Abnehmerverhalten

- Produktkomplexität. 1

Gemeinsamer Zweck dieser Kriterien ist es, sicherzustellen, daß das gewählte Geschäftsfeld eine effiziente oder effektive Bearbeitung erlaubt. ${ }^{2}$ Eine Analyse der Literatur zeigt jedoch, daB die Kriterien der Geschäftsfeldwahl nahezu durchgängig als Kriterien der Auswahl einer bestimmten Geschäfts-

$1 \mathrm{Vgl.Abel1,D.F.,} \mathrm{Defining} \mathrm{the} \mathrm{Business,} \mathrm{a.a.0.,}$ S. $179 \mathrm{ff.;}$ im gleichen Sinne Walters, M., a.a.0., S. 114. Ahnliche Kriterien finden sich bei Corey. R.E., Star, S.H., Organizational Strategy. A Marketing Approach, Harvard Business School, Boston 1971, S. $8 \mathrm{ff}$. sowie Shapiro, B., a.a.0., S. $112 \mathrm{ff} .$.

2 Während die Erzielung von Effizienz dabei auf eine Position der Kostenführerschaft abzielt, verfolgt die Maximierung von Effektivität das Ziel, eine im Vergleich zur Konkurrenz überlegene Bedürfnisbefriedigung herbeizuführen. Vgl. hierzu Abell, D.F., Defining the Business, a.a.0., S. 21 und 178; Porter, M.E., Competitive Strategy, New York, London 1980 , S. 31 ff.; Hinterhuber, H.H., Wettbewerbsstrategie, Beriin, New York 1982, S. 94 ff.; Bauer, E., a.a.0., S. $130 \mathrm{ff}$. 
feldstrategie verstanden und entsprechend auf einer vorgelagerten Stufe der Geschäftsfeldselektion zur Anwendung gebracht werden. 1 Weniger abstrakt und aus entscheidungsorientierter Sicht zweckmäBiger erscheint es demgegenüber, die Kriterien als MaßgröBen konkreter Geschäftsfeldalternativen anzulegen.

Ein solches Vorgehen gestaltet sich allerdings dadurch problematisch, daß die Geschäftsfeldwahl in Stufen zergliedert wird und nicht alle Kriterien auf allen Stufen sinnvolle Maßgrößen darstellen. Aufgrund dieser Oberlegungen wird im folgenden eine Zuordnung einzelner Kriterien zu den jeweiligen Stufen der Geschäftsfeldwahl vorgenommen. ${ }^{2}$ Die Zuordnung folgt dabei relativ einfachen Plausibilitätsuberlegungen hinsichtlich der Relevanz der einzelnen Kriterien in der jeweiligen Auswahlphase. Abbildung 16 gibt das Ergebnis im Oberblick wieder.

\subsection{Bezugsrahmen der Geschäftsfeldwahl}

Einen detaillierten AbriB über die einzelnen Phasen des Auswahlprozesses und die grundsätzlich zu berücksichtigenden Determinanten der Geschäftsfeldwahl gibt der Bezugsrahmen in Abbildung 17. Die Analyse beginnt mit der Auswahl der zu erbringenden Funktion (I). Ihr schliebt sich in einer zweiten Phase die Auswahl geeigneter Technologiealternativen (Formen der Informationsbereitstellung) an (II). Als Kriterium zur Auswahl

$1 \mathrm{Vgl}$. vor allem Abell, D.F., Defining the Business, a.a.0., S. $177 \mathrm{ff.}$, insbes. S. 184; vgl. im gleichen Sinne Walters, M., a.a.0., S. $112 \mathrm{ff}$. , insbes. S. 114 und S. 121.

2 Hierbei werden die "klassischen" Kriterien z. T. ergänzt um weitere Maßgrößen, deren Entscheidungsrelevanz in anderem Zusammenhang in der Literatur Erwähnung findet. 


\begin{tabular}{|l|l|}
\hline $\begin{array}{l}\text { STUFE DES AUSWAHL- } \\
\text { PROZESSES }\end{array}$ & ZENTRALE ENTSCHEIDUNGSKRITERIEN \\
\hline $\begin{array}{l}\text { WAHL DER FUNKTION } \\
\text { (FUNKTIONSBEREICH) }\end{array}$ & - UNTERNEHMENSRESSOURCEN \\
& - UNTERNEHMENSFÄHIGKEITEN \\
& - ABNEHMERBEDARF \\
WAHL DER TECHNOLOGIE & - KONKURRENZSITUATION \\
(FORM DER INFORMATIONSVERSORGUNG) & - TECHNOLOGIEATTRAKTIVITÄT \\
\hline WAHL DER STRATEGISCH RELEVANTEN & - RESSOURCENBEDARF \\
ANGEBOTSKOMBINATIONEN & - SKALENEFFEKT \\
& - KOMPLEXITÄT DER DIENSTLEISTUNG \\
& - ABNEHMERVERHALTEN \\
\hline WAHL DER ABNEHMERGRUPPEN & - DETERMINANTEN DER KAUFVERANLASSUNG \\
& - DETERMINANTEN DER KAUFF̈̈HIGKEIT \\
\hline
\end{tabular}

Abb. 16: Entscheidungskriterien zur iterativen Geschäftsfeldwahl 


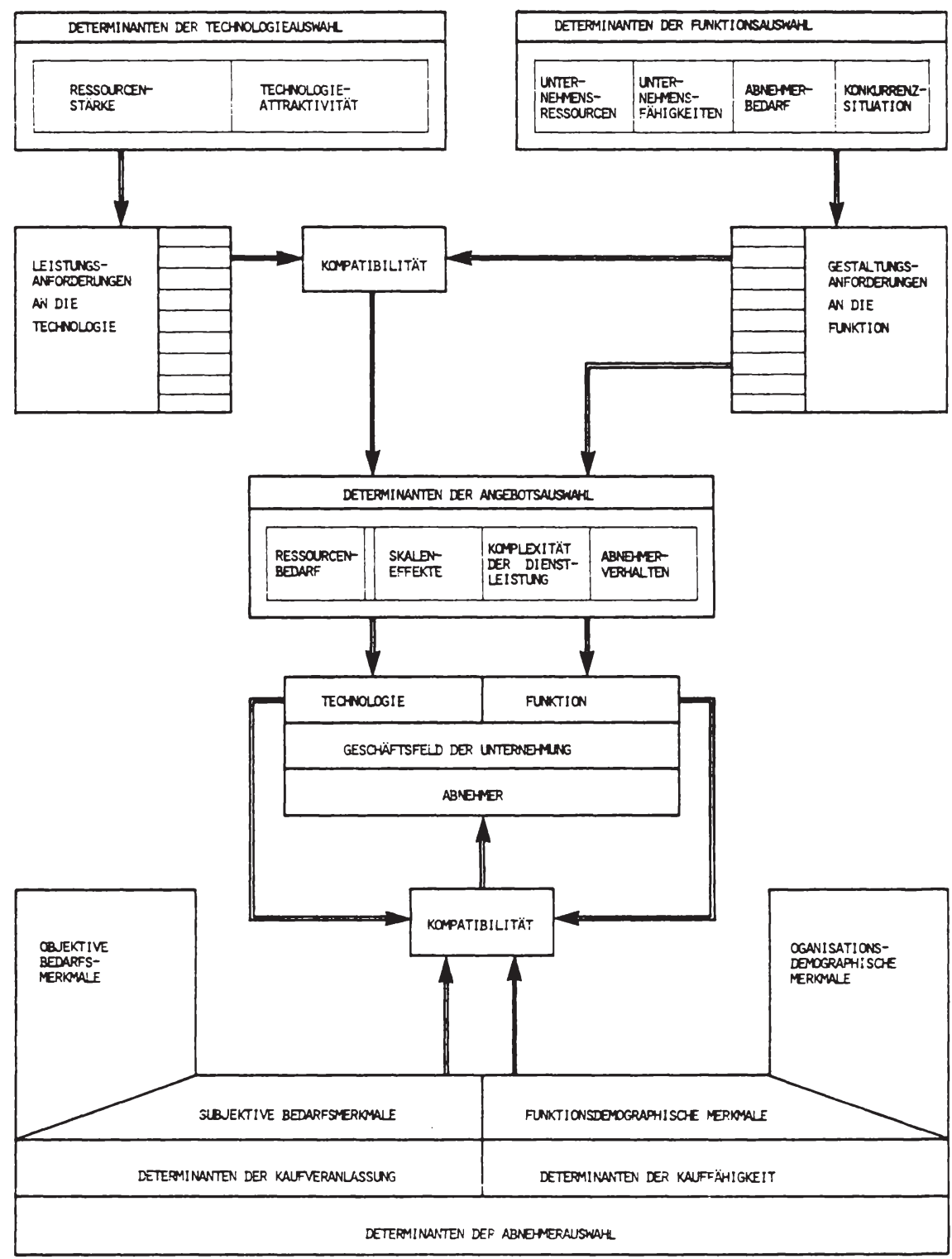

Abb. 17: Bezugsrahmen der Geschäftsfeldwahl 
einer oder mehrerer Technologien ergibt sich in der Folge insbesondere der Grad, in dem eine bestimmte Technologie zur Funktionserfüllung geeignet ist. Der Grad der Eignung drückt sich dabei aus in der Kompatibilität zwischen dem Anforderungsprofil der Versorgung mit bestimmten Informationen und dem Leistungsprofil der jeweiligen Technologie.

Die dritte Auswahlphase ist gekennzeichnet durch die Feinselektion erfolgversprechender Angebotskombinationen (III). In der vierten Auswahlphase werden schlieBlich den selektierten Funktions-/Technologiekombinationen adäquate Abnehmergruppen zugeordnet (IV).

Auf den AuswahlprozeB sei im folgenden detaillierter eingegangen.

\section{Auswahl strategischer Angebotskombinationen}

3.1 Bestimmung der Informationsfunktion

Mit der Auswahl der Informationsfunktion legt das Unternehmen jene Problemlösungen fest, die es im Rahmen seiner geplanten Geschäftsfeldaktivitäten erbringen will. Dabei differenziert es zunächst weder nach Technologien, die sich $u$. U. für die eine oder andere Funktionserfüllung besonders anbieten, noch nach einzelnen Marktsegmenten oder Zielgruppen, die möglicherweise einen besonderen Bedarf an speziellen Funktionen erkennen lassen. Ziel istes, in dieser Stufe lediglich, im Rahmen einer ersten Grobauswahl den Aufgabenradius der geplanten Informationsversorgung abzustecken. Ausgangspunkt bilden hierbei die - im vorliegenden Beispiel acht - Informationsbereiche, die als grundsätzlich infrage kommend entlang der Funktionsachse des strategischen Suchraumes aufgereiht worden sind. Die Aus- 
wahl selbst erfolgt anhand von Kriterien, die sowohl auf die Möglichkeiten des planenden Unternehmens als auch auf zentrale marktbezogene Tatbestände Bezug nehmen. 1

\subsection{Anbieterbezogene Determinanten}

\subsection{Unternehmensressourcen}

Die Ressourcen einer Unternehmung bestimmen maBgeblich seine Fähigkeit, bestimmte Abnehmerfunktionen $z u$ erfiullen bzw. erfolgreich am Markt durchzusetzen. 2 Bei gegebener Unternehmensgröße und Finanzkraft kommt insbesondere dem Grad der Synergie zwischen den bisherigen Aktivitäten und den im Rahmen des neuen Geschäftsfeldes geplanten Aktivitäten eine zentrale Rolle zu. Je enger der Bezug zwischen dem Basisgeschäft (home market) und dem neuen Markt (entry market) gesetzt wird, um so eher können solche Investitionen getätigt werden, die den Charakter von "shared costs" aufweisen. 3

Im Fall eines Informationsanbieters liegt der Kernpunkt des Ressourcenproblems in der Frage, uber welche Informationsbasen das Unternehmen zum Planungszeitpunkt verfügt. Im Sinne einer möglichst umfassenden Ausschöpfung von Synergien versteht es sich von selbst, daB das Unternehmen zunächst solche Informationen für seine Informationsdienstleistung vorsehen sollte, die inm ohnehin vorliegen.

1 Vgl. hierzu auch Abbildung 16, S. 88 dieser Arbeit.

2 Vgl. Abell, D.F., Defining the Business, a.a.0., S. 183 f.; Corey, R.E., Star, S.H., a.a.0., S.

$9 \mathrm{ff}$.; Hubner, H., Produktinnovation, a.a.o., S. $382 \mathrm{ff} .$.

$3 \mathrm{Vgl}$. Porter, M.E., a.a.0., S. $8 \mathrm{f}$.; vgl. zum Konzept der "shared costs" darüber hinaus 0.V., Unternehmensstrategien, Spiel ums Oberleben, in: WW, $\mathrm{Nr} .20,1982, \mathrm{~S} .50$. 
Eine weitere Frage betrifft die Qualität der in Frage kommenden Informationen. Die Beurteilung der Qualität macht eine kritische Analyse dahingehend erforderlich, inwieweit sich die vorhandenen Informationen hinsichtlich ihrer Relevanz, Aktualität, ihres Aufbereitungs grades und ihrer Vollständigkeit als Grundstock für einen neuen Informationsdienst heranziehen lassen, und ob die Informationen eine direkte Abspeicherung auf elektronische Datenträger erlauben. Unter bestimmten Umständen kann es wirtschaftlich sinnvoller sein, eine Datenbank von einem Datenbankverleger in Lizenz $z u$ übernehmen und diese zu vertreiben, als selbst in einem mühsamen AufbereitungsprozeB eigene Informationen zu einer aussagekräftigen Datenbasis zusammenzufügen. 1

Ein plastisches Beispiel für die praktische Relevanz dieser Oberlegungen 1 iefert die Firma Bertelsmann. Der Medienkonzern leitete seinen Einstieg in den Markt elektronischer Informationen 1984 dadurch ein, daB er den Exklusivvertrieb der bei dem Schweizer Host DataStar aufliegenden und online verfügbaren Datenbanken der Bereiche Naturwissenschaft, Technik und Wirtschaftsinformationen in der Bundesrepublik Deutschland übernahm. Die Möglichkeit, das gewichtige Informationspotential des eigenen Hauses auf Datenbanken zu bringen, hatte vor den Vorteilen der Fremdakquisition von Ressourcen erst zweite Priorität. ${ }^{2}$

Eine zweite, eher immaterielle Ressourcenquelle der Unternehmung besteht in ihrem Potential an aktuellen

1 Es stellt sich dem Unternehmen damit das Problem des "make or buy". Vgl. hierzu u. a. Männel, W., Die Wahl zwischen Eigenfertigung und Fremdbezug, 2. Auf1., Stuttgart 1981.

2 Vgl.o.V., Bertelsmann, a.a.0.; Hennemann-Böckels, B., Teil XVII, a.a.0.. 
Abnehmern. 1 Ausgehend von einer Situation gefestigter Kundenbeziehungen und einem in Jahren aufgebauten, positiven Image kann das Unternehmen bestrebt sein, für seine aktuellen Abnehmer ergänzende Informationsfunktionen wahrzunehmen. So bietet zum Beispiel das Verlagshaus Gruner + Jahr den Lesern seines Wirtschaftsmagazins "Capital" gegen ein entsprechendes Entgelt die Möglichkeit an, über Bildschirmtext auf eine Datenbank zuzugreifen, die Auskunft über die Konditionen alternativer KFZ-Versicherungen gibt.

\subsection{Unternehmensfähigkeiten}

Mit den Fähigkeiten der Unternehmung sind die personellen und know how-mäBigen Voraussetzungen des Anbieters im Hinblick auf die Erfüllung unterschiedlicher Funktionen angesprochen. 2 Das Know-How der Unternehmung umfaBt dabei

"alles Wissen .... das notwendig ist, bedarfsgerechte Produkte und/oder Produktionsverfahren wirtschaftlich

- zu erfinden,

- zu entwickeln und konstruieren,

- zu produzieren und

- zu vertreiben

sowie alles Wissen zur Schaffung der strukturellen Voraussetzungen." 3

Notwendige Vorbedingung für die Vermittlung bzw. Bereitstellung von Informationen ist beispielsweise eine profunde Kenntnis der spezifischen Besonderheiten dieser Informationen sowie der Art und Weise, diesen Besonderheiten

1 Nicht zu verwechseln hiermit sind die potentiellen Kunden als konstitutive dritte Dimension des Geschäftsfeldes.

2 Das amerikanische Schrifttum spricht in diesem Zusammenhang von "company skills". Vgl. Abell, D.F., Defining the Business, a.a.0., S. 183.

3 Pfeiffer, W., a.a.0., S. 434 f.. 
Rechnung zu tragen. Es leuchtet unmittelbar ein, daß es anderer Vorkenntnisse und Fähigkeiten bedarf, eine Wirtschaftsdatenbank zu erstellen, zu pflegen und zu vertrejben, als zum Beispiel eine Rechts-oder eine Technologiedatenbank aufzubauen. Letztlich sollte sich das Unternehmen entscheiden, nur solche Informationen anzubieten, hinsichtlich derer es eine "spezifische kompetenz" besitzt.

Neben der Information als solcher kann sich die Fähigkeit einer Unternehmung auch darauf beziehen, Informationen im Wege der Kooperation mit dritten Stellen zu akquirieren. In besonderem Maße gilt dies für statilche und semiöffentliche Informationsstellen. Ein aktuelles Beispiel für eine solche Kooperation liefert in diesem Zusammenhang die Saarbrücker Tageszeitung, die öffentliche Ausschreibungen auf ihrer "TED"-Datenbank zum entgeltlichen Abruf bereithält.'

\subsection{Marktbezogene Determinanten}

\subsection{Abnehmerbedarf}

Als zentrales Entscheidungskriterium bei der Auswahl der Geschäftsfeldfunktion kommt dem Abnehmerbedarf der Stellenwert einer conditio sine qua non zu. Als Maßgröße für das vorhandene Marktpotential entscheidet sich an ihm, ob das entsprechende Geschäftsfeld eine erfolgreiche Erschließung ermöglicht oder nicht. ${ }^{2}$

1 Vgl. Schumacher, D., Venker, K., Der Einsatz von Externen Datenbanken für Import, Export und Geschäftsverbindungen, in: 6. Frühjahrstagung der Online-Benutzergruppe der DGD in Neu-Isenburg vom 8. bis 10. Mai 1984. Vorträge, Hrsg.: Deutsche Gesellschaft fuir Dokumentation e.V., S. $258 \mathrm{f}$.

2 Besondere Bedeutung hat die absolute Marktgröße jedoch be $i$ der Einfihhrung und Anwendung neuer Technologien. Vgl. z. B. Bräunling, G., Harmsen, D.M., Die Förderungsprinzipien und Instrumente der Forschungs- und Technologiepolitik, Göttingen 1975, S. 39; Scholz, L., Innovationspolitik zur Modernisierung der Produktions- und Fertigungstechnik. Wirtschaftliche und technische Entwicklungstendenzen in der Industrie im Zeitraum 19651985, Berlin, München 1977, S. 26. Eine Studie der OECD stellt dazu fest, daB"..., most national markets 
Für einen Informationsanbieter stellt sich der Abnehmerbedarf dabei als der Bedarf potentieller Abnehmerunternehmen nach externen Informationen dar.

Traditionell wird der Informationsbedarf verstanden als "Art, Menge und Qualität der Informationsgiter, die ein Informationssubjekt im gegebenen Informationskontext zur Erfuillung einer Aufgabe in einer bestimmten Zeit und innerhalb eines gegebenen Raumgebietes benötigt bzw. braucht". " Konkreter definiert Brockhaus Informationsbedarf als die Summe der Informationsbedirfnisse aller Informationssubjekte. Informationsbedurfnis beschreibt dabei das Gefühl eines Mangels an Wissen, verbunden mit dem Wunsch, diesen

for new products are not big enough to amortize the costs of original innovation". OECD (Hrsg.), General Report, Gaps in Technology, Paris 1968, S. 35. Vor diesem Hintergrund erstaunt, daß das Kriterium Marktbedarf in der Literatur zur Geschäftsfeldwahl nicht explizit genannt wird. Berücksichtigung findet der Aspekt des Marktpotentials lediglich implizit in der Betrachtung des Marktlebenszyklusses und des Abnehmerverhaltens.

1 Szyperski, N., Informationsbedarf, in: HdO, 2. Aufl., Hrsg.: Grochla, E., Stuttgart 1980, Sp. 904 (im folgenden zitiert als: Szyperski, N., Informationsbedarf). Vgl. im gleichen Sinne auch Grochla, E., Garbe, H., Gillner, R., Gestaltungskriterien für den Aufbau von Datenbanken (Forschungsberichte des Landes NordrheinWestfalen 2292), Opladen 1973, S. 69; Berthel, J., Betriebliche Informationssysteme, Stuttgart 1975, S. 29; Garbe, H., Informationsbedarf, in: HdB, 4. Aufl., Hrsg.: Grochla, E., Wittmann, W., Stuttgart 1975, Sp. 1875; Abel, V., Problemorientiertes Informationsverhalten, Darmstadt 1979, S. 133.

$2 \mathrm{Vgl}$. Brockhoff, K., Informationsverarbeitung in Entscheidungsprozessen: Skizze einer Taxonomie, in:

$\mathrm{ZfB}, 53$. Jg., 1983, H. 1, S. $53 \mathrm{f}$. ( $\mathrm{im}$ folgenden zitiert als: Brockhoff, K., Informationsverarbeitung). 
zu beseitigen. 1 Legt man diese Definition zugrunde, so eröffnet sich die Möglichkeit, Informationsbedarf zu erfragen. Alternative Möglichkeiten, den Informationsbedarf zu operationalisieren, d. h. meBbar zu machen, gibt dazu Abbildung 18 im Oberblick wieder.

Im Rahmen der vorliegenden Arbeit wurde eine empirische Erhebung vorgenommen, in der u. a. am Beispiel der Informationsbereiche "Gesamtwirtschaft/Konjunktur", "Branchen/Märkte" sowie "AuBenhandel/Export" der Informationsbedarf von 182 deutschen Unternehmen gemessen wurde. ${ }^{2}$ Es zeigte sich, daß sowohl hinsichtlich der Häufigkeit von Informationsbedarfssituationen als auch in bezug auf die Zufriedenheit mit der Versorgung mit bestimmten Informationen signifikante Unterschiede in Abhängigkeit der jeweiligen Informationsbereiche auftreten. Dies gilt auch und insbesondere für die synthetisch ermittelte Maßgröße, die den Grad des unbefriedigten Informationsbedarfs wiedergibt (vgl. Tabelle 1). Gerade im Rahmen der Suche nach lukrativen Angebotsfeldern empfiehlt sich diese Maßgröße insofern, als sie über die Messung der Zufriedenheit den EinfluB konkurrierender Informationsangebote implizit mit berücksichtigt.

1 Vgl. hierzu Möhr, M., Einige Begriffe aus der Informationsökonomie und Benutzerforschung, in: $N f D$, 30. Jg., 1979, Nr. 6, S. 240; Garbe, H., a.a.0., Sp. 1875; Szyperski, N., Informationsbedarf, a.a.0., Sp. 905; Brockhoff, K., Informationsverarbeitung, a.a.0., S. 53 .

2 Zum Gegenstand und Aufbau der Untersuchung vgl. die Ausführungen in Anhang 1, S. $273 \mathrm{ff}$. dieser Arbeit. 


\begin{tabular}{|c|c|c|}
\hline Verfasser & $\begin{array}{c}\text { Operational isierungs- } \\
\text { kriterium }\end{array}$ & Skal ierung \\
\hline Brockhoff (1975) & Informationsnutzung & $\%$ \\
\hline DIHT (1979) & Informationsanfragen & $\begin{array}{l}\% \text { der innerhalb von } 5 \text { Tagen } \\
\text { bei } 10 \text { IHKs eingegangenen } \\
\text { Anfragen }\end{array}$ \\
\hline Pfaffhausen (1981) & $\begin{array}{l}\text { - bekundete Bedeutung der } \\
\text { Information (Informa- } \\
\text { tionsbedarf IB) } \\
\text { - bekundete Zufriedenheit } \\
\text { mit dem Informations- } \\
\text { stand (IS) } \\
\text { - errechnetes Informa- } \\
\text { tionsdefizit } \\
\text { (ID }=\text { IB } \times \text { IS) }\end{array}$ & 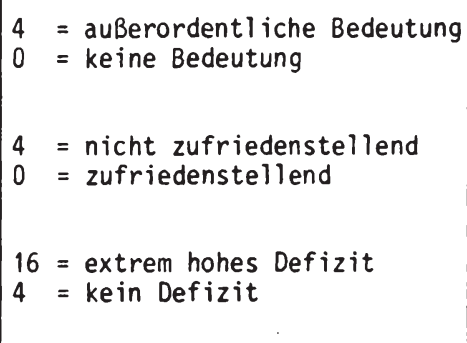 \\
\hline $\begin{array}{l}\text { Schwarting/ } \\
\text { Thoben/ } \\
\text { Wittstock (1982) }\end{array}$ & $\begin{array}{l}\text { Wichtigkeit von Informa- } \\
\text { tionen hins. einer kon- } \\
\text { kreten Entscheidungsal- } \\
\text { ternative (Export) }\end{array}$ & $\begin{array}{l}5=\text { sehr wichtig } \\
1=\text { weniger wichtig }\end{array}$ \\
\hline Kienbaum (1983) & $\begin{array}{l}\text { (vorläufig:) } \\
\text { Vergleich von Wichtig- } \\
\text { keit und Vorhandensein } \\
\text { von Informationen }\end{array}$ & $\%$ \\
\hline $\begin{array}{l}\text { eigene Befragung } \\
(1984)\end{array}$ & $\begin{array}{l}\text { - bekundete Relevanz von } \\
\text { Informationen } \\
\text { - Häufigkeit des wahrge- } \\
\text { nommenen Bedarfs an } \\
\text { Informationen (HI) } \\
\text { - Zufriedenheit mit der } \\
\text { Versorgung mit best imm- } \\
\text { ten Informationen (ZI) } \\
\text { - errechneter Grad des } \\
\text { unbefriedigten Informa- } \\
\text { tionsbedirfnisses } \\
\text { (UI = (5-HI) } \text { - ZI) }\end{array}$ & 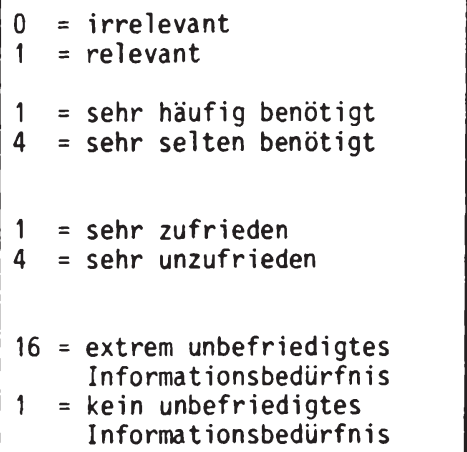 \\
\hline
\end{tabular}

Abb. 18: Alternative Ansätze zur Operationalisierung des Informationsbedarfs 


\begin{tabular}{|l|c|c|c|}
\hline $\begin{array}{l}\text { Informations- } \\
\text { bereich }\end{array}$ & $\begin{array}{l}\text { Häuf igkeit von } \\
\text { Informations- } \\
\text { bedarfssituationen }\end{array}$ & $\begin{array}{c}\text { Zufriedenheit } \\
\text { mit der Informa- } \\
\text { tionsversorgung }\end{array}$ & $\begin{array}{c}\text { Grad des } \\
\text { unbefriedigten In- } \\
\text { formationsbedarfs }\end{array}$ \\
\hline $\begin{array}{l}\text { Gesamtwirt- } \\
\text { schaftliche/ } \\
\text { Konjunktur- } \\
\text { informationen }\end{array}$ & 2.385 & 2.018 & 5.277 \\
$\begin{array}{l}\text { Branchen-/ } \\
\text { Marktinforma- } \\
\text { tionen } \\
\text { Export-/Außen- } \\
\text { handelsinfor- } \\
\text { mationen }\end{array}$ & 1.788 & 2.396 & 7.696 \\
\hline
\end{tabular}

Legende: $1 . \quad 1$ = werden sehr häufig benötigt

$4=$ werden sehr selten benötigt

2. 1 = sehr zufrieden

$4=$ sehr unzufrieden

3. Der Grad des unbefriedigten Informationsbedarfs ergibt sich aus der Multiplikation von "Häufigkeit des Bedarfs" und "Zufriedenheit mit der Versorgung", wobei die Skalierung zuvor gleichgerichtet wird (Häufigkeit $=5-x$ ). Daraus ergibt sich:

$1=$ ke in Bedarf

$16=$ extrem hoher unbefriedigter Bedarf

Tab.1: Quantifizierung des Informationsbedarfs am empirischen Beispiel 


\subsection{Konkurrenzsituation}

Die Wettbewerbssituation findet als Kriterium der Geschäftsfeldwahl in der Literatur nur implizit Berücksichtigung. 1 Dies erstaunt um so mehr, als im Rahmen der Diskussion von Markteintrittsbarrieren diesem Aspekt breite Aufmerksamkeit geschenkt wird. ${ }^{2}$ Da zwischen den Problemen der Geschäftsfeldwahl und der Antizipation von Marktbarrieren enge Beziehungen bestehen ${ }^{3}$, erscheint es konsequent, auch im Rahmen der Marktwahl die Konkurrenzlage explizit mit in das Entscheidungskalkül einzubeziehen. 4

Im vorliegenden Fall des Marktes externer Informationen hat der Konkurrenzaspekt insofern einen hohen Stellenwert, als er die besondere Angebotssituation in der Bundesrepublik Deutschland in den Vordergrund rückt. Stärker als in den meisten hochindustrialisierten Staaten ist hier die Landschaft der unternehmensrelevanten

$1 \mathrm{Vgl}$. U.a. die Ausführungen bei Abell, D.F., Defining the Business, a.a.0., S. $179 \mathrm{ff}$.

$2 \mathrm{Vgl}$. insbesondere Walters, M., a.a.0., S. $94 \mathrm{f}$. und S. $137 \mathrm{ff}$.

$3 \mathrm{Vgl}$. ebenda, S. 123.

4 Eine eingehende Diskussion der Bedeutung, Methoden und Probleme der strategischen Konkurrenzanalyse findet sich bei Allen, M.G., Strategic Planning with a competitive focus, in: The Mckinsey Quarterly, Autumn 1978, S. 6; Hoffmann, K., Die Konkurrenzuntersuchung als Determinante der langfristigen Absatzplanung, Göttingen 1979; Porter, M.E., Competitive Strategy, a.a.0., S. $47 \mathrm{ff} . ;$ Cravens, D.W., Strategic Marketing, Homewood, I11. 1982, S. $38 \mathrm{f}$. und S. 218 f.; Hoffmann, J., Die Konkurrenz-Erkennt$n$ isse für die strategische Führung und Planung, in: Praxis für strategische Unternehmensplanung, Hrsg.: Töpfer, A., Afheldt, H., Frankfurt/M. 1983, S. $183 \mathrm{ff}$. 
Sekundärquellen durch eine relativ geringe Zahl von kommerziellen Informationsanbietern, jedoch ein dichtes Netz von Anbietern unentgeltiicher Informationen gekennzeichnet. 1

Eine Bestandsaufnahme der in der Bundesrepublik Deutschland existierenden Auskunfts- und Beratungsstellen ${ }^{2}$ ermittelte, daß von den insgesamt $r d .230$ vorhandenen Informationsstellen beispielsweise allein über 50 auf das Handwerk fallen. Daneben spielen Handel, Banken und Versicherungen (einschl. Industrie- und Handelskammern), Verbraucher- und Interessenverbände, Schutzverbände sowie Arbeitgeberverbände, Berufsgenossenschaften und freiberufliche Organisationen eine dominante Rolle. 3

Besonders interessant ist in diesem Zusammenhang für das planende Unternehmen die Frage, welche der konkurrierenden Informationsquellen derzeitig in welchem Ausma $B$ in Anspruch genommen werden. Hierzu lieferte die im Rahmen dieser Untersuchung vorgenommene Befragung das in Abbildung 19 wiedergegebene Ergebnis. ${ }^{4}$ Es wird deutlich, daB Fachzeitschriften sowie Wirtschaftszeitungen und -magazine die meistgenutzten Informationsquellen darstellen. Daneben werden in der Rangfolge ihrer Bedeutung der Besuch von Messen, Informationen der jeweiligen Verbände, Kollegengespräche zwischen Unternehmen sowie das Angebot der Industrie- und Handelskammern und kreditinstitute genannt.

1 Vgl. U.a. Beyer, W., a.a.0., S. 31.

2 Vgl. Bernhardt, U., Jungjohann, K., Bestandsaufnahme von Stellen mit Informationsvermittlungs- und -beratungsfunktion in der Bundesrepublik Deutschland, in: Nachrichten für Dokumentation, 31. Jg., 1980, Nr. 415, S. $155 \mathrm{ff}$. sowie Bernhardt, U., a.a.0.

$3 \mathrm{Vgl}$. Bernhardt, U., a.a.0.; genaue Angaben zur Zahl der einzelnen Informationsstelien finden sich ebenda, S. 157.

4 Die Angaben in der zweiten Spalte geben die Ergebnisse einer Befragung von $n=152$ mittelständischen Konsumgiterunternehmen zum gleichen Themenkreis wieder (vgl. Pfaffhausen, A., a.a.0., S. 67. Sie validieren das Resultat der aktuellen Erhebung, wie ein Vergleich der ermittelten Rangfolgen in beiden Spalten belegt. 


\begin{tabular}{|c|c|c|c|c|c|}
\hline INFOR & MATIONSQUELUEN & $\begin{array}{l}\text { NUTZUNGS- } \\
\text { HÄUFIGKEIT } 1) \\
\text { (EIGENE ER- } \\
\text { HEBUNG) } \\
1984\end{array}$ & RANG & $\begin{array}{l}\text { NUTZUNGS- } \\
\text { INTENSITÄT 2) } \\
\text { (PFAFFEN- } \\
\text { HAUSEN) } \\
1981\end{array}$ & RAVG \\
\hline & IHK'S & 2,572 & 6 & 3,9 & 5 \\
\hline & VERBÄNDE & 2,239 & 4 & 5,1 & 2 \\
\hline 夏 & VERLAGE & 3,125 & 8 & & \\
\hline 桌 & KREDITINSTITUTE & 2,657 & 7 & 2,8 & 6 \\
\hline$\frac{1}{2}$ & MESSEN & 2,283 & 3 & 4,5 & 4 \\
\hline 号 & KREDITVERSICHERUNGEN & 3,794 & 14 & & \\
\hline 己 & BOTSCHAFTEN, KONSULATE & 4,191 & 20 & & \\
\hline 亗 & HANDELLSHÄUSER & 4,086 & 18 & & \\
\hline 勇 & ZOUÄMTER & 4,311 & 21 & & \\
\hline 负 & SPEDITIONEN & 4,079 & 17 & & \\
\hline 岃 & NATIONALE/INTERNATIONALE TAGUNGEN & 3,506 & 10 & & \\
\hline & BEFREUNDETE UNTERNEHMER/KOULEGEN & 2,344 & 5 & 1,9 & 7 \\
\hline & (UNIVERSITÄTS-)BIBLIOTHEKEN & 4,100 & 19 & & \\
\hline & WIRTSCHAFTSFORSCHUNGSINSTITUTE & 3,682 & 12 & & \\
\hline 岁 & INFORMATIONSVERMITTLER & 4,006 & 16 & 2,8 & 6 \\
\hline 点 & UNTERNEHMENSBERATUNGS-/WERBEAGENTUREN & 3,511 & 11 & 1,5 & 8 \\
\hline 面翌 & MARKTFORSCHUNGSUNTERNEHMEN & 3,697 & 13 & 1,5 & 8 \\
\hline 岀絰 & FACHZEITSCHRIFTEN & 1,58 & 1 & 5,3 & 1 \\
\hline F & WIRTSCHAFTSIEITSCHRIFTEN/ZEITUNGEN & 1,798 & 2 & 5,0 & 3 \\
\hline 岁 & BUNDESSTELE FUR AUSSENHANDELSINFORMATIONEN & 3,859 & 15 & & \\
\hline & AUSKUNFTEIEN & 3,391 & 9 & & \\
\hline & LEGENDE: & & & & \\
\hline & 1): $\begin{aligned} 1 & =\text { SEHR HÄUFIG } \\
5 & =\text { NIE }\end{aligned}$ & $\begin{array}{l}\text { GELMÄSSIGE NUT } \\
\text { TZNG IM PROBL } \\
\text { LTENE NUTZUNG } \\
\text { INE INTZUNG }\end{array}$ & $\begin{array}{l}\text { ZUNG } \\
\text { EMFALL }\end{array}$ & & \\
\hline
\end{tabular}

Abb. 19: Nutzungshäufigkeit externer Informationsquellen durch Unternehmen 
Wesentlich erscheint, daß mit Ausnahme der Fach-und Wirtschaftspresse alle genannten Informationsstellen ihre Informationen unentgeltlich bereithalten bzw. ihre Dienste lediglich durch einen pauschalen Mitgliedsbeitrag abgelten lassen. Hieraus ergibt sich für einen kommerziellen Informationsanbieter die schwierige Aufgabe, das Entgelt für seine Dienstleistung durch ein erhebliches Mehr an Informationsqualität und Service kompensieren und vertreten zu müssen. Angesichts dieser Situation ist es für die Auswahl einzelner Informationsfunktionen besonders aufschlußreich festzustellen, welcher Grad der Zufriedenheit mit den herkömmlichen Informationsquellen im Hinblick auf die Erfüllung alternativer Informationsfunktionen am Markt besteht. Besondere Marktchancen lassen sich bei solchen Informationsfunktionen vermuten, die der Wettbewerb bislang nur wenig zu befriedigen vermochte.

Zur beispielhaften Aufdeckung solcher relativen Konkurrenzschwächen wurde die funktionsspezifische Zufriedenheit von Entscheidungsträgern mit Quellen entgeltlicher sowie unentgelticher Informationen gemessen (vgl. Tabelle 2). Es zeigte sich, daß die herkömmlichen Quellen wesentlich besser imstande sind, ihrer Informationsfunktion im Bereich gesamtwirtschaftlicher und Brancheninformationen nachzukommen als im Bereich der Exportinformationen. Hieraus kann man schluBfolgern, daB einem leistungsfähigen Informationsdienst für den Exportbereich die vergleichsweise besten Marktchancen einzuräumen sind.

Neben der Identifikation der aktuellen Wettbewerber und ihrer relativen Bedeutung im Hinblick auf alternative Informationsfunktionen ist die Kenntnis des strategischen Verhaltens der Konkurrenz eine kritische Größe. 1 Versprechen bestimmte Informationsfunktionen prima facie

$1 \mathrm{Vgl}$. vor allem Walters, M., a.a.0., S. $139 \mathrm{f}$. 


\begin{tabular}{|c|c|c|c|c|}
\hline & \multicolumn{3}{|c|}{$\begin{array}{l}\text { Besonders zufrieden mit der Leistungs- } \\
\text { fähigkeit externer Informationsquellen } \\
\text { in bezug auf ... }\end{array}$} \\
\hline & & $\begin{array}{l}\text { Gesamtwirtschaft/ } \\
\text { Konjunktur }\end{array}$ & $\begin{array}{l}\text { Branchen / } \\
\text { Märkte }\end{array}$ & $\begin{array}{l}\text { AuBenhandel/ } \\
\text { Export }\end{array}$ \\
\hline \multirow{3}{*}{$\begin{array}{l}\text { externe } \\
\text { Infor- } \\
\text { mations- } \\
\text { quellen }\end{array}$} & $\begin{array}{l}\text { Quellen unent- } \\
\text { geltlicher In- } \\
\text { formationen } \\
\quad \text { (13) }\end{array}$ & $11,43 \%$ & $15,00 \%$ & $5,04 \%$ \\
\hline & $\begin{array}{l}\text { Quellen ent- } \\
\text { geltlicher In- } \\
\text { formationen } \\
\text { (8) }\end{array}$ & $16,26 \%$ & $17,55 \%$ & $3,23 \%$ \\
\hline & $\begin{array}{l}\text { Quellen ins- } \\
\text { gesamt } \\
\qquad(21)\end{array}$ & $13,27 \%$ & $15,97 \%$ & $4,39 \%$ \\
\hline
\end{tabular}

Tab. 2: Zufriedenheit mit externen Informationsquellen in bezug auf alternative Informationsbereiche

gute Marktaussichten, so können diese zukünftig dadurch infrage gestellt werden, daB die Konkurrenz ihren Wettbewerb verschärft oder aber beispielsweise selber Anstrengungen zum Aufbau elektronischer Informationssysteme unternimmt. 1

Vor diesem Hintergrund ist eine eingehende Konkurrenzanalyse dahingehend erforderlich, welche Aktivitäten derzeitig die etablierten Informationsstellen im Hinblick auf das Angebot elektronisch zugreifbarer Infor-

1 Solche Anstrengungen zeichnen sich in jüngster Vergangenheit $z$. B. be $i$ den Industrie- und Handelskammern ab. So haben z. B. die drei IHKS Flensburg, Kiel und Lübeck ein Btx-Programm entwickelt, das die Abfrage von Konjunkturdaten aus Industrie und Mandel für Schleswig-Holstein im Dialog ermöglicht. Vgl. o.V., Konjunkturdaten aus dem externen Btx-Rechner, in: FIBA REPORT, 4. Jg., September 1983, Nr. 17, S. 12 . 
mationen an den Tag legen ${ }^{1}$ und welche künftigen Konkurrenten mit welchen Informationen möglicherweise zu welchem Zeitpunkt auf den Markt stoßen werden. ${ }^{2}$

1 Ein Beispiel solcher (bereits abgeschlossener) Aktivitäten lieferte $z$. B. der Zentralverband der elektrotechnischen Industrie e.V. (ZVEI), der in enger Zusammenarbeit mit der Firma Taylorix Tymshare ein online verfügbares Datenbank-Informationssystem für seine Mitglieder implementierte. Vgl. ZVEI (Hrsg.), Informationsbroschüre zum Datenbank-Informationssystem ELTRODAT, Frankfurt 1984. Bemerkenswert sind in diesem Zusammenhang auch die Maßnahmen der VWD Vereinigte Wirtschaftsdienste in Richtung eines Btx-gestützten "Management-Info-Service" für Entscheidungsträger der Wirtschaft. "Die Zusammenstellung beinhaltet Kurzberichte über Finanz-, Geld- und Kapitalmärkte, Informationen über Großunternehmen und Organisationen, marktbewegende Ereignisse, Geldmengenentwicklung, Konjunktur-Eckdaten sowie auBerordentliche Bewegungen an den in-und ausländischen Märkten." Poppe, R., Zukünftige Incormationswege dar Vereinigten Wirtschaftsdienste (VWD) durch Einsatz von Btx im IBMRechenzentrum, in: Proceedings des Btx-Kongresses ' 83 vom 29. -30. November 1983 in Berlin, K 12, S. 5.

2 Von besonderem Interesse sind dabei $z$. Zt. die an Intensität zunehmenden Aktivitäten ausländischer Informationsanbieter. Verbreitet findet man die Auffassung, daß sich deutsche Anbieter datenbankgespeicherter Informationen beachtlicher Anstrengungen unterziehen muissen, um in der Zukunft dem Dbergewicht ausländischer, insbesondere amerikanischer Hosts ein entsprechendes Gegengewicht aufbauen zu können. Vgl. hierzu insbesondere Schulte-Hillen, J., von Wietersheim, B., I IUD-onl ine-Datenbankbenutzung in der Bundesrepublik Deutschland. Gefahren der Abhängigkeit von aus 1 ändischen Datenbanken für Wissenschaft und Forschung, Wirtschaft und Industrie, München, etc. 1984. 


\subsection{Durchführung der Funktionsauswahl}

Grundlage der Auswahlentscheidung bildet die Bewertung und Verknüpfung der einzelnen Determinanten in bezug auf jede der zur Wahl stehenden Informationsfunktionen. Das Vorgehen entspricht dabei weitgehend den bekannten Punktbewertungsverfahren, die verbreitet bei der Beurteilung von Neuprodikten oder organisatorischen Strukturalternativen ${ }^{2}$ Anwendung finden. Die relevanten Determinanten werden tabellarisch aufgelistet, mit einem ihrer Bedeutung entsprechenden Gewicht versehen und auf eine einheitliche Ratingskala transformiert. Auf jeder dieser Skalen erhält jede einzelne Informationsfunktion einen Punktwert, der ausdrückt, inwieweit die Informationsfunktion dem jeweiligen Entscheidungskriterium entspricht. Die Summe der gewogenen Ratingwerte ergibt schlieBlich den Gesamtwert der Informationsfunktion:

1 Zur Punktbewertung im Rahmen der Neuproduktplanung vgl. Schmitt-Grohé, J., Produktinnovation - Verfahren und Organisation der Neuproduktplanung, Wiesbaden 1972, S. $81 \mathrm{ff}$.; Schlegel, H., Punktbewertungsmodelle, in: Erfolgskontrolle im Marketing, Hrsg.: Böcker, F., Dichtl, E., Berlin 1975, S. $154 \mathrm{ff.}$; Meffert, H., Marketing, a.a.0., S. $365 \mathrm{ff.}$; Brose, P., Planung, Bewertung und Kontrolle technologischer Innovationen, Berlin 1982 .

2 Zur Anwendung der Punktbewertung für die Auswahl einer organisatorischen Strukturalternative vgl. Meffert, H., Die Einführung des Kundenmanagements als Problem des geplanten organisatorischen Wandels, in: Humane Personal- und Organisationsentwicklung, Festschrift für Guido Fischer, Hrsg.: Wunderer, R., Berlin 1979, S. $306 \mathrm{ff}$. 


$$
W F_{i}=\sum_{j=1}^{4} w_{i j} \cdot g_{j}
$$

Hierbei bezeichnen

$$
\begin{aligned}
& W_{i}=\text { den Gesamtwert der Informationsfunktion } i \\
& w_{i j}=\text { den Wert der Informationsfunktion } i \text { in be- } \\
& \text { zug auf die Determinante } j \\
& g_{j}=\text { das (Bedeutungs-) Gewicht der Determinante } j
\end{aligned}
$$

Im einzelnen gilt dabei:

$$
\begin{aligned}
& \mathrm{j}=1 \text { = Unternehmensressourcen } \\
& j=2 \text { = Unternehmensfähigkeit } \\
& j=3=\text { Abnehmerbedarf } \\
& j=4=\text { Konkurrenzsituation } \\
& i=1 \text { = Bereitstellung von Information zum Thema } \\
& \text { "Personal/Aus - und Weiterbildung" } \\
& i=8 \text { = Bereitstellung von Informationen zum Thema } \\
& \text { "Technologie/Produktinnovation" }
\end{aligned}
$$

Entscheidungskriterium für die Auswahl oder Ablehnung einer jeweiligen Informationsfunktion ist schließlich die Höhe des Annahme-/Zurückweisungspunktwertes WF krit . Diese ergibt sich unmittelbar aus der von dem Unternehmen verfolgten Geschäftsfeldstrategie: Plant das Unternehmen ein konzentriertes Vorgehen, so wird es den Punktwert relativ hoch ansetzen, wogegen es bei einer undifferenzierten bzw. einer differenzierten Geschäftsfeldstrategie zunächst einen eher niedrigen Punktwert wählen wird.

Abbildung $2 C$ zeigt die Vorgehensweise der Punktbewertung beispielhaft auf. ${ }^{1}$ Es ergibt sich, daB das exem-

$1 \mathrm{Vgl}$. zu den zentralen Kritikpunkten an Punktbewertungsmodelien z. B. Schmitt-Grohé, J., a.a.0., S. $86 \mathrm{ff} . ;$ Andritzky, K., Der Einsatz von Scoringmodellen für die Punktbewertung, in: DU, 30. Jg., 1976, H. 1, S. 21 ff.; Meffert, H., Marketing, a.a.o., S. 360 . 
plarisch betrachtete Unternehmen als erfolgversprechende Funktionen die Informationsbereiche Gesamtwirtschaft, Branche und Export für sein Geschäftsfeld selektiert $\left(w_{k r i t}>60\right)$. Es stellt sich nunmehr die Frage, unter Einsatz welcher Technologien die Bereitstellung der Informationen erfolgen soll.

\begin{tabular}{|c|c|c|c|c|c|}
\hline \multirow[b]{2}{*}{$\begin{array}{l}\text { Informations- } \\
\text { funktion: } \\
\text { Versorgung mit } \\
\text { Informationen } \\
\text { zum Thema ... }\end{array}$} & \multicolumn{5}{|c|}{ Determinanten der Funktionswahl } \\
\hline & $\begin{array}{c}\begin{array}{c}\text { Unterneh- } \\
\text { mens- } \\
\text { ressourcen }\end{array} \\
g=0,3\end{array}$ & $\begin{array}{l}\text { Unter- } \\
\text { nehmens- } \\
\text { fähigkeit } \\
g=0,2\end{array}$ & $\begin{array}{l}\begin{array}{c}\text { Abnehmer- } \\
\text { bedarf }\end{array} \\
g=0,4\end{array}$ & $\begin{array}{c}\text { Konkurrenz- } \\
\text { situation } \\
g=0,1\end{array}$ & $\begin{array}{c}\text { Gesamt- } \\
\text { wert }\end{array}$ \\
\hline $\begin{array}{l}\text { Personal/ } \\
\text { Aus- und } \\
\text { Weiterbildung }\end{array}$ & 5 & 10 & 80 & 20 & 37,5 \\
\hline $\begin{array}{l}\text { AuBenhandel/ } \\
\text { Export }\end{array}$ & 40 & 80 & 90 & 90 & 73,0 \\
\hline $\begin{array}{l}\text { Wirtschafts- } \\
\text { recht }\end{array}$ & 5 & 10 & 30 & 30 & 18,5 \\
\hline $\begin{array}{l}\text { Gesamtwirt- } \\
\text { schaft l iche } \\
\text { Entwicklung/ } \\
\text { Konjunktur }\end{array}$ & 80 & 80 & 40 & 50 & 61,0 \\
\hline $\begin{array}{l}\text { Branchen/ } \\
\text { Märkte }\end{array}$ & 90 & 80 & 80 & 50 & 80,0 \\
\hline $\begin{array}{l}\text { Anschriften/ } \\
\text { Firmenaus- } \\
\text { künfte }\end{array}$ & 60 & 60 & 60 & 40 & 58,0 \\
\hline $\begin{array}{l}\text { Finanzen/ } \\
\text { Steuern }\end{array}$ & 5 & 10 & 50 & 30 & 26,5 \\
\hline $\begin{array}{l}\text { Technologie/ } \\
\text { Produktinno- } \\
\text { vation }\end{array}$ & 10 & 10 & 70 & 70 & 40,0 \\
\hline
\end{tabular}

Legende: $w_{i j \max }=100$ (sehr günstig) $w_{i j \min }=0 \quad$ (sehr ungünstig)

Abb. 20: Punktbewertungsverfahren zur Auswahl der Geschäftsfeld-Funktion 


\subsection{Zuordnung der Technologiedimension als Feinauswahl}

"Technologien beschreiben alternative Wege, auf denen eine bestimmte Funktion für einen Abnehmer erfüllt werden kann". 1 Dieser Begriffsinterpretation folgend sind nach der Konkretisierung der Funktion im Sinne der "unternehmensexternen Informationsversorgung" die zur Wahl stehenden technologischen Alternativen ${ }^{2}$ als Formen der Informationsversorgung zu bewerten.

Die Auswahl der Technologiealternativen wird im wesentlichen von zwei Gruppen von Determinanten bestimmt. Zum einen sind Faktoren zu berücksichtigen, die Aussagen über die generelle Bedeutung der Technologie - unabhängig von der jeweils zu erbringenden Funktion - beinhalten. Zum anderen kommt im Hinblick auf die Effektivität der Technologie solchen Faktoren eine entscheidende Bedeutung $z u$, die explizit auf die geforderte Funktionserfiillung Bezug nehmen.

\subsection{Funktionsunabhängige Determinanten der Technolo- giewahl}

Den zentralen Ausgangspunkt der Diskussion funktionsunabhängiger Determinanten bildet die Tatsache, daß Technologien dynamischen Prozessen unterliegen. Xhnlich wie Produkte oder Produktsparten durchlaufen Technologien Lebenszyklen, an deren Ende sie durch neue technologische Entwicklungen ganz oder zumindest teilweise abge1 öst werden. 3

1 Abel1, D.F., Defining the Business, a.a.0., S. 172.

2 Zur überschneidungsfreien Begriffsverwendung sollen im folgenden "Technologien" die Obertragungsmedien (z. B. Bildschirmtext), "Technologiealternativen" die Formen der Informationsversorgung unter Einsatz dieser Medien bezeichnen.

$3 \mathrm{Vgl}$. zum Technologielebenszyklus insbesondere Pfeiffer, W., inetze, G., Schneider, W., Amler, R., TechnologiePortfolio zum Management strategischer Zukunftsfelder, Innovative Unternehmensführung, Hrsg.: Pfeiffer, W., Bd. 7, Göttingen 1982, S. 44 ff. (im folgenden zitiert als: Pfeiffer, W. et al., Technologie-Portfolio). 
Diesem Effekt der Technologiesubstitution ist in der jüngeren Literatur zur strategischen Unternehmensfühung verstärktes Interesse zuteil geworden. ${ }^{1}$ Die Einschätzung der Technologie als entscheidende Erfolgskomponente, verbunden mit der Erkenntnis, daB sich die Nachfrage- und Produktzyklen, insbesondere aber auch die Technologiezyklen im Zeitablauf immer mehr verkürzen, hat zu der Forderung nach einem strategischen Technologiemanagement geführt. 2

Abbildung 21 zeigt für den Markt datenbankgestützter Information und Dokumentation den zeitlichen Verlauf von Nachfrage-, Technologie- und Produktlebenszyklen beispielhaft auf.

Der erste Technologiezyklus steht - ausgehend von den späten 60er Jahren - im Zeichen des Auf- und Ausbaus von offline-Information Retrieval-Systemen. Als Produkte entstanden hier die verschiedenen physischen outputs von Datenbankrecherchen, die von geschulten Dokumentaren bzw. Informationsvermittlern durchgeführt wurden (Referatedienste, Bibliographien und Indices, Profildienste, retrospektive Recherchen, Auskiunfte, etc.). ${ }^{3}$

Der zweite Technologiezyklus beginnt mit der Einführung des online-Information Retrieval. Durch die Möglichkeit der Datenfernübertragung wird hierbei der schnelle Zugriff auf verschiedene, auch weit entfernte Datenbasen realisiert. 4 Unterschiediche Produkte ergeben sich im Rahmen dieser Technologie durch die Anwendung unterschiedlicher Suchstrategien (Grobrecherche, Fein-oder

$1 \mathrm{Vgl}$. Abell, D.F., Defining the Business, a.a.0., S. 172 f.; Meffert, H., Strategische Planungskonzepte, a.a.0., S. 197 .

$2 \mathrm{Vgl}$. Meffert, H., Strategische Planungskonzepte, a.a.0., S. 197 .

$3 \mathrm{Vgl}$. GID (Gesellschaft für Information und Dokumentation mbH) (Hrsg.), a.a.0., S. $12 \mathrm{f}$..

$4 \mathrm{Vgl}$. Panyr, I., Lehmann, E., Entwicklungsperspektiven zukünftiger Informationssysteme, in: Entwicklungsperspektiven zukünftiger Informationssysteme, Hrsg.: Fachgruppe Presse-, Rundfunk- und Filmarchivare im Verein Deutscher Archivare, München, etc. 1983, S. $120 \mathrm{f}$.. 


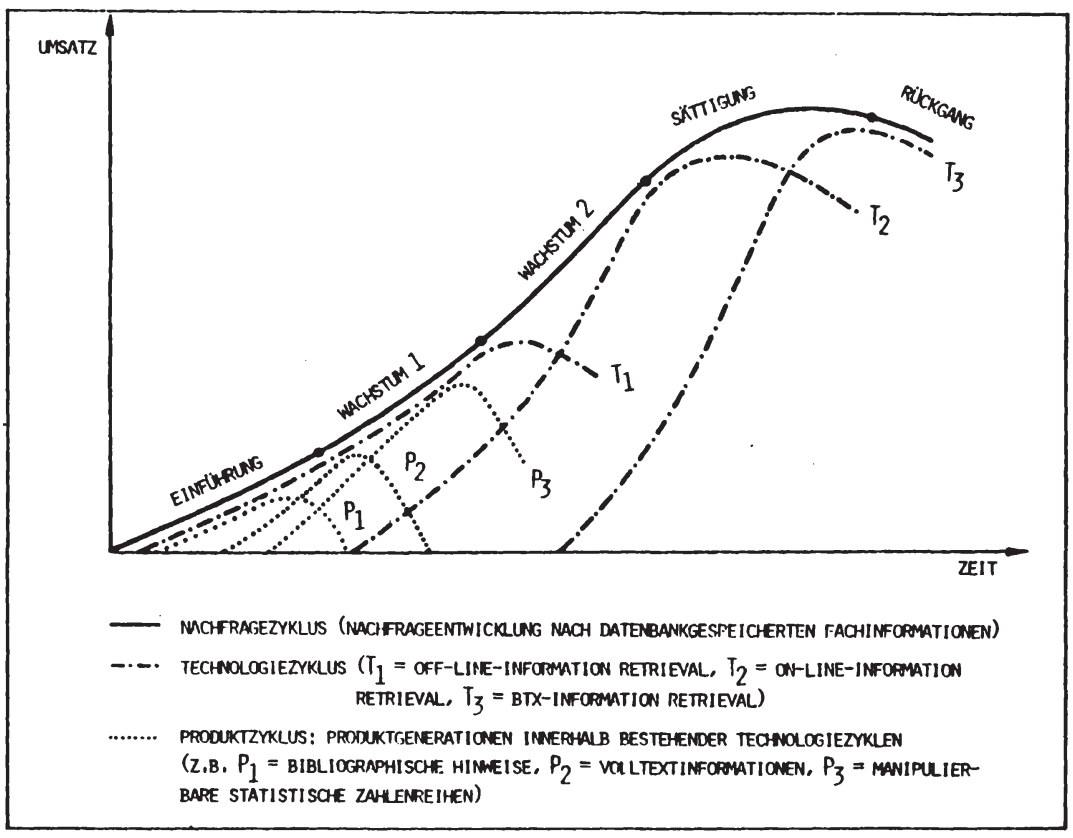

Abb. 21: Nachfrage-Technologie-Zyklus datenbankgespeicherter Fachinformationen

(Quelle: in Analogie zu Meffert, H., Strategische Planungskonzepte, a.a.0., S. 197)

Freitextrecherche) sowie aus der Implementierung von Verfahren unterschiedlicher Intelligenz zur optimierung der Rechercheergebnisse (intellektuelle suchauftragserweiterung vs. Relevanzfeedbackverfahren). 1

Der erst im Entstehen begriffene, dritte Technologiezyklus ist gekennzeichnet durch den Einsatz von Bildschirmtext zur online-Abfrage. ${ }^{2}$ Sowohl durch den AnschluB ex-

1 Vgl. Panyr, I., Lehmann, E., a.a.0., S. $125 \mathrm{ff}$. und die dort angegebene Literatur.

$2 \mathrm{Vgl}$. insbes. Langenbucher, W., Bildschirmtext und die Zukunft von Information und Dokumentation, in: Entwicklungsperspektiven zukünftiger Informationssysteme, Hrsg.: Fachgruppe Presse-, Rundfunk- und Filmarchivare im Verein Deutscher Archivare, München, etc. 1983, S. $33 \mathrm{ff.}$. 
terner Rechner an das Btx-Netz als auch durch direktes Anwählen eines Datenbanknetzwerkes ( $z$. B. Euronet oder Datex $P$ ) wird zukünftig in wachsendem Umfang die Nutzung, von Datenbanken im Btx-online-Bereich stattfinden. 1

Einen innovativen Ansatz zur gezielten Planung des zukünftigen Technologieeinsatzes haben Pfeiffer et al. entwickelt. ${ }^{2}$ Der Ansatz stellt die Größe "Technologieattraktivität", im wesentlichen Weiterentwickelbarkeit und Umfang des Anwendungsspektrums einer Technologie, der GröBe "Ressourcenstärke", als MaB der gegenwärtigen Beherrschung einer Technologie, gegenüber. Aus der Konstellation der beiden Größen leitet er dann für jede im Unternehmen angewandte Technologie differenzierte "Förderungsstrategien" ab. ${ }^{3}$ Die Obertragung dieses Verfahrens auf das Problem der Technologiebestimmung im Rahmen der Geschäftsfeldwahl ermöglicht es, in systematischer Weise eine Determinierung der geschäftsfeldoptimalen Technologie durchzuführen.

$1 \mathrm{Vgl.}$ Langenbucher, W., a.a.0., S. $40 \mathrm{f}$.

$2 \mathrm{Vgl}$. Pfeiffer, W. et al., Technologie-Portfolio, a.a.0., sowie Pfeiffer, W., Amler, R., Schäffner, G.J., Schneider, W., Technologie-Portfolio-Methode des Strategischen Innovationsmanagements, in: $\mathrm{ZfO}$, 52. Jg., 1983, H. 5-6, S. 252 ff. (im folgenden zitiert als: Pfeiffer, $W$. et al., Innovationsmanage-. ment).

$3 \mathrm{Vgl}$. Pfeiffer, W. et al., Technologie-Portfolio, a.a.0., S. $77 \mathrm{ff}$.; Pfeiffer, W. et al., Innovationsmanagement, a.a.0., S. 253. Ahnliche Ansätze finden sich bei Krubasik, E.G., Strategische Waffe, in: Wh, Nr. 25, 18.06.82, S. $28 \mathrm{ff}$. Sowie bei Brose, P., Corsten, H., Technologie-Portfolio als Grundlage von Innovations - und Wettbewerbsstrategien, in: JdAV, 1983, S. $344 \mathrm{ff}$.. 


\subsection{Ressourcenstärke}

Die Ressourcenstärke umfaßt als unternehmensinterne Größe sowohl die Aspekte der unternehmensbezogenen Fähigkeiten als auch der unternehmenseigenen Ressourcen. ' Sie repräsentiert die zur Realisierung des Technologiepotentials nötigen, im Unternehmen bereits vorhandenen Mittel in Relation zur Konkurrenz. ${ }^{2}$ Je mehr die Ressourcen in bezug auf eine Technologie der allgemeinen technischen und wettbewerblichen Entwicklung entsprechen, desto größer ist die Ressourcenstärke.

In Abweichung zu Pfeiffer et al. läßt sich die Ressourcenstärke in bezug auf alternative elektronische Obertragungsmedien durch eine Aufschliusselung in verschiedene Einzelgesichtspunkte ermitteln (vgl. Abbildung 22).

Der Differenzierung folgend bestimmen sowohl der Stand der Anwendung einer Technologie in der Unternehmung als auch die know how-Stärke als konstitutive Merkmale der Ressourcenstärke die Technologieentscheidung. Hat das Unternehmen beispielsweise seit Beginn der Diensteinführung von Bildschirmtext einen $B t x$-Rechnerverbund realisiert (Dauer der Anwendung) und wird dieser durch verschiedene Anwendungen bereits erfolgreich genutzt (Intensität des Einsatzes), so liefert dies einen Hinweis für die vorrangige Eignung der Technologie Bildschirmtext in bezug auf die geplanten Geschäftsfeldaktivitäten. Dies gilt um so mehr, wenn der know how-stand durch die bisherigen Btx-Anwendungen relativ zur Konkurrenz als hoch eingestuft und die Stabilität dieses know hows durch einen Stab spezialisierter Btx-Mitarbeiter als gesichert angesehen werden kann.

1 In modifizierter Form sind beide Aspekte bereits für die Auswahl der Geschäftsfeldfunktion herangezogen worden.

2 Vgl. Pfeiffer, W. et al., Technologie-Portfolio, a.a.0., S. 89 ff.; Pfeiffer, W. et al., Innovationsmanagement, a.a.0., S. 253. Brose und Corsten sprechen in diesem Zusammenhang von Innovationspotential; vgl. Brose, P., Corsten, H., a.a.0., S. $348 \mathrm{ff}$.. 


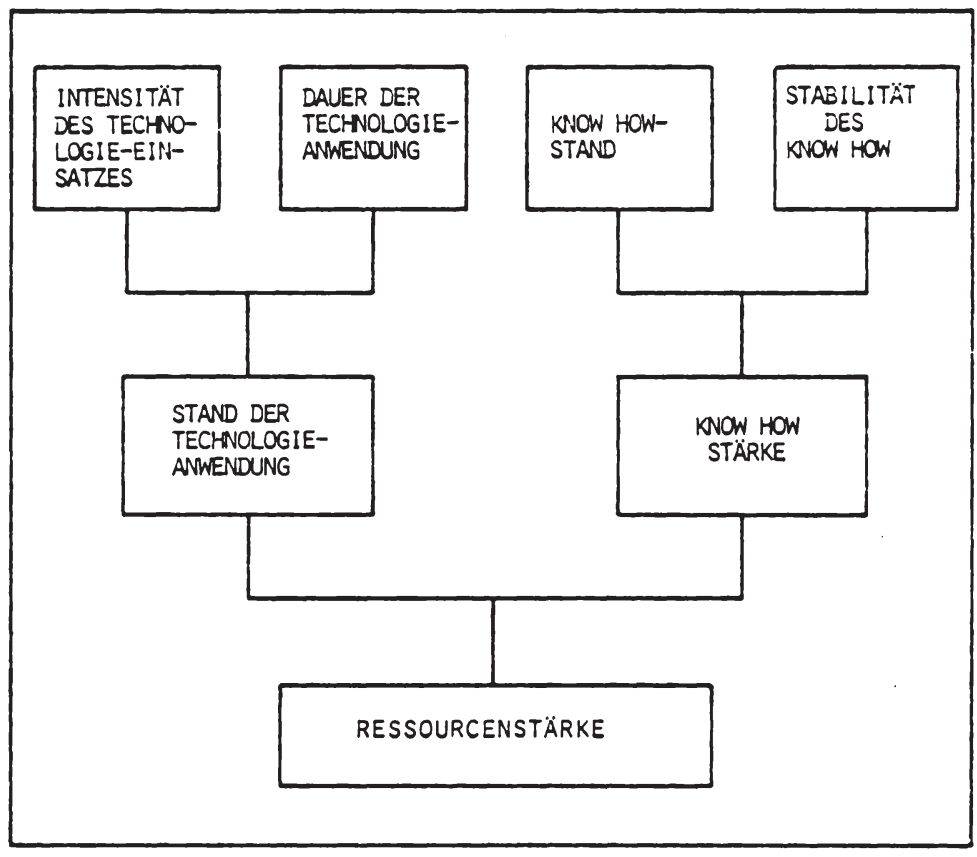

Abb. 22: Ermittlung der Ressourcenstärke

(Quelle: in Anlehnung an Pfeiffer, W. et al. , TechnologiePortefolio-Methode, a.a.0., S. 259)

\subsection{Technologieattraktivität}

Zur Beurteilung der Technologieattraktivität (vgl. Abbildung 23) spielen potentialseitig die Weiterentwickel barkeit der Technologie sowie der Zeitbedarf bis zur nächsten Entwicklungsstufe (Entwicklungsdynamik) eine entscheidende Rolle. 1 Die Stufen der Weiterentwicklung korrespondieren dabei mit dem Stand, den die Technologie zur Zeit in ihrem Lebenszyklus erreicht hat.

$1 \mathrm{Vgl}$. Pfeiffer, W. et al., Technologie-Portfolio, a.a.0., S. $85 \mathrm{ff} . ;$ Pfeiffer, W. et al., Innovationsmanagement, a.a.0., S. 258. Brose und Corsten fassen die Technologieattraktivität unter dem Begriff "Marktfähigkeit" zusammen. 


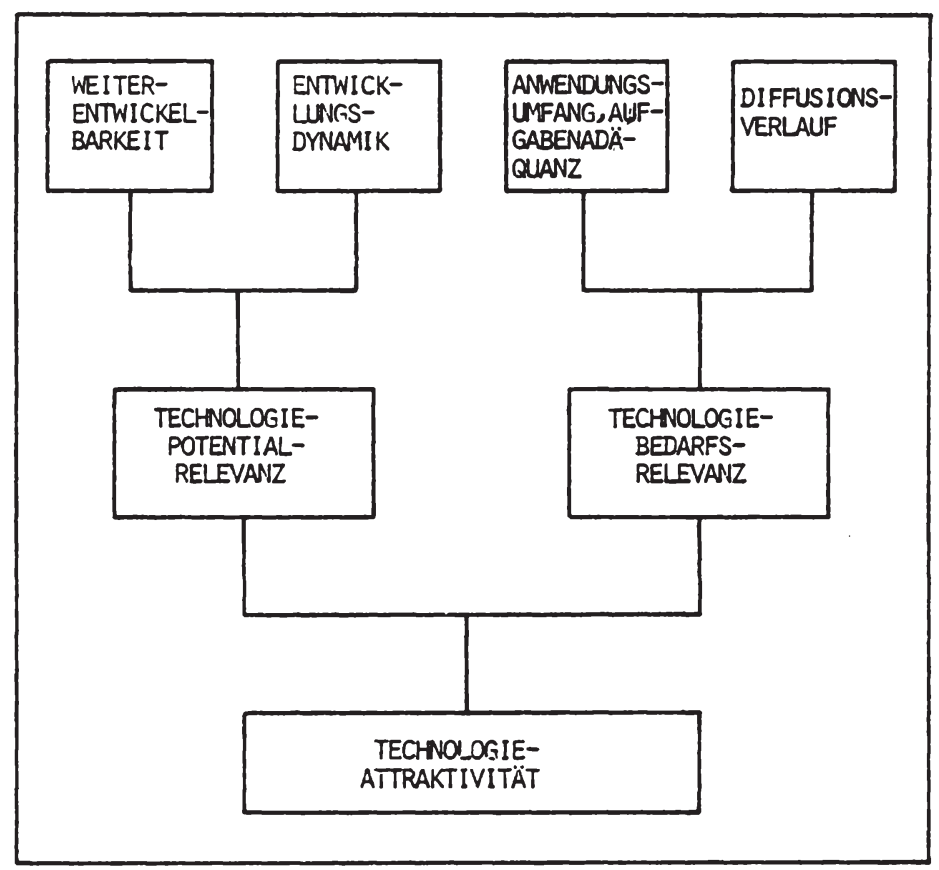

Abb. 23: Ermittlung der Technologieattraktivität

(Quelle: in enger Anlehnung an Pfeiffer, W. et al., Technologie-Portfol io-Methode, a.a.0., S. 259)

Bildschirmtext befindet sich beispielsweise nach der Testphase (Prestel-Standard) seit September 1983 in der Einführungsphase, in welcher es - auf einer höheren Entwicklungsstufe - mit neuen Leistungsmerkmalen (CEPTStandard) einem wachsenden Nutzerkreis zur Verfügung steht. 1 Noch vor dem Obergang von der Einfuhrungs - in die Wachstumsphase durchläuft das neue Medium derzeit mehrere weitere Entwicklungsstufen, die auf die Attraktivität dieser Technologie einen weitreichenden Einfluß

1 Zur technischen Entwicklung des Bildschirmtextsystems in der Bundesrepublik Deutschland vgl. z. B. Drewalowski, H.F., Bildschirmtext - ein Medium steigt aus den Kinderschuhen, in: Praxis der Werbung, 3. Jg., 1983, Nr.4, S. $11 ; 0 . V$., Weltweites Potpourri, in:Wh, Nr. 6, 03.02.84, S. $80 \mathrm{ff} .$. 
haben. Zu nennen ist $z$. B. die Herstellung der Kompatibilität von Bildschirmtext und Microcomputer ${ }^{1}$, die Entwicklung des Miniaturdecoders "EUROM"2, etc. Abbildung 24 gibt zur Veranschaulichung die einzelnen Entwicklungsstufen des Bildschirmtextsystems in einem approximativen Gesamtüberblick wieder.

Aus der Perspektive seiner rapiden technologischen Entwicklung kommt dem Bildschirmtext ein hohes MaB an Technologieattraktivität $z u^{3}$ Die zentrale Frage stellt sich jedoch nicht nur nach der technischen Fortschrittlichkeit, sondern auch nach dem vorhandenen Grad der Technologie-Bedarfsrelevanz. Ein zentraler Aspekt dieser Relevanz betrifft die Frage, in welchem Ausma $B$ das neue Medium von der betrachteten Zielgruppe (institutionelle Anwender) adoptiert wird. ${ }^{4}$ Diesem Aspekt kommt um so gröBere Bedeutung $z u$, als die einzelnen Technologien in partiell konkurrierendem Verhältnis zueinander stehen (so z. B. Telex und Teletex, Bildschirmtext und Datenfernübertragung).

$1 \mathrm{Vgl}$. o.V., Bildschirmtext und Mikrocomputer - Voraus setzungen und Anwendungen, in: Fiba Report, 4. Jg., Oktober 1983, Nr. 19, S. 7; Maciejewski, P.G., Btx und Mikrocomputer, in: MJ, 13. Jg., 1984, Nr. 2, S. 177.

$2 \mathrm{Vgl}$. o.V., Der Eurom ist noch für manche Oberraschung gut, in: Btx-Praxis, Oktober/November 1983, $\mathrm{Nr}$. 5, S. $28 \mathrm{f.;}$ 0.V., Warten auf den Eurom, in: WW, $\mathrm{Nr}$. 6, $03.02 .84, \mathrm{~S} .72 \mathrm{f}$.

3 "Hohe Attraktivität wird dynamischen Technologien beigemessen, reife Technologien sind in diesem Sinne dagegen unattraktiv". Pfeiffer, W. et al., TechnologiePortfolio, S. 85.

$4 \mathrm{Vgl}$. Z. B. Pfeiffer, W. et al., Technologie-Portfolio, a.a.0., S. 88. "Der Nutzen eines Kommunikationsmediums hängt entscheidend davon ab, wieviele Kommunikationspartner über das Medium verbunden sind". "Bis zu einer gewissen Teilnehmerzahl, die als "kritische Masse" bezeichnet werden kann, ist ... der vermutete Kommunikationsnutzen nur gering". Schellhaas, H., Schönecker, H., Kommunikationstechnik und Anwender. Akzeptanzbarrieren, Bedarfsstrukturen, Einsatzbedingungen, Forschungsprojekt Bürokommunikation, Bd. 1, Hrsg.: Picot, A., Reichwald, R., München 1983, S. 35. 
TECHNOLOGISCHER

ENTWICKLUNGSSTAND

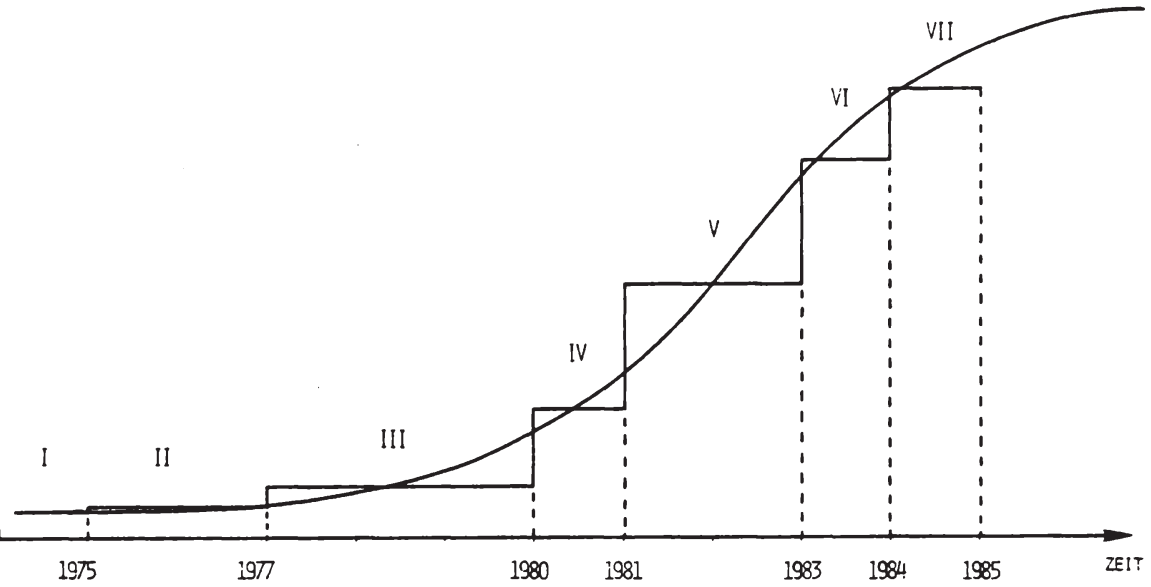

I : ENTWICKUNG DES RRITISCHEN VIEWOATA-SYSTEMS "PRESTEL"

II : ÜBERNAME DES PRESTEL-SYSTEAS DURCH DIE DBP

III : ERSTE NICMTÖFFENTICHE VERSUCHE BDW. TESTPHASE

IV : ZISCHALITNG EXTERNER RECHNER (RECHNERVERBUND.

$V$ : FESTLESUNG DES INTEPPATIONALEN CEPT-STANDARDS, EINRIOTTLNG DES MITTEILUNGSDIENSTES UND ENTWICKLUNG EXTERNER DECODE?

VI : HERSTELLUNG DER KOMPATIBILITÄT MIT MICROCOMPUTERN "UND ENTWICKLLNG DES MINIATUPDECODERS "ELROM"

VII : ENTWICKLUNG NEUER ZUIRIFFSTECHNIKEN UND REALISIERUNG DES MEDIENERBUNOES MIT DER BILDPLATTE

Abb. 24: Technologie-Lebenszyklus und Stufen der Weiterentwicklung des Bildschirmtextsys tems 
Abbildung 25 zeigt in diesem Zusammenhang den prognostizierten Diffusionsverlauf der im vorliegenden Beispiel betrachteten Technologien. Abbildung 26 ergänzt das Bild um den Planungsstand deutscher Unternehmen im Hinblick auf die küftige Nutzung elektronischer Telekommunikationsdienste, der im Rahmen der vorliegenden Untersuchung empirisch erhoben wurde.

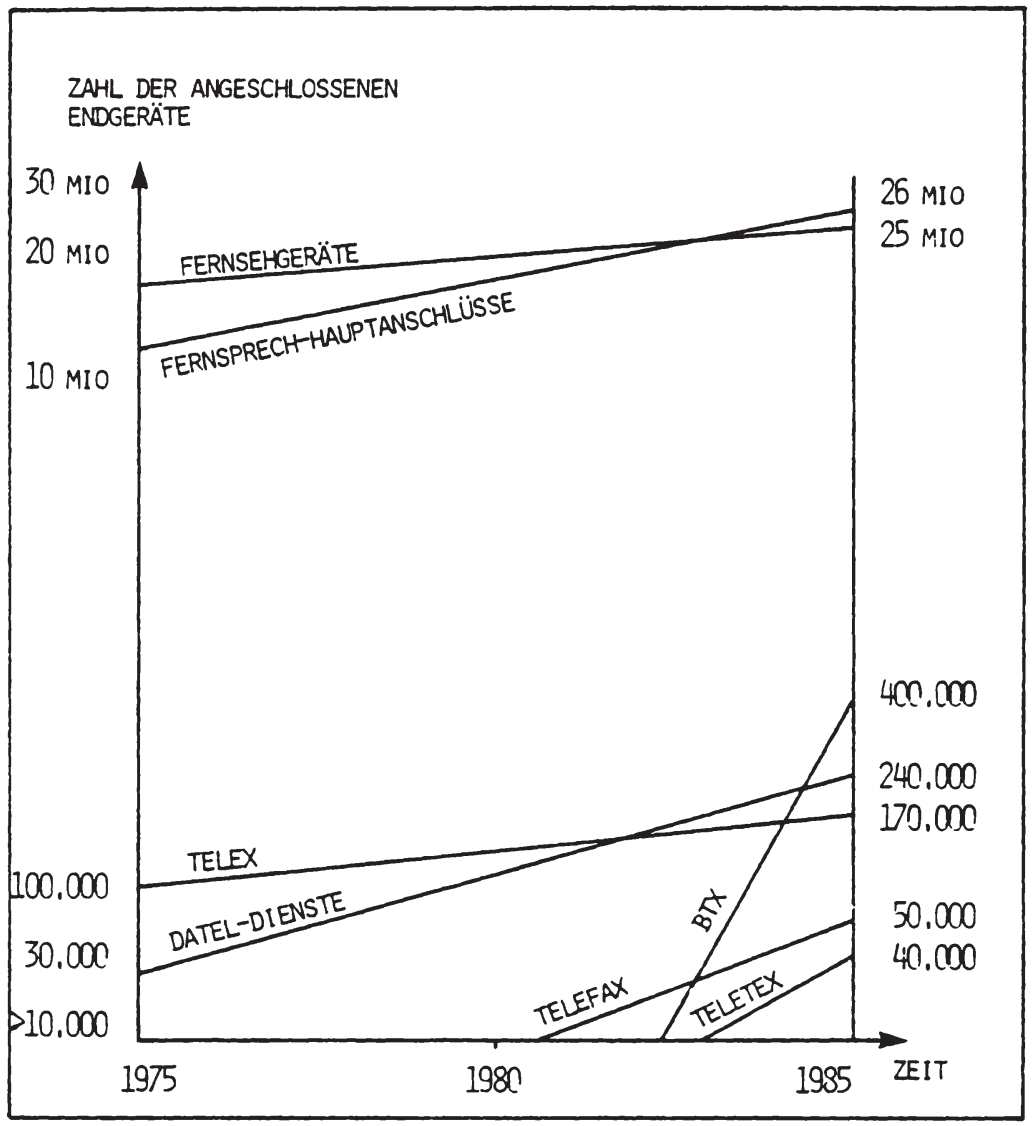

Abb. 25: Diffusionsverlauf telekommunikativer übertragungs formen (1975 - 1985)

(Quelle: SEL, zitiert in Markenartikel, 1983, H. 9, S. 426) 
Bemerkenswert erscheint hier der hohe Anteil der Unternehmen, deren Planungen den Einsatz von Teletex und Bildschirmtext vorsieht. Während sich die Angaben beider Quellen im Fall des Bildschirmtextes wechselseitig bestätigen, zeigt die Diskrepanz in den Voraussagen zu Teletex, daß Prognosen über die Adoption der einzelnen Technologien mit zum Teil erheblichen Unsicherheiten behaftet sind. SchlieBlich ist in der Zukunft auch ohne weiteres vorstellbar, daB ein Funktionsangebot eines Dienstleistungsunternehmens einem interessierten potentiellen Abnehmer den AnstoB dazu gibt, ein bestimmtes, bislang njcht genutztes Obertragungsmedium nunmehr einzusetzen.

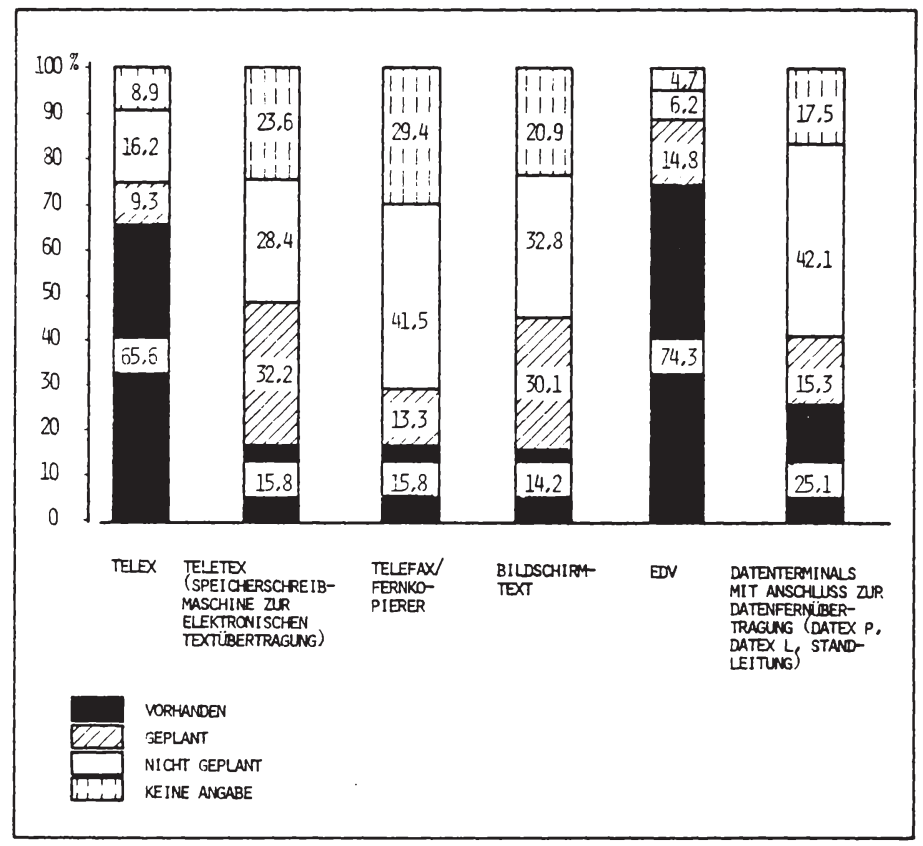

Abb. 26: Ausstattungsstand und Stand der Planung hinsichtlich der Einrichtung von Telekommunikationsanschlüssen in der Bundesrepublik Deutschland

1 Für das englische Prestel-System stellte Bury beispielsweise fest, daß ... "businesses become Prestel users because they have a direct need for the information on some page or pages". Bury, P., Markets for Prestel, in: 4 th International Online Information Meeting, London 9 - 11 December 1980, Hrsg.: Learned Information (Europe) Ltd., oxford, N.J. 1980, S. 102. 
Der Anwendungsumfang sowie die Aufgabenadäquanz einer Technologie können im vorliegenden Sachzusammenhang sowohl aus der Sicht der potentiellen Abnehmerunternehmungen betrachtet als auch aus der Perspektive des Anbieters diskutiert werden. So ist für die Wahl einer Technologie auf der einen Seite entscheidend, daB dem Abnehmer - neben der Inanspruchnahme der angebotenen Informationsfunktion - auch andere Anwendungsmöglichkeiten für die Technologien gegeben sind. DaB dies bei den einzelnen Technologien in unterschiedlichem MaBe der Fall ist, verdeutlicht Abbildung 27.

Auf der anderen Seite spricht es wesentlich für den Einsatz einer bestimmten Technologie, wenn diese hinsichtlich ihres Einsatzspektrums und ihrer Anwendungscharakteristika den Anforderungen des Leistungsangebots möglichst weitgehend genügen. Damit ist die funktionsspezifische Eignung einzelner Technologiealternativen angesprochen.

\subsection{Funktionsbezogene Determinanten der Technologie- wahl}

Eine hohe Priorität im Rahmen der Technologieauswahl gebührt neben der Festlegung von funktionsunabhängigen Determinanten der Suche nach solchen Kriterien, die als Maßgrößen der Effektivität der Informationsversorgung gleichzeitig zur Beurteilung einzelner Technologiealternativen herangezogen werden können. 1

1 "Die Bestimmung des Informationsbedarfs wird damit zum entscheidenden Ansatzpunkt systemanalytischer Betätigung im Rahmen der Entwicklung eines Informationssystems. Erst die Kenntnis des Informationsprofils des Beteiligten bzw. der Gruppen von Beteiligten bildet die Voraussetzung für eine ökonomisch zu rechtfertigende Selektion ... des Lösungspotentials elektronischer Datenverarbeitungsanla-gen". Koreimann, D., Architektur und Planung betrieblicher Informationssysteme, in: Probleme beim Aufbau betrieblicher Informationssysteme, Hrsg.: Hansen, H.R., Wahl, M.P., München 1973, S. 52 f. ( $i m$ folgenden zitiert als: Koreimann, D., Architektur). 


\begin{tabular}{|c|c|c|c|c|c|c|}
\hline PROZESS & TELEFON & $\begin{array}{l}\text { TELEX- } \\
\text { ANLAGE }\end{array}$ & $\begin{array}{l}\text { TELETEX- } \\
\text { ANLAGE }\end{array}$ & $\begin{array}{l}\text { TELEFAX- } \\
\text { ANLAGE }\end{array}$ & $\begin{array}{l}\text { BILD- } \\
\text { SCHIRM- } \\
\text { TEXT- } \\
\text { TERMINAL }\end{array}$ & $\begin{array}{l}\text { DATEN- } \\
\text { FERNÜBER- } \\
\text { TRAGUNGS- } \\
\text { TERMINAL }\end{array}$ \\
\hline \multicolumn{7}{|c|}{ FORMALE ERSTELUNG } \\
\hline \multicolumn{7}{|c|}{ BZW, BEARBEITUNG } \\
\hline \multicolumn{7}{|c|}{$\begin{array}{l}\text { (SCHREIBEN/REPRO- } \\
\text { DUZIEREN) }\end{array}$} \\
\hline & & & & & & \\
\hline \multicolumn{7}{|c|}{ INHALTLICHE BE- } \\
\hline \multicolumn{7}{|l|}{ ARBEITUNG } \\
\hline \multicolumn{7}{|c|}{ SPEICHERUNG BZW. } \\
\hline \multicolumn{7}{|c|}{ DOKLMENTATION } \\
\hline \multicolumn{6}{|c|}{ UBERTRAGUNG: INDI- } & \\
\hline \multicolumn{7}{|c|}{ VIDUELLER VERSAND } \\
\hline \multicolumn{7}{|c|}{ UND EMPFANG VON } \\
\hline \multicolumn{7}{|c|}{ INFORMATIONEN } \\
\hline \multicolumn{7}{|c|}{$\begin{array}{l}\text { RECHERCHE IN EX- } \\
\text { TERNEN INFORMA- } \\
\text { TIONSBESTÄNDEN }\end{array}$} \\
\hline \multirow{3}{*}{\multicolumn{7}{|c|}{$\begin{array}{l}\text { DISPOSITIONEN } \\
\text { (VERANLASSUNGEN, } \\
\text { BESTEULUNGEN) }\end{array}$}} \\
\hline & & & & & & \\
\hline & & & & & & \\
\hline \multicolumn{7}{|c|}{ EOOGLICH } \\
\hline \multicolumn{7}{|c|}{ ZZ DURCH ERNEITERUNGEN MÖGLICH } \\
\hline$\rightarrow$ & NSBEDING & MINDES & 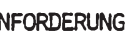 & AN & TECHNIK & \\
\hline
\end{tabular}

Abb. 27: Anwendungsspektrum alternativer Telekommunikationsformen (Quelle: in partieller Anlehnung an Schellhaas, H., Schönecker, H., a.a.0., S. $65 \mathrm{ff} .$. ) 


\subsection{Zentrale Anforderungen an die Informationsver- sorgung als Bestimmungsfaktoren der Technologie}

Es gilt als eine seit langem unbestrittene Erkenntnis, daB die Kriterien der Leistungsfähigkeit eines Informationssystems aus der sicht der (potentiellen) Benutzer festzulegen sind. ${ }^{1}$ Dies gilt um so zwingender, wenn vom Urteil der Benutzer nicht nur die Inanspruchnahme des Systems, sondern auch und insbesondere dessen wirtschaftlicher Erfolg abhängt. Geht man davon aus, daß in erster Linie der wahrgenommene Wert einer Information die Bereitschaft eines potentiellen Nutzers beeinfluBt, diese gegen Entgelt in Anspruch zu nehmen, so fuhrt dies zum Problem der Informationsbewertung.

Die betriebswirtschaftliche Literatur hat sich schon früheitig mit der Frage nach dem Wert einer Information auseinandergesetzt. ${ }^{2}$ Im Mittelpunkt standen zunächst Versuche, den geldwerten Ertrag einer Information mit $\mathrm{Hilfe}$ mathematischer Modelle zu quantifizie-

1 Vgl. Koreimann, D., Architektur, a.a.0., S. 52 f.; vgl. ferner z. B. Balke, S., Benutzerprobleme der Dokumentation und Information, in: $N f D, 24 . \mathrm{Jg} ., 1973$, Nr. 1, S. 8; Wersig, G., Zur Systematik der Benutzerforschung, in: NfD, 24. Jg., 1973, Nr. 1, S. 10; Kirsch, W., Probleme der Unternehmensführung bei der Entwicklung und Implementierung von Management-Informationssystemen, in: DU, 28. Jg., 1974, S. $173 \mathrm{ff}$.; Abel, V., Problemorientiertes Informationsverhalten, Darmstadt 1979, S. 132; Rockart, J.F., Topmanager sollten ihren Datenbedarf selbst definieren, in: HM, 0 . Jg., $1980, \mathrm{Nr} .2$, S. $45 \mathrm{ff}$.; Grochla, E., Die Beteiligung der Unternehmensführung an der Gestaltung computer-gestützter Informationssysteme, in: Führungsprobleme industrieller Unternehmungen, Festschrift fü Friedrich Thomée zum 60. Geburtstag, Hrsg.: Hahn, D., Berlin, New York 1980, insbes. S. 125 und S. 128.

$2 \mathrm{Vgl}$. insbes. Teichmann, H., Informationsbewertung, in: HdB, 4. Auf1., Hrsg.: Grochla, E., Wittmann, W., Stuttgart 1975, Sp. $1894 \mathrm{ff}$.; Wild, J., Zur Problematik der Nutzenbewertung von Informationen, in: $\mathrm{ZfB}, 41 . \mathrm{Jg}$. , 1971 , S. $315 \mathrm{ff}$. Sowie die dort angegebene Literatur. 
ren. ${ }^{1}$ Die Bemühungen endeten jedoch in der Erkenntnis, "daB es nur in wenigen Sonderfällen möglich ist, den Ertrag von Kommunikationsvorgängen zu bestimmen, und daB der Versuch, Informationen unter diesem Gesichtspunkt zu beurteilen, vorläufig als gescheitert betrachtet werden muB".?

Vor diesem Hintergrund konzentrierte sich das aktuelle Interesse zunehmend auf "objektive qualitative MaBstäbe" 3 zur Bewertung von Informationen. Erweitert man das Blickfeld von der Information an sich auf die Versorgung mit Informationen, so haben sich im jüngeren Schrifttum drei - nicht überschneidungsfreie - Dimensionen qualitativer Informationsanforderungen herausgebildet (vgl. Abbildung 28):

- qualitative Anforderungen an die Information 4

- qualitative Anforderungen an externe Informationsquellen 5

1 Hervorzuheben sind vor allem die Arbeiten von 1. Marschak und R. Radner. Vgl. hierzu Bössmann, E., a.a.0., S. $187 \mathrm{ff}$. sowie Domeier, M., Beurteilungskriterien formeller Kommunikationssysteme, Diss. Köln 1969, S. $89 \mathrm{ff}$.

2 Brönimann, Ch., Aufbau und Beurteilung des Kommunikationssystems von Unternehmungen, Schriftenreihe "Führung und Organisation der Unternehmung", Bd. 8, Bern, Stuttgart 1970, S. 111.

3 Ebenda. Die "Qualität" einer Information bemiBt sich dabei in dem Grad, in welchem wesentliche inhaltliche und formale Anforderungen an die Informationen erfuilt werden. Wild spricht in diesem Zusammenhang von "Eigenschaften", "Gütekriterien", einer Information, die unter Berücksichtigung des jeweiligen Verwenders der Information, der Entscheidungssituation und der Phase des Entscheidungsprozesses die Wirkung dieser Information bestimmen. Vgl. Wild, J., a.a.0., S. $326 \mathrm{ff}$. Das Attribut "objektiv" soll klarstellen, daB sich die Beurteilung auf die Information an sich und nicht auf die Eigenschaften des Senders (Status, fachliche Kompetenz) beziehen soll, welche der Empfänger subjektiv wahrnimmt. Vgl. Brönimann, Ch., a.a.0., S. 111.

$4 \mathrm{Vgl}$. z. B. Brönimann, Ch., a.a.0., S. $111 \mathrm{ff}$; in Anlehnung daran Pümpin, C., Information und Marketing, Schriftenreihe "Marketing-Management", Bd. 1, St. Gallen 1973, S. $83 \mathrm{ff}$.; Wild, J., a.a.0., S. 327.

$5 \mathrm{Vgl.z}$. B. Hofer, P., Wolff, H., a.a.0., S. 109 f. 


\begin{tabular}{|c|c|c|}
\hline $\begin{array}{l}\text { INFORMATIONS- } \\
\text { ERFORDERNISSE } \\
\text { Qualitative Anfor- } \\
\text { derungen an die } \\
\text { Information } \\
\text { Brönimann (1970) } \\
\text { im gleichen Sinne } \\
\text { Pümpin (1973) }\end{array}$ & $\begin{array}{l}\text { QUELLEN- } \\
\text { ERFORDERNISSE } \\
\text { Qual itative Anfor- } \\
\text { derungen an exter- } \\
\text { ne Informations- } \\
\text { quellen } \\
\text { Hofer/Wolf (1978) }\end{array}$ & $\begin{array}{l}\text { KOMMUNIKATIONS- } \\
\text { ERFORDERNISSE } \\
\text { Qualitative Anfor- } \\
\text { derungen an die In- } \\
\text { formationsübertra- } \\
\text { gung } \\
\text { Schellhaas/ } \\
\text { Schönecker (1984) }\end{array}$ \\
\hline $\begin{array}{l}\text { Aktualität } \\
\text { Zeitliche Entspre- } \\
\text { chung } \\
\text { sachlicher Bezug }\end{array}$ & $\begin{array}{l}\text { Aktualität } \\
\text { Kurzfristige Ver- } \\
\text { fügbarkeit }\end{array}$ & Schnelligkeit \\
\hline Wahrheitsgehalt & $\begin{array}{l}\text { Objektivität } \\
\text { praxisnahe Sprache } \\
\text { geeignete Darstel- } \\
\text { lungsform } \\
\text { Dokumentierbarkeit } \\
\text { Informationstiefe } \\
\text { Informationsbreite } \\
\text { Ruickfragemöglichkeit }\end{array}$ & $\begin{array}{l}\text { Sicherheit } \\
\text { Repräsentativität; } \\
\text { Bilder/Skizzen; } \\
\text { Anlagen } \\
\text { Ablagefähigkeit/ } \\
\text { Weiterverarbeit- } \\
\text { barkeit } \\
\\
\text { schnelle Registrierung } \\
\text { bzw. Aufnahme }\end{array}$ \\
\hline
\end{tabular}

Abb. 28: Dimensionen der Konkretisierung von Anforderungen an die Informationsversorgung 
- qualitative Anforderungen an die Informationsubertragung. 1

Die Vermutung liegt nahe, daß für die Planung eines externen elektronischen Informationssystems allen drei $\mathrm{Di}$ mensionen gleichermaßen eine bedeutsame Rolle zukommt. Diese Vermutung zu überprïfen war einer der Anlässe, im Rahmen der vorliegenden Untersuchung näher auf dic Frage nach relevanten Anforderungskriterien an die Versorgung mit unternehmensexternen Informationen einzugehen.

\subsection{Bestimmurig relevanter Anforderungskriterien der Informationsversorgung}

Um eine umfassende Abdeckung potentieller Anforderungen an einen externen Informationsdienst zu erreichen, wurde zunächst im Rahmen einer Vorstudie und unter Einbeziehung der in der Literatur vorgeschlagenen Kriterien eine systematische, möglichst vollständige Auflistung denkbarer Anforderungsmerkmale vorgenommen. In Pretests zeigte sich dann, daß aus der Gesamtzahl der Merkmale folgende - wenn auch z. T. schwer voneinander abgrenzbare - Kriterien als grundsätzlich relevant bewertet wurden: ${ }^{2}$

1 Vgl. Schellhaas, H., Schönecker, H., a.a.0., S. $129 \mathrm{ff}$.

2 Zur genauen Formulierung der Items vgl. Abbildung 29 auf S. 127 sowie Frage 6a - 6c des Fragebogens in Anhang 3 , S. $289 \mathrm{ff}$. dieser Arbeit. 
1. VerläBlichkeit der Informationen

2. Vollständigkeit der Informationen

3. Einfachheit des Informationszugriffs

4. Schnelligkeit der Informationsbereitstellung

5. Wirtschaftlichkeit der Informationsversorgung

6. Klarheit der Informationsdarstellung

7. Relevanz der Informationen

8. Aktualität der Informationen

9. Speicherfähigkeit der Informationen

10. Geeigneter Aufbereitungsgrad der Informationen

11. Objektivität der Informationen

12. Exklusivität der Informationen

13. Breite des Informationsspektrums

14. EDV-Verarbeitbarkeit der Informationen

Für die strategische Ausrichtung des geplanten Informationssystems erschien sodann die Frage von Bedeutung, inwieweit Beziehungen zwischen diesen einzelnen Anforderungsmerkmalen bestehen, und ob der Beurteilungsraum sich durch eine Reduzierung der Zahl der Kriterien auf einige wenige Faktoren komprimieren läBt. Zur Beantwortung dieser Frage wurde in Analogie zu der von Kupsch et al. vorgelegten Vorgehensweise ${ }^{1}$ für alternative In-

1 Ziel der Untersuchung von Kupsch et al. war in diesem Zusammenhang die Erfassung relevanter Dimensionen der Beurteilung von Informationsquellen, die Konsumenten vor dem Kauf in Anspruch nehmen. Vgi. Kupsch, P., Hufschmied, P., Mathes, H.D., Schöler, K., Die Struktur von Qualitätsurteilen und das Informationsverhalten von Konsumenten beim Kauf langlebiger Gebrauchsaüter, Forschungsbericht des Landes Nordrhein-Westfalen, 0pladen 1978. Vgl. in Anlehnung daran auch Katz, R., a.a.0., S. $31 \mathrm{ff}$. 
formationsbereiche (-funktionen) eine Faktorenanalyse über die Anforderungskriterien gerechnet. 1

Abbildung 29 gibt beispielhaft die Ergebnisse für den Informationsbereich "Brancheninformation" wieder. ${ }^{2}$ Sie veranschaulicht graphisch, wie sich die einzelnen Faktoren aus den 14 vorgegebenen Kriterien "rekrutieren". 3 Die angegebenen Faktorladungen repräsentieren dabei die Intensität des Zusammenhangs zwischen den Kriterien und den gefundenen Faktoren. Die Anteile der erklärten Varianz sind demgegenüber ein Gradmesser dafür, wie vollständig die den Kriterien innewohnende Information durch die einzelnen Faktoren wiedergegeben wird.

1 Als multivariates statistisches Verfahren findet die Faktorenanalyse Anwendung, wenn es gilt, eine Vielzahl möglicher Variablen auf wenige, wichtige EinfluBfaktoren zurückzuführen. Zum Einsatz der Faktorenanalyse vgl. Oberla, K., Faktorenanalyse, 2. Aufl., Berlin, etc. 1972; Sturm, M., Vajna, T., Grundzüge der Faktorenanalyse, in: Techniken der empirischen Sozialforschung, Hrsg.: Koolwijk, J.V., Wieken-Mayser, M., Bd. 5, Testen und Messen, München 1976, S. $184 \mathrm{ff}$.; Green, P.E., Analyzing Multivariate Data, Hinsdale 1978, S. 342 f.; Stewart, D.W., The Application and Misapplication of Factor Analysis in Marketing Research, in: JoMR, Vol. 18, February 1981, S. $51 \mathrm{ff}$.

2 Sämtliche Auswertungsarbeiten im Rahmen der vorliegenden Untersuchung erfolgten mit Hilfe des SPSS-Programmpaketes (Version 9) des Rechenzentrums der Universität Münster. Zum SPSS-Programmpaket vgl. Nie, N.H., et al., SPSS-Statistical Package for the Social Sciences, New York, etc. 1975; Beute1, P. et al., SPSS, 9. StatistikProgramm-System für die Sozialwissenschaften, 5. Aufl., Stuttgart, New York 1983.

3 Zugrunde gelegt wurde das sog. Kaiser-Kriterium, wonach nur Faktoren mit einem Eigenwert (Erklärungsanteil eines Faktors im Hinblick auf die Varianz aller Einzelkriterien) größer 1 extrahiert werden. Eine (methodisch nicht unbedenkliche) Extraktion weiterer Faktoren liefert nur sehr geringe Zuwächse der erklärten Gesamtvarianz. Auf eine Darstellung dieser Ergebnisse soll deshalb an dieser Stelle verzichtet werden. Zur Problematik der Bestimmung der Faktorenzahl vgl. insbes. Schuchard-Ficher, Chr. et al., Multivariate Analysemethoden. Eine anwendungsorientierte Einführung, Berlin, Heidelberg, New York 1980, S. $237 \mathrm{ff}$.. 
Versucht man, die Faktoren beispielhaft zu interpretieren, so können als zentrale Anforderungskriterien eines externen Informationsdienstes für Brancheninformationen

- die Verläßlichkeit der Informationen,

- die Aktualität der Informationen,

- die Handhabbarkeit der Informationen,

- die Benutzerfreundlichkeit des Informationssystems und

- die Benutzerorientiertheit des Informationsanbieters

unterschieden werden.

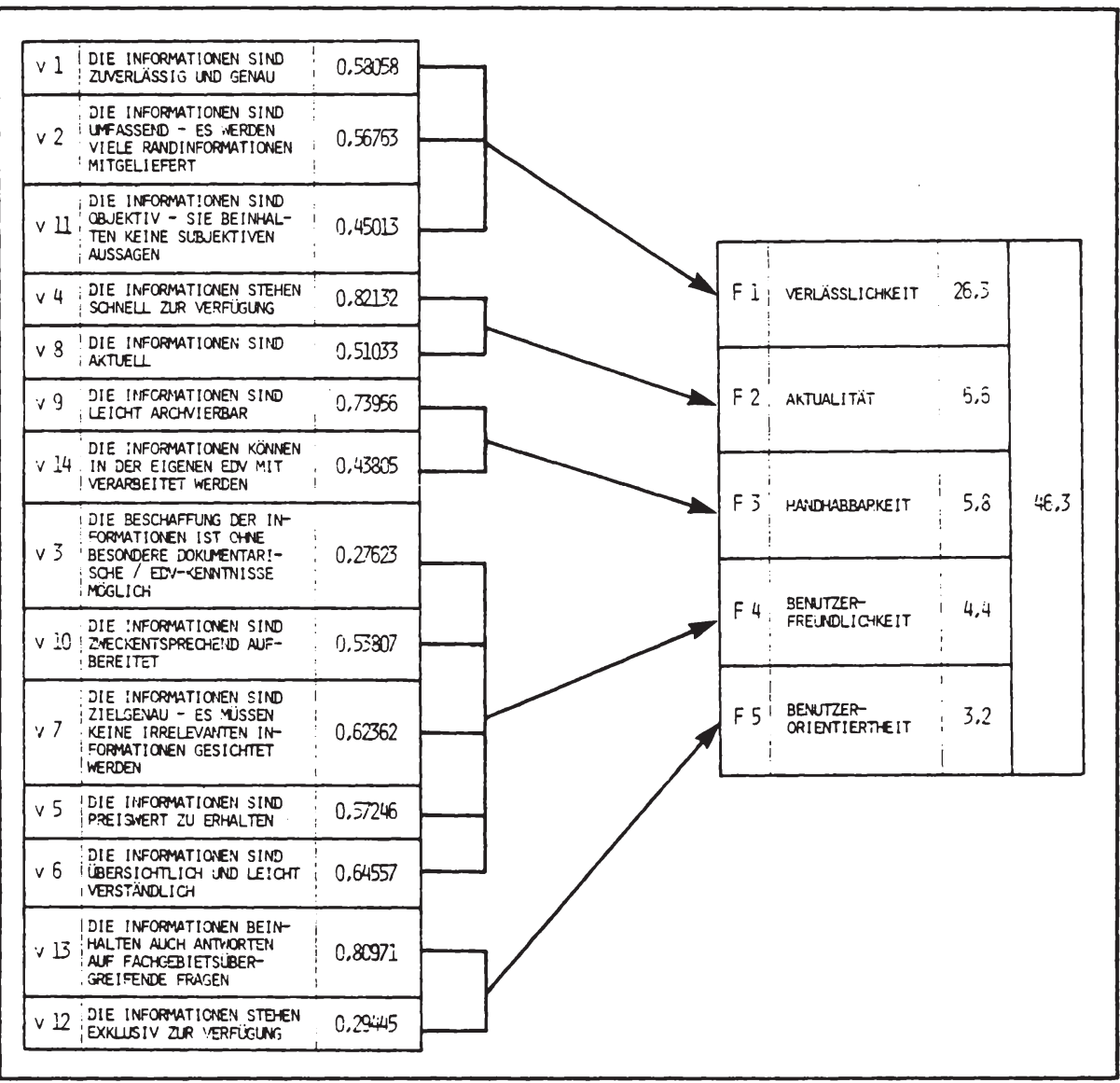

Abb. 29: Bedeutungsinhalt der Faktoren F1 bis F5 
Die "Verläßlichkeit" der Informationen entspricht dem von Brönimann mit "Wahrheitsgehalt" umschriebenen Anforderungsmerkmal, das Authentizität (Echtheit, Objektivität) und Genauigkeit umfaßt. 1

Die "Aktualität" der Information beschreibt die "Diskrepanz zwischen dem Zeitpunkt der Entstehung einer Information und demjenigen ihrer Verwertbarkeit durch den Empfänger". 2 Sie beinhaltet sowohl das "Alter" einer im System gespeicherten Information als auch die Zeitspanne, die vergeht, bis der Anfragende diese Information vom System erhält (Schnelligkeit der Bereitstellung).

Die "Handhabbarkeit" der Information enthält als entscheidende Komponenten die Archivierbarkeit bzw. "Ablagefähigkeit" sowie - eng damit verbunden - die Weiterverarbeitbarkeit der Information in der EDV.

Die "Benutzerfreundlichkeit" beinhaltet eine Vielzahl wichtiger Eigenschaften, die von Informationssystemen typischerweise gefordert werden. Unter Benutzerfreundlichkeit ist dabei vor allem zu verstehen, "daB Informationssysteme die gewuinschten Informationen problemorientiert, d. h. selektiv zur Verfügung stellen, ohne daß durch den Selektionsvorgang die Vollständigkeit der bereitzustellenden Informationen beeinträchtigt wird". 3

1 Vgl. Brönimann, Ch., a.a.0., S. $113 \mathrm{f}$.

2 Ebenda, S. 111.

3 Meindl, U., Oberlegungen zur Informationsbedarfsanalyse bei der Entwicklung von Informationssystemen, Studiengruppe für Systemforschung e.V. Heidelberg, Bericht Nr. 95, München-Pullach, Berlin 1972, S. 3; zur plastischen Kennzeichnung eines "unfreundlichen" Informationssystems vgl. Ackoff, R.L., Management Misinformation Systems, in: MS, Vol. 14, 1967, No. 4, S. B 147 sowie Balke, S., a.a.0., S. 4. 
Als letzter Faktor ist schlieBlich die "Benutzerorientiertheit" des Informationsanbieters von Bedeutung. Benutzerorientiert kann hierbei so interpretiert werden, daß der Anbieter auch fachgebietsübergreifende, speziell auf den Nachfrager ausgerichtete Informationsangebote zusammenstellt und diese exklusiv abgibt.

Im Rahmen der vorliegenden Untersuchung wurden Faktorenanalysen zu den Informationsbereichen "Gesamtwirtschaft", "Branchen" und "Export" gerechnet. Die Ergebnisse sind insbesondere durch folgende übereinstimmende Aspekte gekennzeichnet ( $v g l$. auch Tabelle 3 ):

1. Der erste extrahierte Faktor liefert in allen Fällen einen hohen Erklärungsbeitrag (ca. $26-56 \%$ ). Weitere Faktoren zu extrahieren erweist sich danach nur bedingt als sinnvoll, da ihr Erklärungsbeitrag mit ca. $3-7 \%$ sehr gering ist. Der insgesamt erklärte Varianzanteil bleibt damit relativ niedrig. Er entspricht im vorliegenden Zusammenhang zwar dem methodischen Anspruch, zentrale Klassen von Anforderyngen an das geplante Informationssystem abzugrenzen. 2 Er ist jedoch zu niedrig, als daB bei einem "Weiterrechnen" mit diesen Faktoren ${ }^{3}$ nicht ein beträchtlicher Informationsverlust hingenommen werden mübte. Ein solcher ist jedoch gerade dann nicht vertretbar, wenn es darum geht, aus den gefundenen Anforderungsmerkmalen strategische Entscheidungen hinsichtlich der geschäftsfeldoptimalen Technologie abzuleiten.

$1 \mathrm{Vgl}$. die Ergebnisse in Tab. 15 bis 17 in Anhang 2, S. $279 \mathrm{ff}$. dieser Arbeit.

2 Vgl. Zaltman, G., Burger, P.C., Marketing Research, Hinsdale 1975, S. 504. Der ermittelte Wert bewegt sich zudem auf einem empirisch häufig ermittelten Niveau; vgl. ebenda.

3 Z. B. könnte die Frage gestellt werden, welche Ausprägungen die Faktoren bei alternativen Informationsbereichen annehmen (Berechnung der Faktorwerte). 


\begin{tabular}{|l|ccc|}
\hline $\begin{array}{l}\text { Informations- } \\
\text { bereich }\end{array}$ & $\begin{array}{c}\text { Zahl der } \\
\text { extrahierten } \\
\text { Faktoren }\end{array}$ & $\begin{array}{c}\text { durch den } \\
\text { 1.Faktor } \\
\text { erklärter } \\
\text { Varianzanteil }\end{array}$ & $\begin{array}{c}\text { insgesamt } \\
\text { erklärter } \\
\text { Varianzanteil }\end{array}$ \\
\hline $\begin{array}{l}\text { Gesamtwirt- } \\
\text { schaft/Kon- } \\
\text { junktur } \\
\begin{array}{l}\text { Branchen/ } \\
\text { Markte }\end{array}\end{array}$ & 3 & $29,8 \%$ & $41,3 \%$ \\
$\begin{array}{l}\text { AuBenhandel/ } \\
\text { Export }\end{array}$ & 5 & $26,3 \%$ & $46,3 \%$ \\
\hline
\end{tabular}

Tab. 3: Ergebnisse der faktorenanalytischen Verdichtung von Anforderungen an Informationen alternativer Informationsbereiche

2. Der wichtigste Faktor (im weitesten Sinne "Benutzerfreundlichkeit") lädt grundsätzlich auf viele der vorgegebenen Variablen gleichzeitig (auf $5-12$ von insgesamt 14) und macht dadurch eine sinnvolle Interpretation schwierig (bei Brancheninformationen) bzw. unmöglich (bei Exportinformationen). Das Beispiel der Anforderungen an Exportinformationen zeigt dies be-
sonders deutlich.

Aus den Ergebnissen muß geschlußfolgert werden, daß zum Zweck des Auffindens zentraler Anforderungen an ein externes Informationssystem eine Verdichtung relevanter Merkmale auf wenige Faktoren nicht zweckmäßig ist. Diese Tatsache läßt den Rückschluß zu, daß den einzelnen Kriterien ein hohes Ma $B$ an individueller Relevanz zugesprochen werden kann.

$1 \mathrm{Vgl}$. Tabelle 17 in Anhang 2, S. 282 dieser Arbeit. 


\subsection{Relative Bedeutung relevanter Anforderungs - kriterien}

Zur optimalen Gewichtung der einzelnen Anforderungskriterien im Rahmen der Technologieplanung ist es bedeutsam, zu wissen, welches Gewicht die potentiellen Benutzer des Informationssystems den unterschiedlichen Systemmerkmalen beimessen. Deshalb wurden in der Untersuchung die Befragten aufgefordert, auf einer 5-PunkteSkala von "extrem wichtig" bis "uberhaupt nicht wichtig" die einzelnen Anforderungskriterien einzuordnen. Dabei fand eine Trennung nach Informationen aus den Bereichen Gesamtwirtschaft, Branche und Export statt.

Das Ergebnis in Abbildung 30 zeigt, daB die potentiellen Systemnutzer der Aktualität der Informationen sowie der Schnelligkeit der Informationsbereitstellung offenkundig eine dominante Bedeutung einräumen. Für sehr. wichtig halten die Befragten daneben die Tatsache, daß die Zuverlässigkeit und die Objektivität der Informationen gewährleistet sind. Schließlich nehmen die Kriterien Relevanz und obersichtlichkeit der Informationen einen relativ hohen Rang auf der Wichtigkeitsskala ein. Bemerkenswert ist, da $B$ die Kriterien der Handhabbarkeit (leichte Archivierbarkeit und EDV-Verarbeitbarkeit der Informationen) sowie die Merkmale der Benutzerorientierung (Exklusivität und Informationsbreite) als eher unwichtig eingestuft werden.

1 Zur exakten Formulierung der Fragestellung vgl.

Frage $6 a-6 c$ des Fragebogens in Anhang 3, S. $289 \mathrm{ff}$. dieser Arbeit. 
DIE INFORMATIONEN SIND ZUNERLÄSSIG UND GENAU

DIE INFORMATIONEN SIND UMFASSEND - ES WERDEN VIELE RANDINFOPMATIONEN MITGELIEFERT

DIE BESCHAFFUNG DER INFORMATIONEN IST OHNE BESONDERE DOKLMENTARISCHE EDVKENNTNISSE MÖGLICH

DIE INFORMATIONEN STEHEN SCHNEL ZUR VERFÜELNG

DIE INFORMATIONEN SIND PREISWERT ZU ERHALTEN

DIE INFOPMATIONEN SIND UBBERSICHTLICH UND LEICHT VERSTÄNDLICH

DIE INFORMATIONEN SIND ZIELGENAU - ES MUSSSEN KEINE IRRELEVANTEN INFORMATIONEN GESICHTET WERDEN

DIE INFORMATIONEN SIND AKTUELL

DIE INFORMATIONEN SIND LE:CHT ARCHIVIERBAR

DIE INFOPMATIONEN SIND ZWECKENTSPRECHEND AUFBEREITET

DIE INFORMATIONEN SIND OBJEKTIV - SIE BEINHALTEN KEINE SUBUEKTIVEN AUSSAGEN

DIE INFORMATIONEN STEHEN EXCLUSIV ZUR VERFÜGUNG

DIE INFORMATIONEN BEINHALTEN AUCH ANTWORTEN AUF FACHGEBIETSÜBER GREIFENDE FRAGEN

DIE INFORMATIONEN KÖNNEN MIT DER EIGENEN EDV MIT

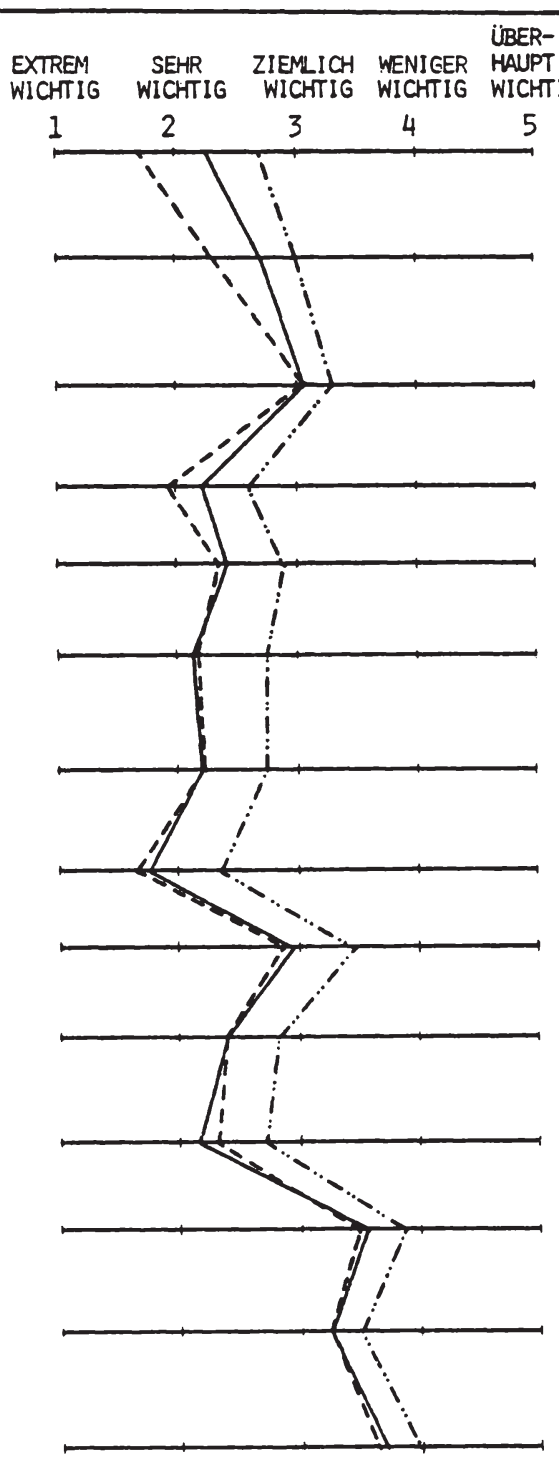
VERARBEITET WERDEN

— GESAMTWIRTSCHAFTLICHE INFORMATION

--D BRANCHENINFORA:ATION

-..-.- EXPORTINFORMATION

Abb. 30: Anforderungen an die Versorgung mit unternehmensexternen Informationen 
Als strategische Implikation für die Technologiewahl kann das Unternehmen aus diesen Ergebnissen den SchluB ziehen, daB das geplante system vor allem

- durch einen hohen Grad der Aktualität der gespeicherten Informationen sowie

- durch eine hohe Reaktionsgeschwindigkeit in der Beantwortung von Anfragen

gekennzeichnet sein muB. Hieraus resultieren konkrete Erfordernisse hinsichtlich eines kurzfristigen Updating der Informationen und eines schnellen Zugriffs auf gewünschte Informationsbestände. Demgegenüber kann das Unternehmen die EDV-Kompatibilität der eingesetzten Technologie sowie den Grad, in dem die Technologie die Exklusivität des Informationsbezugs sicherstellt, als eher nachrangig betrachten.

\subsection{Funktionsabhängigkeit relevanter Anforderungs- kriterien}

Neben der Bedeutung einzelner Kriterien ist für das planende Unternehmen die jeweilige Abhängigkeit dieser Bedeutung von den in Betracht gezogenen (Informations-) Funktionen eine zentrale Größe. Die Ahnlichkeit der Anforderungsprofile in Abbildung 30 legt die Hypothese nahe, da $B$ die einzelnen kriterien eine funktionsunabhängige Giiltigkeit aufweisen. Dies hätte zur Konsequenz, daß die Technologie "lediglich" einem einheitlichen Set von Anforderungen gerecht werden müßte. Eine für eine Funktion als "optimal" erkannte Technologiealternative würde konsequenterweise dann für alle Funktionen Anwendung finden.

Zur Oberprüfung dieser Hypothese wurde im Rahmen der vorliegenden Untersuchung eine Faktorenanalyse über alle $(3 \times 14 \Rightarrow 42$ Anforderungskriterien der drei be- 
trachteten Informationsbereiche gerechnet. Ziel war es, festzustellen, ob die extrahierten Faktoren sich jeweils aus Variablen eines Informationsbereiches oder aus korrespondierenden Variablen aller Informationsbereiche "zusammensetzen". Tritt der erste Fall ein, so kann dies als Hinweis darauf interpretiert werden, daB die Faktoren als Anforderungsbindel sich jeweils auf eine Informationsfunktion beziehen. Anderenfalls kann man darauf schließen, daB die zentralen Anforderungsfaktoren über alle Informationsbereiche hinweg Giultigkeit haben.

Das Beispiel des Faktormusters ${ }^{1}$ in Tabelle 4 läBt erkennen, daB eine eindeutige, zu verallgemeinernde Beantwortung der Eingangshypothese nicht möglich ist. Betrachtet man zunächst lediglich die Bereiche "Gesamtwirtschaft" und "Branche" (GWICHT 1- BWICHT 14), so deuten das identische Faktormuster und die nahezu identischen Faktorladungen eindrucksvoll auf die Existenz bereichsübergreifender Anforderungskriterien hin. Bezieht man jedoch zusätzlich den Bereich "Exportinformationen" (AWICHT 1 - AWICHT 14) ein, verändert sich das Bild (vgl. auch Tabelle 5): Nur noch vier der 14 Variablen laden bereichsübergreifend auf jeweils identische Faktoren (nämlich die Faktoren 4, 5,6 und 9). Gleichzeitig tritt ein neuer Faktor auf, auf den insgesamt 10 Anforderungsvariablen aus dem Bereich "Exportinformationen" hoch laden.

Insgesamt kann folgende Interpretation der Ergebnisse vorgenommen werden:

1 Es handelt sich um die varimaxrotierte Faktormatrix. Zur Bedeutung der Varimax-Rotation vgl. z. B. Schuchard-Ficher, Chr., a.a.0., S. $241 \mathrm{f}$.. 


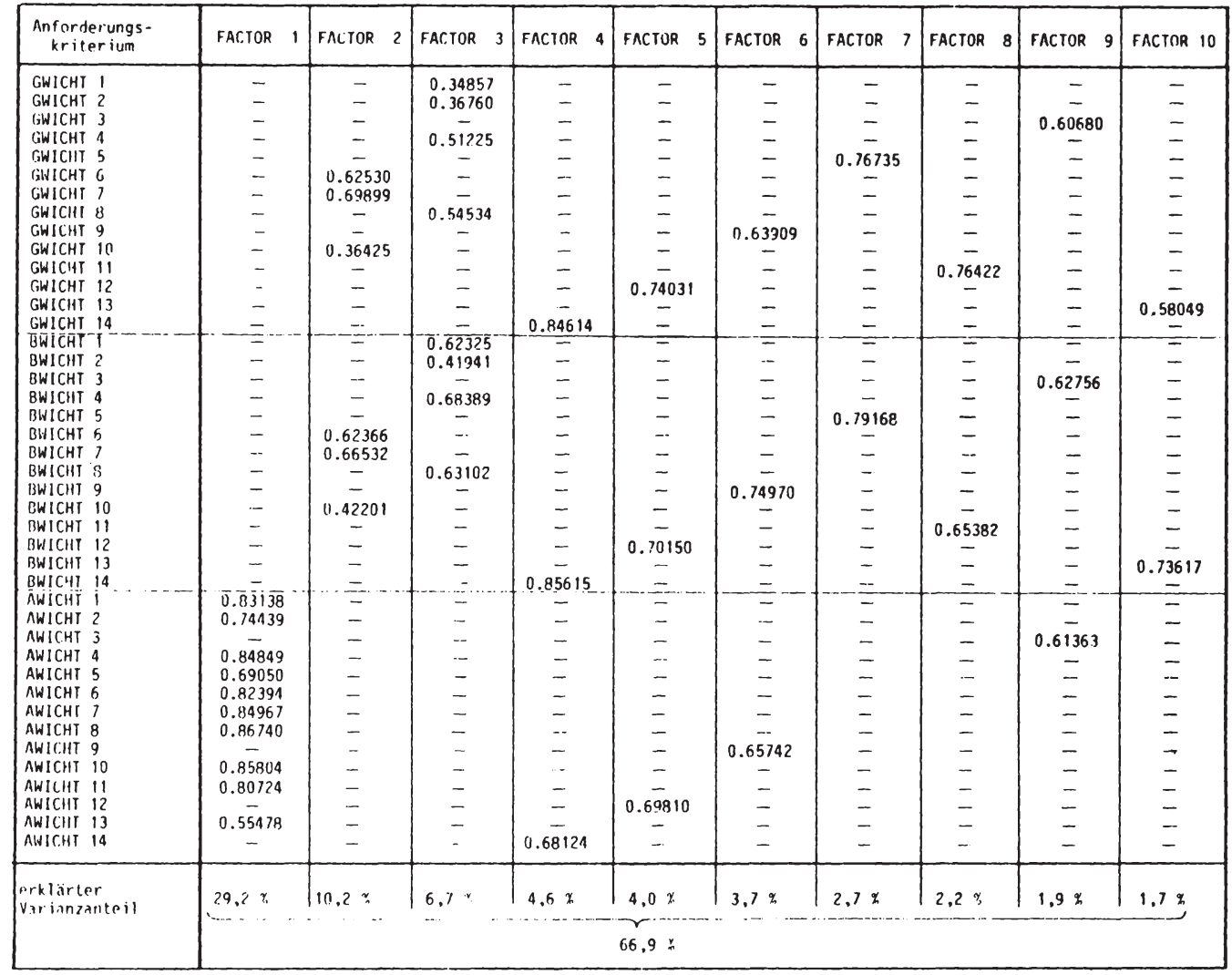

Tab. 4: Faktormuster der Anforderungen an Informationen zum Thema "Gesamtwirtschaft", "Branche" und "Export" 
1. Die Anforderungskriterien "EDV-Verarbeitbarkeit", "Exklusivität", "Speicherfähigkeit" und "Einfachheit des Informationszugriffs" haben eine funktionsunabhängige Guiltigkeit. Ihre Bedeutung ist unabhängig davon, welcher Informationsbereich betrachtet wird.

\begin{tabular}{|c|c|c|c|c|}
\hline \multicolumn{2}{|c|}{$\begin{array}{l}\text { Anforderungs- } \\
\text { Faktoren } F_{j}\end{array}$} & \multicolumn{3}{|c|}{$\begin{array}{l}\text { Funktionsspezifische Anforderungskriterien } \\
\text { (Variablen) } G_{i}, B_{i}, A_{i}\end{array}$} \\
\hline$j=$ & $\begin{array}{l}\text { Bedeutungs- } \\
\text { inhalt }\end{array}$ & $\begin{array}{l}G_{i} l a ̈ d t \text { hoch } \\
\text { auf } F_{j} \text {, wobe } i \\
i=\end{array}$ & $\begin{array}{l}B_{i} \text { lädt hoch } \\
\text { auf } F_{j} \text {, wobe } i \\
i=\end{array}$ & $\begin{array}{l}A_{j} \text { lädt hoch } \\
\text { auf } F_{j} \text {, wobe } i \\
i=\end{array}$ \\
\hline 1 & & & & $\begin{array}{l}1,2,4,5,6,7,8 \\
10,11,13\end{array}$ \\
\hline 2 & Nützl ichkeit & $6,7,10$ & $6,7,10$ & \\
\hline 3 & $\begin{array}{l}\text { Schnellig- } \\
\text { keit/Ver- } \\
\text { läBlichkeit }\end{array}$ & $1,2,4,8$ & $1,2,4,8$ & \\
\hline 4 & $\begin{array}{l}\text { EDV-Verar- } \\
\text { beitbarkeit }\end{array}$ & 14 & 14 & 14 \\
\hline 5 & $\begin{array}{l}\text { Exklusivi- } \\
\text { tät }\end{array}$ & 12 & 12 & 12 \\
\hline 6 & $\begin{array}{l}\text { Speicher- } \\
\text { fähigkeit }\end{array}$ & 9 & 9 & 9 \\
\hline 7 & $\begin{array}{l}\text { Wirtschaft- } \\
\text { lichkeit }\end{array}$ & 5 & 5 & \\
\hline 8 & $\begin{array}{l}\text { Objektivi- } \\
\text { tät }\end{array}$ & 11 & 11 & \\
\hline 9 & $\begin{array}{l}\text { Einfachheit } \\
\text { des Inform. } \\
\text { zugriffs }\end{array}$ & 3 & 3 & 3 \\
\hline 10 & $\begin{array}{l}\text { Informa- } \\
\text { tionsbreite }\end{array}$ & 13 & 13 & \\
\hline
\end{tabular}

Tab. 5: Beziehungszusarimenhang zwischen funktionsspezifischen Anforderungen $(i=1-14)$ und funktionsübergreifenden Anforderungsfaktoren $(j=1-10)$ 
2. Die Informationsbereiche "Branche" und "Gesamtwirtschaft" besitzen identische Anforderungsmuster; alle Anforderungen an die Versorgung und Brancheninformationen gelten in gleichem Maße für die Versorgung mit gesamtwirtschaftlichen Informationen.

3. Die Anforderungen an Exportinformationen zeichnen sich durch ein $z$. T. sehr geringes MaB an Differenziertheit aus. Als Indikator hierfür ist zum einen die geringe Höhe der merkmalspezifischen Einzelrestvarianzen zu werten. 1 Zum anderen deuten hierauf aber auch die $z$. T. sehr hohen Korrelationen $z$ wischen den Bewertungen einzelner Anforderungsmerkmale hin. Die Tatsache, daB die Befragten fur nahezu alle Kriterien jeweils ähnliche oder identische Ausprägungen wählten, kann als Hinweis darauf interpretiert werden, da B noch sehr undifferenzierte Vorstellungen über eine "ideale" Exportinformationsversorgung vorliegen.

Festzuhalten bleibt, da $B$ in bezug auf alle Informationsbereiche die eingangs bestimmten Anforderungskriterien, wenn auch unterschiedlich zu gewichten, so doch grundsätzlich zu berücksichtigen sind. An diesen kriterien gilt es nunmehr, die Leistungsfähigkeit der alternativen obertragungstechnologien zu messen.

\subsection{Leistungscharakteristika alternativer Obertra- gungstechnologien}

Die Leistungsfähigkeit einer Geschäftsfeldtechnologie drückt sich aus in dem Grad ihrer Eignung zur Erfüllung bestimmter Geschäftsfeldfunktionen. Abbildung 31 verdeutlicht diesen Sachverhalt: "'Projiziert' man das Eignungspotential der Technik auf die Aufgaben, dann kennzeichnet diese 'Projektion' die Eignung und Angemessenheit der neuen Technik für die zielgerichtete Abwicklung der Aufgaben". 2 Allgemein bezeichnet man die so verstandene Obereinstimmung der Leistungsmerkmale einer Techno-

1 Mit der Einzelrestvarianz ist der Varianzanteil der Kriterien gemeint, der nicht durch die extrahierten gemeinsamen Faktoren erklärt wird.

2 Schellhaas, H., Schönecker, H., a.a.0., S. 113. 
logie mit den Anforderungen bestimmter Aufgaben als Funk$\frac{\text { tionsadäquanz }}{\text { systems. }}$ oder "Aufgabenbezogenheit" ${ }^{1}$ des Technik-

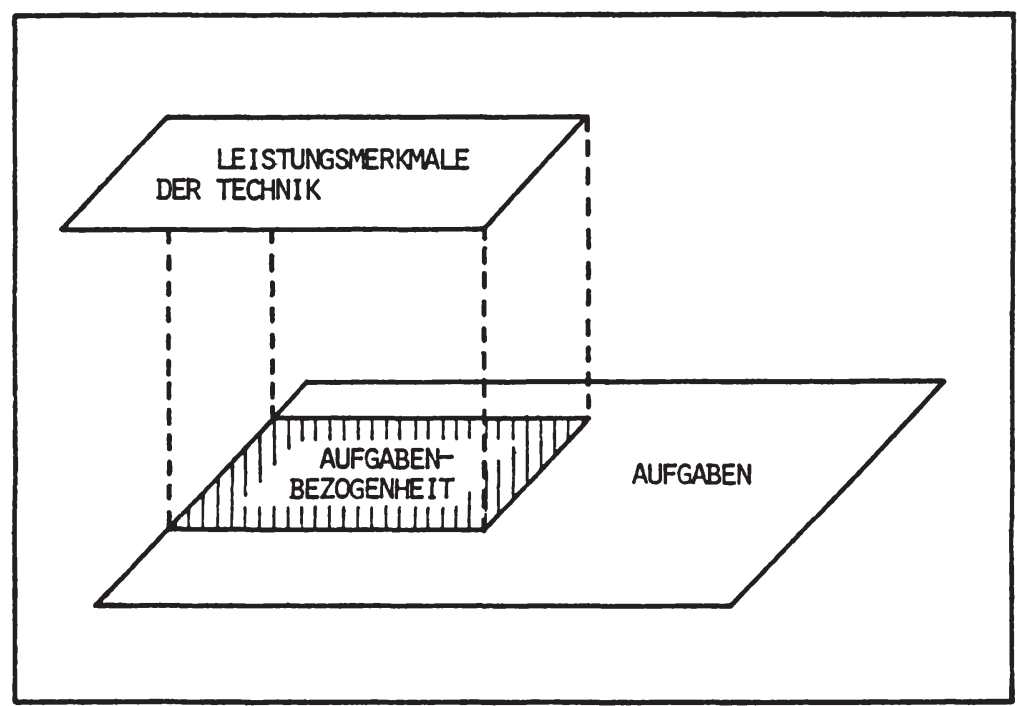

Abb. 31: Aufgabenbezogenheit der Technik

(Quelle: Schellhaas, H., Schönecker, H., a.a.0., S. 114)

1 Schellhaas, H., Schönecker, H., a.a.0., S. 113.

2 Zur grundlegenden Charakterisierung einzelner Leistungsmerkmale der hier betrachteten Telekommunikationsformen vgl. etwa Musiol, A., a.a.0., S. $81 \mathrm{ff}$.; Munter, H., Künftige integrierte Bürokommunikation. Welche Folgen lassen sich bereits übersehen?, in: Zf0, 51.Jg., 1982, H. 7, S. 371 ff.; Kinder, K., Bürokommunikation der Zukunft. Ein Aufgabenfeld für den Organisator, in: ZfO, 51. Jg., 1983, H. 1, S. $11 \mathrm{ff}$.; Mayer, R.-D., Bürokommunikation - Sachstand und Perspektiven, in: ifo-schnelldienst, $34 . \mathrm{Jg}$. , 1982, H. 19, S. 7 ff.; Picot, A., Anders, W., Dienste, a.a.0., S. $275 \mathrm{ff}$.; Wagner, H., a.a.0., S. $8 \mathrm{ff} .$. 


\subsection{Bestimmung relevanter Leistungsmerkmale}

Zur Messung der Aufgabenbezogenheit alternativer Technologielösungen im Rahmen der Geschäftsfeldplanung ist es zunächst erforderlich, für jedes der oben diskutierten, auf die Informationsversorgung bezogenen Anforderungskriterien ein korrespondierendes Leistungskriterium zu definieren. Dies kann dadurch geschehen, daB die Anforderungskriterien in entsprechende technologiebezogene Merkmale "umdimensioniert" werden. Betrachtet man beispielsweise das Kriterium "Schnelligkeit der Informationsbereitstellung", so lautet das korrespondierende Technologiemerkmal zweckmäßigerweise "Obertragungsgeschwindigkeit". Analog entspricht das Anforderungskriterium "Vollständigkeit der Informationen" der Forderung nach einer hohen "Darstellungs-bzw. Obertragungskapazität". "Genauigkeit" beinhaltet schließlich die Maßgabe, daß die Technik die "Exaktheit der Obermittlung" gewährleisten muB.

Projiziert man in dieser Weise alle Anforderungskriterien der Informationsversorgung auf eine technologiebezogene Ebene, so mündet dieses Vorgehen in dem in $A b-$ bildung 32 beispielhaft wiedergegebenen Katalog relevanter Leistungsmerkmale. Es entspricht der Philosophie der dreidimensionalen Geschäftsfeldwahl, daß die Benutzerperspektive auch bei der Konkretisierung von Technologiealternativen im Vordergrund stehen muß. ${ }^{1}$ Dementsprechend enthält der Katalog der Leistungskriterien nicht nur benutzerneutrale Merkmale, die sich in technischen Größen ausdrücken lassen ( $z$. B. Obertragungsgeschwindigkeit in bit/sec, Kommunikationsfähigkeit in

1 "In assessing what are indeed the alternative 'technologies' or ways of fulfilling a particular customer function, a customer perspective is vital". Abell, D.F., Defining the Business, a.a.0., S. 173. 
DIE INFCPMATIONEN SIND ZUNERLÄSSIG LND GENAU

DIE INFORMATIONEN SIND UMFASSEND - ES WERDEN VIELE RANDINFORMATIONEN MITGELIEFERT

DIE BESCHAFFUNG DER INFORMATIONEN IST OHNE BESONDERE DOKUMENTARISCHE EDV-KENNTTNISSE MÖGLICH

DIE INFORMATIONEN STEHEN SCHNELL ZUR VERFIGUNG

DIE INFORMATIONEN SIND PREISWERT ZU ERHALIEN

DIE INFORMATIONEN SIND ÜBERSICHTLICH UND LEICHT VERSTÄNLICH

DIE INFORMATIONEN SIND ZIELGENAU - ES MUSSEN KEINE IRRELEVANTEN INFORMATIONEN GESICH TET WERDEN

DIE INFORMATIONEN SIND AKTUELI

DIE INFORMATIONEN SIND LEICHT ARCHIVIERBAR

DIE INFORMATIONEN SIND ZWECKENTSPRECHEND AUFBEREITET

DIE INFORMATIONEN SINO OBJEKTIV - SIE BEINHALTEN KEINE SUBUEKTIVEN AUSSAGEN

DIE INFORMATIONEN STEHEN EXKUUSIV ZUR VERFÜGUNG

DIE INFORMATIONEN BEINHALTEN AUCH ANTWORTEN AUF FACHGEBIETSÜBERGREIFENDE FRAGEN

DIE INFORMATICNEN KÖNNEN MIT DER EIGENEN EDV MIT VERARBEITET WERDEN

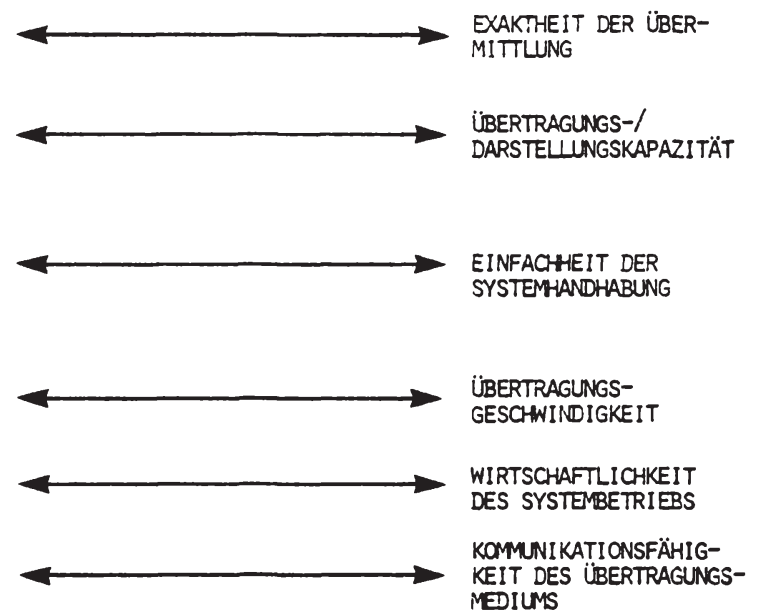

BENUTZUNGSSICHERHEIT/ TREFFSICHERHEIT BEI DER INFORMATIONSSUCHE

UPDATING-STAND DER GESPEICHERTEN INFORMATIONEN

ABLAGEFÄHIGKEIT DER ÜBERMITTELTEN INFORMATIONEN

UBERTRAGBARKEIT VON ZEICHNUNGEN BILDERN

GRAD DER AUTOMATISIERUNG DES SYSTEMS

VERTRAULICHKEIT DES INFORMATIONSTRANSFERS

GRAD DER INDIVIDUALISIERUNG DER INFOPMATIONSBEREITSTELUUNG

EDV-KOMPATIBILITÄT DES EMPFÄNGERSYSTEMS

Abb. 32: Entwicklung von Kriterien der Leistungsfähigkeit alternativer Geschäftsfeldtechnologien 
Betriebsarten (simplex, halbduplex, vollduplex) oder Darstellungskapazität in Zeichenzahl/Seite bzw. Display). Vielmehr finden auch Kriterien Eingang, die aus der Sicht der potentiellen Nutzer auf technologiebedingte Aspekte des Gesamtsystems Bezug nehmen (2. B. Einfachheit der Systemhandhabung; z. B. gemessen in der Zahl der erforderlichen Schulungsstunden), Benutzungssicherheit (gemessen in der Zahl relevanter Informationseinheiten/Abruf ("precision"-Wert), etc.).

Insgesamt weisen die in Abbildung 32 aufgelisteten Technologiemerkmale einen hohen Ahnlichkeitsgrad mit den Anforderungen auf, die - ausgehend vom Konzept des "decision calculus" 1 - in den 70er Jahren an die Gestaltung computergestuitzter Management-Informationssysteme (MIS) gestellt worden sind. ${ }^{2}$ Werden diese Kriterien jedoch im Sinne einer Maximierungsregel in einer höchstmöglichen Ausprägung angestrebt, so sind die hier entwickelten Merkmale als a priori frei variierbare Gestaltungselemente des Informationssystems zu verstehen, welche hinsichtlich ihrer optimalen Ausprägung grundsätzlich keiner normativen Restriktion unterliegen. Die "Soll-Ausprägung" der einzelnen Merkmale richtet sich vielmehr erst im Einzelfall nach den "Soll-Anforderungen", welche durch die jeweilige Funktion bzw. Aufgabe bestimmt werden. Ausgehend von dem empirisch erhobenen Anforderungsprofil der Versorgung mit Brancheninformationen ${ }^{3}$

1 Little, J.D.C., Models and Managers: The Concept of a Decision Calculus, in: MS, Vol. 16, 1970, S. B-466 ff.

$2 \mathrm{Vgl}$. insbes. Amstutz, A.E., Market-Oriented Management Systems: The Current Status, in: JoMR, Vol. 6, November 1969, S. $481 \mathrm{ff}$.; vgl. in Anlehnung hierzu ferner Bartsch, J., Organisatorische Probleme bei der Entwicklung und Handhabung computergestützter ManagementInformationssysteme in divisionalisierten Unternehmungen - dargestellt am Beispiel von Marketing-Informationssystemen, Diss. Münster, 1976, S. 36 sowie Meffert, H., Computergestüzte Marketing-Informationssysteme, a.a.0., S. 24.

$3 \mathrm{Vgl}$. hierzu bereits Abbildung 30 auf $\mathrm{S} .132$ dieser Arbeit. 
soll im folgenden beispielhaft die Bewertung der Leistungsfähigkeit zweier Alternativtechnologien eines externen Informationsdienstes dargestellt werden.

\subsection{Beurteilung relevanter Leistungsmerkmale}

Der Grad der Aufgabenadäquanz einer Technik läBt sich graphisch im Wege einer Gegenüberstellung des Anforderungsprofils der Funktion und des Leistungsprofils der jeweiligen Technologie ermitteln. Unter der Voraussetzung gleicher Skalen (z. B. 1 - 5) drückt sich dann die Funktionsadäquanz der betrachteten Technologie in dem Grad der Obereinstimmung beider Profile aus. Im Idealfalle läge dementsprechend eine Deckungsgleichheit beider Profile vor.

Geht man davon aus, daß das Funktionsprofil durch das Management vorbestimmt oder (wie im vorliegenden Beispiel) empirisch durch eine Befragung der potentiellen Nutzer ermittelt wurde, so hängt die Beurteilung einer Technologie weitgehend von der Beurteilung ihres Leistungsprofils ab. Da diese Beurteilung jedoch notwendig aus dem Blickwinkel der künftigen Systemnutzer erfolgen muß, empfiehlt es sich, keine zu hohen Anspruche an die technische Exaktheit bei der Festlegung einzelner Merkmalsausprägungen zu stellen. ' Vielmehr sollte das Technologierating in einem heuristischen ProzeB,

1 Schellhaas und Schönecker weisen in diesem Zusammenhang darauf hin, daB Nutzer elektronischer obertragungsmöglichkeiten die technischen Merkmale einezlner Medien z. T. anders wahrnehmen, als sie realiter existieren. "So ist zum Beispiel Teletex "technisch" gesehen schneller als zum Beispiel Telex. Teletex wird aber bei diesem Merkmal trotzdem hinter Telex eingestuft, was womöglich mit einer unzulänglichen Einsatzorganisation zusammenhängen mag." Schellhaas, H., Schönecker, H., a.a.0., S. 131. Zur allgemeinen Beurteilung einzelner Telekommunikationsformen aus Nutzersicht vgl. ebenda, S. $65 \mathrm{ff}$.. 
z. B. Unter Zuhilfenahme der Delphi-Methode, erfolgen. ${ }^{1}$ Unter Umständen ist es letztlich sogar zweckmäBig, unter Zugrundelegung eines lediglich ordinalen Skalenniveaus sich auf einen parweisen Vergleich einzelner Technologien zu beschränken. Ein "besseres Abschneiden" einer Technologie, gemessen an den Anforderungen der relevanten Funktion, führt dann zur Bevorzugung dieser technologischen Lösungsalternative.

Abbildung 33 soll beispielhaft die Aussagefähigkeit einer Gegenüberstellung von Leistungs- und Anforderungsprofilen aufzeigen. Es wird unmittelbar deutlich, daB

- wesentliche Unterschiede hinsichtlich der Leistungsfähigkeit der beiden exemplarisch betrachteten Alternativen zutage treten, und

- daB beide technologischen Lösungen in höchst unterschiedlichem Maße imstande sind, den Anforderungen der Funktionserfül?ung zu genügen.

Betrachtet man die zentralen technologischen Stärken und Schwächen, so leuchtet ein, daB der Vorteil der "Teletex/ Post"-Alternative gegenüber der Bildschirmtextlösung in folgenden Leistungsmerkmalen begrüdet 1 iegt:

- die Obertragungs- bzw. Darstellungskapazität ist theoretisch unbegrenzt,

- das Informationssystem läßt sich denkbar einfach handhaben,

- die ubermittelten Informationen sind aufgrund des Trägermediums Papier leicht zu archivieren,

1 Die Delphi-Methode beinhaltet den Versuch, verbalqualitative Items zur Beurteilung einzelner (Technologie-) Alternativen zu quantifizieren. Zur Anwendung der Delphi-Methode vgl.z. B. Strebel, H., Strothmann, K. - H., von Hagen, F., Ergebnisse einer Voruntersuchung zur Anwendung der Delphi-Methode auf das Thema "Hemmende und fördernde Faktoren im DurchsetzungsprozeB der Mikroelektronik", Arbeitspapier des Instituts für Markt- und Verbrauchsforschung der Freien Universität Berlin, Berlin 1982 . 


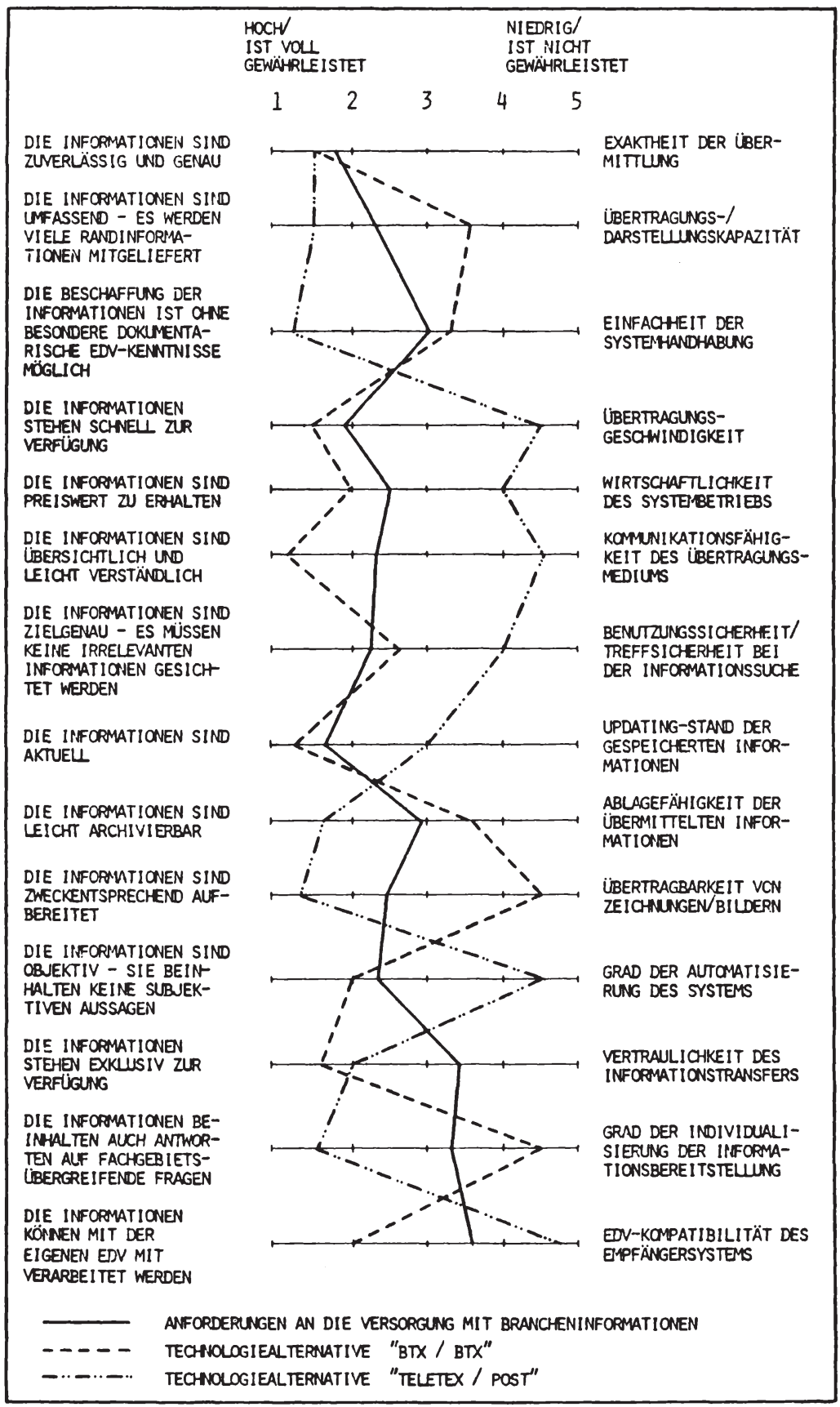

Abb. 33: Gegenüberstellung von Anforderungsmerkmalen der Informationsver-

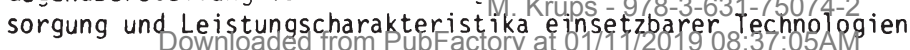


- es können Bilder und Zeichnungen übermittelt werden,

- die Versorgung mit fachgebietsübergreifenden Informationen ist durch den hohen Grad der Individualisierung der Informationsbereitstellung grundsätzlich möglich.

Demgegenüber kommen einer Datenbankrecherche via Bildschirm im Vergleich zur nicht-automatisierten Informationsvermittlung folgende Vorteile zu:

- die Ubertragungsgeschwindigkeit ist ungleich höher,

- die Wirtschaftlichkeit des Informationssystems verspricht durch den geringen Einsatz von Personal eher gewährleistet zu sein,

- das System ist voll kommunikationsfähig,

- die Treffsicherheit der Informationssuche kann durch iterative Suchvorgänge weitgehend optimiert werden in der "Offline"-Lösung muB sich der Informationsvermittler demgegenüber auf die Präzision einer einmal gestellten Anfrage des Abnehmers verlassen,

- der Informationssuchende genieBt den Vorzug, Daten nach einem Updating minutenaktuell zur Verfïgung zu haben, während er bei der offline-Recherche einen Aktualitätsverlust von 1 - 2 Tagen aufgrund des Postversandes hinnehmen muß,

- durch die Automatisierung der Informationsselektion entfällt das subjektive Auswahlermessen eines Informationsvermittlers,

- abgerufene Informationen können auf eigene EDV-Speicher "herabgeladen" und gegebenenfalls weiterverarbeitet werden.

Zentraler Aspekt dieser Gegenüberstellung von Vorteilen ist das Gewicht dieser Vorteile aus Nutzersicht. Hier zeigt Abbildung 33 eindrucksvoll, daB die Btx-OnlineAlternativen im Vergleich die funktionsadäquateren Stärken aufweist.

1 Man spricht auch von "downloading" externer Datenbestände auf eigene elektronische Trägermedien. 


\subsection{Durchführung der Technologieauswahl}

Sind die relevanten funktionsunabhängigen Determinanten der Technologie bestimmt und die einzelnen infrage kommenden Technologiealternativen entsprechend ihrer Funktionsadäquanz in eine Prioritätenrangfolge gebracht, so bleibt in der Folge die Aufgabe, alle einbezogenen Selektionskriterien in einem Punktbewertungsverfahren zu verknüpfen. 1 Abbildung 34 veranschaulicht in diesem Zusammenhang, wie Haupt- und Subfaktoren der Technologieauswahl unterschieden und mit ihrer Bedeutung entsprechenden Gewichten versehen werden können.?

Hinsichtlich der Bewertung der Funktionsadäquanz steht dem Unternehmen prinzipiell die Möglichkeit offen, unterschiedliche Differenzierungsgrade zugrunde zu legen. So kann es entweder globale Werte für den jeweiligen Rangplatz einer Technologie in der Eignungsskala vorsehen, oder aber - wie im Beispiel der Abbildung beispielhaft dargestellt - Einzelwerte für das Abschneiden der Technologie bei jedem Einzelmerkmal berücksichtigen. Diese Einzelwerte sind dann entsprechend der individuellen Bedeutung des jeweils korrespondierenden Anforderungsmerkmals der Funktionserfiullung zu gewichten.

Im Sinne einer konsistenten Fortfühung des Ausgangsbeispiels sei im folgenden unterstellt, da $B$ am Ende des in Abbildung 35 gezeigten Bewertungsprozesses 9 Technologiealternativen verbleiben. Unter der Prämisse, daB diese Alternativen grundsätzlich für jede der drei zu-

1 Im wesentlichen findet hier das gleiche Verfahren Anwendung wie bei der Bewertung einzelner Geschäftsfeldfunktionen; vgl. S. $105 \mathrm{ff}$. dieser Arbeit.

2 Zur mehrstufigen Gewichtung einzelner Bewertungsfaktoren vgl. vor allem das Modell von 0'Meara, J.T., Selecting Profitable Goods, in: HBR, Vol. 39, 1961 , No. $1, \mathrm{~S} .83 \mathrm{ff}$. 


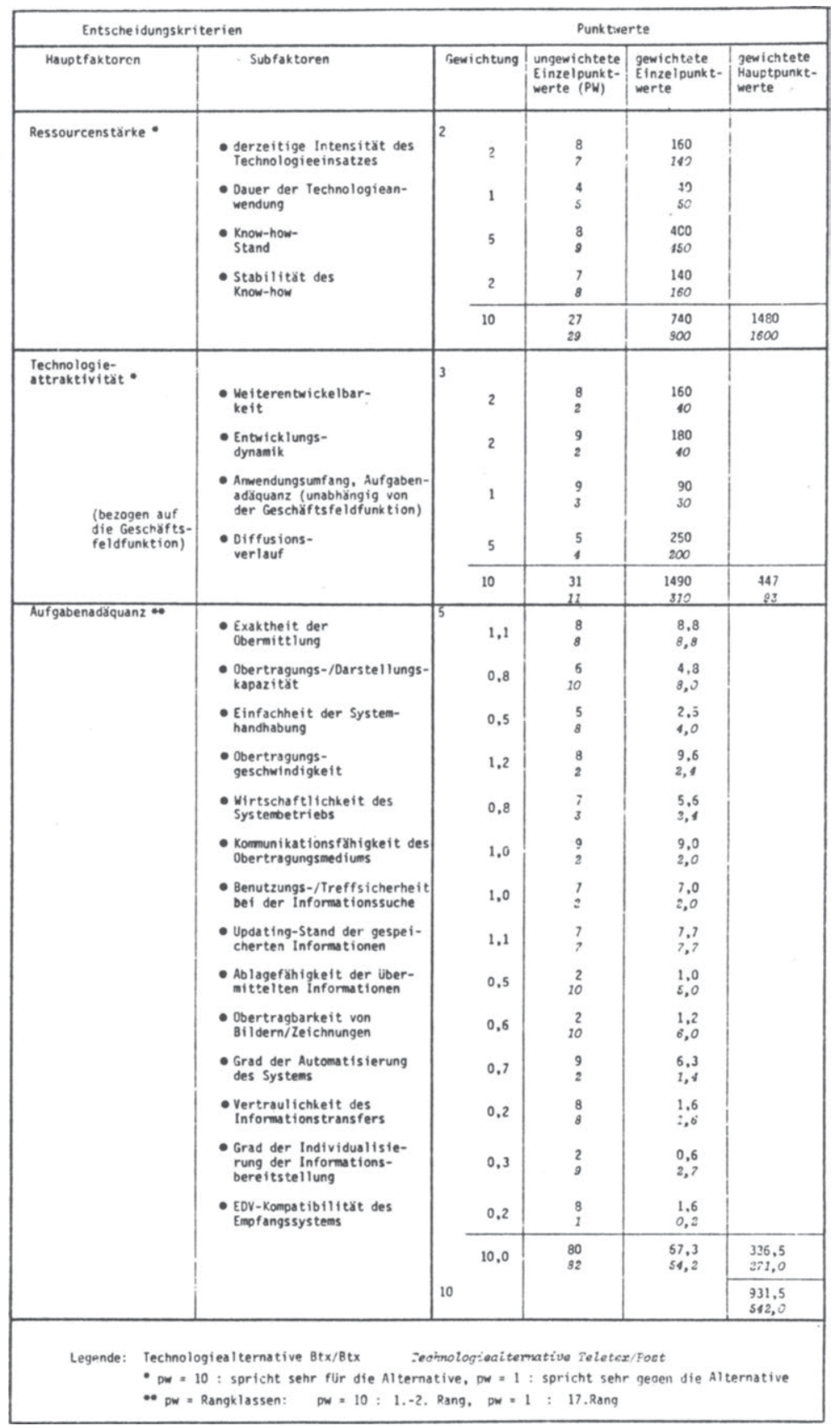

Abb. 34: Punktbewertung zur Auswahl der Geschäftsfeldtechnologie Downloaded from PubFactory at 01/11/2019 08:37:05AM 
vor ausgewählten Geschäftsfeldfunktionen in effizienter Weise zum Einsatz kommen können, setzt sich das Leistungsspektrum dann aus $(3 \times 9=) 27$ Funktions-/Technologiesegmenten zusammen (vgl. Abbildung 35). Für jedes einzelne Segment ist gewährleistet, daB es in leistungswirksamer Weise realisiert werden kann. Die Frage jedoch, ob alle Segmente gleichzeitig, gleichsam als Segment-Mix, in das Geschäftsfeld aufzunehmen sind, ist damit noch nicht entschieden. Vielmehr sind hierzu weitere Entscheidungskriterien heranzuziehen.

\subsection{Selektion geschäftsfeldrelevanter Angebotskombi- nationen}

\section{31 Determinanten der Angebotsauswahl}

\section{311 Ressourcenbedarf}

Der Ressourcenbedarf zur Realisierung einer bestimmten Geschäftsfeldalternative umfaßt das notwendige Potential zur Erstellung und Aufrechterhaltung des Dienstleistungssystems sowie alle mit der Entwicklung und Vermarktung dieses Systems einhergehenden Aktivitäten. Erfordern die in Betracht gezogenen FT-Segmente aufgrund ihrer Differenziertheit einen sehr heterogenen Ressourceneinsatz, so ist dies ein Indiz dafür, im sinne eines konzentrierten Einsatzes der Kräfte beim Vordringen auf den Markt die Zahl der Segmente weiter zu reduzieren. 1

1 Vgl. Abell, D.F., Defining the Business, a.a.0., S. $180 \mathrm{f}$. und S. 184 ; $\mathrm{vgl}$. in Anlehnung daran Walters, M., a.a.0., S. 118 f. und S. 121. 


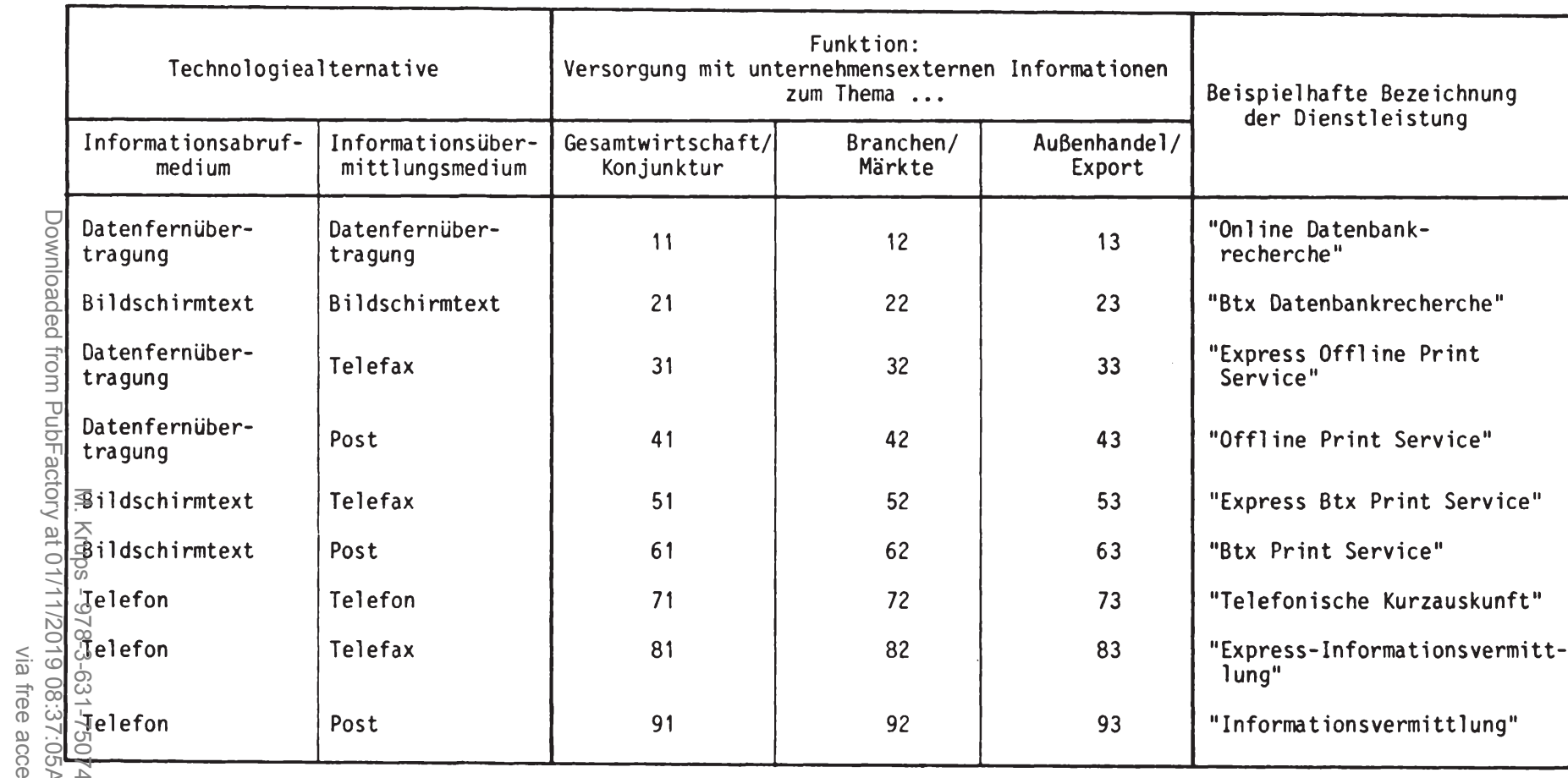

Abb. 35: Vor-ausgewählte Angebotskonstellationen als Funktions-/Technologie-Kombinationen 
Im vorliegenden Beispiel ist unschwer zu erkennen, daB zur gleichzeitigen Realisation aller Teilsegmente ein hoher und uberaus differenzierter Ressourcenbedarf anfallen würde. So sind für die drei geplanten Funktionen einzelne Informationsbasen aufzubauen, auf die zum einen über Datenfernübertragung, zum anderen aber auch über Bildschirmtext die Möglichkeit des Online-Zugriffs geschaffen werden muB. Daneben ist ein Team geschulter Informationsvermittler zusammenzustellen, deren Aufgabe darin besteht, telefonische Informationsanfragen entgegenzunehmen, Recherchen durchzuführen und Auskünfte zu erteilen. Diese wenigen Ausführungen zeigen bereits die Breite und Vielgestaltigkeit des Ressourcenbedarfs und können als Hinweis auf die Notwendigkeit verstanden werden, im vorliegenden Beispiel das Angebotsspektrum weiter zu verdichten.

\subsection{Skaleneffekte}

Ein breites Vordringen auf dem Markt bietet sich stets um so eher an, in je höherem Maße ein solches Vorgehen zur Realisierung von Skaleneffekten beiträgt. 1 Aufbauend auf dem Erfahrungskurvenkonzept ${ }^{2}$ beinhaltet der Skaleneffekt den empirisch belegten Sachverhalt, daß mit zunehmender kumulierter Produktionsmenge die Stückkosten kontinuierlich zurückgehen. ${ }^{3}$

Neben dem Konzept des Produktlebenszyklus ${ }^{4}$, den verschie-

$1 \mathrm{Vgl}$. Abell, D.F. Defining the Business, a.a.0., S. $181 \mathrm{ff}$. und S. 184.

2 Zum Erfahrungskurvenkonzept vgl. Henderson, B.D., Die Erfahrungskurve in der Unternehmensstrategie, Frankfurt, New York 1974; Arbeitskreis langfristige Unternehmensplanung der Schmalenbach-Gesellschaft, Strategische Planung, in: ZfbF, 29. Jg., 1977, S. 9 ff.; Wacker, P.A., Die Erfahrungskurve in der Unternehmensplanung. Analyse und empirische Oberprüfung, Schriftenreihe Wissenschaftliche Forschung und Entwicklung, Bd. 66, Hrsg.: Aschoff, C., Müller-Bader, P., Muinchen 1980.

$3 \mathrm{Vgl}$. Fischer, J., Zimmermann, W., Instrumente der strategischen Planung für Unternehmen mittlerer Größenordnung, in: ZfO, 52. Jg., 1983, H. 3, S. 140.

$4 \mathrm{Vgl}$. hierzu vor allem Meffert, H., Interpretation und Aussagewert des Produktlebenszyklus-Konzeptes, in:

Neuere Ansätze der Marketingtheorie, Festschrift zum 80. Geburtstag von 0tto Schnutenhaus, Hrsg.: Hammann, P., Kroeber-Riel, W., Meyer, C.W., Berlin 1974, S. $85^{\prime} \mathrm{ff}$. und Fischer, J., Zimmermann, W., a.a.0., S. $141 \mathrm{f}$. 
denen Portfolio-Ansätzen ${ }^{1}$ sowie dem PIMS-Mode $11^{2}$ stellt der Skaleneffekt eines der heute bereits als klassisch $z u$ bezeichnenden strategischen Planungsgrößen zur Sicherung der Profitabilität der Gesamtunternehmung dar. Gleichwohl muB einschränkend ergänzt werden, daß die Tauglichkeit dieser Ansätze in bezug auf Dienstleistungsunternehmen bislang nicht unter Beweis gestellt werden konnte. ${ }^{3}$ So unterstreichen zum Beispiel Langeard und Carman, daB "economies of scale are a controversial issue when applied to services with many studies denying that they exist". 4 Ergänzend stellen sie fest: "The effect of learning and experience on total unit cost has never been demonstrated in a service situation". 5

Angesichts dieser Situation ist die grundsätzliche Frage aufgeworfen, ob, und wenn ja, wann Skaleneffekte als sinnvolle Entscheidungskriterien für die Auswahl von Dienstleistungsgeschäftsfeldern in Betracht kommen. Einen wesentlichen Schritt zur Beantwortung dieser Frage hat klaus mit seiner Unterscheidung von "quasi-industriellen" und "interaktionsorientierten" Dienstleistungen ge-

$1 \mathrm{Vgl}$. zur Darstellung der Portfolio-Technik insbesondere Dunst, H.H., Portfolio-Management-Konzeption für eine strategische Unternehmensplanung, Berlin 1979; Engeleiter, H.J., Die Portfolio-Technik als Instrument der strategischen Planung, in: BFuP, 33. Jg., 1981, H. 5, S. $407 \mathrm{ff}$. Einen konzisen Uberblick uber alternative Portfolio-Ansätze liefern Meffert, H., Wehrle, F., a.a.0., S. $53 \mathrm{ff}$.

2 Vgl. zu PIMS ("Profit Impact of Marketing Strategies") vor allem Schoeffler, S., BuzzelT, R.D., Heany, D.F., Impact of Strategic Planning on Profit Performance, in: HBR, Vol. 52, March-April 1974, S. $137 \mathrm{ff}$.; Buzzell, R., Wiersema, F., Erfolgsstrategien im Kampf um Marktanteile, in: HM, O. Jg., 1981, S. $7 \mathrm{ff}$.

3 Langeard, E., Carman, I.M., a.a.0., S. 11.

4 Ebenda.

5 Ebenda. 
tan. 1 Während - so Klaus - sich interaktionsorientierte Dienstleistungen hartnäckig der sinnvollen Anwendung industrieller Managementtechniken widersetzen, lassen sich Unternehmen mit quasi-industriellen Dienstleistungen durchaus erfolgreich mit den Methoden der industriellen Betriebswirtschaftslehre (Standardisierung, Spezialisierung, Massenfertigung, Massenmarketing) führen. 2

Die Obertragung dieser Dichotomisierung der Dienstieistung auf die zur Entscheidung stehenden Angebotsalternativen erlaubt es, erste Anhaltspunkte für die Realisierbarkeit von Skaleneffekten zu gewinnen. Wie Abbildung 36 wiedergibt, sind weitgehend automatisierte online-Information Retrieval systeme als quasi-industrielle Dienstleistungen einzuordnen, be $i$ denen ein $n i c h t$ unbeträchtliches Kostendegressionspotential vermutet werden kann. Vernachlässigt man nämlich die relativ geringen Kosten der laufenden Systemwartung und -pflege, so verteilt sich der einmal investierte Fixkostenblock des Datenbanksystems auf eine wachsende Anzahl von Outputeinheiten (durchgeführten Recherchen); in der Fixkostendegression zeigt sich dabei eine typische Ausprägung des Skaleneffekts.

Aus dieser Perspektive ist dem Unternehmen zu empfehlen, als Kernprodukt eine weitestmöglich automatisierte Dienstleistung anzubieten, personalintensive Angebotsalternativen sollte es demgegenüber eher zurückstellen.

$1 \mathrm{Vgl.Klaus,}$., Dienstleistungen, a.a.0., S. $5 \mathrm{ff}$.

$2 \mathrm{Vgl}$. ebenda, S. 5 sowie insbes. S. $11 \mathrm{f}$. 


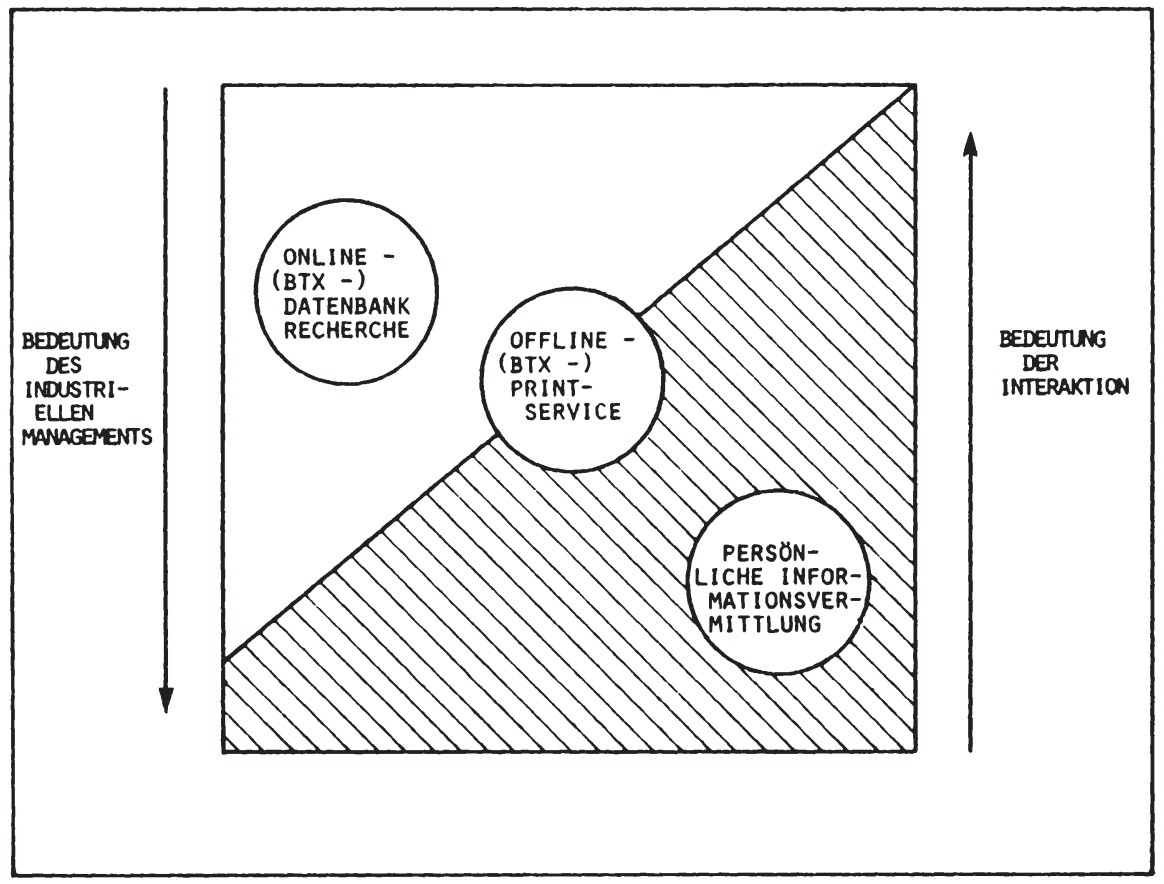

Abb. 36: Managementrelevante Charakterisierung alternativer Informationsdienstleistungen

(Quelle: in Anlehnung an Klaus, P., Dienstleistungen, a.a.0., S. 6)

\subsection{Komplexität der Dienstleistung}

Informationsdienstleistungen, wie sie im vorliegenden Beispiel im Mittelpunkt der Betrachtung stehen, zeichnen sich allein aufgrund ihrer Neuigkeit durch einen hohen Grad der Komplexität aus. Dies gilt um so mehr, je mehr von den Abnehmern erwartet wird, daB sie von bisherigen, gewohnten Verhaltensmustern abweichen. Grundsätzlich gilt die Regel, daB, je komplexer eine Dienstleistung gestaltet ist, desto enger das Geschäftsfeld bemessen sein sollte. ' Wichtig erscheint es dabei nicht

1 Vgl. Walters, M., a.a.0., S. 119 und S. 121. 
zuletzt, zwei Grundsätze zu beachten: ${ }^{1}$

Zum einen sollte nicht ein System angeboten werden, das am "cutting edge of technological progress" 2 angesiedelt ist, dessen Komplexität und Innovationsgrad also die latent interessierte Nutzerschaft abschreckt.

Zum anderen sollten aber auch "faule Kompromisse" vermieden werden ${ }^{3}$, die daraus resultieren können, daß das Management in der Phase des Markteintritts vor den Risiken umfangreicherer Investitionen zurückschreckt. Wichtig ist - gerade im Hinblick auf automatisierte Systeme die Beachtung des Prinzips der MindestgröBe ("principle of magnitude"). 4 Durch dieses Prinzip gelingt es, bereits bei der Definition des Geschäftsfeldes sicherzustelien, da $B$ die angestrebte Funktionserfüllung - nämlich die Versorgung einer größeren Abnehmergruppe mit unternehmensexternen Informationen - auch realisierbar ist. Dabei hat das besondere Augenmerk dem erzielbaren Umsatz zu gelten: "The key point is 'volume" - in a magnitude suf ficient to achieve efficiency and to employ systems and technologies which produce reliable, rapid, and low unitcost service results". 5

1 Levitt spricht hierbei von "Stopgaps" im Dienstleistungsgeschäft. Vgl. Levitt, Th., Production-line approach to service, in: HBR, Vo1. 50, SeptemberOctober 1972, S. $49 \mathrm{ff}$. (im folgenden zitiert als: Levitt, Th., Production -line).

2 Ebenda.

$3 \mathrm{Vgl}$. ebenda, S. $50 \mathrm{ff}$..

$4 \mathrm{Vgl}$. Levitt, Th., The industrialization of service, in: HBR, Vol. 54, September-October 1976, S. $68 \mathrm{ff}$. (im folgenden zitiert als: Levitt, Th., Industrialization).

5 Ebenda, S. 69. 


\section{314 Abnehmerverhalten}

Für die Bestimmung des Geschäftsfeldes haben in bezug auf das Verhalten der potentielien Abnehmer folgende drei Fragen besondere Relevanz: ${ }^{1}$

- Wie stark ist die Preis-bzw. die Qualitätsorientierung der Abnehmer ausgeprägt?

- Wie intensiv zeichnet sich der Wunsch der potentiellen Abnehmer nach Systemlösungen bzw. nach Einzeldienstleistungen ab (Service-bzw. Beratungsaspekt)?

- Wie homogen gestaltet sich der Bedarf der Abnehmer?

Gerade bei innovativen Dienstleistungen, deren Einführung erst bevorsteht, wirft die Beantwortung dieser Fragen nicht unbeträchtliche Probleme auf. So kann z. B. die Preissensitivität nur ansatzweise aus dem bisherigen Verhalten abgeleitet oder mit unbestimmter Zuverlässigkeit durch empirische Untersuchungen erhoben werden.

Im Rahmen der vorliegenden Befragung wurde 2 . B. versucht, die Preissensitivität der potentiellen Abnehmer eines unternehmensexternen elektronischen Informationsdienstes durch die Gegenüberstellung dieses Systems mit der Alternative herkömmlicher Informationsbeschaffung $z u$ erfassen. Das Ergebnis in Abbildung 37 zeigt, daB ab einer Mehrkosten-Höhe von über $10 \%$ die Unternehmen die bisher genutzten Informationsquellen dem neuen Informationsdienst vorziehen. Spricht eine solche konstellation typischerweise für eine breite, undifferenzierte Dienstleistungspalette mit geringen Preisen ${ }^{2}$,

$1 \mathrm{Vgl}$. Abell, D.F., Defining the Business, a.a.0., S. $179 \mathrm{ff}$. und S. 184.

$2 \mathrm{Vgl}$. ebenda, S. 179 und S. 184; vgl. in Anlehnung daran Walters, M., a.a.0., S. 116 und S. 121. 
KOSTEN JAHR

MEHR ALS $10 \%$

MINDERKOSTEN

GLEICHE

KOSTEN

$+10 \%$

MEHRKOSTEN

$+50 \%$

MEHRKOSTEN

$+100 \%$

MEHRKOSTEN

MEHR ALS $100 \%$ MEHRKOSTEN
DER INFORMATIONS- DER INFORMATIONSVERMITTLUNGSDIENST IST

DIENST IST

TENDENZIEUI ZU EINDEUTIG ZU BEVORZIGEN
VERMITTLUNGS-

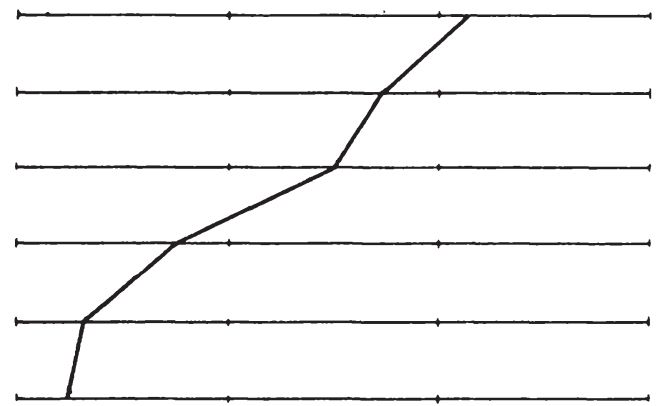

Abb. 37: Kostensensitivität potentieller Nutzer eines elektronischen Informationsdienstes

so steht sie in Konflikt mit der verbreiteten $\mathrm{Klage}$ über die mangelnde Qualität der derzeitigen Informationsversorgung und der Forderung nach einem spezialisierten und leistungsfähigen Informationsservice. Ahnlich konfliktär verhält sich die Strategie einer breiten Angebotsabdeckung mit dem tendenziellen Verzicht der potentiellen Abnehmerunternehmungen auf eine fachgebietsübergreifende Informationsversorgung.

Aus beidem ergibt sich, daB die Prognose spezieller Aspekte des Abnehmerverhaltens gerade bei innovativen Dienstleistungen mit hohen Unsicherheiten behaftet ist. Aufgrund dessen sollte diesen Aspekten kein übergroBes Gewicht im ProzeB der Angebotsauswahl beigemessen wer- 
den. Nicht zuletzt spricht hierfür auch die Tatsache, daB es vielfach das erklärte $Z$ iel der innovativen Dienstleistung ist, das Verhalten der Abnehmer bewuBt zu beeinflussen und zu verändern. 1

\subsection{Darstellung exemplarisch ausgewählter Angebots- kombinationen}

Betrachtet man die Angebotsauswahl in einer Gesamtschau, so fällt auf, daß die Entscheidungskriterien nur in sehr beschränktem Maße einer Operationalisierung zugänglich sind. Dies hat zur Konsequenz, daB die Auswahl letztlich nur nach sehr globalen Heuristiken erfolgen kann. Um so größeres Gewicht kommt in diesem Licht der Tatsache $z u, d a B$ die vorgeschalteten Auswahlstufen folgende drei Erfordernisse erfiullen:

- die Gesamtheit der Alternativen muB auf eine überschaubare $Z a h l$ reduziert werden,

- die Alternativen sollten sich grundsätzlich unabhängig voneinander realisieren lassen ${ }^{2}$,

- jede Alternative sollte für sich Aussicht auf eine erfolgversprechende Verwirklichung haben.

Unter diesen Prämissen ist hinzunehmen, daß sich der abschlieBende AuswahlprozeB in seiner Quintessenz auf eine kurze Kette von Plausibilitätsprüfungen reduziert, an deren Ende das Geschäftsfeld in seinen zwei Angebotsdimensionen determiniert ist.

1 So muß es im vorliegenden Fall das Ziel des Managements sein, das Verhalten der Abnehmer im Sinne einer Vitalisierung der Informationsnachfrage zu beeinflussen. Vgl. hierzu auch die Ausführungen unter Punkt C.3., S.247 ff. dieser Arbeit.

2 Hiervon bleibt die grundsätzliche Möglichkeit von Komplementäreffekten bei der gleichzeitigen Realisation zweier oder mehrerer Alternativen unberijhrt. 
Für die folgenden Betrachtungen sei beispielhaft davon ausgegangen, da $B$ von den zuletzt dargestellten Angebotsalternativen ${ }^{1}$ die personenintensiven und technologisch wenig fortschrittlichen Serviceangebote auf der Basis des telefonischen Informationsabrufs eleminiert werden. Ferner sei unterstellt, daB die Unternehmensfühung die Alternativen des Datel-gestuitzten Online-Retrieval zugunsten der substitutiv wirkenden Bildschirmtextlösung verworfen hat, da die Diffusionsaussichten dieses Mediums ungleich günstigere Penetrationsvoraussetzungen für die geplante Dienstleistung versprechen.

Damit steht für die weiteren oberlegungen ein elektronischer Informationsdienst im Mittelpunkt, der sich ausschließlich auf das neue Medium Btx stützt. ${ }^{2}$ Für diesen Dienst gilt es nunmehr, die Frage der relevanten Abnehmersegmente zu beantworten.

\section{Auswahl angebotsadäquater Abnehmergruppen}

4.1 Marktsegmentierung als Instrument zur Abgrenzung relevanter Abnehmergruppen

Die Lokalisierung und Beschreibung relevanter Abnehmergruppen ist Gegenstand und Aufgabe der Marktsegmentierung. 3 Bezogen auf den Prozeß der iterativen Geschäftsfeldwahl muß es das Ziel der Marktsegmentierung sein, solche Abnehmergruppen herauszufiltern, die als gemeinsame, weithin homogene Eigenschaft die Bereitschaft zum Kauf der geplanten Dienstleistung aufweisen. In diesem Sinne ist es Aufgabe der Marktsegmentierung, solche Kriterien zu finden, die die kaufbereiten Abnehmer von den Nichtbereiten bestmöglich zu trennen imstande sind.

1 Vgl. Abbildung 35 auf $S$. 149 dieser Arbeit.

2 Explizit sind dies die Alternativen 21 bis 23 und 51 bis 63 in Abbildung 35 auf $S$. 149 dieser Arbeit.

$3 \mathrm{Vgl}$. hierzu auch S. $58 \mathrm{ff}$. dieser Arbeit. 
4.11 Ansätze zur Marktsegmentierung im Bereich investiver Dienstleistungen

Eine Analyse der Literatur zur Marktsegmentierung macht deutlich, daB die Marketing-Wissenschaft diesem Problemkreis in bezug auf den Dienstleistungsbereich bislang nur wenig Aufmerksamkeit gewidmet hat. So fehlen bis heute weitgehend Ansätze, die es erlauben würden, das Problem der Marktabgrenzung aus der spezifischen Sicht eines Dienstleistungsanbieters zu lösen. 2

Anders als im Dienstleistungsbereich hat die Literatur auf dem Gebiet des Investitionsgütermarketing erste Hinweise auf Möglichkeiten einer Marktsegmentierung geliefert, die auch auf investive Dienstleistungen übertragen werden können. Besondere Bedeutung kommt hier den

i Dies wird unmittelbar deutlich, wenn man die jüngere Literatur zum Dienstleistungsmarketing heranzieht.

Während A.Meyer beispielsweise in seiner vielbeachteten Ausarbeitung nahezu gänzlich auf die explitite Berücksichtigung des Marktsegmentierungsproblems verzichtet, widmet Scheuch in seinem Standardwerk zum Dienstleistungsmarketing diesem Themenbereich lediglich zwei Seiten. Vgl. Meyer, A., a.a.0., und Scheuch, F., Dienstieistungsmarketing, a.a.0., S. $156 \mathrm{ff}$.. Vereinzelte Spezialuntersuchungen zur segmentierung im Dienstleistungsbereich finden sich lediglich z. B. be i Mazanec, J., Zielgruppenpalnung im Fremdenverkehrs-Marketing. Empirische Ergebnisse einer Segmentationsanalyse des österreichischen Urlaubsreisenden, in: JfB, 1979, H. 3, S. $176 \mathrm{ff}$. Sowie Anderson, W.Th. jr., Cox, E.P., Fulcher, D.G., Bank Selecting Decisions and Market Segmentation, in: JoM, Vol. 40, January 1976, $\mathrm{Nr} .1, \mathrm{~S} .40 \mathrm{ff}$..

2 Gleichzeitig weisen empirische Untersuchungen nach, daB auch im Dienstleistungsbereich das Konzept der Marktsegmentierung eine hohe Priorität genieBt. So ergab eine empirische Erhebung bei 207 schwedischen Dienstleistungsunternehmen, daB rd. $40 \%$ der Firmen die Marktsegmentierung sehr intensiv bzw. intensiv einsetzen. Vgl. Grönroos, Chr., Strategic lianagement, a.a.0., s. $66 \mathrm{ff}$. 
sog. konzeptionellen Ansätzen zu, die versuchen, die für die Trennung einzelner Abnehmergruppen wichtigen Segmentierungskriterien zu ordnen und in ejne sinnvolle Abfolge zu bringen. 1 In ihrem Kern beinhalten die Ansätze die Grundaussage, daB sich die Segmentierung der potentiellen Abnehmerunternehmungen in einem mehrstufigen ProzeB vollziehen sollte.

Aufbauend auf dem zweistufigen Basismodell von Wind und Cordozo empfehlen so z. B. Scheuch und Gröne eine Marktsegmentierung in drei Schritten. Scheuch ${ }^{3}$ schlägt vor, in einem ersten Schritt umweltbezogene Merkmale zugrunde zu legen, um dann auf der zweiten Stufe innerorganisatorische Merkmale heranzuziehen. Auf der dritten Stufe seien dann Merkmale der Mitglieder des Buying Centers zu berücksichtigen.4 Demgegenüber plädiert Gröne für eine Segmentierungsabfolge, an deren Anfang organisationsbezogene Kriterien stehen, gefolgt von Merkmalen des Entscheidungskollektivs und zuletzt Merkmalen der entscheidungsbeteiligten Individuen. 5

Shapiro und Bonoma ${ }^{6}$ entwickeln schließlich den dreistufigen Ansatz weiter, indem sie einen "nested approach" für die Segmentierung industrieller Märkte vorschlagen. Letztlich mündet das Konzept jedoch wiederum in einen Stufenplan, der nunmehr nacheinander nicht mehr drei, sondern fünf Gruppen von Merkmalen als Segmentierungs-

1 Als solche konzeptionellen Ansätze gelten vor allem die Verfahren der sog. Mehrstufen- und Kaufphasensegmentierung. Vgl. vor allem Backhaus, K., a.a.0., S. $80 \mathrm{ff}$. und die dort angegebene Literatur.

2 Vgl. Wind, Y., Cardozo, R., a.a.0. Vgl. im gleichen Sinne Engelhardt, W.H., Günther, B., a.a.0., S. $88 \mathrm{ff}$..

$3 \mathrm{Vgl}$. Scheuch, F., Investitionsgitermarketing, a.a.0.

$4 \mathrm{Vgl}$. ebenda, $S$.

$5 \mathrm{Vgl}$. Gröne, A., a.a.0., insbesondere S. $35 \mathrm{f}$..

$6 \mathrm{Vgl}$. Shapiro, B.P., Bonoma, Th.V., Industrial Market Segmentation: A Nested Approach, Marketing Science Institute, Report No. 83-100, February 1983 (im folgenden zitiert als: Shapiro, B.P., Bonoma, Th.V., Nested Approach). Eine zusammenfassende Darstellung des Ansatzes findet sich bei denselben, How to segment industrial markets, in: HBR, Vol. 62, May-June 1984, S. $104 \mathrm{ff}$. (im folgenden zitiert als: Shapiro, B.P., Bonoma, Th.V., How to segment). 
kriterien nahegelegt. Es sind dies demographische Merkmale der Unternehmung (Branche, Größe, Standort), Handlungsmerkmale der Unternehmung (Technologieeinsatz, Kapazitätsmerkmale, Marken- und Produktnutzung), Beschaffungsmerkmale (Organisation der Einkaufsfunktion, Machtstrukturen, grundsätzliche Einkaufspolitik, Käufer-Einkäufer-Beziehung, Einkaufskriterien), situative Merkmale (Dringlichkeit des Bedarfs, Produktanwendung, Bestellmenge) und individuelle Merkmale des Einkäufers.

Beurteilt man die ZweckmäBigkeit der mehrstufigen Ansätze insgesamt im Hinblick auf den Bereich investiver Dienstleistungen, so verdienen zunächst die generellen Bedenken gegenüber diesen Konzepten Berücksichtigung. So wird vor allem hervorgehoben, daB die Segmentierungskriterien nicht situationsspezifisch konkretisiert und gewichtet werden. Hinzu kommt, daB die kriterien nicht sauber zwischen taktischen unf strategischen Segmentierungsentscheidungen trennen. 1 Noch gravierender erscheint schließlich, daß nur eine vergleichsweise geringe Zahl von Einzelkriterien den grundsätzlichen Anforderungen der Meßbarkeit, der Erfaßbarkeit und der zeitlichen Stabilität genügt.

Vor diesem Hintergrund und unter Berücksichtigung der spezifischen Besonderheiten investiver Dienstleistungen soll im folgenden ein eigenständiger Ansatz zur Abgrenzung der relevanten Abnehmergruppen entwickelt werden. Gegenstand der Segmentierung seien hierbei die potentiellen Abnehmer des betrachteten elektronischen (Btx-) Informationsdienstes.

1 Vgl. vor allem Backhaus, K., a.a.0., S. 85. 


\subsection{Modell der Marktsegmentierung für elektronische} Informationsdienstleistungen

Den zentralen Ausgangspunkt des Modells bildet die Prämisse, daB die Bereitschaft potentieller Abnehmerunternehmungen, eine Dienstleistung in Anspruch zu nehmen, von zwei grundsätzlichen Bedingungen abhängt:

1. Es muB für das Unternehmen eine konkrete Veranlassung geben, die Dienstleistung entgeltlich in Anspruch zu nehmen. "Unter Hervorhebung des zweckrationalen Elements in industriellen Einkaufsentscheidungen kann man annehmen, da $B$ bestimmte Voraussetzungen oder Bedingungen in Form von Bedarfs - und Problemausprägungen gegeben sein müssen, damit ein Unternehmen überhaupt als Käufer eines bestimmten angebotenen Güteroder Problemlösungstyps ... in Betracht kommt." 1 Dabei ist noch keine Aussage daruber getroffen, wie sich dieser Bedarf zu manifestieren hat, $d . h$. in welcher Weise der Bedarf als Veranlassung zum Kauf von den betroffenen Entscheidungsträgern wahrgenommen werden muB.

2. Die zweite Voraussetzung für die Kaufbereitschaft potentieller Abnehmer besteht in dem Vorhandensein einer ausreichenden Kauffähigkeit. ${ }^{2}$ Damit ist die Bedingung angesprochen, daB die Abnehmerunternehmung über die für den Kauf notwendigen finanziellen Mittel verfügt, und $d a B$ bestimmte technische oder infrastrukturelle Voraussetzungen für die Inanspruchnahme des Dienstes vorliegen oder mit vertretbarem Aufwand geschaffen werden können.

1 Gröne, A., a.a.0., S. 180.

2 Zur Bedeutung der Kauffähigkeit vgl. auch Bauer, E., Markt-Segmentierung als Marketing-Strategie, Berl in 1976 , S. 113 und Ahlert, D., Grundzüge des Marketing, 2. Aufl., Düsseldorf 1980, S. $34 \mathrm{f}$. . 
Beiden Bedingungen kommt im Hinblick auf das zu erwartende Verhalten der Abnehmer eine zentrale Rolle zu. Während der Bedarf als notwendige Bedingung für die Bereitschaft zur Inanspruchnahme der Dienstleistung interpretiert werden kann, ist die erforderliche Kauffähigkeit als hinreichende Bedingung zu verstehen. Betrachtet man vor diesem Hintergrund die Kaufbereitschaft der einzelnen Abnehmerunternehmung als abhängige (zu erklärende) Variable und die Kriterien, die die Kaufwahrscheinlichkeit dieser Unternehmung determinieren, als unabhängige (erklärende) Variablen, so nehmen Kaufveranlassung und Kauffähigkeit die Funktion von Brückenvariablen ein. 1 Aus dieser Konstellation folgt, daB alle diejenigen Kriterien, die einen Bezug zu einer oder beiden Brückenvariablen aufweisen, auch einen Bezug zur jeweiligen Ausprägung der Kaufbereitschaft besitzen (vgl. Abbildung 38 ).

Ausgehend von dieser Grundstruktur abhängiger und unabhängiger Variablen sei das in Abbildung 39 entworfene Modell zur Auswahl industrieller Abnehmer eines elektronischen Informationsdienstes vorgeschlagen. Der Bezugsrahmen unterstellt einen Katalog relevanter Segmentierungskriterien, von denen vermutet werden kann, daB sie in signifikanter Weise mit der Bereitschaft potentieller Adopter zur Nutzung des elektronischen Informationssystems zusammenhängen. Damit beinhaltet das Modell die Hypothese, über die Ausprägungen der einzelnen Variablen zu einer Aussage über das zu erwartende Verhalten der potentiellen Abnehmer zu gelangen. Hieraus resultiert schließlich, daB unter Zugrundelegung der einzelnen Variablenausprägungen potentielle Adopter und Nichtadopter als Zielpersonen klassifiziert werden können.

1 Eine abweichende Konkretisierung segmentierungsrelevanter Brückenvariablen geht auf Gröne zurück, der Kriterien der "Kaufvoraussetzung" von Kriterien des "Auswahl - und Bewertungsverhaltens" unterscheidet. Vgl. Gröne, A., a.a.0., S. $32 \mathrm{ff}$. 


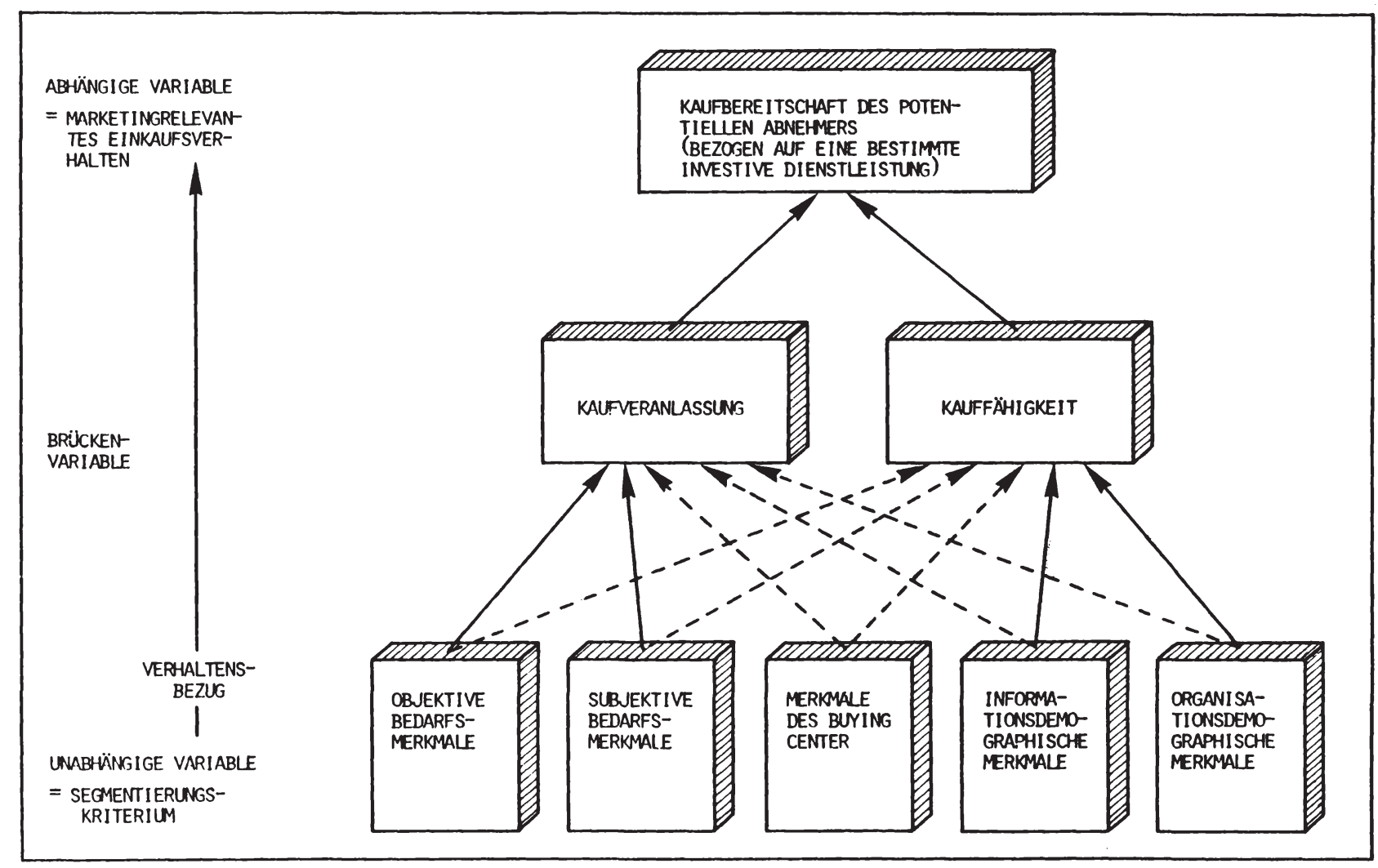

Abb. 38: Hierarchische Grundstruktur von Kriterien zur Abnehmersegmentierung 
Die Auswahl der einzelnen Kriterien erfolgte unter besonderer Bezugnahme auf Erkenntnisse aus der Adoptions- und Diffusionstheorie.' Daneben lieferten Untersuchungen über das industrielle Kaufverhalten ${ }^{2}$ sowie das Informationsnachfrageverhalten in Entscheidungsprozessen ${ }^{3}$ wesenti iche Hinweise auf relevante Merkmale.

Abbildung 39 zeigt die im vorliegenden Modell einbezogenen Variablen im Oberblick. Im Vordergrund stehen zum einen Merkmale, die als Indikatoren des Informationsbedarfs den Grad der Kaufveranlassung der einzelnen Unternehmung widerspiegeln. Hierzu zählen subjektive Bedarfsmerkmale wie die wahrgenommene Zufriedenheit mit der Informationsversorgung und die Intensität des Informationsbedarfs ebenso wie objektive Bedarfsmerkmale, die aus der spezifischen Situation der Unternehmung resultieren. Auf der anderen Seite werden Kriterien berücksichtigt, die als Indikatoren des Dispositionsspielraums auf die Kauffähigkeit der Unternehmung schließen lassen. Wichtige Maßgrößen sind hierbei im folgenden näher zu konkretisierende informationsdemographische Merkmale, die die Situation der Unternehmung in bezug auf die Handhabung

$1 \mathrm{Vgl}$. in diesem Zusammenhang insbesondere Lutschewitz, H., Kutschker, M., Die Diffusion von innovativen Investitionsgiutern. Theoretische Konzeption und empirische Befunde, München 1977.

2 Vgl. hierzu vor allem Webster, F.E., Wind, Y., Organizational Buying Behavior, Englewood Cliffs, N.J. 1972.

3 Besondere Bedeutung kommt hier vor allem den Untersuchungen von Witte zu. Vgl. Witte, E., Das Informationsverhalten in Informationssystemen - Die These von der unvollkommenen Informationsnachfrage -, in: Management-Informationssysteme - Eine Herausforderung an Forschung und Entwicklung -, Hrsg.: Grochla, E., Szyperski, N., Wiesbaden 1971, S. $832 \mathrm{ff}$. (im folgenden zitiert als: Witte, E., Informationsnachfrage); derselbe, Das Informationsverhalten in Entscheidungsprozessen, Tiubingen 1972 (im folgenden zitiert als: Witte, E., Entscheidungsprozesse); derselbe, Informationsverhalten, in: HdB, Hrsg.: Grochla, E., Wittmann, W., Stuttgart 1975, Sp. $1915 \mathrm{ff}$. (im folgenden zitiert als: Witte, E., Informationsverhalten). Zum Informationsverhalten in bezug auf die Nutzung einer Datenbank vgl. Brockhoff, K., Experimente zur Nutzung einer Datenbank, in: $2 \mathrm{fB}, 47$. Jg., 1977, H. 8, S. $509 \mathrm{ff}$. und die dort angegebene Literatur. 


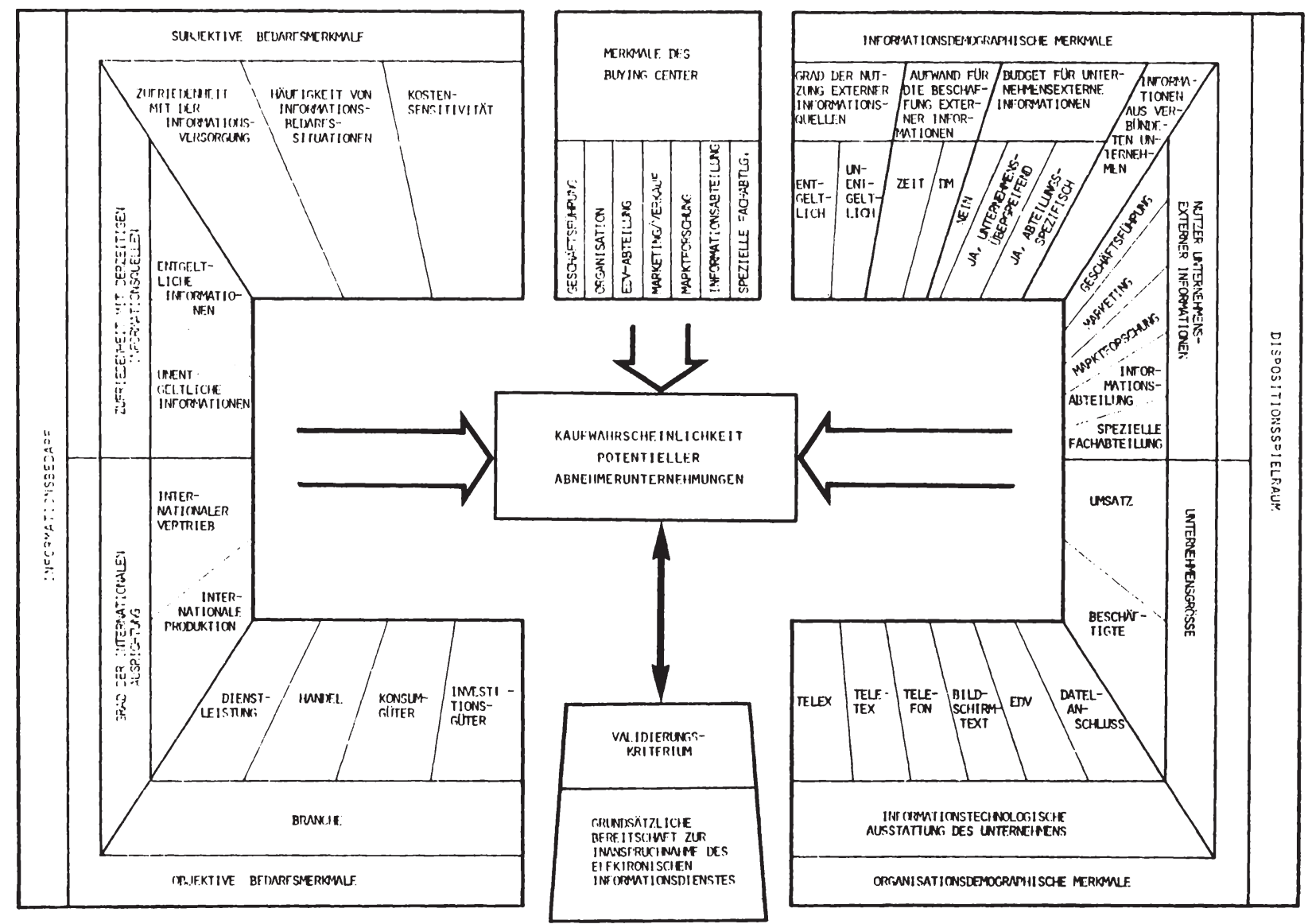

Abb. 39: Bezugsrahmen zur Auswahl relevanter Abnehmergruppen eines elektronischen Informationsdienstes 
und Gewichtung unternehmensexterner Informationen beschreiben. Daneben spielen organisationsdemographische Kriterien eine wesentliche Rolle. Eine gewisse Sonderstellung nehmen schlieBlich die Merkmale des Buying Center ein, die keiner der beiden Brückenvariablen isoliert zugeordnet werden können.

Auf die einzelnen unabhängigen Variablen sei im folgenden näher eingegangen. Dabei soll die Relevanz der Variablen im Hinblick auf die Auswahl von Nachfragern elektronischer Informationsdienste empirisch überprüft werden. Konkret gilt es festzustellen, ob in Abhängigkeit von der Kaufwahrscheinlichkeit (abhängige Variable) die Ausprägungen der einzelnen unabhängigen Variablen signifikante Unterschiede aufweisen. Entsprechend dem jeweils vorliegenden Skalenniveau kommen hierbei die in Abbildung 40 wiedergegebenen statistischen Testverfahren zur Anwendung.

Als Validierungskriterium ${ }^{2}$ für die Relevanz der einbezogenen Auswahlkriterien (unabhängige Variablen) dient die im Rahmen der vorliegenden Untersuchung empirisch erhobene abhängige Variable "Bereitschaft zur Nutzung elektronischer Informationsdienste". Die Messung erfolgt am Beispiel dreier Informationsdienste ${ }^{3}$ zum Thema

- "Gesamtwirtschaft/Konjunktur"

- "Branchen/Märkte" sowie

- "AuBenhandel/Export",

die Informationen aus online zugänglichen Datenbanken bereithalten.

1 Zur Skizzierung der einzelnen Variablen, ihrer Anwendungsvoraussetzungen und ihrer Aussagekraft sowie zur Spezifikation der entsprechenden SPSS-Prozuduren vgl. Beutel, P. et al., a.a.0., S. $176 \mathrm{ff.}, 196 \mathrm{ff}$. , $242 \mathrm{ff}$. Sowie die dort angegebene Literatur.

2 Das Validierungskriterium dient als Hilfsgröße, an dessen Ausprägungen sich die Verhaltensrelevanz einzelner Kriterien in bezug auf die Kaufwahrscheinlichkeit potentieller Abnehmer bemiBt.

3 Zur Reduzierung redundanter Informationen beschränken sich einige vertiefende Analysen $z$. T. exemplarisch auf das Beispiel von Brancheninformat ignsdiems-75074-2 


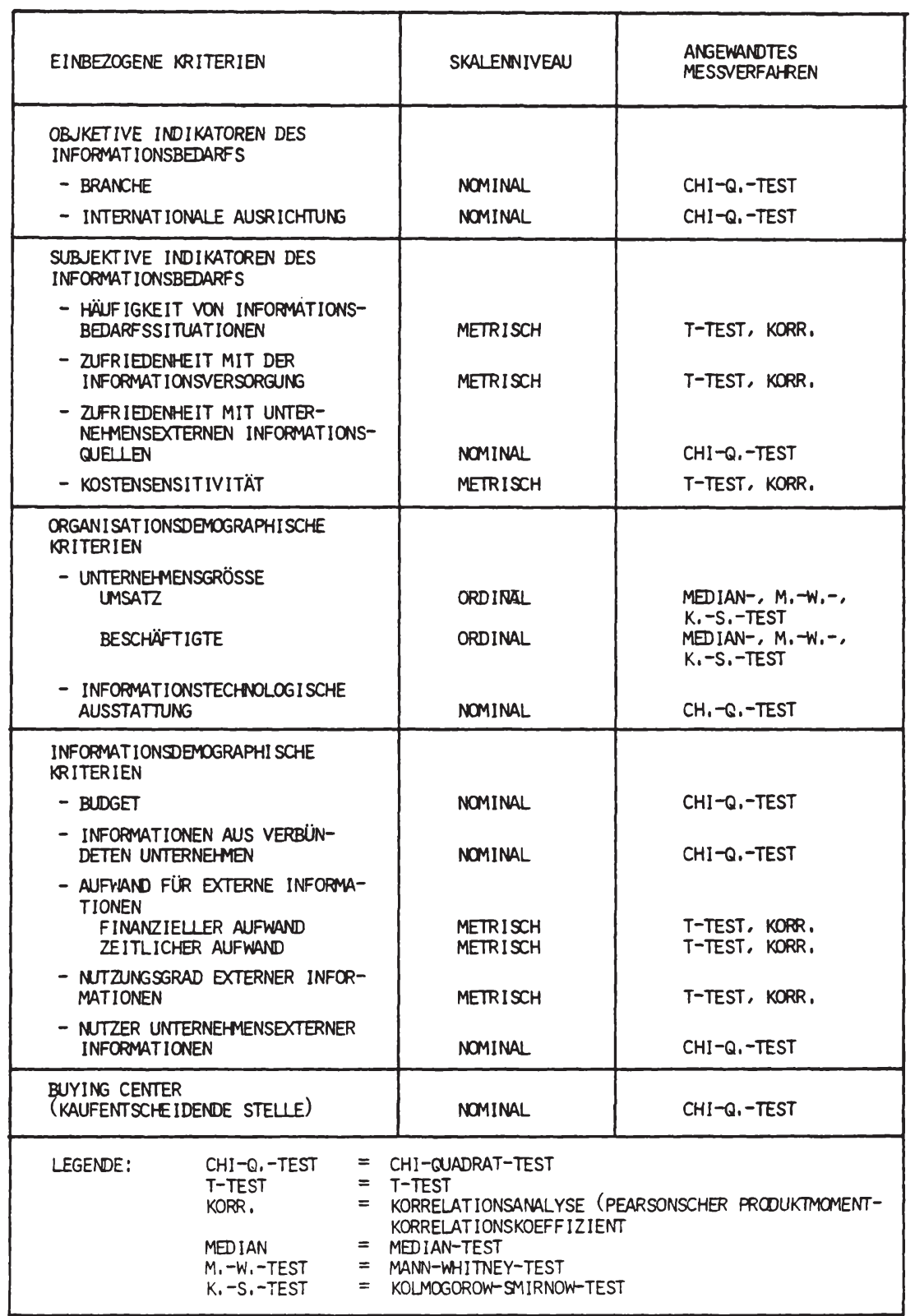

Abb. 40: Angewandte Verfahren zur Überprüfung der Relevanz von Kriterien der Abnehmerauswahl 
4.2 Kriterien zur Auswahl industrieller Abnehmer elektronischer Informationsdienste

4.21 Informationsbedarf als Kriterium der Kaufveranlassung

Ausgehend von der Individualität betrieblicher Entscheidungsprozesse ließe sich grundsätzlich die These vertreten, daß der Bedarf an Informationen im Unternehmen weitgehend unabhängig ist von den Umweltfaktoren, den Merkmalen des Leistungsprogramms oder den Marktbeziehungen der jeweiligen Unternehmung. Dementsprechend wäre für die Nutzung einer unternehmensexternen Informationsstelle entscheidend, in welchem MaBe die individuellen Entscheidungsträger einen aktuellen Informationsbedarf wahrnehmen und diesen in Informationsnachfrage umsetzen. 1 Einer solchen personalisierenden Betrachtungsweise steht die Vermutung gegenüber, daß nicht zuletzt die Eigenschaften einer Unternehmung und ihre Umweltsituation den betrieblichen "Basisbedarf" an externen Informationen und damit die Prädestiniertheit zur Nutzung eines externen elektronischen Informationsdienstes maBgeblich beeinflussen.

Diese Gegenthese, die sich an den sog. situativen oder Kontingenzansatz der Organisationstheorie anlehnt, ${ }^{2}$ geht von der Annahme aus, daB sich der für den Informationsdienst relevante betriebliche Bedarf an externen Informationen auf Unterschiede in der Situation zurückführen lassen, in der sich die Unternehmung jeweils befindet.

$1 \mathrm{Vgl}$. Uebele, H., Heinzelbecker, K., Der situationsgerechte Aufbau betrieblicher Informationssysteme, in: ZfO, 49. Jg., 1980, H. 7, S. 398.

$2 \mathrm{Zu}$ Gegenstand und Bedeutung des Kontingenzansatzes vgl. Dessler, G., Organization and Management - A Contingency Approach, London 1976; Bruns, I., Kritische Analyse des Kontingenz-Ansatzes in der Organisationstheorie, in: DB, $1970, \mathrm{H} .3, \mathrm{~S} .61 \mathrm{ff}$; Staehle, W.H., Deutschsprachige situative Ansätze in der'Managément\}ehre, in: Organisationstheoret ische Ansätze, Hrsg.: Kieser, A., Muinchen 1981, S. $215 \mathrm{ff}$. Zur Operationalisierung der Situation vgl. vor allem Kieser, A., Kubicek, H., Organisation, Berlin 1976, S. 191. 
Solche Kontextfaktoren der Unternehmung sollen im folgenden als objektive Indikatoren des Informationsbedarfs der Unternehmung näher betrachtet werden.

\subsection{Objektive Indikatoren des Informationsbedarfs}

Im Rahmen einer empirischen Untersuchung zur Verbreitung von Management-Informationssystemen (MIS) im Absatzbereich industrieller Unternehmen sind Köhler und Obele der Frage nachgegangen, welche Zusammenhänge zwischen der Umwelt einer Unternehmung und der Existenz eines betrieblichen MIS bestehen. 'Mit Hilfe von Faktorenanalysen konnten sie eine Reihe "objektiver" Merkmale ermitteln, die sich als erklärungsrelevant und gut interpretierbar erwiesen. Vieles spricht dafür, daB einige dieser Merkmale auch zur Unterscheidung von Unternehmen hinsichtlich ihres externen Informationsbedarfs beitragen können.

\subsection{Branche}

Umweltfaktoren der Unternehmung lassen sich grundsätzlich danach ordnen, in welcher Weise sie auf die bei der Planung wahrgenommene UngewiBheit einwirken. 2 Umweltfaktoren, die die wahrgenommene Ungewißheit erhöhen, steigern gleichzeitig den betrieblichen Informationsbedarf und führen zu einer vermehrten Implementierung innerbe-

$1 \mathrm{Vgl}$. Köhler, R., Uebele, H., Planung und Entscheidung im Absatzbereich industrieller Großunternehmen. Ergebnisse einer empirischen Untersuchung, Aachen 1977, S. $226 \mathrm{ff}$. Eine konzise Zusammenfassung ausgewählter Ergebnisse findet sich bei Uebele, H., Heinzelbecker, K., a.a.0., S. $396 \mathrm{ff}$.

2 Vgl. Uebele, H., Heinzelbecker, K., a.a.0., S. 398. 
trieblicher MIS. 1 Oberträgt man diesen Zusammenhang auf die vorliegende Problemstellung und unterstellt, daB die wahrgenommene UngewiBheit in einer Unternehmung auch als MaBstab für ihren Bedarf an unternehmensexterner Informationsversorgung zu interpretieren ist, so folgt daraus, daß die die Ungewißheit auslösenden Umweltfaktoren auch als Einflußgrößen in bezug auf die Nutzung eines unternehmensexternen Informationsdienstes angesehen werden können.

Folgt man diesen Oberlegungen, so liefert die Untersuchung von Köhler und Uebele interessante Ansätze zu einer Konkretisierung positiver (für die Nutzung des Informationsdienstes sprechender) und negativer Umweltfaktoren.

Zu den positiven Umweltfaktoren der anzubietenden Informationsdienstleistung zählt zunächst insbesondere eine hohe Umweltdynamik der betreffenden Abnehmerunternehmung. 2 Je häufiger langfristige Anderungen in der Zusammensetzung des Leistungsprogramms und des absatzpolitischen Instrumentariums vorgenommen werden muissen, und je stärker die Dynamik des technischen Fortschritts, Modetrends, etc. die Unternehmensaktivitäten prägen, um so eher $1 \ddot{a} \beta t$ sich die Bereitschaft zur Nutzung des Informationsdienstes vermuten. 3

1 Bleicher stellt in diesem Zusammenhang fest, daB Planungs- und Informationssysteme in der Wahrnehmung von Entscheidungsträgern neben einer Reduktion von Variabilität und Komplexität der Umwelt ergänzend eine Reduktion der UngewiBheit innerhalb der Organisation herbe iführen können (durch die frühzeitige Fixierung von Planungsalternativen, die Steigerung des Informationsgrades, etc.). Vgl. Bleicher, K., Organisation und Führung der industriellen Unternehmung, in: Industriebetriebslehre in programmierter Form, Bd. III, Organisation und EDV, Hrsg.: Jacob, H., Wiesbaden 1972, S. 147. Vgl. auch Uebele, H., Heinzelbecker, K., a.a.0., S. 398.

$2 \mathrm{Vgl}$. Uebele, H., Heinzelbecker, K., a.a.0., S. 398.

$3 \mathrm{Vgl}$. ebenda, S. $398 \mathrm{f}$. 
Ahnliches gilt für die Komplexität der Produkt/MarktBeziehungen: je größer die Anzahl und die Verschiedenartigkeit der Erzeugnisse des Absatzprogramms, um so eher ist davon auszugehen, da $\beta$ das Unternehmen den Informationsdienst in Anspruch nimmt.

Negative Umweltfaktoren beziehen sich demgegenüber in erster Linie auf die absatzpolitische Abhängigkeit der Unternehmung. 1 Diese kann sich vor allem darin manifestieren, daB das Unternehmen in seinen Planungen und Aktivitäten durch das Verhalten von (GroB-)Abnehmern beeinträchtigt wird. Besonders deutlich tritt eine solche Beeinflussung im Falle der Auftragsfertigung zutage.

Will man die genannten situativen Faktoren zur Segmentierung des vorhandenen Abnehmerpotentials zugrunde legen, so zeigt sich sehr schnell, daß dieser Versuch an der fehlenden Erfaßbarkeit und Meßbarkeit der Kriterien scheitern muß. Auf einen "Ausweg" weist hier die Beobachtung von Köhler und Uebele hin, daB die Branchenzugehörigkeit tendenziell mit bestimmten, jeweils ähnlichen Konstellationen von Einflußgrößen einhergeht. "Während die positiven Einflußgrößen am ehesten in der Verbrauchsguterindustrie vorzufinden sind, können die Negativfaktoren für Unternehmen als typisch gelten, die im industriellen Anlagengeschäft tätig sind". 2

Diese Tatsache läßt auf die Relevanz der Branchenzugehörigkeit ${ }^{3}$ im Hinblick auf die Nutzungsbereitschaft externer Informationsdienste schließen. In Analogie zum

1 Vgl. Uebele, H., Heinzelbecker, K., a.a.0., S. $398 \mathrm{f}$.

2 Uebele, H., Heinzelbecker, K., a.a.0., S. 398.

3 Vor dem Hintergrund der obigen überlegungen sei im folgenden das Auswahlkriterium "Branche" den "objektiven Bedarfsmerkmalen" zugeordnet. Gleichwohl sei darauf hingewiesen, da $B$ die Branche ein typisches organisationsdemographisches Merkmal der Unternehmung darstellt. 
MIS-Fall wäre entsprechend zu erwarten, daB Konsumgüterunternehmen tendenziell eher als Nutzer externer Informationsdienste in Frage kommen, während Investitionsgiterunternehmen weniger Anwendungsmöglichkeiten für den Informationsdienst besitzen. Tatsächlich stellt sich jedoch der Zusammenhang differenzierter dar.

So zeigt sich im Rahmen der vorliegenden empirischen Untersuchung, daß generell keine Rückschlüsse von der Branche auf die Adoptionschancen eines externen elektronischen Informationsdienstes möglich sind (vgl. Tabelle 6). Bezogen auf den Bereich gesamtwirtschaftlicher Information wie auch auf den Bereich der Brancheninformationen lassen sich keine signifikanten Gruppenunterschiede $z w i-$ schen adoptionsbereiten und nicht adoptionsbereiten Unternehmungen hinsichtlich der jeweiligen Branchenzugehörigkeit feststellen.

Anders verhält es sich, wenn man den Bereich der ExportInformationen betrachtet. Hier zeigt sich, daB in Abhängigkeit von der Branche signifikante Unterschiede hinsichtlich der Akzeptanz eines entsprechenden Informationsdienstes bestehen. So sind Dienstleistungsunternehmen eher nicht bereit, eine Export-Datenbank zu nutzen. 1 Demgegenüber ist bei Unternehmen des Konsum- und Investitionsgüterbereichs eine signifikant positive Beziehung zwischen Branchenzugehörigkeit und Nutzungsbereitschaft zu verzeichnen.

1 Dieses Ergebnis leuchtet insofern unmittelbar ein, als die befragten Dienstleistungsunternehmen ihre Aktivitäten nahezu ausschlieBlich auf inländische Märkte konzentrieren. 


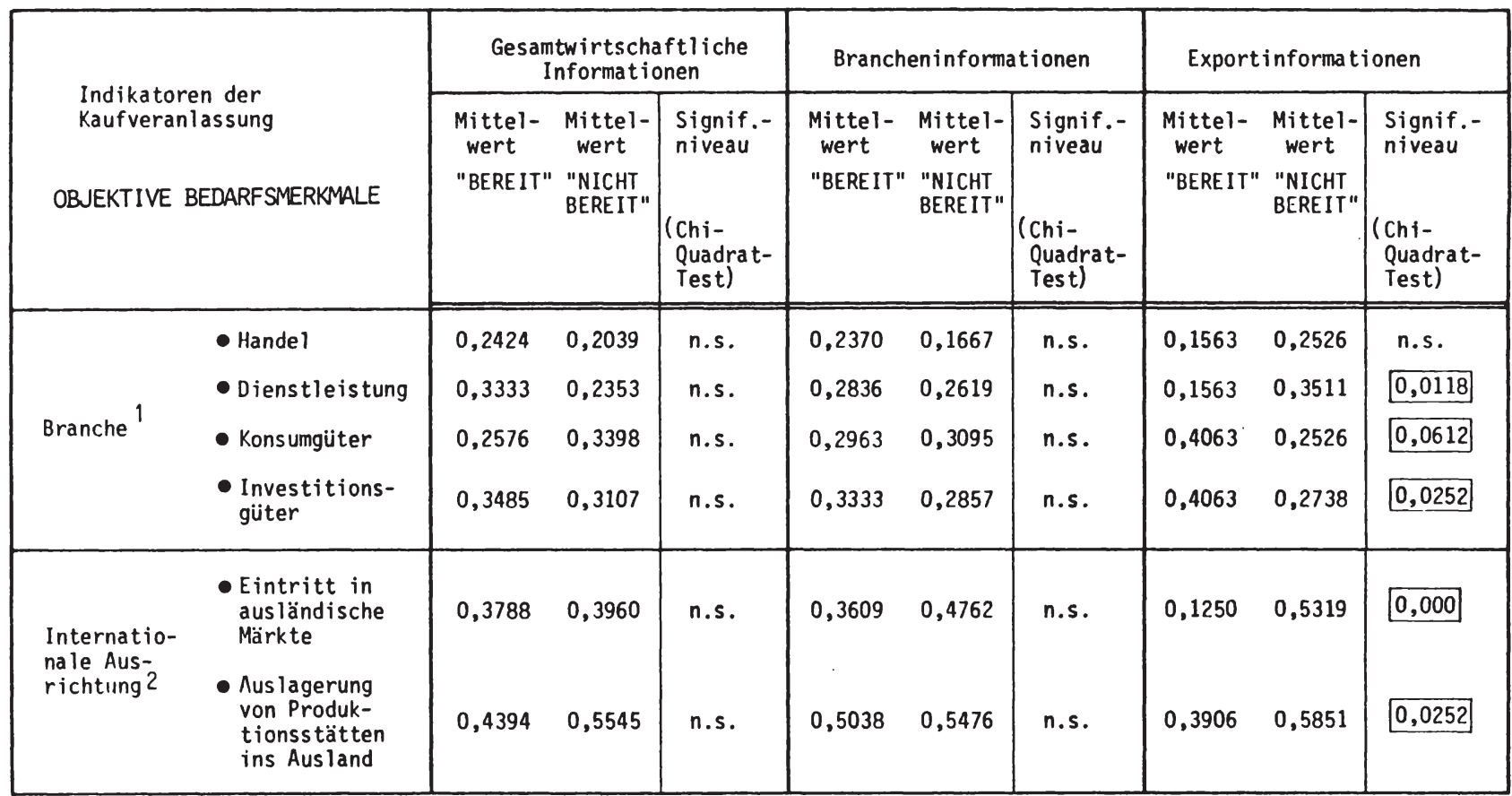

$$
\begin{aligned}
& \text { Legende: Mittelwerte: } \quad 1: 1=\text { trifft zu } \\
& 0=\text { trifft nicht } z u \\
& \text { 2: } 1 \text { = ist irrelevant } \\
& 0=\text { ist nicht irrelevant } \\
& \text { Signifikanzniveau: } \quad \alpha=0,1
\end{aligned}
$$

Tab. 6: Relevanz objektiver Bedarfsmerkmale 


\subsection{Internationale Ausrichtung}

Insbesondere für Informationsdienste zu Exportfragen liegt die Vermutung nahe, daß neben der Branche der Grad der internationalen Ausrichtung als weiterer wichtiger Umweltfaktor die Kaufbereitschaft industrieller Abnehmer beeinflußt. Vor diesem Hintergrund wurde in der vorliegenden Untersuchung gemessen, welchen Einfluß die Internationa1 isierung der Unternehmung auf die Bereitschaft zur Inanspruchnahme externer Informationsdienste nimmt.

Es bestätigte sich die naheliegende Erwartung, daß mit wachsender Bedeutung ausländische Absatzmärkte und mit zunehmender Auslagerung von Produktionsstätten ins Ausland die Bereitschaft zur Nutzung von Exportdatenbanken ansteigt. Deutlich wird dies in den hochsignifikanten Unterschieden zwischen den jeweiligen Gruppen.

\subsection{Subjektive Indikatoren des Informationsbedarfs}

Objektive Indikatoren des Informationsbedarfs beinhalten definitionsgemäß intersubjektiv nachprüfbare, situative Unternehmensmerkmale, die in mehr oder minder indirektem Zusammenhang mit dem Bedarf einer Unternehmung nach unternehmensexternen Informationen insgesamt stehen. Vieles spricht für die These, daß darüber hinaus eine Reihe direkter Einflußfaktoren wirksam werden, die ihren Ursprung in der subjektiven Wahrnehmung eines Informationsmangels durch die betroffenen Individuen im Unternehmen haben. Es steht zu erwarten, daB dieses individuell empfundene Informationsversorgungsdefizit als aktivierendes Element für die Nutzungsentscheidung aus der Sicht des betroffenen Entscheidungsträgers von zentraler Bedeutung ist. 1

1 Meffert spricht in diesem Zusammenhang von "prozeßaktivierenden Faktoren". Vgl. Meffert, H., ProduktivguiterMarketingforschung im System des Marketing, in: Der Markt, 13. Jg., 1974, Nr. 49, S. 12 (im folgenden zitiert als: Meffert, H., Produktivguiter-Marketingforschung). 
Zur empirischen Oberprüfung dieser Hypothese geht die vorliegende Untersuchung davon aus, da $B$ der subjektive Informationsbedarf durch eine Zerlegung in folgende Komponenten operationalisiert werden kann:

- die Häufigkeit von Informationsbedarfssituationen,

- die Zufriedenheit mit der gegebenen Informationsversorgung,

- die Zufriedenheit mit externen Informationsquellen sowie

- die Kostensensitivität hinsichtlich unternehmensexterner Informationen.

\subsection{Häufigkeit von Informationsbedarfssituationen}

Die Ergebnisse der Untersuchung bestätigen eindrucksvoll, daß die subjektiv wahrgenommene Bedarfshäufigkeit ein dominanter Indikator der Bereitschaft ist, ein entsprechendes unternehmensexternes Informationssystem zu nutzen (vgl. Tabelle 7). Bei allen drei betrachteten Informationsbereichen konnten hohe positive Korrelationswerte zwischen Bedarfshäufigkeit und Nutzungsbereitschaft festgestellt werden. Die durchschnittliche Bedarfshäufigkeit derer, die sich zur Nutzung des externen Informationsdienstes bereitfanden, war durchweg signifikant höher als der Bedarf derer, die die Bereitschaft zur Nutzung des Dienstes verweigerten. 


\begin{tabular}{|c|c|c|c|c|c|c|c|c|}
\hline \multirow{2}{*}{\multicolumn{2}{|c|}{$\begin{array}{l}\text { Indikaturen der } \\
\text { Kaufveranlassing } \\
\text { SUB.IF KIIVE BE OARI SME RMULE. }\end{array}$}} & \multirow[b]{2}{*}{ Legende } & \multicolumn{2}{|c|}{$\begin{array}{l}\text { Gesamtwirtschaftliche } \\
\text { Informationen }\end{array}$} & \multicolumn{2}{|c|}{ Branchen informationen } & \multicolumn{2}{|c|}{ Exportinformationen } \\
\hline & & & $\begin{array}{|cc|}\begin{array}{c}\text { Mittel- } \\
\text { wert }\end{array} & \text { Mittel- } \\
\text { "BERt } \\
\text { "BEREIT" } & \text { "MICHT } \\
\text { BERE IT" } \\
\begin{array}{c}\text { (Signifikanz- } \\
\text { niveau) }\end{array} \\
\end{array}$ & $\begin{array}{l}\text { Korre- } \\
\text { lation } \\
\text { (Signif.- } \\
\text { niveau) }\end{array}$ & 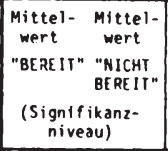 & \begin{tabular}{|l}
$\begin{array}{l}\text { Korre- } \\
\text { lation }\end{array}$ \\
(Signif.- \\
niveau)
\end{tabular} & 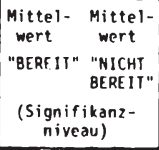 & $\begin{array}{l}\text { Korre- } \\
\text { lation } \\
\text { (Signif.- } \\
\text { niveau) }\end{array}$ \\
\hline $\begin{array}{l}\text { Hiuf igkeit vo } \\
\text { bedarfssituat }\end{array}$ & $\begin{array}{l}\text { on Informations- } \\
\text { tionen }\end{array}$ & $\begin{array}{l}\text { 1-sehr häurig } \\
\text { 4-sehr selten }\end{array}$ & $2,000, \frac{2,5631}{(0,000)}$ & $\frac{0.313]}{(0,000)}$ & $\left.{ }_{0.001}^{1,6692}\right]^{1220}$ & $\begin{array}{l}0.2459 \\
(0.001)\end{array}$ & ${ }^{2,1429}(0,000)^{3}, 1613$ & $\frac{0.4688}{(0.000)}$ \\
\hline $\begin{array}{l}\text { Zufriedenhe it } \\
\text { Informationsv }\end{array}$ & $\begin{array}{l}\text { mit der } \\
\text { jersorgung }\end{array}$ & $\left\{\begin{array}{l}1=\text { sehr } \\
\text { zufrieden } \\
\text { 4=sehr un- } \\
\text { zufrieden }\end{array}\right.$ & 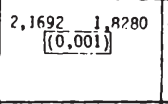 & $\begin{array}{c}-0,0016 \\
(n . s .)\end{array}$ & ${ }_{\text {(n.s.) }}^{2.2231} 1,9722$ & $\begin{array}{c}-0,0051 \\
(n .5 .)\end{array}$ & ${ }_{(\text {n.s.) }}^{2.4516}$ & $\frac{-6, \overline{3850}}{(0,000)}$ \\
\hline $\begin{array}{l}\text { Rosten- } \\
\text { sensitivitat }\end{array}$ & $\begin{array}{l}\text { - } 10 \% \text { Minder- } \\
\text { kosten } \\
\text { - gleiche } \\
\text { Kosten } \\
\begin{array}{l}+10 \% \text { Mehr- } \\
\text { knsten }\end{array} \\
\text { + } 50 \& \text { Mehr- } \\
\text { kosten } \\
\text { + } 100 \% \text { Mehr- } \\
\text { kosten } \\
\text { - > } 100 \% \text { Mehr- } \\
\text { kosten }\end{array}$ & 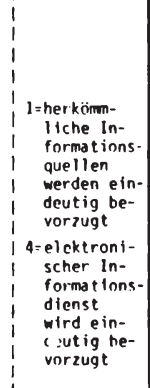 & 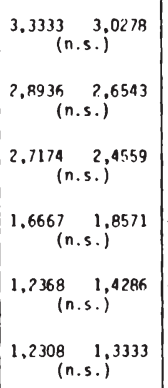 & $\begin{array}{l}{\left[0, \frac{2067}{(0,013)}\right.} \\
{\left[0, \frac{17}{13}\right]} \\
(0,027) \\
{\left[0, \frac{1554}{10}\right.} \\
(0,049) \\
-0,0997 \\
(n .5 .) \\
-0.1384 \\
(n .5 .) \\
-0.0745 \\
(n .5 .)\end{array}$ & 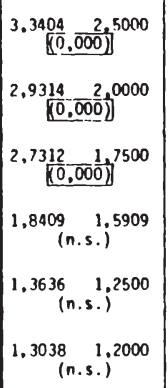 & $\begin{array}{l}(0,3769 \\
(0,000) \\
0,4692 \\
(0,000) \\
0,4810 \\
(0,060) \\
0.2527 \\
(0,004) \\
0.1521 \\
(0,069) \\
0.1106 \\
(\text { n.s. })\end{array}$ & 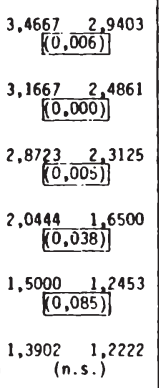 & $\begin{array}{l}0,2599 \\
(0,003) \\
0,3482 \\
(0,000) \\
\frac{0,2581}{(0,003)} \\
0, \overline{0,2046} \\
(0,018) \\
0,1848 \\
(0,038) \\
0,1226 \\
(\text { n.s. })\end{array}$ \\
\hline & & $\begin{array}{l}1 \\
1 \\
1 \\
1 \\
1 \\
1 \\
1 \\
1\end{array}$ & 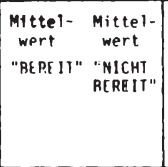 & $\begin{array}{l}\text { Signif.- } \\
\text { niveav } \\
\text { (Chi- } \\
\text { Quadrat- } \\
\text { Test) }\end{array}$ & $\begin{array}{|cc|}\text { Mittel- } & \text { Mittel- } \\
\text { wert } & \text { wert } \\
\text { "BERE II" } & \text { "NICHT } \\
& \text { BEREIT" }\end{array}$ & $\begin{array}{l}\text { Signif.- } \\
\text { niveau } \\
\text { (Chi- } \\
\text { Quadrat- } \\
\text { Test) }\end{array}$ & $\begin{array}{|cc|}\text { Mittel- } & \text { Mittel- } \\
\text { wert } & \text { wert } \\
\text { "BEREIT" "NICHT } & \text { BEREIT" } \\
& \text { BERE T }\end{array}$ & $\begin{array}{l}\text { Signif.- } \\
\text { niveau } \\
\text { (Chi- } \\
\text { Quadrat- } \\
\text { Test) }\end{array}$ \\
\hline $\begin{array}{l}\text { 7ufriednnipit } \\
\text { mit unter- } \\
\text { nehumensextep- } \\
\text { men informa- } \\
\text { ticinsquellen }\end{array}$ & $\begin{array}{l}\text { - Ourllen entgelt- } \\
\text { lichnr informa- } \\
\text { tionen } \\
\text { - oellen iment- } \\
\text { grltlicher ln- } \\
\text { fnimiationen }\end{array}$ & $\begin{array}{c}\text { 1-besonders } \\
\text { zurrteden } \\
\text { A=nirht } \\
\text { besonders } \\
\text { zuifrieden }\end{array}$ & $\begin{array}{ll}0.275 .1 & 0.1262 \\
0.11801 & 0.0911\end{array}$ & {$[0.0156$} & $\begin{array}{ll}0.1857 & 0.1369 \\
0.1926 & 0.139 ?\end{array}$ & n.s. & $\begin{array}{ll}0,0391 & 0,0368 \\
0,0625 & 0,0453\end{array}$ & n.s. \\
\hline
\end{tabular}




\subsection{Zufriedenheit mit der Informationsversorgung}

Es 1 iegt nahe, zu vermuten, daß neben der Intensität des Informationsbedarfs die Zufriedenheit mit der aktuellen Informationsversorgung wesentlich die Einstellung zu neuen externen Informationsangeboten allgemein und gegenüber neuen elektronischen Informationsdiensten im speziellen beeinflußt und formt. Die Vermutung konnte im Rahmen der Untersuchung jedoch nur bedingt erhärtet werden (vgl. Tabelle 7). Der Mittelwerttest zeigt zwar, daß in der Tat der durchschnittliche Zufriedenheitsgrad tendenziell bei der Gruppe derjenigen niedriger ist, die die Nutzung des neuen Informationsdienstes befürworteten. Gleichwohl nimmt diese Tendenz nur im Falle gesamtwirtschaftlicher Informationen ein signifikantes Niveau an. Die Korrelationsanalyse kann demgegenüber nur im Falle der Exportdatenbank einen signifikanten, negativen Zusammenhang zwischen Informationszufriedenheit und Nutzungsbereitschaft ermitteln.

Damit ist die Schlußfolgerung zu ziehen, daß weniger die wahrgenommenen Dissonanzen der Entscheidungsträger im Hinblick auf ihre $u$. U. wenig befriedigende Informations situation den Ausschlag für die Nutzung innovativer Informationsmöglichkeiten geben. Vielmehr scheinen eher die dezidierten Nutzenerwartungen der potentiellen Abnehmer vor dem Hintergrund entsprechend ausgeprägter Informationsbedarfe für die Nutzungsentscheidung bestimmend zu sein.

Eine Bestätigung dieser Ergebnisinterpretation ergibt sich, wenn man auf der einen Seite den aktuellen Grad der Erfiullung bestimmter Informationsanforderungen aus Unternehmenssicht, auf der anderen Seite die bekundete Wichtigkeit dieser Informationsanforderungen mit der Bereitschaft zur Nutzung des elektronischen Informationsdienstes in Beziehung setzt. Tabelle 8 gibt das Ergebnis beider Korrelationen exemplarisch am Beispiel der 
"Brancheninformationen" wieder. Spalte 1 drückt aus, wie intensiv die (negative) Beurteilung der aktuellen Informationsversorgung mit einer positiven Einstellung zu dem neuen Informationsdienst verbunden ist. Ein hoher negativer Korrelationswert bedeutet dabei, daß die Nichterfiil1 ung der jeweiligen Informationsanforderung in starkem Maße dazu führt, den neuen Informationsdienst zu adoptieren. Der Spalte 2 ist demgegenüber zu entnehmen, wie sehr eine hohe (positive) Gewichtung einzelner (durch den Informationsdienst gewährleisteter) Informationsanforderungen zu einer positiven Adoptionsentscheidung führt. Ein Vergleich der Häufigkeit und der Höhe der signifikanten Korrelationen macht deutlich, daB die positiven Nutzenerwartungen erheblich enger mit der Adoptionsbereitschaft der entsprechenden potentiellen Abnehmer verbunden sind als die aktuell wahrgenommenen, qualitativen Informationsdefizite.

\subsection{Zufriedenheit mit unternehmensexternen Informa- tionsquellen}

Ein wichtiger kompetitiver Aspekt wird angesprochen mit der Frage nach der Bedeutung, cie der Zufriedenheit mit bisher genutzten Informationsquellen im Hinblick auf die Nutzungsbereitschaft eines neuen Dienstes zukommt. Durchaus plausibel erscheint prima facie die These, daß, je höher die Zufriedenheit, desto geringer die Bereitschaft zur Adoption eines neuen Dienstes sein müßte.

Die Untersuchung ergab, daß das Gegenteil der Fall ist. Mit der Zufriedenheit der Entscheidungsträger im Hinblick auf die bisher genutzten unternehmensexternen Quelien steigt auch tendenziell ihre offenheit für neue Informationsmöglichkeiten ( $v g l$. Tabelle 7 ). Besonders deutlich zeigt sich dieser Effekt in bezug auf Quellen entgeltlicher Informationen. 


\begin{tabular}{|c|c|c|}
\hline Informationsanforderungen & $\begin{array}{c}\text { Korrelation } \\
\text { "Erfüllungsgrad/ } \\
\text { Adopt ionsbereitschaft" } \\
\text { (Signifikanzniveau) }\end{array}$ & $\begin{array}{c}\text { Korrelation } \\
\text { "Bedeutung/ } \\
\text { Adopt ionsbereitschaft" } \\
\text { (Signifikanzniveau) }\end{array}$ \\
\hline $\begin{array}{l}\text { 1. Verläßlichkeit der } \\
\text { Informationen }\end{array}$ & $\begin{array}{r}-0,1419 \\
(0,030)\end{array}$ & $\begin{array}{r}0,2312 \\
(0,001)\end{array}$ \\
\hline $\begin{array}{l}\text { 2. Vollständigkeit der } \\
\text { Informationen }\end{array}$ & $\begin{array}{c}-0,0674 \\
\text { (n.s.) }\end{array}$ & $\begin{array}{r}0,2061 \\
(0,088)\end{array}$ \\
\hline $\begin{array}{l}\text { 3. Einfachheit des In- } \\
\text { formationszugriffs }\end{array}$ & $\begin{array}{l}-0,0054 \\
(\text { n.s.) }\end{array}$ & $\begin{array}{l}0,0988 \\
\text { (n.s.) }\end{array}$ \\
\hline $\begin{array}{l}\text { 4. Schnelligkeit der } \\
\text { Informationsbereit- } \\
\text { stellung }\end{array}$ & $\begin{array}{c}-0,0894 \\
(\text { n.s. })\end{array}$ & $\begin{array}{l}0,1956 \\
(0,006)\end{array}$ \\
\hline $\begin{array}{l}\text { 5. Wirtschaftl ichkeit } \\
\text { der Informations- } \\
\text { versorgung }\end{array}$ & $\begin{array}{r}-0,1216 \\
(0,053)\end{array}$ & $\begin{array}{r}0,1117 \\
(0,078)\end{array}$ \\
\hline $\begin{array}{l}\text { 6. Klarheit der Infor- } \\
\text { mationsdarstellung }\end{array}$ & $\begin{array}{l}-0,0912 \\
(\text { n.s.) }\end{array}$ & $(0,008)$ \\
\hline $\begin{array}{l}\text { 7. Relevanz der Infor- } \\
\text { mationen }\end{array}$ & $\begin{array}{r}-0,1398 \\
(0,032)\end{array}$ & $\begin{array}{l}0,2019 \\
(0,005)\end{array}$ \\
\hline $\begin{array}{l}\text { 8. Aktualität der In- } \\
\text { formationen }\end{array}$ & $\begin{array}{r}-0,1057 \\
(0,081)\end{array}$ & $\begin{array}{r}0,2101 \\
(0,003)\end{array}$ \\
\hline $\begin{array}{l}\text { 9. Speicherfähigkeit } \\
\text { der Informationen }\end{array}$ & $\begin{array}{r}-0,1062 \\
(0,080)\end{array}$ & $\begin{array}{l}0,1241 \\
(0,057)\end{array}$ \\
\hline $\begin{array}{l}\text { 10. Geeigneter Aufbe- } \\
\text { reitungsgrad der } \\
\text { Informationen }\end{array}$ & $\begin{array}{r}-0,1713 \\
(0,011)\end{array}$ & $\begin{array}{l}0,1238 \\
(0,059)\end{array}$ \\
\hline $\begin{array}{l}\text { 11. Objektivität der } \\
\text { Informationen }\end{array}$ & $\begin{array}{l}-0,0755 \\
(\text { n.s. })\end{array}$ & $\begin{array}{r}0,1566 \\
(0,022)\end{array}$ \\
\hline $\begin{array}{l}\text { 12. Exklusivität der } \\
\text { Informationen }\end{array}$ & $\begin{array}{c}-0,0511 \\
(\text { n.s. })\end{array}$ & $\begin{array}{l}0,0918 \\
(\text { n.s.) }\end{array}$ \\
\hline $\begin{array}{l}\text { 13. Breite des Infor- } \\
\text { mationsspektrums }\end{array}$ & $\begin{array}{l}-0,0319 \\
(\text { n.s. })\end{array}$ & $\begin{array}{r}0,1228 \\
(0,058)\end{array}$ \\
\hline $\begin{array}{l}\text { 14. EDV-Verarbeitbar- } \\
\text { keit der Informa- } \\
\text { tionen }\end{array}$ & $\begin{array}{c}-0,0025 \\
(n . s .)\end{array}$ & $\begin{array}{r}0,2 \leq 59 \\
(0,001)\end{array}$ \\
\hline
\end{tabular}

Tab. 8: Relevanz einer geringen informationszufriedenheit vs. hoher

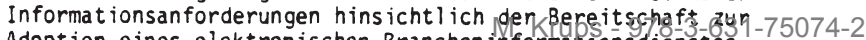
Adoption eines elektron ischen Branchen informat ons ienstes
Downloaded from PubFactory at 01/11/2019 08:37:05AM 
Eine Erklärung für diesen Zusammenhang läßt sich dahingehend geben, daß sich in der Zufriedenheit auch die Aufgeschlossenheit gegenüber externen Informationsquelien dokumentiert. Es leuchtet dann ein, daB sich diese Aufgeschlossenheit als ein relevanter Indikator für die Bereitschaft zur Nutzung neuer Informationsmöglichkeiten herausstellt, dies schon deshalb, weil die bisherigen guten Erfahrungen auch auf den neuen Dienst übertragen werden. 1

\subsection{Kostensensitivität}

Wesentliches EinfluBkriterium für das Verhalten potentieller Abnehmer ist die individuelle Gewichtung von Nutzen und Kosten des betreffenden Dienstleistungsangebots. Von vorrangigem Interesse kann im vorliegenden Zusammenhang nicht die offensichtliche Tatsache sein, daß, j.e höher die Kostensensitivität, desto höher auch der Kaufwiderstand ${ }^{2}$ eines potentiellen Abnehmers ausgeprägt ist. Vielmehr interessiert vor allem die absolute Höhe der

1 Eine Bestätigung dieser Vermutung im Sinne einer Negativ-Argumentation 1 ieferte eine Delphi-Befragung zur aktuellen Bedeutung von Informations - und Dokumentationsdienstleistungen aus Unternehmenssicht. Danach begründen diejenigen Experten, die eine eher zurückhaltende Auffassung gegenuiber IUD-Diensten vertreten, ihre vergleichsweise negative Prognose mit der Tatsache, daB viele Unternehmen bereits heute durch verwirrende und teilweise auch unseriöse Informationsdienste abgeschreckt werden ( $93 \%$ der zur Abschwächung der positiven Argumente führenden Einwände; das statistische Gruppenurteil fiel mit $+0,5$ auf einer Skala von - 3 bis + 3 eher positivaus). Vgl. Schertler, W., Popp, W., Attraktivitätsanalyse von Dienstleistungen. Ein empirischer Ansatz zur Entwicklung von Ausbaustrategien fur Unternehmen und Staat, München 1983, S. 99 f.

2 Zum Konzept des Kaufwiderstands vgl. vor allem Walters, M., a.a.0., S. $151 \mathrm{ff}$. 
Kostensensitivität. Fällt diese bei einem Informationsbereich hoch aus, so ist im Rahmen der Marktsegmentierung diesem Aspekt eine vergleichsweise stärkere Aufmerksamkeit zu widmen als im Falle durchschnittlicher Kostensensitivität.

Vor diesem Hintergrund liefert die Untersuchung das interessante Ergebnis, da $\beta$ die Kostenorientiertheit im Bereich gesamtwirtschaftlicher Informationen besonders hoch ausgeprägt ist. Ab über $10 \%$ Mehrkosten spricht sich die Mehrzahl der Unternehmen gegen den neuen Dienst und für die herkömmlichen Informationsquellen aus (vgl. Tabelle 7). Weniger stark ausgeprägt ist diese Einstellung im Fall der Brancheninformationen.

Bei Exportinformationen nimmt die Kostensensitivität schließlich ein sehr niedriges Niveau an: selbst bei einer Unterstellung von 100 \% Mehrkosten korreliert die Bevorzugung des neuen Dienstes noch signifikant positiv mit der bekundeten Nutzungsbereitschaft. Eine Erklärung für dieses Ergebnis läßt sich zum einen darin finden, da $\beta$ in dem betrachteten Bereich die Informationsversorgung noch die vergleichsweise größten Lücken aufweist und daß damit die Zahl der Alternativen zu dem neuen Dienst relativ gering ist. Zum anderen sind gerade Exportentscheidungen mit hohen Risiken behaftet, die im Unternehmen zur Forderung nach einer bestmöglichen Informationsgrundlage führen.

Schließlich ist nicht auszuschließen, daß die absolute Höhe der bisherigen Aufwendungen für Exportinformationen als Basis der Mehrkosten-Beurteilung entsprechend niedrig ausfällt.

$1 \mathrm{Vgl}$. S. 177 dieser Arbeit. 


\subsection{Dispositionsspielraum als Kriterium der Kauffähig- keit}

Gerade im Dienstleistungsbereich stellt sich häufig die Frage, ob ein Unternehmen bestimmte Leistungen selber zu erbringen imstande und gewillt ist, oder ob es diese Leistungen fremd bezieht. Vielfältige Beispiele sind geeignet, das Entscheidungsproblem zu verdeutlichen. So ist es ohne weiteres denkbar, daß ein Unternehmen bestimmte Aufgaben wie Wartungs-, Reparatur-, Reinigungs-oder Transportleistungen in die Hände spezialisierter Dienstleistungsanbieter legt. Ebenso zeigt sich in der Praxis häufig, daß diese Aufgaben intern ohne Unterstuizung Dritter abgewickelt werden. Engelhardt und Schwab prägten in diesem Zusammenhang den Begriff "Dispositionsspielraum"."

Bezogen auf die vorliegende Problemstellung beschreibt der Dispositionsspielraum das Möglichkeitsfeld einer Unternehmung, bestimmte betrieblich benötigte, unternehmensexterne Informationen eigenständig zu beschaffen, aufzubereiten und abzuspeichern. Der Dispositionsspielraum läßt sich dabei auf mehrere Faktoren zurückführen: Kostenüberlegungen, Ertragsgesichtspunkte, Kapitalgründe und Risikoaspekte spielen hier eine wesentliche Rolle.?

Während der Dispositionsspielraum in diesem Sinne die Fähigkeit beschreibt, auf die Inanspruchnahme einer Dienstleistung von seiten Dritter zu verzichten, interessiert im Rahmen der Marktsegmentierung vor allem der Dispositionsspielraum, der sich auf die Fähigkeit potentieller Abnehmer bezieht, eine angebotene Dienstleistung in Anspruch zu nehmen. ${ }^{3}$ In diesem Sinne sei im folgenden

1 Vgl. Engelhardt, W.H., Schwab, W., a.a.0., S. 505.

$2 \mathrm{Vgl}$. ebenda, S. 506.

3 In diesem Sinne soll der Dispositionsspielraum im folgenden als Indikator der Kauffähigkeit einer potentiellen Abnehmerunternehmung verstanden werden. 
nach Merkmalen gesucht, die den Dispositionsspielraum einer Unternehmung kennzeichnen. Die vorliegende Untersuchung unterstellte dabei vor allem die Relevanz innerorganisatorischer Kontextfaktoren.

\subsection{Organisationsdemographische Kriterien}

Zentrale Merkmale der Unternehmung, die in nahezu jedem Ansatz zur Segmentierung industrieller Abnehmer Anwendung finden, stellen die sog. organisationsdemographischen Merkmale dar. 1 Ihr besonderer Vorzug ist es, auf der Basis von Sekundärdaten relativ leicht erfaßbar und problemlos meBbar zu sein. Vielfach üben Experten jedoch auch Kritik an ihrem geringen Bezug zum Verhalten der betreffenden Unternehmung. ${ }^{2}$

Im Hinblick auf die Inanspruchnahme elektronischer Informationsdienste wurde in der vorliegenden empirischen Untersuchung näher auf die Relevanz der Unternehmensgröße und der informationstechnologischen Ausstattung eingegangen.

\subsection{Unternehmensgröße}

"... die Absatzchancen eines Abnehmers /Werdeñ vielfach sowohl in quantitativer als auch in qualitativer Hinsicht durch die jeweilige Größe des Abnehmers beeinflußt". 3 Im

1 Vgl. U.a. Wind, Y., Cardozo, R., a.a.0., S. 156; Scheuch, F., Investitionsgiuter-Marketing, a.a.0., S. $71 \mathrm{f}$.; Gröne, A., a.a.0., S. $51 \mathrm{ff}$. Zur (ausschließlichen!) Anwendung organisationsdemographischer Merkmale im Rahmen der Segmentierung investiver Dienstleistungsmärkte vgl. Kaufmann, E.J., a.a.0., S. $98 \mathrm{ff}$.

$2 \mathrm{Vgl}$. insbesondere die synoptische Bewertung organisationsdemographischer Merkmale bei Gröne, A., a.a.0., S. 75 .

3 Gröne, A., a.a.0., S. 55. 
allgemeinen geht man gerade im Zusammenhang innovativer, komplexer Güterarten davon aus, daB GroBunternehmen, u.a. bedingt durch ihre breitere Kapitalbasis, eher bereit und in der Lage sind, die mit der Adoption verbundenen Risiken zu tragen. 1 Andererseits findet man häufig das Argument, daB das niedrigere technologische Niveau, die geringere Professionalisierung von Entscheidungsprozessen sowie weniger komplizierte Entscheidungsstrukturen in kleineren Unternehmen als adoptionsfördernde Faktoren wirken. 2

In bezug auf investive Dienstleistungen streicht vor allem Kaufmann die Bedeutung der UnternehmensgröBe als wichtiges Selektionskriterium heraus. ${ }^{3}$ "Konzentriert sie Lëine Anbieterunternehmung, Anm.d.Verfassers $\bar{J}$ sich auf kleinere Kundenunternehmungen, kann sie davon ausgehen, da $B$ ein breites Leistungsspektrum nachgefragt wird. Je größer jedoch die Kundenunternehmung ist, desto mehr kann davon ausgegangen werden, da $B$ diese Unternehmen fur allgemeine, oft anfallende Bedurfnisse eigene Mitarbeiter eingesetzt haben, die mit der entsprechenden Leistungserstellung durchgängig ausgelastet werden können". 4

Vor diesem Gesamthintergrund erscheint hinsichtlich der Inanspruchnahme unternehmensexterner Informationsdienste die Rolle der Unternehmensgröße vor allem deshalb interessant, weil ihre Wirkungsrichtung auf den ersten Blick weitgehend offen ist. Ebenso, wie ein GroBunternehmen z. B. aufgrund entsprechender finanzieller Spielräume zur Inanspruchnahme der Dienstleistung neigen könnte, ist der Fall

$1 \mathrm{Vgl}$. zu dieser Auffassung insbesondere O'Neal, C.R., Thorelli, H.B., Utterbach, I.M., Adoption of Innovations by Industrial Organizations, in: IMM, Vol. 3, 1973, Nr. 2, S. 246.

2 Vgl. vor allem Gröne, A., a.a.0., S. 55 und die dort angegebene Literatur.

$3 \mathrm{Vgl}$. Kaufmann, E.J., a.a.0., S. 102.

4 Ebenda. 
denkbar, daß es die Dienstleistung ablehnt, da innerbetriebliche Stellen die betreffende Funktion bereits zufriedenstellend erbringen.

In der vorliegenden Untersuchung wurde zur empirischen Vertiefung dieser Frage die Unternehmensgröße zum einen durch den Umsatz, zum anderen durch die Zahl der Beschäftigten operationalisiert. Während die Ergebnisse in ihrer Einflußrichtung bei beiden Maßgrößen gleich sind, fällt auf, daß die Stärke des Einflusses z. T. Unterschiede aufwe ist.

Insgesamt ergibt sich, daB die Bereitschaft zur Nutzung eines elektronischen Informationsdienstes sich nicht generell aus der Unternehmensgröße ableiten 1 äßt. Als entscheidende Prämisse ist vielmehr die inhaltliche (funktionsbezogene) Dimension des Dienstes zu berücksichtigen. So geht aus Tabelle 9 hervor, daB z. B. in bezug auf Exportinformationen die Nutzungsbereitschaft mit wachsender Unternehmensgröße ansteigt. Das gleiche gilt für gesamtwirtschaftliche Informationen, wenngleich hier die ermittelten Zusammenhänge kein signifikantes Niveau aufweisen.

Demgegenüber scheint sich die Einflußwirkung im Bereich der Brancheninformationen umzukehren: hier ist eine tendenziell (wiederum nicht signifikant) abnehmende Nutzungsbereitschaft bei steigender Unternehmensgröße zu registrieren. Offenbar zeigt sich hier, daß gerade die gröBeren Unternehmen ihren Bedarf an Brancheninformationen bereits laufend und in weitgehend zufriedenstellender Weise decken können. 


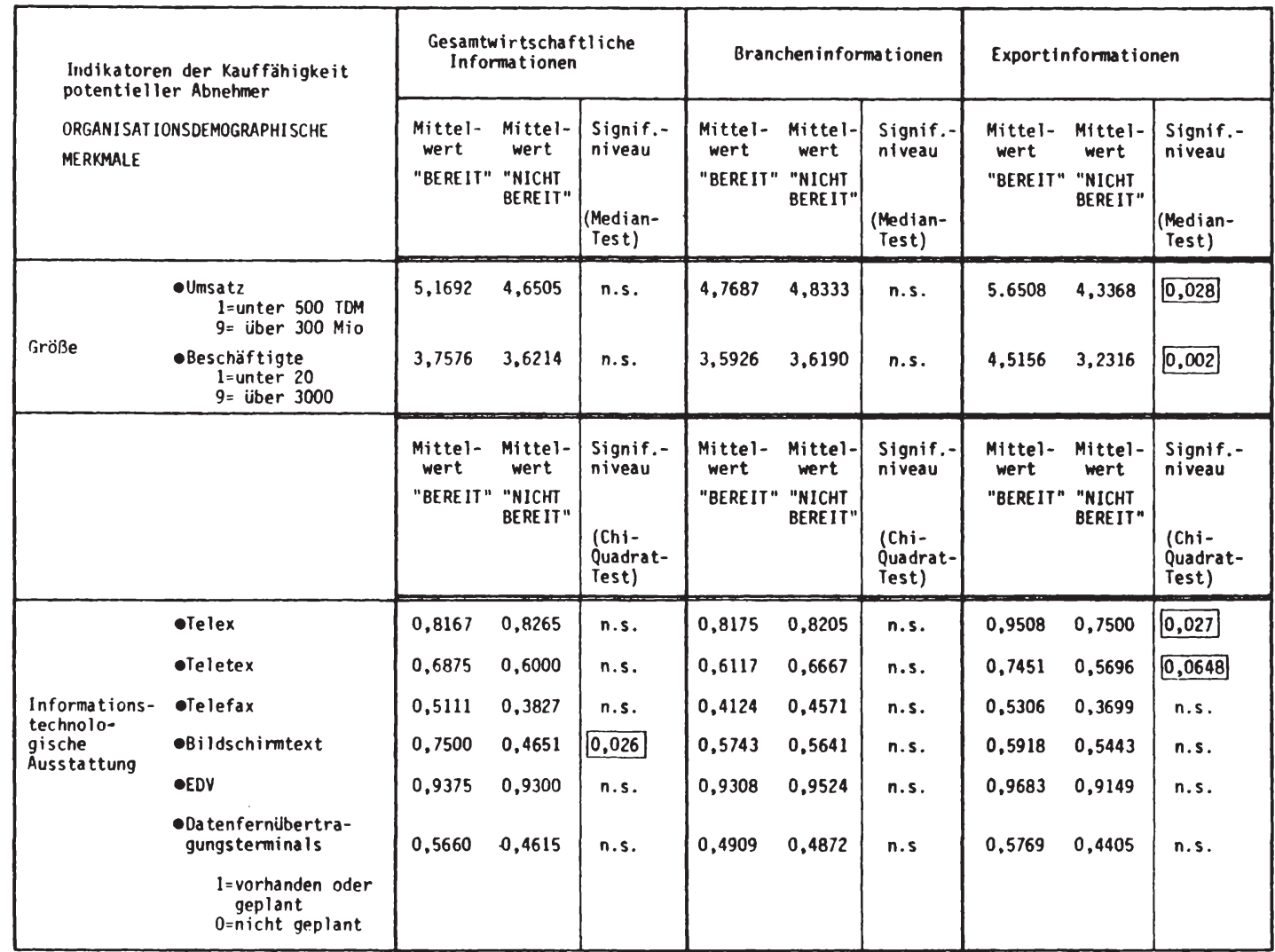




\subsection{Informationstechnologische Ausstattung}

Besonderes Interesse gebührt der Frage, welche Rolle die informationstechnologische Ausstattung eines potentiellen Abnehmerunternehmens in bezug auf seine Bereitschaft zur Nutzung einer auf neuen Informationstechnologien basierenden Dienstleistung spielt. Die Untersuchung liefert in diesem Zusammenhang wiederum sehr differenzierte Ergebnisse. So zeigt sich eine bemerkenswert enge Beziehung zwischen dem Einsatz von Bildschirmtext und der Bereitschaft zur Nutzung einer Datenbank mit gesamtwirtschaftlichen Informationen ( $v g l$. Tabelle 9). Der offenkundige, positive EinfluB der Btx-Nutzung auf die Adoptionsbereitschaft ist um so bemerkenswerter, als er nur für diesen Informationsbereich gilt. Die zur Nutzung einer Exportdatenbank bereiten Abnehmer weisen demgegenüber im Vergleich zu den "Nicht-Bereiten" eine höhere Ausstattung mit Telex- und Teletex-Endgeräten auf. Keine signifikanten Aussagen sind schlieBlich im Bereich der Brancheninformationen möglich.

\subsection{Informationsdemographische Kriterien}

Als zweite wesentliche Gruppe von Kriterien, die den Dispositionsspielraum der potentiellen Abnehmerunternehmung determinieren, sind im Rahmen der Marktabgrenzung die informationsdemographischen Merkmale zu berücksichtigen. Sie beschreiben die ablauforganisatorischen Regeln und Usancen, die sich in einer jeden potentiellen Abnehmerunternehmung zur Sicherstellung der Versorgung mit unternehmensexternen Informationen im Laufe der Zeit etablieren. Die Oberlegungen, die zur Einbeziehung dieser Merkmale führen, haben ihren Ursprung im Konzept des geplan- 
ten organisatorischen Wandels. ${ }^{1}$ Dieses Konzept weist auf die empirisch belegte Erkenntnis hin, daB Veränderungen im Unternehmen vielfach mit organisatorischen Anpassungswiderständen verbunden sind. Mit Blick auf diese Widerstände verbinden sich mit dem "planned organizational change" verschiedene Ansätze, deren gemeinsames $Z$ iel es ist, den organisatorischen Wandel bewuBt und gezielt vor zubereiten, einzuleiten, zu steuern und zu kontrollieren. 2

Vieles spricht dafür, daB auch die innerbetriebliche Adoption einer innovativen Form der Informationsbeschaffung gewisse ablauforganisatorische Anpassungsprozesse im Unternehmen hervorruft. Aufgabeninhalte, Erwartungshaltungen des Managements und Kommunikationsbeziehungen werden Veränderungen unterworfen. ${ }^{3}$ Gegenuiber diesen Veränderungen treten in der Folge möglicherweise Widerstände auf, die der Anbieter der Informationsdienstleistung im Rahmen seiner Abnehmerselektion zu berücksichtigen hat.

Grundsätzlich ist davon auszugehen, daB Anpassungswiderstände in Gestalt mangelnder Anpassungsfähigkeit oder mangelnder Anpassungsbereitschaft der Betroffenen zutage treten können. ${ }^{4}$ Elemente beider Ursachen möglicher Adoptionswiderstände finden sich in den nachfolgend exemplarisch analysierten Faktoren der Abnehmerauswahl.

$1 \mathrm{Vgl}$. zu diesem Konzept vor allem Leavitt, H.J., Applied Organizational Change in Industry: Structural, Technological and Humanistic Approaches, in: Handbook of Organizations, Hrsg.: March, J.G., Chicago 1965, S. $1144 \mathrm{ff}$. ; Dienstbach, H., Dynamik der Unternehmensorganisation - Anpassung auf der Grundlage des "planned organizational change", Wiesbaden 1972; Kirch, W., Betriebswirtschaftspolitik und geplanter Wandel betriebswirtschaft 1 icher Systeme: in: Unternehmensführung und Organisation, Hrsg.: Kirsch, W., Wiesbaden 1973 , S. $15 \mathrm{ff.}$; Kirch, W., Esser, W.-M., Gabele, E., a.a.0..

$2 \mathrm{Vgl}$. Meffert, H., Die Durchsetzung von Innovationen in der Unternehmung und am Markt, in: ZfB, 46. Jg., 1977, $\mathrm{Nr} .2$, S. 80 .

$3 \mathrm{Vgl.z}$. B. Witte, E., Informationsverhalten, a.a.0., $\mathrm{Sp} .1921$.

$4 \mathrm{Vgl}$. vor allem Böhnisch, W., Personale Widerstände bei der Durchsetzung von Innovationen, Stuttgart 1979 . 5 .
$36 \mathrm{ff}$. und $51 \mathrm{ff} .$. 


\subsection{Existenz eines Budgets für unternehmensexterne Informationen}

Als ein naheliegender Indikator (mangelnder) Anpassungsfähigkeit kann das (fehlende) Vorhandensein eines Budgets für unternehmensexterne Informationen angesehen werden. Ein Entscheidungsträger, der über keine planmäßig festgelegten Mittel für die Inanspruchnahme solcher Leistungsangebote wie der eines elektronischen Informationsdienstes hat, wird sich ohne Zweifel weniger imstande sehen, den Dienst zu nutzen als ein Entscheidungsträger, der über ein solches Budget verfügt.

Die vorliegende Untersuchung bestätigte diese Vermutung inhaltlich voll, begrenzt jedoch auf den Bereich gesamtwirtschaftlicher sowie Exportinformationen. Tabelle 10a macht deutiich, daß hier die Adoptionsbereitschaft derjenigen Befragten, die über ein entsprechendes Informationsbudget verfügen, signifikant höher ist als die Bereitschaft derer, die nicht mit einem solchen Budget ausgestattet sind.

Interessant erscheint, $d a B$ bei der Beschaffung gesamtwirtschaftlicher Informationen die Existenz eines unternehmensübergreifenden Budgets entscheidend ist, während bei Exportinformationen eher das Vorhandensein eines abteilungsspezifischen Informationsbudgets zur Inanspruchnahme des Dienstes führt. Die Erklärung hierfür liegt in der Person des für den jeweiligen Informationsbereich zuständigen Entscheidungsträgers begründet. Im Falle gesamtwirtschaftlicher Informationen, wo in erster Linie die Geschäftsführung über die Nutzung des Informationsdienstes entscheidet ${ }^{1}$, ist es wesentlich, daß diesem ein (unternehmensuibergreifendes) Informationsbudget zur Disposition steht. Demgegenüber ist es im Falle der Export-

1 Vgl. hierzu Tabelle 11 auf S. 198 dieser Arbeit. 


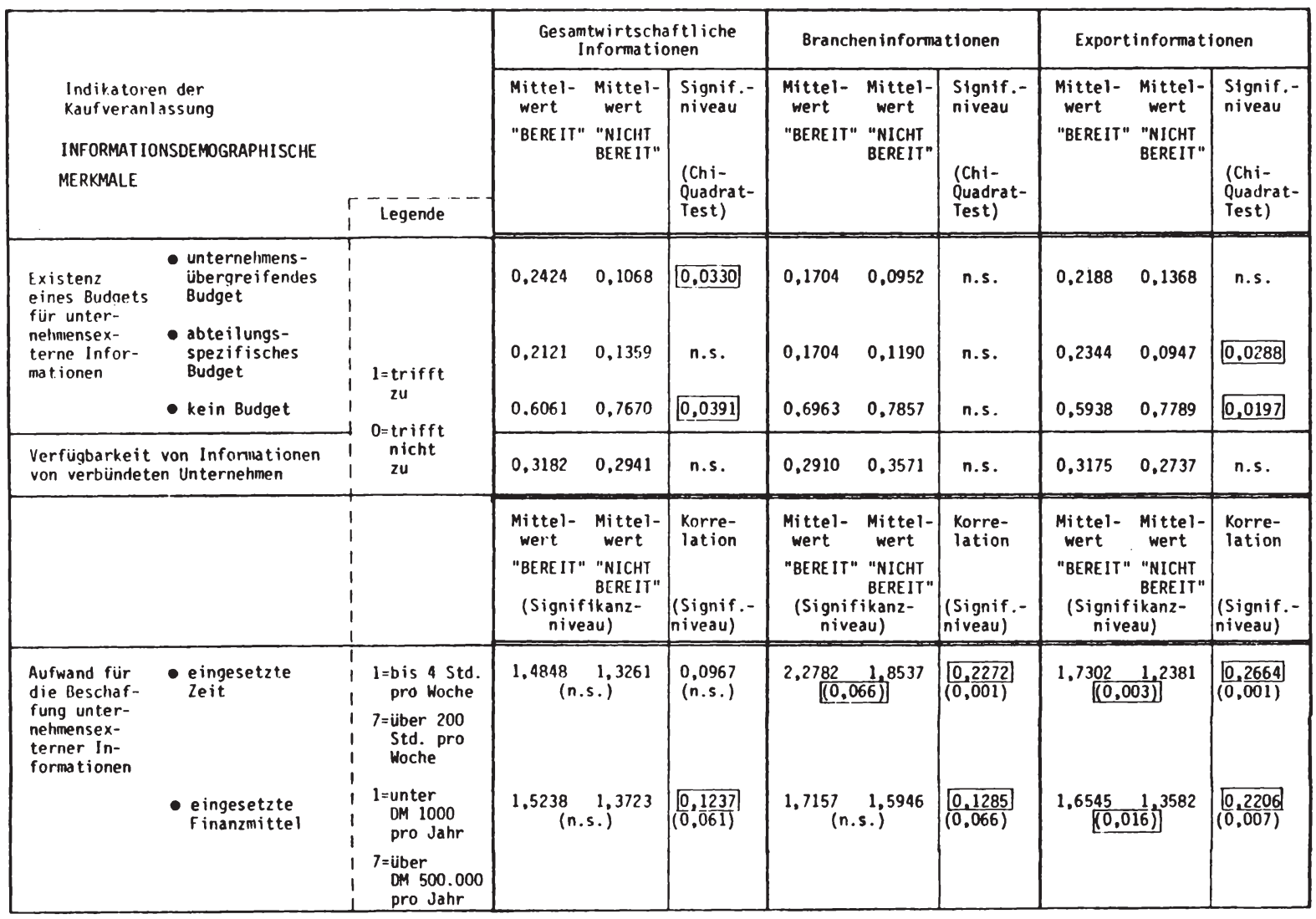

Tab. 10a: Relevanz informationsdemographischer Merkmale (I) 
information vor allem wichtig, daß die in der Regel kaufentscheidende Marketingabteilung ${ }^{1}$ über ein (abteilungsspezifisches) Budget verfügt.

Hieraus ergibt sich, daß die Relevanz eines Budgets nur in Verbindung mit der Frage zu beurteilen ist, ob dieses Budget auch dem relevanten Entscheidungsträger zur direkten Verfügung steht.

Bemerkenswert erscheint schlieBlich, daB die Entscheidung zur Adoption eines Brancheninformationsdienstes offenbar weitgehend losgelöst von dem Vorhandensein eines entsprechenden Budgets gesehen werden kann. Aus dem Fehlen eines gesonderten Budgets erwachsen demzufolge ceteris paribus keine Adoptionswiderstände gegen den neuen Informationsdienst.

\subsection{Verfügbarkeit von Informationen aus verbundenen Unternehmen}

Im Hinblick auf einen zweiten anpassungshemmenden Faktor wurde im Rahmen der Untersuchung die Hypothese aufgestellt, daß die Verfügbarkeit von Informationen aus verbundenen Unternehmen tendenziell dazu führe, den Informationsdienst nicht in Anspruch zu nehmen. Hinter dieser Hypothese stand die Vermutung, daß ein konzernubergreifendes system der Informationsversorgung sich für den geplanten Informationsdienst als Adoptionswiderstand auswirken müBte.

Diese Vermutung konnte die Untersuchung nicht bestätigen. Wie aus Tabelle 10a hervorgeht, lassen sich keine signifikanten Zusammenhänge zwischen der Verfügbarkeit von Informationen aus verbundenen Unternehmen und der Bereitschaft zur Nutzung einer der drei betrachteten Informationsdienste herstellen.

1 Vgl. hierzu Tabelle 11, S. 198. 


\subsection{Aufwand für die Beschaffung unternehmensexterner Informationen}

Auf die Intensität der aktuellen Informationsbemihungen und ihre Bedeutung für die Adoptionsbereitschaft stellt die Frage nach dem von der potentiellen Abnehmerunternehmung betriebenen Aufwand (finanzielle Mittel, Zeit in Mannstunden) für die Beschaffung externer Informationen ab. Die Hypothese, daB mit wachsendem Aufwand auch die Bereitschaft zur Inanspruchnahme des Informationsdienstes steigt, konnte durch die Untersuchung verifiziert werden. Es bestätigte sich damit, daß eine innovative Informationsdienstleistung gerade bei solchen Unternehmen auf Erfolg hoffen kann, welche bereits der Informationsbeschaffung eine hohe Priorität einräumt. Unterschiede ergeben sich - je nach Informationsbereich - lediglich insofern, als einmal die eingesetzte Zahl von Mannstunden, ein andermal die Höhe der aufgewendeten Finanzmittel der entscheidende EinfluBfaktor ist (vgl. Tabelle 10a).

Bemerkenswert erscheint, da $B$ die positive Korrelation zwischen aktuellem Zeitaufwand und Adoptionsbereitschaft auch im Bereich der Brancheninformationen auftritt. Damit verliert der Einwand an Bedeutung, da $B$ im Grunde der Informationsaufwand lediglich ein Substitutionskriterium der Unternehmensgröße darstellt. ' Tatsächlich korreliert die Adoptionsbereitschaft nicht mit der Unternehmensgröße (vgl. Tabelle 9), wohl aber mit der im Unternehmen für die Informationssuche eingesetzten Zeit (vgl. Tabelle 10a).

1 Dieser Einwand erscheint zunächst insofern berechtigt, als der Umsatz der befragten Unternehmen auffällig hoch mit dem jeweiligen Zeitaufwand für die Beschaffung externer Informationen korreliert $(r=0,4074$, Signifikanzniveau $=0,000$ ). 


\subsection{Nutzungsgrad unternehmensexterner Informationen}

Ahnlich dem Aufwand stellt das Kriterium "Nutzungsgrad unternehmensexterner Informationen" die aktuellen Informationsgewohnheiten der Unternehmung in den Mittelpunkt. AnlaB zur Berücksichtigung dieses Kriteriums liefert die Vermutung, daB mit zunehmender Nutzung externer Informationsquellen auch die Aufgeschlossenheit gegenüber innovativen Informationsdiensten ansteigt.

Diese Vermutung bestätigte die Untersuchung uneingeschränkt in bezug auf die Nutzung von Quellen entgeltlicher Informationen (Agenturen, Wirtschaftsforschungsinstitute, Informationsvermittler, etc.). Darüber hinaus zeigt Tabelle 10b, daß bemerkenswerterweise auch mit der zunehmenden Inanspruchnahme von Quellen unentgeltlicher Informationen (Verbände, Handelskammern, etc.) eine graduell wachsende Bereitschaft zur Nutzung eines neuen, entgeltlichen Informationsangebots einhergeht. Dieser im Bereich wirtschaftlicher Entscheidungen höchst untypische Zusammenhang kann als besonders starkes Indiz dafür interpretiert werden, da $B$ die bisherigen Informationsquellen bislang nur sehr unzureichend den Bedarf der Unternehmen an externen Informationen zu decken vermögen.

\subsection{Nutzer unternehmensexterner Informationen}

Ein letzter hier zu betrachtender EinfluBfaktor der Adoptionsbereitschaft zielt auf die Person des Informationsnutzers im Unternehmen ab. Die Annahme, daß sich in Abhängigkeit vom jeweiligen Informationsnutzer die Bereitschaft zur Inanspruchnahme des Informationsdienstes verändert, erweist sich aufgrund der vorliegenden Untersuchung generell als falsch. Tabelle $10 b$ veranschaulicht, daß lediglich im Bereich der Exportinformationen signifikante Zusammenhänge zwischen der Person des Informationsnutzers und der jeweiligen Adoptionsbereitschaft bestehen. So 


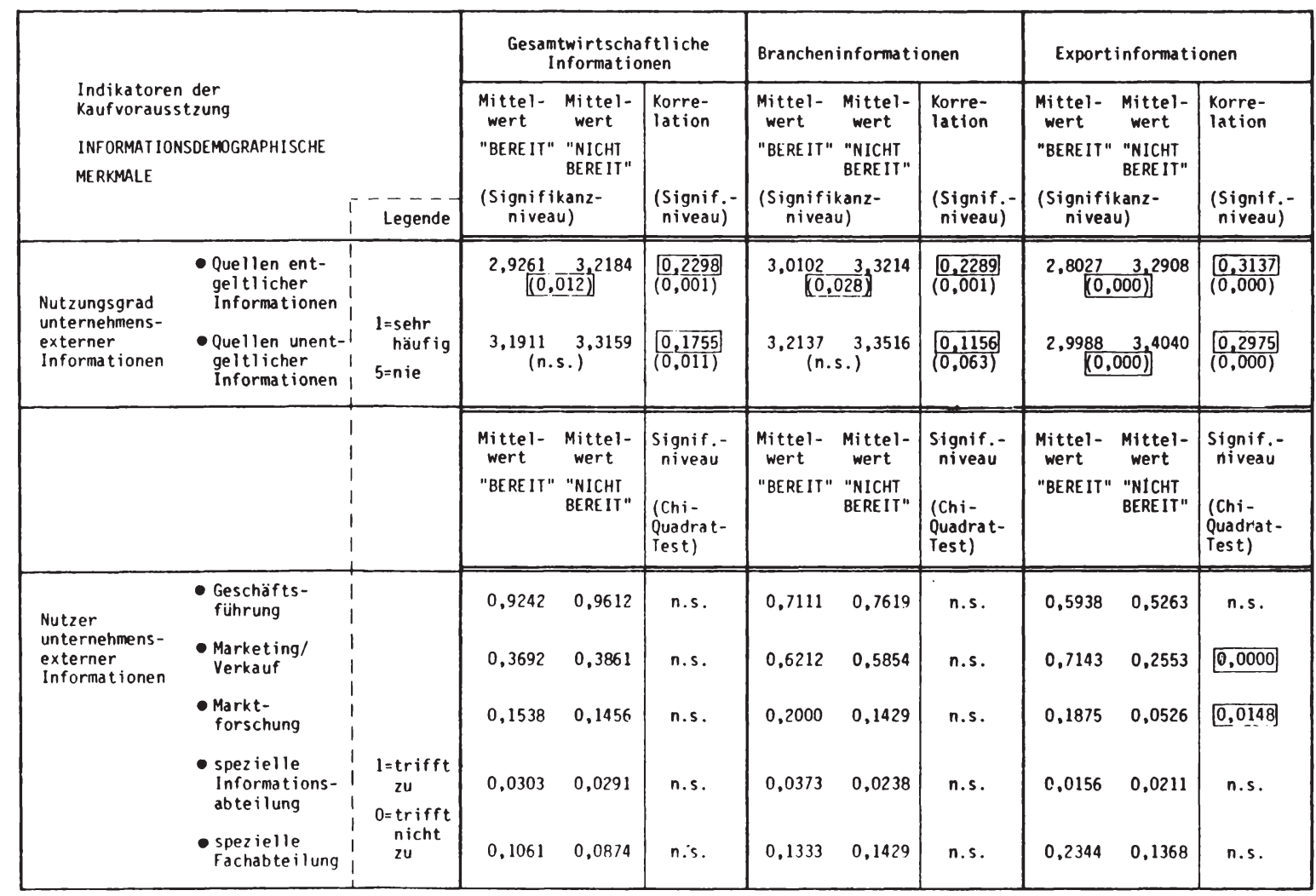

Tab. 10b: Relevanz informationsdemographischer Merkmale (II) 
korreliert die Bereitschaft, eine Exportdatenbank in Anspruch zu nehmen, signifikant mit dem Umstand, daB im Unternehmen vor allem die Marketingabteilung Exportinformationen nutzt. Deutlich geringer, aber auch noch signifikant, ist der fördernde EinfluB einer Situation, in der sich vor allem die Marktforschung oder eine spezielle Fachabteilung ( $z$. B. eine Auslandsabteilung) mit Exportinformationen befassen.

\subsection{Merkmale des Buying Center}

Neben den Variablen, die sich auf die Kaufveranlassung potentieller Kunden beziehen, und den Faktoren, die die Fähigkeit der betreffenden Kunden zur Realisierung des Kaufvorhabens zum Ausdruck bringen, gilt es zuletzt, solche Merkmale zumindest ansatzweise zu berücksichtigen, die auf die eigentliche Durchführung der Kaufentscheidung Bezug nehmen. 1 Hierzu wurde im Rahmen der empirischen Erhebung die Frage untersucht, inwiefern die Person des Kaufentscheiders für die Adoptionsbereitschaft von Bedeutung ist.

1 Zur Eingrenzung des Fragebogenumfangs und aufgrund der Vermutung, da $B$ be $i$ hochinnovativen und komplexen Dienstleistungen noch mit sehr wenig gefestigten Strukturen eines Buying Centers gerechnet werden kann, wurde auf eine Abfrage detaillierterer Angaben (Größe, Zusammensetzung des Buying Centers, etc.) verzichtet. Vgl. zu solchen Kriterien z. B. Gröne, A., a.a.0., S. $77 \mathrm{ff}$.

2 Eine detaillierte Systematisierung relevanter Einzeldimensionen eines Buying Centers beim Einkauf von Dienstleistungen geht auf Johnston und Bonoma zurück. Diese unterscheiden im einzelnen die vertikale (hierarchische) Zusammensetzung (vertical involvement), die horizontale (abteilungsuibergreifende) Zusammensetzung (lateral involvement) sowie den Umfang des Buying Centers (extensivity), ferner die Enge der Entscheidungsabstimmung (connectedness) sowie die Zentralisierung des Entscheidungsprozesses in bezug auf den Einkaufsmanager (centrality). Vgl. Johnston, W.I., Bonoma, Th.V., Purchase Process for Capital Equipment and Services, in: IMM, Vol. 10, 1981, S. $253 \mathrm{ff}$., insbes. S. $254 \mathrm{f}$. 
Das Ergebnis in Tabelle 11 zeigt, daB eine positive Adoptionsentscheidung in bezug auf die Nutzung einer Gesamtwirtschaftsdatenbank signifikant beeinflußt wird durch die Frage, ob die Adoptionsentscheidung in der Hand der Geschäftsleitung liegt. In ähnlicher, wenn auch nicht signifikant ausgeprägter Weise ist eine positive Adoptionsentscheidung in bezug auf eine Branchendatenbank dann zu beobachten, wenn der Marketingabteilung die Verantwortung zufällt. Schließlich wird ersichtlich, daß, wenn immer die Adoptionsentscheidung bei der Marketingabteilung liegt, tendenziell mit einer Befürwortung der Nutzung einer Exportdatenbank gerechnet werden kann.

\subsection{Zusammenfassende Darstellung relevanter Auswahl- kriterien}

Eine synoptische Darstellung aller signifikant verhaltensrelevanter EinfluBfaktoren erlaubt es, die Gesamtheit der für die Abnehmerselektion entscheidenden Auswahlkriterien auf einen Blick zu erfassen. Abbildung 41 beinhaltet eine solche Zusammenstellung für den beispielhaft betrachteten elektronischen Informationsdienst. Aus der Abbildung geht hervor, mit den Ausprägungen welcher Einzelkriterien die Adoptionsbereitschaft jeweils signifikant verbunden ist. Anders formuliert zeigt die Abbildung auf, anhand welcher Variablen die potentiellen Abnehmerunternehmungen im Rahmen der Zielgruppenbestimmung zu bewerten sind.

Es wird deutlich, daß die Zahl der relevanten Variablen in bezug auf Exportinformationen relativ hoch ist, wogegen bei isolierter Betrachtung die Zahl der Variablen in bezug auf gesamtwirtschaftliche und Brancheninformationen wesentlich geringer ausfällt. Eine Interpretation dieser Tatsache hat vermutich an der erhöhten Situationsabhängigkeit der Relevanz von Exportinformationen anzusetzen. 


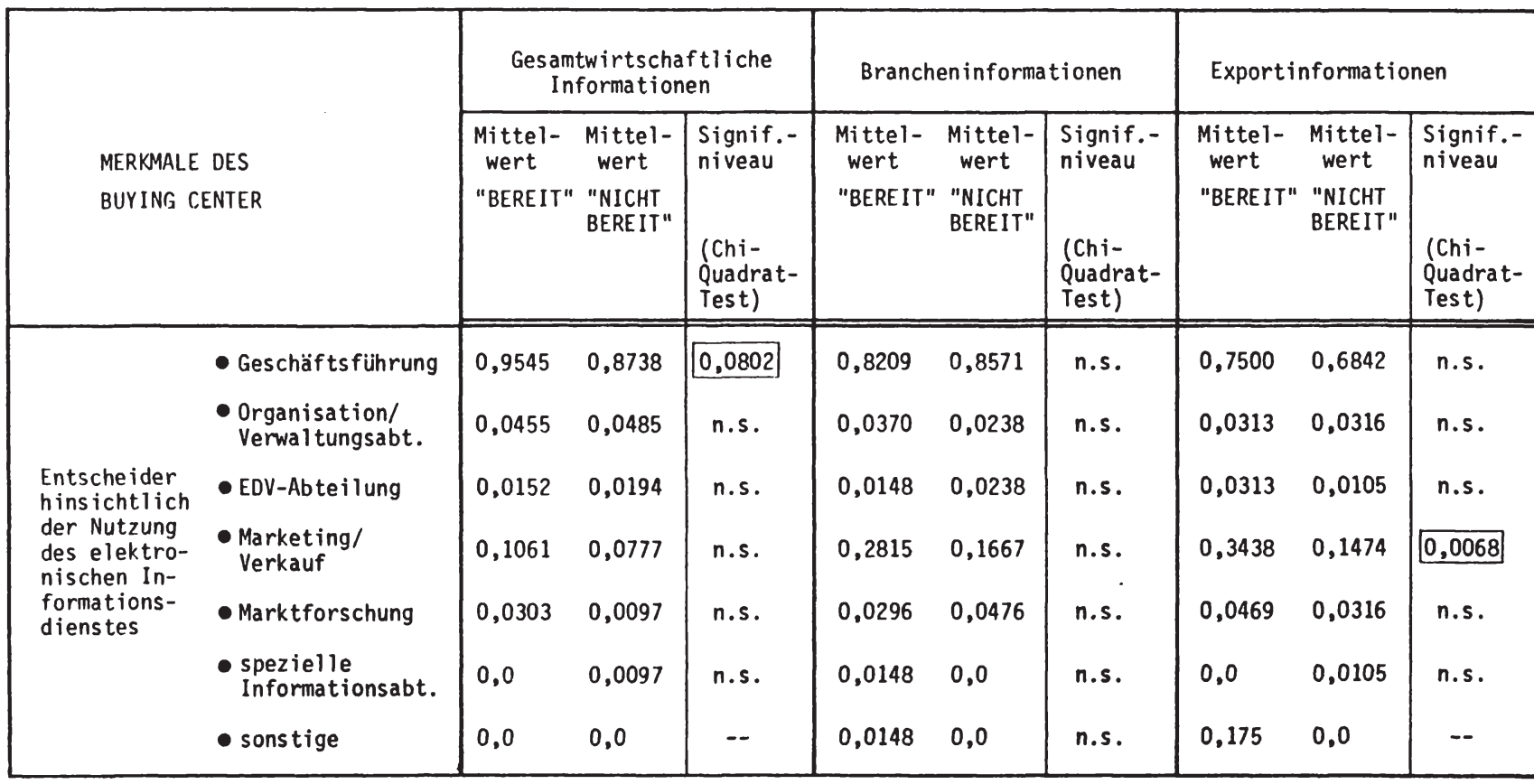

Legende: Mittelwerte $\quad: \begin{aligned} 1 & =\text { trifft } z \mathrm{u} \\ 0 & =\text { trifft } \text { nicht }\end{aligned}$

$0=$ trifft nicht $z u$

Signifikanzniveau: $\alpha=0,1$

Tab. 11: Relevanz von Merkmalen des Buying Center 


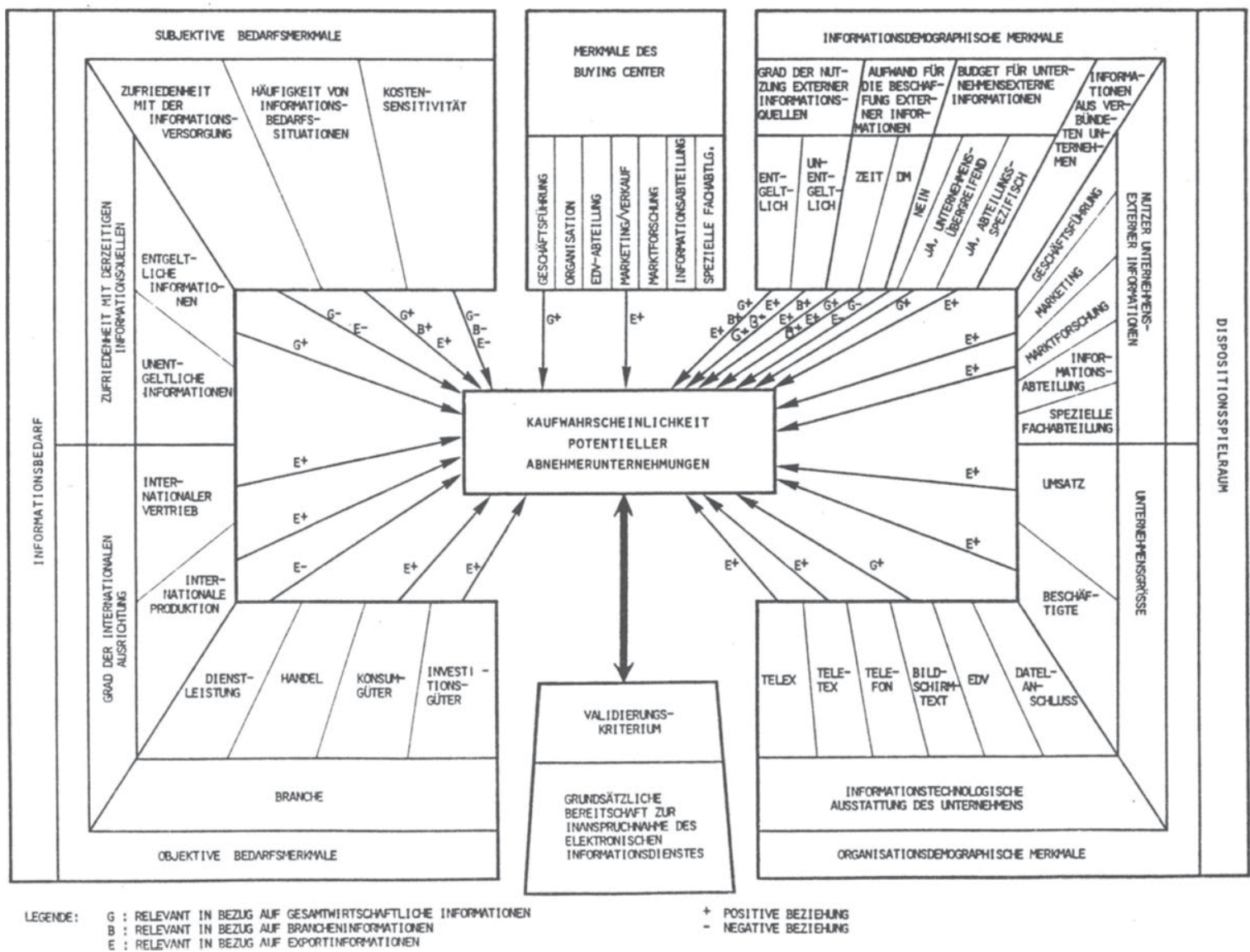


Da in nahezu jedem Unternehmen ein gewisser Bedarf an gesamtwirtschaftlichen und Brancheninformationen besteht, werden sich die Unternehmen in diesem Punkt tendenziell wenig unterscheiden. Umgekehrt wird es - von der (vorhandenen bzw. fehlenden) Adoptionsbereitschaft ausgehend tendenziell schwierig sein, die Unternehmen nach verschiedenen Kriterien wie GröBe, Branche etc. zu trennen.

Anders verhält es sich bei Exportinformationen. Ein Blick in die Praxis macht deutlich, daB sich Unternehmen in ihrem Bedarf an Exportinformationen $z$. T. wesentlich unterscheiden. Entsprechend ist es umgekehrt eher möglich, von der Adoptionsbereitschaft eines Exportinformationsdienstes ausgehend auf die Ausprägungen einzelner Merkmale der betrachteten Unternehmen zurückzuschlieBen.

Am Beispiel eines Branchendatenbankdienstes sei im folgenden abschließend gezeigt, wie die Auswahl der relevanten Abnehmer vorgenommen werden kann.

\subsection{Durchführung der Abnehmerauswahl am Beispiel eines elektronischen Brancheninformationsdienstes}

Bei der Durchführung der Abnehmerselektion stellt sich dem Planenden das Problem, die modellhaft ermittelten Auswahlkriterien gesamtheitlich in einem Entscheidungskalkül zusammenzufügen. Als geeignetes statistisches Verfahren bietet sich hier die Diskriminanzanalyse an. ${ }^{1}$

1 Bei der Diskriminanzanalyse handelt es sich um ein mathematisch-statistisches Verfahren zur Trennung (Klassifikation) einer Menge von objekten oder Personen und deren Zuordnung zu vorgegebenen Teilgruppen. Zur Methode der Diskriminanzanalyse und deren Anwendungsvoraussetzungen vgl. insbesondere Schuchard-Ficher, Chr. et al., a.a.0., S. $153 \mathrm{ff}$. Eine differenzierte Zusammenstellung der Anwendungsmöglichkeiten der Diskriminanzanalyse im Marketing findet sich u.a. bei: Hammann, P., Zur Integration multivariater Marketinginformationen in Marketingentscheidungsmodellen, in: Marketing - Neue Ergebnisse aus Forschung und Praxis, Hrsg.: Topritzhofer, E., Wiesba- 
Sie ermöglicht bei Kenntnis der Ausprägungen aller relevanten EinfluBgrößen bestimmter Unternehmen eine Vorhersage darüber, ob diese Unternehmen jeweils zur Nutzung des geplanten Informationsdienstes grundsätzlich bereit sein werden oder nicht. Darüber hinaus läBt sich aus den Ergebnissen einer solchen Analyse die relative Bedeutung einzelner Kriterien für das Vorhandensein des abhängigen Merkmals (Adoptionsbereitschaft/keine Adoptionsbereitschaft) ersehen.

Im Rahmen der vorliegenden Untersuchung wurden auf der Grundlage alternativer Variablenkombinationen Diskriminanzanalysen in bezug auf die Adoptionsbereitschaft von Branchen-, Gesamtwirtschafts- und Exportinformationsdiensten gerechnet. Da die Beschränkung auf die reduzierte Zahl der zuvor als signifikant ermittelten Variablen zu relativ starken EinbuBen an wichtiger Trenninformation führte, wurden letztlich alle Kriterien des Segmentierungsmodells in die Diskriminanzfunktion mit einbezogen. 1 Die so durchgeführte Diskriminanzanalyse erlaubte in bezug auf alle drei Informationsbereiche eine hochsignifikante Gruppentrennung.

den 1968, S. $29 \mathrm{ff}$. sowie Green, P.E., Tull, D.S., Research for Marketing Decisions, 3. Aufl., Englewood Cliffs, N.J. 1975, insbes. S. $521 \mathrm{ff.}$ Zur Anwendung der Diskriminanzanalyse im Rahmen der industriellen Marktsegmentierung vgl. vor allem Choffray, J.M., Lilien, G.L., Industrial Market Segmentation by the Structure of the Purchasing Process, in: IMM, Vol. 9, 1980, S. $331 \mathrm{ff}$.

1 Nichtsdestoweniger war es aus methodischen Gründen unvermeidlich, einzelne untereinander hoch korrelierende Variablen aus der Berechnung auszuschlieBen, da sonst die Analyse aufgrund von Multikollinearität zu verzerrten Ergebnissen geführt hätte. Vgl. hierzu z. B. Schuchard-Ficher, Chr. et al., a.a.0., S. $92 \mathrm{ff}$. 
Tabelle 12 zeigt als Ergebnis der Diskriminanzanalyse ${ }^{1}$ die Kriterien, die entsprechend der Rangfolge ihres Beitrages zur Gruppentrennung in die Diskriminanzfunktion einbezogen worden sind. ${ }^{2}$ Die absolute Höhe der standardisierten Diskriminanzfunktionskoeffizienten gibt dabei das Bedeutungsgewicht der einzelnen Kriterien hinsichtlich des Vorhandenseins bzw. Fehlens einer Adoptionsbereitschaft wieder.

Im einzelnen zeigt sich, daß Kriterien aller Merkmalsgruppen zur Diskriminierung von adoptionsbereiten und nicht-adoptionsbereiten Unternehmungen beitragen. Eine wesentliche Rolle spielen vor allem die subjektiven Bedarfsmerkmale. Hier liefern allein die Kriterien, die den Grad der Kostensensitivität der potentiellen Abnehmer zum Ausdruck bringen, über 20 Prozent der gesamten Trenninformation. Als weitere trennstarke Kriterien treten daneben die Merkmale des Buying Center sowie informationsdemographische Kriterien in den Vordergrund.

Die "Güte" bzw. Zuverlässigkeit der gefundenen Diskriminanzfunktion $1 \ddot{a} B t$ sich überprüfen, indem man die $Z u-$ gehörigkeit der befragten 182 Unternehmen zur Gruppe der adoptionsbereiten bzw. nicht-adoptionsbereiten potentiellen Abnehmer auf der Basis der jeweiligen Diskriminanzwerte prognostiziert und anschließend mit der tatsächlichen Gruppenzugehörigkeit vergleicht. Der Erfolg der

1 Aus Gründen der Redundanzminimierung soll sich im folgenden die Darstellung der Ergebnisse beispielhaft auf den Fall des betrachteten Brancheninformationsdienstes beschränken.

2 Zugrunde gelegt wurde die Stufenmethode, die über eine sequentielle Auswahl der jeweils "nächstbesten" Variablen einen "optimalen" Satz unabhängiger Variablen ermittelt. Auswahlkriterium zur sequentiellen Einbeziehung der unabhängigen Variablen war Wilks' Lambda, das auf eine "bestmögliche" Trennung der beiden vorliegenden Gruppen abzielt. 


\begin{tabular}{|c|c|c|c|c|}
\hline \multicolumn{5}{|c|}{$\begin{array}{l}\text { ADOPTIIONSBEREITE VS. NICHT ADOPTIONSBEREITE POTENTIELE ABNEHER } \\
\text { EINES ELEKTRONISCHEN BRANCEENINFORMATIONSDIENSTES }\end{array}$} \\
\hline MEROALFAUPPEN & DISKRIMINIEREDE VARIABLEN & $\begin{array}{l}\text { STANAROISIERTER } \\
\text { DISRIMINARE- } \\
\text { FUNKIIONS- } \\
\text { KOEFF IZIENT }\end{array}$ & $\begin{array}{l}\text { PROZEN } \\
\text { AN DER } \\
\text { TREWII }\end{array}$ & $\begin{array}{l}\text { ER ANTEIL } \\
\text { MATEN } \\
\text { MATION }\end{array}$ \\
\hline $\begin{array}{l}\text { OBNEKTIVE } \\
\text { BEDARF STERMALE }\end{array}$ & 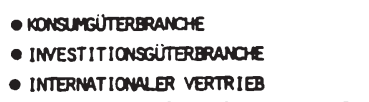 & $\begin{array}{l}+0.31250 \\
+0.95969 \\
-0.42178\end{array}$ & $\begin{array}{l}1.8 \% \\
5.6 \% \\
2.5 \%\end{array}$ & 9.96 \\
\hline $\begin{array}{l}\text { SUBNEKTIVE } \\
\text { BEDARF SMERIOMALE }\end{array}$ & 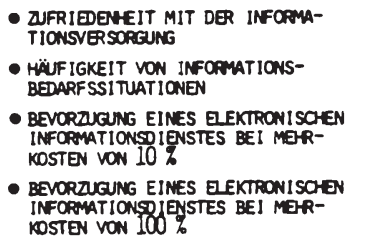 & $\begin{array}{l}-0.39392 \\
+1.1019 \\
+1.99716 \\
-1.88480\end{array}$ & $\begin{array}{l}2.3 \% \\
6.5 \% \\
11.7 \% \\
11.0 \%\end{array}$ & $31.5 \%$ \\
\hline $\begin{array}{l}\text { ORGANISATIONSDERO- } \\
\text { GRAPHISCHE MRROMUE }\end{array}$ & $\begin{array}{l}\text { - umsatz } \\
\text { - tedeax-ausstattung } \\
\text { - bTx-Ausstatung } \\
\text { - datentepminal-ausstattung }\end{array}$ & $\begin{array}{l}-0.45558 \\
-0.52980 \\
+0.60982 \\
-1.04009\end{array}$ & $\begin{array}{l}2.7 \% \\
3.1 \% \\
3.6 \% \\
6.1 \%\end{array}$ & $15.5 \%$ \\
\hline $\begin{array}{l}\text { INFORMATIONSDEMO- } \\
\text { GRAPHISCHE MERIOMALE }\end{array}$ & 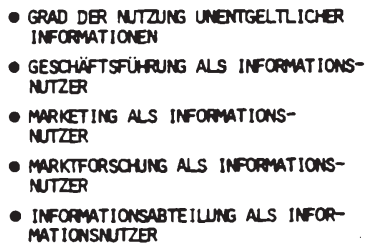 & $\begin{array}{r}-0.74999 \\
-0.83205 \\
+0.45952 \\
+1.27995 \\
+0.69994\end{array}$ & $\begin{array}{l}4.4 \% \\
4.9 \% \\
2.7 \% \\
7.1 \% \\
4.1 \%\end{array}$ & $\mathcal{B} .2 \%$ \\
\hline $\begin{array}{l}\text { MERPMALE DES } \\
\text { BUYING CENTER }\end{array}$ & 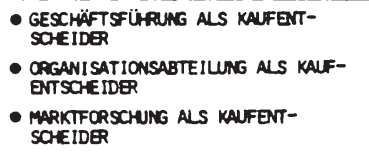 & $\begin{array}{l}-1.02422 \\
+0.90949 \\
+1.47409\end{array}$ & $\begin{array}{l}6.0 \% \\
5.3 \% \\
8.6 \%\end{array}$ & $19,9 \%$ \\
\hline
\end{tabular}

Tab. 12: Ergebnis der Diskriminanzanalyse zur Ermittlung relevanter Kriterien zur Auswahl potentieller Abnehmer eines elektronischen Brancheninformationsdienstes 
Diskriminierung bemiBt sich dann am Anteil der korrekten klassifikation. Abbildung 42 gibt in zahlenmäßiger und graphischer Darstellung das Ergebnis der Klassifikation der befragten Unternehmen als potentielle Adopter eines Brancheninformationsdienstes wieder.

Es wird deutlich, daB auf der Grundlage der ermittelten Variablen-Kombination annähernd $70 \%$ der Unternehmen der jeweils richtigen Gruppe (Adoptionsbereitschaft positiv (1) / negativ (2)) zugeordnet werden. Berücksichtigt man, $d a \beta$ bei einer Zufallsklassifikation eine richtige Gruppenzuordnung von lediglich 50 Prozent zu erwarten gewesen wäre, so spricht das Ergebnis für die relativ hohe prognostische Relevanz der Diskriminanzfunktion. Dies bedeutet in seiner Konsequenz, daß es möglich ist, aufgrund der ausgewählten Kriterien eine relativ genaue Auswahl der erfolguersprechenden Abnehmer zu treffen.

Insgesamt kann festgehalten werden, daß die Diskriminanzanalyse sich als taugliches Verfahren zur Abnehmerselektion im Rahmen der Geschäftsfeldwahl anbietet. Gleichwohl ist zu dem vorliegenden Beispiel anzumerken, da $B$ den Ergebnissen aus methodischer Sicht nur eine bedingte Gültigkeit zugesprochen werden kann. So versagt vor allem die gegebene geringe Stichprobengröße die Ermittlung einer methodisch zuverlässigen Diskriminanzfunktion. 1 Zudem ist ein Restma $B$ an Verzerrungen durch das Auftreten von Abhängigkeiten der Kriterien untereinander nicht auszuschließen.?

1 Der Stichprobenumfang betrug im vorliegenden Fall 50 . Bedingt durch die Vielzahl der einbezogenen Variablen wurden durch die SPSS-Prozedur "DISCRIMINANT" 132 der ursprünglich 182 Fälle aufgrund von "missing values" aus der Berechnung eliminiert.

2 Verzerrungen und damit Fehlinterpretationen können insbesondere dadurch auftreten, daß eine an sich trennschwache Variable aufgrund ihrer hohen Korrelation mit einer anderen trennstarken Variablen einen überproportional hohen Diskriminanzkoeffizienten erhält, der dann fälschlich als Indiz für eine besondere diskriminanzstarke Variable angesehen wird. 
CLASSIF ICATION RESULTS -

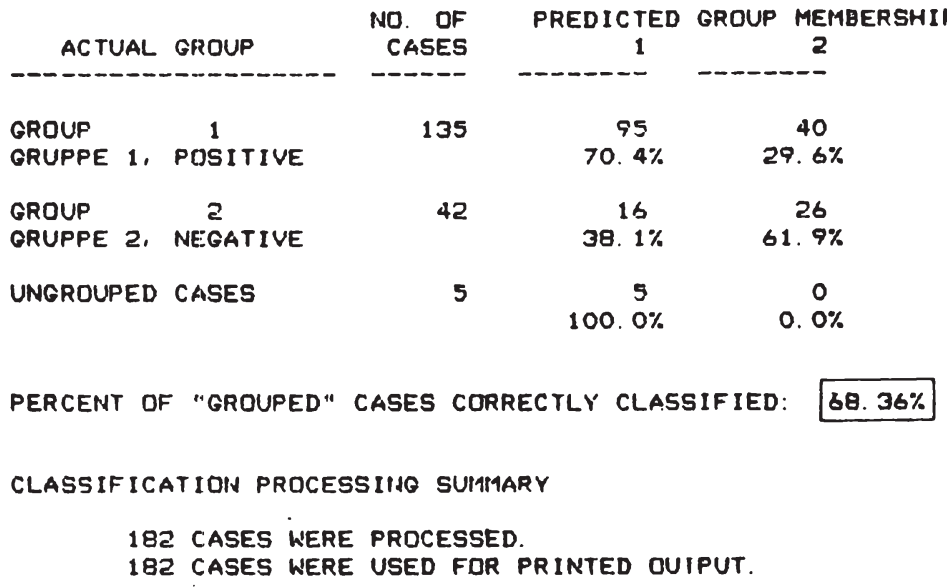

SIIARDL USEN IN RLOTS

SYMACK. GADIP LABEL

-... - .......

1 I GRITFE 1. POSIIIVE

- GRITFE 2. IHGAIIVE

ALL UNGRDUFED CASES

ALL - GROUPS STACKE, HISTOGRAM

16 .

- CAIMHICAL DISCAIMIMANT FUHCTION 1 -.

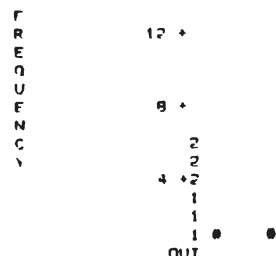

מוז

1. 10 in 1111111112121 11 1212 2 1121

1112111112

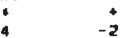
CLASSIFICATIOH $-4$

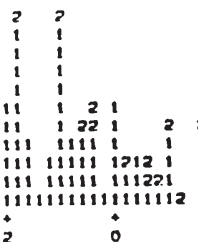

111 $\begin{array}{lll}2 & 2 & 2 \\ 12 & 22 & 2 \\ 11 & 1212 & 2\end{array}$

112112222112122 $+$ 4 4

Abb. 42: Ergebnis der diskriminanzanalytischen Klassifikation der befragten Unternehmen als Adopter/Nichtadopter eines elektronischen Brancheninformationsdienstes 
5. Darstellung und Würdigung des ausgewählten Geschäftsfeldes

Nachdem Abnehmerfunktionen, Technologien und Abnehmergruppen festgelegt wurden, kann das planende Unternehmen sein zukünftiges Geschäftsfeld durch die Kombination aller drei Dimensionen verbindlich definieren. Im vorliegenden Beispiel resultiert das Geschäftsfeld schließlich im

- Angebot von gesamtwirtschaftlichen, Branchen- und Exportinformationen,

- die aus elektronisch gespeicherten Datenbeständen per Bildschirmtext

- online bzw.

- offline als postalisch oder

per Telefax übermitteltes Informationspaket

- von bestimmten, nachfragebereiten Abnehmerunternehmungen

abgerufen werden können.

Abbildung 43 versucht, das Dienstleistungs-Geschäftsfeld graphisch zu veranschaulichen. Kernbestandteil des Dienstleistungsangebots bilden drei Datenbanken, die die Gesamtheit der bereitzustellenden Information speichern. Die zentrale Dienstleistung ("core service") ${ }^{1}$ des Unternehmens besteht im Verkauf von (einzelnen oder pauschalen) Zugriffsberechtigungen auf diese Datenbanken, verbunden mit dem Leistungsversprechen, daß die gewünschten Informationen auch "geliefert" werden können. Darüber hinaus bietet das Unternehmen als zusätzliche Dienstleistung ("peripherical service") seinen potentiellen Abnehmern an, per Bildschirmtext eintreffende Informationswiusche offline durch den Versand von hardcopies zu erfüllen.

$1 \mathrm{Vgl}$. zur Unterscheidung von "core services" und "peripherical services" vor allem Carman, J.M., Langeard, E., a.a.0., S. $9 \mathrm{f}$. 


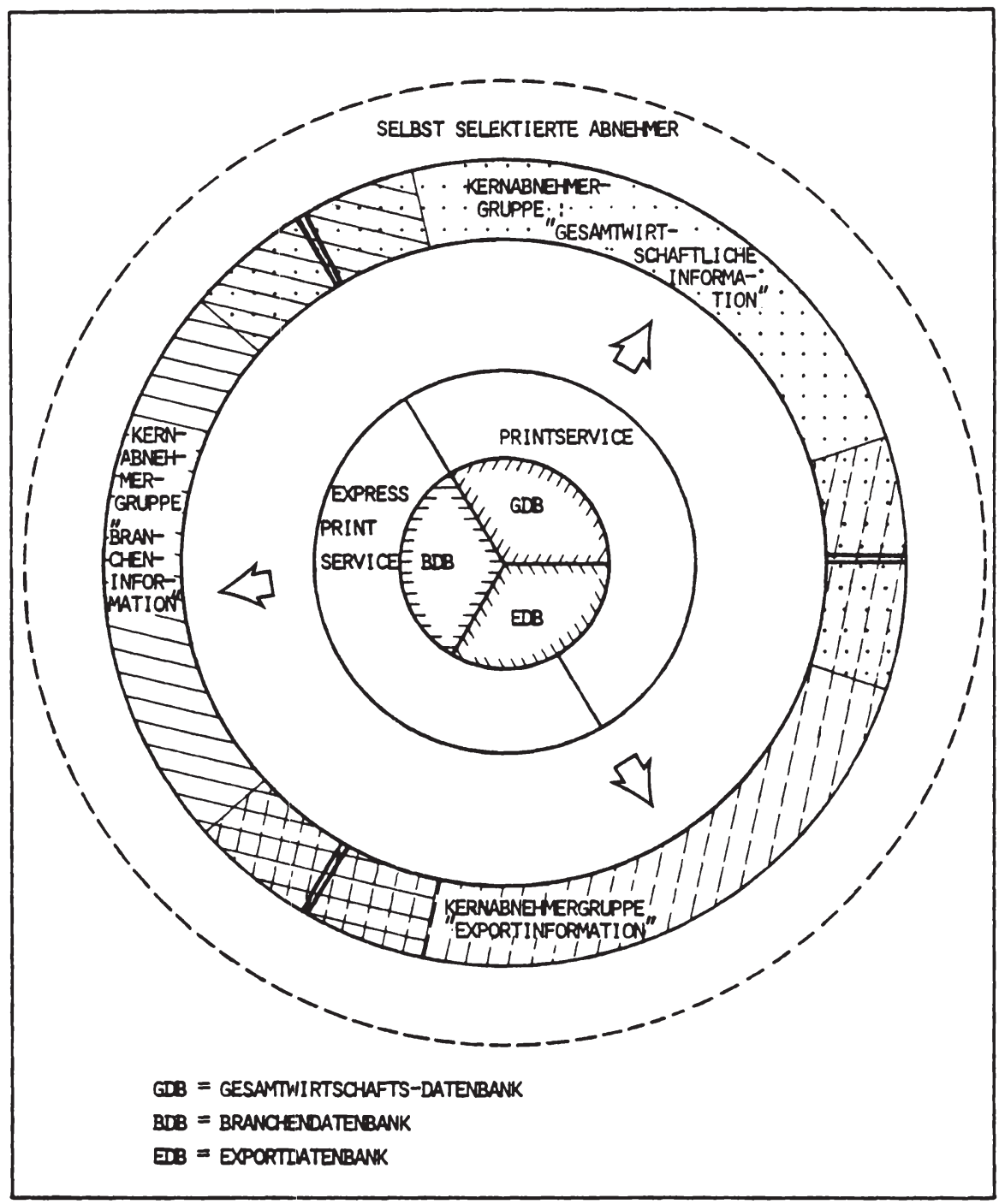

Abb. 43: Schematische Darstellung des ausgewählten Dienstleistungsgeschäftsfeldes 
Während hinsichtlich dieses Dienstleistungspaketes die Funktions- und Technologiedimension zumindest mittelfristig festgeschrieben sind, kennzeichnet das Geschäftsfeld eine gewisse Flexibilität in bezug auf die Abnehmerdimension. So ist die Auswahl der adäquaten Abnehmer nicht im Sinne einer exkludierenden Selektion zu verstehen. Vielmehr soll die Bestimmung von "Kernabnehmergruppen" die Konzentration der Marktbearbeitungsaktivitäten auf erfolgversprechende Segmente erleichtern. Eine Oberschneidung zwischen diesen Kernabnehmergruppen ist dabei ebenso möglich, wie es für die Unternehmung wünschenswert ist, daB im Wege der Selbstselektion neue Abnehmergruppen sich zu den Kernsegmenten hinzugesellen.

Im folgenden sei nun der Frage nach geeigneten Strategien der Marktbearbeitung für die beispielhaft entwickelte investive Dienstleistung nachgegangen. 
C. Strategien der Geschäftsfeldbearbeitung im Bereich elektronischer Informationsdienste

1. Besonderheiten der Geschäftsfeldbearbeitung bei investiven Dienstleistungen

Ein Konzept zur Bearbeitung investiver Dienstleistungsmärkte hat notwendig von den spezifischen Besonderheiten der angebotenen Marktleistung auszugehen. Trotz zum Teil divergierender Auffassungen in der Literatur dominiert in den letzten Jahren in der Marketingwissenschaft die Auffassung, daß für ein erfolgreiches Dienstleistungsmarketing die Beriucksichtigung der besonderen Charakteristika des Dienstleistungsabsatzes eine unabdingbare Voraussetzung darstellt. 1

Als herausragende Besonderheiten verdienen vor allem folgende Aspekte Aufmerksamkeit: ${ }^{2}$

- die Immaterialität der Dienstleistung,

- die Notwendigkeit der Integration eines Fremdfaktors,

- die Simultaneität von Dienstleistungsproduktion und -konsumtion sowie

- die engen Interdependenzen zwischen Dienstleistungsmarketing und Dienstleistungserstellung.

$1 \mathrm{Vgl}$. hierzu insbesondere Grönroos, Chr., Applied Service Marketing Theory, a.a.0., S. 31; Lovelock, Chr.H., Why Marketing Management Needs To Be Different For Services, in: Marketing of Services, Hrsg.: Donnelly, I.H., George, W.G., Chicago, I11., 1981, S. 5 (im folgenden zitiert als: Lovelock, Chr.H., marketing management); Datesuí, J.L.G., a.a.O., S. 34 ff.; Uhl, K.P., Upah, G.D., The marketing of services: why and how is it different, Faculty Working Papers, College of Commerce and Business Administration, University of Illinois at UrbanaChampaign, July 1979. Im Gegensatz dazu vgl. Enis, B.M., Roering, U.J., Services marketing: different products, similar strategy, in: Marketing of Services, Hrsg.: Donnelly, J.H., George, W.G., Chicago, Ill. 1981, S. 1 ff.

2 Vgl. z. B. Kulhavy, E., Dienstleistung, in: HWA, Hrsg.: Tietz, B. et al., Stuttgart 1974, Sp. $455 \mathrm{ff}$.; Lovelock, Chr.H., marketing management, a.a.0., S. $5 \mathrm{f}$.; Grönroos, Chr., A service-oriented approach to marketing of services, in: EJoM, Vol. 12, 1978, H.8,S.591(im folgenden zitiert als: Grönroos, Chr., service-oriented approach); Meyer, A., a.a.0., S. $17 \mathrm{ff}$. 
Am Beispiel elektronischer Wirtschaftsinformationsdienste seien diese Merkmale im folgenden kurz skizziert.

\subsection{Zentrale Merkmale des Absatzes investiver Dienst- leistungen}

1.11 Immaterialität der Dienstleistung

Elektronische Informationsdienste stellen eine immaterielle Marktleistung dar. Als solche sind sie zunächst und vor allem physisch unberührbar ("that cannot be touched, impalpable" ${ }^{1}$ ). Aufgrund inrer Unstofflichkeit haben sie keinen "want appeal", d. h. sie strahlen keinen Kaufanreiz aus. ${ }^{2}$ Während schon der Anblick eines Sachgutes ( $z$. B. das funktionale Styling einer Maschine) vielfach eine Kaufentscheidung positiv beeinfluBt, muß der Kaufimpuls bei Dienstleistungen notwendig vom wahrgenommenen Bedarf ausgehen. Auch ein Betrachten oder Prüfen des objektes vor dem Kauf ist bei investiven Dienstleistungen grundsätzlich nicht möglich.

Neben die physische Unstofflichkeit tritt die gedankliche Immaterialität. Viele, insbesondere innovative Dienstleistungen, lassen sich oft nur als abstrakte, gedankliche Gebilde beschreiben, deren konkrete Ausgestaltung sich der Vorstellungskraft potentieller Abnehmer entzieht ("that cannot be grasped, mentally" ${ }^{3}$ ). Elektronische wirtschaftsinformationsdienste liefern hierzu ein anschauliches Beispiel. Aufgrund ihrer Unstofflichkeit haben diese Dienste heute noch mit höchst ungenauen und diffusen Vorstellungen seitens der potentiellen Nutzer zu kämpfen.

1 Bateson, J.E.G., a.a.0., S. 139.

2 Vgl. Maleri, R., Grundzüge der Dienstleistungsproduktion, Berlin, Heidelberg, New York 1973, S. 45.

3 Bateson, J.E.G., a.a.0., S. 139. 
Tatsächlich ist die Leistungsfähigkeit der Dienste aus der Sicht potentieller Nachfrager kaum abschätzbar, da die Informationsversorgung ja lediglich als Leistungsversprechen angeboten wird.

\subsection{Notwendigkeit der Integration eines Fremdfaktors}

Die Effizienz, d. h. die nutzenstiftende Wirkung eines elektronischen Wirtschaftsinformationsdienstes ist untrennbar mit der Person dessen verbunden, die die Dienstleistung in Anspruch nimmt. ${ }^{1}$ Orne die Anfrage erfolgt keine Informationsvermittlung. Mehr noch: Die Qualität (Präzision) der Anfrage entscheidet wesentlich über die Qualität (Relevanz) der gelieferten Information. Die zentrale Bedeutung dieser Tatsache leuchtet unmittelbar ein, wenn man sich vergegenwärtigt, daß Entscheidungsträger im Unternehmen vielfach weniger Informationen nachfragen und ihre Informationswünche weniger präzise formulieren, als dies ihrer jeweiligen Entscheidungssituation entspräche. ${ }^{2}$

\subsection{Simultaneität von Dienstleistungsproduktion und -konsumtion}

Ein wesentliches Merkmal eines elektronischen Informationsdienstes besteht darin, daB die Erstellung der Dienstleistung (Informationsgenerierung) und ihre Inanspruchnahme durch den Nachfrager (Informationsaufnahme) simultan erfolgen. Sobald die angeforderte Information auf dem Display des Bildschirmtextterminals erscheint,

1 Zur zentralen Bedeutung des Fremdfaktors im Rahmen der Dienstleistungserstellung vgl. insbesondere Hilke, W., a.a.0., S. $11 \mathrm{ff}$.; Meyer, A., a.a.0., S. $21 \mathrm{ff.}$.

$2 \mathrm{Vgl}$. vor allem Witte, E., Informationsnachfrage, a.a.0., S. 838; Witte, E., Entscheidungsprozesse, a.a.0., S. $71 \mathrm{f}$.. 
nimmt sie der Informationssuchende auf und befriedigt damit seinen Informationsbedarf. Diese Tatsache hat zweierlei Konsequenzen:

\section{- Fehlende Lagerfähigkeit der Dienstleistung}

Die Versorgung eines Individuums mit spezifischen Informationen vollzieht sich als ProzeB. Dieser ProzeB der Dienstleistungserstellung beginnt ad hoc mit dem Abruf bestimmter Informationen und endet damit, daB der Informationssuchende die Informationssuche abbricht. Eine Lagerhaltung der Informationsdienstleistungen ist mithin aus Anbietersicht nicht möglich.

\section{- Fehlen von Distributionskanälen}

Der ProzeB der Informationsversorgung vollzieht sich an dem Ort, wo Dienstleistungssystem und Fremdfaktor zusammentreffen. Insofern sind Abrufinformationen in ihrer Eigenschaft als investive Dienstleistungen nicht "handelbar". Der VersorgungsprozeB kann vielmehr nur durch beiderseitigen, unmittelbaren Kontakt zustandekommen. Dieses direkte Zusammentreffen geschieht im Falle elektronischer Informationssysteme durch Telekommunikationskanäle, denen eine raumüberbrückende Funktion zukommt. ". services directed at the customers mind -. such as advice, education, entertainment, and information -can be telecommunicated through such channels as radio, television, the telephone, telecopying, or microwave relays".

1 Lovelock, Chr.H., marketing management, a.a.0., S. 6 . 


\subsection{Interdependenzen zwischen Dienstleistungserstellung} und -marketing

Ein fundamentaler Unterschied zwischen dem Absatz investiver Sach- und Dienstleistungen betrifft schlieBlich die Funktionen, die in Abhängigkeit von der jeweiligen Marktleistung dem Marketing zufallen ${ }^{1}$ (vgl. Abbildung 44).

Im Investitionsguterbereich besteht das Ziel des Marketing im wesentlichen darin, eine Brücke zwischen Produktion und Investition (Kauf) zu schlagen. In diesem Sinne kommt dem Marketing in erster Linie eine auBengerichtete Funktion zu, die darin besteht, den Absatz der produzierten Güter durch die Akquisition von Nachfrage sicherzustellen ("traditionelles Marketing").

Im investiven Dienstleistungsbereich geht die Aufgabe des Marketing weiter. Bedingt durch die Zeitgleichheit von Produktion und Konsumtion wird das Nachfragerverhalten in Sonderheit die Bereitschaft zum Zweitkauf und zum Aufbau fester Lieferantenbeziehungen - in starkem Maße von dem Ablauf und dem Ergebnis der Interaktion zwischen Anbieter und Nachfrager beeinfluBt. Diese Interaktion gezielt zu stevern ist eine zweite zentrale Funktion des Marketing. ${ }^{2}$ Bezogen auf den Fall elektronischer Informationsdienste kann diese interaktionsgerichtete Funktion $z$. B. darin bestehen, die optimale Bearbeitung der eintreffenden Anfragen sicherzustellen, die jederzeitige Auskunftsbereitschaft des Datenbanksystems zu überwachen, Hilfestellung bei der Datenbankabfrage zu leisten ( $z$. B. durch Einrichtung eines "Help Desk"), etc.

$1 \mathrm{Vgl}$. insbesondere Grönroos, Chr., Applied Theory I, a.a.0., S. 32 f.; derselbe, Applied Theory II, a.a.0., S. 49 .

2 Vgl. Grönroos, Chr., Applied Theory I, a.a.0., S. 49 ff.; derselbe, Applied Theory II, a.a.0., S. 32 . 


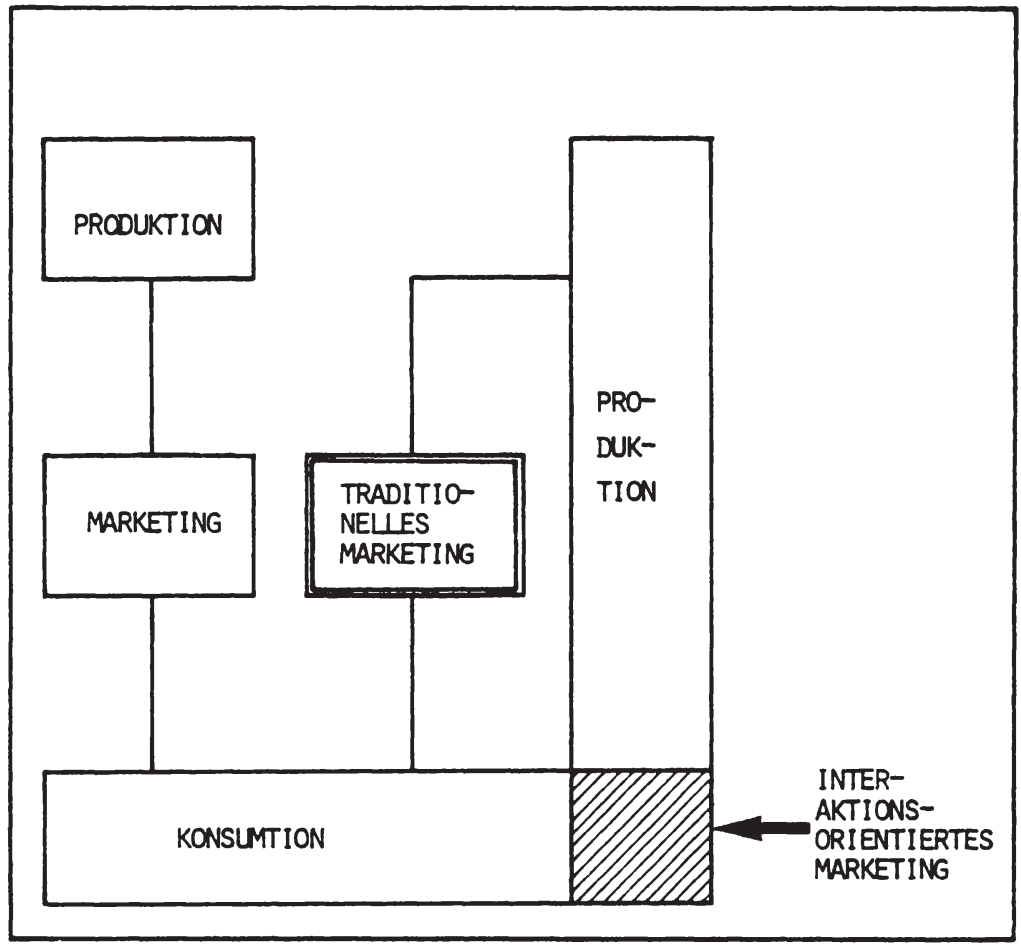

Abb. 44: Zusammenhang zwischen Leistungserstellung, Marketing und Konsumtion

(Quelle: Grönroos, Chr., Applied Theory II, a.a.0., S. 32) 
Eine dritte Funktion des Marketing im Rahmen der Bearbeitung investiver Dienstleistungsmärkte richtet sich schlieB1 ich nach innen und trägt dafür Sorge, da B ein hohes Niveau der Leistungsbereitschaft des Kontaktpersonals wie auch der automatisierten Systeme stets gewährleistet ist.

Mit den drei Funktionen sind bereits wesentliche Eckpunkte des Marktbearbeitungsprozesses umrissen.

\subsection{ProzeB der Bearbeitung investiver Dienstleistungs- märkte}

Abbildung $45 \mathrm{gibt}$ in schematischer Darstellung den Ablauf des Marktbearbeitungsprozesses wieder, wie er beim Absatz von investiven Dienstleistungen idealtypisch abläuft. 1

Den Ausgangspunkt bildet die Herstellung der Leistungsbereitschaft. In dieser Vorphase ("pre-stage") geht es darum, das Leistungsprogramm zu erstellen und die relevanten Zielgruppen zu determinieren. Im wesentlichen entspricht diese Phase dem oben detailliert entwickelten MarktwahlprozeB, ergänzt um die physische Integration der erforderlichen internen Faktoren (Aufbau der Datenbank, Herstellung des Rechnerverbunds, etc.).

Die aus traditioneller Marketingsicht interessanteste Phase beginnt sodann mit der Akquisition potentieller Abnehmer. Dem Marketing muB es hier unter Einsatz geeigneter Strategien und Instrumentekombinationen gelingen, Interessenten als externe Faktoren für eine Interaktion mit dem servuctionssystem zu gewinnen.

1 Vgl. hierzu vor allem Meyer, P.W., Trostmann, Th., Dienstleistungsmarketing. Genese einer Dienstleistungstheorie und der systeminhärenten Implikationen für das Marketing, in: JdAV, 24. Jg., 1978, H. 4, S. $292 \mathrm{ff}$. (im folgenden zitiert als: Meyer, P.W., Trostmann, Th., Dienstleistungsmarketing). 


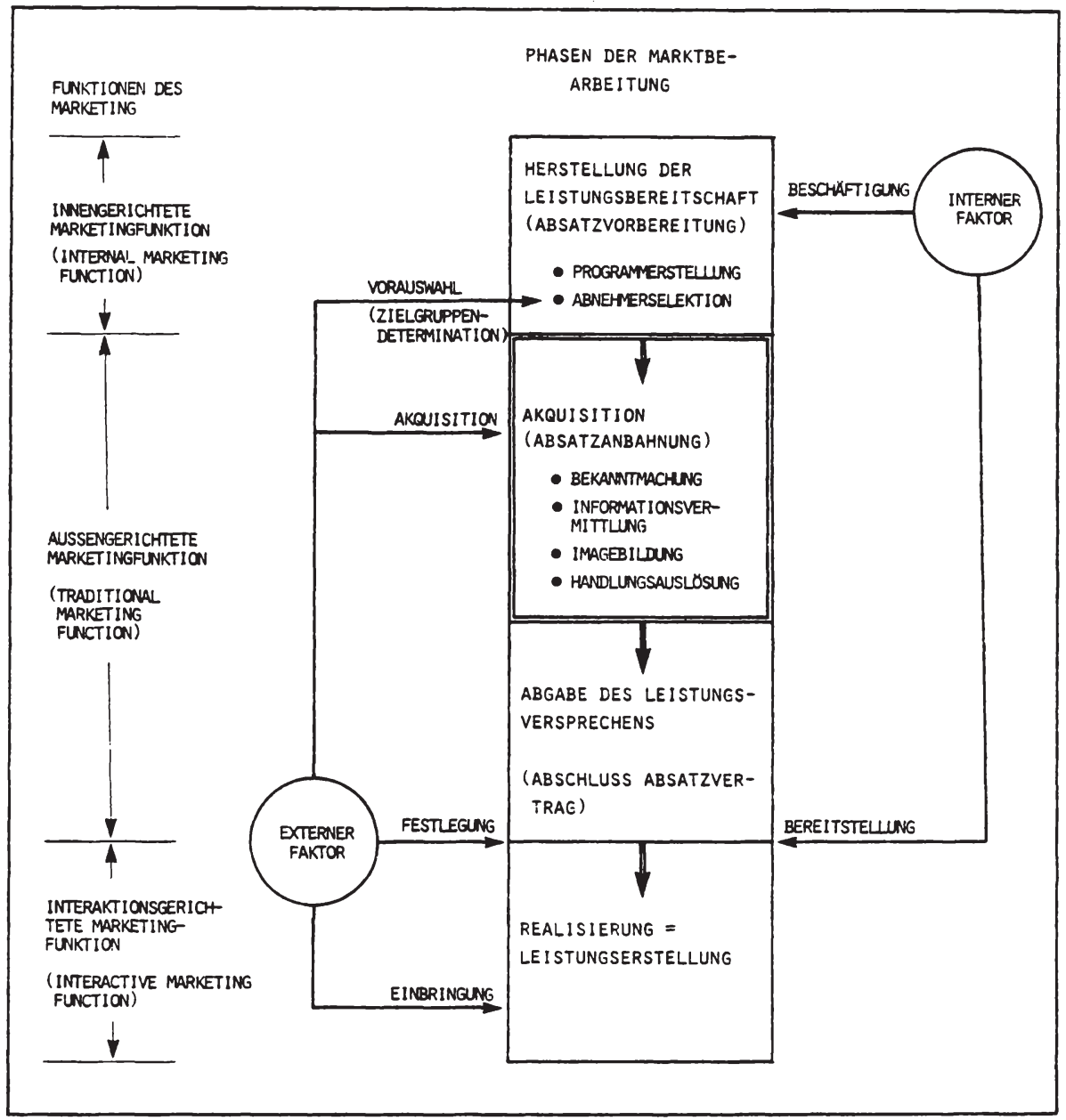

Abb. 45: Phasen des Marktbearbeitungsprozesses bei investiven Dienstleistungen (Quelle: in Anlehnung an Meyer, P.W., Trostmann, Th., Dienstleistungsmarketing, a.a.0., S. 293) 
Ist den Akquisitionsbemühungen des Anbieters Erfolg beschieden, so wird in der Phase des Absatzvertragsabschlusses der Absatz in der Form der Abgabe eines Leistungsversprechens vollzogen. Von diesem Zeitpunkt an stellt das Unternehmen seine internen Faktoren zur Verfügung. Mit der Einbringung des Informationswunsches erfolgt dann jeweils die eigentliche Leistungserstellung.

Im folgenden soll näher auf die Phase der Akquisition eingegangen werden. Wenn damit schwerpunktartig eine Konzentration auf die außengerichtete Funktion der Marktbearbeitung erfolgt, so soll dies nicht als Hinweis auf die geringere Relevanz der internen und interaktionsgerichteten Funktion verstanden werden. Vielmehr erscheint die Beobachtung gerechtfertigt, da $B$ bei innovativen Dienstleistungen, wie elektronische Wirtschaftsinformationsdienste sie darstellen, sich gerade mit der Nachfrageakquisition besondere Herausforderungen an das Marketing verbinden.

\section{Segmentierung der ausgewählten Abnehmer als Grundlage} von Marktbearbeitungsstrategien

Ausgangspunkt einer jeden zielgerichteten Marktbearbeitung bildet die Unterteilung der anvisierten Abnehmerschaft in Gruppen möglichst homogenen Kaufverhaltens. Um eine auf den gezielten Einsatz der Marketinginstrumente gerichtete taktische Marktsegmentierung ${ }^{2}$ vorzunehmen, ist es notwendig, sich Kenntnis über die grundsätzlichen Kaufverhaltensmuster in bezug auf die betrachtete Dienstleistung zu verschaffen. Erste Anhaltspunkte zur Erfas-

1 Walters spricht in diesem Zusammenhang von "Zielgruppenfeinauswahl", die der taktischen Marketingplanung (Bildung von Prioritätsklassen der Marktbearbeitung) dient. Vgl. Walters, M., a.a.0., S. $221 \mathrm{ff.}$. 
sung dieser Verhaltensmuster 1 iefert eine klassifizierende Beschreibung der Beschaffungssituation, in der der potentielle Abnehmer seine Kaufentscheidung zu treffen hat.

\subsection{Beschaffungsverhalten bei elektronischen Informa- tionssystemen als Ausgangspunkt}

\subsection{Typologisierung der Beschaffungsentscheidung}

Ein zentrales Erkenntnisziel der industriellen Kaufverhaltensforschung richtet sich seit jeher auf die Frage, welche zentralen Determinanten die Kaufentscheidung in einer Unternehmung am stärksten beeinflussen. In der Literatur stellte man in diesem Zusammenhang schon frühzeitig fest, daß die Dauer, der Verlauf und die Teilnehmer der organisationalen Kaufentscheidung weit mehr mit dem Typ der Entscheidungssituation als mit der Art des Investitionsgutes variieren.

Aufbauend auf dieser Grunderkenntnis entwickelte Kutschker einen ersten Ansatz zur Typologisierung von Investitionsentscheidungen. ${ }^{2}$ Der Ansatz sieht drei Kriterien vor, die in besonderem Maße geeignet sind, Investitionsentscheidungen nach dem Grad ihrer Komplexität zu differenzieren (vgl. Abbildung 46):

- der relative Wert des Investitionsgutes,

- die Neuartigkeit der Problemdefinition und

- das Ausmaß der durch das Investitionsobjekt ausgelösten organisatorischen Wandels.

$1 \mathrm{Vgl}$. Kutschker, M., Verhandlungen als Elemente eines verhaltenswissenschaftlichen Bezugsrahmens des Investitionsgütermarketing, Diss. Mannheim 1972, S. 45.

$2 \mathrm{Vgl}$. ebenda, S. $47 \mathrm{ff}$; $v g l$. ebenso Meffert, H., Produktivguiter-Marketingforschung, a.a.0., S. $11 \mathrm{f}$. 


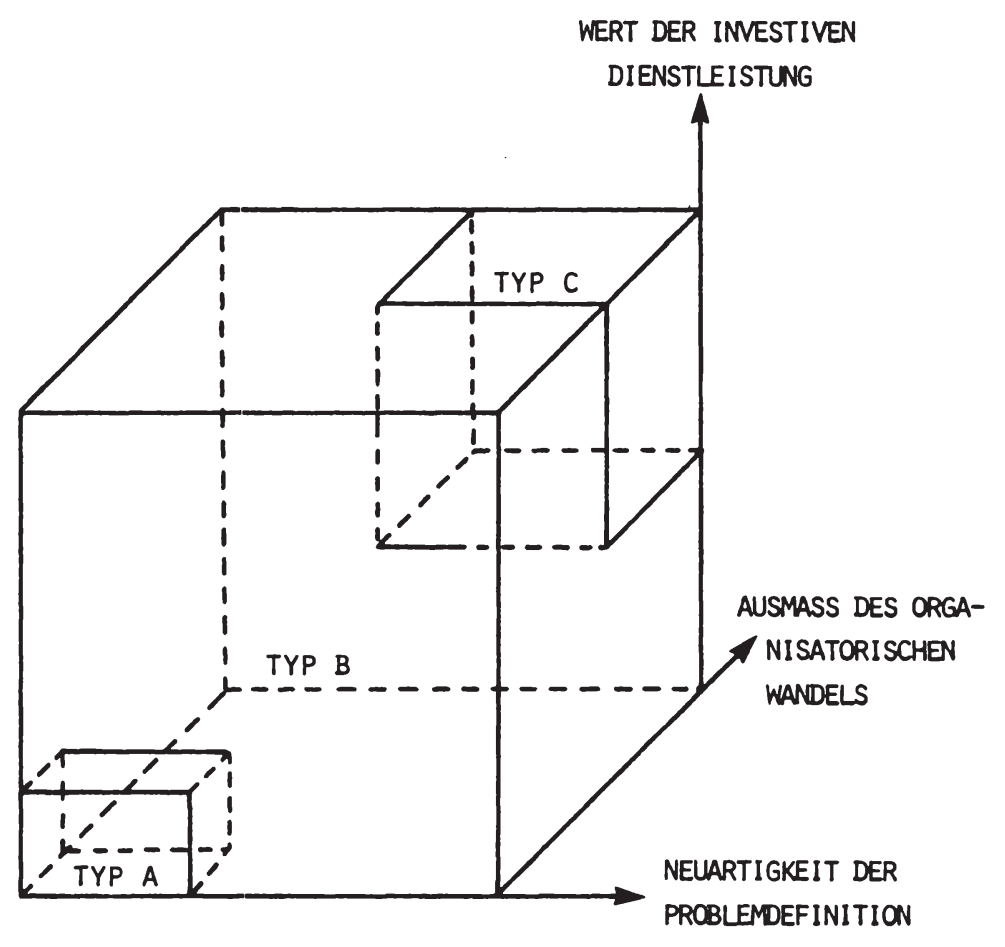

Abb. 46: Typologie von Investitionsentscheidungen

(in Anlehnung an Kutschker, M., a.a.0., S. 47)

Unter Zugrundelegung dieser Kriterienkombination ${ }^{1}$ lassen sich investive Dienstleistungen des Typs A, B und c unterscheiden.

1 Kritisch ist an dieser Stelle anzumerken, daB die Kriterien nicht streng unabhängig voneinander sind und insofern die drei Achsen nicht orthogonal aufeinanderstoßen. 
Versucht man, elektronische Wirtschaftsinformationsdienste in dieses Raster investiver Dienstleistungen einzuordnen, so wird man aus der Sicht der überwiegenden Mehrzahl der Unternehmen eine Zuordnung zu den Dienstleistungen des Typs $C$ vornehmen müssen. Als neuartige Marktleistung zielen diese Dienste nämlich darauf $a b$, eine bisher nicht am Markt angebotene Problemlösung zu erbringen. Ihr relativer Marktpreis ist vergleichsweise hoch, wenn man bedenkt, daß ähnlich geartete, auf konventionellem Wege erbrachte Dienste heute von anderen Stellen kostenfrei zur Verfügung gestellt werden. Schließlich implizieren sie u. U. erhebliche ablauforganisatorische Anpassungserfordernisse.

Auf der Grundlage dieser Typologisierung ist zu schlußfolgern, daß in den meisten Unternehmen in bezug auf die entgeltiche Nutzung eines elektronischen Informationsdienstes noch kein vorgeprägtes Programm vorherrscht, welches in einer bestimmten, prognostizierbaren Art und Weise die Kaufentscheidungen steuert. Insofern gilt es, im Rahmen einer disaggregierenden Analyse die einzelnen Phasen des Beschaffungsprozesses näher zu untersuchen.

\subsection{Darstellung des Entscheidungsprozesses}

Zur gezielten Beeinflussung der Kaufentscheidung ist es notwendig, alle informationsverarbeitenden Vorgänge in den Abnehmerunternehmungen vor, während und nach dem Kauf zu analysieren. 1 Da sich das Informationsverhalten der Nachfragerorganisationen im Zeitablauf ändert, hat es sich als zweckmäßig erwiesen, den Kaufentscheidungspro$z e B$ in einzelne Phasen zu zerlegen.

1 Vgl. Meffert, H., Produktivguiter-Marketingforschung, a.a.0., S. 12 . 
Versucht man, die in der Literatur zum Investitionsgutermarketing entwickelten Phasenabgrenzungen auf investive Dienstleistungen zu übertragen, so gelangt man zu folgendem Entscheidungsverlauf (vgl. Abbildung 47):

ERKENNEN EINES BEDARFS UND

DER GEEIGNETEN DIENSTLE I STUNG

ALS PROBLEMLÖSUNG

SUCHE UND AUSWAHL DES INFRAGE KOMMENDEN EXTERNEN DIENST-

\section{LE I STUNGSANBIETERS}

KAUF EINES ANRECHTES AUF

"PURCHASING

PROCESS"

LEI STUNGS INANSPRUCHNAHME

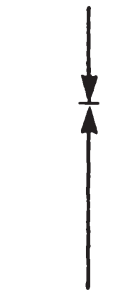

"CONSUMPTION

PROCESS"

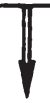

INANSPRUCHNAHME DER LEISTUNG

UNTER WEITGEHENDER BETEILIGUNG

AN DER LEISTUNGSERSTELLUNG

$$
1
$$

NACHTRÄGLICHE BEWERTUNG

DER DIENSTLEISTUNG

Abb. 47: Phasen des Kaufentscheidungsprozesses bei der Beschaffung investiver Dienstleistungen 
Jeder Phasenabschnitt des Entscheidungsprozesses erfordert vom Marketing bestimmte, auf die jeweilige Situation der Abnehmerunternehmung zugeschnittene Strategien und Maßnahmenbindel.

Im Rahmen des ersten Phasenabschnitts ("initial stage") muß es dem Marketing darum gehen, dem potentiellen Nachfrager seine Problemstellung deutlich zu machen und das Interesse an der angebotenen Dienstleistung zu wecken.

Im zweiten Stadium des Beschaffungsprozesses ("purchasing process") kommt dem Marketing die Aufgabe zu, dieses Interesse auf die Anbieterunternehmung zu lenken und einen Kaufabschluß herbeizuführen.

Die dritte Phase ("consumption process") erfordert vom Marketing schließlich, über die Gewährleistung einer erfolgreichen Interaktion zwischen Nachfrager und Dienstleistungssystem eine anhaltende Kundenzufriedenheit und die Entwicklung fester Kundenbeziehungen sicherzustellen.

Auf der Grundlage dieses ProzeBablaufs kommt hinsichtlich der Marktbearbeitung zunächst und insbesondere der Frage eines "geeigneten" Problembewußtseins des potentiellen Nachfragers eine kardinale Bedeutung zu. Der zentrale Stellenwert dieses ProblembewuBtseins erklärt sich aus seiner Auslöserfunktion bei der Initialisierung des Kaufentscheidungsprozesses. Erkennt nämlich der potentielle Nachfrager nicht sein Problem und/oder die geeignete Dienstleistung als Problemlösung, so wird der KaufentscheidungsprozeB entweder überhaupt nicht zustande kommen oder aber anders verlaufen, als wenn die "geeignete" Problembewußtseinslage gegeben wäre. Damit ist als zentraler Bestimmungsfaktor des Kaufverhaltens bei investiven Dienstleistungen der Aspekt der Problemevidenz angesprochen.

1 Vgl. zum folgendem vor allem Grönroos, Chr., Applied Theory I, a.a.0., S. $46 \mathrm{ff}$. 


\subsection{Problemevidenz als zentrale Determinante des Be- schaffungsverhaltens}

\subsection{Begriff und Abgrenzung der Problemevidenz}

"Unter Evidenz soll die Einsicht eines Nachfragers in die Tatsache verstanden werden, daB er eine bestimmte Dienstleistung zur Lösung seiner Probleme benötigt". 1 Folgt man dieser Definition, so bedeutet Problemevidenz die Wahrnehmung eines auf eine bestimmte Dienstleistung gerichteten Bedarfs.

Engelhardt und Schwab, die den Begriff der Problemevidenz in die wissenschaftliche Diskussion eingebracht haben, grenzen die Problemevidenz einer Dienstleistung uber ein zweistufiges Bedingungsraster ab ${ }^{2}$ (vgl. Abbildung 48). Diesem Raster folgend impliziert Problemevidenz beim potentiellen Nachfrager zunächst die Erkenntnis, daB überhaupt eine Dienstleistung benötigt wird. Der Nachfrager muB demnach zunächst einmal überhaupt ein Problem im Sinne eines wie auch immer gearteten Mangelzustandes wahrnehmen, der ihn veranlaßt, nach einer Problemlösung zu suchen. Einfach formuliert ist damit gefordert, daß der potentielle Abnehmer einen (kaufauslösenden) Bedarf wahrnimmt.

Zusätzlich knüpft sich die Problemevidenz sodann an die Bedingung, daB der Nachfrager Kenntnis darüber besitzt, welche Dienstleistung zur Beseitigung des Bedarfs und damit zur Lösung seines Problems nötig ist.

1 Engelhardt, W.H., Schwab, W., a.a.0., S. 506.

$2 \mathrm{Vgl}$. ebenda, S. $506 \mathrm{ff}$. 


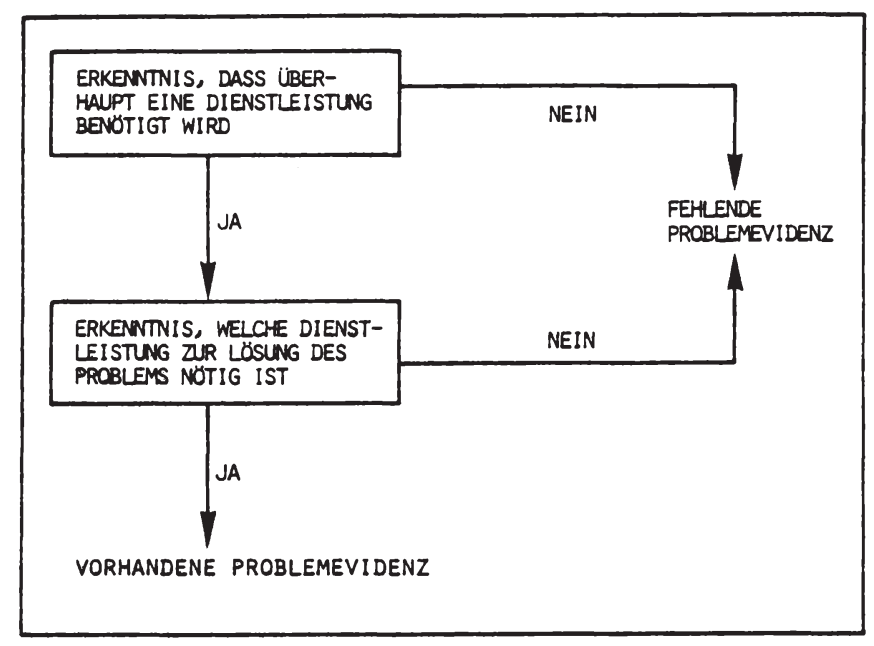

Abb. 48: Abgrenzung der Problemevidenz einer Dienstleistung

(Quelle: Engelhardt, W.H., Schwab, W., a.a.0., S. 507)

Zur Veranschaulichung der beiden Voraussetzungen für das Vorhandensein von Problemevidenz und zur Charakterisierung der denkbaren Ausprägungen von Situationen fehlender Problemevidenz unterscheiden Engelhardt und Schwab verschiedene "Problembewußtseinslagen" beim potentiellen Abnehmer. ${ }^{1}$ Sie lassen sich - unter Heranziehung des Beispiels elektronischer Informationsdienste - wie folgt skizzieren:

a. Der Abnehmer nimmt im Fehlen bestimmter Informationen ein Problem wahr, verkennt jedoch, daß die grundsätzliche Inanspruchnahme externer Informationsdienstleistungen ihm bei der Lösung des Informationsproblems helfen könnte. Entsprechend identifiziert er den elektronischen Wirtschaftsinformationsdienst auch nicht als geeignete Problemlösungsalternative.

1 Vgl. Engelhardt, W.H., Schwab, W., a.a.0., S. 507. 
b. Der Abnehmer erkennt zwar grundsätzlich den Nutzen externer Informationsdienste, vermag jedoch die Informationsdienstleistung, die er zur Bewältigung seiner Informationsprobleme benötigt, nicht oder nur unzureichend zu präzisieren. Folgende Einzelfälle sind denkbar:

- Der Abnehmer erkennt seinen Informationsmangel, ist jedoch auBerstande zu präzisieren, welche Informationen er benötigt. So ist denkbar, daB der potentielle Nachfrager die Notwendigkeit der Einbeziehung gesamtwirtschaftlicher Rahmen- und Konjunkturdaten in seine Planungen einsieht, jedoch nicht zu konkretisieren vermag, ob nun zeitpunktbezogene, aktuelle Informationen oder Entwicklungsdaten in Form von zeitreihen für ihn die gröBere Relevanz besitzen.

- Der Abnehmer ist subjektiv der Auffassung, die zur Lösung seines Informationsproblems geeignete Informationsdienstleistung zu kennen, unterliegt jedoch aufgrund einer falschen oder mangelhaften Problemanalyse darin einem Irrtum. Ein solcher Fall ist beispielsweise gegeben, wenn der betreffende Entscheidungsträger meint, eine unzureichende Versorgung mit Brancheninformationen erkannt zu haben, tatsächlich jedoch die im Unternehmen vorhandenen Brancheninformationen von den verantwortlichen Stellen aufgrund eines gewissen "information overload" lediglich nicht gründich genug ausgewertet und systematisch archiviert werden.

- Der Abnehmer nimmt sein Problem "richtig" wahr, leitet jedoch aus diesem die falschen Maßnahmen ab. In diesem Fall erkennt der Nachfrager z. B., da $B$ es in seiner Unternehmung an einer prompten Versorgung mit Informationen aus den wichtigsten aktuellen und potentiellen Exportländern mangelt. 
Statt jedoch die erforderlichen Informationen ad hoc von einer tagesaktuellen Exportdatenbank abzufragen, stellt er einen Mitarbeiter mit der Aufgabe ab, turnusmäBig die Veröffentlichungen der BfAI, der Kreditinstitute und anderer Informationsquellen zu sichten und in regelmäßigen Abständen Länderberichte zu verfassen.

c. Der Abnehmer kennt seinen Informationsbedarf und ist imstande, diejenige Dienstleistung zu beschreiben, die diesen Informationsbedarf zu befriedigen bestmöglich imstande ist. In diesem und nur in diesem Falle liegt eine Problemevidenz der betreffenden Dienstleistung vor.

Grundsätzlich trägt die Analyse der Problemevidenz wesentlich dazu bei, die relevante Ausgangssituation potentieller Dienstleistungsabnehmer zu erfassen und geeignete Maßnahmen zur Handlungsbee influssung abzuleiten. 'Gleichwohl muß an dem Konstrukt in der von Engelhardt/Schwab beschriebenen Ausgestaltung bemängelt werden, daß es in keiner Weise operational ist. So besteht weder die Möglichkeit, zwischen einzelnen Abnehmergruppen ein Mehr oder Weniger an vorhandener Problemevidenz festzustellen, noch gibt es einen konkreten Maßstab, der es erlaubt, den Grad der Problemevidenz einzelner Nachfrager zu quantifizieren.

Angesichts der Heterogenität der Probleme und Problemlösungen, die sich mit investiven Dienstleistungen verbinden, spricht vieles dafür, daß die Entwicklung eines allgemeinen Operationalisierungsansatzes für die Problemevidenz nicht möglich ist. In bezug auf die einzelne Dienstleistung hingegen erscheint es realistisch, daB sich ein Maßstab zur Bemessung einer dienstleistungs-

1 Vgl. Engelhardt, W.H., Schwab, W., a.a.0., S. $508 \mathrm{ff}$. 
typspezifischen Problemevidenz finden $1 \ddot{a} \beta t$. Im folgenden soll der Versuch unternommen werden, einen solchen Maßstab für die Problemevidenz in bezug auf das Angebot eines elektronischen Wirtschaftsinformationsdienstes zu entwickeln.

\subsection{Ansatz zur Operationalisierung der Problemevidenz in bezug auf elektronische Informationsdienste}

Ausgehend von der oben dargestellten Abgrenzung kann die Problemevidenz als zweistufiges Konstrukt modellhaft abgebildet werden. Auf der ersten stufe knüpft sich an das Vorhandensein von Problemevidenz eine notwendige Bedingung, die vom potentiellen Abnehmer verlangt, daB er ein bestimmtes Problem (richtig) wahrnimmt. Auf der zweiten Stufe erfordert eine hinreichende Bedingung, da $B$ er die "richtige" Dienstleistung als Problemlösung identifiziert. Sowohl im Falle mangelnder Problemwahrnehmung als auch im Falle mangelnder Dienstleistungsidentifikation liegt definitionsgemäß keine Problemevidenz vor.

Die Frage stellt sich nunmehr, auf welche Weise die Wahrnehmung des Problems und die Identifikation der Dienstleistung operationalisiert werden kann.

\subsection{Operational isierung der Problemwahrnehmung}

Von einer Wahrnehmung eines Informationsproblems kann dann ausgegangen werden, wenn der Abnehmer feststellt, daß bestimmte, von ihm als wichtig erachtete Informationsanforderungen nicht erfüllt werden. Folgt man dieser Grundhypothese, so bestimmen der Erfiillungsgrad und der Grad der Wichtigkeit relevanter Informationsanforderungen das AusmaB an Problemwahrnehmung. Entsprechend wird ein Problem vom Nachfrager stets um so stärker wahrgenommen, 
- je weniger die von dem Entscheidungsträger als wichtig erachteten Informationsanforderungen erfiult werden bzw.

- je größer die Wichtigkeit ist, die der Entscheidungsträger den nicht oder nur unzureichend erfuillten Informationsanforderungen einräumt.

Aus dieser Perspektive kann als Hilfsgröße für die Problemwahrnehmung die Einstellung des potentiellen Abnehmers zu seiner gegenwärtigen Informationsversorgung herangezogen werden. Problemwahrnehmung liegt demzufolge dann vor, wenn der potentielle Abnehmer eine nicht hinreichend positive Einstellung gegenuiber der gegenwärtigen Informationsversorgung an den Tag legt.

Die Operationalisierung dieser (negativen) Einstellung kann analog zum Vorgehen multidimensionaler Einstellungsmodelle erfolgen. ${ }^{2}$ Aus der Vielzahl unterschiedlicher Modellvarianten scheint dabei das sog. "adequacy-importance"-Modell in besonderem Maße der vorliegenden Problemstellung zu entsprechen. ${ }^{3}$

Das "adequacy - importance"-Modell stellt darauf ab, in welchem Ausmaß (mengenmäBige Ausprägung) einzelne Eigenschaften an einem objekt vorhanden sind (belief). Die einzelnen Eigenschaften sind dabei nach ihrer Bedeutung zu gewichten (importance weight). 4 Interpretiert man die

1 Unter Einstellung soll dabei die gefühlsmäBige Wertung oder Einschätzung eines objektes verstanden werden.

Vgl. Freter, H., Interpretation und Aussagewert mehrdimensionaler Einstellungsmodelle im Marketing, in: Konsumentenverhalten und Information, Hrsg.: Meffert, H., Steffenhagen, H., Freter, H., Wiesbaden 1979, S. 163 (im folgenden zitiert als: Freter, H., Einstellungsmodelle).

2 Vgl. hierzu vor allem Freter, H., Einstellungsmodelle, S. $164 \mathrm{ff}$. sowie die dort angegebene Literatur.

3 Zum adequacy-importance"-Modell vgl. Freter, H., Einstellungsmodelle, a.a.0., S. 165; vgl. ferner Bettmann, J.R., Capon, N., Lutz, R.J., Cognitive Algebra in MultiAttribute Attitude Models, UCLA Working Papers No. 10, February 1974; Mazis, M.B., Athola, 0.T., Klippel, R.E., A Comparison of Four Multi-Attribuie Models in the Prediction of Consumer Attitudes, in: JoCR, Vol. 2, 1975, No. 1, S. 40 .

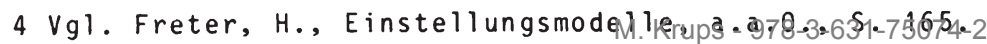


Eigenschaften als Merkmale (Anforderungen) der Informationsversorgung, so verbindet das Modell als zentrale Komponenten

- den Eindruck über den Grad, in dem die geforderten Eigenschaften der Informationsversorgung derzeit erfiilt werden, mit

- der Wichtigkeit, die diesen Eigenschaften im einzelnen zugesprochen wird.

Die mathematische Struktur des so konkretisierten Einstellungsmodells kann wie folgt charakterisiert werden:

PROD $_{j}$ (eroblem detection) $\frac{\sum_{i=1}^{n} \text { ANWICHT }_{i j} \cdot \sum_{i=1}^{n} \text { ANERF }_{i j}}{n}$

Dabei ist PROD ${ }_{j} \quad=$ Problemeinstellung (Mas für die Intensität der Problemwahrnehmung in bezug auf die Informationsart $j$ )

ANWICHT $_{i j}=$ transformiertes MaB für die bekundete Wichtigkeit eines Anforderungsmerkmáts in bezug auf die Informationsart $j$

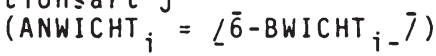

ANERF $_{i j}=$ Eindruck, inwieweit die derzeitige Informationsversorgung ein Anforderungsmerkmal $i$ in bezug auf die Informationsart $j$ erfuilit

$j=$ Informationsart

$j=G=$ Gesamtwirtschaftliche Information

$j=B=$ Brancheninformation

$j=E=$ Exportinformation

$i=$ Anforderungsmerkmal

$i=1$ = Schnelligkeit

$i=2=$ Aktualität

i

$i=n=14=$ EDV -Verarbeitbarkeit 
Mit dem Einstellungsmaß PROD ("eroblem detection") ist die erste Bedingung der Problemevidenz operationalisiert. Die Frage, ob ein potentieller Abnehmer Einsicht in die Notwendigkeit einer Dienstleistung besitzt, kann demzufolge anhand der Höhe seiner jeweiligen Problemerkenntnis beantwortet werden. Als Bedingung der Problemerkennt$\mathrm{n}$ is gilt

PROD $\geqslant x_{k r i t}$, wobei $x_{k r i t}$ einen vorgegebenen kritischen Wert darstellt, der die Grenze zwischen "hoher" Problemwahrnehmung" (negative Einstellung) und "geringer Problemwahrnehmung" (keine negative Einstellung) kennzeichnet.

Abbildung 49 macht beispielhaft deutlich, wie die Abgrenzung zwischen hoher und geringer Problemwahrnehmung, bezogen auf den konkreten Fall der Versorgung mit Brancheninformationen, vorgenommen werden kann.

\begin{tabular}{|c|c|c|c|c|c|c|}
\hline & & \multicolumn{5}{|c|}{$\begin{array}{l}\text { WICHTIGKEIT DER INFORMATIONS- } \\
\text { ANFORDERLNGEN }\end{array}$} \\
\hline & & $\begin{array}{l}\text { GAR } \\
\text { NICHT } \\
\text { WICHTIG } \\
\text { (I) }\end{array}$ & $\begin{array}{c}\text { WENIGER } \\
\text { WICHTIG } \\
(2)\end{array}$ & $\begin{array}{c}\text { ZIEMLICH } \\
\text { WICHTIG } \\
\text { (3) }\end{array}$ & $\begin{array}{c}\text { SEHR } \\
\text { WICHTIG } \\
(4)\end{array}$ & $\begin{array}{c}\text { EXTREM } \\
\text { WICHTIG } \\
(5)\end{array}$ \\
\hline \multirow{5}{*}{$\begin{array}{l}\text { ERFULUNGS- } \\
\text { GRAD DER IN- } \\
\text { FORMATIONS- } \\
\text { ANFORDERUNGEN }\end{array}$} & $\begin{array}{l}\text { VOL } \\
\text { ERFULTT } \\
\text { (1) }\end{array}$ & 1 & 2 & 3 & 4 & 5 \\
\hline & $\begin{array}{l}\text { ÜBER- } \\
\text { WIEGEND } \\
\text { ERFiULT } \\
\text { (2) }\end{array}$ & 2 & $\begin{array}{c}4 \\
\text { INGE PROB }\end{array}$ & Ler 6 & 8 & 10 \\
\hline & $\begin{array}{l}\text { ETWAS } \\
\text { ERFÜLT } \\
\text { (3) }\end{array}$ & 3 & & 9 & 512 & 15 \\
\hline & $\begin{array}{l}\text { KALM } \\
\text { ERFULT } \\
\text { (4) }\end{array}$ & 4 & 8 & 512 & \multirow{2}{*}{$\begin{array}{l}\text { DOHE PROBLE } \\
\text { HOHE } \\
\text { WAHPREMMNG } \\
\mid 20\end{array}$} & $\begin{array}{r}20 \\
+\end{array}$ \\
\hline & $\begin{array}{l}\text { GAR } \\
\text { NICHT } \\
\text { ERPULLT } \\
\text { (5) }\end{array}$ & 5 & 10 & $k^{15}$ & & 25 \\
\hline
\end{tabular}

Abb. 49: Abgrenzung der Problemwahrnehmung hinsichtlich der Versorgung mit Brancheninformationen 
2.222 Operationalisierung der Dienstleistungsidentifikation

Die zweite Bedingung der Problemevidenz erfordert von dem potentiellen Abnehmer, daB er die Dienstleistung, die das von ihm wahrgenommene Problem zu lösen imstande ist, als eine solche Problemlösung identifiziert. Die Operationalisierung dieser Dienstleistungsidentifikation wirft nicht unerhebliche methodische Schwierigkeiten auf, gilt es doch, ohne direkte Bezugnahme auf die wahrgenommenen Probleme festzustellen, ob der Abnehmer die Dienstleistung als Problemlösung identifiziert. Ein praktikabler Lösungsweg könnte an der intentionalen Komponente der Einstellung des Abnehmers gegenüber der Dienstleistung, d. h. an seiner Handlungstendenz, ansetzen: ${ }^{1}$ Identifiziert ein Nachfrager eine Dienstleistung als Lösung seiner Probleme, so wird er - zweckrationales Handeln unterstellt - dieser Dienstleistung gegenüber eine grundsätzliche Nutzungsbereitschaft aufweisen.

Unter Bezugnahme auf das Beispiel eines elektronischen Brancheninformationsdienstes könnte damit die Bedingung fur die Identifizierung der Dienstleistung als Problemlösung wie folgt charakterisiert werden: ${ }^{2}$

BBEREIT $\stackrel{!}{=} 1$, d. h. die Bereitschaft, einen elektronischen Brancheninformationsdienst zu nutzen, wird "wahrscheinlich" oder "bestimmt" bejaht

1 Zur Drei-Komponentenlehre in der Einstellungsforschung vgl. McGuire, W.J., The Nature of Attitudes and Attitude Change, in: The Handbook of Social Psychology, Vol. III, Hrsg.: Lindzey, G., Aronson, E., Reading, Mass. 1969. Vgl. ferner Meffert, H., Marketing, a.a.0., S. 122 ; Freter, H., Marktsegmentierung, a.a.0., S. 64.

2 Zur genauen Fragestellung vgl. Frage 9 des Fragebogens in Anhang 3, S.293. Ein Ankreuzen von "ja, bestimmt" und "ja, wahrscheinlich" wurde hierbei mit 1, "nein, wahrscheinlich nicht" und "nein, bestimmt nicht" mit 2 kodiert. 


\subsection{Verknüpfung von Problemwahrnehmung und Dienst- leistungsidentifikation}

Verbindet man die beiden nunmehr operationalisierten Komponenten der Problemevidenz, so lassen sich - wie in Abbildung 50 veranschaulicht - vier Konstellationen vorhandener bzw. fehlender Problemevidenz unterscheiden. In den ersten beiden Quadranten ist die notwendige Bedingung der Problemevidenz nicht erfiult. Während in Quadrant I angesichts fehlender Problemwahrnehmung konsequenterweise keine Nutzungsbereitschaft (i.e. Dienstleistungsidentifikation) vorliegt, resultiert die Bereitschaft zur Nutzung des Dienstes in Quadrant II u. U. aus einer gewissen Innovationsorientiertheit. Im

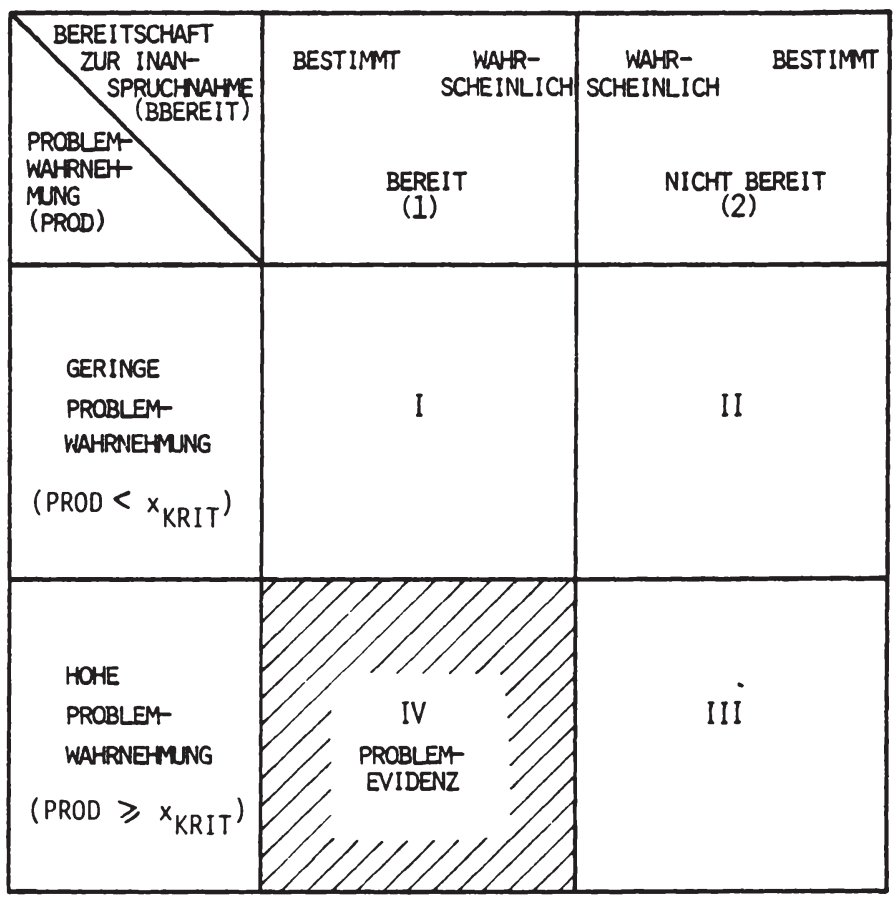

Abb. 50: Konstellationen vorhandener und fehlender Problemevidenz 
Falle des Quadranten III erkennen die Abnehmer ihr Informationsproblem, sind jedoch nicht imstande, die (geeignete) Dienstleistung zu identifizieren. Die hieraus resultierende Unsicherheit führt zur Ablehnung des elektronischen Informationsdienstes. Im Quadranten IV schlieBlich - und nur hier - liegt Problemevidenz vor.

Abbildung 50 zeigt abschließend das Vorgehen zur Abgrenzung der Problemevidenz im Oberblick. Inwieweit eine auf dieser Operationalisierung aufbauende empirische Analyse der Problemevidenz zu sinnvollen Ergebnissen führt, sei im folgenden untersucht.

\subsection{Messung der Problemevidenz am Beispiel eines elek- tronischen Brancheninformationsdienstes}

Unter Zugrundelegung der im Rahmen der vorliegenden Untersuchung erhobenen Daten folgt die Quantifizierung der Problemevidenz dem in Abbildung 51 vorgegebenen Ablaufplan. In einem ersten Schritt gilt es zunächst, das Ausmaß der Problemwahrnehmung zu ermitteln. Dies ist möglich, indem man für jeden der 182 Befragten den Eindruck hinsichtlich der derzeitigen Erfuillung bestimmter Informationsanforderungen der individuell empfundenen Wichtigkeit dieser Anforderungen gegenüberstellt. Beide Einstellungskomponenten wurden in der empirischen Erhebung mit Hilfe von bipolaren Ratingskalen gemessen. 1 Die Punktwerte der einzelnen Items wurden dabei addiert und durch die Itemzahl dividiert.

1 Zur genauen Fragestellung vgl. Fragen $6 a$ bis $6 \mathrm{c}$ des Fragebogens in Anhang 3, S. 289-291 dieser Arbeit. 


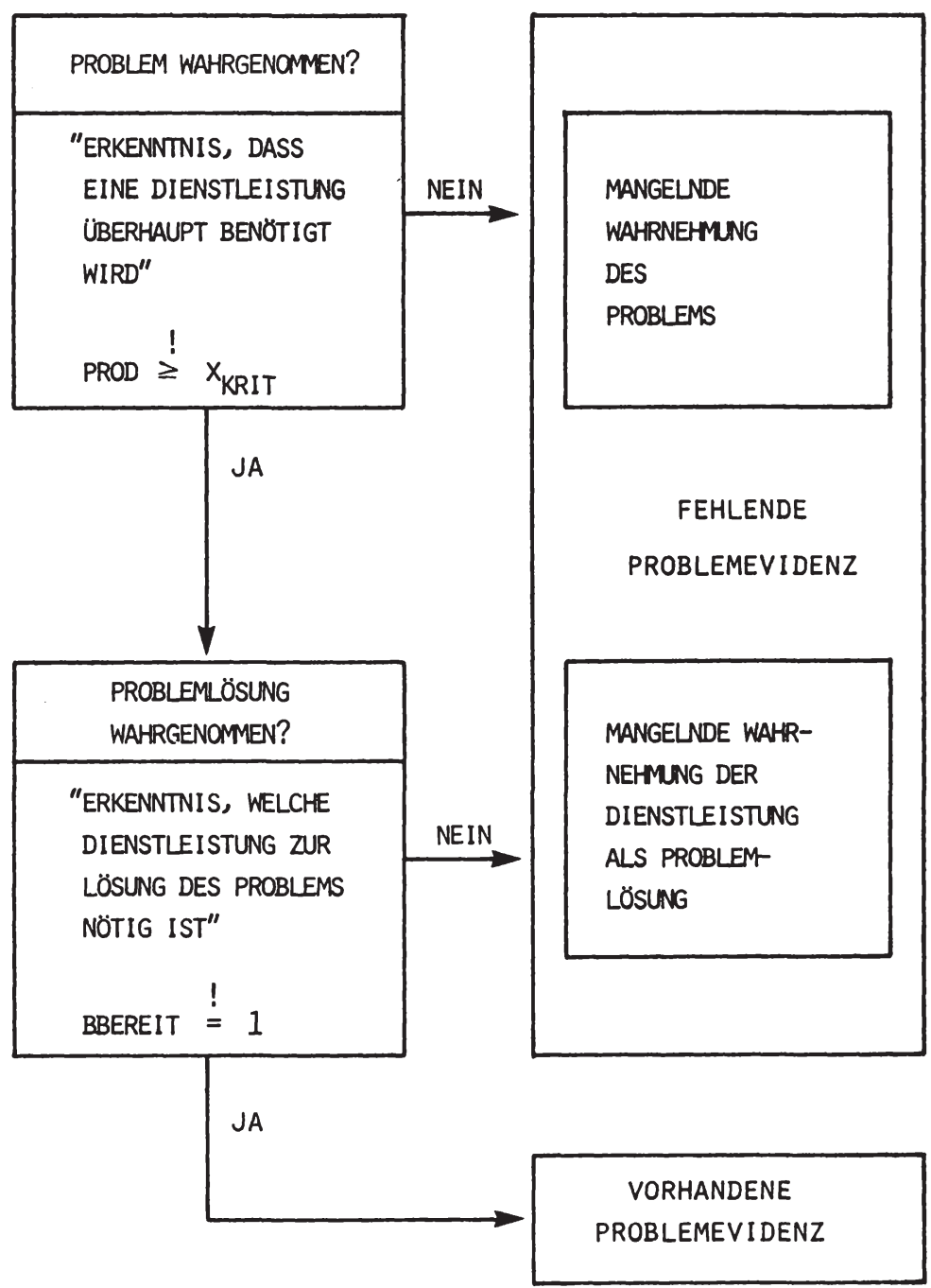

Abb. 51: Operationalisierte Abgrenzung der Problemevidenz 
Tabelle 13 gibt das Ergebnis der kreuztabellarischen Gegenüberstellung der so aggregierten Einstellungskomponenten wieder. ${ }^{1}$ Unterstellt man, daß das notwendige Ausma $B$ an Problemwahrnehmung mindestens einen Punktwert von 12 aufweisen muB (vgl. auch Abbildung 49), so ergibt sich, daß 84 der 182 Befragten über ein für das Vorhandensein von Problemevidenz notwendiges Problembewußtsein verfügen.

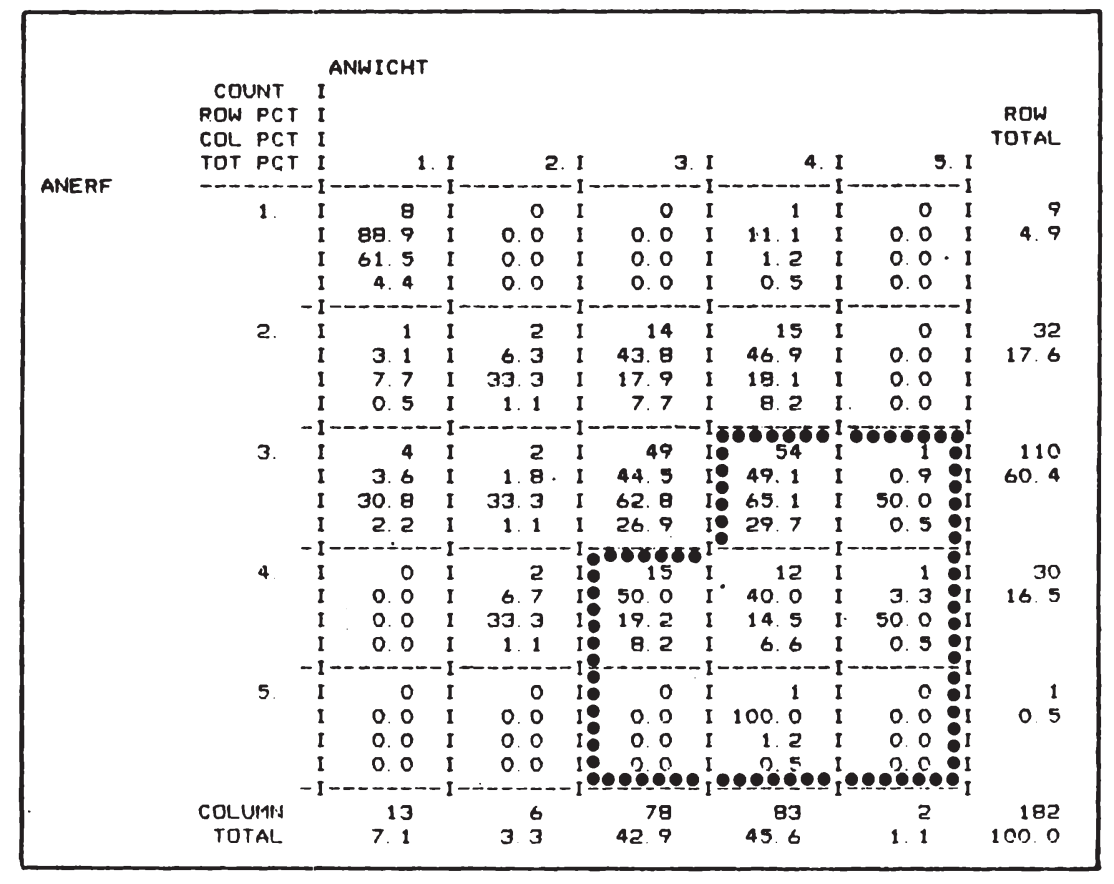

Tab. 13: Kreuztabellarische Gegenüberstellung der Wichtigkeit (ANWICHT) und des Erfüllungsgrades (ANERF) von Anforderungen an die Versorgung mit Brancheninformationen

1 Die Kreuztabulierung erfolgte mit dem SPSS-Programm "CROSSTABS". 
Der zweite Abgrenzungsschritt unterscheidet diese $84 \mathrm{Ab}-$ nehmer weiter nach Maßgabe ihrer grundsätzlichen Bereitschaft zur Nutzung eines elektronischen Brancheninformationsdienstes (i.e. Dienstleistungsidentifikation).

Tabelle 14 gibt das Ergebnis wieder ${ }^{1}$ und zeigt gleichzeitig die mengenmäBige Besetzung der in Abbildung 50 dargestellten Quadranten auf.

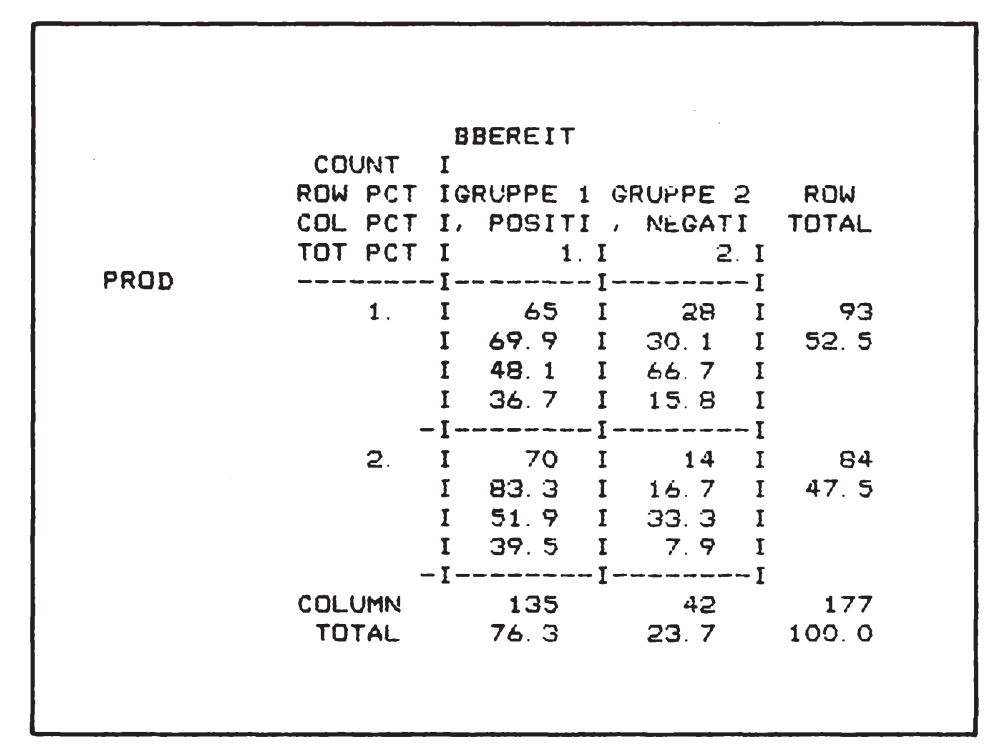

Tab. 14: Kreuztabellarische Gegenüberstellung der Wahrnehmung eines Informationsversorgungsproblems (PROD) und der Bereitschaft, einen elektronischen Branchen informationsdienst in Anspruch zu nehmen (Dienstleistungsidentifikation: BBEREIT)

1 Die Kreuztabulierung erfolgte ebenfalls mit dem SPSSProgramm "CROSSTABS". 
Es wird deutlich, daB für lediglich 70 von 177 Abnehmern $(39,5 \%)$ elektronische Brancheninformationsdienste problemevident sind. ${ }^{1} 65$ potentiellen Abnehmern $(36,7 \%)$ mangelt es an der ausreichenden Problemwahrnehmung, 14 Nachfragern fehlt es an der ausreichenden Dienstleistungskenntnis, für 28 Abnehmer $(15,8 \%)$ gilt schlieblich beides.

Insgesamt ist diesen Zahlen zu entnehmen, da $\beta$

- der zentrale Grund für die Ermangelung von Problemevidenz offenbar im Fehlen einer ausreichenden Problemwahrnehmung zu suchen ist und $d a B$

- eine bemerkenswert hohe Zahl von Abnehmern die Dienstleistung als geeignete Problemlösung identifiziert hat, ohne daB die sich darin zeigende Aufgeschlossenheit auf einen besonderen Bedarf zurückzuführen ist.

Zusammenfassend kann festgestellt werden, da $B$ die vorgeschlagene operationalisierung der Problemevidenz in bezug auf elektronische Informationsdienste zu plausiblen Ergebnissen führt. ${ }^{2}$ Dennoch erscheint es angezeigt, bei der Zugrundelegung dieses Konstrukts im Rahmen der Abnehmersegmentierung die Prämissen und Grenzen der Problemevidenzanalyse klar herauszustreichen.

1 Dieser Wert enthält insofern einen Bias, als durch die Darstellung der Dienstleistung ( $v g l$. Fragebogen in Anhang 3, S. 290) dem Befragten der Nutzen dieser Dienstleistung relativ stark suggeriert wurde. Tatsächlich ist ein geringerer Wert für die Zahl derer, denen elektronische Informationsdienste problemevident sind, realistischer.

2 Gleichwohl erscheint es in diesem Zusammenhang verfrüht, von einer Validität des Problemevidenz-Konstrukts zu sprechen. Zur Problematik der Beurteilung der Konstruktvalidität vgl. vor allem Breitung, A., Kritische Analyse der Validitätsproblematik von Verfahren der Einstellungsmessung. Ein methodologischer Beitrag zum Design von Einsteliungsskalierungen, in: Meffert, H., Steffenhagen, H., Freter, H., a.a.0., S. $209 \mathrm{ff.}$, hier insbes. S. 212. 


\subsection{Leistungsfähigkeit und Grenzen des Konzepts der} Problemevidenz

Die Beurteilung der Tauglichkeit der Problemevidenz als Kriterium zur Unterscheidung ausgewählter Abnehmergruppen hat an zweierlei Maßstäben anzusetzen:

- Realitätsgehalt des Problemevidenzkonstrukts,

- Aussagewert der Problemevidenz für die Marktsegmentierung.

Die Oberprüfung des Realitätsgehaltes dient dem Zweck, festzustellen, in welchem Umfang das die Problemevidenz abbildende Modell die Wirklichkeit entspricht. Mit dem Aussagewert der Problemevidenz für die Marktsegmentierung ist demgegenüber die Frage verbunden, inwieweit die Problemevidenz die relevanten Anforderungskriterien an die Marktsegmentierung erfüllt.

\subsection{Realitätsgehalt des Problemevidenzkonstrukts}

Die Wirklichkeitsnähe der Problemevidenz bestimmt sich aus dem Grad, in welchem die bei der Operationalisierung gesetzten Prämissen in der Realität erfuillt werden. Ausgehend von der zugrunde gelegten Art der Messung und der Verknüpfung der Modellkomponenten sind folgende Prämissen des Problemevidenzkonstrukts zu prüfen: ${ }^{1}$

\section{a. Multiplikativitätsprämisse}

Die Multiplikation von Eigenschaftsbedeutung (Gewichtung einzelner Informationsanforderungen) und subjektiv wahrgenommener, objektspezifischer Eigenschaftsausprägung (Erfüllungsgrad der Anforderungen) setzt Unabhängigkeit dieser beiden Elemente voraus. Sonst kommt es zu Verzerrungen.

$1 \mathrm{Vgl}$. zum folgenden vor allem Freter, H., Einstellungsmodelle, a.a.0., S. 166 f.. 
b. Additivitätsprämisse

Die Addition der gewichteten Eindruckswerte impliziert, da $B$ die Beurteilung der einzelnen Anforderungen unabhängig voneinander erfolgt.

\section{c. Linearitätsprämisse}

Die Ermittlung der Problemwahrnehmung folgt einem linearen Modell. Das bedeutet, daß bei einer Anderung einer Anforderungsausprägung um eine Einheit sich die Gesamtproblemwahrnehmung proportional verändert.

\section{d. Kompensationsprämisse}

In seiner ersten Stufe ist das Modell der Problemevidenz kompensatorisch gestaltet, $d$. h. eine niedrige Problemwahrnehmung bei einer Informationsanforderung kann durch eine hohe Problemwahrnehmung bei einer anderen Informationsanforderung kompensiert werden.

Die empirische Oberprüfung dieser Prämissen am Beispiel der Problemevidenz eines elektronischen Brancheninformationsdienstes zeigt, daß die Multiplikativitätsprämisse weitgehend der Wirklichkeit entspricht. Mit Hilfe der Korrelationsanalyse konnte im Rahmen der vorliegenden empirischen Untersuchung nachgewiesen werden, daß der Grad, in dem die Befragten bestimmte Anforderungen an die Informationsversorgung als erfillt beurteilten, weitgehend unabhängig von der Wichtigkeit war, die sie diesen Anforderungen zuwiesen. Lediglich bei zwei Anforderungskriterien ("Exklusivität" und "EDV-Verarbeitbarkeit") zeigten sich signifikante Beziehungen zwischen dem jeweiligen Bedeutungsgehalt und dem Erfuillungsgrad.

Demgegenuiber ergaben tiefergehende Analysen, daB die Additivitätsprämisse eine wenig realistische Bedingung unterstellt. So machte die Korrelationsarialyse deutlich, daß die Eindrücke hinsichtlich der Erfüllung einzelner Informationsanforjerungen $z$. T. hoch untereinander korrelieren, d. $h$. es herrscht unter den befragten Unternehmen entweder ein tendenziell positiver oder 
ein tendenziell negativer Gesamteindruck hinsichtlich der Informationsversorgung vor. Während über die Realitätsnähe der Linearitätsprämisse keine konkreten Aussagen möglich sind, spricht vieles dafür, daß die Kompensationsprämisse in bezug auf elektronische Informationsdienste sehr kritisch zu betrachten ist. So ist zu vermuten, daß beispielsweise die mangelnde Genauigkeit bestimmter Informationen sich nicht oder nur sehr begrenzt durch die hohe Schnelligkeit der Informationsbereitstellung kompensieren 1 äßt.

Insgesamt erfuilt das Konstrukt der Problemevidenz im vorliegenden Beispiel elektronischer Informationsdienste nur bedingt die Prämissen, die der Modellentwicklung zugrunde liegen. Insofern liefert es nur ein relativgrobes Abbild der Wirklichkeit. Gleichwohl erscheint seine Anwendung aus methodischer sicht als heuristische Entscheidungshilfe gegenüber der Unterlassensalternative dann als vorteilhaft, wenn folgende Anforderungen erfuillt werden: ${ }^{1}$

- Gegenstand der Beurteilung sind jeweils die subjektiven Wahrnehmungsdimensionen und nicht direkt meßbare, objektive Eigenschaften.

- Die Eigenschaften (hier Anforderungskriterien) sind unabhängig voneinander. ${ }^{2}$

1 Vgl.dazu z. B. Bass, F.M., Wilkie, W.L., A Comparative Analysis of Attitutional Predictions of Brand Preference, in: JoMR, Vol. 10, 1973, S. 262 ff.; Freter, H., Einstellungsmodelle, a.a.0., S. 172.

2 Die Unabhängigkeit der Eigenschaften (Anforderungskriterien) konnte in der vorliegenden empirischen Untersuchung im Wege der Faktorenanalyse nachgewiesen werden. Vgl. hierzu Punkt 3.2211, S. 124 dieser Arbeit. 


\subsection{Aussagewert der Problemevidenz für die ilarktsegmen- tierung}

Die Anwendung des Problemevidenzkriteriums für die Unterscheidung von Abnehmergruppen im Rahmen der Marktbearbeitung kann nur dann sinnvoll sein, wenn die zentralen Anforderungen an die Marktsegmentierung in hinreichendem Umfang erfuillt sind. 1

Die Forderung nach Kaufverhaltensrelevanz verlangt, daB die Problemevidenz als Segmentierungskriterium möglichst enge Beziehungen zu den Bestimmungsfaktoren des Kaufverhaltens potentieller Dienstleistungsabnehmer hat. Diese Beziehungen sind angesichts der zentralen Bedeutung der jeweiligen Problembewußtseinslage für den Ablauf des Entscheidungsprozesses ohne Zweifel gegeben. ${ }^{2}$ So wird ein Nachfrager in einer Situation vorhandener Problemevidenz seinen KaufprozeB relativ einfach gestalten; in Abhängigkeit von der Art und/oder dem finanziellen Wert der Dienstleistung wird er U. U. ein habituelles Kaufverhalten an den Tag legen ${ }^{3}$ (z. B. bei der Erteilung kleinerer Reparatur- oder Transportaufträge durch den Fuhrparkleiter). Demgegenüber hat eine Situation fehlender Problemevidenz zur Konsequenz, daB entweder der potentielle $A b-$ nehmer gar nicht erst als Nachfrager auftritt oder aber daB der Nachfrager, nicht wissend, welche Dienstleistung seine Probleme zu lösen vermag, entsprechend unsicher in seinem Kaufverhalten ist. 4

$1 \mathrm{Vgl}$. hierzu S. 60 dieser Arbeit.

2 Vgl. vor allem Engelhardt, W.H., Schwab, W., a.a.0., S. 508 .

$3 \mathrm{Vgl}$. ebenda, S. 509.

$4 \mathrm{Vgl}$. ebenda, S. $510 \mathrm{f}$. 
Insgesamt kann damit mit hoher Plausibilität von der Kaufverhaltensrelevanz der Problemevidenz ausgegangen werden. Demzufolge ist es auch möglich, Ansatzpunkte für den differenzierten Einsatz der Marketinginstrumente abzuleiten. In diesem Sinne trägt das Problemevidenzkriterium wesentlich dazu bei, im Rahmen der Marktbearbeitung den verschiedenen unternehmensspezifischen Entscheidungsvoraussetzungen und Kaufverhaltensweisen Rechnung zu tragen.

Das Kriterium der Zugänglichkeit stellt auf das Ausma $a b$, in dem eine Unternehmung ihre Marketinganstrengungen über Kommunikations- und Distributionskanäle auf die einzelnen, zuvor unterschiedenen segmente ausrichten kann. 1 In diesem Punkt zeigt sich relativ offenkundig die besondere Schwachstelle des Problemevidenzkonstrukts. So ist es faktisch nicht möglich, Unternehmen nach Maßgabe der vorhandenen Problemevidenz gezielt oder gar isoliert anzusprechen. In dieser Situation hat der Dienstleistungsanbieter nur die Möglichkeit, bestimmte unternehmensdemographische Merkmale als Hilfskriterien heranzuziehen, die es ihm erlauben, potentielle Abnehmer zumindest näherungsweise in Abhängigkeit vom Grad der Problemevidenz zu erreichen. Als ein solches Kriterium könnte beispielsweise die Unternehmensgröße fungieren. 2

Verzichtet die Anbieterunternehmung auf diese approximative Steuerung der Marktbearbeitungsaktivitäten, so bleibt ihr nur die Möglichkeit, die jeweiligen Marktbearbeitungs -

1 Vgl. Freter, H., Marktsegmentierung, a.a.0., S. 44.

2 Vgl. Engelhardt, W.H., Schwab, W., a.a.0., S. 508. Gleichwohl ist ein Rückgriff auf solche relativ groben Kriterien wenig befriedigend, wenn man berücksichtigt, daB im Rahmen der Marktwahl bereits eine wesentlich feinere Beschreibung der potentiellen Abnehmer vorgenommen wurde. 
programme breit zu streuen und auf die sog. Strategie der Kundenselbstselektion zu setzen. ${ }^{1}$

Die MeBbarkeit eines Segmentierungskriteriums impliziert die quantitative Erfaßbarkeit von Segmenten unter Zuhilfenahme der verfügbaren Marktforschungsmethoden. ${ }^{2}$ Grundsätzlich ist die Meßbarkeit der Problemevidenz bei einzelnen Nachfragern dann gewährleistet, wenn eine valide Form der Operationalisierung gefunden wird. In diesem Sinne kann - ausgehend von dem oben entwickelten operationalisierungsansatz für elektronische Informationsdienstleistungen - prinzipiell von einer dienstleistungs typspezifischen Erfïllung des MeBbarkeitskriteriums ausgegangen werden.

Das Kriterium der zeitlichen Stabilität fordert, daß die Problemevidenz über einen längeren Zeitraum hinweg ihre Aussagefähigkeit behält. ${ }^{3}$ Hinter dieser Forderung steht die Oberlegung, daß sowohl die Planung segmentspezifischer Marketingmaßnahmen als auch die Durchdringung der einzelnen Segmente zeit erfordern. Grundsätzlich ist in diesem

1 "Die Strategie der Selbstauswahl bzw. der passiven Segmentansprache geht von einem Zielsegment aus, das sich zwar iber bestimmte Kaufverhaltensweisen, nicht aber uber bestimmte unmittelbar beobachtbare Merkmale bezeichnen läßt. Da der Anbieter die jeweiligen Kaufverhaltensweisen (... L V Vorhandensein von Problemevidenz, Ergänzung des Verfassers /) nicht bestimmten Abnehmern im Markt zuordnen kann, ist er gezwungen, zunächst einmal einen größeren Teil des Marktes anzusprechen und damit bewuBt Streuverluste hinzunehmen. Dabei geht man davon aus, daB die Mitglieder des Zielsegments als Verhaltenssegment reagieren und das spezifisch gestaltete Marketingprogramm als "die" von ihnen präferierte Alternative erkennen, um dann in einen näheren kontakt zur Anbieterunternehmung zu treten". Gröne, A., a.a.0., S. $41 \mathrm{f}$..

$2 \mathrm{Vgl}$. Freter, H., Marktsegmentierung, a.a.0., S. 44.

$3 \mathrm{Vgl}$. ebenda. 
Zusammenhang festzustellen, daB sich die Problemevidenz einer Dienstleistung im Zeitablauf ändert. " "Tritt eine Schwierigkeit zum erstenmal auf, und ist sie dazu noch komplex, wird die Dienstleistungsevidenz i.d.R. geringer sein als bei wiederholt auftretenden Problemen. Im Zeitablauf nimmt das Erfahrungspotential des Nachfragers zu und somit seine Fähigkeit, Dienstleistungen als Instrument zur Lösung bestimmter Probleme zu kennen". 2

Strenggenommen wird die Problemevidenz dem Kriterium der zeitlichen Stabilität damit nicht gerecht. Es spricht jedoch vieles dafür, daß über die durchschnittliche Zeitdauer eines Marktbearbeitungsprogramms die Problemevidenz einer Dienstleistung in der Wahrnehmung einzelner Zielpersonen keine wesentlichen Veränderungen erfährt. Aus dieser Warte kann die zeitliche Stabilität als hinreichend angesehen werden.

"Segmentierungskriterien sollen Segmente abgrenzen helfen, deren Bearbeitung lohnt. Die sich aufgrund der Segmentierungsmaßnahmen ergebenden Kosten (Kosten der Informationsgewinnung und -verarbeitung sowie Kosten, die durch eine differenzierte Marktbearbeitung entstehen) muissen durch den Nutzen der Segmentierung (höhere Erlöse, sichere Marktposition) kompensiert werden". 3 Inwieweit das Problemevidenzkriterium in der Lage ist, diese ubergreifende Anforderung der Wirtschaftlichkeit zu gewährleisten, kann aus theoretischer Sicht nicht allgemeinguiltig und abschließend beantwortet werden. Praktische Erfahrungen mit der Anwendung dieses Kriteriums mussen hier erste verläBliche Hinweise liefern. Die enge Beziehung des Problemevidenzaspekts zum Kaufverhalten und zur Marktbearbeitung sprechen jedoch dafür, daß unter Zugrundelegung

1 Vgl. Engelhardt, W.H., Schwab, W., a.a.0., S. 507.

2 Ebenda.

3 Freter, H., Marktsegmentierung, a.a.0., S. 44. 
dieses Kriteriums die Effizienz der Marktbearbeitung sich wesentlich steigern läßt und daß somit von der Wirtschaftlichkeit des Segmentierungsansatzes ausgegangen werden kann.

\subsection{Zusammenfassende Bewertung des Problemevidenzkonzepts}

Abbildung 52 faBt die Einzelbewertungen der Problemevidenz in einer Gesamtschau zusammen. Als Fazit kann dabei festgehalten werden, daB sich mit der expliziten Einbeziehung des Problemevidenzaspekts die grundsätzliche Chance verbindet, die Maßnahmenplanung bei der Bearbeitung investiver Dienstleistungsmärkte nach einer sinnvollen Grundstruktur auszurichten. Die aus der Anwendung des Problemevidenzkriteriums resultierenden "objektivierten" Erkenntnisse erlauben es dabei, auf eine rein qualitativ-intuitive Form der Marktbearbeitung zu verzichten und den. PlanungsprozeB rationaler und zielgerechter zu gestalten.

Andererseits weist der Problemevidenz-Ansatz in der skizzierten form zwei zentrale Schwächen auf:

1. Das zugrunde gelegte Meßkonzept ist kompensatorisch gestaltet und spiegelt damit das Bild der Realität wenig treffend wider.

2. Die potentiellen Abnehmer sind nach Maßgabe der Problemevidenz nicht erfaßbar. Damit werden die Vorteile des relativ genauen Zugriffs auf die relevanten Abnehmer nicht genutzt, die aufgrund der Beschreibung der potentiellen Adopter im Rahmen der Marktwahl möglich gewesen wäre. 1

Wenn im folgenden trotzdem an dem Aspekt der Problemevidenz festgehalten werden soll, so deshalb, weil im Rahmen der Neueinfühung einer komplexen Dienstleistung die

$1 \mathrm{Vgl}$. vor allem Abbildung 41, S. 199 dieser Arbeit. 


\begin{tabular}{|c|c|c|c|}
\hline \multicolumn{2}{|c|}{$\begin{array}{l}\text { Realitätsgehalt des } \\
\text { Meßkonzepts }\end{array}$} & \multicolumn{2}{|c|}{$\begin{array}{l}\text { Aussagewert für die } \\
\text { Marktsegmentierung }\end{array}$} \\
\hline \multirow{2}{*}{$\begin{array}{l}\text { Multiplikativitäts- } \\
\text { prämisse (Unabhän- } \\
\text { gigkeit von Bedeu- } \\
\text { tung und Erfüllungs- } \\
\text { grad) }\end{array}$} & \multirow{2}{*}{$\begin{array}{l}\text { weitgehend } \\
\text { erfüllt }\end{array}$} & $\begin{array}{l}\text { Kaufverhaltens- } \\
\text { relevanz }\end{array}$ & gegeben \\
\hline & & $\begin{array}{l}\text { Marktbearbei- } \\
\text { tungsrelevanz }\end{array}$ & gegeben \\
\hline \multirow{2}{*}{$\begin{array}{l}\text { Additivitätsprä- } \\
\text { misse (Unabhängig- } \\
\text { keit von Eigen- } \\
\text { schaftsbewertungen } \\
\text { untereinander) }\end{array}$} & \multirow[t]{2}{*}{$\begin{array}{l}\text { in weiten } \\
\text { Teilen } \\
\text { nicht } \\
\text { erfiillt }\end{array}$} & Zugängl ichkeit & $\begin{array}{l}\text { nicht gegeben } \\
\text { (nur über Hilfskri- } \\
\text { terien oder im Wege } \\
\text { der Selbstselektion) }\end{array}$ \\
\hline & & Meßbarkeit & gegeben \\
\hline \multirow[t]{2}{*}{$\begin{array}{l}\text { Linearitäts- } \\
\text { prämisse }\end{array}$} & \multirow{2}{*}{$\begin{array}{l}\text { keine Aus- } \\
\text { sage mög- } \\
\text { lich, aber } \\
\text { allgemeine } \\
\text { Konvention }\end{array}$} & $\begin{array}{l}\text { zeitliche Stabi- } \\
\text { lität }\end{array}$ & $\begin{array}{l}\text { grundsätzlich nicht } \\
\text { gegeben, i.d.R. je- } \\
\text { doch hinreichend }\end{array}$ \\
\hline & & \multirow[t]{2}{*}{$\begin{array}{l}\text { Wirtschaftlich- } \\
\text { keit }\end{array}$} & \multirow[t]{2}{*}{ keine Aussage möglich } \\
\hline $\begin{array}{l}\text { Kompensations- } \\
\text { prämisse }\end{array}$ & $\begin{array}{l}\text { z. T. pro- } \\
\text { blematisch }\end{array}$ & & \\
\hline
\end{tabular}

Abb. 52: Bewertung des Problemevidenzkonzeptes in der Anwendung auf elektronische Informationsdienste

aus diesem Aspekt resultierenden zwei strategischen Stoßrichtungen der Marktbearbeitung das Spektrum des möglichen Instrumenteeinsatzes relativ umfassend und zielgenau widerspiegeln. Vor diesem Hintergrund sei im folgenden am Beispiel elektronischer Informationsdienste aufgezeigt, wie nach Maßgabe vorhandener bzw. fehlender Problemevidenz differenzierte Marktbearbeitungsstrategien entworfen und ein entsprechender Instrumenteeinsatz geplant werden kann. 
3. Bearbeitung ausgewählter Abnehmergruppen am Beispiel elektronischer Informationsdienste

3.1 Ableitung von Basisstrategien der Marktbearbeitung

Folgt man der These, daB das AusmaB der Problemevidenz einen entscheidenden Ansatzpunkt zur Bearbeitung einzelner Abnehmergruppen liefert, so können im Sinne einer idealtypischen Dichotomisierung aus dem Fehlen bzw. dem Vorhandensein von Problemevidenz situationsspezifische Basisstrategien der Marktbearbeitung abgeleitet werden. Ein solches Vorgehen hat den Vorzug, daB die einzelnen Abnehmergruppen auf relativ hohem Aggregationsniveau unterschieden werden können, gleichzeitig jedoch bereits zentrale gruppenspezifische Merkmale des Kaufverhaltens in den Marktbearbeitungsaktivitäten Berücksichtigung finden.

Im Einzelfall ist es bei diesem Planungsansatz u. U. nicht einmal erforderlich, die exakte stärke der Segmente "mit" bzw. "ohne Problemevidenz" empirisch zu erheben. Aus dem Innovationsgrad und der Komplexität der angebotenen Marktleistung können vielmehr bereits relativ zuverlässige Hinweise auf die jeweiligen evidenzspezifischen Abnehmerpotentiale abgeleitet werden. So bestätigte sich im Fall elektronischer Wirtschaftsinformationsdienste die Vermutung, daB nur eine Minderzahl von Unternehmen von vornherein diese Dienste als wesentliche $\mathrm{Hilfe}$ bei der Lösung ihrer Informationsprobleme erkennt. 1 Insofern hat das Schwergewicht zunächst auf der Entwicklung von Strategien für den Fall fehlender Problemevidenz zu liegen.

1 Vgl. die beispielhafte Quantifizierung der Problemevidenz eines Erancheninformationsdienstes auf $\mathrm{S} .233 \mathrm{ff}$. dieser Arbeit. 


\section{11 Strategien bei fehlender Problemevidenz}

Zentrale Zielsetzung der Marktbearbeitung im Falle fehlender Problemevidenz muß es sein, diese Problemevidenz herbeizuführen. Pointiert läßt sich die Zielrichtung mit dem Schlagwort "managing the evidence" ${ }^{1}$ umschreiben. Das Management der Dienstleistungsunternehmung hat dieser Zielrichtung folgend darauf abzuzielen, daß den relevanten Zielpersonen das Problemlösungspotential des Angebots in überzeugender Weise nahegebracht wird. Dabei gilt es, die bereits oben beschriebenen Ausgangslagen zu berücksichtigen:

a. Der potentielle Nachfrager erkennt gar nicht, daB er vor bestimmten Problemen steht, bei deren Lösung ihm die angebotene Dienstleistung helfen kann.

b. Der potentielle Nachfrager erkennt wohl, daB er bestimmte Probleme zu lösen hat und daB er hierzu unternehmensexterne Unterstützung benötigt, vermag jedoch nicht zu präzisieren, welche Dienstleistung ihm diese Unterstützung sichern kann.

Im Falle der Ausgangslage "a" muß es dem Dienstleistungsmanagement darum gehen, dem potentiellen Nachfrager die Problemsituation in seinem Unternehmen transparent zu machen. "Das wichtigste ist dabei die Verdeutlichung der Dienstleistung als Problemlösungsinstrument mit dem Ziel, Dienstleistungsbedarf bei diesem Unternehmen zu wecken".?

1 Berry, L.L., Service Marketing is Different, in: Business, May-June 1980, S. 24, zitiert in: Levitt, Th., Marketing intangible products and product intangibles, in: HBR, Vol. 59, May-June 1981, S. 101 (im folgenden zitiert als: Levitt, Th., Marketing intangible products).

2 Engelhardt, H.W., Schwab, W., a.a.0., S. 510. 
In bezug auf elektronische Informationsdienste erhält damit die Forderung Witte's nach der "Vitalisierung der Informationsnachfrage" eine neue Bedeutung. "Witte kommt als Ergebnis umfassender Experimente zum Informationsverhalten von Entscheidungsträgern zu dem SchluB, "daB ein Informationssystem nicht passiv auf seine Benutzung warten darf, sondern durch ein werbendes Nutzungsangebot aktiv werden muB. Sonst muB damit gerechnet werden, daB auch eine reichhaltig ausgerüstete Datenbank, die durch eine moderne Software objektiv leistungsfähig ist, doch tatsächlich an den Verhaltensbarrieren der 'Nichtbenutzer' scheitern und damit zum automatisierten Zahlenfriedhof degeneriert". 2

Erkennt der Nachfrager nun, daB er eine Dienstleistung benötigt, ohne jedoch sie präzisieren zu können, so wird die hieraus resultierende Unsicherheit tendenziell zum Verzicht auf die Inanspruchnahme der angebotenen Marktleistung führen. Diese Ausgangslage "b" ist dadurch gekennzeichnet, da $B$ der Dienstleistungsnachfrager oftmals nicht genau weiB, was er eigentlich will. ${ }^{3}$ Er hat insbesondere keine genauen Vorstellungen über die relevanten Qualitätsdimensionen der erforderlichen Dienstleistung und ist dadurch nicht imstande, die Leistungsmerkmale der angebotenen Dienstleistung zu würdigen.

In dieser Situation muB die Strategie des Anbieters vor allem auf eine Reduzierung der Problemunsicherheit abzielen. Dies gelingt in nicht unerheblichem MaBe bereits

1 Vgl. Witte, E., Informationsnachfrage, a.a.0., S. $839 \mathrm{ff.}$; derselbe, Entscheidungsprozesse, a.a.0., S. $74 \mathrm{ff}$.

2 Witte, E., Informationsnachfrage, a.a.0., S. 840.

$3 \mathrm{Vgl.Rathmell,} \mathrm{J.M.,} \mathrm{Marketing} \mathrm{in} \mathrm{the} \mathrm{Service} \mathrm{Sector,}$ Cambridge, Mass. 1974, S. $190 \mathrm{ff}$. Vgl. im gleichen Sinne Engelhardt, H.W., Schwab, W., a.a.0., S. 511. 
durch eine Problemverdeutlichung und -strukturierung. 1 Im Falle des elektronischen Informationsdienstes kann der Anbieter z. B. Typen von Informationsbedarfssituationen (Planung des Markteintritts in Auslandsmärkte, Beurteilung der Unternehmenssituation im Branchendurchschnitt, Planung längerfristiger Anlageinvestitionen) aufzeigen, für die die angebotene Dienstleistung jeweils spezifische Problemlösungen bereithält.

Darüber hinaus muB es dem Anbieter daran gelegen sein, die Leistungsfähigkeit der Dienstleistung dem Nachfrager transparent zu machen. Levitt spricht in diesem Zusammenhang von "making tangible the intangible". 2 Surrogate, die als Indikatoren der Dienstleistungsqualität dienen, sind vielfach das geeignetste Mittel, die mangelnde "Faßbarkeit" der Dienstleistung zu kompensieren. 3 Solche Surrogate können im Fall elektronischer Informationsdienste $z$. B. die Personen sein, die die Anfragen bearbeiten. Mehr noch spielt vermutlich der Eindruck eine Rolle, den das eingesetzte materielle Trägermedium beim Nachfrager erweckt.

So können z. B. Referenzrecherchen, auf Computerausdrucken dokumentiert, die Vorstellungen über die Leistungsfähigkeit des Dienstes wesentlich unterstützen.

Zusammenfassend hat die Marktbearbeitungsstrategie letztlich in zwei Schritten dem Fehlen von Problemevidenz Rechnung zu tragen ( $v g l$. Abbildung 53 ). In einem ersten

1 Vgl. Engelhardt, H.W., Schwab, W., a.a.0., S. 511.

2 Levitt, Th., Marketing intangible products, a.a.0., S. 101 .

$3 \mathrm{Vgl}$. Z. B. Schonberger, R.J., Purchasing Intangibles, in: JPMM, Vol. 16, 1980, Nr. 3, S. $25 \mathrm{ff}$.; Engelhardt, H.W., Schwab, W., a.a.0., S. 508. Zur Nutzungshäufigkeit von Informationsmöglichkeiten beim Kauf von Standard-Softwareprogrammen (Aufsätze in Fachzeitschriften, Forschungsberichte, Kataloge, Referenzl isten, Seminare etc.) vgl. Englert, G., Marketing von Standard-Anwendungssoftware, München 1977, S. 259. 
Schritt gilt es, die Aufmerksamkeit des Nachfragers auf das noch nicht hinreichend wahrgenommene Problem zu lenken und die Suche nach einer Problemlösung zu initialisieren. In einem zweiten Schritt kommt es dann darauf an, dem Nachfrager die angebotene Dienstleistung grundsätzlich als die geeignete Problemlösung deutlich zu machen. Keine Aussage ist in diesem Stadium darüber getroffen, ob der Nachfrager damit auch die Dienstleistung des spezifischen Anbieters gegenüber Konkurrenzangeboten präferiert. Diese Präferenz. zu schaffen ist zentrale Zielsetzung der Marktbearbeitung bei gegebener Problemevidenz.

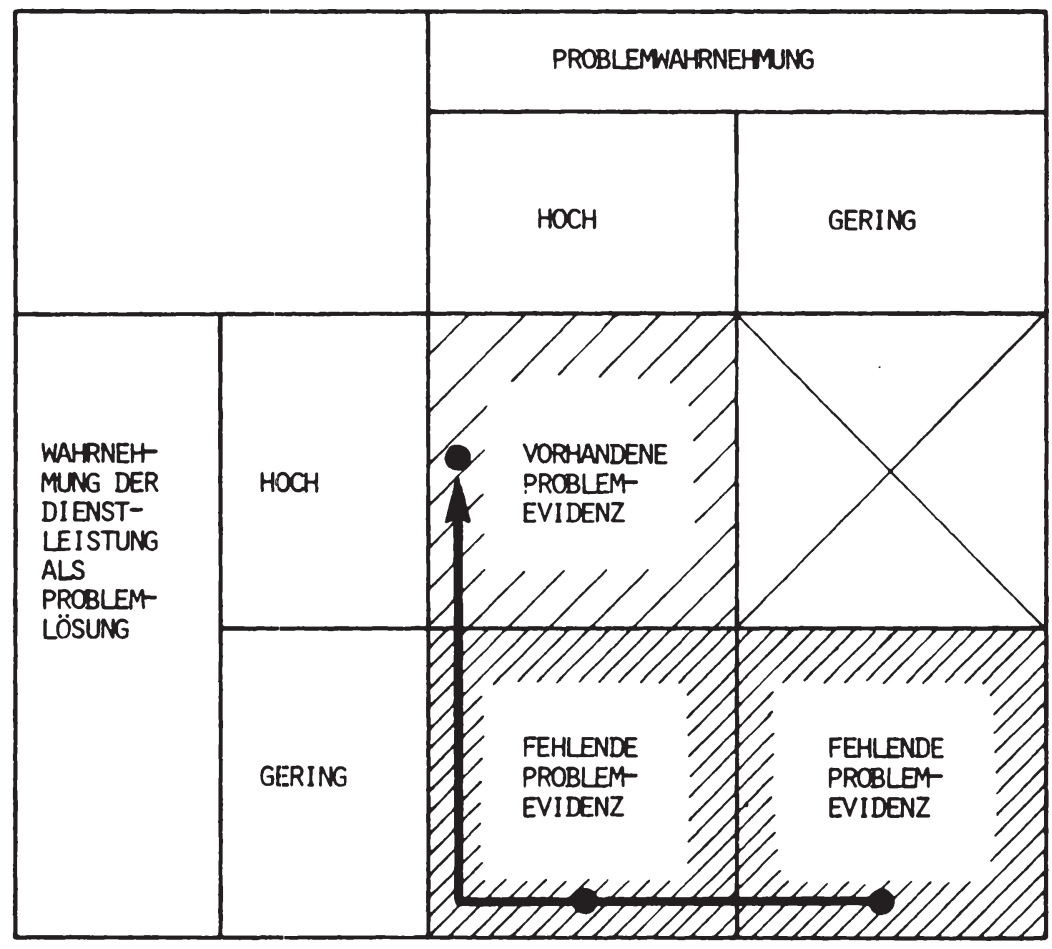

Abb. 53: Strategie der Marktbeeinflussung im Fall fehlender Problemevidenz 


\subsection{Strategien bei vorhandener Problemevidenz}

"Der Nachfrager, der die zur Lösung seines Problems geeignete Dienstleistung kennt, hält diese für abgrenzbar sowie spezifizierbar und fühlt sich in der Lage, eine qualitative Beurteilung der Dienstleistung, zumindest in Teilbereichen, vornehmen zu können". " Vor diesem Hintergrund muB die Marktbearbeitung durch den Anbieter zunächst und vor allem darauf abzielen, die Qualität seiner Dienstleistung überzeugend zu kommunizieren. Drei Komponenten der Qualität einer Dienstleistung gebührt hierbei besondere Beachtung: ${ }^{2}$

- die technische Qualität,

- die funktionale Qualität sowie

- die affektive Qualität.

Die technische Qualität der Dienstleistung stellt darauf $a b$, was der Abnehmer im Sinne einer grundsätzlichen Problemlösung erhält. Hier muB der Anbieter eines externen Datenbankdienstes den Nachfrager davon überzeugen, daB seine Dienstleistung die gewünschten Informationen beizubringen tatsächlich imstande ist. Die funktionale Qualität betrifft sodann das "wie" der Informationsbereitstellung. Kurze Antwortzeiten, eine benutzerfreundliche Suchsprache und fachliche Unterstützung durch geschultes Personal können zu einem hohen Niveau der funktionalen Qualität beitragen und damit als wichtiges Argument für die systemnutzung eingebracht werden.

SchlieBlich kommt auch der affektiven Komponente der Dienstleistungsqualität eine nicht zu unterschätzende Bedeutung zu. Die damit angesprochene gefühlsmäßige Einschätzung der Dienstleistung durch den potentiellen Abnehmer hängt wesentlich vom Image des Anbieters ab. "If

1 Engelhardt, H.W., Schwab, W., a.a.0., S. 508.

2. Vgl. zum folgenden vor allem Grönroos, Chr., Applied Theory II, a.a.0., S. $33 \mathrm{f}$.; derselbe, Strategic Management, a.a.0., S. $23 \mathrm{ff}$.. 
the image is unattractive, the customers may not even expose themselves to the marketing and operational activities of the firm, which, therefore, never will be allowed to prove its high technical and functional quality. Moreover, an outstanding image will be an excuse for minor problems in the other quality components".

Ist es gelungen, den Abnehmer von der Dienstleistungsqualität zu uberzeugen und findet in der Folge eine erste Interaktion zwischen Dienstleistungssystem und Nachfrager statt, so muß die Marktbearbeitungstrategie des Anbieters darauf ausgerichtet sein, diese Interaktion im Hinblick auf eine optimale Kundenzufriedenheit gezielt zu steuern und zu kontrollieren. Die generelle Zielrichtung dieser Verhaltensweise läBt sich mit dem Schlagwort "managing the interaction" umreiBen.

Abbildung 54 gibt das Beziehungsgefüge zwischen Kunden, Dienstleistung und Anbieter im Falle eines elektronischen Informationsdienstes wieder. Ausgehend von diesem Beziehungsgefüge muß es dem Anbieter daran gelegen sein, auf drei Interaktionen EinfluB zu nehmen: ${ }^{2}$

(1) Interaktion Abnehmer - Datenbank,

(2) Interaktion Abnehmer - Auskunftspersonal,

(3) Interaktion Abnehmer - Abnehmer.

Die Interaktion Abnehmer - Datenbank kann der Anbieter vor allem durch eine Strategie der Systemoptimierung positiv beeinflussen. Je reibungsloser der Informationsabruf sich gestaltet, je höher die Trefferquote der einzelnen Abfragen und je zuverlässiger die jederzeitige Zugänglichkeit des Systems gesichert ist (Minimierung der Ausfallzeiten), um so eher wird der Nachfrager auch zukünftig den Informationsdienst in Anspruch nehmen.

1 Grönroos, Chr., Applied Theory II, a.a.0., S. 33.

$2 \mathrm{Vgl}$. zum folgenden insbes. ebenda, S. $34 \mathrm{ff.}$ 


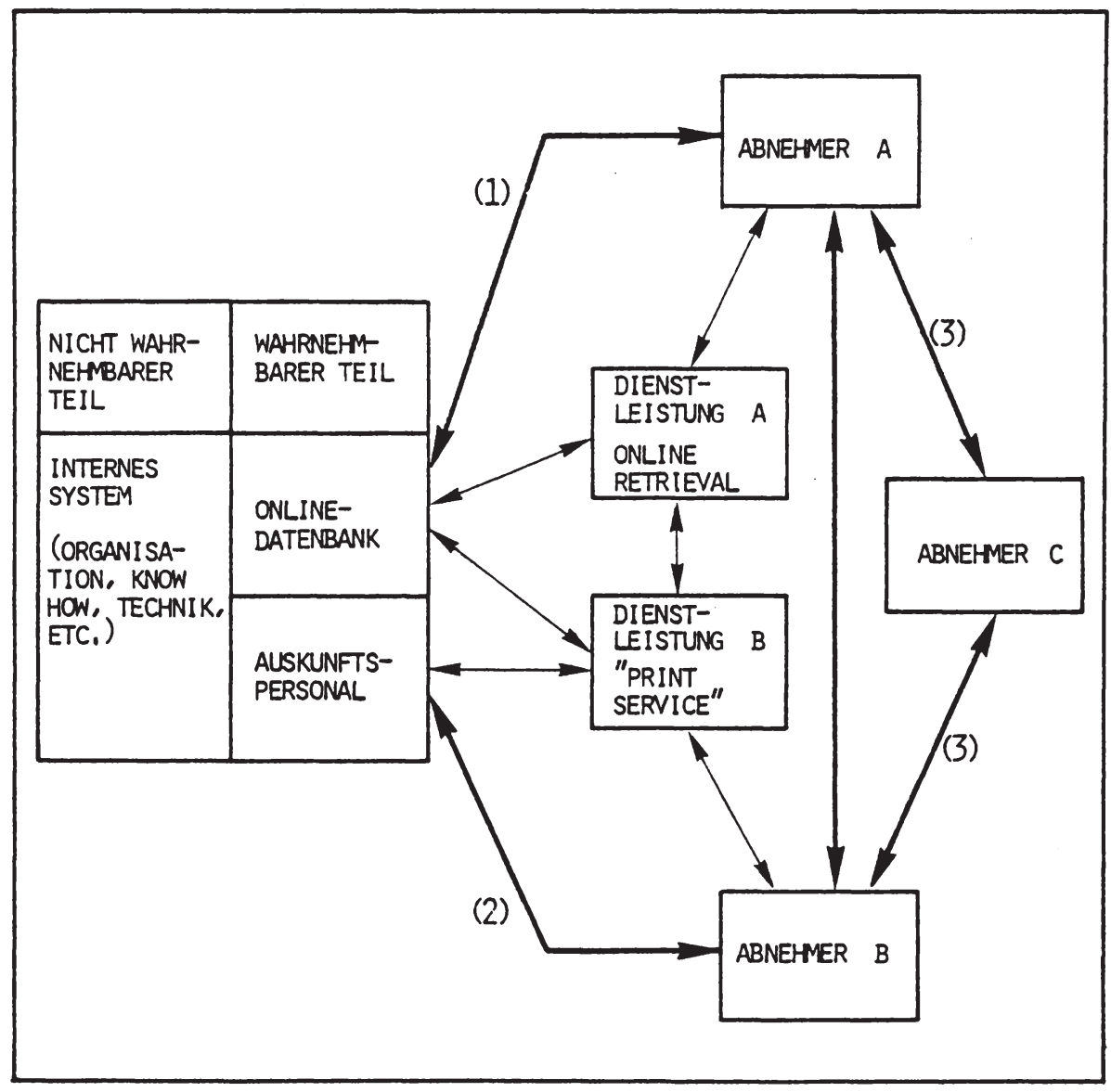

Abb. 54: Modell der Beziehungen im Servuctionssystem eines elektronischen Informationsdienstes

(Quelle: in Anlehnung an Eiglier, P., Langeard, E., Principes de politique marketing pour les enterprises de services, L'Institut d'Administration des Enterprises, Université d'Aix-Marseille, Dezember 1976, S. 11) 
Auf die Interaktion zwischen Abnehmer und Auskunftspersonal kann vor allem durch eine gezielte Schulung der eingesetzten Fachkräfte Einfluß genommen werden. Im Sinne einer umfassenden "Customer Relations"-Strategie muB es das Anliegen des Anbieters sein, auch die nicht-automatisierte Informationsversorgung (Print Service, Informationsberatung) auf einem hohen qualitativen Niveau zu halten.

Vielfach übersehen werden die Interaktionen zwischen den einzelnen aktuellen oder potentiellen Abnehmern, denen hinsichtlich der Durchsetzung der Dienstleistung am Markt oft eine zentrale Rolle zukommt. Das Ziel des Anbieters muB es in diesem Zusammenhang sein, die aktuellen Systemnutzer zur Weitervermittlung ihrer positiven Erfahrungen an andere Interessenten zu bewegen. Die Anwendung der Meinungsfürerstrategie liegt hier unmittelbar nahe. So kann das Unternehmen beispielsweise versuchen, insbesondere in der Einfihrungsphase die relevanten Fach- und Interessensverbände, Handelskammern $u$. ä. für seine Zwecke einzusetzen.

Zusammenfassend kommt es bei vorhandener Problemevidenz im Rahmen der Marktbearbeitung darauf an, die weitgehend "orientierten" Abnehmer von der Leistungsfähigkeit der angebotenen Dienstleistung zu uberzeugen und dauerhaft an die Unternehmung zu binden. Auf den Einsatz des hierfür geeigneten Marketinginstrumentariums sei im folgenden abschließend eingegangen. 


\subsection{Umsetzung der Basisstrategien durch Einsatz der Marketing-Matrix}

Für die Realisierung der situationsspezifischen Marktbearbeitungsstrategien steht dem Anbieter investiver Dienstleistungen ein breites Set von Einzelmaßnahmen zur Verfiugung. Klassischerweise versucht die Marketingwissenschaft, dieses Maßnahmenset als Summe der verfügbaren absatzpolitischen Aktionsparameter nach bestimmten Gesichtspunkten zu ordnen und gegeneinander abzugrenzen. 1 Eine Analyse der Marketingliteratur macht deutlich, daß sich bei diesen Bemühungen in den vergangenen zwei Dekaden nahezu durchgängig die "4P"-Systematik von McCarthy durchgesetzt hat. ${ }^{2}$ Diese Systematik sieht die Unterteilung der Marketinginstrumente in Produktvariablen (product), Plazierungsvariablen (place), Promotionsvariablen (promotion) und Preisvariablen (price) vor.

War dieser Instrumente-Katalog zunächst für den Konsumguiterbereich konzipiert, so wurde er aufgrund seiner Einfachheit und der Einprägsamkeit der verwendeten Terminologie mit oft nur geringfügigen Modifikationen auf den Investitionsgüterbereich ${ }^{3}$ und schlieBlich auch auf den Dienstleistungsbereich ${ }^{4}$ übertragen. Bei einer intensiveren Auseinandersetzung mit der besonderen Problemstellung

$1 \mathrm{Vgl.Z}$. B. Gutenberg, E., Grundlagen der Betriebswirtschaftslehre, 2. Bd.: Der Absatz, 16. Aufl., Berlin, etc. 1979, S. 123 ff.; Nieschlag, R., Dichtl, E., Hörschgen, H., Marketing. Ein entscheidungstheoretischer Ansatz, 11. Aufl., Berlin 1980, S. $122 \mathrm{ff}$.; Meffert, H., Marketing, a.a.0., S. $81 \mathrm{ff}$.

2 Vgl. McCarthy, J.E., Basic Marketing: A Managerial Approach, rev. ed., Homewood, I1l. 1964, S. $28 \mathrm{ff}$.

$3 \mathrm{Vgl}$. Z. B. Engelhardt, H.W., Ginther, B., a.a.0., insbes. S. $155 \mathrm{ff}$. und $166 \mathrm{ff}$.; Strothmann, K.H., Investitionsgutermarketing, München 1979, S. $111 \mathrm{ff}$. , insbes. S. $118 \mathrm{ff}$.

4 Vgl. z. B. Rathmell, J.M., a.a.0., S. $55 \mathrm{ff}$.; Scheuch, F., Dienstleistungsmarketing, a.a.0., S. $162 \mathrm{ff} . ;$ Hilke, W., a.a.0., S. $11 \mathrm{ff}$. 
des Dienstleistungsabsatzes zeigt es sich jedoch, daB der Vier-Instrumente-Katalog wenig geeignet ist, auf den Dienstleistungsbereich angewendet zu werden. 1 So gelingt es weder, das breite Spektrum der marktbeeinflussenden Variablen in erschöpfender Weise zu erschließen, noch liefert der Ansatz konkrete normative Hinweise auf die Gestaltung des Marketingmix.

Lovelock konstatiert insbesondere drei generelle Schwächen des 4P-Ansatzes: ${ }^{2}$ Seine irrefuihrende Semantik, seine Konzentration auf die Perspektive des Marktbeeinflussers statt auf die des zu Beeinflussenden sowie seine fehlende Einbeziehung des Konsumtions - und Dispositionsaspektes. Speziell in bezug auf den Bereich investive Dienstleistungen sind ergänzend folgende zentrale Schwächen hervorzuheben: ${ }^{3}$

- die Fiktion der physischen Bewegung der Gïter durch den Absatzkanal,

- die übergewichtige Konzentration der Beeinflussungsmaßnahmen auf die Phase vor, nicht jedoch während oder nach dem Kauf,

- die mangelnde Trennung von Kauf- und Eigentumsübertragungsaspekt.

$1 \mathrm{Vgl}$. zur Kritik an der 4P-Systematik der Marketinginstrumente in bezug auf den Dienstleistungsbereich vor allem Lovelock, Chr.H., Theoretical Contributions from Services and nonbusiness marketing, in: Conceptual And Theoretical Developments in Marketing, Hrsg.: Ferrell, 0.C., Brown, St.W., Lamb, C.W., Chicago, Ill. 1979, S. $147 \mathrm{ff}$, insbes. S. $159 \mathrm{ff}$. (im folgenden zitiert als: Lovelock, Chr., Contributions).

$2 \mathrm{Vgl}$. Lovelock, Chr., Contributions, a.a.0., S. $159 \mathrm{ff}$.

$3 \mathrm{Vgl}$. ebenda, S. 161: "After a while, one begins to tire of trying to force square application pegs into round conceptual holes and to look for something better". 
Die Summe der Kritikpunkte gibt AnlaB, nach einer neuen Instrumentesystematik zu suchen, die den Besonderheiten des investiven Dienstleistungsbereichs in angemessener Weise Rechnung trägt. 1 Als richtungweisender Ansatz soll in diesem Zusammenhang die Marketing-Matrix Lovelocks zur Anwendung gebracht werden. 2

\subsection{Struktur und Merkmale der Marketing-Matrix}

Ausgangspunkt für die Entwicklung der Marketing-Matrix bildet eine Systematik der zentralen Nutzenaspekte, die generell mit dem Kauf einer jeden Marktleistung untrennbar verbunden sind: ${ }^{3}$

- der physische Nutzen ("form utility"), der aus der physischen Ausgestaltung (Design, Farbe eines Fahrzeugs) sowie den physischen Auswirkungen der erworbenen bzw. in Ansprch genommenen Marktleistung resultiert (Lackierung eines Fahrzeugs);

- der psychische Nutzen ("psychic utility"), der sich in Form unterschiedlichster Affektionen einstellt, so z. B. Befriedigung, Genugtuung, Stolz, etc., der aber auch dadurch entsteht, daß eine Marktleistung leicht zu begreifen und ohne größere intellektuelle Anstrengungen in Anspruch genommen werden kann (Leichtigkeit der Handhabung eines Mikrocomputers).

1 Vgl. Lovelock, Chr., Contributions, a.a.0., S. 159.

$2 \mathrm{Vgl}$. ebenda, S. $161 \mathrm{ff}$.

$3 \mathrm{Vgl}$. ebenda, S. 155. Mit nahezu identischen Begriffen kategorisiert Kaufmann die "Gestaltungsmittel" des investiven Dienstleistungsmarketing ("Material" - das einen physischen Nutzen vermittelt, "Personen" und "Zeichen" - von denen ein psychischer Nutzen ausgeht, "Zeit" und "Ort"). Vgl. Kaufmann, E.J., a.a.O., S. $133 \mathrm{ff}$. 
- der räumliche Nutzen ("place utility"), den der Abnehmer aus der Tatsache zieht, daß die Marktleistung an einem inm genehmen ort erworben bzw. genutzt werden kann, sowie der Nutzen, durch die Marktleistung Platz zu sparen;

- der zeitliche Nutzen ("time utility"), der darin besteht, die Marktleistung zur gewïnschten Zeit kaufen oder nutzen zu können, sowie der Nutzen, der aus einer durch die Leistung bewirkten Zeitersparnis resultiert sowie

- der monetäre Nutzen ("monetary utility"), der z. B. mögliche finanzielle Gewinnaussichten umfaBt (Lottogewinn), der U. U. auch in Form geringer Betriebskosten oder eines hohen WiederveräuBerungswertes (Pkw) zutage tritt.

Auf jeden dieser Nutzenfaktoren kann der Anbieter durch den Einsatz dreier zentraler Marketingparameter einwirken: 1

- die Gestaltung des Angebots,

- die Gestaltung des Preises sowie

- die Gestaltung der Kommunikation.

1 Vgl. Lovelock, Chr., Contributions, a.a.0., S. $161 \mathrm{ff}$. Die Aussparung des Marketingparameters "Distribution" ist insofern konsequent, als es eine physische Distribution nicht bei jeder Angebotskategorie gibt. Die distributionspolitischen Aufgaben bei investiven Dienstleistungen allgemein liegen 2 . B. in der Reduzierung der räumlichen Distanz zwischen Dienstleistungsanbieter und -nachfrager. Diese Aufgabe wird jedoch im Sinne eines "place utility" durch die Dienstleistung selbst erbracht. Das Zweigstellennetz einer Bank, Filialen einer Fast-Food-Kette bzw. die Online-Verfügbarkeit eines Informationsdienstes sind Beispiele für solche "place utilities", die in sich Merkmale der alternativen Dienstleistungsangebote verkörpern. Vgl. hierzu auch Abbildung 54 auf S. 266 dieser Arbeit. 
Stellt man nunmehr die einzelnen Marketingparameter den fünf Nutzenkomponenten gegenüber, so erhält man als Ergebnis die von Lovelock vorgeschlagene Marketing-Matrix. ${ }^{1}$ Bezogen auf das Marketing elektronischer Informationsdienste ist die Matrix - beispielhaft für den Gesamtbereich investiver Dienstleistungen - wie folgt zu interpretieren (vgl. Abbildung 55):

Im Rahmen der Angebotsgestaltung (1. Zeile) geht es darum, den aus der Inanspruchnahme des Informationsdienstes resultierenden physischen, psychischen, räumlichen, zeitlichen und monetären Nutzen des Abnehmers zu maximieren. Hierzu gehört es, daB die Datenbank inhaltlich und von der Gestaltung der Outputs her den Erwartungen des Nachfragers entspricht, daB die Nutzung des Systems das Gefühl der Informationssicherheit vermittelt, daß das System von jedem ort aus und zu jeder stunde erreichbar ist, und daB effektive Einsparungen bei der Informationsbeschaffung erzielt werden.

Demgegenüber gilt es, im Rahmen der - im weitesten sinne zu verstehenden - Preisgestaltung (2. Zeile) die mit der Inanspruchnahme des Informationsdienstes verbundenen Kosten ("disutilities") des Abnehmers zu minimieren. Hierzu gehört es wiederum, angemessene Zahlungsmodalitäten anzubieten (physischer (form-bezogener) Nutzen), Frustrationen der Datenbanknutzung zu vermeiden (psychischer Nutzen), Kosten des Raums und der Raumüberbrückung zu minimieren (Kostenbeteiligung bei Anschaffung eines Terminals, DIN A4-Verkleinerung von Computer-outputs), zeitbedingte Kosten (Obertragungsgebuihren der Bundespost) durch kurze Log-on- und Responsezeiten gering zu halten sowie die wirtschaftliche Vertretbarkeit der systemnutzung durch die Forderung angemessener Preise sicherzustellen.

1 Vgl. Lovelock, Chr., Contributions, a.a.0., S. 162. 


\begin{tabular}{|c|c|c|c|c|c|}
\hline \multirow[b]{2}{*}{$\begin{array}{l}\text { MARKETING- } \\
\text { PARAMETER }\end{array}$} & \multicolumn{5}{|c|}{ ABNEMERNUTIEN } \\
\hline & $\begin{array}{l}\text { PHYSISCCER } \\
\text { NUTZEN }\end{array}$ & $\begin{array}{l}\text { PSYCHISCHER } \\
\text { NUTZEN }\end{array}$ & $\begin{array}{l}\text { RALUMICAER } \\
\text { NUTZEN }\end{array}$ & $\begin{array}{l}\text { ZEITLIOAER } \\
\text { MUTZEN }\end{array}$ & $\begin{array}{l}\text { MONETÄRER } \\
\text { MUIZEN }\end{array}$ \\
\hline ANGEBOT & 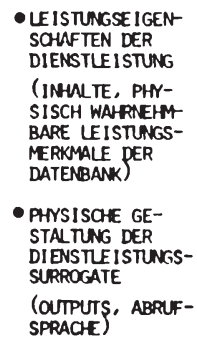 & 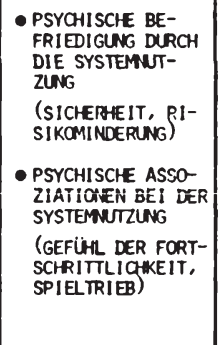 & $\begin{array}{l}\text { ๑ORT DER SYSTEM } \\
\text { NUTZUNG } \\
\text { (ARBEITSSTẌTTE, } \\
\text { BURO) } \\
\text { - ORT DES ERUERBS } \\
\text { DER NUTZUNGSBE- } \\
\text { RECITIGUNG } \\
\text { (ARBEITSSTÄTTE, } \\
\text { BURO) }\end{array}$ & $\begin{array}{l}\text { - MUTZUNGSZEITEN } \\
\text { (24 STUNDEN) } \\
\text { - WARTEZEITEN } \\
\text { (ZEIT BIS ZIR } \\
\text { SYSTEMEEREIT- } \\
\text { SCHAT, LEIT BIS } \\
\text { ZUR INFOPMATIONS- } \\
\text { LIEFERLNG) }\end{array}$ & 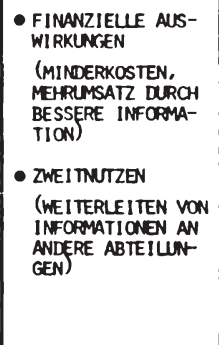 \\
\hline PREIS & $\begin{array}{l}\text { - FOPA DER ZAHLUNG } \\
\text { (ZEITLICHE PAU } \\
\text { SCHWL/KOSTENBE- } \\
\text { RECWUNG PRO AB- } \\
\text { RUF) } \\
\text { - KONDITIONENGE- } \\
\text { STAL TUNG } \\
\text { (ABRUFKOSTEN- } \\
\text { DEGRESSION) }\end{array}$ & $\begin{array}{l}\text { - PSYGHISCHE KO- } \\
\text { STEN } \\
\text { (FRUSTRAIION, } \\
\text { DISSONANZEN, BE- } \\
\text { LATENEE BIL- } \\
\text { SCHIPUARBEIT) } \\
\text { - PSYCHOLOGISCAE } \\
\text { KONDITIONENGE- } \\
\text { STAL TUNG } \\
\text { (GEBROCHENE PREI- } \\
\text { SE, GED-ZURUCK- } \\
\text { GARANIIE) }\end{array}$ & 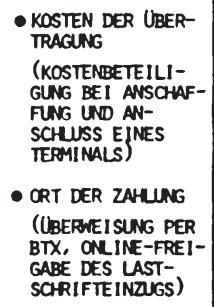 & 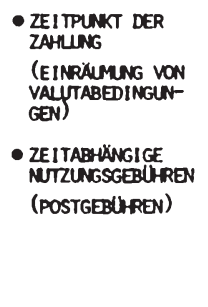 & $\begin{array}{l}\text { - ABSOWTE HÖHE DER } \\
\text { KOSTEN } \\
\text { - KOSTENERTEILUNG } \\
\text { AUF DIE PROFITIE- } \\
\text { RENDEN ABTEILUN- } \\
\text { GEN }\end{array}$ \\
\hline KOMMUNIIKATION & $\begin{array}{l}\text { DARSTELUNS DER } \\
\text { - LEISTUNGSFNHIG- } \\
\text { KEIT DES SYSTEMS } \\
\text { - MUTZUNGSMOOALI- } \\
\text { TÁTEN }\end{array}$ & $\begin{array}{l}\text { UBERZEUGEN VON DER } \\
\text { - FORTSCHRITLICH } \\
\text { KEIT DER SYSTEA } \\
\text { NUTZER } \\
\text { - RISIKOVERIINGE- } \\
\text { RUNG DURCH FUN } \\
\text { DIERTE INFOPAA- } \\
\text { TIONSGRLNDLGE. }\end{array}$ & 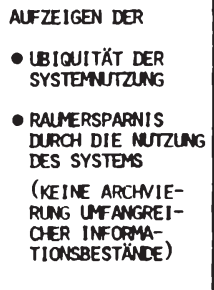 & $\begin{array}{l}\text { BETONUNG DER } \\
\text { - JEDERZEITIGEN } \\
\text { NUTZWNGSBEREIT- } \\
\text { SCAFT DES } \\
\text { SYSTENS } \\
\text { - ZEITERSPAFNIS } \\
\text { DURA DIE NUT- } \\
\text { ZNG DES SYSTES } \\
\text { (KEINE LANGWIE- } \\
\text { RIGE INFORMA- } \\
\text { TIONSSUOHE) }\end{array}$ & $\begin{array}{l}\text { HINWEIS AUF DIE } \\
\text { - KOSTEN DER SYSTEH } \\
\text { NUTZUNG } \\
\text { - EINSPARUNGEN UND } \\
\text { GEWINGOWNCEN } \\
\text { DORCH DIE SYSTEA } \\
\text { NUTZWGG }\end{array}$ \\
\hline
\end{tabular}

Abb. 55: Marketing-Matrix für elektronische Wirtschafts informationsdienste 
Im Rahmen der Kommunikation (3. Zeile) geht es schlieBlich darum, die mit der Inanspruchnahme des Informationsdienstes verbundenen Nutzen und die relative Geringfügigkeit der Kosten überzeugend und umfassend darzustel1 en.

Welches konkrete Aussehen die Marketing-Matrix im Einzelfall annehmen kann, sei Gegenstand einer abschlieBenden Erörterung. Dabei ist getrennt auf die Fälle vorhandener bzw. fehlender Problemevidenz einzugehen.

\subsection{Marketing-Matrix bei fehlender Problemevidenz}

Die Bearbeitung von Abnehmersegmenten, für die elektronische Wirtschaftsinformationsdienste keine Problemevidenz besitzen, muB darauf ausgerichtet sein, diese $A b$ nehmersegmente gezielt an das für sie neuartige Marktangebot heranzuführen. Dabei gilt es, sowohl der möglichen Unwissenheit um die eigenen Informationsprobleme als auch der möglichen Unsicherheit hinsichtlich der mit der Inanspruchnahme des Iiformationsdienstes verbundenen Nutzen- und Risikogesichtspunkte Rechnung zu tragen.

Eine beispielhafte Ausgestaltung der Marketing-Matrix zur Umsetzung dieser strategischen Vorgehensrichtung gibt Abbildung 56 a wieder. Grundsätzlich spricht jede der aufgeführten Einzeimaßnahmen für sich. Hervorzuheben ist jedoch die gemeinsame Zielsetzung aller Aktivitäten, die nämlich darin besteht, die generelle Zurückhaltung gegenuber elektronischen Informationsdiensten abzubauen. Dies gelingt vor allem darin, Produktwissen zu schaffen und die bestehende Bedarfsunsicherheit abzubauen. Bedeutsam ist darüber hinaus, daß die Abnehmerrisiken bei einer Erst-Nutzung des Informationsdienstes minimiert werden. 


\begin{tabular}{|c|c|c|c|c|c|}
\hline \multirow[b]{2}{*}{$\begin{array}{l}\text { MARKETING- } \\
\text { PARAMETER }\end{array}$} & \multicolumn{5}{|c|}{ ABNEHMERNUIZEN } \\
\hline & $\begin{array}{l}\text { PFYSISQHER } \\
\text { NUTZEN }\end{array}$ & $\begin{array}{l}\text { PSYCHISCHER } \\
\text { NUZZEN }\end{array}$ & $\begin{array}{l}\text { RALUMICHER } \\
\text { NUTZEN }\end{array}$ & $\begin{array}{l}\text { ZEITLICAER } \\
\text { NUTZEN }\end{array}$ & $\begin{array}{l}\text { MONETÄRER } \\
\text { NUTZEN }\end{array}$ \\
\hline ANGEBOT & $\begin{array}{l}\text { - INHALTLICH BE- } \\
\text { GRENZIE, GUT } \\
\text { STRUKTURIERTE } \\
\text { INFOPMATIONSBA- } \\
\text { SEN } \\
\text { - BENUTZBARKEIT } \\
\text { AUCH FIR DATEN } \\
\text { VERARBE ITUNGS- } \\
\text { LAIEN } \\
\text { - UBERSICHTICHE } \\
\text { OUTPUTGESTALTUNG }\end{array}$ & 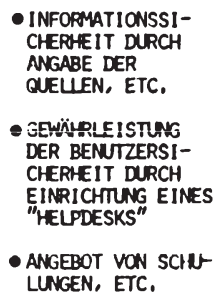 & $\begin{array}{l}\text { - EIIRICHTUNG RE- } \\
\text { GIONALER MUSTER- } \\
\text { INSTALLATIOEN } \\
\text { - VERSAND VON OUT- } \\
\text { PUTS WEEN KEIN } \\
\text { DRUCKER VORHAN } \\
\text { DEN) } \\
\text { - TELEFONISCHE BE- } \\
\text { RATUNG BEI } \\
\text { SYSTEMSCHWIERIG- } \\
\text { KEITEN }\end{array}$ & 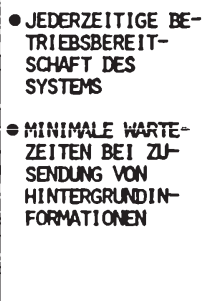 & $\begin{array}{l}\text { - REDUZIERUNG DES } \\
\text { PERSONALAUFWANDES } \\
\text { FUR DIE INFOFMA- } \\
\text { TIONSBESCHAFFUNG }\end{array}$ \\
\hline PREIS & $\begin{array}{l}\text { - PAUSCHALPREIS- } \\
\text { REGELUNG FUR DIE } \\
\text { ERSTEN X MONATE } \\
\text { DER NUZUNG }\end{array}$ & $\begin{array}{l}\text { - EINRALMUNG EINER } \\
\text { PARTIEUEN KO- } \\
\text { STENERSATIUNG, } \\
\text { WENW WENIGER ALS } \\
\text { Y BRAUCHBARE IN } \\
\text { FOPMATIONSEIN } \\
\text { HEITEN PRO Z RE- } \\
\text { CHERCHEN GELIE- } \\
\text { FERT WERDEN }\end{array}$ & $\begin{array}{l}\text { - KOSTENBETEILIGUNG } \\
\text { BEIM KAUF BZW, } \\
\text { LEASING ODER VER- } \\
\text { MIETUNG VON UBER- } \\
\text { TRAGUNGSTERMINALS }\end{array}$ & $\begin{array}{l}\text { - EINRAUMMNG WEITER } \\
\text { ZAHUNGSZIELE } \\
\text { (X MONATE NACH } \\
\text { VERTRAGSAB- } \\
\text { SCHUSS) }\end{array}$ & $\begin{array}{l}\text { - MODERATE PREIS- } \\
\text { FORDERUNG } \\
\text { - PREISAUSSCAREIBEN }\end{array}$ \\
\hline KOMMNIKATION & $\begin{array}{l}\text { - DARSTELUNG DER } \\
\text { NOTWENDIGKEIT UND } \\
\text { LEISTUNGSF ̈̈HIG- } \\
\text { KEIT DES SYSTEMS } \\
\text { (PROSPEKTE: } \\
\text { DIRECT MAIL ODER } \\
\text { ALS ZEEITSCIRIF- } \\
\text { TENBEILAGE) }\end{array}$ & $\begin{array}{l}\text { - AUFZEIGEN DES } \\
\text { STELUENWERTS GE- } \\
\text { EIGNETER INFOFMA- } \\
\text { TIONEN UND DER } \\
\text { GEFAREN, DIE AUS } \\
\text { INFOPMATIONSDEFI- } \\
\text { ZITEN RESULTIEREN } \\
\text { - VERMITTUNG DES } \\
\text { GEFUHLS DES "MIT } \\
\text { DER ZEIT-GEENS" }\end{array}$ & $\begin{array}{l}\text { - HINWEIS AUF ORTS- } \\
\text { UNGEBUNDENE NUTZ- } \\
\text { BARKEIT } \\
\text { (ZEITSCRIFIENAH- } \\
\text { ZEEGEN, PRODUKT- } \\
\text { DEMONSTRATIONEN) }\end{array}$ & $\begin{array}{l}\text { - HINWEIS AUF TA- } \\
\text { GESZEITUNABHLNGI- } \\
\text { GE NUTBAAREIT } \\
\text { (ZEITSCRRIFTENAH- } \\
\text { ZEIGEN, PRODUT- } \\
\text { DEMONSTRATIONEN) }\end{array}$ & $\begin{array}{l}\text { - HINWEIS AUF } \\
\text { MARKTCHANCEN, } \\
\text { DIE DURCH BESSERE } \\
\text { INFOPMAIONS- } \\
\text { GRUNDLAGEN GE- } \\
\text { SCHAFFEN WERDEN } \\
\text { KÖWNEN } \\
\text { (ERFOLGSSTORIES } \\
\text { IN WIRTSCHAFTS- } \\
\text { JOURULIEN) }\end{array}$ \\
\hline
\end{tabular}

Abb. 56a: Ausgestaltung der Marketing-Matrix für elektronische Informationsdienste im Fall fehlender Problemevidenz 


\subsection{Marketing-Matrix bei vorhandener Problemevidenz}

Kann der Anbieter elektronischer Wirtschaftsinformationsdienste von vorhandener Problemevidenz seiner Dienstleistung ausgehen, so muB der Einsatz seiner Marketing-Matrix vor allem darauf abzielen, den Kunden zur Inanspruchnahme seines Marktangebots zu bewegen und dauerhafte Kundenbeziehungen aufzubauen (vgl. Abbildung $56 \mathrm{~b}$ ). Dies gelingt insbesondere durch ein überzeugendes Leistungsangebot und Kontrahierungsbedingungen, die eine regelmäßige Nutzung des Dienstes fördern. SchlieBlich kommt auch der Kommunikation - in Sonderheit der Werbung - ein zentraler Stellenwert zu. "Ein werbendes - im Grenzfall sogar aggressives - Informationsangebot vitalisiert die Nachfrage sowohl hinsichtlich der Menge als auch hinsichtlich der Präzision". ${ }^{1}$ Als besonders wirksames Werbemittel bietet sich dabei die Streuung von Prospekten an, die zu jeweils abgegrenzten Sachbereichen sämtliche im Informationssystem vorhandenen Informationen anzeigt.

\section{3 Bewertung der Geschäftsfeldbearbeitung}

Zusammenfassend ist festzuhalten, daß eine erfolgversprechende Strategie der Marktbearbeitung für elektronische Informationsdienste sich jeweils am vorhandenen Grad der Problemevidenz dieses Dienstleistungstyps zu orientieren hat. Geht man davon aus, daß zum Zeitpunkt des Markteintritts bei der Mehrzahl der potentiellen Abnehmer die Problemevidenz noch wenig ausgeprägt ist, so hat dies zur Konsequenz, daß das anbietende Unternehmen sich zunächst auf die Strategie der Bedarfsweckung und der Dienstleistungsbekanntmachung konzentrieren sollte. Mit einer Zunahme der allgemeinen Problemevidenz - infolge eines auf breiter Front fortschreitenden Lernprozesses - liegt

1 Witte, E., Informationsnachfrage, a.a.0., S. 842 . 


\begin{tabular}{|c|c|c|c|c|c|}
\hline \multirow[b]{2}{*}{$\begin{array}{l}\text { MARKETING- } \\
\text { PARAMETER }\end{array}$} & \multicolumn{5}{|c|}{ ABNEMERANTZEN } \\
\hline & $\begin{array}{l}\text { PAYSISCAER } \\
\text { MUTZEN }\end{array}$ & $\begin{array}{l}\text { PSYOHISCHER } \\
\text { MUIZEN }\end{array}$ & $\begin{array}{l}\text { RÄLMLIOAER } \\
\text { NUTEN }\end{array}$ & $\begin{array}{l}\text { ZEITLICHER } \\
\text { NUTZEN }\end{array}$ & $\begin{array}{l}\text { MONETXRER } \\
\text { NUIZEN }\end{array}$ \\
\hline ANGEBOT & $\begin{array}{l}\text { - INWLTLICH TRANS- } \\
\text { PARENTES, UNFANG- } \\
\text { REICHES INFOAMA- } \\
\text { TIONSANGEBOT } \\
\text { - HINWEISE AUF } \\
\text { WEITERFUHRENDE } \\
\text { INFOPMATIONS- } \\
\text { OUEUEN }\end{array}$ & $\begin{array}{l}\text { - INFOPMATIONS- } \\
\text { SICHEATEIT DURCH } \\
\text { VOLSTANNDIGKEIT } \\
\text { DER INFORMA- } \\
\text { IIOAEN } \\
\text { - ANPASSUNGSF̈̈HIG- } \\
\text { KEIT AN BENUT- } \\
\text { ZERKENWNISISSE }\end{array}$ & 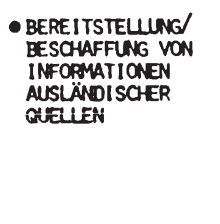 & $\begin{array}{l}\text { - MINIMIERUNG DER } \\
\text { SUCHZLIT DURCH } \\
\text { INTELIIGENTE } \\
\text { SUCHNERFAHREN }\end{array}$ & $\begin{array}{l}\text { - REDUZIERUNG DES } \\
\text { PERSONWLAUFWANDS } \\
\text { FUR DIE INFOPMA- } \\
\text { TIONSBESCHAFFUNG: } \\
\text { ERMÖGLICHNG } \\
\text { EINER EFFIZIEN } \\
\text { TEREN PLANUNG }\end{array}$ \\
\hline PREIS & $\begin{array}{l}\text { - PREIS PRO AH- } \\
\text { SOHLISS-STUNOE } \\
\text { - ABSCHLUSS VON } \\
\text { RAMENERTRÄGEN } \\
\text { - MONATLICHE } \\
\text { RECHERCHEKOSTEN- } \\
\text { UBERSICHIEN }\end{array}$ & $\begin{array}{l}\text { - SETZEN GEBRgCGE- } \\
\text { NER PREISE (Z.B. } \\
\text { DM } 39,90 \text { PRO ANt } \\
\text { SCHUUSS-STUNOE) }\end{array}$ & $\begin{array}{l}\text { - AUTOMATISCHER } \\
\text { GEBLHRENEINZIG } \\
\text { MAC FREIGABE } \\
\text { VIA TERMINAL }\end{array}$ & $\begin{array}{l}\text { - AUSGLEICH DER } \\
\text { POSTALISOISEN } \\
\text { VERBINOUNGSGE- } \\
\text { BUHREN DUROH } \\
\text { IEITICHE } \\
\text { RABATISTAFELW }\end{array}$ & $\begin{array}{l}\text { - ANGEMESSENE } \\
\text { PREI SFESTSETZUNG }\end{array}$ \\
\hline KOMMNIKATION & 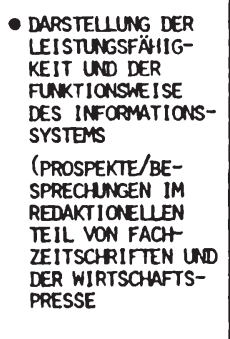 & 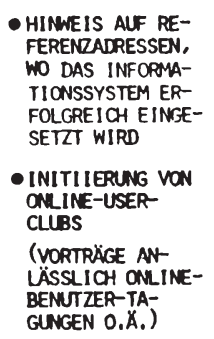 & $\begin{array}{l}\text { - HINWEIS ALF } \\
\text { RAUMERSPAPNIS } \\
\text { DURCH BEGRENZING } \\
\text { DER INFOPMA- } \\
\text { TIONSFUT } \\
\text { (ZEITSCRRIFTEN- } \\
\text { ANZEIGEN) }\end{array}$ & $\begin{array}{l}\text { - HINWEIS AUF } \\
\text { TEITERSPAPNIS } \\
\text { DUROH KARZE } \\
\text { RESPONSEAEITEN } \\
\text { (ZEITSCARIFTEN- } \\
\text { ANZEIGEN) }\end{array}$ & $\begin{array}{l}\text { - MUSTERRECHUNGEN } \\
\text { ZUM MACWEIS DER } \\
\text { EINSPARUNGEN BEI } \\
\text { NUTZNG DES IN } \\
\text { FORAMTIONSSY- } \\
\text { STEMS } \\
\text { (PROSPEKT) }\end{array}$ \\
\hline
\end{tabular}

Abb. 56b: Ausgestaltung der Marketing-Matrix für elektronische Informationsdienste im Fall vorhandener Problemevidenz 
es dann nahe, die Abnehmersegmente getrennt nach der jeweiligen Ausprägung der Problemevidenz zu bearbeiten. Langfristig wird sich das Unternehmen schlieblich primär auf eine Marktbearbeitungsstrategie stiutzen, die von der vorhandenen Problemevidenz ihrer Dienstleistung ausgeht. 


\section{Implikationen der Untersuchung}

Ausgehend von einem wenig befriedigenden Stand der theoretischen und empirischen Forschung im Bereich investiver Dienstleistungen wurde in der vorliegenden Arbeit versucht, das strategische Problem des Markteintritts in innovative Dienstleistungsmärkte aufzuarbeiten und Hinweise auf eine "optimale" Gestaltung des Marktwahlund Marktbearbeitungsprozesses zu liefern. Die Untersuchungsergebnisse sind in diesem Sinne sowohl für die Praxis des Marketing investiver Dienstleistungen als auch für die weiteren Forschungen im Bereich des strategischen Dienstleistungsmanagements relevant.

1. Zusammenfassende Wurdigung der Untersuchungsergebnisse

Unter Bezugnahme auf die eingangs formulierten Zielsetzungen der Untersuchung läBt sich das vorgelegte Konzept der Marktwahl und Marktbearbeitung abschlieBend würdigen.

(1) Die Gestaltung der Geschäftsfeldwahl als iterativer SelektionsprozeB hat sich grundsätzlich als zweckmäßig erwiesen. Durch die problemadäquate Zuordnung von Auswahlkriterien gelingt es, die Zerschneidung bestehender Interdependenzen zwischen Funktion, Technologie und Abnehmergruppen trotz der getrennten Analyse aller drei Aspekte zu vermeiden.

(2) Für die Auswahl der zu erbringenden Dienstleistungsfunktion konnten wichtige in der Literatur vorgeschlagene Kriterien der Geschäftsfeldwahl zur Anwendung gebracht werden. Daneben erwies es sich als zweckmäBig, die Auswahl der Technologie vor allem unter dem zentralen Aspekt der Aufgabenbezogenheit zu diskutieren. 
(3) Für die Auswahl geeigneter Abnehmergruppen konnte am Beispiel elektronischer Wirtschafts informationsdienste unter Zugrundelegung empirischer Untersuchungsergebnisse eine Reihe von Bestimmungsfaktoren herausgearbeitet werden, die sich für die grundsätzliche Nutzungsbereitschaft als erklärungsrelevant erwiesen haben.

(4) Die Entwicklung geeigneter Marktbearbeitungsstrategien stand im Zeichen des Problemevidenzaspektes. Die Operationalisierung dieses zentralen Bestimmungsfaktors der Beschaffung investiver Dienstleistungen erfolgte nach dem Muster multidimensionaler Einstellungsmodelle. Die beispielhafte Anwendung des zweistufigen Operationalisierungsansatzes auf elektronische Brancheninformationsdienste lieferte weitgehend plausible Ergebnisse.

(5) Die Kenntnis des vorhandenen AusmaBes der Problemevidenz versetzte schlieBlich in die Lage, konkrete Maßnahmen in bezug auf einzelne Abnehmergruppen zu formulieren. Hierbei bewährte sich die Einordnung der Einzelaktivitäten in eine Marketing-Matrix, die durch ihre Struktur explizit die einzelnen Nutzenaspekte der einzuführenden Dienstleistung berücksichtigt.

Zusammenfassend hat sich das Konzept der Marktwahl und Marktbearbeitung in seiner operationalisierten Form für das Marketing innovativer Investivdienstleistungen als tragfähiger Ansatz erwiesen. Die generalisierende Obertragung des am Beispiel elektronischer Informationsdienste dargestellten iterativen Planungsprozesses auf den gesamten Bereich investiver Dienstleistungen erlaubt es, fur die Praxis einige zentrale Hinweise zur Gestaltung des Markteintritts an die Hand zu geben. 
2. SchluBfolgerungen für die Praxis

(1) Als Quintessenz der vorliegenden Arbeit empfiehlt sich bei der Erschließung innovativer Investivdienstleistungsmärkte ein Planungsansatz, der die drei Dimensionen "Funktion", "Technologie" und "Abnehmergruppen" explizit berücksichtigt. Zur Reduzierung der mit dem Markteintritt verbundenen Risiken sollte die Geschäftsfeldwahl dabei von dem spezifischen Möglichkeitsfeld der Unternehmung ausgehen. In diesem Sinne sollte zunächst die Frage beantwortet werden, zur Erfüllung welcher Abnehmerfunktionen das Unternehmen sich vor dem Hintergrund seiner vorhandenen Ressourcen und know how-Stärken mittelfristig in der Lage sieht. In jedem Falle sollte es vermeiden, die Besetzung solcher Geschäftsfelder zu planen, für die es keine originäre Kompetenz besitzt.

(2) Die Definition des geplanten Geschäftsfeldes vollzieht sich zweckmäBigerweise in der Abfolge Funktion - Technologie - Abnehmergruppe. Diese Vorgehensweise widerspricht nun scheinbar dem Prinzip einer marktorientierten Planung. Tatsächlich bestimmit der Marktaspekt durch die Zugrundelegung abnehmerbezogener Auswahlkriterien bereits in starkem MaBe die Auswahl der zu erbringenden Funktion und der einzusetzenden Technologie. Im Rahmen der Abnehmerauswahl ist schlieBlich dafür Sorge zu tragen, daB die Ausgestaltung der Marketinginstrumente (einschlieBlich der Detailkonzeption des Dienstleistungsangebots) sich an der relevanten Zielgruppe orientiert.

(3) Die Bearbeitung der relevanten Abnehmergruppe hat von der Frage auszugehen, inwieweit welchen Teilsegmenten innerhalb der vorgesehenen Abnehmerschaft die angebotene Dienstleistung problemevident ist. Nach Maßgabe der vorhandenen Problemevidenz ist sodann die Marketing-Matrix zu gestalten, die für die einzelnen Segmente einen integrierten Maßnahmenkatalog beinhaltet. 
Die empirische Untersuchung belegt am Beispiel elektronischer Informationsdienste die Anwendungstauglichkeit des vorgeschlagenen Markteintrittskonzepts. Gleichzeitig liefert sie Anregungen für weiterfürende Forschungen in methodischer Hinsicht sowie im Hinblick auf das strategische Marketing investiver Dienstleistungen.

\section{Auswirkungen auf die weitere Forschung}

Entsprechend ihrer theoretischen und empirischen Anlage kommt der vorliegenden Untersuchung der Charakter einer Basisstudie zu, die den grundlegenden Rahmen eines investiven Dienstleistungsmarketing aufspannt. Die Bestätigung der getroffenen Tendenzaussagen sowie die Beantwortung differenzierterer Fragestellungen muß weiteren, darauf aufbauenden Detailstudien vorbehalten bleiben. Ausgehend von dieser Einordnung zeichnen sich verschiedene Ansatzpunkte für vertiefende Analysen ab.

(1) Von kardinalem Interesse für den Anbieter innovativer Dienstleistungen ist die Prognose der Akzeptanz dieser Dienstleistung am Markt, und zwar mit einer Genauigkeit, die die ( $z$. T. immensen) Investitionen in das neue Marktangebot rechtfertigt. Die zentrale Schwierigkeit besteht hierbei in dem Problem, dem geringen Wissensstand der potentiellen Abnehmer Rechnung zu tragen. 1 Hier gilt es, Methoden zu entwickeln, um die Bestimmungsfaktoren der Adoptionsbereitschaft in Abhängigkeit von dem sich verändernden Bild der Dienstleistung in der Wahrnehmung der potentiellen Adopter zu prognostizieren. 2 Zugleich ist damit auf

1 Vgl. Pessemier, E., Wilton, P., Pretesting the acceptance of innovations, Paper No. 696, Institute for Research in the Behavioral, Economic, and Management Sciences, Krannert Graduate School of Management, Purdue University, West Lafayette, Indiana 1979, S. 1.

$2 \mathrm{Vgl}$. ebenda, S. $6 \mathrm{ff}$. 
das weite Feld der Forschung hingewiesen, das sich der Marketing-Wissenschaft im Zusammenhang mit der Erklärung und Messung der Problemevidenz auftut.

(2) Ein zweiter Ansatzpunkt zukünftiger Forschung betrifft die optimale Gestaltung des geplanten investiven Dienstleistungsangebots. Der Einsatz der Conjoint-Analyse kann sich hier als hilfreiches Instrument erweisen, um auf alternative Abnehmergruppen abgestimmte optimale Sets von Dienstleistungseigenschaften zu lokalisieren.

(3) Ein dritter Forschungsbereich zeichnet sich in dem Problem der planmäßigen Entwicklung investiver Dienstleistungen ab. Mit Blick auf die hohen Qualitätsanforderungen an immaterielle Marktleistungen kommt hier dem Einsatz eines perfektionierten ServiceBlueprinting ein zentraler Stellenwert zu. ${ }^{2}$

(4) Schließlich eröffnet sich in der Konzeptionierung eines "zwei-seitigen Dienstleistungsmarketing" ${ }^{3}$ ein vierter Forschungsbereich. Hier gilt es, das Absatzmarketing und das Beschaffungsmarketing der investiven Dienstleistung zu einer wohlkoordinierten Einheit zusammenzufuigen. 4

$1 \mathrm{Vgl.z}$. B. den Einsatz der Conjoint Analyse zur optimalen Gestaltung wissenschaftlich-technischer Informationsdienste. Vgl. Wind, Y., Grashof, J.F., Goldhar, J.D., Market-based guidelines far design of industrial products. A new application of conjoint analysis to scientific and technical information (STI) services, in: JoM, Vol. 42, July 1978, S. $27 \mathrm{ff}$.

$2 \mathrm{Vgl}$. hierzu vor allem die Arbeiten von Shostak, G.L., How to Design a Service, in: EJoM, Vol. 16, 1982, $\mathrm{Nr}$. 1, S. $49 \mathrm{ff}$. Sowie dieselbe, Service Design in the operating environment, in: Developing New Services, Hrsg.: George, W.R., Marshall, C.E., Chicago, I1l. 1984 , S. $27 \mathrm{ff}$.

3 Hilke, W., a.a.0., S. 17.

$4 \mathrm{Vgl}$. ebenda, S. $17 \mathrm{f}$. 
Insgesamt zeichnet sich ab, daß das Marketing für Anbieter investiver Dienstleistungen in Zukunft sprunghaft an Bedeutung gewinnen wird. Neue Dienstleistungen werden in wachsender Zahl auf den Markt drängen, und dienstleistungsfremde Firmen werden zunehmend im Dienstleistungssektor neue Wachstumschancen suchen. Vor diesem Hintergrund sollte sich die Praxis darauf einstellen,... "that 'marketing muscle' is becoming a survival factor for many service firms in the $80 \mathrm{~s} " . "$ Die Marketing-Wissenschaft ist indessen aufgerufen, ihrer entscheidungsunterstützenden Funktion gegenüber den Dienstleistungsfirmen durch die verstärkte Berücksichtigung dieses noch nahezu unergründeten Forschungsgebietes gerecht zu werden.

1 Parasuraman, A., Berry, L.L., Zeithaml, V.A., Service Firms Need Marketing Skills, in: BH, Vol. 26, November - December 1983, S. 31. 


$$
\text { A } n \text { h a } n \text { g 1: }
$$

\section{Anlage der empirischen Untersuchung zur Akzeptanz elektronischer Wirtschaftsinformationsdienste in der Bundesrepublik Deutschland}




\section{Ziele der Untersuchung}

Aufbauend auf umfangreichen Sekundäranalysen und der theoretischen Entwicklung eines strategischen Konzepts zur Marktabgrenzung und Marktbearbeitung im Bereich elektronischer Wirtschaftsinformations dienste verfolgte die empirische Untersuchung vor allem folgende Zielsetzungen:

1. Ermittlung des Marktbedarfs nach elektronischen Wirtschaftsinformationsdiensten,

2. Herausarbeitung von Bedarfsspezifika (Anforderungen an die Versorgung mit unternehmensexternen Sekundärinformationen),

3. Ermittlung akzeptanzbestimmender Faktoren (Determinanten der grundsätzlichen Adoptionsbereitschaft).

Aufgrund der Komplexität und des hohen Innovationsgrades der Fragestellung strebte die Untersuchung keine repräsentativen Ergebnisse an. Vielmehr versteht sie sich als explorative Erhebung, die erste zentrale Hinweise, jedoch keine allgemeingültigen Aussagen zu liefern vermag.

\section{Untersuchungsdesign und Stichprobenstruktur}

Die Untersuchung stützt sich auf eine empirische Erhebung, die zu Beginn des Jahres 1984 durchgeführt wurde. Nach zwei Testbefragungen wurden Anfang Januar 1984 insgesamt 3.600 vollstrukturierte Fragebögen verschickt. Davon richteten sich 
- 1.600 Fragebögen an zufällig ausgewählte Abonnenten eines großen deutschen Wirtschaftsmagazins,

- 2.000 Fragebögen an zufällig ausgewählte Industrieunternehmen mit mehr als 50 Beschäftigten.

Der Rücklauf ergab eine Gesamtzahl auswertbarer Fragebögen von 182. Damit wurde eine Rücklaufquote von 5,1 \% realisiert, die zwar nicht ganz zu befriedigen vermochte, sich aber noch im Bereich bisheriger Erfahrungen bewegte. Als wesentliches Kriterium erfiulte der Rücklauf die wichtige Anforderung, eine Auswertung unter Anwendung multivariater statistischer Verfahren zu ermöglichen.

Die Auswertung selbst erfolgte unter Einsatz des statistischen Programmpaketes SPSS ("statistical package for the social sciences") in der Version 9 im Rechenzentrum der Westfälischen Wilhelms-Universität Muinster.

Die Stichprobe zeichnet sich durch eine annähernde Gleichverteilung der Unternehmen hinsichtlich ihrer Branchenzugehörigkeit aus. Bei der Beschäftigtenstruktur zeigt sich ein gewisses ubergewicht der kleinstunternehmen, das aus der für das Wirtschaftsmagazin typischen Abonnentenstruktur (Handwerk, Steuerberater ect.) resultiert. Hinsichtlich der Umsatzstruktur uberwiegen ebenfalls die klein-bis Mittelunternehmen, in Sonderheit solche mit einem Umsatz von 5 - 50 Mio. DM (Abbildung 57).

Hinsichtlich der Position der Fragebogenbeantworter ergab sich, daB der persönlichen Adressierung der Fragebögen an die "Geschäftsführer" ein recht guter Erfolg beschieden war: $62 \%$ der Beantworter waren Geschäftsführer oder Inhaber der angeschriebenen Unternehmen (vgl. Abbildung 58). 

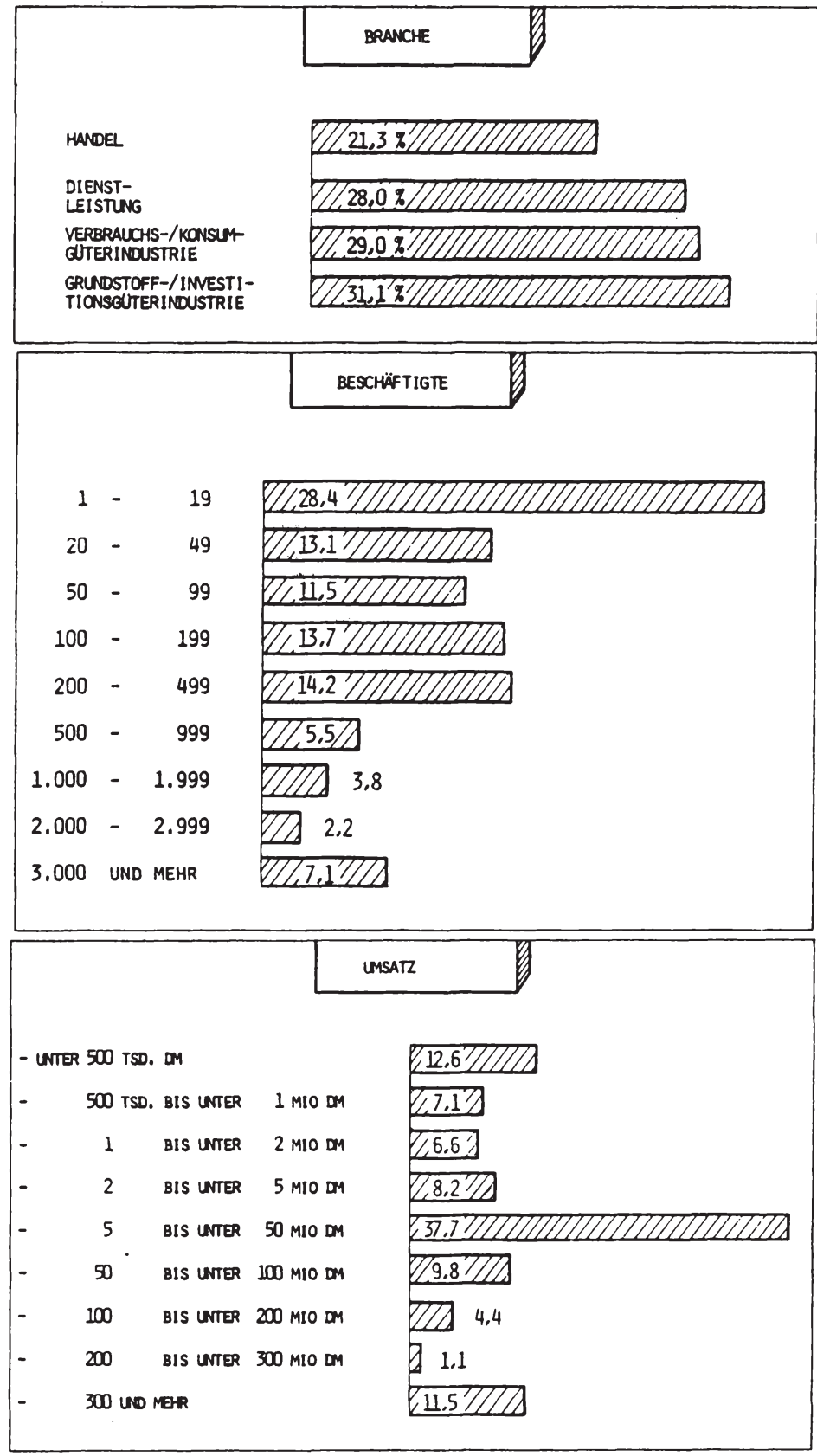

Abb. 57: Organisationsdemographische Merkmale der Stichprobe 

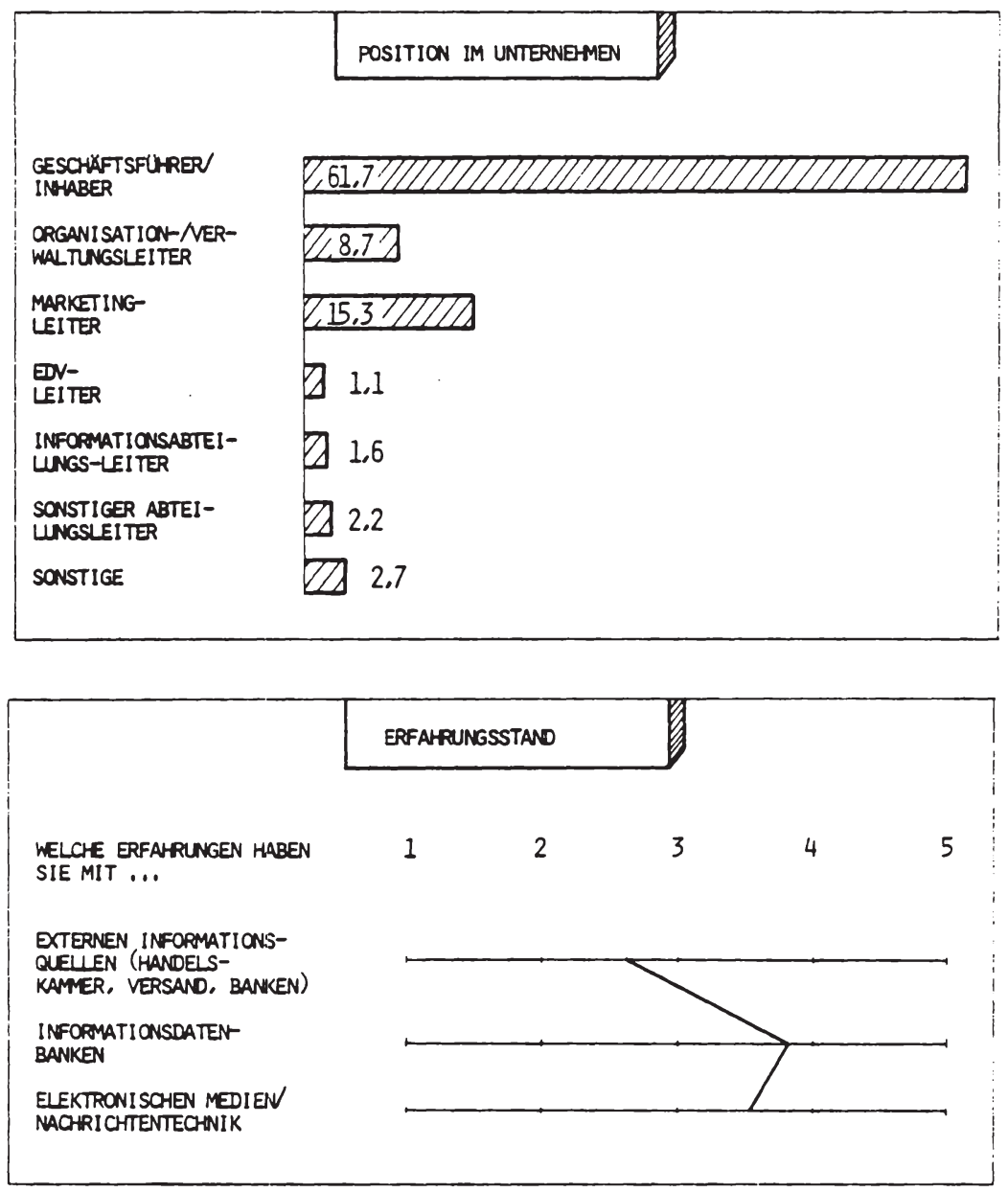

Abb. 58: Merkmale der Fragenbeantworter 
Damit wurde in zwei Drittel der Fälle die Person erreicht, die in der überragenden Mehrzahl der Unternehmen ggfs. auch die Entscheidung über die Nutzung eines elektronischen Informationsdienstes fällen würde. Die Frage zum Erfahrungsstand der Fragebogenbeantworter lieferte schlieBlich folgendes Ergebnis (Abbildung 58): während die Kenntnisse zu externen Informationsquellen allgemein als relativ hoch eingestuft wurden $(1,63 \%)$, gestand sich die Mehrzahl der Befragter eher geringe Erfahrungen mit Informationsdatenbanken und elektronischen Medien ein. 
A $n h$ a $n g$ 2: Tabellen

M. Krups - 978-3-631-75074-2

Downloaded from PubFactory at 01/11/2019 08:37:05AM

via free access 


\begin{tabular}{|c|c|c|c|c|c|}
\hline & FAKTOR 1 & FAKTOR 2 & FAKTOR 3 & FAKTOR 4 & FAKTOR 5 \\
\hline $\begin{array}{l}\text { DIE INFORMATIONEN SIND } \\
\text { ZUNERLÄSSIG UND GENAU }\end{array}$ & 0,03962 & 0,46641 & 0,28172 & 0,58058 & 0,03543 \\
\hline $\begin{array}{l}\text { DIE INFORMATIONEN SIND } \\
\text { UMFASSEND - ES WERDEN } \\
\text { VIEIE RANDINFORMATIONEN } \\
\text { MITGELIEERT }\end{array}$ & 0,09362 & 0,19738 & 0.02128 & 0,56763 & 0,15197 \\
\hline $\begin{array}{l}\text { DIE BESCHAFFUNG DER INFOR- } \\
\text { MATIONEN IST OHNE BESONDERE } \\
\text { DOKUMENTAR ISCHE } 7 \text { EDV- } \\
\text { KENWTNISSE MÖGLICH }\end{array}$ & 0,27623 & $-0,01658$ & $-0,05601$ & 0,17124 & 0,17163 \\
\hline $\begin{array}{l}\text { DIE INFORMATIONEN STEHEN } \\
\text { SCHNELL ZUR VERFÜGUNG }\end{array}$ & 0,23843 & $\underline{0,82132}$ & 0,09874 & 0,13794 & 0,07354 \\
\hline $\begin{array}{l}\text { DIE INFOPMATIONEN SIND } \\
\text { PREISWERT } Z U \text { ERHALTEN }\end{array}$ & 0,57246 & 0,23517 & $-0,03185$ & $-0,01586$ & 0,17908 \\
\hline $\begin{array}{l}\text { DIE INFORMATIONEN SIND } \\
\text { ÜBERSICHTLICH UND LEICHT } \\
\text { VERSTÄNDLICH }\end{array}$ & 0,64557 & 0,16478 & 0,10664 & 0,09430 & 0.07469 \\
\hline $\begin{array}{l}\text { DIE INFORMATIONEN SIND } \\
\text { ZIELGENAU - ES MÜSSEN } \\
\text { KEINE IRRELEVANTEN INFOR- } \\
\text { MATIONEN GESICHIET WERDEN }\end{array}$ & 0,62362 & 0.13909 & 0,29868 & 0,13094 & $-0,00662$ \\
\hline $\begin{array}{l}\text { DIE INFORMATIONEN SIND } \\
\text { AKTUEL }\end{array}$ & 0,34896 & 0,51033 & 0,05570 & 0,30089 & 0,17498 \\
\hline $\begin{array}{l}\text { DIE INFOPMATIONEN SIND } \\
\text { LEICHT ARCHIVIERBAR }\end{array}$ & 0,30090 & $-0,00367$ & 0,73956 & 0,08123 & 0,08857 \\
\hline $\begin{array}{l}\text { DIE INFOPMATIONEN SIND } \\
\text { ZWECKENTSPRECHEND AUFBE- } \\
\text { REITET }\end{array}$ & 0,53807 & 0,11800 & 0,36347 & 0,19173 & 0,11200 \\
\hline $\begin{array}{l}\text { DIE INFORMATIONEN SIND } \\
\text { OBUEKTIV - SIE BEINHALTEN } \\
\text { KEINE SUBJEKTIVEN AUSSAGEN }\end{array}$ & 0,36914 & $-0,04682$ & 0,19757 & 0,45013 & 0,04226 \\
\hline $\begin{array}{l}\text { DIE INFOPMATIONEN STEHEN } \\
\text { EXKUSIV ZLR VERFUUGUNG }\end{array}$ & 0,12470 & 0,10411 & 0,09426 & 0,02504 & 0,29445 \\
\hline $\begin{array}{l}\text { DIE INFORMATIONEN BEIN- } \\
\text { HALTEN AUCH ANTWORTEN } \\
\text { AUF FACHGEBIETSÜBER- } \\
\text { GREIFENDE FRAGEN }\end{array}$ & 0,08230 & 0,00435 & 0,14904 & 0,15238 & 0,80971 \\
\hline $\begin{array}{l}\text { DIE INFORMATIONEN KÖNNEN } \\
\text { IN DER EIGENEN EV MIT } \\
\text { VERARBEITET WERDEN }\end{array}$ & $-0,02598$ & 0,15310 & 0,43805 & 0,09210 & 0,23164 \\
\hline \multirow[t]{2}{*}{ ERKLÄRTER VARIANZANTEIL } & 26,38 & 6,68 & 5,88 & 4,48 & 3,28 \\
\hline & \multicolumn{5}{|c|}{46,38} \\
\hline
\end{tabular}

Tab. 15: Faktorenstruktur der Anforderungen an die Versorgung mit Branchen informationen 


\begin{tabular}{|c|c|c|c|}
\hline $\begin{array}{l}\text { ANFORDERUNGS- } \\
\text { RRITERIEN }\end{array}$ & FAKTOR 1 & FAKTOR 2 & FAKTOR 3 \\
\hline $\begin{array}{l}\text { DIE INFORMATIONEN SIND } \\
\text { ZUVERLÄSSIG UND GENAU }\end{array}$ & 0,31955 & 0,16320 & 0,55944 \\
\hline $\begin{array}{l}\text { DIE INFORMATIONEN SIND } \\
\text { UMFASSEN - ES WERDEN } \\
\text { VIEE RANDINFCRMATIONEN } \\
\text { MITGEIEEET }\end{array}$ & 0,15169 & 0,14319 & 0,65712 \\
\hline $\begin{array}{l}\text { DIE BESCHAFFUNG DER INFOR- } \\
\text { MATIONEN IST OHNE BESONDERE } \\
\text { DOKUMENTARISCHE } \text { EDV } \\
\text { KENNTNISSE MÖ́GLICH }\end{array}$ & $\underline{0,44538}$ & 0.07546 & 0,21111 \\
\hline $\begin{array}{l}\text { DIE INFORMATIONEN STEHEN } \\
\text { SCHNEU ZUR VERFÜGUNG }\end{array}$ & 0,62561 & 0,19656 & 0,40698 \\
\hline $\begin{array}{l}\text { DIE INFORMATICNEN SINO } \\
\text { PREISWERT ZU ERHALTEN }\end{array}$ & 0,59892 & 0,01632 & 0,17146 \\
\hline $\begin{array}{l}\text { DIE INFORMATIONEN SIND } \\
\text { ÜBERSICHMICH UND LEICHT } \\
\text { VERSTANOLICH }\end{array}$ & $\underline{0,73535}$ & 0,20944 & $-0,02052$ \\
\hline $\begin{array}{l}\text { DIE INFORMATICNEN SIND } \\
\text { ZIEIGENAU - ES MUSSEN } \\
\text { KEINE IRRELEVANTEN INFOR- } \\
\text { MATIONEN GESICHIET WERDEN }\end{array}$ & 0,60241 & 0,29852 & 0,07974 \\
\hline $\begin{array}{l}\text { DIE INFORMATIONEN SIND } \\
\text { AKTUEL }\end{array}$ & 0,54455 & 0,13876 & 0,33216 \\
\hline $\begin{array}{l}\text { DIE INFORMATIONEN SIND } \\
\text { LEICHT ARCHIVIERBAR }\end{array}$ & 0,37558 & 0,64843 & 0,01285 \\
\hline $\begin{array}{l}\text { DIE INFORMATIONEN SIND } \\
\text { ZWECKENTSPRECHEND AUFBE- } \\
\text { REITET }\end{array}$ & 0,37298 & 0,52573 & 0,05694 \\
\hline $\begin{array}{l}\text { DIE INFORMATIONEN SIND } \\
\text { OBUEKTIN - SIE BEINHALTEN } \\
\text { KEINE SUBJEKTIVEN AUSSAGEN }\end{array}$ & $\underline{0.43150}$ & 0.26626 & 0,16705 \\
\hline $\begin{array}{l}\text { DIE INFORMATIONEN STEHEN } \\
\text { EXKLSIV ZUR VERFÜGUNG }\end{array}$ & 0,12401 & $\underline{0,47127}$ & 0,07684 \\
\hline $\begin{array}{l}\text { DIE INFORMATIONEN BEIN- } \\
\text { HALTEN AUCH ANTWORTEN } \\
\text { AUF FACHGEBIETSUBBER- } \\
\text { GREIFENDE FRAGEN }\end{array}$ & $-0,04569$ & 0,53277 & 0,25892 \\
\hline $\begin{array}{l}\text { DIE INFORMATIONEN KÖNNEN } \\
\text { IN DER EIGENEN EDV MIT } \\
\text { VERARBEITET WERDEN }\end{array}$ & 0,08987 & $\underline{0,52768}$ & 0,09625 \\
\hline \multirow[t]{2}{*}{ ERKLÄRTER VARIANZANTEIL } & 29,88 & 6,68 & $4,9 \&$ \\
\hline & \multicolumn{3}{|c|}{41,38} \\
\hline
\end{tabular}

Tab. 16: Faktorenstruktur der Anforderungen an die Versorgung mit Gesamitwirtschaftl ichen / Konjunkturinformationen 


\begin{tabular}{|c|c|c|}
\hline $\begin{array}{l}\text { ANFORDERUNGS- } \\
\text { KRITERIEN }\end{array}$ & FAKTOR 1 & FAKTOR 2 \\
\hline $\begin{array}{l}\text { DIE INFCRMATIONEN SIND } \\
\text { ZNERLÄSSIG UND GENAU }\end{array}$ & 0,77385 & 0,35497 \\
\hline $\begin{array}{l}\text { DIE INFORMATIONEN SIND } \\
\text { UMFASSEND - ES WERDEN } \\
\text { VIEE RANDINFORMATIONEN } \\
\text { MITGEIIEFERT }\end{array}$ & 0,66918 & 0,38590 \\
\hline $\begin{array}{l}\text { DIE BESCHAFFUNG DER INFOR- } \\
\text { MATIONEN IST OHWE BESONDERE } \\
\text { DOLMENTARISCHE } 7 \text { EDV- } \\
\text { KENNTIISSE MÖGLICH }\end{array}$ & 0,55675 & 0,40138 \\
\hline $\begin{array}{l}\text { DIE INFORMATIONEN STEFEN } \\
\text { SCHNEU ZUR VERFULGUNG }\end{array}$ & 0,81853 & 0,28935 \\
\hline $\begin{array}{l}\text { DIE INFORMATIONEN SIND } \\
\text { PREISWERT ZU ERHALTEN }\end{array}$ & 0,69430 & 0,25198 \\
\hline $\begin{array}{l}\text { DIE INFORMATIONEN SIND } \\
\text { ÜBESICHMLICH UND LEICHT } \\
\text { VERSTÄNDLICH }\end{array}$ & 0,84667 & 0,23121 \\
\hline $\begin{array}{l}\text { DIE INFORMATIONEN SIND } \\
\text { ZIELGENAU - ES MUSSSEN } \\
\text { KEINE IRRELEVANTEN INFOR- } \\
\text { MATIONEN GESICHTET WERDEN }\end{array}$ & 0,88531 & 0,14909 \\
\hline $\begin{array}{l}\text { DIE INFORMATIONEN SIND } \\
\text { AKTUEU }\end{array}$ & 0,85305 & 0,23530 \\
\hline $\begin{array}{l}\text { DIE INFOPMATIONEN SIND } \\
\text { LEICHT ARCHIVIERBAR }\end{array}$ & 0,36325 & 0,70395 \\
\hline $\begin{array}{l}\text { DIE INFORMATIONEN SIND } \\
\text { ZWECKENTSPRECHEND AUFBE- } \\
\text { REITET }\end{array}$ & 0,80016 & 0,39359 \\
\hline $\begin{array}{l}\text { DIE INFORMATIONEN SIND } \\
\text { OBJEKTIV - SIE BEINHALTEN } \\
\text { KEINE SUBJEKTIVEN AUSSAGEN }\end{array}$ & 0,76494 & 0,28052 \\
\hline $\begin{array}{l}\text { DIE INFOPMATIONEN STEHEN } \\
\text { EXKUSIV ZUR VERFUGGUNG }\end{array}$ & $\underline{0,39056}$ & 0,34171 \\
\hline $\begin{array}{l}\text { DIE INFORMATIONEN BEIN- } \\
\text { HALTEN AUCH ANTWORTEN } \\
\text { AUF FACHGEBIETSUIBER- } \\
\text { GREIFENDE FRAGEN }\end{array}$ & $\underline{0,46263}$ & 0,44085 \\
\hline $\begin{array}{l}\text { DIE INFORMATIONEN KÖNNEN } \\
\text { IN DER EIGENEN EDV MIT } \\
\text { VERARBEITET WERDEN }\end{array}$ & 0,06549 & $\underline{0,69670}$ \\
\hline \multirow[t]{2}{*}{ ERKLÄRTER VARIANZANTEIL } & 56,58 & 5,78 \\
\hline & & \\
\hline
\end{tabular}

Tab. 17: Faktorenstruktur der Anforderungen an die Versorgung mit Exportinformationen 
A $n h$ a $n g$ 3: Fragebogen

M. Krups - 978-3-631-75074-2

Downloaded from PubFactory at 01/11/2019 08:37:05AM

via free access 


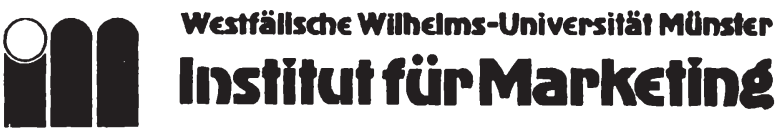

Direkfor:Prof.Dr.H.Meffert

Institut für Marketing - Universitätsstraße 14 - 16 - 4400 Münster

Universitätsstraße 14-16

4400 Münster

Telefon (0251) 832931

9. Januar 1984

Sehr geehrte Damen und Herren,

das Institut für Marketing beschätigt sich seit geraumer zeit mit den Auswirkungen neuer Informationstechnologien auf die Informationsbeschaffung von Unternehmen der Wirtschaft. Einen besonderen Schwerpunkt der Forschung bildet die Untersuchung der Akzeptanz elektronischer Wirtschaftsinformationsdienste.

Mit dem Ihnen vorliegenden Fragebogen möchten wir Ihre Einschätzung hinsichtlich der zukinftigen Bedeutung und des praktischen Nutzens solcher neuartiger Informationsdienste erfahren. Fur unsere Untersuchung stützen wir uns auf eine nach einem statistischen Stichprobenverfahren speziell ausgewählte Anzahl von Unternehmen. Selbstverständlich erfolgt die Untersuchung streng vertraulich und anonym. Ruckschlusse auf Ihre Person oder Ihr Unternehmen sind ausgeschlossen.

Sollten Sie persönlich nicht uber die notwendigen Informationen zur Ausfüllung des Fragebogens verfügen, bitten wir Sie, den Bogen an einen Experten in Ihrem Hause weiterzuleiten.

Den Rücksendern des ausgefullten Fragebogens steht auf Wunsch gerne eine Dokumentation der Untersuchungsergebnisse zur Verfügung. Fullen Sie hierzu bitte den belliegenden Anforderungsbogen aus und senden sie diesen mit getrennter post ein.

Für Ihre Mithilfe an unserem Forschungsprojekt bedanke 1ch mich $1 \mathrm{~m}$ voraus.

Mit freundlichen GrüBen<smiles>C=C(C)OCC(C)CC(C)CC(C)CC(C)C</smiles>

Prof. Dr. H. Meffert

Anlage 


\section{WAS SIND ELEKTRONISCHE WIRTSCHAFTSINFORMATIONSDIENSTE?}

- In dein vorliegenden Fragebogen geht es um das Diensteangebot kommerzieller Informationsantieter 'In for formationsbroker).

Das Angebot beinhaltet aufbereitete Sekundär-Informationen zu den Themenbereichen

- Gesamtwirtschaft/Konjunktur

- Branchen/Märkte

- Außenhandel/Export

Die Informationen entstammen umfassenden Wirtschafts-. Marktforschungs- und Pressedatenbanken, so 2.B. der OECD-Datenbank, der IFO-Datenbank, etc.

- Der Nutzer des Informationsdienstes hat die Möglichkeit, eine bestimmte Informationsanfrage zu stelier. die dann in der gewünschten Form dem Anfrager beantwortet wird.

- Die Informationsanfrage und ubermittlung erfolgt über ein vom Anfragenden bestimmies elektronshas Medium.

Auf Wunsch ist es möglich, die abgerufenen Informationen in textlicher und graphischer Form darzustel. len, unterschiedliche Daten miteinander zu koppeln oder individuell in Beziehung zueinander zu setzen und Berechnungen an gelieferten Daten vorzunehmen.

For die beantwortete Informationsanfrage zahlt der Anfrager ein vereinbartes Entgelt.

\section{BEISPIELE}

\section{Beispiel 1:}

Ein Unternehmen möchte expandieren und wünsche eine Analyse des Außenhandels mit den betreffenden Produk. ten, bezogen auf eine $Z_{2}$ hl vorgegebener Länder.

\section{Beispiel 2:}

Ein Unternehmen sucht nach Informa tionen über Handelspartner und $\mathrm{Ab}$ satzmärkte in bestimmren Ländern.

Beispiel 3:

Ein Unternehmen der Investitionsgüterbranche möchte regelmäßig über alle inländischen und ausländischen Aus. schreibungen unterrichtet sein.
Der Informationsdienst stellt eine datenbankgespeicherte Liste der Exporte (Menge und Wert) der betreffenden Pro. dukte zur Verfügung ind berechnet eine Prognose furr die näch. sten Jahre. Das Unternehmen wählt entwedet den Direktzugang zur Datenbank uber Datenfernubertragung bzw. Bild. schirmtext (Unline Recherche, Kosten: ca. DM 200,--) oder läBt sich die Daten über einen Computerausdruck via Fernko. pierer zuschicken (Kosten: ca. DM 250,--).

Der Informationsdienst stellt Marktpotentialdaten, Sättigungs. raten, Kontaktadressen etc. uber seine AuBenhandelsdaten. bank via Bildschirmtext zu Verfügung. Die Informationen sind z.T. direke zu entnehmen, teilweise können Zusatzinformationen per Bildschirmtext angefordert werden.

(Kosten: ca. DM 400,--)

Der Informationsdienst stellt seine (tagesaktuelle) Ausschreibungsdatenbank via Bildschirmtext zu Verfügung.

(Kosten: ca. DM 550,- pro Jahr) 
FRAGEBOGEN

1 In weichem Umfang werden folgende Informationen in Ihrem Hause benötigt?

\begin{tabular}{|l|c|c|c|c|}
\hline $\begin{array}{l}\text { Informationen } \\
\text { über... }\end{array}$ & $\begin{array}{l}\text { werden } \\
\text { sehr häufig } \\
\text { benötigt }\end{array}$ & $\begin{array}{l}\text { werden } \\
\text { eher häuflig } \\
\text { benötigt }\end{array}$ & $\begin{array}{l}\text { werden } \\
\text { eher selten } \\
\text { benötigt }\end{array}$ & $\begin{array}{l}\text { werden } \\
\text { sehr selten } \\
\text { benb́tigt }\end{array}$ \\
\hline $\begin{array}{l}\text { Gesamiwirtschaftliche } \\
\text { Entwicklung/Konjunktur }\end{array}$ & 0 & 0 & 0 & 0 \\
Außenhendel/Export & 0 & 0 & 0 & 0 \\
Branchen/Märkte & 0 & 0 & 0 & 0 \\
\hline
\end{tabular}

2 Betrachten Sie bitte einmal die im folgenden aufgelisteten Einzelinformationen. Wie beurteilen Sie - bezogen auf Ihr Unternehmen - die Versorgung mit diesen Informationen?

\begin{tabular}{|c|c|c|c|c|c|c|}
\hline & & \multicolumn{4}{|c|}{ Mit der Informationsversorgung bin lch... } & \multirow{2}{*}{$\begin{array}{l}\text { Dle Infor. } \\
\text { motionen } \\
\text { sind für } \\
\text { uns irrele- } \\
\text { vant }\end{array}$} \\
\hline & & $\begin{array}{l}\text { sehr } \\
\text { zufrieden }\end{array}$ & $\begin{array}{l}\text { ziemlich } \\
\text { zufrieden }\end{array}$ & $\begin{array}{l}\text { weniger } \\
\text { zufrieden }\end{array}$ & $\begin{array}{l}\text { sehr } \\
\text { unzufrieden }\end{array}$ & \\
\hline $\begin{array}{l}\text { Gesaminirtschaft/ } \\
\text { Konjunktur } \\
\text { (bezogen auf die } \\
\text { Bundesrepublik } \\
\text { Deutschland) }\end{array}$ & $\begin{array}{l}\text { insgesamt } \\
\text { Preise/Zinsen } \\
\text { Stimmungslage } \\
\text { Umsätze } \\
\text { Auftragse ingänge }\end{array}$ & $\begin{array}{l}0 \\
0 \\
0 \\
0 \\
0\end{array}$ & $\begin{array}{l}0 \\
0 \\
0 \\
0 \\
0\end{array}$ & $\begin{array}{l}0 \\
0 \\
0 \\
0 \\
0\end{array}$ & $\begin{array}{l}0 \\
0 \\
0 \\
0 \\
0\end{array}$ & $\begin{array}{l}0 \\
0 \\
0 \\
0 \\
0\end{array}$ \\
\hline $\begin{array}{l}\text { Branchen /Märkte } \\
\text { (bezogen ouf die } \\
\text { relevantel(n) } \\
\text { Branche(n)l) }\end{array}$ & $\begin{array}{l}\text { insgesamit } \\
\text { allgemeine Marktentwicklung (Umsätze, Sätti- } \\
\text { gungsraten, etc.) } \\
\text { inländische Produktion } \\
\text { Außenhandel } \\
\text { Branchenstruktur/Absatzmittlerdaten } \\
\text { Entwicklung der Verbraucherpreise } \\
\text { Nachfrage der privaten Haushalte } \\
\text { Verbraucherdaten } \\
\text { Werbeaufwand } \\
\text { Wettbewerbsdaten }\end{array}$ & $\begin{array}{l}0 \\
0 \\
0 \\
0 \\
0 \\
0 \\
0 \\
0 \\
0 \\
0\end{array}$ & $\begin{array}{l}0 \\
0 \\
0 \\
0 \\
0 \\
0 \\
0 \\
0 \\
0 \\
0\end{array}$ & $\begin{array}{l}0 \\
0 \\
0 \\
0 \\
0 \\
0 \\
0 \\
0 \\
0 \\
0\end{array}$ & $\begin{array}{l}0 \\
0 \\
0 \\
0 \\
0 \\
0 \\
0 \\
0 \\
0 \\
0\end{array}$ & $\begin{array}{l}0 \\
0 \\
0 \\
0 \\
0 \\
0 \\
0 \\
0 \\
0 \\
0\end{array}$ \\
\hline $\begin{array}{l}\text { Außenhandel/ } \\
\text { Export (bezogen } \\
\text { auf einzelne } \\
\text { Länder/Länder. } \\
\text { gruppen) }\end{array}$ & $\begin{array}{l}\text { insgesamt } \\
\text { Absatzchancen (Marktpotentiale, Sättigungsraten) } \\
\text { Finanzielle Abwicklung (Zahlungsverkehr, Doku- } \\
\text { mente, Währungen) } \\
\text { Vertriebswege/Transport (Absatzkanäle/Logistik) } \\
\text { Adressen (Geschäfts-/Ansprechpertner, Service. } \\
\text { unternehmen) } \\
\text { Rechtsinformationen (Normen, Bestimmungen, Ver. } \\
\text { tragsgestaltung). Steuern, Versicherungen } \\
\text { Ausschreibungen }\end{array}$ & $\begin{array}{l}0 \\
0 \\
0 \\
0 \\
0 \\
0 \\
0\end{array}$ & $\begin{array}{l}0 \\
0 \\
0 \\
0 \\
0 \\
0 \\
0\end{array}$ & $\begin{array}{l}0 \\
0 \\
0 \\
0 \\
0 \\
0 \\
0\end{array}$ & $\begin{array}{l}0 \\
0 \\
0 \\
0 \\
0 \\
0 \\
0\end{array}$ & $\begin{array}{l}0 \\
0 \\
0 \\
0 \\
0 \\
0 \\
0\end{array}$ \\
\hline
\end{tabular}

3 Welche Stellen in Ihrem Unternehmen nutzen schwerpunktmäßig folgende Informationen?

\begin{tabular}{|l|c|c|c|c|c|}
\hline $\begin{array}{l}\text { Informationen } \\
\text { über... }\end{array}$ & $\begin{array}{l}\text { Geschäfts. } \\
\text { führung }\end{array}$ & $\begin{array}{l}\text { Marketing/ } \\
\text { Verkauf }\end{array}$ & $\begin{array}{l}\text { Markt- } \\
\text { forschung }\end{array}$ & $\begin{array}{l}\text { spezielle } \\
\text { Informationsabteilung }\end{array}$ & $\begin{array}{l}\text { spezielle } \\
\text { Fachabteilung }\end{array}$ \\
\hline $\begin{array}{l}\text { Gesamtwirtschaft/ } \\
\text { Konjunktur }\end{array}$ & 0 & 0 & 0 & 0 & 0 \\
\hline Außenhandel/Export & 0 & 0 & 0 & 0 & 0 \\
\hline Branchen/Märkte & 0 & 0 & 0 & 0 & 0 \\
\hline
\end{tabular}


4 Zu welchen Anlässen bzw. in welchen Entscheidungssituationen stellt sich Ihnen das Problem, Informationen zu den unten genannten Bereichen von außerhalb des Unternehmens zu beschaffen?. Gehen Sie bitte zeilenweise vor und kreuzen Sie die Ziffer an, die Ihr Urteil am besten wiedergibt.

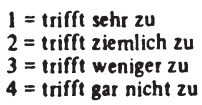

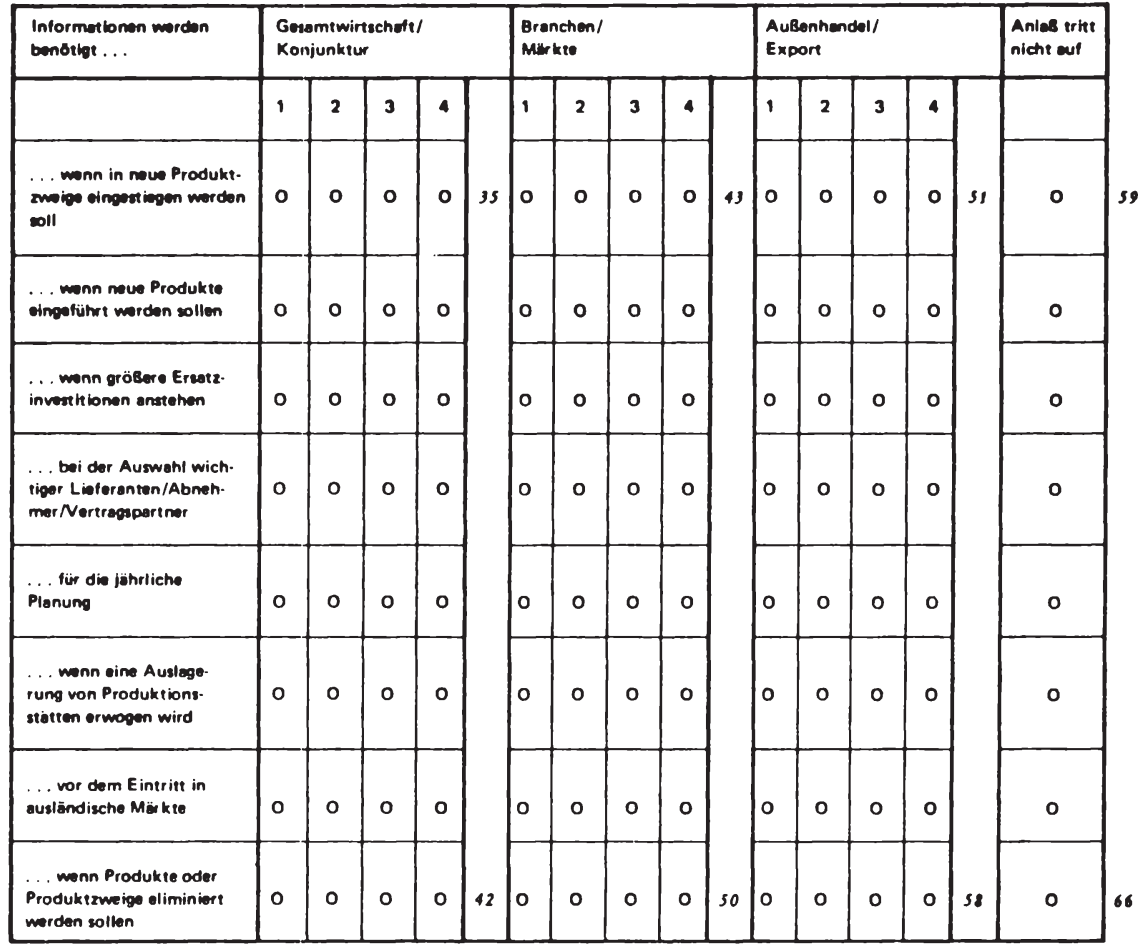


53. In welchem Umfang weruen die folgenden Informationsquellen in Ihrem Unternehmen in Anspruch yommen? Bitte kreuzen Sie unter Spalte 5a an!

\begin{tabular}{|c|c|c|c|c|c|c|c|c|c|}
\hline & \multicolumn{6}{|c|}{5.} & \multicolumn{3}{|c|}{$5 \mathrm{~b}$} \\
\hline & $\begin{array}{l}\text { sohr } \\
\text { häufig }\end{array}$ & $\begin{array}{l}\text { ônc: } \\
\text { häufíg }\end{array}$ & $\begin{array}{l}\text { oner } \\
\text { selten }\end{array}$ & wolten & nite & & $\begin{array}{l}\text { Gesamtwirtsch./ } \\
\text { Konjunktur }\end{array}$ & $\begin{array}{l}\text { Branchen/ } \\
\text { Mörkte }\end{array}$ & $\begin{array}{l}\text { Aubenhandel/ } \\
\text { Export }\end{array}$ \\
\hline IHK, & 0 & 0 & 0 & 0 & 0 & 7 & 0 & 0 & 0 \\
\hline Vorboundo & 0 & 0 & 0 & 0 & 0 & & 0 & 0 & 0 \\
\hline Verleose & 0 & 0 & 0 & 0 & 0 & & 0 & 0 & 0 \\
\hline Kreditinntitute & 0 & 0 & 0 & 0 & 0 & & 0 & 0 & 0 \\
\hline Mossen & 0 & 0 & 0 & 0 & 0 & & 0 & 0 & 0 \\
\hline Kreditversicherungen & 0 & 0 & 0 & 0 & 0 & & 0 & 0 & 0 \\
\hline Informationsvermittler & 0 & 0 & 0 & 0 & 0 & & 0 & 0 & 0 \\
\hline Untern.beratungs- Merbeapenturen & 0 & 0 & 0 & $\mathbf{0}$ & 0 & & o & 0 & $\mathbf{0}$ \\
\hline Marktforschungsunternehmen & 0 & 0 & 0 & 0 & 0 & & 0 & 0 & 0 \\
\hline Fachzeitschriften & 0 & 0 & 0 & 0 & 0 & & 0 & 0 & 0 \\
\hline Wirtschaftszeitschriften/-zoitungen & 0 & 0 & 0 & $\mathbf{0}$ & 0 & & 0 & 0 & 0 \\
\hline Bundesstelle für Aubenhandelsinform. & 0 & 0 & 0 & 0 & 0 & & 0 & 0 & 0 \\
\hline Auskunfteien & 0 & 0 & o & o & 0 & & 0 & o & o \\
\hline Botschaften, Konsulate & 0 & 0 & 0 & 0 & 0 & & 0 & 0 & 0 \\
\hline Handelahäuser & 0 & 0 & 0 & 0 & 0 & & 0 & 0 & 0 \\
\hline Zollämter & 0 & o & 0 & o & 0 & & 0 & 0 & 0 \\
\hline Speditionen & o & 0 & 0 & 0 & 0 & & 0 & o & 0 \\
\hline nationale/internationale Tegungen & 0 & 0 & 0 & 0 & 0 & & 0 & 0 & 0 \\
\hline Befreundete Unternehmer/Kollegen & 0 & 0 & 0 & 0 & 0 & & 0 & 0 & 0 \\
\hline Win tschaftiorschungsinstitute & 0 & o & 0 & 0 & 0 & & 0 & 0 & 0 \\
\hline IUniversitärs-) Bibliotheken & 0 & o & 0 & 0 & 0 & 27 & 0 & 0 & 0 \\
\hline
\end{tabular}

Bitte kreuzen Sie unter Spalte 5 b zusätzlich die Informationsquellen an, mit deren Leistungsfähigkeit Sie in bezug auf Informationen zum Thema „Gesamtwirtschaft”, „Branchen” oder "Außenhandel" besonders zufrieden sind! 
6 Wenn Sie einmal die Situation in Ihrem Unternehmen betrachten, dann können Sie die Versorgung mit unternehmensexternen Informationen unter verschiedenen Blickwinkeln beurteilen. In mancher Hinsicht wird sie lhren Anforderungen entsprechen, in mancher hingegen weniger.

Im folgenden legen wir Ihnen einige Anforderungsmerkmale der Informationsversorgung vor. Wir möchten von Ihnen wissen, welche dieser Merkmale für Ihr Unternehmen zutreffen, und für wie wichtig Sie die einzelnen Merkmale halten.

6 Informationen über Gesamtwirtschaft/Konjunktur

\begin{tabular}{|c|c|c|c|c|c|c|c|c|c|c|c|}
\hline & \multicolumn{6}{|c|}{ o IST es } & \multicolumn{5}{|c|}{ so WICHTIG istes } \\
\hline & $\begin{array}{l}\text { triffet } \\
\text { zehr }\end{array}$ & $\begin{array}{l}\text { erifft } \\
\text { überwiegend } \\
\text { zu }\end{array}$ & $\begin{array}{l}\text { erifft } \\
\text { etwea } \\
\text { zu }\end{array}$ & $\begin{array}{l}\text { trifft } \\
\text { koum } \\
\text { zu }\end{array}$ & $\begin{array}{l}\text { erifft } \\
\text { nicht } \\
\text { zu }\end{array}$ & & $\begin{array}{l}\text { ist } \\
\text { oxtrem } \\
\text { wichtig }\end{array}$ & $\begin{array}{l}\text { ist } \\
\text { sehr } \\
\text { wichtig }\end{array}$ & $\begin{array}{l}\text { ist } \\
\text { ziemlich } \\
\text { wichtig }\end{array}$ & $\begin{array}{l}\text { ist } \\
\text { wonnger } \\
\text { wichtrg }\end{array}$ & $\begin{array}{l}\text { ist uberhaupt } \\
\text { nicht } \\
\text { wichtig }\end{array}$ \\
\hline $\begin{array}{l}\text { Die Informationen sind zuver. } \\
\text { lätasio und genau }\end{array}$ & 0 & 0 & o & o & 0 & 49 & 0 & 0 & 0 & o & 0 \\
\hline $\begin{array}{l}\text { Die Informationen sind um- } \\
\text { fassend - os werden viele } \\
\text { Randinformationen mitge- } \\
\text { liefort }\end{array}$ & 0 & 0 & 0 & 0 & 0 & & 0 & 0 & 0 & 0 & 0 \\
\hline $\begin{array}{l}\text { Die Beschaffung der Infor- } \\
\text { mationen ist ohne bessondere } \\
\text { dokumentarische EDV. } \\
\text { Kenntnisse moglich }\end{array}$ & o & 0 & 0 & 0 & 0 & & 0 & 0 & 0 & 0 & 0 \\
\hline $\begin{array}{l}\text { Die Informationen stehen } \\
\text { schnell zur Verfügung }\end{array}$ & 0 & 0 & 0 & 0 & 0 & & 0 & 0 & 0 & 0 & 0 \\
\hline $\begin{array}{l}\text { Die Informationen sind preis. } \\
\text { wort zu erhalten }\end{array}$ & 0 & 0 & 0 & 0 & 0 & & 0 & 0 & 0 & 0 & 0 \\
\hline $\begin{array}{l}\text { Die Informationen sind über. } \\
\text { sichtlich und leicht verständ. } \\
\text { lich }\end{array}$ & 0 & 0 & 0 & 0 & 0 & & 0 & 0 & 0 & 0 & 0 \\
\hline $\begin{array}{l}\text { Die Informationen sind ziel. } \\
\text { genau - es mussen keine irre. } \\
\text { levanten Informationen ge- } \\
\text { sichtet werden }\end{array}$ & 0 & o & 0 & 0 & 0 & & 0 & 0 & 0 & 0 & 0 \\
\hline $\begin{array}{l}\text { Die Informationen sind } \\
\text { aktuell }\end{array}$ & 0 & 0 & 0 & 0 & 0 & & 0 & 0 & 0 & 0 & 0 \\
\hline $\begin{array}{l}\text { Die Informationen sind leicht } \\
\text { archivierbar }\end{array}$ & 0 & 0 & 0 & o & 0 & & o & 0 & o & 0 & 0 \\
\hline $\begin{array}{l}\text { Die Informationen sind zweck. } \\
\text { ontsprechend aufbereitet }\end{array}$ & 0 & 0 & 0 & 0 & 0 & & 0 & 0 & 0 & 0 & 0 \\
\hline $\begin{array}{l}\text { Die Informationen sind objek. } \\
\text { tiv - sie be inhalten keine sub. } \\
\text { jektiven Aussagen }\end{array}$ & 0 & 0 & 0 & 0 & 0 & & 0 & 0 & o & 0 & 0 \\
\hline $\begin{array}{l}\text { Die Informationen stehen ex. } \\
\text { klusiv zur verfugung }\end{array}$ & 0 & 0 & 0 & 0 & 0 & & 0 & 0 & 0 & 0 & 0 \\
\hline $\begin{array}{l}\text { Die Informationen beinhal. } \\
\text { ten auch Antworten auf fach. } \\
\text { gebietsubergreifende Fragen }\end{array}$ & 0 & 0 & 0 & 0 & 0 & & 0 & 0 & 0 & 0 & 0 \\
\hline $\begin{array}{l}\text { Die Informationen konnen in } \\
\text { der eigenen EDV mit verar. } \\
\text { beitet werden }\end{array}$ & 0 & 0 & 0 & 0 & 0 & 622 & 0 & 0 & 0 & 0 & 0 \\
\hline
\end{tabular}


$6 b$ Informationen über Branchen/Märkte

\begin{tabular}{|c|c|c|c|c|c|c|c|c|c|c|c|}
\hline & \multicolumn{6}{|c|}{ so IS T es } & \multicolumn{5}{|c|}{ so WICHTIG istes } \\
\hline & $\begin{array}{l}\text { trifft } \\
\text { sehr } \\
2 u\end{array}$ & $\begin{array}{l}\text { trifft } \\
\text { ubterwiegend } \\
\text { zu }\end{array}$ & $\begin{array}{l}\text { triffe } \\
\text { etwas } \\
\text { zu }\end{array}$ & $\begin{array}{l}\text { trifft } \\
\text { kaum } \\
\text { zu }\end{array}$ & $\begin{array}{l}\text { trifft } \\
\text { nicht } \\
\text { zu }\end{array}$ & & $\begin{array}{l}\text { ist } \\
\text { extrem } \\
\text { wicheig }\end{array}$ & $\begin{array}{l}\text { ist } \\
\text { whr } \\
\text { wichtig }\end{array}$ & $\begin{array}{l}\text { ist } \\
\text { ziemlich } \\
\text { wichtig }\end{array}$ & $\begin{array}{l}\text { ist } \\
\text { weniger } \\
\text { wichtig }\end{array}$ & $\begin{array}{l}\text { ist überhoupt } \\
\text { nicht } \\
\text { wichtig }\end{array}$ \\
\hline $\begin{array}{l}\text { Die Informationen sind zuver- } \\
\text { lässig und genau }\end{array}$ & 0 & 0 & 0 & 0 & 0 & 7 & 0 & 0 & 0 & 0 & 0 \\
\hline $\begin{array}{l}\text { Die Informationen sind um } \\
\text { fasend - es werden viele } \\
\text { Randinformationen mitgo- } \\
\text { liefort }\end{array}$ & 0 & 0 & 0 & 0 & 0 & & 0 & 0 & 0 & 0 & 0 \\
\hline $\begin{array}{l}\text { Die Beschaffung der Infor- } \\
\text { metionen ist oonne besondere } \\
\text { dokumentar ische EDV. } \\
\text { Kenntnisee möglich }\end{array}$ & 0 & 0 & 0 & 0 & 0 & & 0 & 0 & 0 & 0 & 0 \\
\hline $\begin{array}{l}\text { Die Informationen stehen } \\
\text { schnell zur Verfügung }\end{array}$ & o & 0 & 0 & 0 & 0 & & 0 & 0 & $\mathbf{0}$ & $\mathbf{0}$ & 0 \\
\hline $\begin{array}{l}\text { Die informationen sind preis- } \\
\text { wert zu erhalten }\end{array}$ & 0 & 0 & 0 & 0 & 0 & & 0 & 0 & 0 & 0 & 0 \\
\hline $\begin{array}{l}\text { Die Informationen sind über. } \\
\text { sichtlich und leicht verständ- } \\
\text { lich }\end{array}$ & 0 & 0 & 0 & 0 & 0 & & 0 & 0 & 0 & 0 & 0 \\
\hline $\begin{array}{l}\text { Die Informationen sind ziel- } \\
\text { genau - os müssen keine irre- } \\
\text { levanten Informmotionen ge- } \\
\text { sichtet werden }\end{array}$ & 0 & 0 & 0 & 0 & 0 & & 0 & 0 & 0 & 0 & 0 \\
\hline $\begin{array}{l}\text { Die Informationen sind } \\
\text { aktuell }\end{array}$ & 0 & 0 & o & 0 & o & & 0 & o & o & 0 & 0 \\
\hline $\begin{array}{l}\text { Die Informationen sind leicht } \\
\text { archivierbar }\end{array}$ & o & o & o & o & o & & 0 & 0 & 0 & 0 & 0 \\
\hline $\begin{array}{l}\text { Die Informationen sind zweck- } \\
\text { entsprechend aufbereitet }\end{array}$ & 0 & o & 0 & 0 & o & & 0 & 0 & 0 & 0 & 0 \\
\hline $\begin{array}{l}\text { Die Informationen sind objek- } \\
\text { tiv - sie beinhatten keine sub- } \\
\text { jektiven Aussagen }\end{array}$ & 0 & 0 & 0 & 0 & 0 & & 0 & 0 & 0 & 0 & 0 \\
\hline $\begin{array}{l}\text { Die Informationen stehen ex. } \\
\text { ktusiv zur Verfügung }\end{array}$ & 0 & 0 & 0 & 0 & 0 & & 0 & 0 & 0 & 0 & 0 \\
\hline $\begin{array}{l}\text { Die Informationen beinhal- } \\
\text { ten auch Antworten ayf fach. } \\
\text { gebietsibergreifonde Fragen }\end{array}$ & 0 & 0 & 0 & 0 & 0 & & 0 & 0 & 0 & 0 & 0 \\
\hline $\begin{array}{l}\text { Die Informationen können in } \\
\text { der eigenen EDV mit verar- } \\
\text { beitet werden }\end{array}$ & 0 & 0 & 0 & 0 & 0 & 20 & 0 & 0 & 0 & 0 & 0 \\
\hline
\end{tabular}




\begin{tabular}{|c|c|c|c|c|c|c|c|c|c|c|c|}
\hline & \multicolumn{6}{|c|}{ so I S T es } & \multicolumn{5}{|c|}{ s WICHTIG wes } \\
\hline & $\begin{array}{l}\text { trifft } \\
\text { su }\end{array}$ & $\begin{array}{l}\text { eriffe } \\
\text { iberwiegend } \\
\text { zu }\end{array}$ & $\begin{array}{l}\text { trifft } \\
\text { otwes } \\
\text { zu }\end{array}$ & $\begin{array}{l}\text { telffe } \\
\text { koum } \\
\text { zu }\end{array}$ & $\begin{array}{l}\text { trifft } \\
\text { nichit } \\
20\end{array}$ & & $\begin{array}{l}\text { ist } \\
\text { extrem } \\
\text { wichtio }\end{array}$ & $\begin{array}{l}\text { let } \\
\text { whr } \\
\text { wichtip }\end{array}$ & $\begin{array}{l}\text { ist } \\
\text { ziomlich } \\
\text { wichtio }\end{array}$ & $\begin{array}{l}\text { ist } \\
\text { wonioer } \\
\text { wehtig }\end{array}$ & $\begin{array}{l}\text { Int überheupt } \\
\text { nicht } \\
\text { wichtio }\end{array}$ \\
\hline $\begin{array}{l}\text { Die Informotionen sind zuver- } \\
\text { thesio und geneu }\end{array}$ & 0 & $\mathbf{0}$ & o & 0 & 0 & is & 0 & 0 & 0 & 0 & 0 \\
\hline $\begin{array}{l}\text { Die Informetionon sind um } \\
\text { fassend - es werden viale } \\
\text { Randinformationen mitge- } \\
\text { liefert }\end{array}$ & 0 & 0 & 0 & 0 & 0 & & 0 & 0 & o & 0 & 0 \\
\hline $\begin{array}{l}\text { Die Bescheffune der Infor. } \\
\text { mationen lat ohne besondere } \\
\text { dokumenterhche EDV. } \\
\text { Kenntnises móglich }\end{array}$ & 0 & 0 & 0 & 0 & 0 & & 0 & 0 & 0 & 0 & 0 \\
\hline $\begin{array}{l}\text { Die Informotionen stehen } \\
\text { schnell zur Verfioung }\end{array}$ & 0 & 0 & 0 & 0 & 0 & & 0 & 0 & 0 & 0 & 0 \\
\hline $\begin{array}{l}\text { Die Informationen alnd preis. } \\
\text { wert zu erhalten }\end{array}$ & 0 & 0 & 0 & 0 & 0 & & 0 & 0 & 0 & 0 & 0 \\
\hline $\begin{array}{l}\text { Die Informationen sind iber- } \\
\text { sichtlich und leicht verstïnd- } \\
\text { lich }\end{array}$ & $\mathbf{0}$ & $\mathbf{0}$ & $\mathbf{0}$ & 0 & 0 & & 0 & o & 0 & O & o \\
\hline $\begin{array}{l}\text { Die Informationen sind ziel. } \\
\text { genau - es müsen keine lrre- } \\
\text { levanten Informationen ge- } \\
\text { sichtet worden }\end{array}$ & c & 0 & 0 & 0 & 0 & & 0 & 0 & 0 & 0 & 0 \\
\hline $\begin{array}{l}\text { Die Informationen sind } \\
\text { oktuell }\end{array}$ & 0 & 0 & 0 & 0 & 0 & & 0 & 0 & 0 & 0 & 0 \\
\hline $\begin{array}{l}\text { Die Informationen sind loicht } \\
\text { archivierber }\end{array}$ & 0 & 0 & 0 & 0 & 0 & & 0 & 0 & 0 & 0 & 0 \\
\hline $\begin{array}{l}\text { Die Informationen sind zweck- } \\
\text { ent sprechend oufber eitet }\end{array}$ & 0 & 0 & 0 & 0 & 0 & & 0 & 0 & 0 & 0 & 0 \\
\hline $\begin{array}{l}\text { Die Informationen sind objek. } \\
\text { tin - sie beinhalten keine sub. } \\
\text { jektiven Aussagen }\end{array}$ & 0 & 0 & 0 & 0 & 0 & & 0 & 0 & 0 & 0 & 0 \\
\hline $\begin{array}{l}\text { Die Informationen stehen ox- } \\
\text { klugiv zur Verfïgung }\end{array}$ & 0 & 0 & 0 & 0 & 0 & & 0 & 0 & 0 & 0 & 0 \\
\hline $\begin{array}{l}\text { Die Informationen beinhal. } \\
\text { ten auch Antworten auf foch. } \\
\text { gebietsubergreifende Fragen }\end{array}$ & 0 & 0 & 0 & 0 & 0 & & 0 & 0 & 0 & 0 & 0 \\
\hline $\begin{array}{l}\text { Die informationen können in } \\
\text { der eigenen EDV mit verar. } \\
\text { beitet werden }\end{array}$ & 0 & 0 & 0 & 0 & 0 & 48 & 0 & 0 & 0 & 0 & 0 \\
\hline
\end{tabular}


7 Denken Sie bitte einıal an die Praxis Ihres Unternehmens. Wieviel Zeit wird nach Ihren Erfahrungen pro Woche für die Beschaffung, Sichtung, Auswertung und Archivierung von Informationen verwandt. die von außerhalb des eigenen Unternehmens beschafft werden müssen:
8 Denken Sie bitte einmal an die Informationen, die im Bereich "Gesamt wirtschaft", "Branchen/Märkte" und "Außenhandel/Export" jedes Jahr von Ihrem Unternehmen käuflich erworben werden. Welche Größsenordnung nehmen die Kosten solcher Informationen im Durchschnitt (ohne Personalkosten) ein?

\begin{tabular}{|c|c|c|c|}
\hline & 63 & & 65 \\
\hline & $\begin{array}{l}\text { Gorememewireschoft/ } \\
\text { Konjunktur }\end{array}$ & $\begin{array}{l}\text { Brontron/ } \\
\text { Mutrkio }\end{array}$ & $\begin{array}{l}\text { Aubenthendol/ } \\
\text { Export }\end{array}$ \\
\hline $\begin{array}{l}\text { bis } 4 \text { Stunden } \\
\text { (1/2 Menntag Nochel }\end{array}$ & 0 & 0 & 0 \\
\hline $\begin{array}{l}4 \text { bis unter } 8 \text { Stunden } \\
\text { (1 Manntag Wochel }\end{array}$ & 0 & 0 & 0 \\
\hline $\begin{array}{l}8 \text { bis unter } 20 \text { Stundon } \\
\text { 11 Holbtegskreft) }\end{array}$ & o & o & 0 \\
\hline $\begin{array}{l}20 \text { bis unter } 40 \text { Stunden } \\
\text { i1 Vollzeitkrofte) }\end{array}$ & o & o & 0 \\
\hline $\begin{array}{l}40 \text { bls unter } 80 \text { Stundon } \\
12 \text { Vollzeliksöftel }\end{array}$ & 0 & o & o \\
\hline $\begin{array}{l}80 \text { bh unter } 200 \text { Stunden } \\
\text { (5 Vollzoitkrüfte) }\end{array}$ & 0 & 0 & 0 \\
\hline Uber 200 Stunden & 0 & 0 & 0 \\
\hline
\end{tabular}

66

\begin{tabular}{|c|c|c|c|}
\hline & $\begin{array}{c}\text { Gesamtwirtschaft/ } \\
\text { Konjunktur }\end{array}$ & $\begin{array}{l}\text { Branchen/ } \\
\text { M̈̈rte }\end{array}$ & $\begin{array}{c}\text { AuBsenhandel/ } \\
\text { Export }\end{array}$ \\
\hline unter 1.000 DM & 0 & 0 & 0 \\
\hline 1.000 bis unter 5.000 DM & 0 & 0 & 0 \\
\hline 5.000 bis unter 10.000 DM & 0 & 0 & 0 \\
\hline 10.000 bis unter 50.000 DM & 0 & 0 & 0 \\
\hline 50.000 bis unter 100.000 DM & 0 & 0 & 0 \\
\hline 100.000 bis unter 500.000 DM & 0 & 0 & 0 \\
\hline über 500.000 DM & 0 & 0 & 0 \\
\hline
\end{tabular}

Besteht in Ihrem Unternehmen ein spezielles Budget für den Erwerb unternehmensexterner Informationen?

\begin{tabular}{|l|c|}
\hline \multicolumn{2}{|c|}{69} \\
\hline$-j a:$ unternehmensibergreifend & 0 \\
\hline$-j a$ : abteilungsspezifisch & 0 \\
\hline- nein & 0 \\
\hline
\end{tabular}


9 Wären Sie grundsätzlich bereit, die Dienste eines kommerziellen elektronischen Informationsdienstes bei der Beschaffung von Informationen aus den unten genannten Bereichen in Anspruch zu nehmen?

\begin{tabular}{|c|c|c|c|c|}
\hline & $\begin{array}{l}\text { ja. } \\
\text { bestimmt } \\
\text { berelit }\end{array}$ & $\begin{array}{l}\text { Jo. } \\
\text { wahrecheinlich } \\
\text { berait }\end{array}$ & $\begin{array}{l}\text { noin, } \\
\text { wahricheinlich } \\
\text { niche bereit }\end{array}$ & $\begin{array}{l}\text { nein, } \\
\text { bestimmt } \\
\text { niche bereite }\end{array}$ \\
\hline $\begin{array}{l}\text { Gevaminirtechoft/ } \\
\text { Konjunktur }\end{array}$ & 0 & 0 & 0 & 0 \\
\hline $\begin{array}{l}\text { Branchen/ } \\
\text { Mirkte }\end{array}$ & 0 & 0 & 0 & 0 \\
\hline $\begin{array}{l}\text { Aubsenhondel/ } \\
\text { Export }\end{array}$ & 0 & 0 & 0 & 0 \\
\hline
\end{tabular}

10 Uns interessiert die Bedeutung, die in Ihrem Unternehmen den Kosten für die Beschaffung von Informationen beigemessen wird. Gehen Sie bitte einmal von unterschiedlichen Kostengrößenklassen für die Nutzung eines elektronischen Informationsdienstes aus, wie er idealerweise Ihren Ansprüchen entspräche. Bis zu welcher Kostengrößenklasse würden Sie diesen Dienst dem Weg der herkömmlichen Informationsquellennutzung vorziehen? Bitte geben Sie Ihre Entscheidung für jede Kostengrößenklasse an und berücksichtigen Sie dabei bitte Ihre bisherigen Personalkosten ( $\mathrm{Vgl}$. Frage 8).

\begin{tabular}{|c|c|c|c|c|}
\hline $\begin{array}{l}\text { Kosten/Jahr für die Inan. } \\
\text { spruchnahme des Informetions- } \\
\text { vermlttlungedienntes }\end{array}$ & $\begin{array}{l}\text { herkömmliche Quellen } \\
\text { sind eindeutig zu bo- } \\
\text { vor zugen }\end{array}$ & $\begin{array}{l}\text { herkömmliche Quellion } \\
\text { sind tendenziell zu } \\
\text { bevor zugen }\end{array}$ & $\begin{array}{l}\text { der Informetions. } \\
\text { ver mittlungydienst } \\
\text { ist tendenzioll zu } \\
\text { bevorzugen }\end{array}$ & $\begin{array}{l}\text { der Informatiom. } \\
\text { vermitllungedienst } \\
\text { ist eindeutio zu } \\
\text { bevorzugen }\end{array}$ \\
\hline mohr als $10 \%$ Minderkosten & 0 & 0 & 0 & 0 \\
\hline gleiche Kosten & 0 & 0 & 0 & 0 \\
\hline$+10 \%$ Mehrkosten & 0 & 0 & 0 & 0 \\
\hline + 50\% Mehrkosten & 0 & 0 & 0 & 0 \\
\hline$+100 \%$ Mohrkoston & 0 & 0 & o & 0 \\
\hline mehr als $100 \%$ Mehrkosten & 0 & 0 & 0 & o \\
\hline
\end{tabular}


11 Stellen Sie sich bitte vor, in Ihrem Unternehmen stehe die Inanspruchnahme eines elektronischen Informa. tionsdienstes zur Diskussion. Welche Stelle wlirde die Entscheidung fallen?

\begin{tabular}{|c|c|c|c|c|c|c|c|c|}
\hline $\begin{array}{l}\text { Informationen } \\
\text { ther... }\end{array}$ & $\begin{array}{l}\text { Geachäfte- } \\
\text { fohrung }\end{array}$ & $\begin{array}{l}\text { Organizations-/ } \\
\text { Verwaltung- } \\
\text { ebtoilung }\end{array}$ & $\begin{array}{l}\text { EDV-Ab- } \\
\text { tollung }\end{array}$ & $\begin{array}{l}\text { Verkaufs-I } \\
\text { Marketing- } \\
\text { abteilung }\end{array}$ & $\begin{array}{l}\text { Markt. } \\
\text { forschungs. } \\
\text { ebteilung }\end{array}$ & $\begin{array}{l}\text { Informe- } \\
\text { tonteb- } \\
\text { tellune }\end{array}$ & Sonst & \\
\hline $\begin{array}{l}\text { Gesemtwireschoft/ } \\
\text { Konjunktur }\end{array}$ & 0 & 0 & 0 & 0 & 0 & 0 & 0 & 7 \\
\hline $\begin{array}{l}\text { Branchen/ } \\
\text { Markte }\end{array}$ & 0 & 0 & 0 & 0 & 0 & 0 & 0 & 8 \\
\hline $\begin{array}{l}\text { Aubenhandel/ } \\
\text { Export }\end{array}$ & 0 & 0 & 0 & 0 & 0 & 0 & 0 & , \\
\hline
\end{tabular}

I1b wie würde die Entscheidung gefällt?

\begin{tabular}{|l|l|}
\hline & 10 \\
\hline- Unter Elnschaltumg eines Beroters & 0 \\
\hline - Hewsintorn ohne externe Unterstüzzuns & 0 \\
\hline
\end{tabular}

12 In welcher Branche ist Ihr Unternehmen tätig?

\begin{tabular}{|c|c|}
\hline - Mander & $\mathbf{0}$ \\
\hline - Dienstleistung & 0 \\
\hline - Verbreuchs-/Konsumgüter industrio & 0 \\
\hline - Grundatoff-/Investitionmütterindustrie & 0 \\
\hline
\end{tabular}

13 Besteht die Möglichkeit, Infonnationen von einem verbundenen Unternehmen (Konzernmutter o.ä.) zu beziehen?

\begin{tabular}{|c|c|}
\hline \multicolumn{1}{c}{12} & 12 \\
\hline- nein & 0 \\
\hline
\end{tabular}

14 Welche technische Ausstattung ist in Ihrem Unternehmen vorhanden?

\begin{tabular}{|c|c|c|c|}
\hline & $\begin{array}{l}\text { vor- } \\
\text { handen }\end{array}$ & $\begin{array}{l}\text { ge- } \\
\text { plant }\end{array}$ & $\begin{array}{l}\text { nicht } \\
\text { geplant }\end{array}$ \\
\hline - Telex & 0 & 0 & 0 \\
\hline $\begin{array}{l}\text { - Toletex (Speicherschreibmaschine zur obok- } \\
\text { wonischen Textübertragung) }\end{array}$ & o & 0 & o \\
\hline - Tolefox/Fornkoplerer & 0 & 0 & 0 \\
\hline - Bildschirmtext & 0 & 0 & $\mathbf{0}$ \\
\hline - EDV & 0 & 0 & 0 \\
\hline $\begin{array}{l}\text { - Datenterminals mit AnschluB zur Daten- } \\
\text { fernubertragung (Dotex P. Dotex L. Stand- } \\
\text { loitung) }\end{array}$ & 0 & o & 0 \\
\hline
\end{tabular}


15 Wie viele Beschastigte arbeiten in Ihrem Unternehmen?

\begin{tabular}{|c|c|c|}
\hline 1 & 19 & 0 \\
\hline 20 & 48 & 0 \\
\hline 60 & 99 & 0 \\
\hline 100 & 199 & 0 \\
\hline 200 & 499 & 0 \\
\hline 500 & - 890 & 0 \\
\hline 1.000 & $-\quad 1.989$ & 0 \\
\hline 2.000 & $-\quad 2.899$ & 0 \\
\hline 3.000 & und mothr & 0 \\
\hline
\end{tabular}

$16 \mathrm{Zu}$ welcher Umsatz-Kategorie gehörte Ihr Unternehmen im letzten Jahr?

\begin{tabular}{|ccccc|}
\hline - unter 500 Tsd. DM & & 20 \\
- & 500 Ted. bis unter & 1 Mlo OM & 0 \\
- & 1 & bls unter & 2 Mlo DM & 0 \\
- & 2 & bls unter & 5 Mlo DM & 0 \\
- & 5 & bls unter 50 Mlo DM & 0 \\
- & 50 & bls unter 100 Mlo DM & 0 \\
- & 100 & bis unter 200 Mlo DM & 0 \\
- & 200 & bls unter 300 Mlo DM & 0 \\
- & 300 und mehr & Mlo DM & 0 \\
\hline
\end{tabular}

17 Welche Position haben Sie in Ihrem Unternehmen?

\begin{tabular}{|c|c|}
\hline - Geschäftsfiuhrer/Inhobar & 0 \\
\hline - Orgenizations./Nonwel tungedeiter & 0 \\
\hline - Merketingletter & o \\
\hline - EOV.Leiter & 0 \\
\hline - Informetionsebteilungs-Leiter & 0 \\
\hline - Sonstiper Abtollungsileiter & o \\
\hline - Sonstige & o \\
\hline
\end{tabular}

18 Welche Erfahrungen haben Sie mit ...

\begin{tabular}{|c|c|c|c|c|c|}
\hline & $\begin{array}{l}\text { Speziet. } \\
\text { kenntnise }\end{array}$ & $\begin{array}{l}\text { umisosendo } \\
\text { Kenntnizese }\end{array}$ & $\begin{array}{l}\text { durchschnittilcho } \\
\text { Kenntnizes }\end{array}$ & geringe & $\begin{array}{l}\text { keine } \\
\text { Kenntnise }\end{array}$ \\
\hline $\begin{array}{l}\text { - externen Informetions } \\
\text { quellen (Handelakemmer } \\
\text { Verbend, Banken) }\end{array}$ & $\mathbf{0}$ & $\mathbf{0}$ & 0 & $\mathbf{0}$ & 0 \\
\hline - Informationsdrembenken & 0 & 0 & 0 & 0 & 0 \\
\hline $\begin{array}{l}\text { - olektronischen Modion/ } \\
\text { Nechr ichtentechnik }\end{array}$ & 0 & o & 0 & 0 & 0 \\
\hline
\end{tabular}

19 Anmerkungen zum Thema der Befragung 


\section{Literaturverzeichnis}

Abell, D.F.

Abell, D.F.

Abell, D.F., Hammond, J.S.

Ackoff, R.L.

Ahlert, D.
Abel, V.

Alber, A.F.

Problemorientiertes Informationsverhalten, Darmstadt 1979.

Strategic Windows, in: JoM, Vol. 42 , July 1978, No. 3, S. 21-26.

Defining the Business. The Starting Point of Strategic Planning, Englewood Cliffs, N.J. 1980.

Strategic Planning, Englewood Cliffs, N.J. 1979.

Management Misinformation Systems, in: MS, Vol. 14, 1967, No. 4, S. B $147 \mathrm{ff}$.

Grundzüge des Marketing, 2. Auf1., Düs seldorf 1980 .

Get Ready for Electronic Publishing, in: BH, Vol. 26, January-February 1983, S. $51-55$.

Albers, W. et al. Handwörterbuch der Wirtschaftswissen(Hrsg.) schaft, Stuttgart, New York etc. 1978.

Allen, M.G.

Strategic Planning with a competitive focus, in: The Mckinsey Quarterly, Autumn 1978.

Amstutz, A.E. Market-Oriented Management Systems: The Current Status, in: JoMR, Vol. 6, November 1969, S. 481-496.

Anderson, W.Th.Jr.,Bank Selecting Decisions and Market

Cox, E.P.,

Segmentation, in: JoM, Vol. 40, January

Fulcher, D.G. 1976, Nr. 1, S. 40-45

Andritzky, $k$.

Der Einsatz von Scoringmodellen für die Punktbewertung, in: DU, 30. Jg., 1976, H. 1 , S. 21-37

Ansoff, H.J. Corporate Strategy, New York u.a. 1965.

Arbeitskreis langfristige Unternehmensplanung der Schmalenbach-Gesellschaft, Strategische Planung, in: ZfbF, 29. Jg., 1977, S. $1-20$. 


\begin{tabular}{|c|c|}
\hline Backhaus, $K$. & $\begin{array}{l}\text { Investitionsgüter-Marketing, München } \\
1982 \text {. }\end{array}$ \\
\hline Balke, S. & $\begin{array}{l}\text { Benutzerprobleme der Dokumentation und } \\
\text { Information, in: NfD, } 24 . \mathrm{Jg} ., 1973 \text {, } \\
\mathrm{Nr} .1, \mathrm{~S} .2-9 .\end{array}$ \\
\hline Bartsch, J. & $\begin{array}{l}\text { Organisatorische Probleme bei der Ent- } \\
\text { wicklung und Handhabung computergestütz- } \\
\text { ter Management-Informationssysteme in } \\
\text { divisionalisierten Unternehmungen - dar- } \\
\text { gestellt am Beispiel von Marketing-In- } \\
\text { formationssystemen, Diss. Mijnster } 1976\end{array}$ \\
\hline $\begin{array}{l}\text { Bass, F.M., } \\
\text { Wilkie, W.L. }\end{array}$ & $\begin{array}{l}\text { A Comparative Analysis of Attitutional } \\
\text { Predictions of Brand Preference, in: } \\
\text { JoMR, Vol. 10, August 1973, Nr. 3, S. 262-268. }\end{array}$ \\
\hline Bateson, J.E.G. & $\begin{array}{l}\text { Why we need service marketing, in: } \\
\text { Ferrell, 0.C., Brown, St.W. Lamb, C.W. } \\
\text { jr. (Hrsg.), Conceptual And Theoretical } \\
\text { Developments in Marketing, Chicago, } \\
\text { Illinois } 1979 \text {, S. } 131-146 \text {. }\end{array}$ \\
\hline Bauer, E. & $\begin{array}{l}\text { Markt-Segmentierung als Marketing-Stra- } \\
\text { tegie, Berlin } 1976 \text {. }\end{array}$ \\
\hline Bearchell, C.A. & $\begin{array}{l}\text { Information handling - as product or as } \\
\text { service, in: MJ, } 13 . \mathrm{Jg} ., 1980, \mathrm{H} .3 \text {, } \\
\text { S. } 226 .\end{array}$ \\
\hline Bearchel1, C.A. & $\begin{array}{l}\text { Information as Resource - Its Importance } \\
\text { is Growing Ever Greater, in: MJ, 16. Jg., } \\
1983 \text {, H. 1, S. } 24 \text {. }\end{array}$ \\
\hline Bellomy, F.o. & $\begin{array}{l}\text { Die privatwirtschaftliche Informations- } \\
\text { versorgungsszene (information brokerage) } \\
\text { in den USA, in: NfD, 30. Jg., 1979, } \\
\text { Nr. 1, S. 17-20. }\end{array}$ \\
\hline Berekoven, L. & $\begin{array}{l}\text { Die Dienstleistungs-Generation - Erbin } \\
\text { der Industriegesellschaft?, in: asw, } \\
\text { Sonderausgabe, 1979, Nr. 10, S. 6-15. }\end{array}$ \\
\hline Berekoven, $L$. & $\begin{array}{l}\text { Dienstleistungsmärkte - Die Wachstums- } \\
\text { hoffnung der } 80 \text { er Jahre?, in: Marken- } \\
\text { artikel, } 1983, \mathrm{H} .6, \mathrm{~S} .314-316 .\end{array}$ \\
\hline Bernhardt, $U$. & $\begin{array}{l}\text { Bestandsaufnahme der in der Bundesrepu- } \\
\text { blik Deutschland existierenden Stellen } \\
\text { mit Informationsvermittlungs- und -be- } \\
\text { ratungsfunktion, Forschungsbericht } \\
10 \text { 80-019 des Bundesministeriums furr } \\
\text { Forschung und Technologie, August } 1980 \text {. }\end{array}$ \\
\hline
\end{tabular}


Bernhardt, $U_{\dot{K}}$,

Berthel, J.

Berthel, J.

Bettmann, J.R.,

Capon, N.,

Lutz, R.J.

Beutel, P. et al.

Beyer, $W$.

Bleicher, $K$.
Bestandsaufnahme von Stellen mit Informationsvermittlungs - und -beratungsfunktion in der Bundesrepublik Deutschland, in: NfD, 31. Jg., 1980, Nr. 415, S. $155-159$.

Information, in: Handwörterbuch der Betriebswirtschaft, 4. Aufl., Hrsg.:

Grochla, E., Wittmann, W., Stuttgart 1975, Sp. 1865-1873.

Betriebliche Informationssysteme, Stuttgart 1975.

Cognitive Algebra in Multi-Attribute Models, UCLA Working Papers, February 1974, No. 10.

SPSS, 9. Statistik-Programm-System für die Sozialwissenschaften, 5. Aufl., Stuttgart, New York 1983.

Informationsvermittlung in der Bundesrepublik Deutschland. Ein Oberblick, Bd. 12 der Reihe Aktuelle Beiträge und Berichte, Hrsg.: Gesellschaft für Information und Dokumentation mbH (GID), Frankfurt am Main 1982.

Organisation und Führung der industriellen Unternehmung, in: Industriebetriebslehre in programmierter Form, Bd. III, Organisation und EDV, Hrsg.: Jacob, H., Wiesbaden 1972 .

BMFT (Bundesministerium für Forschung und Technologie) (Hrsg.), Programm der Bundesregierung zur Förderung der Information und Dokumentation (IUD-Programm) 1974-1977, Bonn 1974 .

Böcker, F., Erfolgskontrolle im Marketing, Berlin Dichtl, E. (Hrsg.) 1975.

Böhler, H.

Methoden und Modelle der Marktsegmentierung, Stuttgart 1977.

Böhnisch,W.

Personale Widerstände bei der Durchsetzung von Innovationen, Stuttgart 1979.

Bössmann, E.

Information, in: HdW, Hrsg.: Albers, W. et al., Stuttgart, New York etc., 1978, S. 184-200. 

Bräunl ing, G.,
Harmsen, D.M.

Breitung, A.

Breuer, N., Bromann, P.

Briggadike, R.

Brockhoff, $K$.

Brockhoff, $K$.

Brönimann, Ch.

Brose, $P$.

Brose, P., Corsten, H.

Bruns, J.

Bues, M.

Bury, P.
Die Förderungsprinzipien und Instrumente der Forschungs- und Technologiepolitik, Göttingen 1975 .

Kritische Analyse der Validitätsproblematik von Verfahren der Einstellungsmessung. Ein methodologischer Beitrag zum Design von Einsteliungsskalierungen, in: Konsumentenverhalten und Information, Hrsg.: Meffert, H., Steffenhagen, H., Freter, H., Wiesbaden 1979, S. 209-224.

Leerlauf der Systeme? Unzureichende Informationsbas is für Marketing-Entscheider, in: asw, 26. Jg., 1983, H. 8, S. 59-64.

The risky business of diversification, in: HBR, Vol. 47, May-June 1979, S. 103-111.

Experimente zur Nutzung einer Datenbank, in: ZfB, 47. Jg., 1977, H. 8, S. 509-530.

Informationsverarbeitung in Entscheidungsprozessen: Skizze einer Toxonomie, in: $Z f B, 53 . J g ., 1983, H .1$, S. 53-61.

Aufbau und Beurteilung des Kommunikationssystems von Unternehmungen, Schriftenreihe "Führung und Organisation der Unternehmung", Bd. 8, Bern, Stuttgart 1970 .

Planung, Bewertung und Kontrolle technologischer Innovationen, Berlin 1982.

Technologie-Portfolio als Grundlage von Innovations - und Wettbewerbsstrategien, in: JAV, 1983, S. 344-369.

Kritische Analyse des Kontingenz-Ansatzes in der Organisationstheorie, in: $D B$, 1977, H. 3, S. 61-64.

Wirtschaftsinformatik der 90 er Jahre, in: Büro + Verkauf, 53: Jg., Februar 1984, Nr. 2, S. 18-20.

Markets for Prestel, in: 4th International Online Information Meeting, London 9 - 11 December 1980, Hrsg.: Learned Information (Europe) Ltd., Oxford - New Jersey 1980, S. 101-105. 


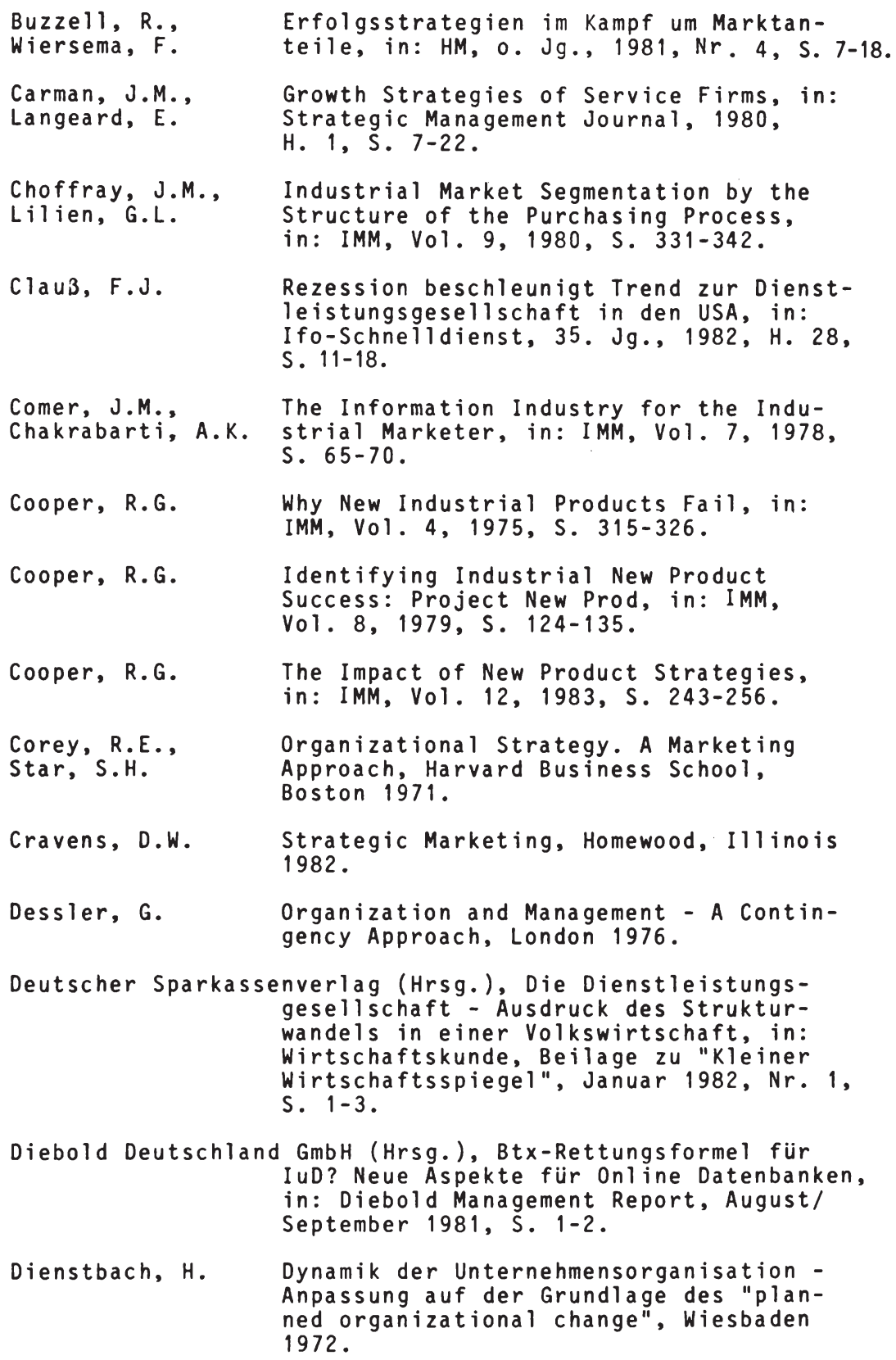

Erfolgsstrategien im Kampf um Marktanteile, in: HM, o. Jg., 1981, Nr. 4, S. 7-18.

Growth Strategies of Service Firms, in: Strategic Management Journal, 1980, H. 1, S. 7-22.

Industrial Market Segmentation by the Structure of the Purchasing Process, in: IMM, Vol. 9, 1980, S. 331-342.

Rezession beschleunigt Trend zur Dienstleistungsgesellschaft in den USA, in: Ifo-Schnelldienst, 35. Jg., 1982, H. 28, S. 11-18.

The Information Industry for the Industrial Marketer, in: IMM, Vol. 7, 1978, S. 65-70.

Why New Industrial Products Fail, in: IMM, Vol. 4, 1975, S. 315-326.

Identifying Industrial New Product Success: Project New Prod, in: IMM, Vol. 8, 1979, S. 124-135.

The Impact of New Product Strategies, in: IMM, Vol. 12, 1983, S. 243-256.

Organizational Strategy. A Marketing Approach, Harvard Business School, Boston 1971.

Strategic Marketing, Homewood, Illinois 1982 .

Organization and Management - A Contingency Approach, London 1976.

Deutscher Sparkassenverlag (Hrsg.), Die Dienstleistungsgesellschaft - Ausdruck des Strukturwandels in einer Volkswirtschaft, in: Wirtschaftskunde, Beilage zu "Kleiner Wirtschaftsspiegel", Januar 1982, Nr. 1 , S. 1-3.

Diebold Deutschland GmbH (Hrsg.), Btx-Rettungsformel für I UD? Neue Aspekte für Online Datenbanken, in: Diebold Management Report, August/ September 1981, S. 1-2.

Dienstbach, H. Dynamik der Unternehmensorganisation Anpassung auf der Grundlage des "planned organizational change", Wiesbaden 1972 . 
Diller, H.

Dirlam, H.-W.,

Langheim, J.

Domeier, $M$.

Donnelly, J.H., George, W.G. (Hrsg.) 1981.

Drewalowski, H.F. Bildschirmtext - ein Medium steigt aus

Drucker, P.F.

Dunst, H.H.

Ehrlicher, W.

Esenwe in-Rothe Jürgensen, H., Rose, K. (Hrsg.)

Eiglier, P., Langeard, E.

Ela, J.D., Irwin, M.R.

Engeleiter, H.J.

Engelhardt, W.H., Guinther, $B$.

Engelhardt, W.H., Schwab, W.

Englert, G.

Enis, B.M., Roering, U.J.

Ettel, W. den Kinderschuhen, in: Praxis der Werbung, 3. Jg., 1983, Nr. 4, S. 11.

Marketing-Informationssysteme, in: WiSt, 5. Jg., 1976, $\mathrm{Nr} .3, \mathrm{~S} .97-104$.

Anforderungen an die Informationsvermittlung in der Fleischwirtschaft, in: NfD, 31. Jg., 1980, S. 177-182.

Beurteilungskriterien formeller Kommunikationssysteme, Diss. Köln 1969.

Marketing of Services, Chicago, Illinois The Effective Executive, London 1966.

Portfolio-Management-Konzeption für eine strategische Unternehmensplanung, Berlin 1979.

Kompendium der Volkswirtschaftslehre, ,5., uiberarbeitete und ergänzte Aufl., Bd. 1, München 1975.

Principes de politique marketing pour les enterprises de services, L'Institut d'Administration des Enterprises, Université d'Aix-Marseille, Dezember 1976.

Technology Changes Market Boundaries, in: IMM, Vol. 12, 1983, S. 153-156.

Die Portfolio-Technik als Instrument der strategischen Planung, in: BFuP, 33. Jg., 1981, H. 5, S. 407-420.

Investitionsgiutermarketing, Stuttgart u.a. 1981 .

Die Beschaffung von investiven Dienstleistungen, in: DBW, 42. Jg., 1982, $\mathrm{Nr} .4$, S. 503-512.

Marketing von Standard-Anwendungssoftware, München 1977.

Services marketing: different products, similar strategy, in: Marketing of Services, Hrsg.: Donnelly, J.H., George, W.G., Chicago, I11. 1981, S. 1-4.

Informationsvermittlung in den Vereinigten Staten, in: NfD, 31. Jg., 1980, $\mathrm{Nr} .4 / 5$, S. 185-189. 
Fellbaum, K.-R. Telekommunikation von $A-Z$, Ausgabe 1981/82, Berlin 1981 .

Ferre11, 0.C.,

Brown, St.W., Lamb, C.W.Jr. (Hrsg.) 1979.

Conceptual And Theoretical Developments in Marketing, Chicago, I11.

Fischer, J.,

Zimmermann, W.

Instrumente der strategischen Planung für Unternehmen mittlerer GröBenordnung, in: $Z f 0,52 . \mathrm{Jg} ., 1983, H .3$, S. 139-144.

Fouratiè, J.

Die große Hoffnung des 20. Jahrhunderts (deutsche obersetzung von: Le grand expoir du XXe siécle, Paris 1963), 2. Aufl., Köln 1969.

Frank, R.E.,

Market Segmentation, Englewood Cliffs, Massy, W.F., Wind, $Y$. N.J. 1972 .

Freter, $\mathrm{H}$.

Interpretation und Aussagewert mehrdimensionaler Einstellungsmodelle im Marketing, in: Konsumentenverhalten und Information, Hrsg.: Meffert, H., Steffenhagen, H., Freter, H., Wiesbaden 1979, S. 163-184.

Freter, $H$.

Marktsegmentierung, Stuttgart usw. 1983.

Gälweiler, A.

Unternehmensplanung, Frankfurt, New York 1974.

Garbe, $H$.

Informationsbedarf, in: $H d B, 4$. Aufl., Hrsg.: Grochla, E., Wittmann, W., Stuttgart 1975, Sp. 1873-1882.

Geist, M. , Die Führung des Betriebes, Festschrift Köhler, R. (Hrsg.) für Curt Sandig zum 80 . Geburtstag, Stuttgart 1981 .

George, W.R., Developing New Services, Chicago, Ill. Marsha11, C.E. 1984. (Hrsg.)

GID (Gesellschaft für Information und Dokumentation m.b.H.)

(Hrsg.), Zugang zur Fachinformation für jedermann, Informationsbroschüre, Frankfurt am Main 1981.

Gore, W.J.

Decision-Making Research: Some Prospects and Limitations, in: Concepts and Issues in Administrative Behaviour, Hrsg.: Mailick, S., von Ness, E.H., Englewood Cliffs, N.J. 1962, S. 49-65. 


\begin{tabular}{|c|c|}
\hline Graumann, J. & $\begin{array}{l}\text { Die Dienstleistungsmarke, München } \\
1983 \text {. }\end{array}$ \\
\hline Green, P.E. & $\begin{array}{l}\text { Analyzing Multivariate Date, Hinsdale } \\
1978 .\end{array}$ \\
\hline $\begin{array}{l}\text { Green, P.E., } \\
\text { Tull, D.S. }\end{array}$ & $\begin{array}{l}\text { Research for Marketing Decisions, } 3 \text {. } \\
\text { Auf1., Englewood Cliffs, N.J. } 1975 .\end{array}$ \\
\hline Greiner, $P$. & $\begin{array}{l}\text { Zentrale oder dezentrale Informations- } \\
\text { versorgung?, in: HM, O. Jg., 1984, } \\
\text { H. 1, S. 70-77. }\end{array}$ \\
\hline Grochla, E. (Hrsg.) & $\begin{array}{l}\text { Handwörterbuch der Organisation, } 2 \text {. } \\
\text { Aufl., Stuttgart } 1980 \text {. }\end{array}$ \\
\hline Grochla, E. & $\begin{array}{l}\text { Die Beteiligung der Unternehmensfüh- } \\
\text { rung an der Gestaltung computer-ge- } \\
\text { stützter Informationssysteme, in: } \\
\text { Fürungsprobleme industrieller Unter- } \\
\text { nehmungen, Festschrift für Friedrich } \\
\text { Thomée zum 60. Geburtstag, Hrsg.: } \\
\text { Hahn, D., Berlin, New York 1980, S. } \\
125-136 \text {. }\end{array}$ \\
\hline $\begin{array}{l}\text { Grochla, E., } \\
\text { Garbe, H. } \\
\text { Giliner, R. }\end{array}$ & $\begin{array}{l}\text { Gestaltungskriterien für den Aufbau } \\
\text { von Datenbanken (Forschungsberichte } \\
\text { des Landes Nordrhein-Westfalen } 2292 \text { ), } \\
\text { Opladen } 1973 \text {. }\end{array}$ \\
\hline $\begin{array}{l}\text { Grochla, E., } \\
\text { Szyperski, N. } \\
\text { (Hrsg.) }\end{array}$ & $\begin{array}{l}\text { Management-Informationssysteme - Eine } \\
\text { Herausforderung an Forschung und Ent- } \\
\text { wicklung, Wiesbaden } 1971 \text {. }\end{array}$ \\
\hline $\begin{array}{l}\text { Grochla, } E_{\text {Wittmann, }} \text {.'(Hrsg.) }\end{array}$ & $\begin{array}{l}\text { Handwörterbuch der Betriebswirtschaft, } \\
\text { 4. Auf1., Stuttgart } 1975 \text {. }\end{array}$ \\
\hline Gröne, A. & $\begin{array}{l}\text { Marktsegmentierung bei Investitions- } \\
\text { guitern, Schriftenreihe "Unternehmens- } \\
\text { führung und Marketing", Bd. 9, Hrsg.: } \\
\text { Meffert, H., Wiesbaden } 1977 \text {. }\end{array}$ \\
\hline Grönroos, Chr. & $\begin{array}{l}\text { A Service-Oriented Approach to Marke- } \\
\text { ting of Services, in: EJoM, Vol. } 12 \text {, } \\
1978 \text {, H. 8, S. 588-601. }\end{array}$ \\
\hline Grönroos, Chr. & $\begin{array}{l}\text { An Applied Theory For Marketing Indu- } \\
\text { strial Services, in: IMM, Vol. } 8 \text {, } \\
1979 \text {, S. } 45-50 \text {. }\end{array}$ \\
\hline Grönroos, Chr. & $\begin{array}{l}\text { An Applied Service Marketing Theory, } \\
\text { in: EJoM, Vol. 16, 1982, H. 7, S. } \\
30-41 \text {. }\end{array}$ \\
\hline
\end{tabular}


Grönroos, Chr.

Gummesson, E.

Gutenberg, E.

Hahn, D. (Hrsg.)

Hammann, P., Kroeber-Riel, W., Meyer, C.W. (Hrsg.) Hammann, P.

Hansen, H.R., Wahl, M.P. (Hrsg.) Heil, G.

He inzelbecker, $K$.

He inzelbecker, $K$.

He inzelbecker, $K$.

Helmstädter, E. Henderson, B.D.
Strategic Management and Marketing in the Service Sector, Report No. 83-104 des Marketing Science Institute (Hrsg.), Cambridge, Massaghusetts, May 1983.

Toward a Theory of Professional Service Marketing, in: IMM, Vol. 7, 1978, S. 89-95.

Grundfragen der Betriebswirtschafts 1ehre, 2. Bd.: Der Absatz, 16. Auf1., Berlin etc. 1979.

Führungsprobleme industrieller Unternehmungen, Festschrift für Friedrich Thomée zum 60. Geburtstag, Berlin, New York 1980.

Neuere Ansätze der Marketingtheorie, Festschrift zum 80 . Geburtstag von otto Schnutenhaus, Berlin 1974.

Zur Integration multivariater Marketinginformationen in Marketingentscheidungsmodellen, in: Marketing - Neue Ergebnisse aus Forschung und Praxis, Hrsg.: Topritzhofer, E., Wiesbaden 1968, S. 29-

Probleme beim Aufbau betrieblicher Informationssysteme, München 1973.

Die Dienstleistungsmarke - erste Erfahrungen und geplante Praxis, in: Markenartike 1, 1979, H. 5, S. 237-245.

Marketing-Informationssysteme heute, in: MJ, 11. Jg., 1978, Nr. 2, S. 133138 .

Ausbaustufen eines EDV-Marketing-Informationssystems, in: ManagementZeitschrift io, 47. Jg., 1978, Nr. 9, S. 403-408.

Aufbau und Implementierung eines Planungs - und Informationssystems für das Marketing in der Investitionsgijterindustrie (POM), Forschungsbericht ID 83-005 des BMFT, Mannheim, Mai 1983.

Wirtschaftstheorie, Bd. 1, München 1974.

Die Erfahrungskurve in der Unternehmensstrategie, Frankfurt, New York 1974. 


\begin{abstract}
Hennemann-Böckels, B. Ausländische Hosts teilen sich den Markt auf. Noch Lücken im Angebot, Teil III der Beitragsreine zum Umgang mit Datenbanken, in: BddW, $0 . N r ., 29.08 .83,0.5$.
\end{abstract}

Hennemann-Böckels, B. Bertelsmann will auch "DatenbankVerleger" werden, Teil XVII der Beitragsreihe zum Umgang mit Datenbanken, in: BddW, 0.Nr., 15.02.84, S. 5 .

Henssler, R. Die Information als Produktionsfaktor, Teil 2 einer Beitragsreihe unter dem Titel "Die Informationselite", in: BddW, O.Nr., 02.03.83, 0.S.

Hilke, W. Dienstleistungs-Marketing aus der Sicht der Wissenschaft, gekürzte Fassung des Grundsatzreferates vom 28. Nov. 1983 im Rahmen des Workshops "Dienstleistungs-Marketing", durchgeführt von der Wissenschaftlichen Gesellschaft für Marketing und Unternehmensführung e.V., Münster/Westfalen.

Hinterhuber, H.H. Wettbewerbsstrategie, Berlin, New York 1982.

Hofer, P., Wolff, H. et al.

Wirksamkeitskriterien einer branchenorientierten Informationsvermittlungs - und Beratungsstelle für den Maschinenbau, Forschungsbericht ID 78-01 des BMFT, Basel, April 1978.

Hoffmann, K.

Die Konkurrenzuntersuchung als Determinante der langfristigen Absatzplanung, Göttingen 1979.

Hoffmann, J.

Die Konkurrenz - Erkenntnisse für die strategische Führung und Planung, in: Praxis für strategische Unternehmensplanung, Hrsg.: Töpfer, A., Afheldt, H., Frankfurt/M. 1983, S. $183 \mathrm{ff}$.

Höring, $K$.

Organisation der Informationsdienstleistungsbetriebe, in: Handwörterbuch der Organisation, 2. Aufl., Hrsg.: Grochla, E., Stuttgart 1980 , Sp. $914-920$. 
Hübner, $H$.

Hupe, R.

IBM (Hrsg.)

Jacob, H. (Hrsg.)

Johnston, W.J., Bonoma, Th.V.

Judd, R.

Ka iser, W.

Katz, R.

Kaufmann, E.J.

Kieser, A. (Hrsg.)

Kieser, A.,

Kubicek, H.
Produktinnovation und Produktion. Möglichkeiten und Grenzen der Nutzung von Erfolgsfaktoren der Produktion für die marktorientierte Innovation, in: Marktorientierte Unternehmensfuhrung: Wiss. Tagung des Verbandes der Hochschullehrer für Betriebswirtschaft, Wirtschaftsuniversität Wien 1983, Hrsg.: Mazanec, I., Scheuch, F., Wien 1984, S. 377-397

Wettlauf um die Super-Märkte, in: MM, 13. Jg., 1983, Nr. 6, S. 112-120.

Das Management des Informationssystems: BISM (Business Information Systems Management), Informationsbroschüre, Frankfurt 1981 .

Industriebetriebslehre in programmierter Form, Wiesbaden 1972.

Purchase Process for Capital Equipment and Services, in: IMM, Vol. 10, 1981 , S. 253-264.

The Case of Redefining Services, in: JoM, Vol. 28, 1964, H. 1, S. 58-59.

Kabeltext und Kabeltextabruf, in: Elektronische Textkommunikation. Vorträge des vom 12. - 15. Juni 1978 in München abgehaltenen Symposiums, Hrsg.: Kaiser, W., Berlin, Heidelberg, New York 1978, S. 70-87.

Informationsquellen der Konsumenten. Eine Analyse der Divergenzen $z$ wischen der Beurteilung und Nutzung, Schriftenreihe Unternehmensführung und Marketing, Bd. 17, Hrsg.: Meffert, H., Steffenhagen, H., Freter, H., Wiesbaden 1983.

Marketing für Produktivdienstleistungen, Frankfurt und Zürich 1977.

Organisationstheoretische Ansätze, München 1981.

Organisation, Berlin 1976. 
Kinder, $k$.

Kirsch, W.

Kirsch, W. (Hrsg.)

Kirsch, W.

Kirsch, w.

Kirsch, W.,

Esser, W. -M.,

Gabele, E.

Klaus, P.

Köhler, R.

Köhler, R.,

He inzelbecker, $K$.

Köhler, R.,

Uebele, H.

Koolwijk, J.V., Wieken-Mayser, M. (Hrsg.)

Koreimann, D.
Bürokommunikation der Zukunft. Ein

Aufgabenfeld fur den Organisator,

in: Zfo, 51. Jg., 1983, H. 1, S.

$11-19$.

Betriebswirtschaftspolitik und geplanter Wandel betriebswirtschaft-

licher Systeme, in: Unternehmens-

führung und Organisation, Hrsg.:

Kirsch, W., Wiesbaden 1973, S. 15-40.

Unternehmensführung und Organisation, Wiesbaden 1973.

Probleme der Unternehmensführung bei der Entwicklung und Implementierung von Management-Informationssystemen, in: DU, 28. Jg., 1974, S. 173-185

Die Handhabung von Entscheidungsproblemen, München 1978.

Das Management des geplanten Wandels von Organisationen, Stuttgart 1979.

Auf dem Weg zu einer Betriebswirtschaftslehre der Dienstleistungen: Der interaktionsansatz, Entwurf eines Beitrags für die DBW, vorgelegt im Februar 1984.

Grundprobleme der strategischen Marketingplanung, in: Die Führung des Betriebes, Festschrift für Curt Sandig zum 80 . Geburtstag, Hrsg.: Geist, M., Köhler, R., Stuttgart 1981, S. 261-291.

Informationssysteme für die Unternehmensführung, in: DBW, $37 . \mathrm{Jg}$. , 1977, Nr. 2, S. 267-282.

Planung und Entscheidung im Absatzbereich industrieller GroBunternehmen. Ergebnisse einer empirischen Untersuchung, Aachen 1977.

Techniken der empirischen Sozialforschung, München 1976.

Architektur und Planung betrieblicher Informationssysteme, in: Probleme beim Aufbau betrieblicher Informationssysteme, Hrsg.: Hansen, H.R., Wahl, M.P., München 1973, S. $49-82$. 


\author{
Kotler, Ph. \\ Kotler, Ph., \\ Connor, R.A.Jr. \\ Krubasik, E.G. \\ Krups, M.
}

Krups, M.,

Schubert, $F$.

Kulhavy, E.

Kupsch, P., Hufschmied, P., Mathes, H.D., Schöler, $K$.

Kutschker, M.

Langeard, E.

Langeard, E.,

Bateson, J.E.G., Lovelock, Chr.H., Eiglier, P.
Marketing-Management: Analyse, Planung und Kontrolle, Stuttgart 1977.

Marketing Professional Services, in: JoM, Vol. 41, January 1977, Nr. 1, S. $71-76$.

Strategische Waffe, in: WW, Nr. 25, 18.06 .82 , S. 28-33.

Situationsanalyse zum Markt für Wirtschaftsinformationen. Eine Bestandsaufnahme auf der Basis von Sekundärinformationen, Arbeitspapier des Instituts für Marketing der Westfälischen Wilhelms-Universität Münster, Münster 1982 .

Zum Stand des Bildschirmtextes in der Bundesrepublik Deutschland, Arbeitspapier Nr. 31 des Instituts für Marketing der Universität Münster, Hrsg.: Meffert, H., Münster 1983.

Dienstleistung, in: HWA, Hrsg.: Tietz, B. et al., Stuttgart 1974, Sp. 455458 .

Die Struktur von Qualitätsurteilen und das Informationsverhalten von Konsumenten beim Kauf langlebiger Gebrauchsgüter, Forschungsbericht des Landes Nordrhein-Westfalen, Opladen 1978.

Verhandlungen als Elemente eines verhaltenswissenschaftlichen Bezugsrahmens des Investitionsgütermarketing, Diss. Mannheim 1972.

Grundfragen des Dienst leistungsmarketing (ins Deutsche übertragen und bearbeitet von Pardey, R.), in: Marketing-ZFP, 3. Jg., 1981, H. 4, S. $233-240$.

Service Marketing: New Insights from Consumers and Managers, Report No. 81-104 des Marketing Science Institute, Cambridge, Massachusetts, August 1981. 
Langenbucher, $W$.

Leavitt, H.J.

Levitt, Th.

Levitt, Th.

Levitt, Th.

Levitt, Th.

Levitt, Th.

Little, J.D.C.

Logica Ltd. (Hrsg.)

Lovelock, Chr.H.
Bildschirmtext und die Zukunft von Information und Dokumentation, in: Entwicklungsperspektiven zukünftiger Informationssysteme, Hrsg.: Fachgruppe Presse-, Rundfunk- und Filmarchivare im Verein Deutscher Archivare, München etc. 1983, S. 33-45.

Learned Information Ltd. (Hrsg.), Information Industry Market Place 1981. An International Directory of Information Products and Services, New York - London 1980.

Applied Organizational Change in Industry: Structural, Technological and Humanistic Approaches, in: Handbook of Organizations, Hrsg.: March, J.G., Chicago 1965, S. 1144-1170.

Marketing Myopia, in: HBR, Vol. 38 , July-August 1960 , S. 45-56.

Production-line approach to service, in: HBR, Vol. 50, September/October 1972 , S. 41-52.

The industrialization of service, in: HBR, Vol. 54, September-october 1976 , S. 63-74.

Marketing success through differentiation - of anything, in: HBR, Vol. 58, January-February 1980, S. 83-91.

Marketing intangible products and product intangibles, in: HBR, Vol. 59, May-June 1981, S. 94-102.

Models and Managers: The Concept of a Decision Calculus, in: MS, Vol. 16,1970, S. B-466 ff.

Eurodata'79, study synopsis (Zusammenfassung der Eurodata Reports über Datenkommunikation in West Europa 1979-1987), London 1979.

Theoretical Contributions from Services and nonbusiness marketing, in: Conceptual And Theoretical Developments in Marketing, Hrsg.: Ferrell, 0.C., Brown, St.W., Lamb, C.W., Chicago, 111. 1979, S. 147-165. 
Lovelock, Chr.H.

Luhn, H.P.

Lutschewitz, H., Kutschker, M.

Maciejewski, P.G.

McCarthy, J.E.

Mag, W.

Mailick, S., von Ness, E.H.

Maleri, R.

Männel, W.

Mayer, R. $-D$.

Mazanec, J.

Mazanec, J., Scheuch, F. (Hrsg.)
Why Marketing Management Needs To Be Different For Services, in: Marketing of Services, Hrsg.: Donnelly, J.H., George, W.G., Chicago, Ill. 1981, S. 5-9.

Automation und Feedback in einem umfassenden wissenschaft lichen Kommunikationssystem, NfD, 10. Jg., 1959, H. 4, S. 173-179.

Die Diffusion von innovativen Investitionsgutern. Theoretische Konzeption und empirische Befunde, München 1977.

Btx und Mikrocomputer, in: MJ, 13. Jg., 1984, Nr. 2, S. 177.

Basic Marketing: A Managerial Approach, rev. ed., Homewood, I11. 1964.

Informationsbeschaffung, in: $H d B, 4$. Aufl., Hrsg.: Grochla, E., Wittmann, W., Stuttgart 1975, Sp. 1882-1894.

Concepts and Issues in Administrative Behaviour, Englewood Cliffs, N.J. 1962.

Grundzüge der Dienstleistungsproduktion, Berlin, Heidelberg, New York 1973 .

Die Wahl zwischen Eigenfertigung und Fremdbezug, 2. Auf1., Stuttgart 1981.

Bürokommunikation - Sachstand und Perspektiven, in: ifo-schnelldienst, 34. Jg., 1982, H. 19, S. 6-9.

Zielgruppenplanung im Fremdenverkehrs-Marketing. Empirische Ergebnisse einer Segmentationsanalyse des österreichischen Urlaubsreisenden, in: JfB, 1979, H. 3, S. 176-189.

Marktorientierte Unternehmensführung: Wissenschaftliche Tagung des Verbandes der Hochschullehrer für Betriebswirtschaft, Wirtschaftsuniversität Wien, 1984 . 


\begin{tabular}{|c|c|}
\hline $\begin{array}{l}\text { Mazis, M.B., } \\
\text { Athola, O.ं', } \\
\text { Klippel, R.E. }\end{array}$ & $\begin{array}{l}\text { A Comparison of Four Multi-Attribute } \\
\text { Models in the Prediction of Consumer } \\
\text { Attitudes, in: JoCR, Vol. } 2,1975 \text {, } \\
\text { No. } 1,5.38-55 \text {. }\end{array}$ \\
\hline Meffert, $H$. & $\begin{array}{l}\text { Modelle des Käuferverhaltens und ihr } \\
\text { Aussagewert für das Marketing, in: } \\
\text { Zeitschrift für die gesamten Staats- } \\
\text { wissenschaften } 1971 \text {, S. } 326-353 \text {. }\end{array}$ \\
\hline Meffert, $H$. & $\begin{array}{l}\text { Produktivguiter-Marketingforschung im } \\
\text { System des Marketing, in: Der Markt, } \\
13 . \mathrm{Jg}, 1974, \mathrm{Nr} .49, \text { S. 6-17. }\end{array}$ \\
\hline Meffert, $H$. & $\begin{array}{l}\text { Interpretation und Aussagewert des } \\
\text { Produktlebenszyklus-Konzeptes, in: } \\
\text { Neuere Ansäze der Marketingtheorie, } \\
\text { Festschrift zum } 80 \text {. Geburtstag von } \\
\text { otto Schnutenhaus, Hrsg.: P. Hammann, } \\
\text { W. Kroeber-Riel, C.W. Meyer, Berlin } \\
1974 \text {, S. 85-134. }\end{array}$ \\
\hline Meffert, $H$. & $\begin{array}{l}\text { Informationssysteme - Grundbegriffe } \\
\text { der EDV und Systemanalyse, Tübingen } \\
\text { und Duisseldorf } 1975 \text {. }\end{array}$ \\
\hline Meffert, $H$. & $\begin{array}{l}\text { Computergestiutzte Marketing-Informa- } \\
\text { tionssysteme - Konzeptionen, Modell- } \\
\text { anwendungen, Entwicklungsstrategien, } \\
\text { Schriftenreihe "Unternehmensfuhrung } \\
\text { und Marketing, Bd. 1, Hrsg.: Meffert, } \\
\text { H., Wiesbaden } 1975 \text {. }\end{array}$ \\
\hline Meffert, H. (Hrsg.) & $\begin{array}{l}\text { Marketing heute und morgen. Entwick- } \\
\text { lungstendenzen in Theorie und Praxis, } \\
\text { Wiesbaden } 1975 \text {. }\end{array}$ \\
\hline Meffert, $H$. & $\begin{array}{l}\text { Die Durchsetzung von Innovationen in } \\
\text { der Unternehmung und am Markt, in: } \\
\mathrm{ZfB}, 46 . \mathrm{Jg} ., 1977, \mathrm{Nr} .2, \mathrm{~S} .77-99 \text {. }\end{array}$ \\
\hline Meffert, $H$. & $\begin{array}{l}\text { Marktsegmentierung und Marktwahl im } \\
\text { internationalen Marketing, in: DBW, } \\
37 . \mathrm{Jg} ., 1977, \mathrm{Nr} \cdot 3, \mathrm{~S} .433-446 .\end{array}$ \\
\hline Meffert, H. & $\begin{array}{l}\text { Die Einfürung des Kundenmanagements } \\
\text { als Problem des geplanten organisa- } \\
\text { torischen Wandels, in: Humane Perso- } \\
\text { nal- und Organisationsentwicklung, } \\
\text { Festschrift für Guido Fischer, Hrsg.: } \\
\text { Wunderer, R., Berlin } 1979,5.285-320 .\end{array}$ \\
\hline Meffert, $H$. & $\begin{array}{l}\text { Perspektiven des Marketing in den } \\
80 \text { er Jahren, in: DBW, } 40 . \mathrm{Jg} ., 1980 \text {, } \\
\text { H. 1, S. 59-80. }\end{array}$ \\
\hline
\end{tabular}


Meffert, $H$.

Meffert, $H$.

Meffert, $H$.

Meffert, $H$.

Meffert, $H$.

Meffert, H.,

Steffenhagen, H.,

Freter, H. (Hrsg.)

Meffert, H.,

Bruhn, M.

Middelhoff, Th.

Meffert, H.,

Althans, J.

Meffert, H.,

Ohlsen, G.T.

Meffert, H., unter

Mitarbeit von

Althans, J.,

Krups, M.,

Middelhoff, Th.,

Schnetkamp, G.

Meffert, H.,

Wehrle, F.
Marketing. Einführung in die Absatzpolitik, 6. durchges. Aufl., Wiesbaden 1982 .

Neue Medien im Management-Urteil, in: asw, 25. Jg., 1982, H. 6, S. 72-81.

Stichwort Bildschirmtext, in: $D B W$, 43. Jg., 1983, H. 2 , S. 315-316.

Strategische Planungskonzepte in stagnierenden und gesättigten Märkten, in: DBW, 43. Jg., 1983, H. 2, S. 193-209.

Marketingstrategien in stagnierenden und schrumpfenden Märkten, in: Betriebswirtschaftliche Entscheidungen bei Stagnation, Festschrift für Edmund Heinen zum 65. Geburtstag, Hrsg.: Pack, L., Börner, D., Wiesbaden 1984, S. 37-72.

Konsumentenverhalten und Information, Wiesbaden 1979.

Neue Medien. Situation und Stand der Diskussion in der Bundesrepublik Deutschland, Arbeitspapier $\mathrm{Nr} .2$ der Wissenschaftlichen Gesell schaft für Marketing und Unternehmensführung e.V., Hrsg.: Meffert, H., Wagner, H., Muinster 1981.

Internationales Marketing, Stuttgart, Berlin, Köln, Mainz 1982.

Was Sie beim Marktein- und -austritt beachten müssen, in: asw, 25. Jg., 1982 , H. 10, Sonderausgabe, S. 178190 .

Bildschirmtext als Kommunikationsinstrument. Einsatzmöglichkeiten im Marketing, Stuttgart 1983.

Strategische Unternehmensplanung, in: $\mathrm{HM}, 0 . \mathrm{Jg} ., 1983, \mathrm{Nr} .2$, S. 50-60. 
Meffert, H., Schubert, $F$.

Me indl, $K$.

Messing, H.W.

Meyer, A.

Meyer, P.W., Trostmann, Th.

Meyer, P.W., Trostmann, Th.

Middelhoff, Th., Walters, M.

Möhr, M.

Miiller, W., Nickel, H.
Der EinfluB von Telekommunikationsmedien, insbesondere Bildschirmtext auf die Non-Store-Marketinginstrumente von Hersteller-, Handels- und Dienstleistungsunternehmen in der Bundesrepublik Deutschland, Arbeitspapier Nr. 32 des Instituts für Marketing der Universität Münster, Hrsg.: Meffert, H., Münster 1984.

Oberlegungen zur Informationsbedarfsanalyse bei der Entwicklung von Informationssystemen, Studiengruppe für Systemforschung e.V., Bericht $\mathrm{Nr}$. 95, München, Pullach, Berlin 1972.

"Dienstleistungsmarke" - eine neue Variante im Markenangebot, in: Markenartikel, 1983, H. 10, S. 496-500.

Dienstleistungs-Marketing. Erkenntnisse und praktische Beispiele, Schriftenreihe Schwerpunkt Marketing, Hrsg.: Meyer, P.W., Bd. 20, Augsburg 1983.

Die Revolution findet nicht stat, in: asw, 22. Aufl., 1979, Nr. 10, Sonderheft, S. 22-27.

Dienstleistungsmarketing - Genese einer Dienstleistungstheorie und der systeminhärenten Implikationen für das Marketing, in: Jahrbuch der $A B-$ satz- und Verbrauchsforschung, 1978 , H. 4, S. 286-294.

Akzeptanz neuer Medien - Eine empirische Analyse aus Unternehmersicht. Arbeitspapier $\mathrm{Nr} .27$ des Instituts für Marketing der Universität Münster, Hrsg.: Meffert, H., Münster 1981.

Einige Begriffe aus der Informations ökonomie und Benutzerforschung, in: $\mathrm{NfD}, 30 . \mathrm{Jg} ., 1979, \mathrm{Nr} .6, \mathrm{~S} .237-241$.

Das Marketing von Informationsprodukten, am Beispiel von Versicherungen, in: Marktorientierte Unternehmensführung: Wissenschaftliche Tagung des Verbandes der Hochschullehrer für Betriebswirtschaft, Wirtschaftsuniversität Wien 1983, Hrsg.: Mazanec, J., Scheuch, F., Wien 1984, S. 731-752 
Munter, $H$.

Musiol, A.

Nie, N.H. et al.

Nieschlag, R., Dicht1, E., Hörschgen, $H$.

Nüssle, G.

Ockenfeld, M.

OECD (Hrsg.)

Ohem, K. (Hrsg.)

O'Meara, J.T.

O'Neal, C.R., Thorelii, H.B., Utterbach, I.M.

ott, A.E.
Küntige integrierte Bürokommunikation. Welche Folgen lassen sich bereits übersehen?, in: $Z \mathrm{fO}, 51 . \mathrm{Jg}$., 1982 , H. 7, S. 370-374.

Einheit der Burroarbeit und Vielfalt der Büromaschinen - eine Analyse der heutigen und eine Prognose der künftigen Bürosituation, in: $Z \mathrm{fO}, 50$. Jg., 1981, H. 2, S. 75-83 (Teil 1) und H. 3, S. 163-173 (Teil 2).

SPSS-Statistical Package the Social Sciences, New York etc. 1975.

Marketing. Ein entscheidungstheoretischer Ansatz, 11. Auf1., Berlin 1980 .

Goldene Zeiten für Dienstleister?, in: asw, 22. Auf1., 1979, H. 10, Sonderausgabe, S. 28-37.

Einsatz von Videotex (Bildschirmtext) fuir Information und Dokumentation - drei Beispiele, in: NfD, 31. Jg., 1980, Nr. 1, S. 27-36.

General Report, Gaps in Technology, Paris 1968.

Mit Ideen zum Erfolg, Frankfurt/Main 1980 .

Selecting Profitable Goods, in: HBR, Vol. 39, 1961, No. 1, S. 83-89.

Adoption of Innovations by Industrial Organizations, in: IMM, Vol. 3, 1973, $\mathrm{Nr} .2$, S. 235-250.

Preistheorie, in: Kompendium der Volkswirtschaftslehre, 5., überarbeitete und ergänzte Aufl., Hrsg.: Ehrlicher, W., Esenwe in-Rothe, I., Jürgensen, H., Rose, K., Bd. 1, Göttingen 1975 , S. 114-182.

Informationen ohne Grenzen, in: asw, 25. Jg., 1982, H. 9, S. 80-85.

Unternehmensstrategien, Spiel ums Oberleben, in: WW, 1382, Nr. 20, S. 46-61. 
o.V.

o.V.

o.V.

o.V.

o.V.

o.V.

o.V.

Pack, L.,

Börner, D. (Hrsg.)

Panyr, J., Lehmann, E.

Parasuraman, A., Berry, L.L., Zeithaml, V.A.

Pessemier, E., Wilton, P.
Bertelsmann bietet Informationsdienste an. Datenbankdienste GmbH können eine Marktlücke schlieBen, in: $F A Z$, $\mathrm{Nr} .284,07.12 .83$, S. 16.

Konjunkturdaten aus dem externen BtxRechner, in: FIBA REPORT, 4. Jg., September 1983, $\mathrm{Nr} .17, \mathrm{~S} .12$.

Der Eurom ist noch für manche Oberraschung gut, in: Btx-Praxis, Oktober/November 1983, Nr. 5, S. 28-29.

Bildschirmtext und Microcomputer Voraussetzungen und Anwendungen, in: FIBA REPORT, 4. Jg., Oktober 1983, $\mathrm{Nr} .19, \mathrm{~S} .7$.

Warten auf den Eurom, in: WW, Nr. 6 , 03.02 .84 , S. 72-73.

Weltweites Potpourri, in: WW, Nr. 6 , 03.02 .84 , S. 80-86.

Aufbruch in die Zukunft, in: WW, $\mathrm{Nr} .17,20.04 .84$, S. 40-56.

Betriebswirtschaftliche Entscheidungen bei Stagnation, Festschrift für Edmund Heinen zum 65. Geburtstag, Wiesbaden 1984.

Entwicklungsperspektiven zukünftiger Informationssysteme, in: Entwicklungsperspektiven zukünftiger Informationssysteme, Hrsg.: Fachgruppe Presse-, Rundfunk - und Filmarchivare im Verein Deutscher Archivare, München etc. 1983, S. 117-154.

Service Firms Need Marketing Skills, in: BH, Vol. 26, November-December 1983, S. 28-31.

Pretesting the acceptance of innovations, Paper No. 696, Institute for Research in the Behavioral, Economic, and Management Sciences, Krannert Graduate School of Management, Purdue University, West Lafayette, Indiana 1979. 
Pfaffhausen, A.

Pfeiffer, W.

Pfeiffer, W.,

Amler, R.,

Schäffner, G.J.,

Schneider, W.

Pfeiffer, W.,

Metze, G.,

Schneider, W.,

Amler, R.

Picot, A.,

Anders, $W$.

Picot, A.,

Anders, $W$.

Poppe, R.

Porter, M.E.

Potter, A.R.
Zur Informationsversorgung der $A b$ satzführung in mittelständischen Betrieben - Eine empirische Analyse in ausgewählten Bereichen der Konsumgüterindustrie -, Beiträge zur Mittelstandsforschung, Hrsg.: Institut für Mittelstandsforschung - Forschungsgruppe Bonn -, H. 80 , Göttingen 1981.

Innovationsmanagement als know-HowManagement, in: Führungsprobleme industrielier Unternehmungen, Festschrift für Friedrich Thomée zum 60 . Geburtstag, Hrsg.: Hahn, D., Berlin, New York 1980, S. 421-452.

Technologie-Portfolio - Methode des Strategischen Innovationsmanagements, in: Zfo, 52. Jg., 1983, H. 5-6, S. 252-261.

Technologie-Portfol io zum Management strategischer Zukunftsfelder, Innovative Unternehmensführung, Hrsg.: Pfeiffer, W., Bd. 7, Göttingen 1982.

Telekommunikationsnetze als Infrastruktur neuer Entwicklungen der geschäftlichen Kommunikation, in: WiSt, 12. Jg., April 1983, H. 4, S. 183-189.

Telekommunikationsdienste für den Geschäftsbereich, in: WiSt, 12 . Jg., Juni 1983, H. 6 , S. 275-281.

Zukünftige Informationswege der Vereinigten Wirtschaftsdienste (VWD) durch Einsatz von Btx im IBM-Rechenzentrum, in: Proceedings des IBM-BtxKongresses ' 83 vom 29.-30. November 1983 in Berlin, K 12 .

Competitive Strategy, New York. London 1980.

Information Brokers and their Role in the Online Industry, in: 4 th International online Information Meeting, London 9 - 11 December 1980, Hrsg.: Learned Information (Europe) Ltd., Oxford, N.J. 1980, S. 295-301. 


\author{
Pümpin, C. \\ Pimpin, C. \\ Raffeè, H. \\ Rasmussen, Th. \\ Rathme11, J.M. \\ Rauch, W., \\ Rüdel, W., \\ Simon, C., \\ Zimmermann, H. \\ Regan, W.J. \\ Rieth, D., \\ Eckert, G.
}

Rockart, J.F.

Rockart, J.F.,

Treacy, M.

Rosenthal, $K$.

Rupp, M.

Seeger, Th.,

Strauch, D.
Information und Marketing, Schriftenreihe "Marketing-Management", Bd. 1, St. Gallen 1973 .

Management strategischer Erfolgspositionen, Bern, Stuttgart 1982.

Marketingperspektiven der 80 er Jahre, in: Marketing.ZFP, 4. Jg., 1982, H. 2, S. 81-90.

Aspekte einer verschlafenen Revolution, in: asw, 22. Jg., 1979, Nr. 10, Sonderausgabe, S. 16-21.

Marketing in the Service Sector, Cambridge, Mass. 1974.

Zum Einsatz von Bildschirmtext im IUD-Bereich (Teil I.), in: Das Inforum, 1980, Nr. 6, 0.S.

The Service Revolution, in: JoM, Vol. 27, July 1963, S. 57-62.

Erhebung bei faktischen und potentiellen Informationsanbietern und Terminalbetreibern, Teilbericht 4 der ODIN-SYSTEMANALYSE. Systemanalyse, Konzeption und technische Realisierung des nationalen Informationsverbundes mit AnschluB an EURONET, Teilprojekt GID/SfS, Heidelberg 1979.

Topmanager sollten ihren Datenbedarf selbst definieren, in: HM, O. Jg., $1980, \mathrm{Nr} .2$, S. 45-58.

Der Chef arbeitet on-line, in: HM, o. Jg., 1983, Nr. 2, S. 14-19.

Bildschirmtext: Datenbank mit Mickymaus-Image?, in: Onl ine-adi-nachrichten, 1980, Nr. 11, S. 905-907.

Produkt/Markt-Strategien, 2. Auf1., Zürich 1983.

Aufgaben und Möglichkeiten von Informationsunternehmen, in: $N f D$, 30. Jg., 1979, Nr. 1, S. 5-11. 
Shapiro, B.P.

Shapiro, B.P., Bonoma, Th.V.

Shapiro, B.P., Bonoma, Th.V.

Shostack, G.L.

Shostack, G.L.

Shostack, G.L.

Szyperski, N.

Szyperski, N.

Szyperski, N.

Scheer, A. $-W$.

Schellhaas, H., Schönecker, H.

Schertler, W., Popp, $W$.
Industrial Products Policy, Marketing Science Institute, Cambridge, Mass. 1977.

Industrial Market Segmentation: A Nested Approach, Marketing Science Institute, Report No. 83-100, February 1983.

How to segment industrial markets, in: HBR, Vol. 62, May-June 1984, S. 104-110.

Breaking Free from Product Marketing, in: JoM, 41. Jg., April 1977, S. 73-80.

How to Design a Service, in: EJoM, Vol. 16, 1982, H. 1, S. 49-63.

Service Design in the operating environment, in: Developing New Services, Hrsg.: George, W.R., Marshal1, C.E., Chicago, I11. 1984, S. 27-43.

Informationssysteme, in: $H d B, 4$. Aufl., Hrsg.: Grochla, E., Wittmann, W., Stuttgart 1975, Sp. 1900-1910.

Computergestuitzte Informationssysteme, in: HdO, 2. Auf1., Hrsg.: Grochla, E., Stuttgart 1980, Sp. 920-933.

Informationsbedarf, in: HdO, 2 . Aufl., Hrsg.: Grochla, E., Stuttgart 1980, Sp. 904-913.

Datenbanksysteme im Marketing, in: Marketing.ZFP, 2. Jg., März 1980, H. 1, S. 33-4i (Teil I) und Juni, H. 2, S. 103-111 (Teil II).

Kommunikationstechnik und Anwender. Akzeptanzbarrieren, Bedarfsstrukturen, Einsatzbedingungen, Forschungsprojekt Bürokommunikation, Bd. 1, Hrsg.: Picot, A., Reichwald, R., München 1983.

Attraktivitätsanalyse von Dienstleistungen. Ein empirischer Ansatz zur Entwicklung von Ausbaustrategien für Unternehmen und Staat, Muinchen 1983. 
Scheuch, F.

Scheuch, F.

Schlegel, H.

Schmitt-Grohé, J.

Schoeffler, S., Buzzell, R.D., Heany, D.F.

Scholz, L.

Schonberger, R.J.

Schreiner, R.

Schuchard-Ficher, Chr. et al.

Schuh, C.F.

Schumacher, D., Venker, $K$.

Schulte-Hillen, J., von Wietersheim, $B$.

Schwab, H.
Investitionsgüter-Marketing, Opladen 1975.

Dienstleistungsmarketing, München 1982 .

Punktbewertungsmodelle, in: Erfolgskontrolle im Marketing, Hrsg.: Böcker, F., Dichtl, E., Berl in 1975, S. 147164 .

Produktinnovation - Verfahren und Organisation der Neuproduktplanung, Wiesbaden 1972 .

Impact of Strategic Planning on Profit Performance, in: HBR, Vol. 52, March-April 1974, S. 137-145.

Innovationspolitik zur Modernisierung der Produktions - und Fertigungs technik. Wirtschaftliche und technische Entwicklungstendenzen in der Industrie im Zeitraum 1965 - 1985, Berlin, München 1977.

Purchasing Intangibles, in: JPMM, Vol. 16, 1980, Nr. 3, S. 25-27.

Die Dienstleistungsmarke, in: Markenartikel, 1982, H. 10, S. 436-440.

Multivariate Analysemethoden. Eine anwendungsorientierte Einführung, Berlin, Heidelberg, New York 1980.

Die Buiroautomation wartet auf neue Dienstleistungsangebote, in: asw, 22. Auf1., 1979, H. 10, Sonderausgabe, S. 120-130.

Der Einsatz von Externen Datenbanken für Import, Export und Geschäftsverbindungen, in: 6. Frühjahrstagung der Onl ine-Benutzergruppe der DGD in NeuIsenburg vom 8. bis 10. Mai 1984. Vorträge, Hrsg.: Deutsche Gesellschaft für Dokumentation e.V., S. 251-262.

IuD-online-Datenbankbenutzung in der Bundesrepublik Deutschland. Gefahren der Abhängigkeit von ausländischen Datenbanken für Wissenschaft und Forschung, Wirtschaft und Industrie, Miunchen etc. 1984.

Das IuD-Programm aus heutiger sicht, Voraussetzungen, Mängel, positive Ergebnisse, in: NfD, 32. Jg., 1981, $\mathrm{Nr} .415$, S.M.16/5 $\$ 7278-3-631-75074-2$ 
Schwarting, U., Thoben, C., Wittstock, M.

Schwerdtner, H.E.

Staehle, W.H.

Statistisches Bundesamt (Hrsg.)

Statistisches Bundesamt (Hrsg.)

Steffenhagen, $H$.

Steffenhagen, H.

Stewart, D.W.

Strauch, D., Vowe, G.
Nachfrageverhalten kleiner und mittlerer Unternehmen nach AuBenhandelsinformation und -beratung. Eine Umfrage im Bereich der Industrie- und Handelskammer zu Muinster, Beiträge zur Mittelstandsforschung, Hrsg.: Institut für Mittelstandsforschung Forschungsgruppe Bonn -, Göttingen 1982, H. 83 .

Schlüsseltechnologien / Weg zu neuem Wachstum. Chancen auch für kleinere und mittlere Unternehmen, in: Handelsblatt, Nr. 125, 4. Juli 1983, S. B 1 und B4.

Deutschsprachige situative Ansätze in der Managementlehre, in: Organisationstheoretische Ansätze, Hrsg.: Kieser, A., Muinchen 1981, S. 215-226.

Statistisches Jahrbuch für die Bundesrepublik Deutschland, Wiesbaden 1983.

Umsatzsteuerstatistik, Wiesbaden 1983.

Industrielle Adoptionsprozesse als Probleme der Marketingforschung, in: Marketing heute und morgen. Entwicklungstendenzen in Theorie und Praxis, Hrsg.: Meffert, H., Wiesbaden 1975, S. 107-125.

Der Strategiebegriff in der Marketingplanung. Ein literaturkritisch gestutzter Vorschlag zur Verwendung des Ausdrucks "Marketing-Strategie", Arbeitspapier $\mathrm{Nr} .29$ des Instituts für Marketing der Universität Münster, Hrsg.: Meffert, H., Münster 1983.

The Application and Misapplication of Factor Analysis in Marketing Research, in: JoMR, Vol. 18, February 1981 , S. 51-62.

Bildschirmtext und Zwe iwegkabelkommunikation als neue Medien fiur Informationssysteme. Relevanz für den IuD-Bereich, in: Erstes deutsches Onl ine-Informationstreffen, Köln, 5. - 8. Mai 1980 . 
Strebel, H., Strothmann, K. -H., von Hagen, $F$.

Strothmann, K. $-H$.

Stukenbröker, B.

Sturm, M.,

Vajna, T.

Taylor, K.H.

Teichmann, H.

Thomas, D.R.E.

Tietz, B. (Hrsg.)

Töpfer, A.,

Afheldt, H. (Hrsg.)

Topritzhofer, E.

(Hrsg.)

Turner, E.B.

Oberla, $k$.

Uebele, H., He inzelbecker, $k$.
Ergebnisse einer Voruntersuchung zur Anwendung der Delphi-Methode auf das Thema "Hemmende und fördernde Faktoren im DurchsetzungsprozeB der Mikroelektronik", Arbeitspapier des Instituts für Markt- und Verbrauchsforschung der Freien Universität Berlin, Berlin 1982 .

Investitionsgütermarketing, München 1979 .

Zur Angebotssituation der OnlineDienste in Deutschland, in: $N f D$, 30. Jg., 1979, Nr. 1, S. 2-6.

Grundzuge der Faktorenanalyse, in: Techniken der empirischen Sozialforschung, Hrsg.: Koolwijk, J.V., Wieken-Mayser, M., Bd. 5, Testen und Messen, München 1976.

Online Business Databases and Viewdata, in: 3rd International onl ine Information Meeting, London $4-6$ December 1979, Hrsg.: Learned Information (Europe) Ltd., Oxford, New York 1979.

Informationsbewertung, in: $H d B, 4$. Aufl., Hrsg.: Grochla, E., Wittmann, W., Stuttgart 1975, Sp. 1894-1900.

Strategie in Dienstleistungsunternehmen, in: HM, O. Jg., 1983, H. 2, S. $42-48$.

Handwörterbuch der Absatzwirtschaft, Stuttgart 1974 .

Praxis fur strategische Unternehmensplanung, Frankfurt/M. 1983.

Marketing - Neue Ergebnisse aus Forschung und Praxis, Wiesbaden 1968.

Marketing Professional Services, in: JoM, Vol. 33, October 1969, S. 56-61.

Faktorenanalyse, 2. Aufl., Berlin etc. 1972 .

Der situationsgerechte Aufbau betrieb1 icher Informationssysteme, in: $Z \mathrm{fo}$, 49. Jg., 1980, H. 7, S. 396-404. 
Uh1, K.P., Upah, G.D.

Uh1mann, L.

Ulrich, H.

Wacker, P.A.

Wagner, $H$.

Wagner, H.

Walters, M.

Webster, F.E.Jr.

Webster, F.E., Wind, $Y$.

Wehrle, F.
The marketing of services: why and how is it different, Faculty Working Papers, College of Commerce and Business Administration, University of Illinois at Urbana-Champaign, July 1979.

Typen industrieller Innovationsprozesse, in: ifo-schnelldienst, 30 . Jg., 1978, Nr. 33, S. 5-18.

Unternehmenspolitik, Bern, Stuttgart 1978.

Die Erfahrungskurve in der Unternehmensplanung. Analyse und empirische Oberprüfung, Schriftenreihe Wissenschaftliche Forschung und Entwicklung, Bd. 66, Hrsg.: Aschoff, C., MiillerBader, P., München 1980.

Unternehmensführung und neue Informationstechnologien. Auswirkungen auf Unternehmensstrategie und Unternehmensorganisation, Arbeitspapier $\mathrm{Nr} .13$ der Wissenschaftlichen Gesellschaft für Marketing und Unternehmensführung e.V., Hrsg.: Meffert, H., Wagner, H., Muinster 1983.

Personal - und Organisationsentwicklung als Ansätze zur Handhabung des Interface-Gap zwischen Systemspezia1 isten und Benutzern computergestüzter Informationssysteme, in: Humane Personal - und Organisationsentwicklung, Festschrift für G. Fischer, Hrsg.: Wunderer, R., Berlin 1979, S. 255-272.

Marktwiderstände als Problem der Marketingplanung, dargestellt am Beispiel der Marktwahl und Marktbearbeitung im Bereich der Textverarbeitung, Diss. Muinster 1983.

Industrial Marketing Strategy, New York u.a. 1979.

Organizational Buying Behavior, Englewood Cliffs, N.J. 1972.

Strategische Marketingplanung in Warenhäusern. Anwendung der PortfolioMethode, Schriften zum Marketing, Hrsg.: Meffert, H., Bd. 1, Frankfurt und Bern 1981. 
Wersig, $G$.

Widmer, S.

Wild, J.

Wilson, A.

Wind, Y.,

Cardozo, R.

Wind, $Y$. ,

Grashof, J.F.,

Goldhar, J.D.

Witte, E.

Witte, E.

Witte, E.

wittmann, w.

Wittreich, W.J.

Wunderer, R. (Hrsg.)

Wyckham, R.G.,

Fitzroy, P.T.,

Mandry, G.D.
Zur Systematik der Benutzerforschung, in: NfD, 24. Jg., 1973, Nr. 1, S. 1014 .

Erfolg mit Lizenzen, Zürich 1980.

Zur Problematik der Nutzenbewertung von Informationen, in: $Z f B, 41 . \mathrm{Jg}$., 1971, S. 315-334.

Marketing of Professional Services, London 1972.

Industrial Market Segmentation, in:

IMM, Vol. 3, 1974, S. 153-165.

Market-based guidelines for design of industrial products. A new application of conjoint analysis to scientific and technical information (STI) services, in: JoM, Vol. 42 , July 1978, S. 27-37.

Das Informationsverhalten in Informationssystemen - Die These von der unvollkommenen Informationsnachfrage -, in: Management-Informationssysteme - Eine Herausforderung an Forschung und Entwicklung -, Hrsg.: Grochla, E., Szyperski, N., Wiesbaden 1971, S. 832-842.

Das Informationsverhalten in Entscheidungsprozessen, Tübingen 1972.

Informationsverhalten, in: HdB, Hrsg.: Grochla, E., Wittmann, W., Stuttgart 1975, Sp. 1915-1924.

Information, in: Hdo, 2. Auf1., Hrsg.: Grochla, E., Stuttgart 1980, Sp. 894-904.

How to Buy/Sell Professional Services, in: HBR, March-April 1966, S.

Humane Personal - und Organisationsentwicklung, Festschrift für Guido Fischer, Berlin 1979.

Marketing of Services. An Evolution of the Theory, in: EJoM, 9. Jg., $1975, \mathrm{Nr} .1$, S. 59-67. 
Zahn, E.

Zaltman, G., Burger, P.C.

ZVEI (Hrsg.)
Mikroelektronik in der Informationsgesellschaft, in: HM, 0. Jg., 1983, H. 3, S. 7-13.

Marketing Research, Hinsdale 1975.

Informationsbroschüre zum DatenbankInformationssystem ELTRODAT, Frankfurt 1984 . 
M. Krups - 978-3-631-75074-2

Downloaded from PubFactory at 01/11/2019 08:37:05AM

via free access 
M. Krups - 978-3-631-75074-2

Downloaded from PubFactory at 01/11/2019 08:37:05AM

via free access 
Band 1 Friedrich Wehrle: Strategische Marketingplanung in Warenhäusern. Anwendung der Portfolio-Methode. 1981. 2. Auflage. 1984.

Band 2 Jürgen Althans: Die Ubertragbarkeit von Werbekonzeptionen auf internationale Märk. te. Analyse und Exploration auf der Grundlage einer Befragung bei europaweit tätigen Werbeagenturen. 1982.

Band 3 Günter Kimmeskamp: Die Rollenbeurteilung von Handelsvertretungen. Eine empirische Untersuchung zur Einschätzung des Dienstleistungsangebotes durch Industrie und Handel. 1982.

Band 4 Manfred Bruhn: Konsumertenzufriedenheit und Beschwerden. Erklärungsansätze und Ergebnisse einer empirischen Untersuchung in ausgewählten Konsumbereichen. 1982.

Band 5 Heribert Meffert (Hrsg.): Kundendienst Management. Entwicklungsstand und Entscheidungsprobleme der Kundendienstpolitik. 1982.

Band 6 Ralf Becker: Die Beurteilung von Handelsvertretern und Reisenden durch Hersteller und Kunden. Eine empirische Untersuchung zum Vergleich der Funktionen und Leistungen. 1982.

Band 7 Gerd Schnetkamp: Einstellungen und Involvement als Bestimmungsfaktoren des sozialen Verhaltens. Eine empirische Analyse am Beispiel der Organspendebereitschaft in der Bundesrepublik Deutschland. 1982.

Band 8 Stephan Bentz: Kennzahlensysteme zur Erfolgskontrolle des Verkaufs und der Marketing-Logistik. Entwicklung und Anwendung in der Konsumgüterindustrie. 1983.

Band 9 Jan Honsel: Das Kaufverhalten im Antiquitätenmarkt. Eine empirische Analyse der Kaufmotive, ihrer Bestimmungsfaktoren und Verhaltenswirkungen. 1984.

\section{SCHRIFTEN ZU MARKETING UND MANAGEMENT}

Band 10 Matthias Krups: Marketing innovativer Dienstleistungen am Beispiel elektronischer Wirtschaftsinformationsdienste. 1985. 
Wehrle, Friedrich

\section{STRATEGISCHE MARKETINGPLANUNG IN WARENHÄUSERN}

Anwendung der Portfolio-Methode

Frankfurt/M., Bern, 1981. XI, 301 S.

Schriften zum Marketing. Bd. 1

ISBN 3-8204-6954-0

br. sFr. 68.-

Die strategische Marketingplanung im Handel muss auch heute noch als vergleichsweise wenig beachtetes Problemfeld angesehen werden. Vielmehr stehen sowohl in Theorie als auch in Praxis Fragestellungen der taktisch-operativen Planung im Mittelpunkt. Die vorliegende Arbeit analysiert die planerischen Probleme des Marlieting im Handel aus strategischer Sicht und zeigt am Beispiel der Betriebsform Warenhaus die systematische Entwicklung eines strategischen Marketing-Planungssystens auf, in dessen Mittelpunkt die Anwendung der aktuell stark diskutierten Portfolio-Methode steht.

Aus dem Inhalt: Strategisches Planungssystem im Handel - Diskussion der Anwendung der Portfolio-Methode in Warenhäusern - Ziele und Zielsysteme in Warenhäusern - Entwicklung von Marketing-Strategien in Warenhäusern - Einsatz der Marketinginstrumente in Warenhäusern - Fragen der organisatorischen Umsetzung von Marketingstrategien.

Meffert, Heribert (Hrsg.)

KUNDENDIENST-MANAGEMENT

Entwicklungsstand und Entscheidungsprobleme der Kundendienstpolitik

Frankfurt/M., Bern, 1982. VIII, 308 S.

Schriften zum Marketing. Bd. 5

ISBN 3-8204-5764-X

br. sFr. 70.-

Die Entwicklung des Kundendienstes zu einem bedeutenden Instrument der Marketingpolitik ist vor dem Hintergrund gesättigter Märkte, differenzierter Kundenbedürfnisse und verstärktem Konkurrenzdruck zu sehen. Das Angebot an Kundendienstleistungen ist zu einem wesentlichen Kaufentscheidungsfaktor geworden. Die erheblichen Koordinationserfordernisse kundendienstpolitischer Entscheidungen im Rahmen des gesamten Marketingmix legen es nahe, von einem Kundendienst-Management zu sprechen. Der Sammelband umfasst neun Beiträge kompetenter Praktiker und Wissenschaftler zu zentralen Problembereichen der Kundendienstpolitik. Es handelt sich dabei um die Ergebnisse eines Workshops über Kundendienst-Management an der Universität Münster.

\section{Verlag Peter Lang Bern · Frankfurt a.M. New York \\ Auslieferung: Verlag Peter Lang:AG, Jupiterstr.15,CH-3000 Bern 15 Telefon $(0041 / 31) 321122$, Telex verlch 32420}

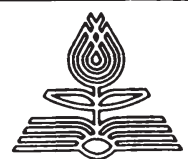


M. Krups - 978-3-631-75074-2

Downloaded from PubFactory at 01/11/2019 08:37:05AM

via free access 
M. Krups - 978-3-631-75074-2

Downloaded from PubFactory at 01/11/2019 08:37:05AM

via free access 